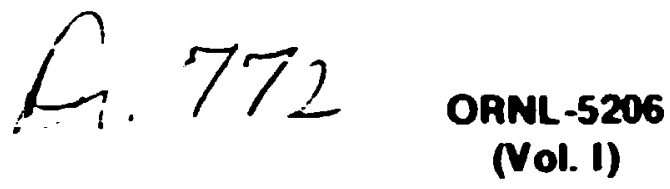

\title{
Atomic Data for Controlled Fusion Research
}

\author{
C. F. Bamett \\ J. A. Ray \\ E. Ricci \\ M. I. Wilker \\ E. W. McDaniel \\ E. W. Thomas \\ H. B. Gilbody
}


$-\cdots$

\section{BLANK PAGE}




\section{Printed in the United Statws of America. Available from}

National Technical Intarmation Service

U.S. Department of Commerce

This rupat wes prepered as en eccount of work sponsored by the Unied Stems

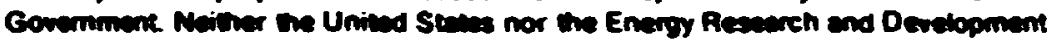

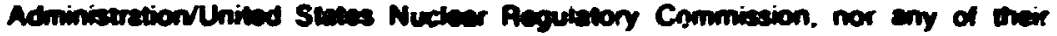

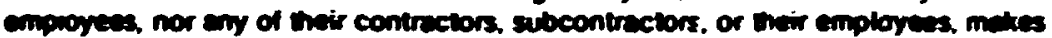

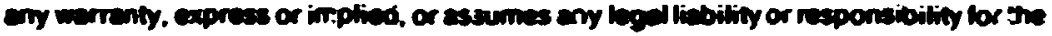

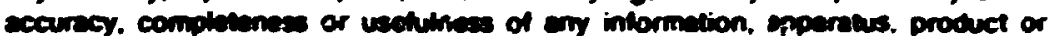

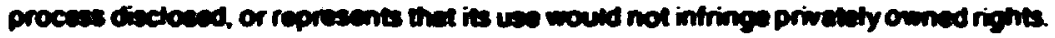




$$
\begin{aligned}
& \text { Canl-5zO6 } \\
& \text { (Vol. I) } \\
& \text { Dist. Category UC-2C }
\end{aligned}
$$

Contract No. w-7405-eng-26

PHYSICS DIVISION

ATUMIC DATA FOR CONTROLLED FUSION RESEARCH

C. F. Barnett, J. A. Ray, E. Ricci, M. I. Hilker Dak Pidge Hational Laboratory

E. H. McDaniel, E. W. Thomas Georgia Institute of Technolocy

and

H. B. Gilboäy

Queens University, belfast

Dale Publithed: Februoy 197

Oak Ridge lational Laluratory

Oak Ridge, Tennessee 37830 operated by

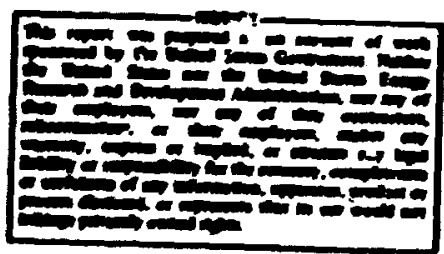

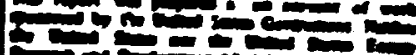

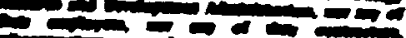

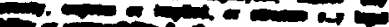

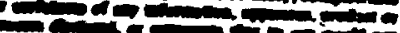
$\rightarrow$ anding

\section{Union Carbide Corporation for Energy Research and Development Administration


Abstract ................................ xi

Forewozd............................. xv

introduction ......................... xvi . . .

A. Feavy Particle Dollisions

A.I Heavy Particle Scattering

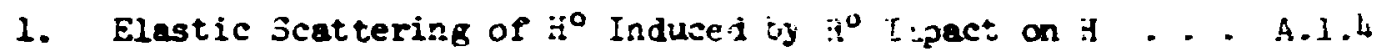

2. Elastic Scattering of $\mathrm{H}^{+}$Induced by $\mathrm{H}^{+}$I $\mathrm{s}_{-3}$ ect on $\mathrm{H}$. . A.1.6

3. Angular Distribution of $\mathrm{i}^{+}$Formed $2 \mathrm{~g} \mathrm{i}^{\mathrm{t}}$ Pro erses a

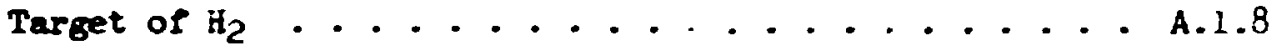

4. Scattering of $\mathrm{i}^{\circ}$ Formed by Charge in insfer Neutralization of $\mathrm{H}^{+}$in $\mathrm{H}_{2} \ldots \ldots . . . \ldots$ A.1.10

5. Angular Distribution of $\mathrm{H}^{+}$Formed by i. .sociat in

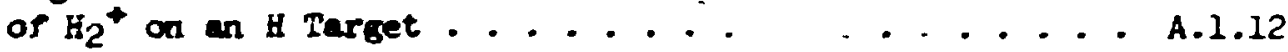

6. Angular Distribution of $\mathrm{H}^{\circ}$ and $\mathrm{H}^{+}$Foripd oy Dissociation of $\mathrm{H}_{2}^{+}$on $\mathrm{H}_{2}$ Target .......... A.1.14

7. Scattering of $i^{t}$ and $H^{\circ}$ Induced by $H^{+}$: s.ace on He . . A.1.16

8. Angurar Distribution of $1^{\circ}$ and $\mathrm{H}^{+}$Pormec $15 \%^{-a}$ Treverses a Target of He ................18

9. Angular Distributior of $\mathrm{h}^{3}$ and $\mathrm{H}^{+}$Fonned by Drssociation of $\mathrm{H}_{2}{ }^{+}$on an He Target.......... A.1.20

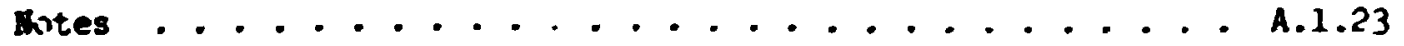

$\therefore .2$ Excitation by heary Particies

1. Excitation Cross Sections for the A. lons

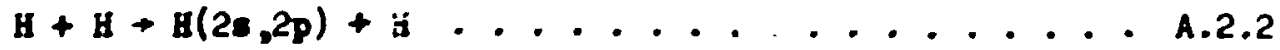

2. Excitation Croas Sections for the Reece ins $\mathrm{H}+\mathrm{H}_{2} \rightarrow \mathrm{H}(2 \mathrm{~s}, 3 \mathrm{~s}, 2 \mathrm{p}, 3 \mathrm{p}, 3 \mathrm{~d})+\mathrm{H}_{2} \ldots . . \ldots . . . . . .2 .4$

3. Excitation Cross Sections for the Ree tions $\mathrm{H}+\mathrm{H}_{2} \rightarrow \mathrm{H}+\mathrm{H}+\mathrm{H}(2 \mathrm{2s}, 2 \mathrm{p}) \ldots . . . \ldots . . . . . .2 .6$

4. Excitation Cross Sec;ions for the Reac: ion: $\mathrm{H}^{\circ}+\mathrm{He}+\mathrm{H}^{\circ}+\mathrm{He}\left(4 \mathrm{I}_{\mathrm{S}}, 4 \mathrm{l}_{\mathrm{P}}, 43 \mathrm{~S}, 43 \mathrm{P}\right) \ldots . . . . . \mathrm{A} .2 .8$

5. Excitation Cross Sections by Electron Cagtivin for the Reactions $\mathrm{H}^{+}+\mathrm{H} \rightarrow \mathrm{H}(2 \mathrm{~s}, 2 \mathrm{p})+\mathrm{H}^{+}$........ A.2.10 
6. Excitation Cross Sections for the Reactions

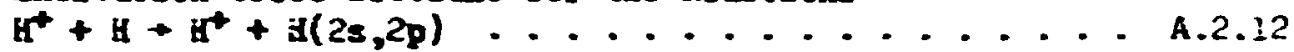

7. Cross Sections for Excitation by Electron Capture for the Reections $\mathrm{H}^{+}+\mathrm{H}_{2}+\mathrm{H}(2 \mathrm{~s}, 3 \mathrm{~s}, 4 \mathrm{~s})+\mathrm{H}_{2}^{+} \ldots \ldots . . .14$

8. Cross Sections for ixcitation by jlectron Capture for the Peactions $\mathrm{I}^{+}+\mathrm{H}_{2}+\mathrm{H}(2 \mathrm{p}, 3 \mathrm{p}, 3 \mathrm{~d})+\mathrm{H}_{2}^{+} \ldots \ldots . . .16$

9. Excitation Cross Sections for the Reactions $\mathrm{H}^{+}+\mathrm{H}_{2}+\mathrm{H}^{+}+\mathrm{H}+\mathrm{H}(2 \mathrm{~s}, 2 \mathrm{p}, 3 \mathrm{p}) \ldots \ldots .2 .18$

10. Cross Sections for Bdission of Balner-Nlphe Radiation for the Reections $\mathrm{H}^{+}+\mathrm{H}_{2}+\mathrm{H}^{+}+\mathrm{H}+\mathrm{H}(\mathrm{n}=3+2) \ldots . .20$

1). Cross Sections for Bassion of the $1606 R$ and $4180 R$

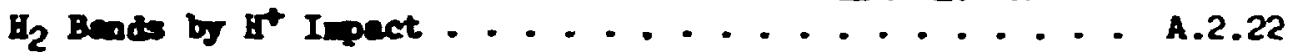

12. Becitation Cross Sections for Renctions $\mathrm{H}+\mathrm{He}+\mathrm{H}(2 \mathrm{~s}, 2 \mathrm{p})+\mathrm{He}+\mathrm{e} \ldots \ldots .2 .24$

13. Rreftation Cross Sections for the Reactions $B+\mathrm{He} \rightarrow \mathrm{H}(2 \mathrm{~s}, 3 \mathrm{~s}, 2 \mathrm{p}, 3 \mathrm{p}, 3 \mathrm{~d})+\mathrm{He}$........... A.2.26

14. Bratetion Cross Sections by Blectron Cepture for the Rection $\mathrm{H}^{+}+\mathrm{He} \rightarrow \mathrm{H}(2 \mathrm{p}, 3 \mathrm{p}, 3 \mathrm{~d})+\mathrm{He}^{+} \ldots \ldots .2 .28$

15. Breitetion Cross Sections by Electron Copture for

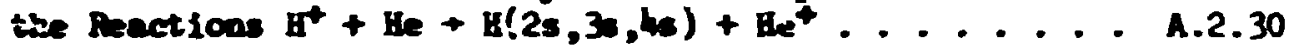

16. Breitation Cross Section for the Resctions

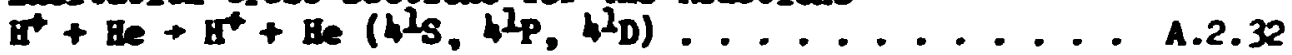

17. Cross Sections for Pornetion of 8 Atom in Hich

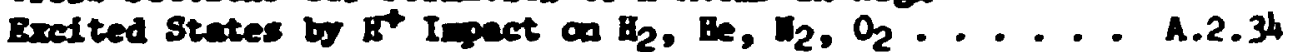

18. Breltation Cross Sections for the Renctions

$$
\mathrm{B}^{+}+\left(\mathrm{H}_{2}, \mathrm{O}_{2}, \mathrm{CO}_{2}\right)+\mathrm{H}(2 \mathrm{~s})+\left(\mathrm{H}_{2}^{+}, \mathrm{O}_{2}^{+}, \mathrm{CO}_{2}^{+}\right) \ldots . . .36
$$

19. Bucitation Croos Sections for the Reactions $\mathrm{H}^{+}+\left(\mathrm{H}_{2}, \mathrm{O}_{2}, \mathrm{CO}_{2}\right) \rightarrow \mathrm{B}(3 \mathrm{3})+\left(\mathrm{H}_{2}^{+}, \mathrm{O}_{2}^{+}, \mathrm{CO}_{2}^{+}\right) \ldots \ldots .2 .38$

20. Breftation Cross Sections for the Reactions

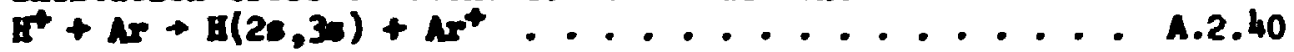

21. Bxeltation Cross Sections for the Benctions

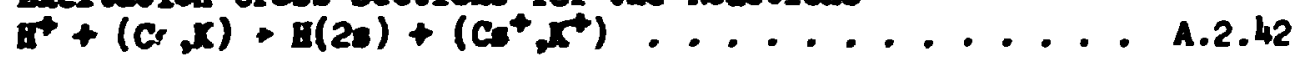

22. Croses S. :tions for Pormation of B Atan in Hich

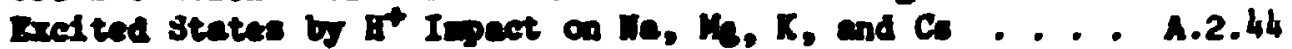

23. Ireitetion Crowe Sections for the Reactions $\mathrm{B}_{2}^{+}+\mathrm{B}_{2} \rightarrow \mathrm{H}(2 \mathrm{~s}, 3 \mathrm{~B}, 2 \mathrm{p}, 3 \mathrm{p}, 3 \mathrm{~d})+\mathrm{B}^{+}+\mathrm{H}_{2} \ldots \ldots .2 .46$ 
24. Bxcitation Cross Sections for the Reactions

$\mathrm{H}_{2}^{+}+\mathrm{H}_{2}+\mathrm{H}_{2}^{+}+\mathrm{H}+\mathrm{H}(2 \mathrm{~s}, 2 \mathrm{p}, 3 \mathrm{p})$

$\mathrm{in}_{2}^{+}+\mathrm{H}_{2}+\mathrm{H}_{2}^{+}+\mathrm{H}+\mathrm{H}(\mathrm{n}=3 \rightarrow 2) \ldots \ldots .2 .48$

25. Excitation Cross Sections for the Reactions

$\mathrm{H}_{2}^{+}+\mathrm{He} \rightarrow \mathrm{H}(2 \mathrm{~s}, 3 \mathrm{se}, 3 \mathrm{p}, 3 \mathrm{~d}) \ldots \ldots . . . \ldots \ldots$

26. Excitation Cross Sections for the Reaction

$\mathrm{H}_{2}^{+}+\mathrm{He} \rightarrow \mathrm{H}_{2}{ }^{+}+\mathrm{He}^{\mathrm{E}}(\mathrm{nL}) \ldots . . . . . . . . . . .2 .52$

27. Excitation Cross Secticns for the Reactions

$\mathrm{H}_{3}^{+}+\mathrm{H}_{9} \rightarrow \mathrm{H}(3 \mathrm{~B}, 3 \mathrm{~d})+\mathrm{H}_{2}^{+}+\mathrm{He}$ end

$\mathrm{H}_{3}^{+}+\mathrm{H}_{2}+\mathrm{H}(36)+\mathrm{H}_{2}^{+}+\mathrm{H}_{2} \ldots \ldots . . . . . . . . . . . . .44$

28. Excitation Cross Sections for the Reaction

$\mathrm{H}_{3}{ }^{+}+\mathrm{He}+\mathrm{H}_{3}{ }^{+}: \mathrm{He}^{\mathrm{E}}(\mathrm{nl}) \ldots . . . . . . . . . . .2 .56$

25. Excitation Cross Sections for the Reaction

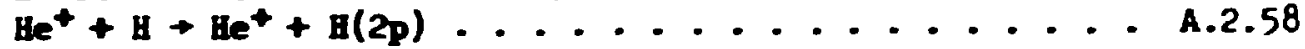

30. Croes Sections for Balmer-Niphe Buissica Prom

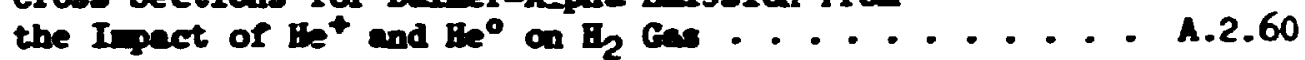

31. Exeitation Cross Sectious for the Renctisns

$\mathrm{He}^{+}+\mathrm{H}_{2} \rightarrow \mathrm{He}^{+}+\mathrm{H}+\mathrm{H}(2 \mathrm{~s}, 3 \mathrm{~s}, 2 \mathrm{p}, 3 \mathrm{p}) \ldots . . . . . . . .2 .62$

32. Excitation Cross Sections for the Reactions

$\mathrm{be}^{+}+\mathrm{Be}+\mathrm{Be}^{+}+\mathrm{Be}\left(\mathrm{H}_{\mathrm{S}}, \mathrm{H}_{\mathrm{P}}, \mathrm{H}_{\mathrm{D}}\right) \ldots \ldots . . . . . . .64$

33. Excltation Cross Sections for the Reactions

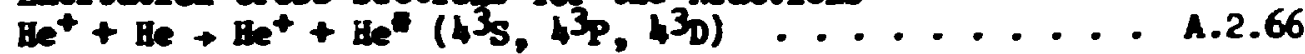

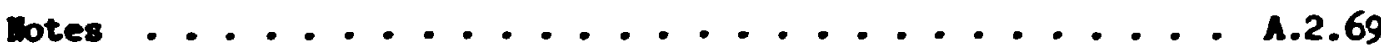

\section{A.3 Heang Particle Dissociation}

1. Cross Sections for the Conversion of Past $\mathrm{H}_{2}+$ Ions

Into Protons in Pasesere Throuth $\mathrm{B}_{2}$ and $\mathrm{E}$...... 4.3 .2

2. Dissocietion Cross Section for the Poryation of

past $\mathrm{I}^{+}$and $\mathrm{B}^{\circ}$ for $\mathrm{B}_{2}$ kolecules in $\mathrm{H}_{2} \ldots . . . . . .4 .3 .4$

3. Partial Cross Sections for Dissocietios of Past

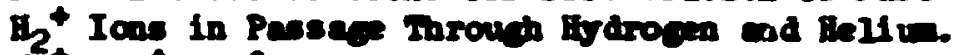

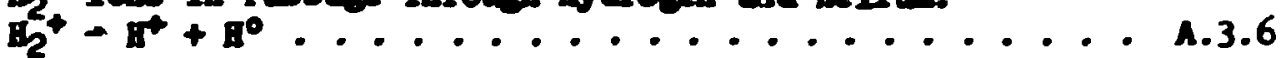

4. Dissoclation Crons Sections for the Fornution of Protons for $\mathrm{g}^{+}$in ile and the Formation of Past $\mathrm{H}^{\circ}$

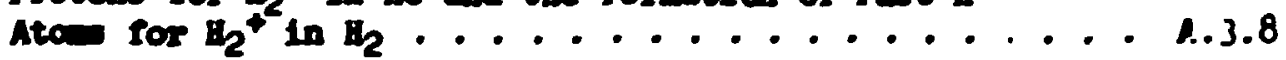

5. Dissociation Cross Secticas for the Pormation of

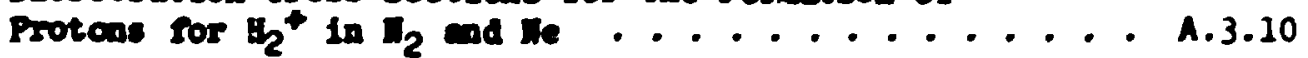


6. Cross Sections for the Production Protons and $H$

Atas for $\mathrm{H}_{2}{ }^{+}$in $\mathrm{Y}_{6}$ and $\mathrm{y}_{2} \ldots . . . . . . . . . .3 .12$

7. Dissociation Cross Sections for the Production of Protons for the Reaction Products $\left(\mathrm{H}^{+}+\mathrm{H}^{\circ}\right)$ for

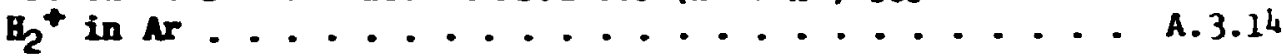

8. Cross Sections for the Formation of Electronically Bxelted $\mathrm{I}$ itoms from the Iissociation of $\mathrm{E}_{2}{ }^{+}$and

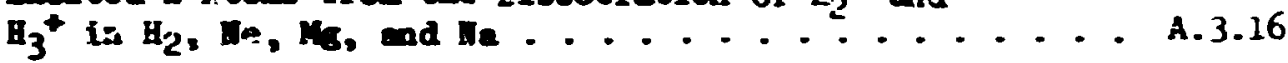

9. Dissociation Cross Sections for HDt Ions in tie and

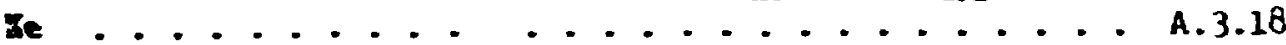

10. Dissociation Cross Sections for the Production of

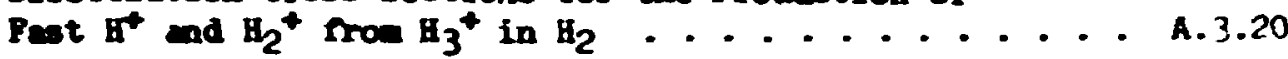

11. Dissociation Cross Sections for the Production of

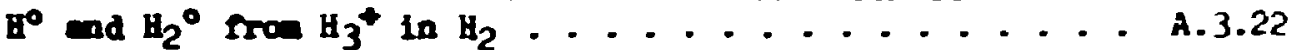

12. Cross Sections for the Pornation of Fast $\mathrm{H}^{+}$and $\mathrm{H}_{2}^{+}$ Tron the Dissociation of $\mathrm{H}_{3}{ }^{+}$in He ad be ....... A.3.24

13. Cross Sections for the Foriation of $\mathrm{H}^{+}, \mathrm{H}_{2}^{+}$, and $\left(\mathrm{H}_{2}^{\circ}+\mathrm{H}^{\circ}\right)$ fron the Dissociation of $\mathrm{H}_{3}^{+}$in Weter

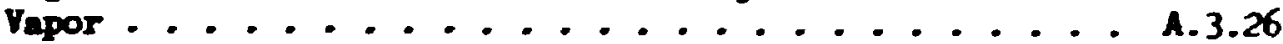

14. Total ad Partial Cross Sections for the Dissociation

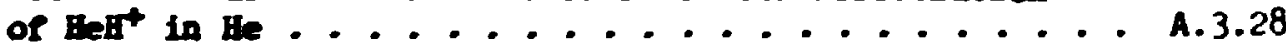

15. Total and Partial Cross Sections for the Dissociation

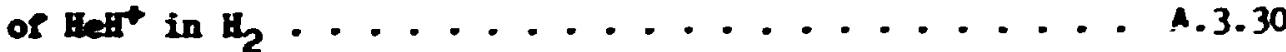

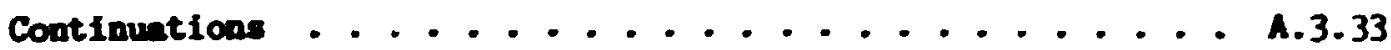

A.4 Bectron Capture Cross Sections

1. Electron Copture Cross Sections for $\mathbf{H}^{-}, \mathrm{H}^{\circ}$ and $\mathrm{H}^{+}$ Paselog throur ktcide Hodrogen .......... A.4.2

2. Mectron Cepture Cross Sections for $\mathrm{H}^{t}$ and $\mathrm{h}^{\circ}$ Peasing mrourh $\mathrm{H}_{2}$................. A.h.h

3. Blectron Ceptwe Crose Sections for gt and $\mathrm{I}^{\circ}$

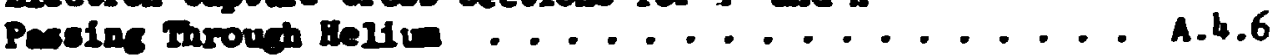

4. Hectron Cepture Crose sections for It and $\mathrm{I}^{\circ}$ Pessing throum $\mathrm{t}_{2}$.....................8

5. Electron Cogture Crose sections for $\mathrm{I}^{+}$and $\mathrm{H}^{\circ}$ Peasing mround 0 and $\mathrm{O}_{2} \ldots \ldots . . . . . . . .4 .10$ 
6. Electron Cepture Cross Sections for $\mathrm{H}^{+}$and $\mathrm{H}^{\circ}$

Passine Through te Gess . . . . . . . . . . A.í.12

7. F ectron Capture Cross Sections for $\mathrm{H}^{+}$and $\mathrm{H}^{\circ}$

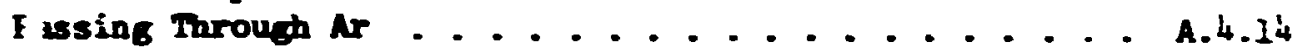

3. Electron Cepture Cross Sections for $\mathrm{H}^{*}$ in $\mathrm{H}_{2} \mathrm{O}$ ad

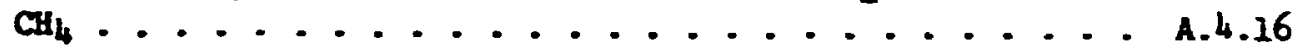

9. Electron Capture Cross Sections for $\mathrm{H}^{*}$ in co ad

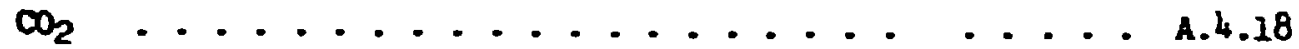

10. Electron Cepture Cross sections of $\mathrm{H}^{+}$in Li Vepor ... A.4.20

11. Electron Cepture Cross Sections for $H^{+}$in Ia Vepor . A.4.22

12. Electron Cepture Cross Sections for it in I Vepor ... A.4.24

13. Electron Copture Cross Sections ror $\mathrm{B}^{+}$in Ms Vepor . . A.4.j6

14. Electron Capture cross Sections for $\mathrm{H}^{t}$ in Co Vepor . . A.4.28

15. Electron Capture Cross Sections for the Reactions

$\mathrm{H}_{2}^{+}+\mathrm{H}_{2}$ and $\mathrm{H}_{2}^{+}+\mathrm{H} \ldots \ldots . . . . . . . . . . . .30$

16. Electron copture crous Sections for $\mathrm{Be}^{+}$in $\mathrm{H}_{2}$ ad

Be .................... 4.4 .32

17. Bectron Cepture croes Sectiors sor Bet in $O_{2}$ and

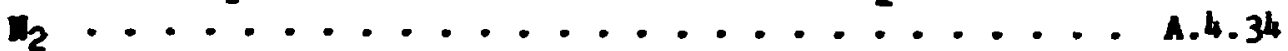

18. Bectron Cepture Cross Sections for Bett in 1

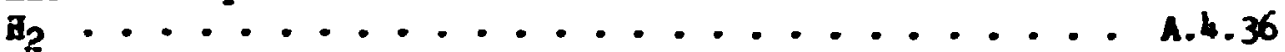

19. Bectron Cepture croses sections for bet in De .... A.4.38

20. Blectron Cepture Crose Sections for Bet in $\mathrm{H}_{2}$ ad

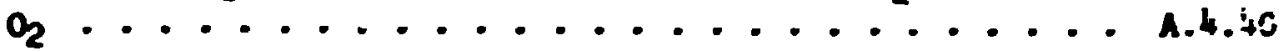

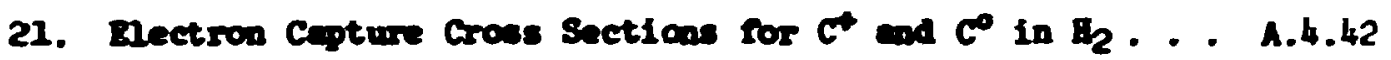

22. Blectron Copture Grons Sections for the Beactions

$\omega^{t}+\mathrm{B}_{2}$ and $\mathrm{w}^{t}+\mathrm{H} . . . . . . . . . . . . .9 .4 .44$

23. Bection Cepture Croas Sections for the Bections

$0^{+}+B \rightarrow 0^{\circ}, 0^{+}+\mathrm{H}_{2} \rightarrow 0^{\circ}$, ad $\mathrm{O}^{+}+\mathrm{B}_{2}+\mathrm{O}^{-} \ldots \ldots . . .4 .46$

2h. Eectron capture crose sections for $0 \circ$ and ont in

$\mathrm{B}_{2} \ldots \ldots . . . . . . . . . . . . . . . .4 .48$

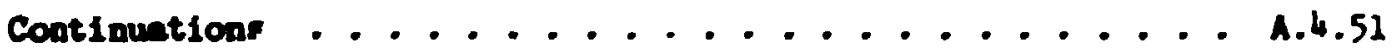




\section{A.5 Ionization and Stripping by Heavy Particles}

1. Cross Sections for Ionization of Atonic H by it . . . A.5.2

2. Cross Sections for the Production of Positive Charges in $\mathrm{H}_{2}$ and He by $\mathrm{H}^{+} \ldots \ldots . . . . . . . . . . .4 .4$

3. Cros: Sections for Production of Free Elestrons in $\mathrm{H}_{2}$ ad He by Protons ............. A.5.6

4. Cross Sections for the Production of Slou $\mathrm{H}_{2}^{+}$and $\mathrm{H}^{+}$

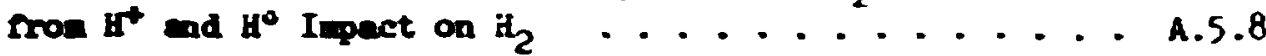

5. Cross Sections for Positive Ion Production in $\mathbf{v}_{2}$

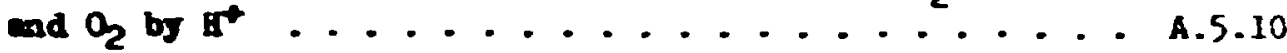

6. Cross Sections for Zlectron Production in $D_{2}$ and

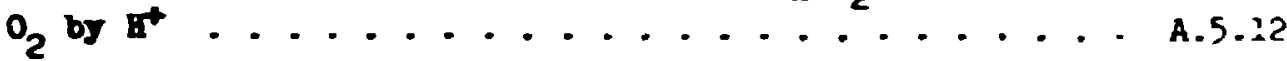

7. Cross Sections for Production of $\mathrm{I}_{2}^{+}, x^{+}$, and $\mathrm{I}^{2+}$ Ions by Protons in $\mathrm{H}_{2}$ Cas............... A.5.14

8. Cross Sections for Production of $\mathrm{O}_{2}^{+}, \mathrm{O}^{+}$and $\mathrm{O}^{2+}$

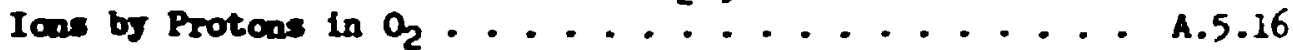

9. Angular Distribution of Blectrons from 300-keV

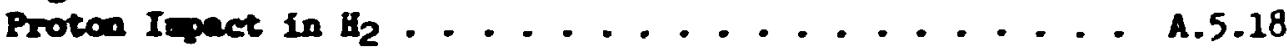

-0. Ancular Distribution of Electrons of Various Bnerdes rrom 300-keV Proton Inpacts in $\mathrm{H}_{2} \ldots \ldots$

11. Differentiel Cross Sections sor Bjection of Electrons fron $\mathrm{H}_{2}$ iblecules by 50 -keV $\mathrm{H}^{+}$Projectiles . A.5.22

12. Differential Cross Sections for Bjection of Blectrons from die atom by so-keV ht Projectiles . . A.5.24

13. Cross Sections for Production of Slow Electrons in Callisions of $\mathrm{H}_{2}^{+}$and $\mathrm{H}_{3}^{+}$in $\mathrm{H}_{2} \ldots \ldots . . . . . . . . . . .26$

14. Cross Sections for Electron Loss or Stripping of

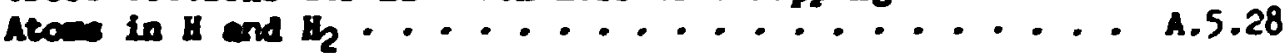

15. Totel Apparent Cross Sections for the Production of Positive Iens bs $\mathrm{B}^{\circ}$ Atans Incident on $\mathrm{H} 2$ ad be . . A.5.30

16. Totel Cross sections for the Production of Pree

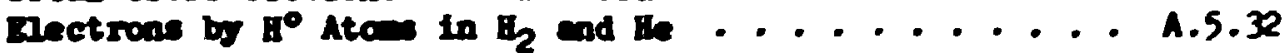


17. Cross Sictions for Electron Loss or Stripping for

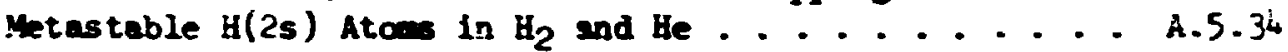

18. Cross Sections for Electron Stripping, H Atons in

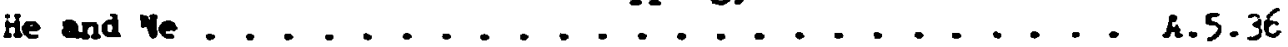

19. Cross Sections for Electron Loss of 1 Atoms in $d_{2}$,

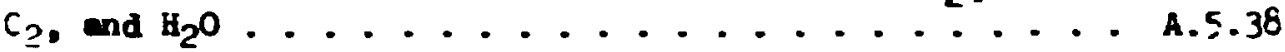

20. Cross Sections for Electron Production in $\mathrm{d}_{2}$ and

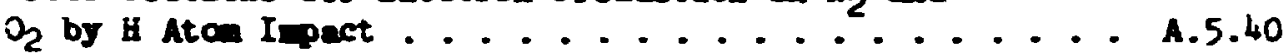

21. Cross Sections for Cue Electron Loss of H htoms in

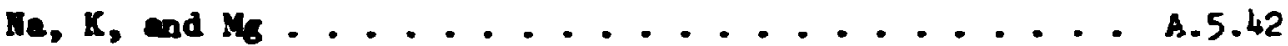

22. Cross Sectious for ane Ellectron Loss of $\mathrm{H}_{2}$ and $\mathrm{D}_{2}$

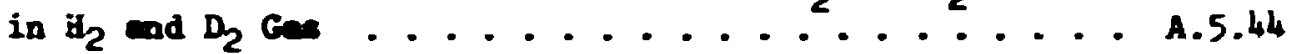

23. Cross Sections for Blectron Production in Collisions of IF with H Aton .............. A.5.46

24. Crose Sections for one Blectron Lose or Stripping for $\mathrm{H}^{-}$In $\mathrm{H}_{2}$ ad He ................ A.5.48

25. Cross Sections for Loss of Two Electrons in Collisions of $\mathrm{H}^{-}$wth $\mathrm{H}_{2}$ and $\mathrm{BE} . \ldots . . . . . . . .4 .50$

26. Cross Sections for Ose Electron Lass or Stripping

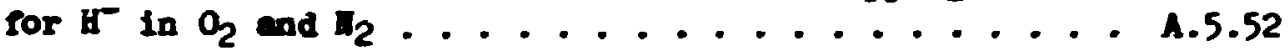

a7. Cross Sections for Loss of Two Blectrons by $\mathrm{H}^{-}$in

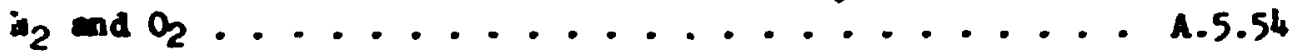

28. Coss Sections for single Blectron Loss or Stripping

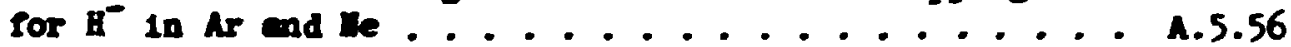

29. Cross Sections for One Blectroc Loss of Het Ions In $\mathrm{B}_{2}$ and $\mathrm{E}$.......................... A.58

30. Cross Sections for the Production of Slow plectrons by $\mathrm{He}^{+} \mathrm{I}$ cons in $\mathrm{H}_{2}$ and be ............. A.5.60

31. Cross Sections for the Production of Positive Ioss by $\mathrm{He}^{+}$Ions in B ad He ............ A.5.62

32. Cross Sections for Production of $m_{2}+, m^{+}$, and $\pi^{2+}$

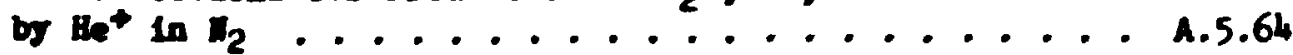

33. Croas sections for the Production of $\mathrm{O}_{2}+\mathrm{O}^{+}$, and $0^{2+}$ Ions by $\mathrm{Be}^{+}$in $\mathrm{O}_{2}$. 
3h. Cross Sections for Positive Ion Production by

Hett Ions in H2 and lle ............... A. 68

35. Croes Sections for the Production of Pree Blectrons

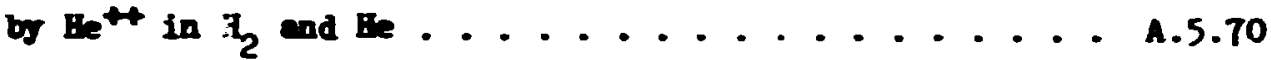

36. Cross Sietions for One and Two Electron Loss for He Atons in $\mathrm{H}_{2}$ and be .............. A.5.72

37. Cross Sections for Ionization of $\mathrm{H}_{2}$ and He by

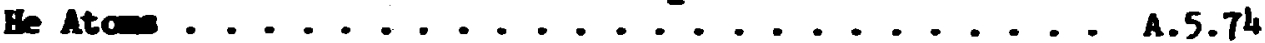

\section{A.6 Equilibrive Prections}

1. Equilibriun Practions of a Hodrogen Been in $B$ and

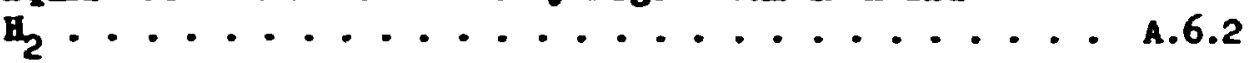

2. Equilibriu Practions of a Harogen Been in He and s. . . . . . . . . . . . . . A.64

3. Bquflibriug Practions of a ifdrogen Bean in $\mathrm{I}_{2}$ and

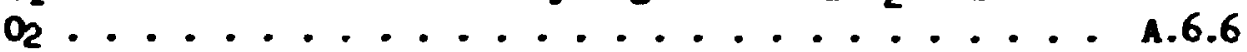

4. Equilibriv Practions of a Harogen Bean in $\mathrm{H}_{2} \mathrm{O} \ldots \ldots .6 .8$

5. Equilibriu Fractions of a Hdrogen Been in hi and

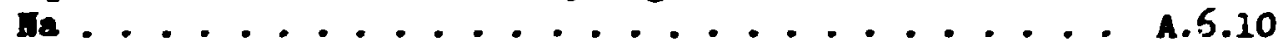

6. Equilibriu Practions of a fudrogien Bean in $K$ and

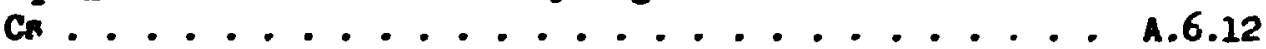

7. Equilibriu Breited State Fractions for fodrogen Esem Traversing Sodiu and Potassiu ....... A.6.14

8. Equilibriun Practions of a Hydrogen Been in

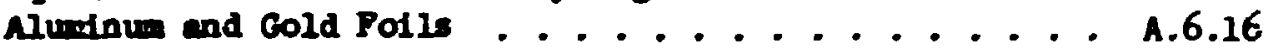

9. Yield of HO and H-Observed for an $\mathrm{H}_{2}^{+}$Bean

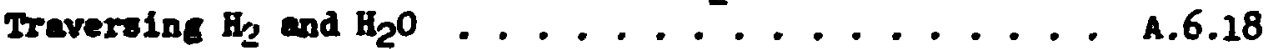

10. Yield of $\mathrm{H}^{\circ}$ and $\mathrm{y}$ - Obaerved for an $\mathrm{H}_{3}+$ Been

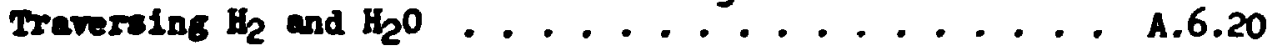

11. Practions of $\mathrm{H}_{3}^{+}, \mathrm{H}_{2}^{+}, \mathrm{H}_{2}, \mathrm{H}^{+}$, and $\mathrm{H}$ as a Punction of Target Thiciness for IoO-keV $\mathrm{H}_{3}^{+}$Traversing $\mathrm{B}_{2}$. . A.6.22

12. Equilibriu Fractions of a Helium beem in $\mathrm{H}_{2}$ and

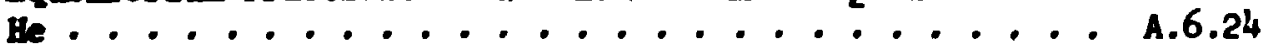

13. Equilibriu Prections of Beliu Been in $H_{2}$ and $\mathrm{O}_{2}$. 
14. Equilibriun Practions of a Beliu Bear in Ar ..... A.6.28

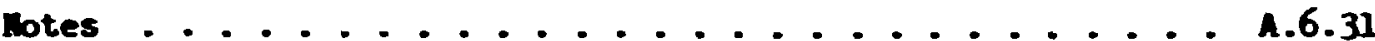

A.7 Stopping Cross Sections and Particle Range for Heary

Particles in Gases and Metals

1. Stopping Cross Sections for Protons and H Atom

in Atonic dydrogen ............... A.7.2

2. Stopping Cross Sections for Proton in Gases

$\left(\mathrm{H}_{2}, \mathrm{He}, \mathrm{N}_{2}\right) \ldots \ldots . \ldots . . \ldots . . . \ldots$

3. Stopping Cross Sections for Protons in Curbon

and Iron . . . . . . . . . . . . . A.7.6

4. Stopping Cross Sections for Frotons in Copper,

Holybdenue, Tunesten, and Gold .......... A.7.8

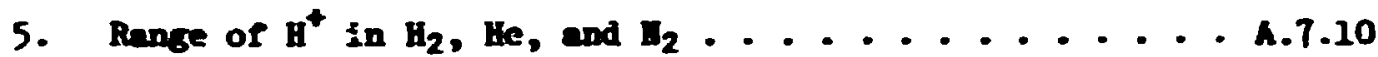

6. Range of $\mathrm{H}^{+}$in Solid $\mathrm{C}$, Su, and Alu ......... A.7.12

7. Ranges of Heliu Ions in Gases $\left(\mathrm{H}_{2}, \mathrm{He}, \mathrm{N}_{2}\right) \ldots \ldots .7 .14$

8. Ranges of Heliun Ions in Solids (C, Cu, and Au) . . . A.7.I6

9. Conversion Pactors for Enero Loss Meesurements .... A.T.18

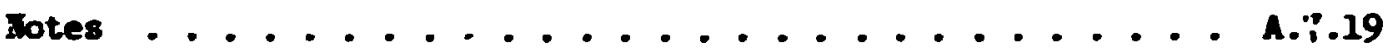

A.8 Positive-Negative Ion Neutralization Cross Section

1. Croas Sections for the Tro-Body Recomination of

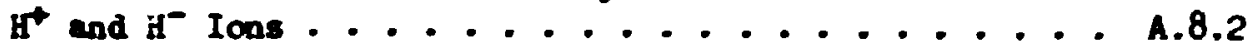

2. Cross sections for the Tro-Bod Recosbination of $\mathrm{He}^{+}$with $\mathrm{H}^{-}$Ions and of $\mathrm{He}^{+}$with $\mathrm{D}^{-}$Ions...... A.8.4

3. Cross Sections for the Tro-Body Recombination of $\mathrm{B}^{+}$ulth $\mathrm{O}^{-}$Ions, of $\mathrm{O}^{+}$with $\mathrm{O}^{-}$Ions, and of $\mathrm{l}_{2}^{+}$

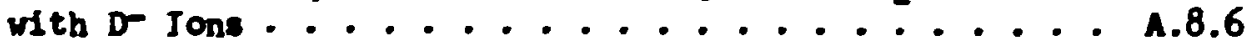

4. Cross Sections for the Tro-Bod Recombination of $\mathrm{O}_{2}^{+}$with $\mathrm{O}_{2}^{-}$Ions and of $\mathrm{N}_{2}^{+}$with $\mathrm{O}_{2}^{-}$Ions ..... A.8.8

5. Cross Sections for the Tro-Body Reconbination of $\mathrm{O}_{2}{ }^{+}$with $\mathrm{O}^{-}$Ions and of $\mathrm{O}^{+}$with $\mathrm{O}^{-}$Ions........ A.8.10

A.9 Ion-Molecule and Atom-Molecule Interchange Reactions

i. Cross Section for the Ion-Holecule Reaction

$\mathrm{H}_{2}^{+}+\mathrm{H}_{2}+\mathrm{H}_{3}^{+}+\mathrm{H}$....................2 
2. Cross Sections for the Production of Dt, EDt, and $\mathrm{D}_{2}{ }^{+}$by Proton I pect on $\mathrm{D}_{2} \ldots \ldots . . . . . . .4 .4$

3. Rate Coefficients, $k$, of Thernal Berer IonVolecule Reactions at Roon Tepereture . . . . . . 4.9 .6

4. Rate Coefficients, k, of Thermil Berer Beutral-Weutral Reactions ............ A.9.7

B. Photan collisions

B.1 Fhotoebsorption an Photolonization

1. Fhotonbsorption and ohotoionieation in Atonie fudrogen ................... B.1.4

2. Fhotonbeoption and Photolonization in Molecular Apdrogen .................... \$.1.6

3. Photolonization of Molecular Holrogen; the Batic of Discociated to Undissociated Product Ions . . . . B.1.8

4. Photorbsorption ad Photolonizetion in Belifin .... B.1.10

5. Photolonisation and Photosberption in Atcale C

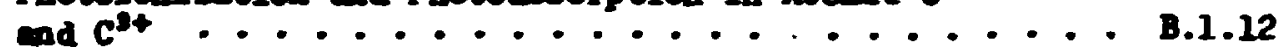

6. Photonbsopition and Photofoniention of Atonic Molvodenue, Tuaten, and cold .......... B.1.14

Hotes ......................... B.1.17

B.2 Photodiscoeletion

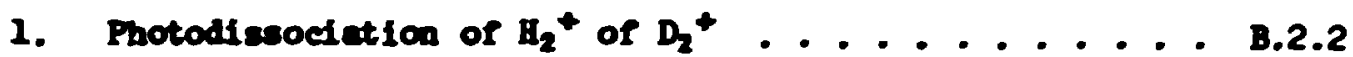

2. Photodisocoletion of $\mathrm{H}_{2}{ }^{+}$from selected Vibretional States (Theortical) ............... B.2.h

3. Photodissoefetion of $\mathrm{D}_{2}$ * from selected vibrational States (Theoretical) . . . . . . . . . . B.2.6

B.3 Photodetechnent

1. Crose sections for Photodetechnent of en Blectron

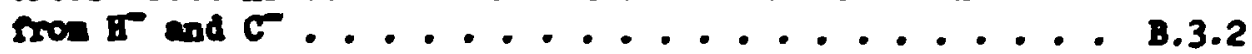

2. Crose sections for Photodstachneat of en Electron Fron $0^{-}$and $\mathrm{O}_{2}^{-} \ldots . . . . . . . . . . . .3 .4$ 
Presented is an eveluated craphicel and tebular copilation of atonic and nolecular croes sections of interest to controlled thernonuclear research. The cross sections are tabuleted and oraphed as a ruction of ebercs for collision processes imrolvin bens particles, electrons, and photons vith atos ad ions. Also ibeluded are sections on date for particle penctiretion throun meroscopic ntter, particle trensport properties, particle interections vith surfeces, and pertinent chrred particle muclear cross sections and reaction retes. In nost cases estimtes here been ande of the dite securacy. 
Fo:Grow

The work deseribed in this repart wes spossored by the Berto Research and Developent Mhinistration and is pert of a series of oull reports on stonic and wolecular processes of interest in rusion eners techolog. The reports in this series are: OSiL-3113,

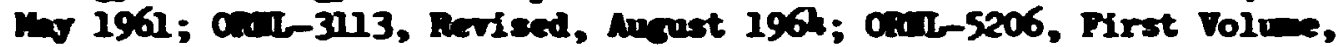
Pebrung 1977; and 0in-5207, Second Volve of OAII-5206, Pebruary 1977. 
This report is an expaded revision of a previous copilation of atoric and nolecular cross sections issued i i 196h as ain-30132. One difricults encountered in using the rrevious edition ves the problen of obtaining the date fros graphs covering severel orders of monitude. This proble hes been solvied by presenting the date in both tabular and craphicel form. Por each set of date, references are diven fron which the data vere obtained. All data vere plotted and a best-rit to the date wes ande resulting in a sincle curve. Botimates beve been ade of the eccuracy or confidence level of the date.

The cross section notation used is that in current vace. The cross section $\sigma_{\text {if }}$ rep-esents the croas section of an energetic particle of initial charge state 1 and final chroe state $f$. 111 cross sections are plotted in terws of $\mathrm{cm}^{2} /$ olecule or $\mathrm{cm}^{2} / \mathrm{etan}$ for a notome ons. Particular attention should be diven to the explanetory notes foud in eech section.

A diligent effort has been ande to enowre the sccuracy of the publication process. Howerer, in an effort of this monitude errors will exdst. The autbors would greatis appreclate the veers bringing these to our attention. An waval up-iating of the data is planoed. 
A. Dears Particle Collision

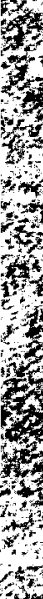




\subsection{Dowy Particle seattering}




\section{1.h}

Anotic Seatteriog of $\mathrm{i}^{\circ}$ Indeed is $\mathrm{i}^{\circ}$ Inpet on $\mathrm{E}$

$\mathbf{n}+\mathbf{n}+\mathbf{n}+\mathbf{n}$

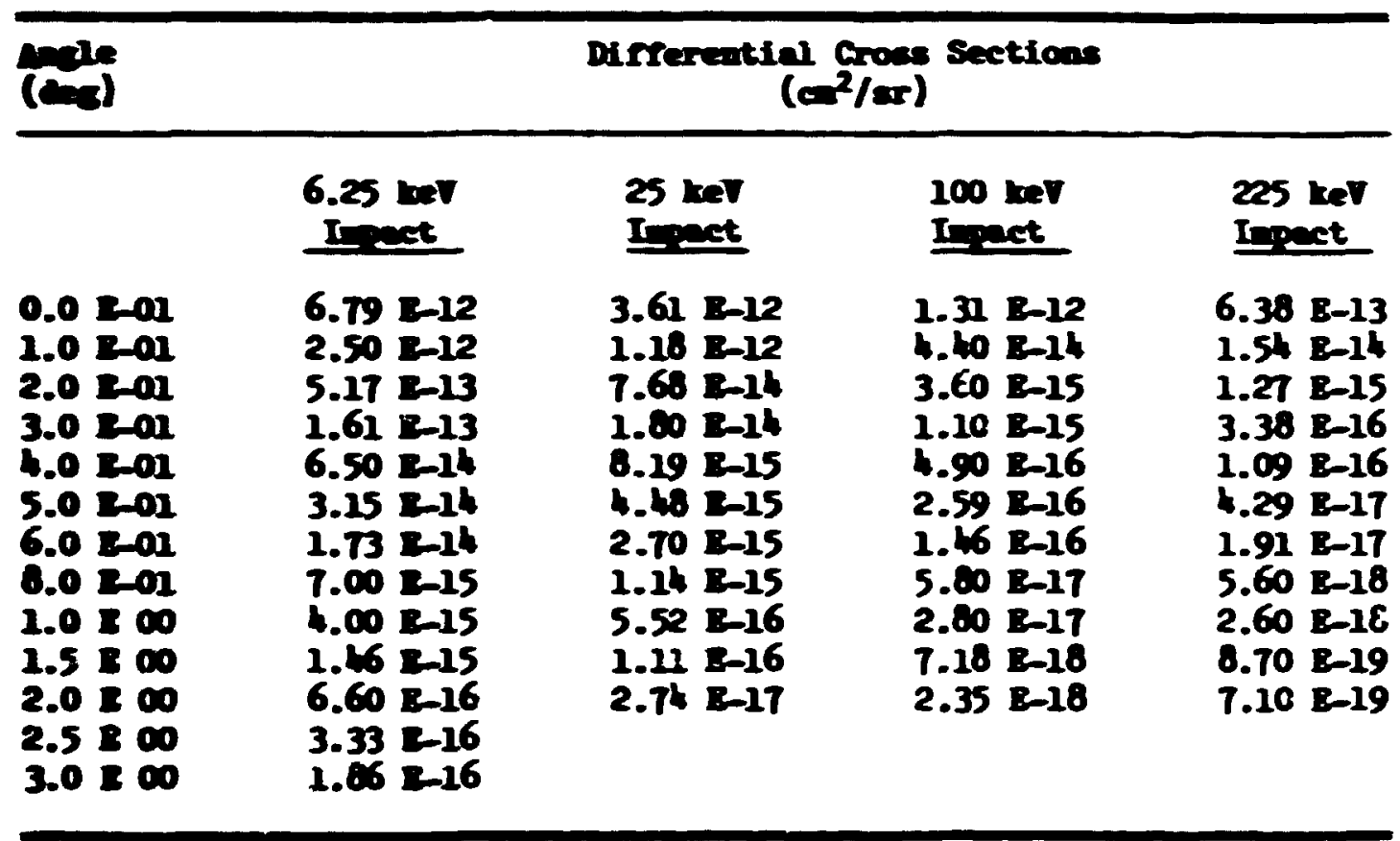

\section{Prices:}

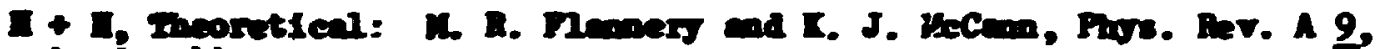
IST (IOT).

\section{Saine:}

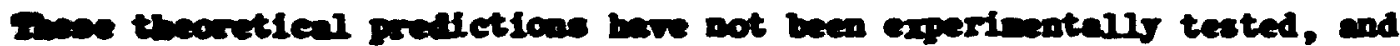

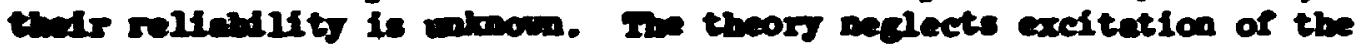
terct, so the erose sections are cortalnj 10w; this ofll be nost chilow et the hicher ingact enerdes. 


\section{A.1.5}

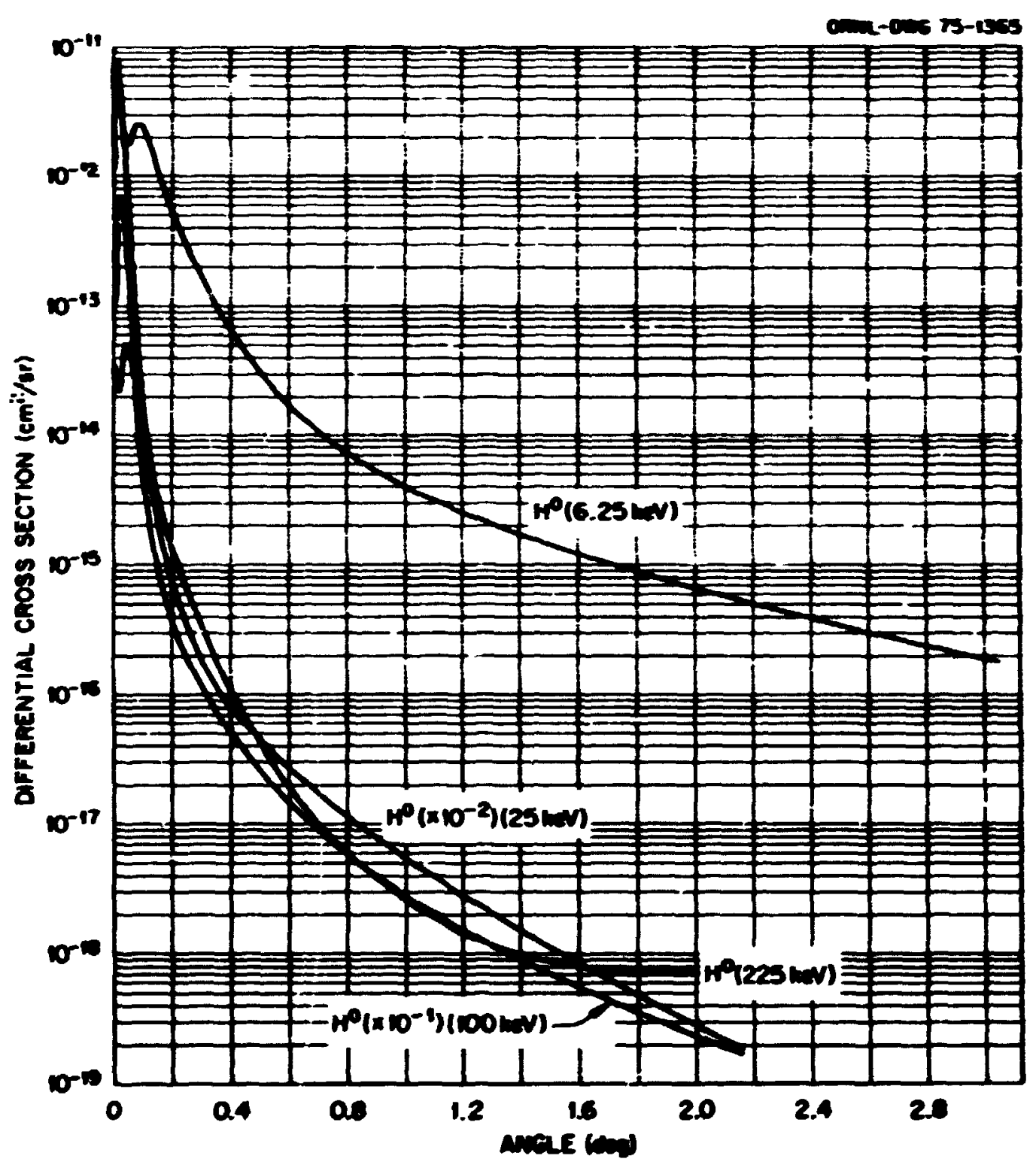




\section{A.1.6}

Blestic Senteriog of $\mathrm{A}^{*}$ Induced by $\mathrm{H}^{*}$ Irpact on $\mathrm{a}$

$$
\mathbf{a}^{+}+\mathbf{a}+\mathbf{a}^{+}+\mathbf{z}
$$

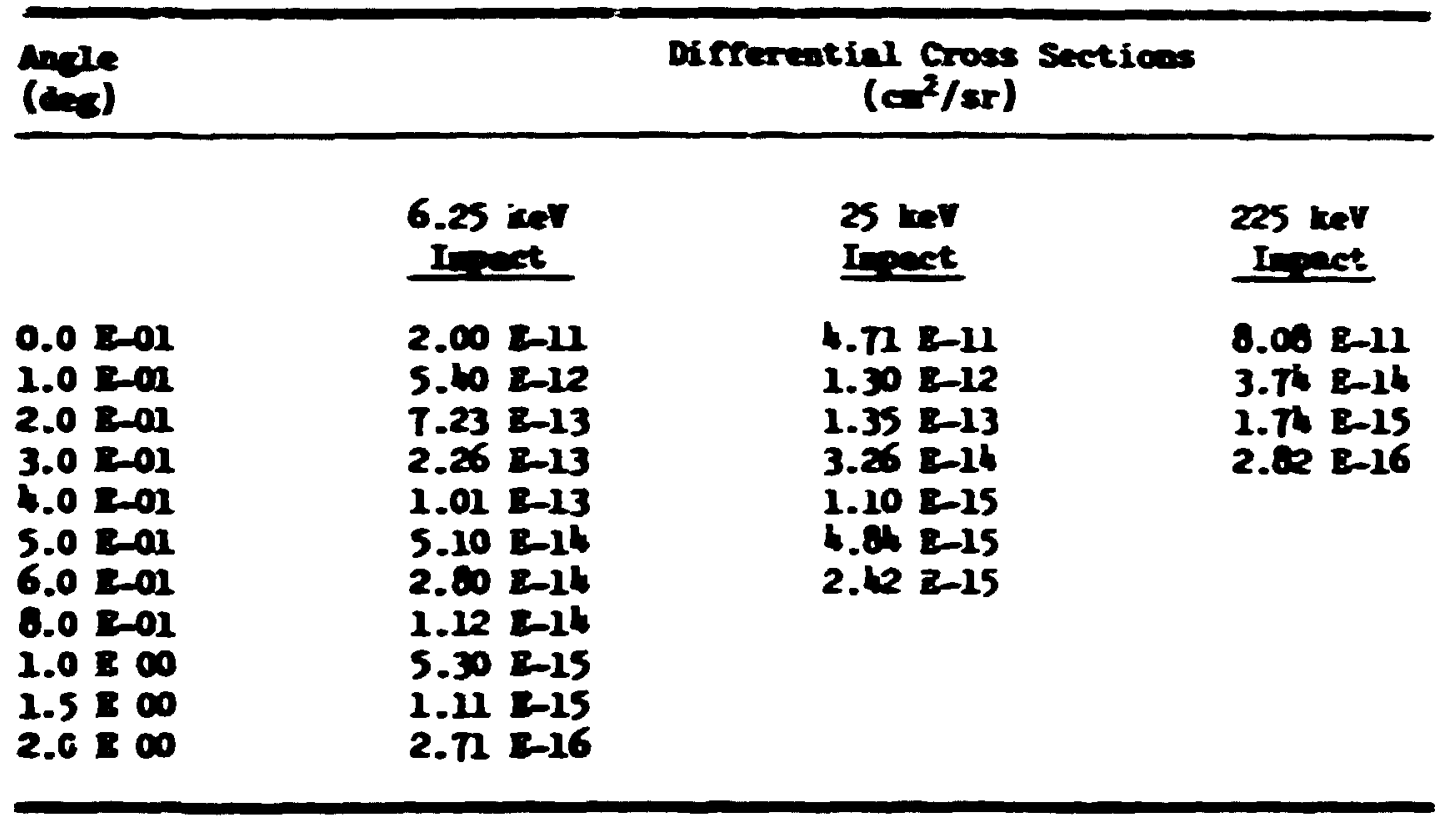

\section{Defencian:}

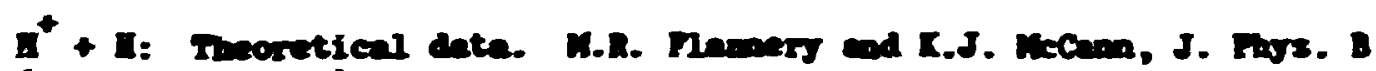
(to be poblisted). Ihis prer discusees ouls the bosis for the

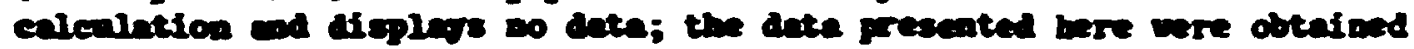
b. private compieation with the cuthors.

\section{seeres:}

These theoretieal prodicticas bov sot bea experimeatelly tested, ad

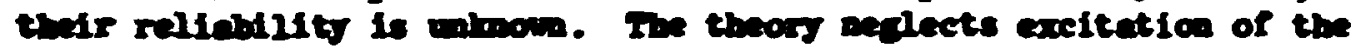
target so the eroes sections are certafing 10\%; this will be wost serficus at the hicher inget enerdes.

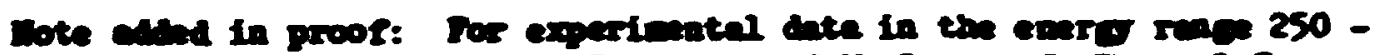

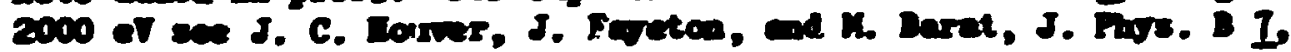
2350 (297t). 


\section{A.1.?}

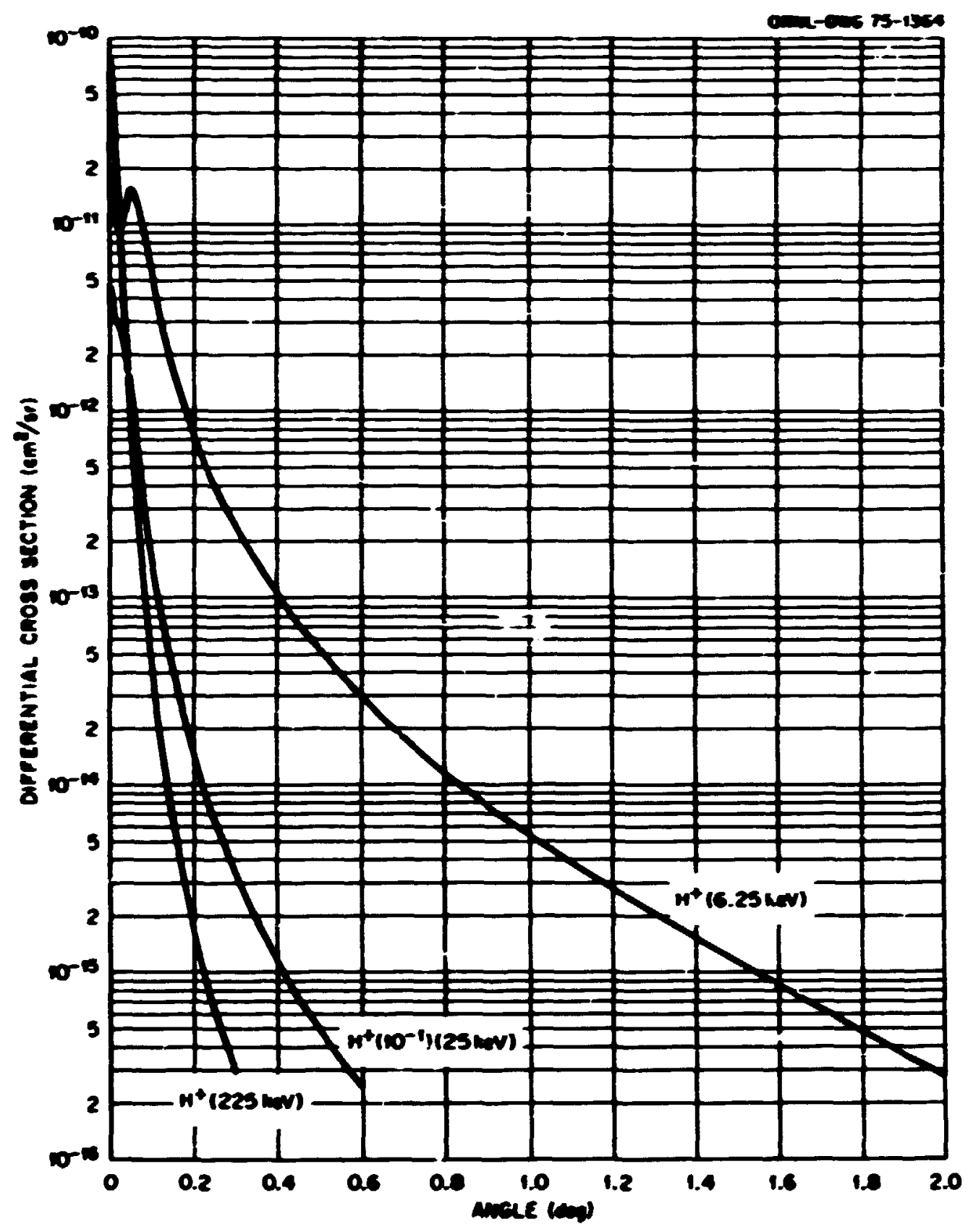




\section{A.1.8}

Angular Distribution of $\mathrm{I}^{+}$Formed as $\dddot{I}^{0}$ Trererses a Target of $\ddot{z}_{2}$

$$
z^{0}+z_{2}+i^{+}+e+z_{2}
$$

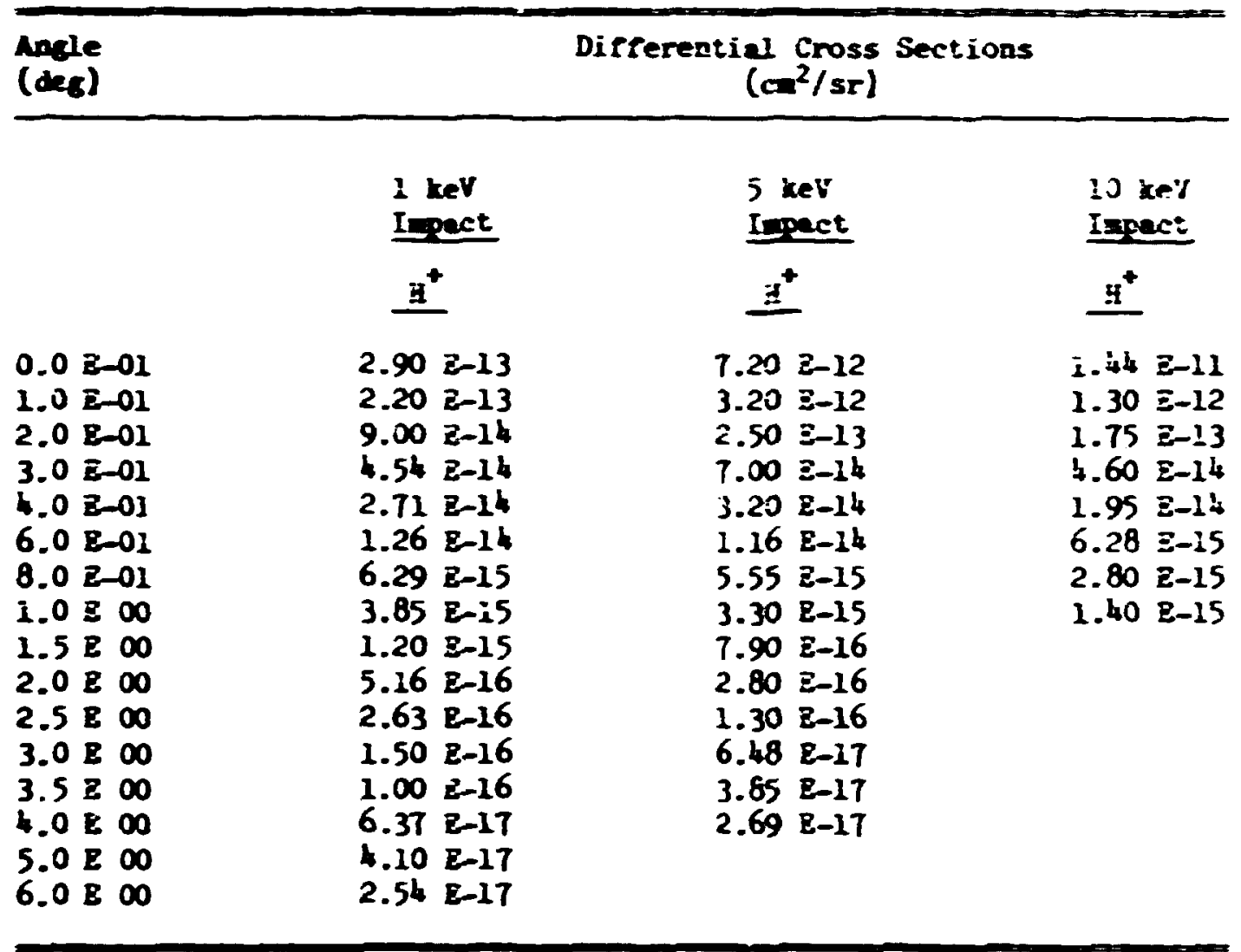

\section{Pefereace:}

B + B2, Bperimental: H. H. Fleischnon, C. P. Barnett, and J. A. Ras, Pro. Ret. A 10, 569 (1974).

\section{Accuract:}

Systenatic error $< \pm 13 \%$. Randon error $< \pm 5 \%$. 
A.1.9

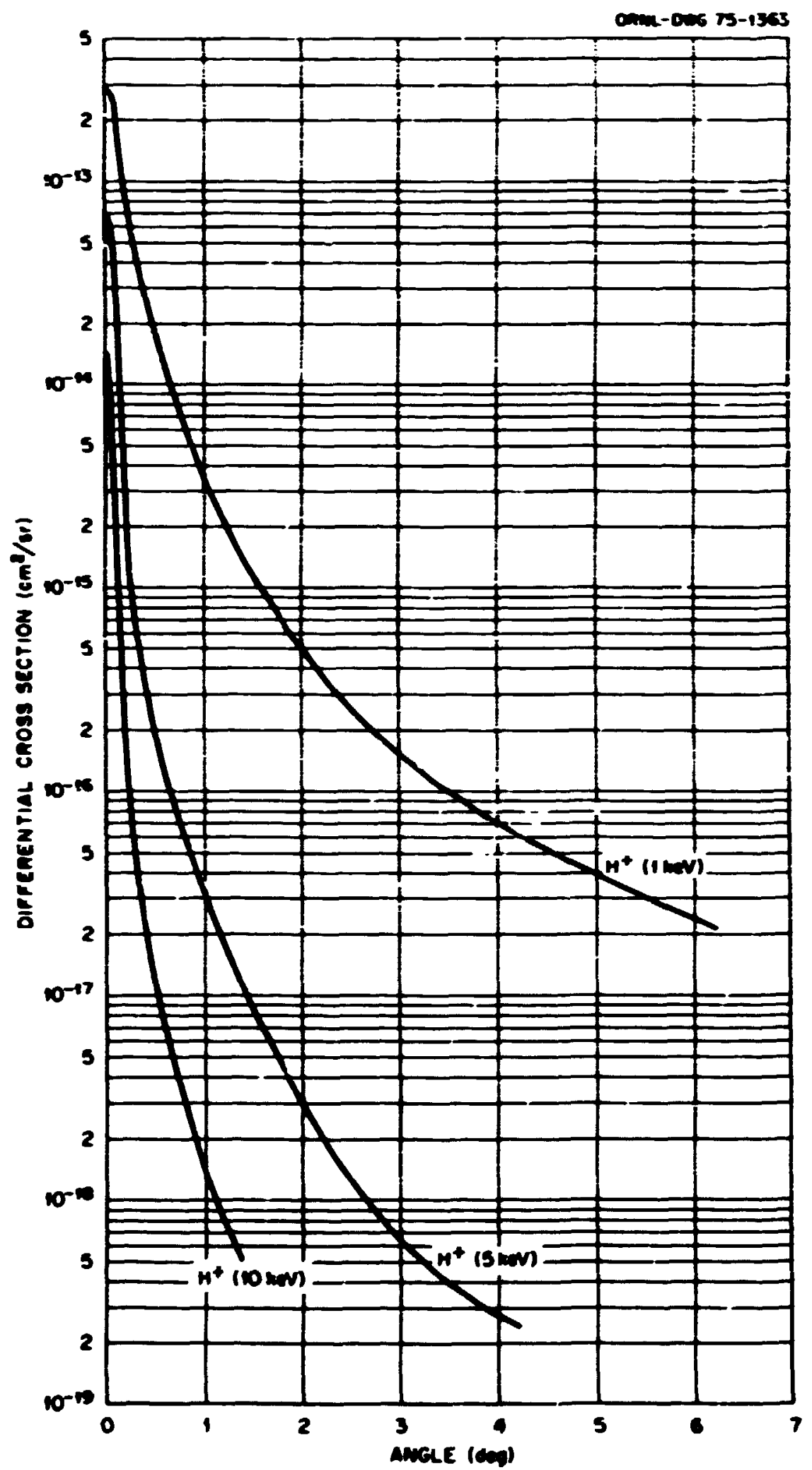




\section{A.1.10}

Scattering of $\mathrm{H}^{\mathrm{O}}$ Formed by Charge Transfer

$$
\text { Neutralization of } \mathrm{H}^{+} \text {in } \mathrm{H}_{2}
$$

\begin{tabular}{ll}
\hline $\begin{array}{c}\text { Energy } \\
\text { (kev) }\end{array}$ & $\begin{array}{c}\text { Half Angle } \\
\text { (deg) }\end{array}$ \\
& \\
$1.0 \mathrm{E} 00$ & $2.40 \mathrm{E}-02$ \\
$1.5 \mathrm{E} 00$ & $1.90 \mathrm{E}-02$ \\
$2.0 \mathrm{~F} 00$ & $1.69 \mathrm{E}-02$ \\
$3.6 \mathrm{E} \mathrm{O0}$ & $1.50 \mathrm{E}-02$ \\
$4.2 \mathrm{E} 00$ & $1.43 \mathrm{E}-02$ \\
$5.5 \mathrm{E} 00$ & $1.43 \mathrm{E}-02$ \\
\hline
\end{tabular}

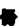

There are no published cross sections for this process. He present here the half angle of the scattered $\mathrm{H}^{\circ}$ distribution (i.e. the leviation angle at which the HO flux is half its maximum value).

\section{Reference:}

$\mathrm{H}^{+}+\mathrm{H}_{2}$, Experimental: A.B. Wittkover, P.H. Rose, P.P. Bastide, and M.B. Brooks, Phys. Rev. 136, A1254 (1964).

\section{Accuracy:}

Unspecified. 


\section{A.1.11}

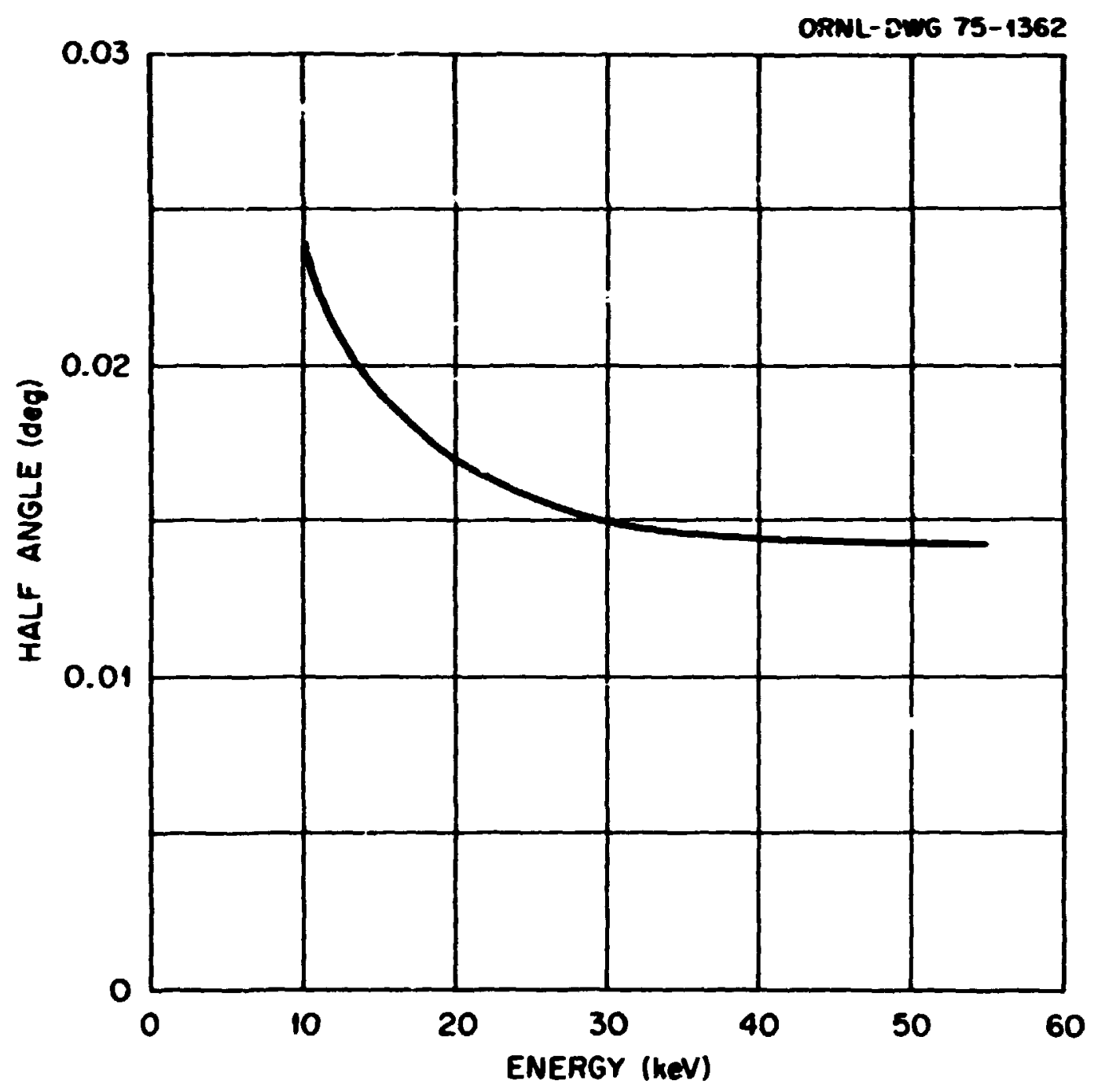




\section{A.1.12}

Angular Distribution of $\mathrm{H}^{+}$Forned by Dissociation

$$
\begin{aligned}
& \text { of } \mathrm{H}_{2}^{+} \text {on an } \mathrm{H} \text { Target } \\
& \mathrm{H}_{2}^{+}+\mathrm{H} \rightarrow \mathrm{H}^{+}+\mathrm{H}^{\circ}+\mathrm{H}
\end{aligned}
$$

\begin{tabular}{l}
\hline $\begin{array}{l}\text { Angle } \\
(\text { deg })\end{array}$ \\
\hline
\end{tabular}

$10 \mathrm{keV}$

Inpact

0.0 E-01

$1.0 \mathrm{E}-01$

$2.0 \mathrm{~B}-01$

$3.0 \mathrm{E}-\mathrm{Cl}$

$4.0 \mathrm{~B}-01$

$6.0 \mathrm{E}-01$

$8.0 \mathrm{E}-01$

$1.0 \mathrm{E} 00$

$1.2 \mathrm{E} 00$

$1.4 \mathrm{E} 00$

$1.6 \mathrm{E} 00$

$1.8 \mathrm{~B} 00$
$2.80 \mathrm{E}-13$

$2.14 \quad \mathrm{E}-13$

1.87 E-13

1.74 E-13

1.64 E-13

1.54 E-13

1.49 E-13

$1.06 \mathrm{E}-13$

7.63 E-14

4.32 E-14

2.70 E-14

1.37 E-14
$80 \mathrm{keV}$

Impact

$6.00 \mathrm{E}-13$

$5.40 \mathrm{E}-13$

5.14 E-13

4.99 E-13

4.54 E-13

$3.16 \mathrm{E}-13$

$5.90 \mathrm{E}-14$

$1.00 \mathrm{E}-14$

\section{Reference:}

Bxperimental Data: According to G.H. McClure, Phys. Rev. 153, 182 (1967), the cross section for this procese is essentially equal to the corresponding cross section F.or $\mathrm{H}_{2}{ }^{+}$dissociation on $\mathrm{H}_{2}$ as measured in G.H. McClure, Phys. Rer. 140, A769 (1965). Equality within $\pm 10 \%$ is indicated and the accuracy of the lata is no better than this. The data presented here are actually the cross sections for dissociation in $\mathrm{H}_{2}$ from McClure's work.

\section{Ascuracy:}

Systematic error $< \pm 8 \%$. Random error $< \pm 8 \%$. 


\section{A.1.13}

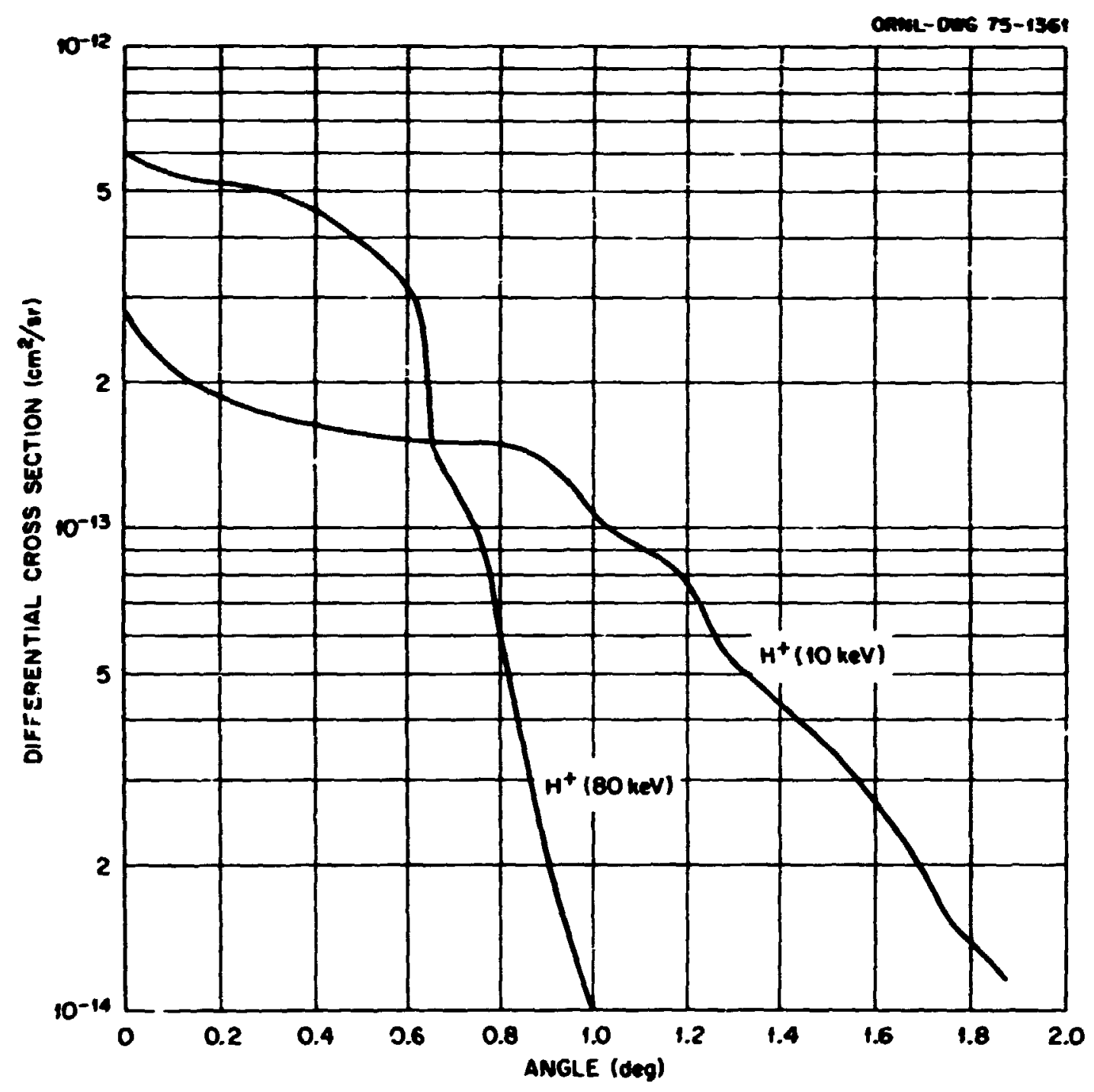




\section{A.1.14}

Angular Distribution of $\mathrm{H}^{\circ}$ and $\mathrm{H}^{*}$ Porsed by

Dissociation of $\mathrm{H}_{2}^{+}$on $\mathrm{H}_{2}$ Target

$$
\mathrm{B}_{2}^{+}+\mathrm{H}_{2}+\mathrm{H}^{+}+\mathrm{H}^{0}+\mathrm{H}_{\hat{C}}
$$

\section{$10 \mathrm{keV}$ Inpect}

$$
\underline{\mathbf{H}}^{\mathbf{0}}
$$

$3.50 \mathrm{E}-12$

$1.40 \mathrm{E}-12$

8.00 E-13

$5.62 \mathrm{E}-13$

3.70 E-13

2.35 E-13

$1.43 \mathrm{E}-13$

8.45 B-14

4.74 E-14

$2.50 \mathrm{~B}-14$

9.70 B-15

4.67 E-15

1.79 E-15
$80 \mathrm{keV}$ Impect

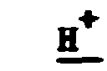

$2.40 \mathrm{E}-13$

$2.02 \mathrm{~B}-13$

$1.82 \mathrm{E}-13$

$1.62 \mathrm{~B}-13$

1.26 B-13

$9.35 \mathrm{E}-14$

$6.62 \mathrm{~B}-14$

$4.60 \mathrm{E}-14$

3.10 E-14

1.94 B-14

9.23 E-15

4.95 E-15

2.01 E-15

\section{Reference:}

$\mathrm{H}_{2}^{\dagger}+\mathrm{H}_{2}$, Bxperimental: I. Sauers, R.L. Fitzwilson, J.C. Ford, and B.H. Thosas, Phys. Rev. A 6, 1418 (1972); G.W. McClure, Phys. Rev. 140, A 769 (1965).

Accuracy:

Systemtic error $< \pm 87$. Rendon error $< \pm 87$.

\section{Dotes:}

See lote (1) at end of chapter. 


\section{A.1.15}

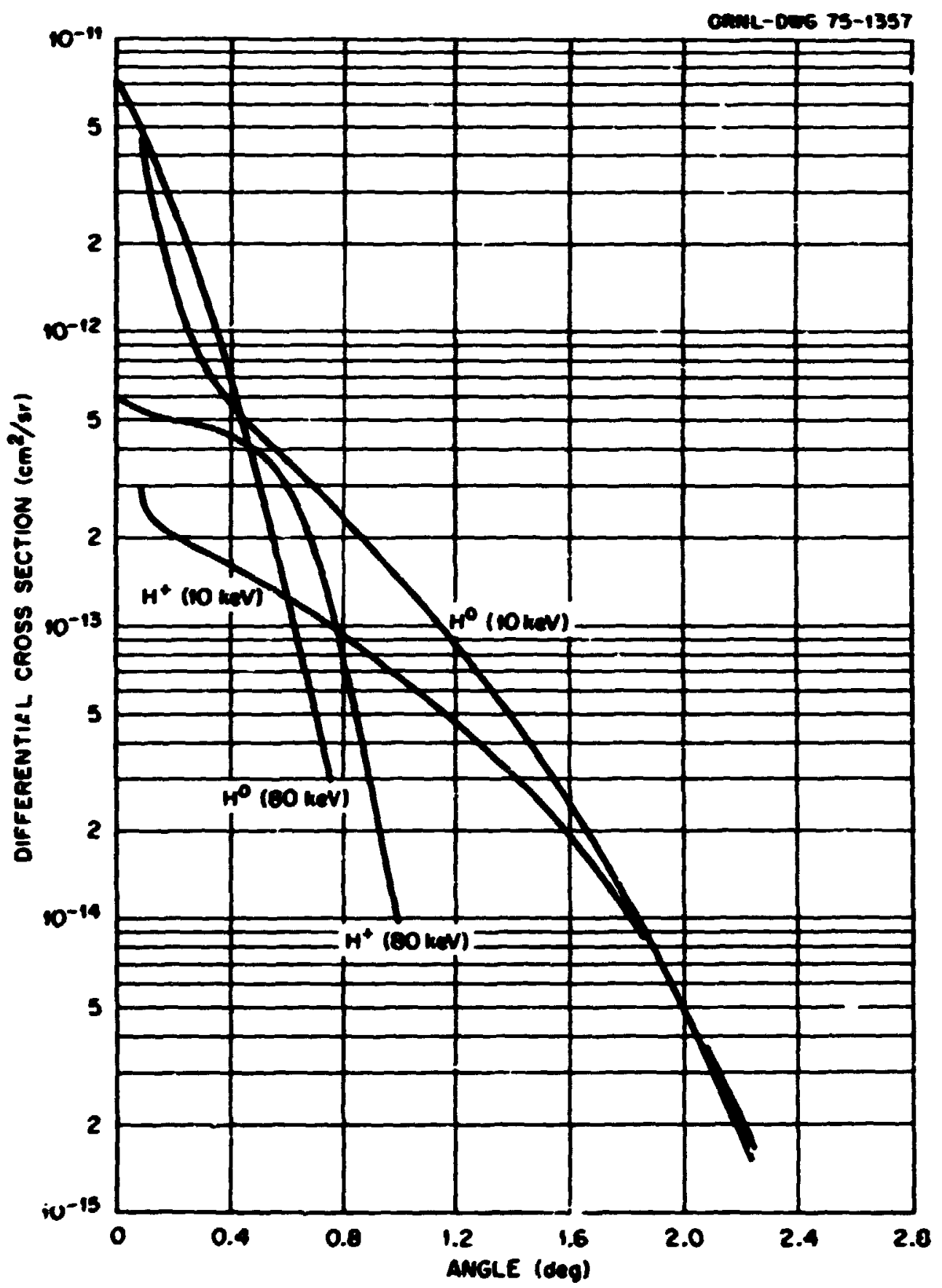




\section{A.1.16}

Scettering of $\mathrm{H}^{+}$and $\mathrm{B}^{\circ}$ Induced by

$$
\begin{aligned}
& \mathrm{H}^{+} \text {Impact on } \mathrm{He} \\
& \mathrm{H}^{+}+\mathrm{He}+\mathrm{H}^{\circ}+\mathrm{He}^{+} \\
& \mathrm{H}^{+}+\mathrm{He} \rightarrow \mathrm{H}^{+}+\mathrm{He}
\end{aligned}
$$

\begin{tabular}{|c|c|c|c|c|}
\hline $\begin{array}{l}4.0 \mathrm{~B}-01 \\
6.0 \mathrm{~B}-01 \\
8.0 \mathrm{~B}-01 \\
1.0 \mathrm{~B} 00 \\
1.5 \mathrm{~B} 00\end{array}$ & $\begin{array}{ll}2.55 & \mathrm{~B}-15 \\
1.10 & \mathrm{~B}-15 \\
5.78 & \mathrm{~B}-16\end{array}$ & $\begin{array}{ll}2.41 & \mathrm{~B}-14 \\
1.10 & \mathrm{~B}-14 \\
6.20 & \mathrm{~B}-15\end{array}$ & $\begin{array}{ll}2.64 & E-15 \\
1.01 & E-15 \\
4.25 & E-16 \\
9.40 & E-17\end{array}$ & $\begin{array}{ll}1.07 & \mathrm{E}-14 \\
4.68 & \mathrm{E}-15 \\
2.11 & \mathrm{E}-15 \\
1.06 & \mathrm{E}-15 \\
2.40 \mathrm{E}-16\end{array}$ \\
\hline
\end{tabular}

$\begin{array}{r}\begin{array}{l}\text { Angle } \\ (\text { deg })\end{array} \\ \hline\end{array}$

$\underline{\mathrm{H}}^{\frac{5 \mathrm{keV} \text { Imact }}{\mathrm{H}^{+}}} \quad \underline{\mathrm{H}}^{\frac{20 \mathrm{keV} \text { Impact }}{\mathrm{H}^{+}}}$

\section{Beference:}

R.L. P1tzorilson and E.W. Thonas, Phys. Rer. A 6, 1054 (1972).

\section{Accurac:}

Systematic error $< \pm 6 \%$. Randon error $< \pm 7 \%$.

Lotes:

See Dote (2) at end of chapter. 


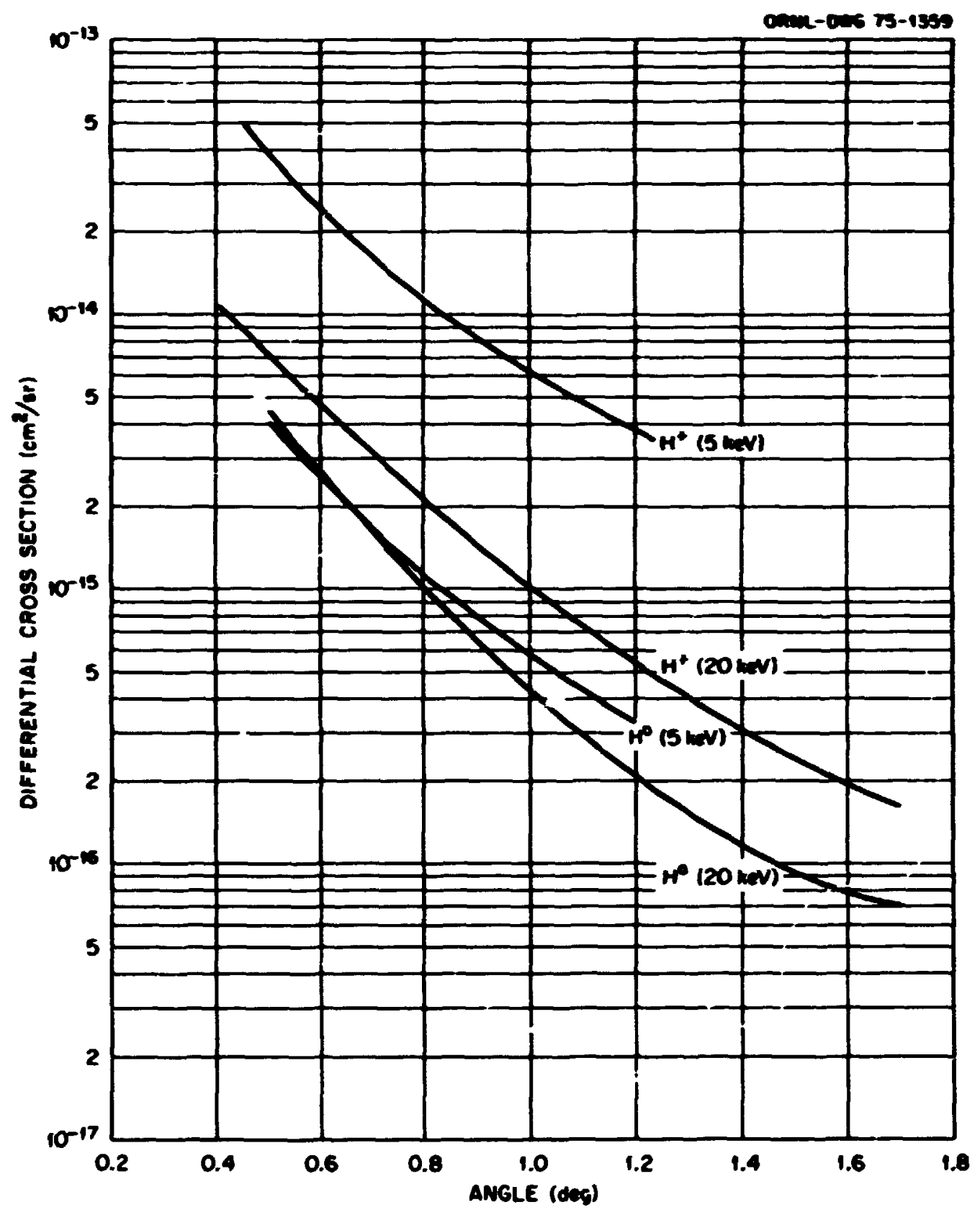




\subsection{6}

Angular Distribution of $\mathrm{H}^{\circ}$ and $\mathrm{H}^{+}$Porned

es $\mathrm{H}^{\circ}$ Treverses a Target of the

$$
\begin{gathered}
\mathrm{B}^{0}+\mathrm{Be}+\mathrm{B}^{0}+\mathrm{He} \\
\mathrm{H}^{0}+\mathrm{He}+\mathrm{H}^{+}+\mathrm{e}+\mathrm{He}
\end{gathered}
$$

\begin{tabular}{|c|c|c|c|c|}
\hline \multirow[t]{3}{*}{$\begin{array}{l}\text { Angle } \\
\text { (des) }\end{array}$} & \multicolumn{4}{|c|}{$\begin{array}{l}\text { Differential Cross Sections } \\
\left(\mathrm{cm}^{2} / \mathrm{sr}\right)\end{array}$} \\
\hline & $\begin{array}{l}1 \text { keV } \\
\text { I paet }\end{array}$ & $\begin{array}{l}5 \mathrm{keV} \\
\text { Inect }\end{array}$ & & \\
\hline & $\underline{\mathbf{A}^{+}}$ & $\underline{\mathbf{H}^{+}}$ & $\underline{\mathbf{H}^{\mathbf{0}}}$ & $\underline{\mathbf{H}^{+}}$ \\
\hline 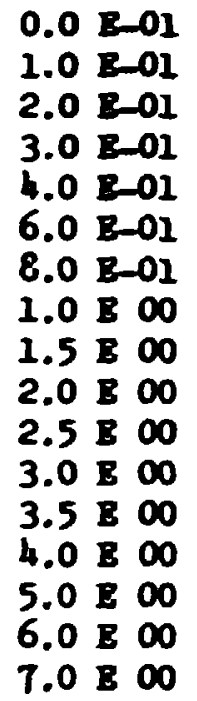 & $\begin{array}{ll}2.50 & \mathrm{E}-13 \\
1.95 & \mathrm{E}-13 \\
7.40 & \mathrm{E}-14 \\
4.00 & \mathrm{E}-14 \\
2.59 & \mathrm{E}-14 \\
1.30 & \mathrm{E}-14 \\
7.13 & \mathrm{E}-15 \\
4.20 & \mathrm{E}-15 \\
1.50 & \mathrm{E}-15 \\
7.00 & \mathrm{E}-16 \\
3.90 & \mathrm{E}-16 \\
2.20 & \mathrm{E}-16 \\
1.40 & \mathrm{E}-16 \\
1.00 & \mathrm{E}-16 \\
5.20 & \mathrm{E}-17 \\
3.19 & \mathrm{E}-17 \\
2.00 & \mathrm{E}-17\end{array}$ & $\begin{array}{ll}7.00 & E-12 \\
2.10 & E-12 \\
3.05 & E-13 \\
1.10 & E-13 \\
5.23 & E-14 \\
1.99 & E-14 \\
9.30 & E-15 \\
5.00 & E-15 \\
1.57 & E-15 \\
6.35 & E-16 \\
3.30 & E-16 \\
1.82 & E-16 \\
1.10 & E-16 \\
7.30 & E-17\end{array}$ & $\begin{array}{ll}1.20 & \mathrm{E}-14 \\
3.52 & \mathrm{E}-15 \\
1.58 & \mathrm{E}-15 \\
8.50 & \mathrm{E}-16 \\
2.30 & \mathrm{E}-16 \\
8.30 & \mathrm{E}-17 \\
3.90 & \mathrm{E}-17 \\
2.10 & \mathrm{E}-17 \\
1.20 & \mathrm{E}-17 \\
8.55 & \mathrm{E}-18\end{array}$ & $\begin{array}{ll}2.38 & \mathrm{E}-11 \\
2.60 & \mathrm{E}-12 \\
2.30 & \mathrm{E}-13 \\
6.50 & \mathrm{E}-14 \\
2.80 & \mathrm{E}-14 \\
8.63 & \mathrm{E}-15 \\
4.38 & \mathrm{E}-15 \\
2.37 & \mathrm{E}-15 \\
6.55 & \mathrm{E}-16 \\
2.21 & \mathrm{E}-16 \\
9.70 & \mathrm{E}-17 \\
4.55 & \mathrm{E}-17 \\
2.40 & \mathrm{E}-17 \\
1.50 & \mathrm{E}-17 \\
7.34 & \mathrm{E}-18\end{array}$ \\
\hline
\end{tabular}

\section{References:}

H + He, Experimental: H. H. Pleischman, C. P. Barnett, and J. A. Rav, Puys. Rev. A 10, 569 (1974). I. Savers and W. B. Thomas, Phys. Rev. A 10, $822(1974)$.

\section{Accuracy:}

Systenatic error $< \pm 10 \%$. Randon error $< \pm 5 \%$. 
A.1.19

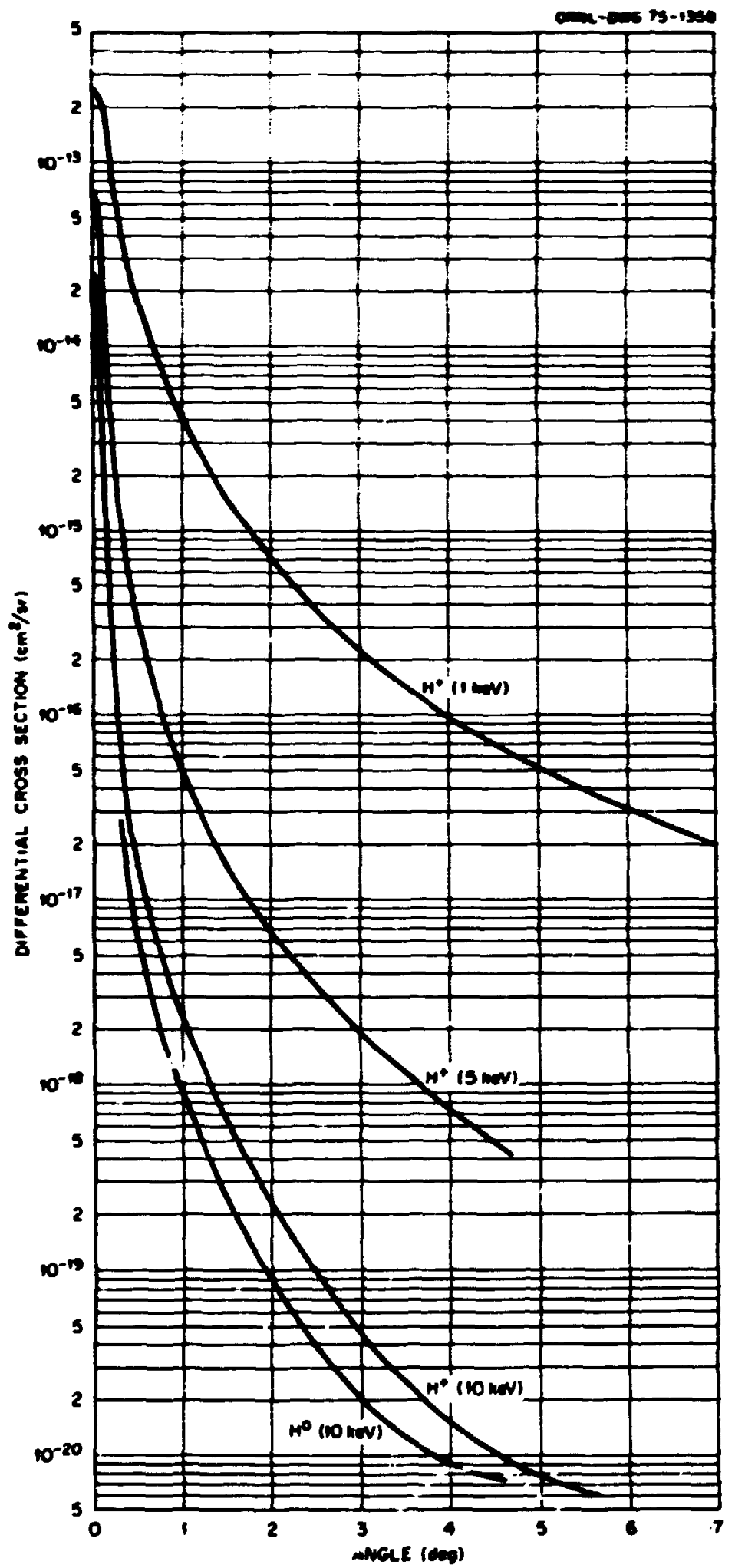




\section{A.1.20}

Angular Distribution of $\mathrm{H}^{\circ}$ and $\mathrm{H}^{+}$Forned bs Dissociation of $\mathrm{H}_{2}^{+}$on an He Target

$$
\mathrm{H}_{2}^{+}+\mathrm{He} \rightarrow \mathrm{B}^{+}+\mathrm{H}+\mathrm{He}
$$

\section{$4 \mathrm{keV}$ I pact}

$\underline{\mathbf{B}^{\circ}}$

\section{$1.50 \mathrm{E}-13$}

$4.0 \mathrm{E}-01$

$6.0 \mathrm{~B}-01$

$8.0 \mathrm{E}-01$

$1.0 \mathrm{~B} 00$

$1.2 \mathrm{~B} \mathrm{00}$

$1.4 \mathrm{~B} 00$

$1.6 \mathrm{E} 00$

$1.8 \mathrm{E} 00$

$2.0 \mathrm{~B} 00$

$2.2 \geq 00$

$2.4 \mathrm{E} 00$

$2.6 \mathrm{E} 00$

$2.8 \mathrm{E} 00$
$6.70 \mathrm{E}-14$

$6.20 \mathrm{~B}-14$

5.65 E-14

$5.08 \mathrm{E}-14$

$4.40 \mathrm{E}-14$

3.67 E-14

2.94 E-14

2.31 B-14

1.76 B-14

$1.26 \mathrm{E}-14$

8.48 E-15

5.42 E-15
8.55 E-14

$6.16 \mathrm{~B}-14$

$4.90 \mathrm{~B}-14$

$4.20 \mathrm{~B}-14$

$3.70 \mathrm{E}-14$

3.12 E-14

$2.50 \mathrm{E}-14$

$1.93 \mathrm{~B}-14$

$1.42 \mathrm{E}-14$

$1.00 \mathrm{~B}-14$

7.21 B-15

4.35 B-15
12 keV I pact

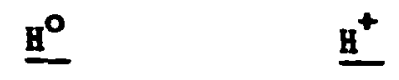

1.21 E-13

$9.64 \mathrm{E}-14$

$7.20 \mathrm{E}-14$

$5.00 \mathrm{E}-14$

3.21 E-14

$1.89 \mathrm{E}-14$

$9.06 \mathrm{~B}-15$

3.45 E-15

$1.10 \mathrm{E}-15$

$1.05 \mathrm{E}-13$

$8.51 \mathrm{E}-14$

$6.88 \mathrm{E}-14$

$5.38 \mathrm{E}-14$

$3.95 \mathrm{E}-14$

2.64 E-14

$1.50 \mathrm{E}-14$

6.90 R-15

$2.60 \mathrm{E}-15$

\section{Reference:}

$\mathrm{H}_{2}^{+}+\mathrm{He}$, Bxperimental: I. Sauers, R.L. Pitzwilson, J.C. Pord, and B.H. Thomas, Phys. Rev. A 6, 1418 (1972).

\section{Accuracy:}

Systematic error $< \pm 8 \%$. Random error $< \pm 8 \%$. 


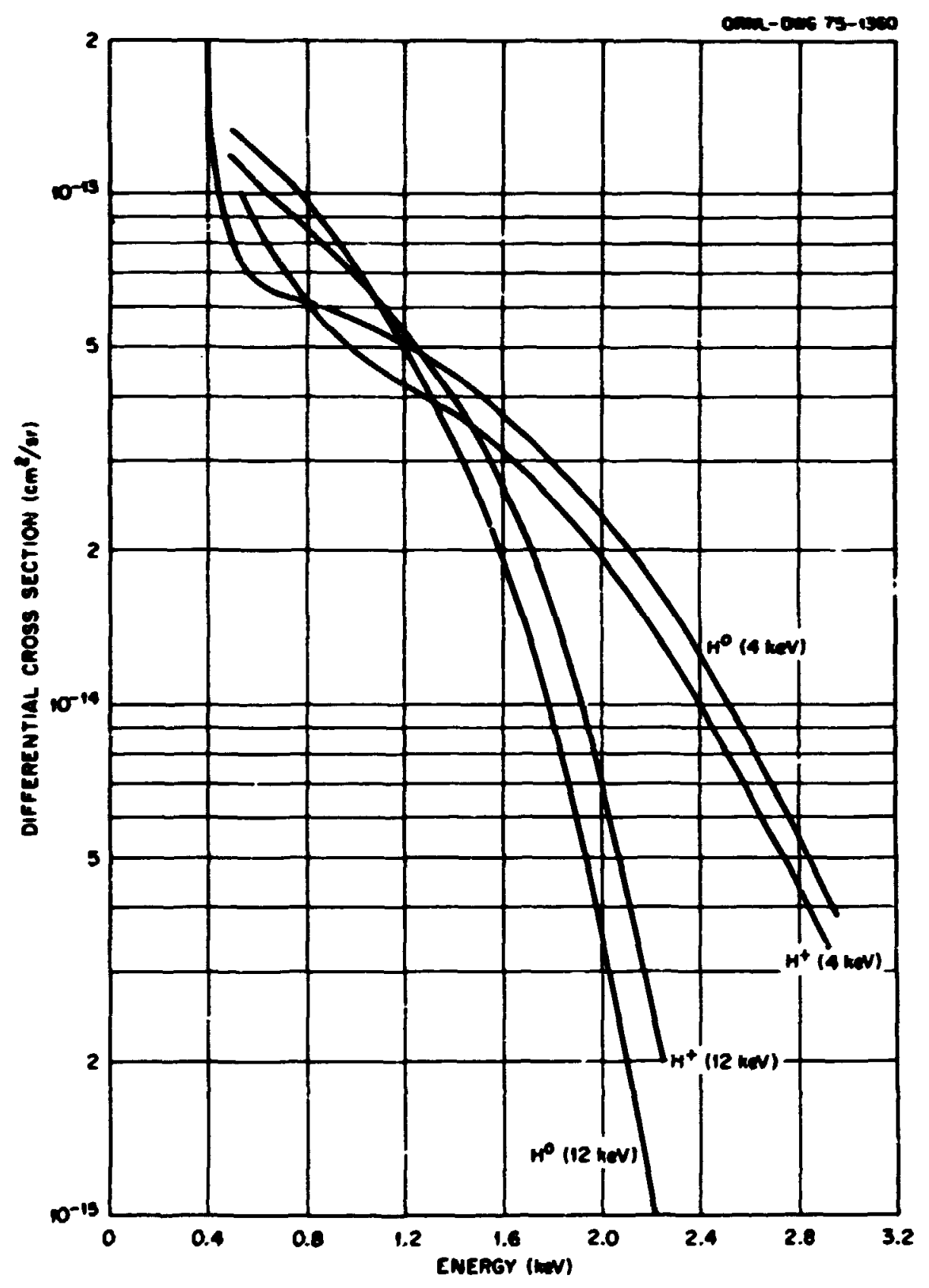




\section{A.1.23}

\section{Motes}

(1) References cited provide dete for other energies fron 4 to $80 \mathrm{keV}$. The vidth of the $\mathrm{B}^{+}$distribution has been mesured to $200 \mathrm{reV}$ by C.F. Barnett and J.A. Ray, Atonic Collision Processes, (North Hollani Publ. Co. Ansterda, 1964) P. 743. Also see D.R. Sueetma, Proc. Ros. Soc. A 256, 416 (1960).

(2) There are some studies of $\mathrm{a}^{\circ}$ angular distributions induced by $\mathrm{H}^{\dagger}$ on He where only the half ancle (the deriation angle at wich the $\mathrm{B}^{\circ}$ flux is half its maxime ralue) is given. According to A.B. Hittkower, P.H. Rose, R.P. Bestide, and M.B. Brooks, Phys. Rev. 136, Al254 (1964), the balf angle varjes from 0.35 milliradians at $20 \mathrm{keV}$ to 0.25 milliradians at $55 \mathrm{keV}$. 
A.2 Bucitation by Heary Particles 


$$
\text { A.2.2 }
$$

Excitation Cross Sections for the Reactions

$$
\mathrm{H}+\mathrm{H} \rightarrow \mathrm{H}(2 \mathrm{~s}, 2 \mathrm{p})+\mathrm{H}
$$

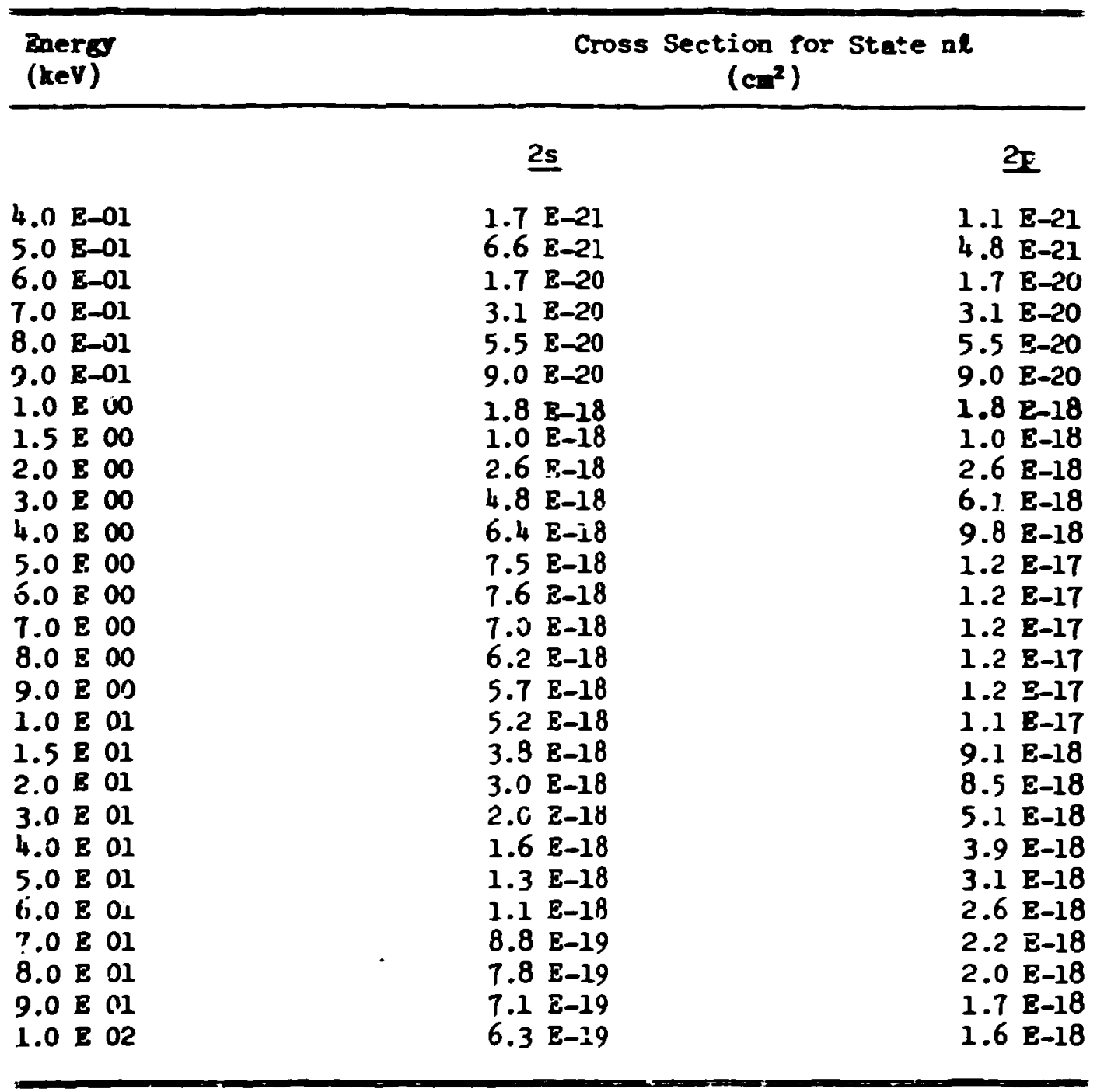

References:

$H+H \rightarrow H(2 s, 2 p)+H:$ M.R. Flannery, Phys. Rev. 183, 241 (1969).

\section{Notes:}

See Note (22) at end of chapter. 


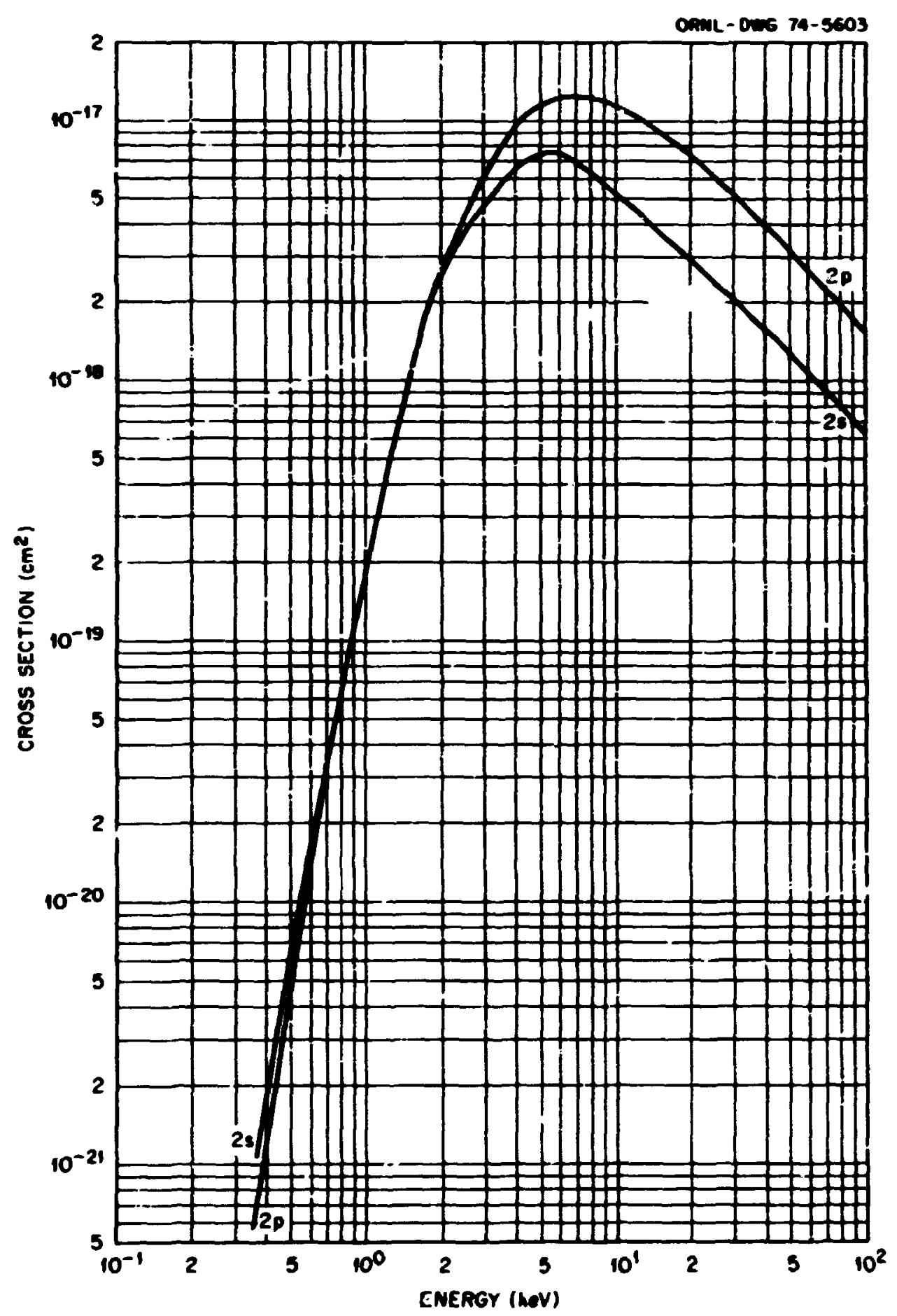




$$
\text { A.2.4 }
$$

Excitation Cross Sections for the Reactions

$$
\mathrm{H}+\mathrm{H}_{2} \rightarrow \mathrm{H}(2 \mathrm{~s}, 3 \mathrm{~s}, 2 \mathrm{p}, 3 \mathrm{p}, 3 \mathrm{~d})+\mathrm{H}_{2}
$$

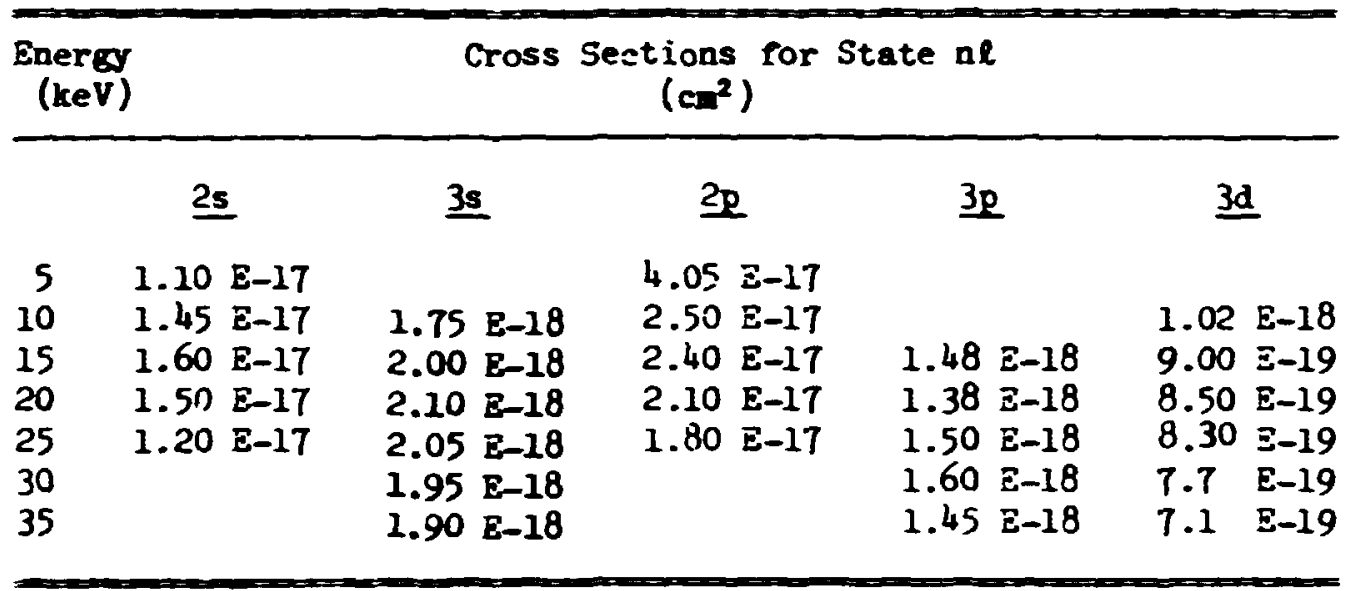

Reierences:

$\mathrm{H}+\mathrm{H}_{2} \rightarrow \mathrm{H}(2 \mathrm{~s}, 2 \mathrm{p})+\mathrm{H}_{2}:$ J.H. Birely and R.J. Mciteal, Phys. Rev. A 5 , $692(1972)$

$\mathrm{H}+\mathrm{H}_{2} \rightarrow \mathrm{H}(3 \mathrm{~s}, 3 \mathrm{p}, 3 \mathrm{~d})+\mathrm{H}_{2}:$ R.Y. Hughes, H.M. Peterish, and H. Kisner, Phys. Rev. A 5, 2103 (1972).

Notes:

See Note (23) at end of r:hapter.

\section{Accuracy:}

Systematic error $<50 \%$ for és, 2p state; systematic error < 20 for $3 s, 3 p, 3 d$ state. Random error < $10 \%$. 
A.2.5

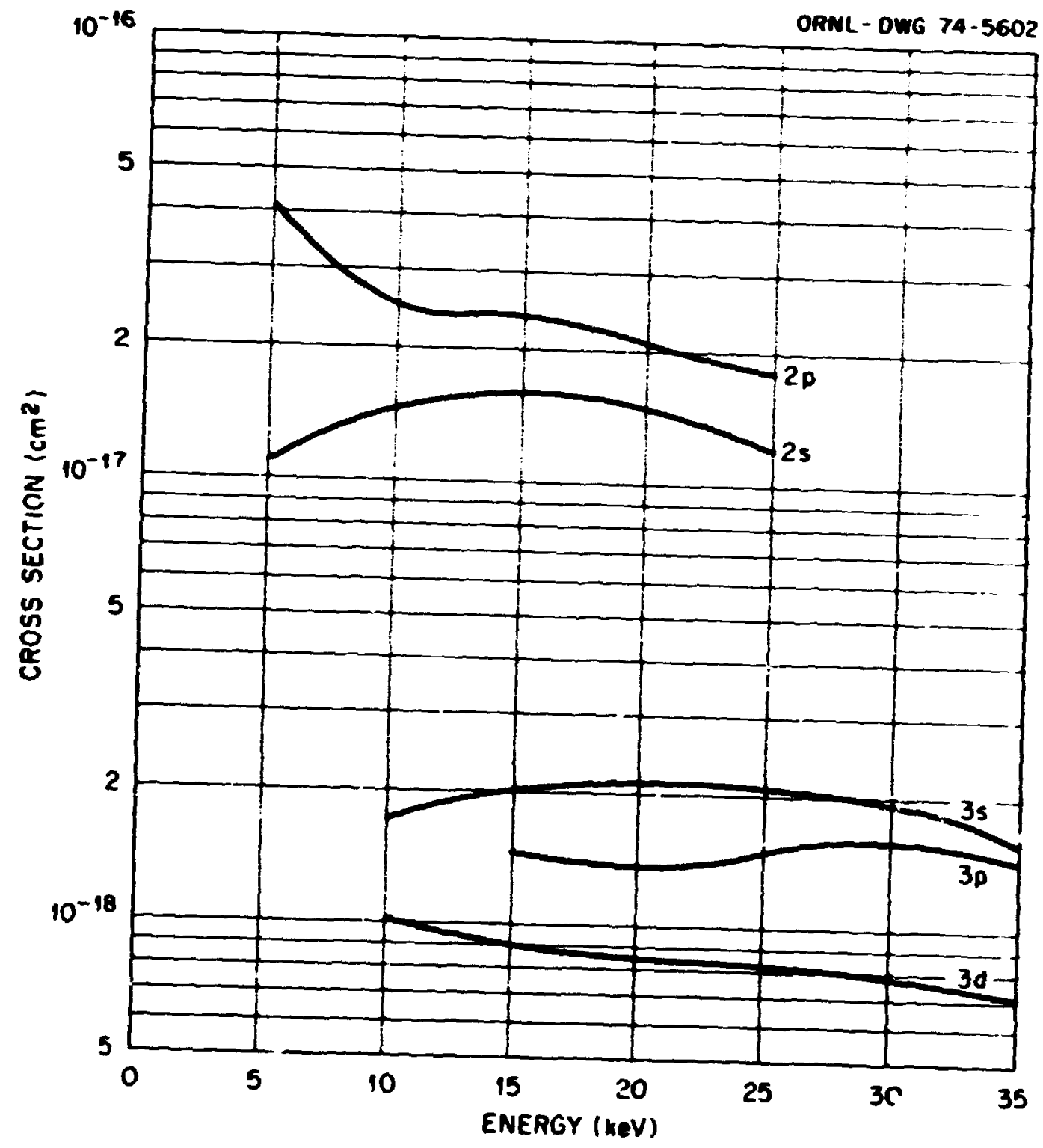




$$
\text { A.2.6 }
$$

Excitation Cross Sections for the Feactions

$$
H+H_{2}+H+H+H(2 s, 2 D)
$$

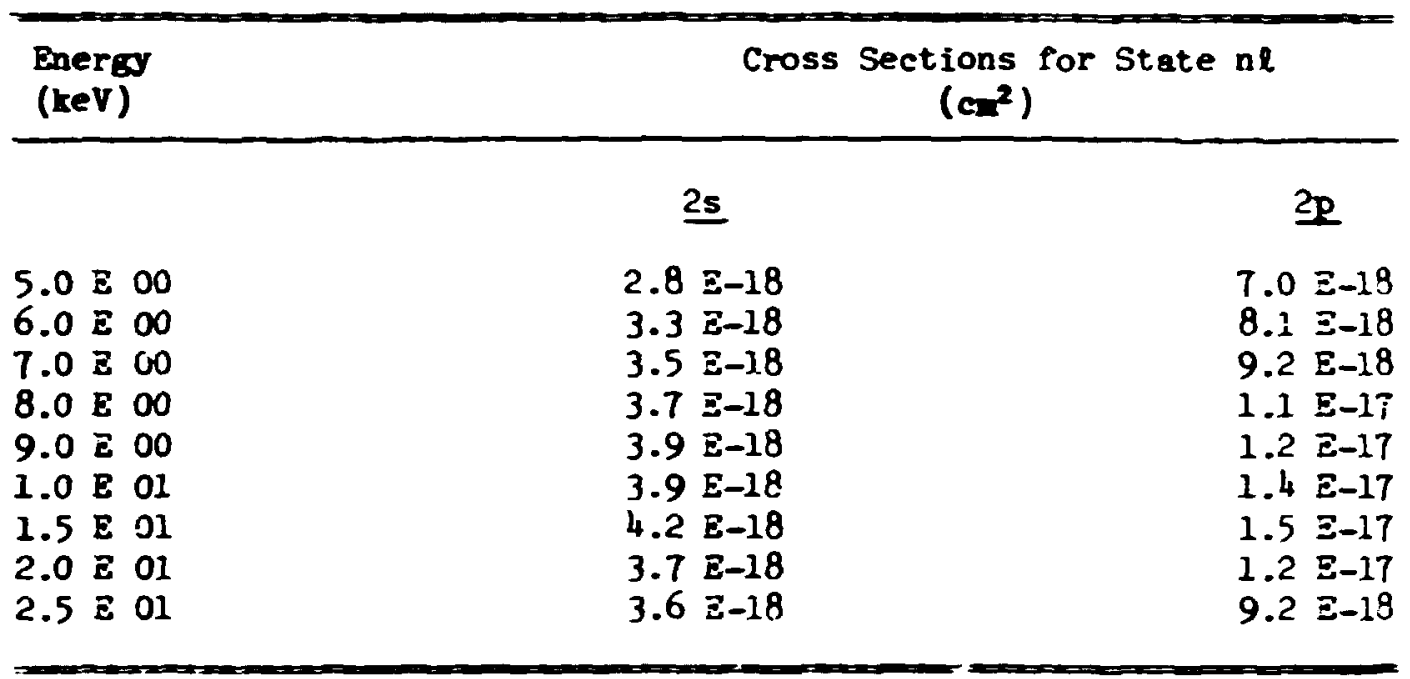

\section{Reference:}

J.H. Birely and R.J. Mcleal, Phys, Rev. A 5, 692 (1972).

Notes:

See Iote (24) at end of chapter.

Accuracy:

Systematic error $<50 \%$. Random error $<10 \%$. 
A.2.?

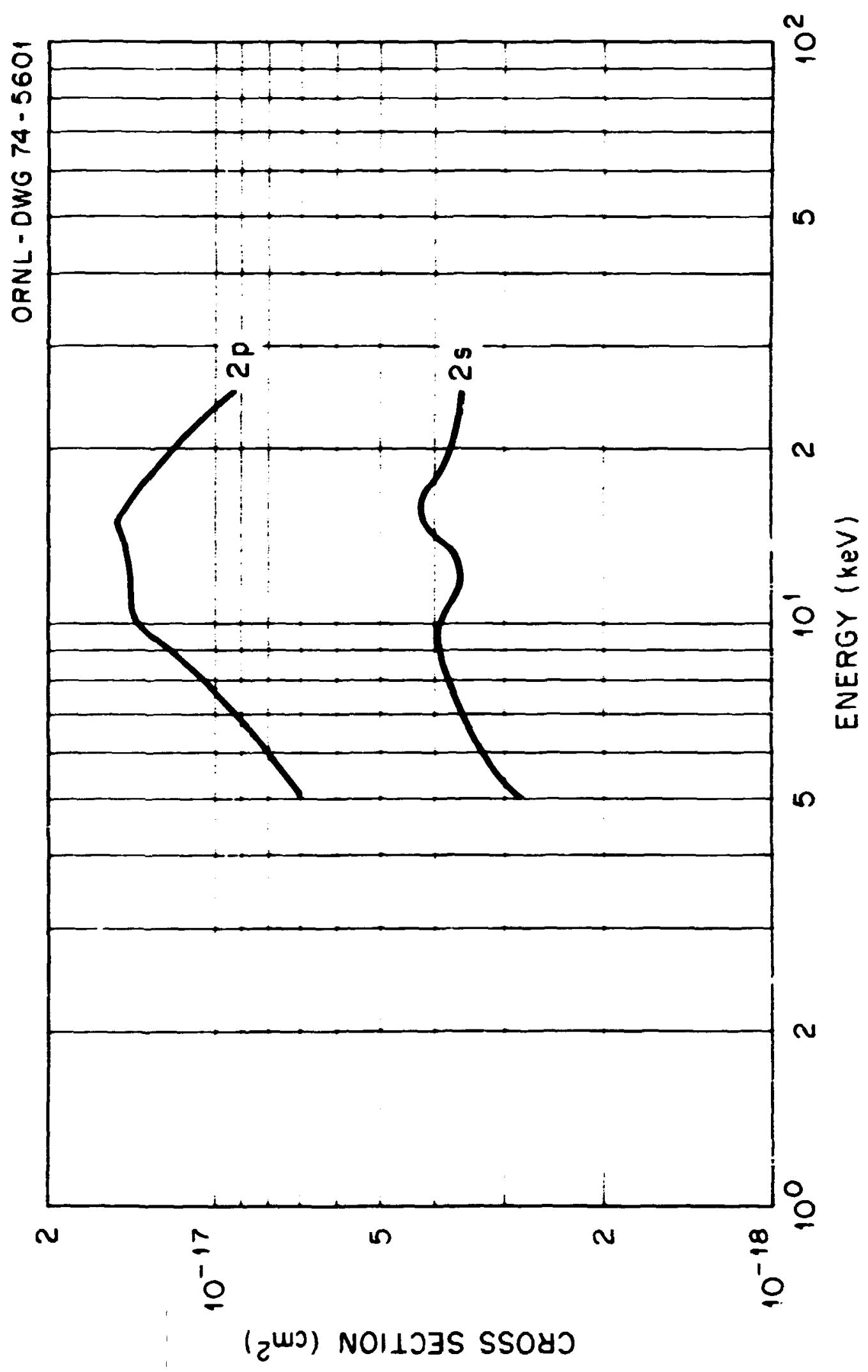


Excitation Cross Sections for the Reactions

$$
\mathrm{H}^{\mathrm{O}}+\mathrm{He}+\mathrm{H}^{0}+\mathrm{iHe}\left(4^{1} \mathrm{~S}, 4^{1} \mathrm{P}, 4^{3} \mathrm{~S}, 4^{3} \mathrm{P}\right)
$$

\begin{tabular}{|c|c|c|c|c|}
\hline \multirow[t]{2}{*}{$\begin{array}{l}\text { Buergor } \\
\text { (keV) }\end{array}$} & \multicolumn{4}{|c|}{ Cross Sections } \\
\hline & $4 \frac{1}{5}$ & LI $^{1}$ & $4^{3} s$ & $4^{3} \mathrm{P}$ \\
\hline $\begin{array}{lll}1.0 & \mathrm{E} & 01 \\
1.5 & \mathrm{E} & 01 \\
2.0 & \mathrm{E} & 01 \\
2.5 & \mathrm{E} & 01 \\
3.0 & \mathrm{E} & 01 \\
3.5 & \mathrm{E} & 01\end{array}$ & $\begin{array}{ll}1.30 & E-19 \\
1.63 & E-19 \\
1.85 & E-19 \\
1.96 & E-19 \\
1.82 & E-1.9 \\
1.58 & E-19\end{array}$ & $\begin{array}{ll}2.60 & \text { E-20 } \\
7.90 & E-20 \\
1.25 & E-19 \\
1.47 & E-19 \\
1.86 & E-19 \\
2.12 & \text { E-19 }\end{array}$ & 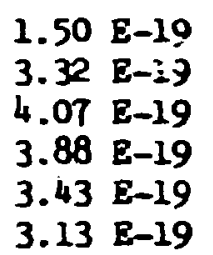 & $\begin{array}{ll}3.64 & E-19 \\
4.71 & E-19 \\
3.68 & E-19 \\
2.50 & E-19 \\
1.71 & E-19 \\
1.25 & E-19\end{array}$ \\
\hline
\end{tabular}

\section{References:}

J. Van Bck, P. J. de Heer, and J. Kistenaker, Physica 30, 1171 (1964), as revised by J. Van den Bos;(private comunication from F. J. de Ieer).

\section{Motes:}

See lotes (41) and (42) at end of chapter. 


\section{A.2.9}

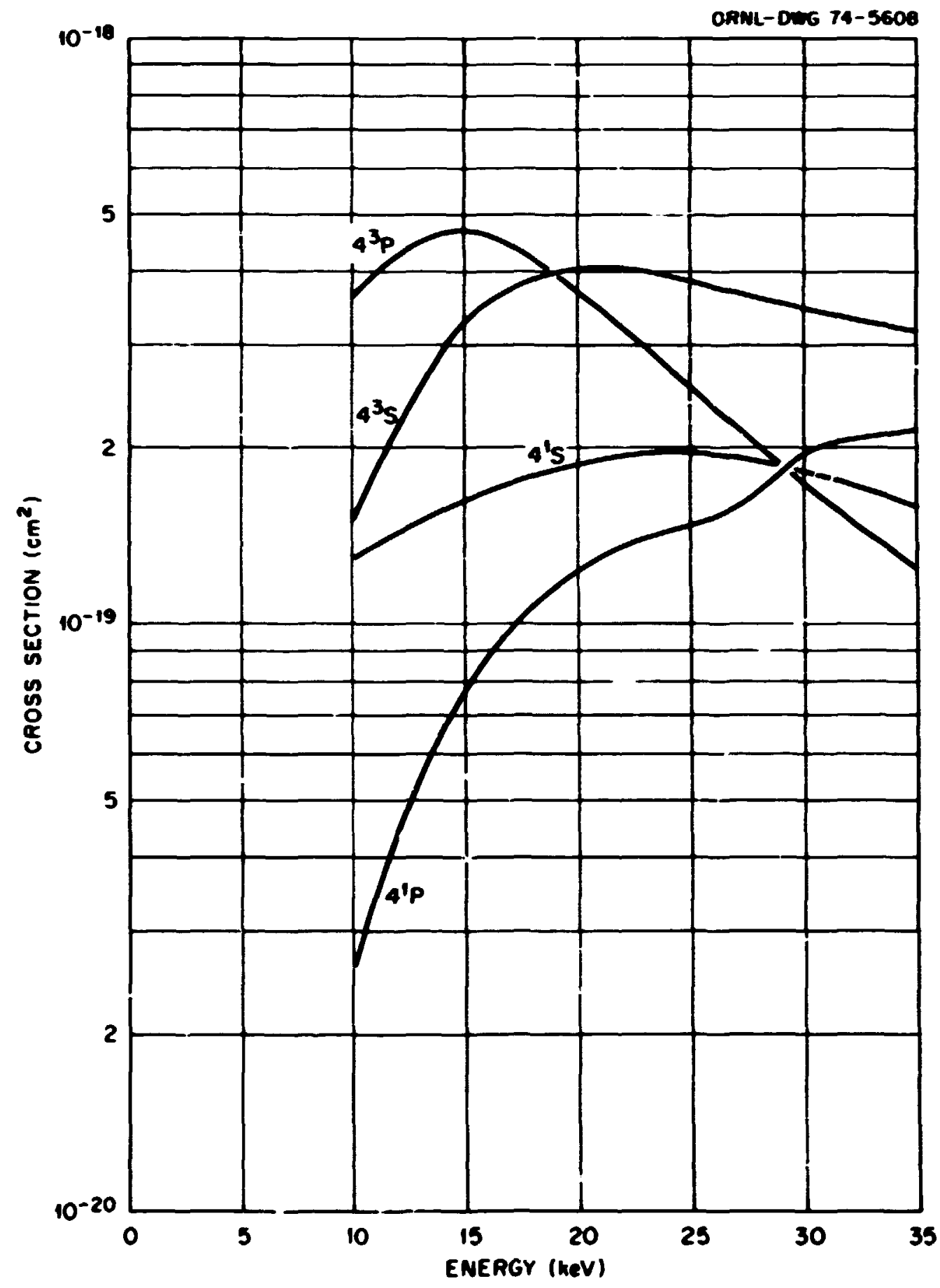


rcitation Cross Sections by Electron Capture for the React.

$$
\underline{u}^{+}+H \rightarrow H(2 s, 2 p)+H^{+}
$$

\begin{tabular}{|c|c|c|c|c|}
\hline \multirow[t]{3}{*}{$\begin{array}{l}\text { Enerey } \\
\text { (hev) }\end{array}$} & \multicolumn{4}{|c|}{$\begin{array}{l}\text { Cross Sections for State nl } \\
\left(\mathrm{cm}^{2}\right)\end{array}$} \\
\hline & \multicolumn{2}{|c|}{ Experimental } & \multicolumn{2}{|c|}{ Theoretical } \\
\hline & $\underline{2 s}$ & $2 p$ & $2 s$ & $2 p$ \\
\hline $\begin{array}{l}2.3 \mathrm{E} 00 \\
3.0 \mathrm{E} 00 \\
4.0 \mathrm{E} 00 \\
5.0 \mathrm{E} 00 \\
6.0 \mathrm{E} 00 \\
7.0 \mathrm{E} 00 \\
8.0 \mathrm{E} 00 \\
9.0 \mathrm{E} 00 \\
1.0 \mathrm{E} 01 \\
1.5 \mathrm{E} 01 \\
2.0 \mathrm{E} 01 \\
3.0 \mathrm{E} 01 \\
4.0 \mathrm{E} 01 \\
5.0 \mathrm{E} 01 \\
6.0 \mathrm{E} 01 \\
7.0 \mathrm{E} 01 \\
8.0 \mathrm{E} 01 \\
9.0 \mathrm{E} 01 \\
1.0 \mathrm{E} 02 \\
1.5 \mathrm{E} 02 \\
2.0 \mathrm{E} 02 \\
3.0 \mathrm{E} 02 \\
1.0 \mathrm{E} 03\end{array}$ & $\begin{array}{ll}4.6 & \mathrm{E}-19 \\
1.5 & \mathrm{E}-18 \\
3.6 & \mathrm{E}-18 \\
6.6 & \mathrm{E}-18 \\
1.0 & \mathrm{E}-17 \\
1.5 & \mathrm{E}-17 \\
\mathrm{\Xi} .9 & \mathrm{E}-17 \\
2.3 & \mathrm{E}-17 \\
3.2 & \mathrm{E}-17 \\
3.4 & \mathrm{E}-17 \\
2.8 & \mathrm{E}-17 \\
2.0 & \mathrm{E}-17 \\
1.3 & \mathrm{E}-17 \\
7.4 & \mathrm{E}-18 \\
4.2 & \mathrm{E}-18\end{array}$ & $\begin{array}{ll}2.8 & E-17 \\
2.9 & E-17 \\
3.3 & E-1, \\
3.4 & E-17 \\
3.3 & E-17 \\
3.3 & E-17 \\
3.2 & E-17 \\
3.1 & E-17 \\
3.0 & \Xi-17 \\
2.3 & E-17 \\
1.5 & E-17 \\
\text { S.J } & \Xi-18\end{array}$ & $\begin{array}{ll}3.2 & \mathrm{E}-17 \\
2.4 & \mathrm{E}-17 \\
2.6 & \mathrm{E}-17 \\
1.1 & \mathrm{E}-17 \\
7.6 & \mathrm{E}-18 \\
5.3 & \mathrm{E}-18 \\
3.8 & \mathrm{E}-18 \\
2.7 & \mathrm{E}-18 \\
5.8 & \mathrm{E}-19 \\
1.8 & \mathrm{E}-19 \\
3.2 & \mathrm{E}-20 \\
7.6 & \mathrm{E}-23\end{array}$ & 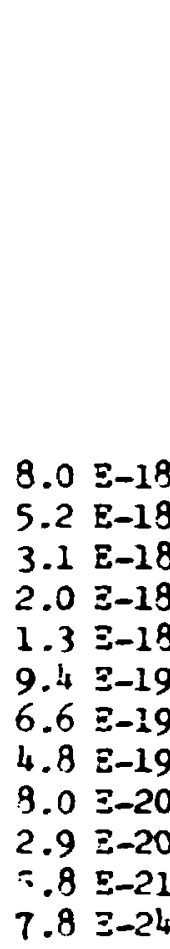 \\
\hline
\end{tabular}

References:

$\mathrm{H}^{+}+\mathrm{H} \rightarrow \mathrm{H}(2 \mathrm{~s})+\mathrm{H}^{+}$Exp.: T.J. Morgan, J. Geddes, and H.B. Gilbods, J. Phys. B 6, 2118 (1973); J.E. Bay field, Phys. Rev. 185, 105 (1969).

$\mathrm{H}^{+}+\mathrm{H} \rightarrow \mathrm{H}(2 \mathrm{p})+\mathrm{H}^{+}$Exp.: T.J. Morgan, J. Geddes, and H.B. Gilbod, J. Ptys. B K. 2118 (1973).

$\mathrm{H}^{+}+\mathrm{H} \rightarrow \mathrm{H}(2 \mathrm{~s}, 2 \mathrm{p})$ Theoretical: I.M. Cheshire, D.P. Gallaher, and A. Joanna Taylor, J. Phys. B 3, 813 (1970).

Motes:

See notes (1) and (2) at end of chapter.

Aicuracy:

Systenatic error < 50\%. Randon error < $10 \%$. 


\section{A.2.11}

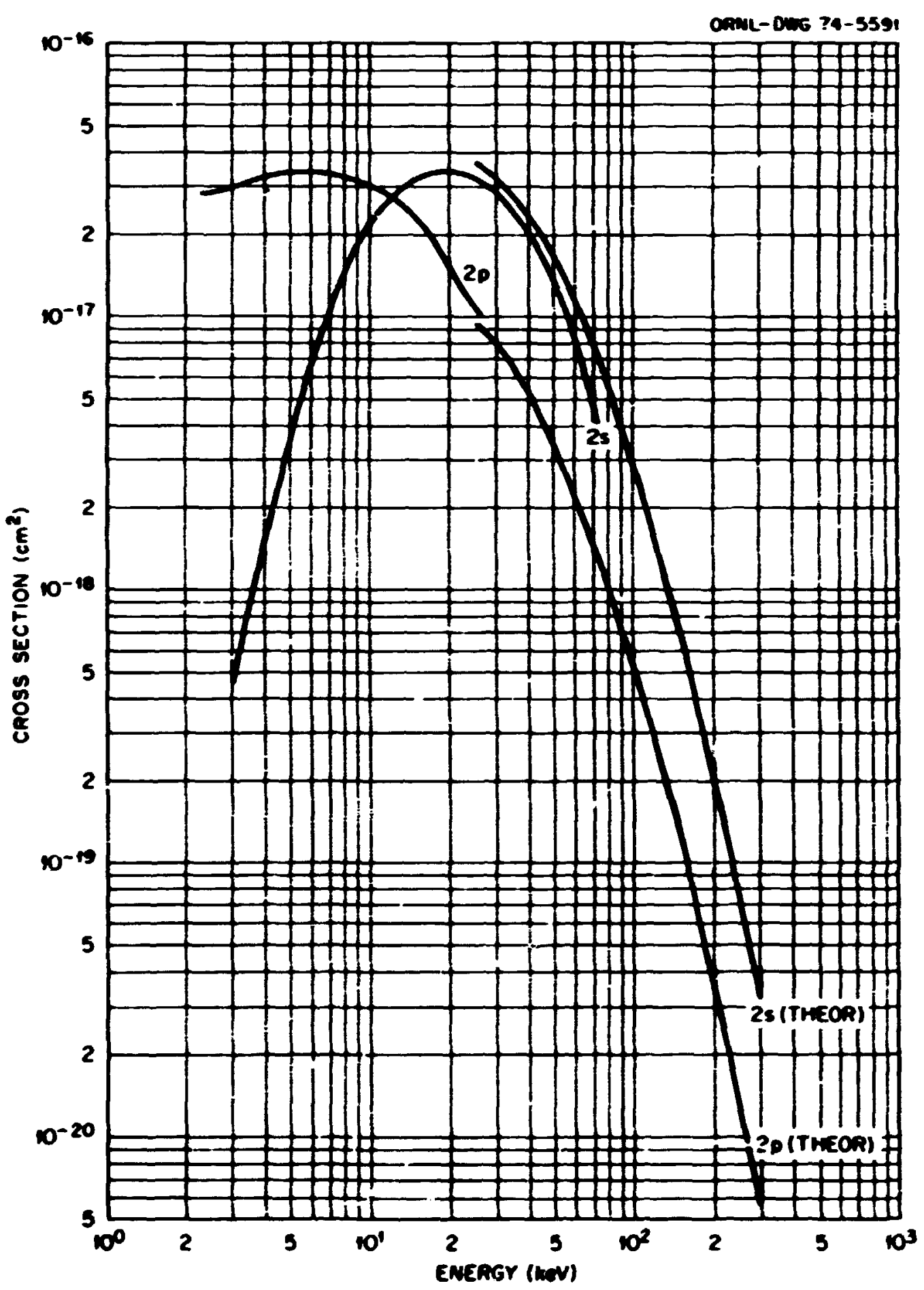


Excitation Cross Sections for the Reactions

$$
\underline{u}^{+}+\mathrm{H} \rightarrow \mathrm{H}^{+}-\mathrm{H}(2 \mathbf{s}, 2 \mathbf{p})
$$

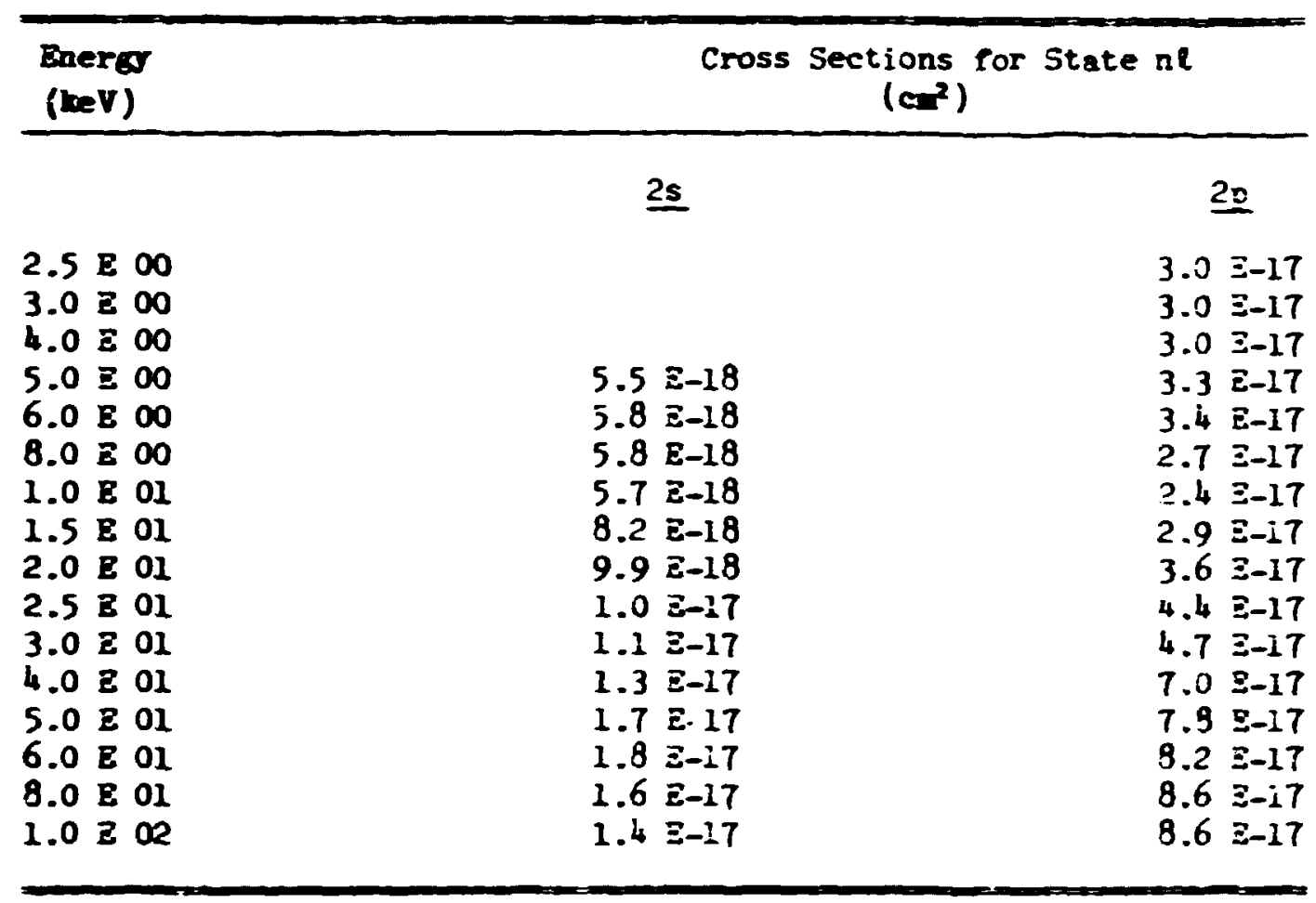

\section{References:}

Experimental (2 - $26 \mathrm{keV})$ : T.J. Morgan, J. Geddes, and 4.B. Gilbody, J. Phys. B 6, 2118 (1973).

Theoretical (25 - $100 \mathrm{keV})$ : I.M. Cheshire, D.P. Gallaher, and A.J. Taylor, J. Phys. B 3, 813 (1970).

Xotes:

See Jotes (3) and (4) at end of chafter.

Accurect:

Experimentel data: Systeastic error $<50 \%$. Random error $<10 \%$. 


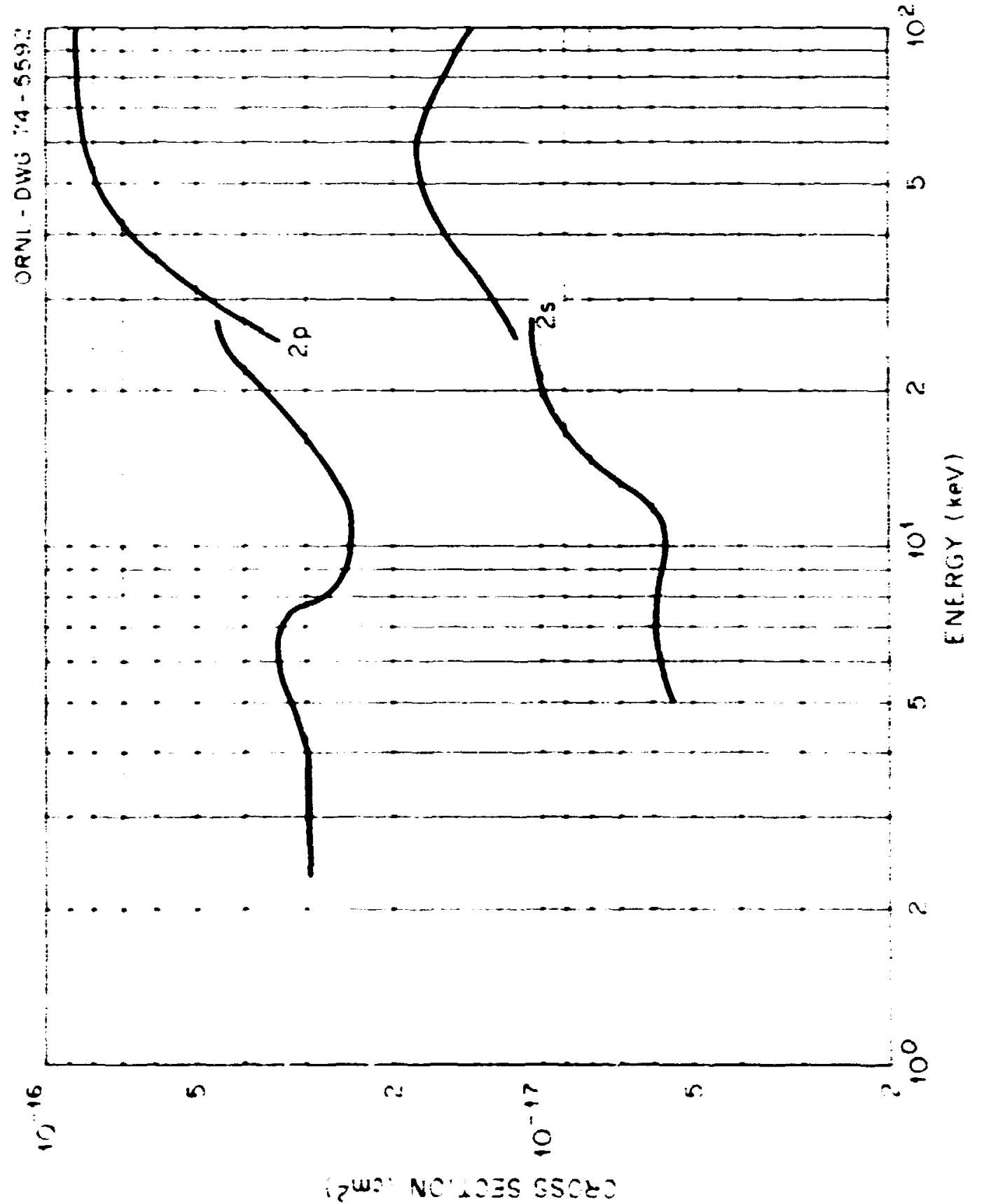




\section{A.2.14}

Cross Sections for Excitaticn by Electron Capture for the Reactions

$$
\mathrm{H}^{+}+\mathrm{H}_{2}+\mathrm{i}(2 \mathrm{~s}, 3 \mathrm{~s}, 4 \mathrm{~s})+\mathrm{z}_{2}^{+}
$$

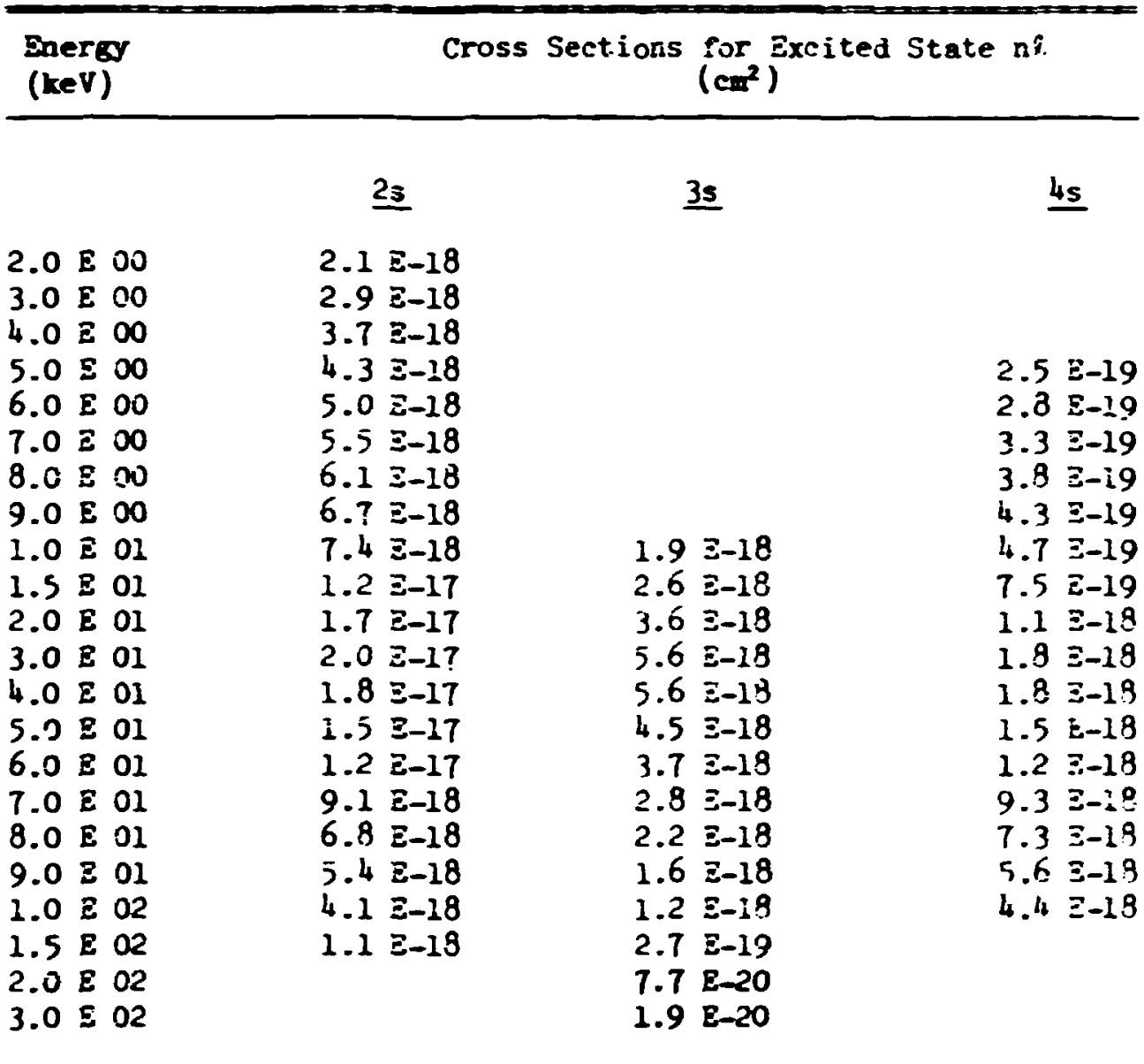

\section{References:}

$\mathrm{H}^{+}+\mathrm{H}_{2} \rightarrow \mathrm{H}(2 \mathrm{~s})+\mathrm{H}_{2}^{+}:$R.H. Hughes, Z.D. Stokes, Song-Sih Choe, and T.J. King, Phys. Rev. A 4, 1453 (19i1); G. Pyding, A.3. dittkower, and H.B. Gilbody, Proc. Phys. Soc., London 89,547 (1966); i. Baytield, Phys. Rev. 192, 115 (1969).

$\mathrm{H}^{+}+\mathrm{H}_{2} \rightarrow \mathrm{H}\left(3 \mathrm{~s}:+\mathrm{H}_{2}^{+}:\right.$R.H. Hughes, C.A. Stigers, B.M. Doughty, E.D. stokes, Phys. Rev. A I, 2424 (1970); J.C. Pord and E.y. Thomns, Phys. Rev. A 1,1701 (2972).

$\mathrm{H}^{+}+\mathrm{H}_{2} \rightarrow \mathrm{H}\left(\mathrm{H}_{\mathrm{s}}\right)+\mathrm{H}_{2}^{+}:$R.H. Hughes, H.R. Dawson, ant 3.Y. Doughty, Phys. Rer. 164, 166 (1967).

\section{Motes:}

See Notes (6) and (7) at and of chapter.

\section{Accurecy:}

Systenatic error < 50\%. Random error < i5\%. 
6.2.15

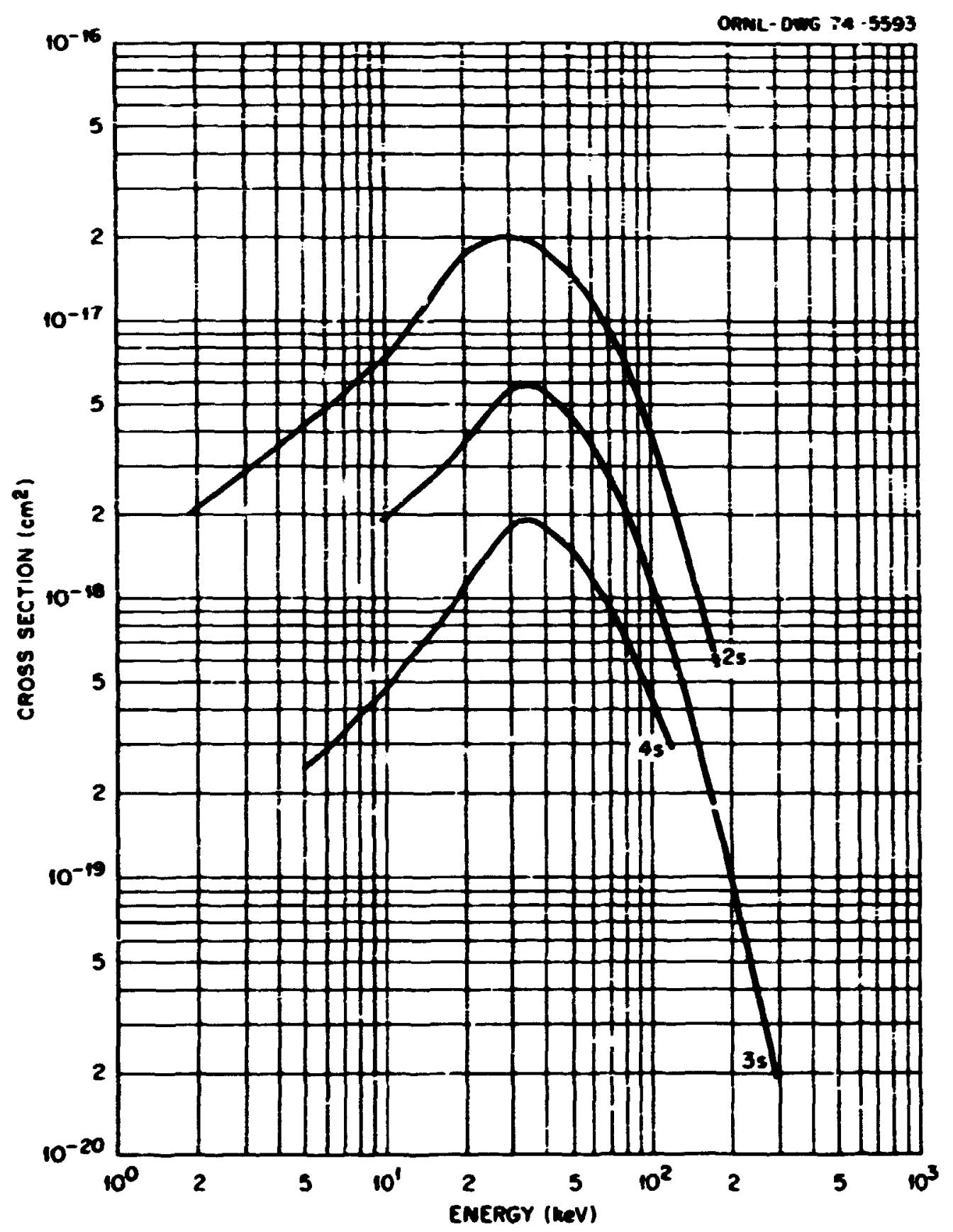




\begin{tabular}{|c|c|c|c|}
\hline \multirow[t]{2}{*}{$\begin{array}{l}\text { Energy } \\
\text { (keV) }\end{array}$} & Cross Sections & 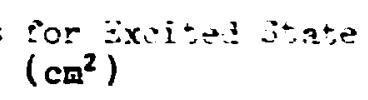 & $\mathrm{ni}$ \\
\hline & $\underline{2 p}$ & $3 n$ & 31 \\
\hline $4.0=00$ & $2.3 \overrightarrow{\mathrm{E}}-\mathrm{i} 7$ & & \\
\hline $5.0 \equiv 20$ & $2.0 \quad \Xi-17$ & & \\
\hline $5.6 \geq 0$ & $2.4 \geqq-17$ & & \\
\hline$i .0 \equiv 0$ & $2.3 \Xi-i \vec{i}$ & & \\
\hline נט: & $2.3 \Xi-17$ & & \\
\hline $9.0 \equiv 00$ & $2.5 \equiv-1.7$ & & \\
\hline $1.0 \equiv 0$ & $2.7 \quad 3-17$ & $1.2=38$ & $1.9 \equiv-1^{2}$ \\
\hline $1.5 \mathrm{E} u 1$ & $2.9 \bar{ت}-17$ & $2.1 \equiv-18$ & $1.3 \equiv-19$ \\
\hline $2.0 \equiv 01$ & $2.5 \ddot{E}-17$ & $3.2=-18$ & $9.7 \equiv-19$ \\
\hline $3.0 \Xi 0_{1}$ & $1.5 \mathrm{E}-27$ & $1.7 \equiv-18$ & $0.5 \quad 3-19$ \\
\hline $4.0 \Xi 01$ & $9.0 \Sigma-19$ & $9.5 \Xi-19$ & $4.5 \quad \vec{z}-19$ \\
\hline $5.0 \equiv 01$ & $6.0 \because-8$ & $0.0 \equiv-19$ & $3.02-1 n$ \\
\hline $6.0 \mathrm{E} 01$ & $4.5 \div-28$ & $3.5 \mathrm{E}-29$ & 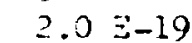 \\
\hline $7.0 \equiv 01$ & $3.9 \Xi-16$ & $2.5=-19$ & $1.3 \equiv-19$ \\
\hline $8.0 \equiv 01$ & $2.7 \equiv-18$ & $1.9:-19$ & $9.9 \equiv-20$ \\
\hline $9.0 \equiv 01$ & $2.2 \bar{E}-1.8$ & $1.6 \equiv-10$ & $7.2 \equiv-20$ \\
\hline $1.0 \equiv 02$ & $1.9 \Xi-19$ & $1.32-19$ & $5.0 \quad 3-20$ \\
\hline $1.2 \mathrm{\Xi} 02$ & $1.7 \mathrm{E}-18$ & & \\
\hline
\end{tabular}

\section{References:}

$\mathrm{H}^{+}+\mathrm{H}_{2} \rightarrow \mathrm{H}(2 \mathrm{p})+\mathrm{H}_{2}^{+}: \mathrm{F} . \mathrm{H}$. Fughes, $\mathrm{T} . \mathrm{T}$. King, and sonp-sik Choe, Phys. Riv. A 5 , 644 (1972); J.H. Birely and R.J. Mcleol, Phys. Rev. A 2, 692 (1972); Z.P. Andreev, V.A. Ankudinov, and S.V. Bnbashev, 5i th International Conference on the Physics of Electronic \& Atoric Collisions: Abstract of Papers, p.309, Fublishing Slouse Vaukz, Ieningrad, Us.3R (1967).

$4^{+}+\mathrm{H}_{2} \rightarrow \mathrm{I}(3 \mathrm{p})+\mathrm{H}_{2}^{+}:$E.F. Andreev, V.A. Ankudinov, and $3 . V$. Bobashev, Fiftin Internatiwal Conference on the Physics of Electronic \& Atomic Collisions: Abstract of Papers, p.309, Publishing Hounie Vauka, Leningrad, USSR (1967); R.H. Haghes, C.A. Stigers, B.M. Dought.y, and Z.D. 3to.ies, Phys. Rev. A 1,1424 (1970).

$\mathrm{H}^{+}+\mathrm{H}_{2} \rightarrow \mathrm{H}(3 \mathrm{~d})+\mathrm{H}_{2}^{+}:$R.H. Hughes, C.A. Stigers, 3.4. Doughty, and F.D. 3tokes, Phys. Rev. A 1,1424 (1970).

\section{Notes:}

See Note (j) at end of chapter.

Accurac; :

iystematic error $<50$, Random error $<15, \overrightarrow{0}$ 
A.?.17

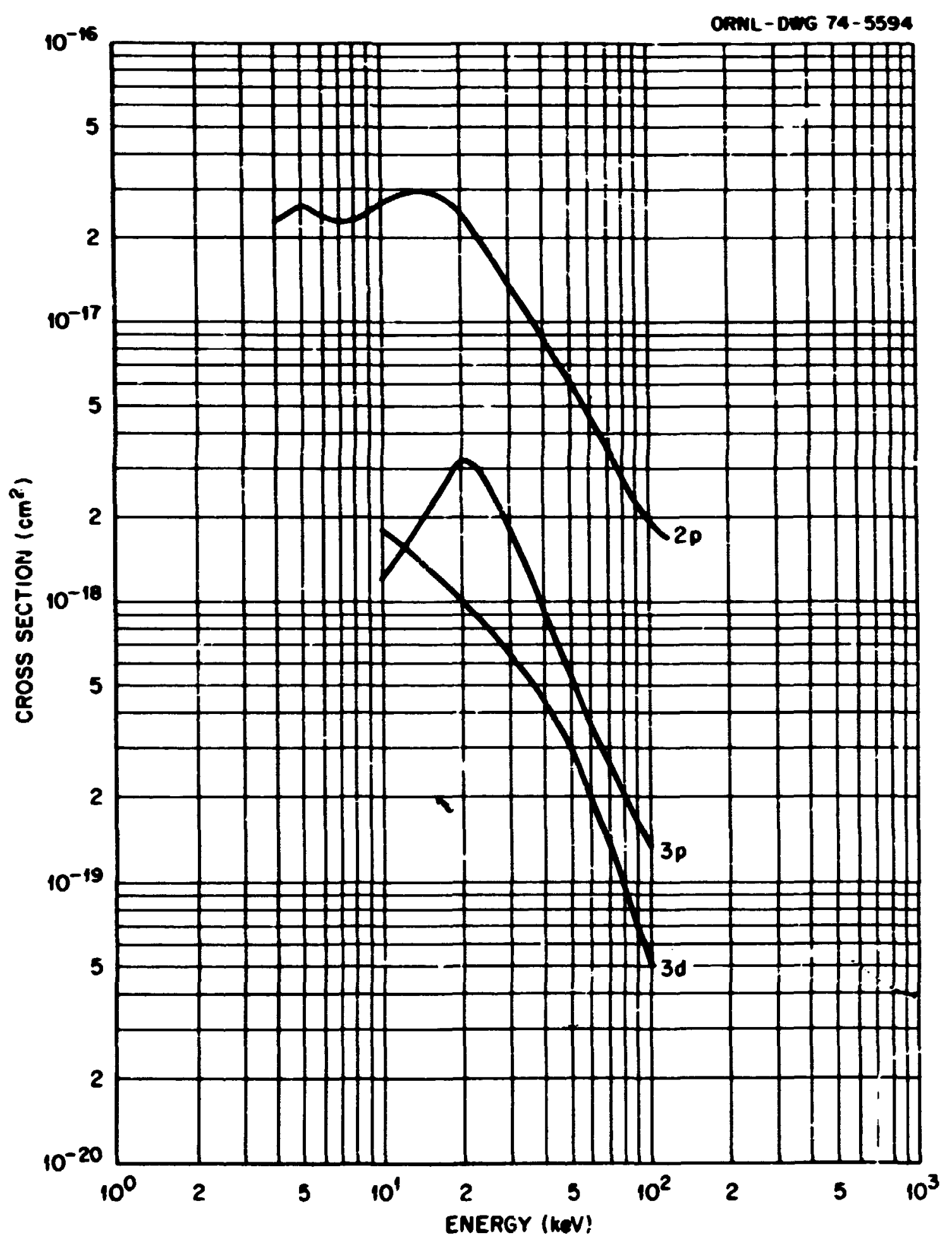




$$
\text { A.2.18 }
$$

Excitation Cross Sections for the Renctions

$$
\mathrm{H}^{+}+\mathrm{H}_{2}+\mathrm{H}^{+}+\mathrm{H}+\mathrm{H}(25,2 \mathrm{~F}, 30)
$$

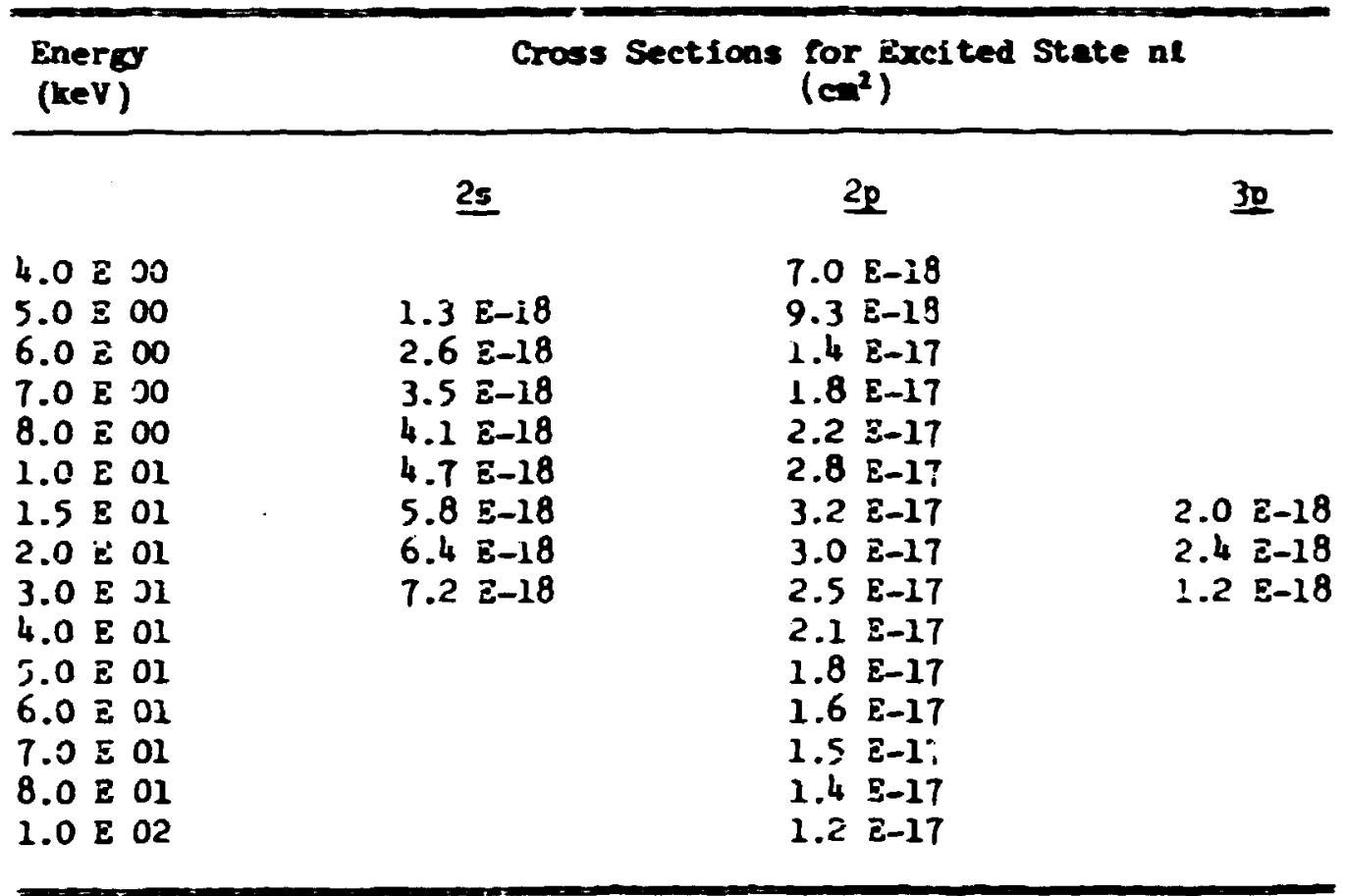

\section{Rererences:}

$\mathrm{H}^{+}+\mathrm{H}_{2} \rightarrow \mathrm{H}^{+}+\mathrm{H}+\mathrm{H}(2 \mathrm{~s}):$ J.H. Birely and R.J. Melleal, Phys. Rev. A. , 692 (1972); E.F. Andreev, V.A. Ankudinov, and S.V. Bobasher, Fi fth

International Conference on the Physics of Electronic Atomic Collisions: Abstract of Papers, p.30y, Publishing House Nauka, Leningred, USSR (1967).

$\mathrm{H}^{+}+\mathrm{H}_{2} \rightarrow \mathrm{H}^{+}+\mathrm{H}+\mathrm{H}(2 \mathrm{p}):$ J.H. Birely, R.J. Mcklesl, Phys. Rev. A S, 692 (1972); R.H. Hughes, T.J. King, and Song-Sik Choe, Phys. Rev. A $\underline{5}$, $644(1972)$.

$\mathrm{H}^{+}+\mathrm{H}_{2} \rightarrow \mathrm{H}^{+}+\mathrm{H}+\mathrm{H}(3 \mathrm{P}):$ E.P. Andreev, V.A. Ankudinov, and $\mathrm{J} . \mathrm{V}$. Bobasher, Fifth International Conference on the Physics of EI satronic \& Atomic Collisions: Abstract of Papers, p.309, Publishing House Iauka, Leningrad, USSR (1967).

\section{utes:}

See lotes (8), (9), and (10) at end of chapter.

\section{Accirgey:}

Sysiematic error < 50\% for $H(28)$ and $H(20)$; systematic error < $20 \%$ for $H(3 p)$. Randoz error $<10 \%$. 


\section{A.2.19}

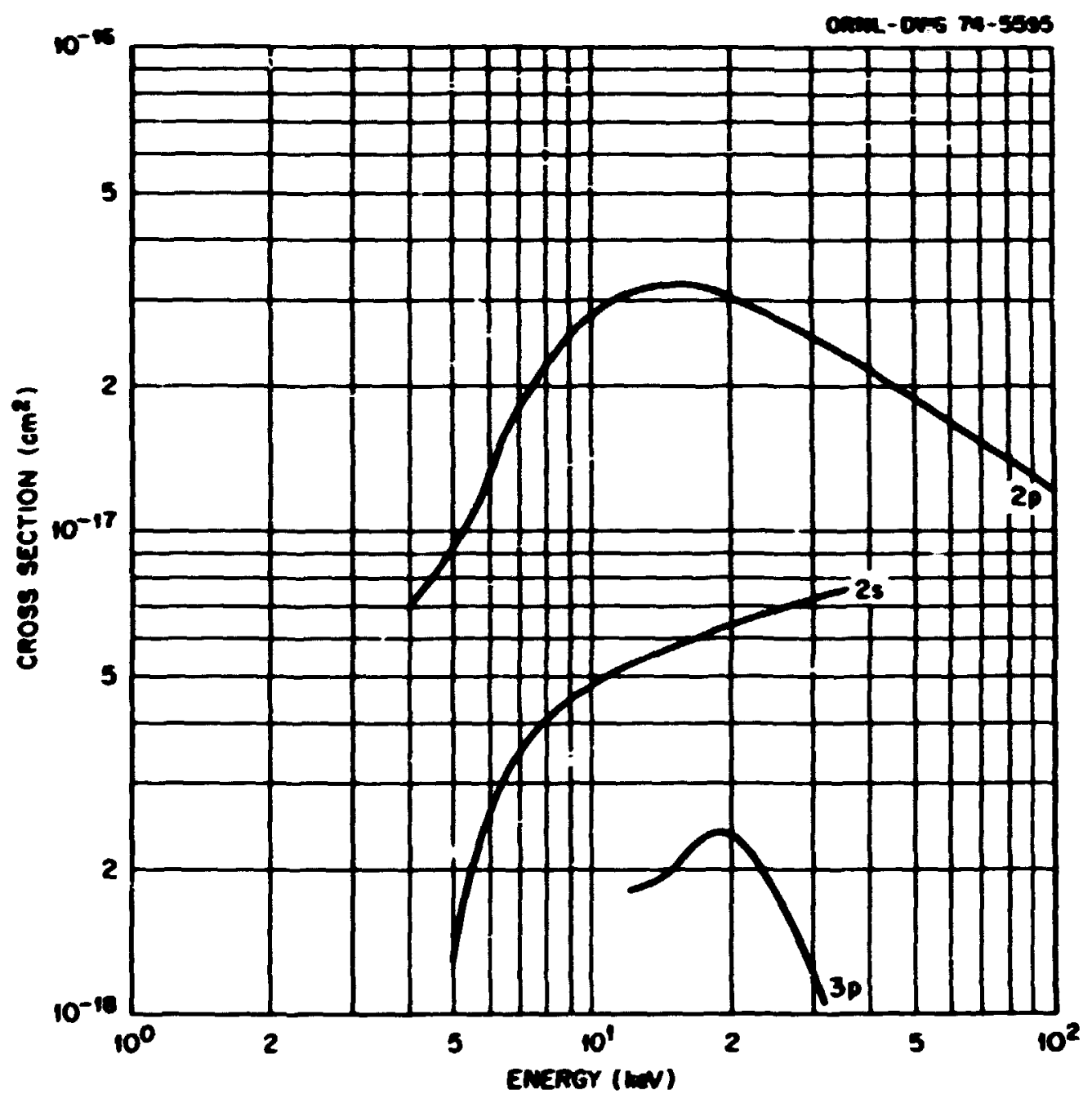




$$
\text { A. } 2.20
$$

Cross jections for jeission : Balaer-Aiona Ralintis: Our the Reactions

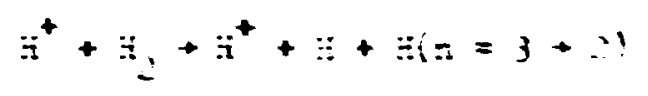

$\begin{array}{ll}\begin{array}{l}\text { Snergy } \\ \text { (keV) }\end{array} & \text { Jaission } \\ \left(\mathrm{cm}^{2}\right)\end{array}$

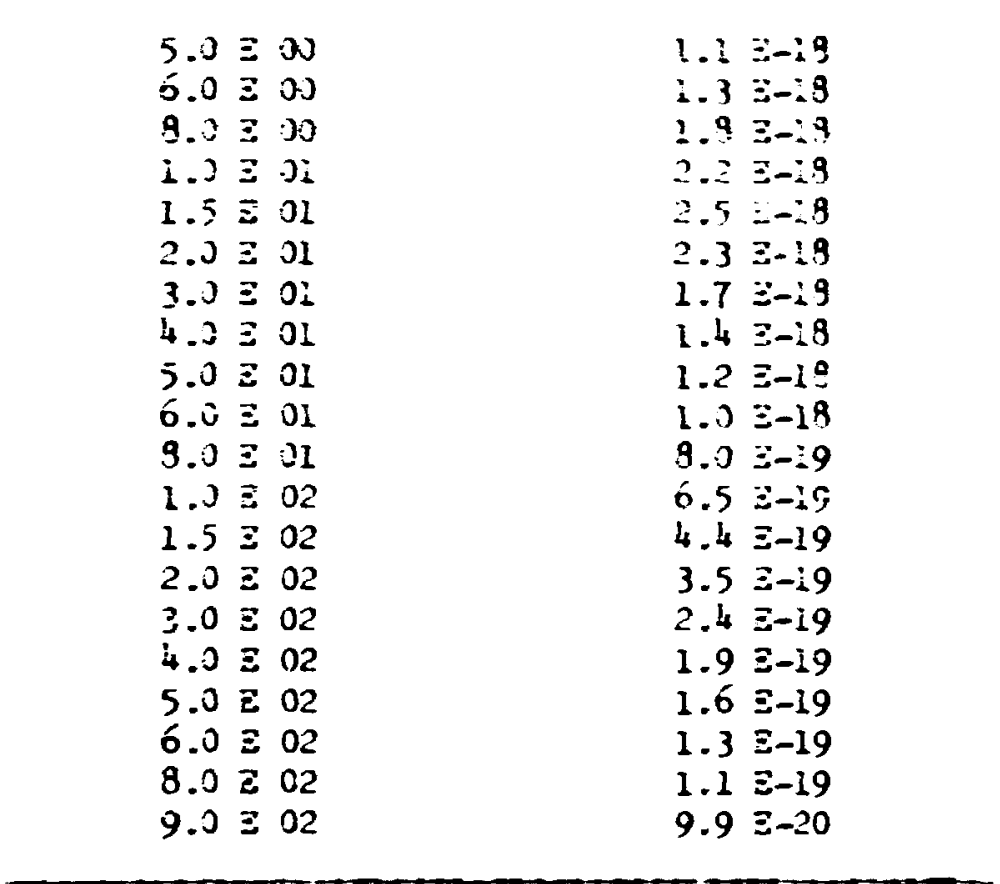

\section{References:}

J.L. Edvards, and $\Xi . W$. Thomas, Phys. Rev. 165,16 (1968); R.太. Enghes, S. Lin, and L.L. Hatfield, Phys. Rev. 130, $23: 8$ (1963) (normalized to Edwards et al.).

Accuracy:

Systematic error $<20 ;$. Random error $<10:$. 


\section{A.2.22}

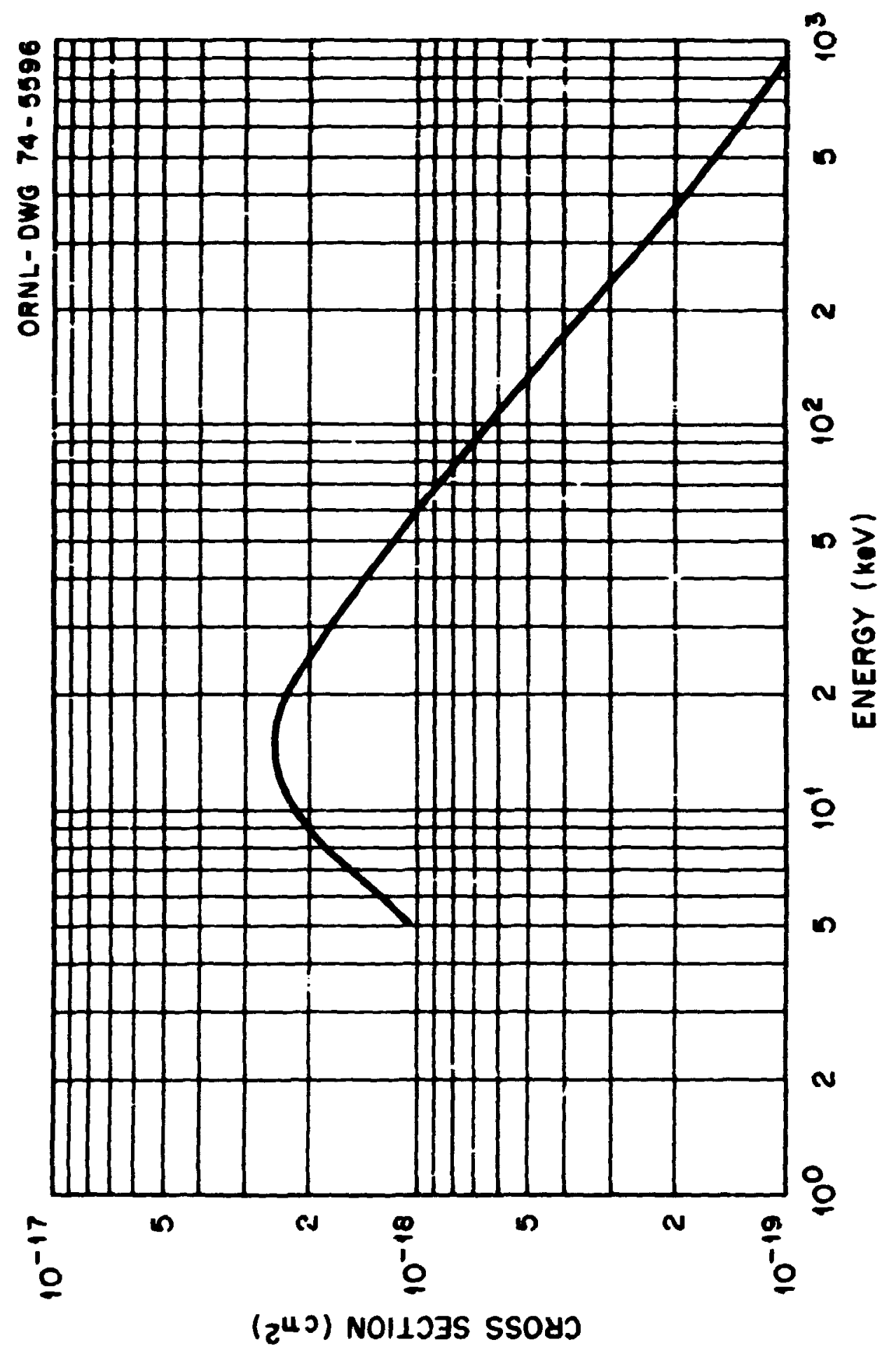




\section{A.2.22}

Cross Sections for anission of tine $1606 \Omega$ and $4190 R$

$\mathrm{H}_{2}$ Bands by $\mathrm{H}^{*}$ Impace

\begin{tabular}{|c|c|c|}
\hline \multirow[t]{2}{*}{$\begin{array}{l}\text { Deero } \\
\text { (heV) }\end{array}$} & Znission & $\begin{array}{l}\text { Cross Section } \\
\text { (cal) }\end{array}$ \\
\hline & 1606 R Rend & 41828 gend \\
\hline $\begin{array}{l}2.0 \mathrm{E} 01 \\
3.0 \mathrm{E} 01 \\
4.0 \mathrm{E} 01 \\
5.0 \mathrm{E} 01 \\
6.0 \mathrm{E} 01 \\
8.0 \mathrm{E} 01 \\
1.0 \mathrm{E} 02 \\
1.5 \mathrm{E} 02 \\
2.0 \mathrm{E} 02 \\
3.0 \mathrm{E} 02 \\
4.0 \mathrm{E} 02 \\
5.0 \mathrm{E} 02 \\
6.0 \mathrm{E} 02 \\
8.0 \mathrm{E} 02 \\
9.0 \mathrm{E} 02\end{array}$ & $\begin{array}{ll}3.3 & \mathrm{E}-18 \\
4.5 & \mathrm{E}-18 \\
5.0 & \mathrm{E}-18 \\
5.2 & \mathrm{E}-18 \\
5.2 & \mathrm{E}-18 \\
5.0 & \mathrm{E}-18 \\
4.7 & \mathrm{E}-18\end{array}$ & $\begin{array}{ll}8.0 & \mathrm{E}-21 \\
6.3 & \mathrm{E}-2 \mathrm{I} \\
1.3 & \mathrm{~g}-21 \\
3.2 & \mathrm{E}-21 \\
2.5 & \mathrm{E}-21 \\
2.1 & \mathrm{E}-21 \\
1.6 & \mathrm{~g}-21 \\
1.4 & \mathrm{E}-21\end{array}$ \\
\hline
\end{tabular}

References:

1606 R Band: D.A. Dahlbers, D.K. Anderson, and I.E. Dayton, Phys. Rev. 170, $12 T$ (1968).

4180 R Band: J.L. Zdwards, and E.H. Thome, Phyz. Rev. 165, 16 (1968).

Dotes:

See Jotes (11) and (12) at end of chapter.

Accuracy:

Systematic error < $50 \%$ for $1606 R$ bend; systematic error < 20 for $4180 \AA$ band. Randow error $<10 \%$. 


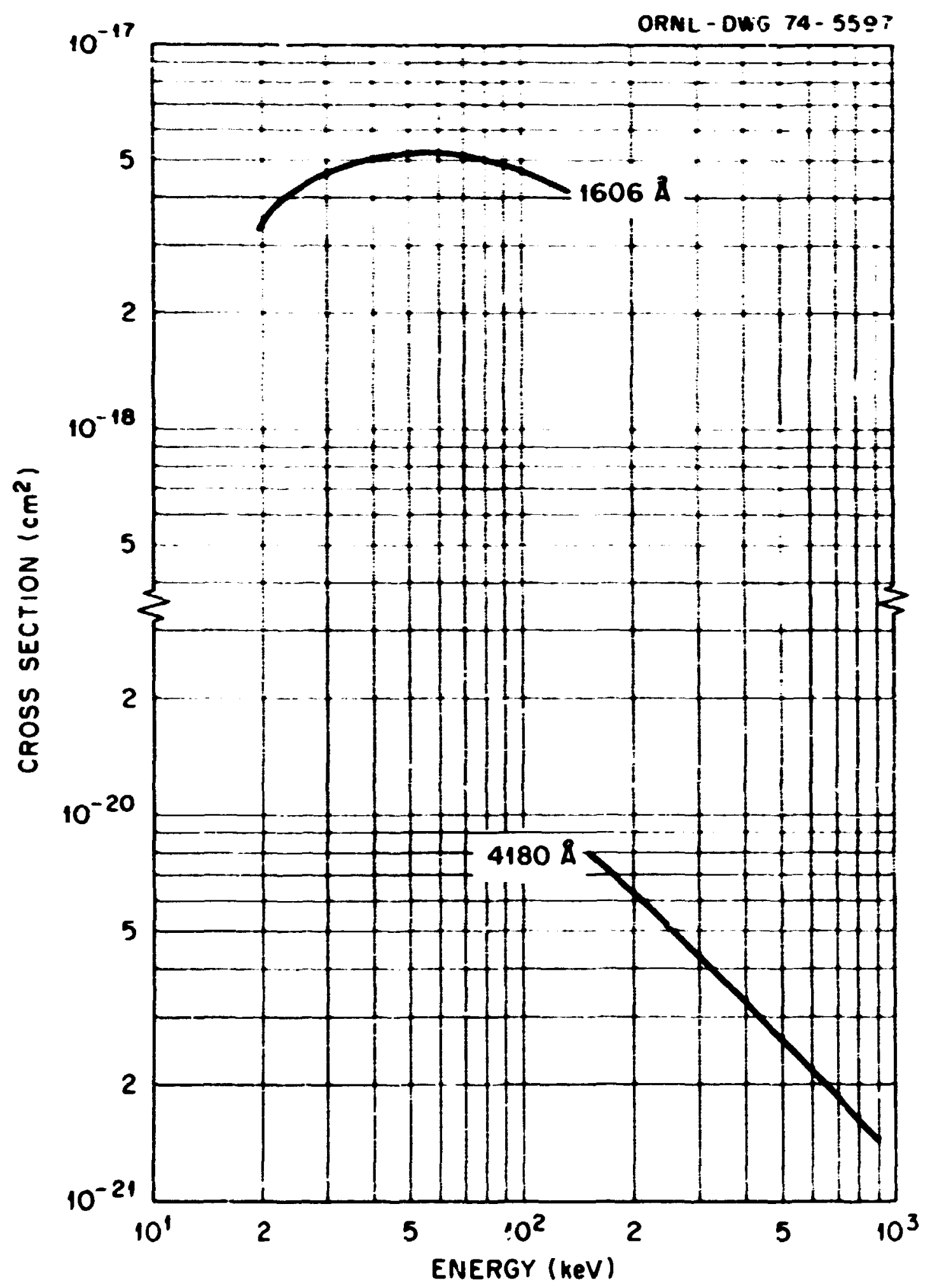




\section{2 .25}

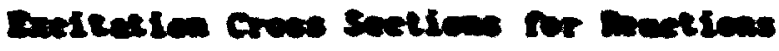

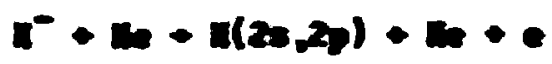

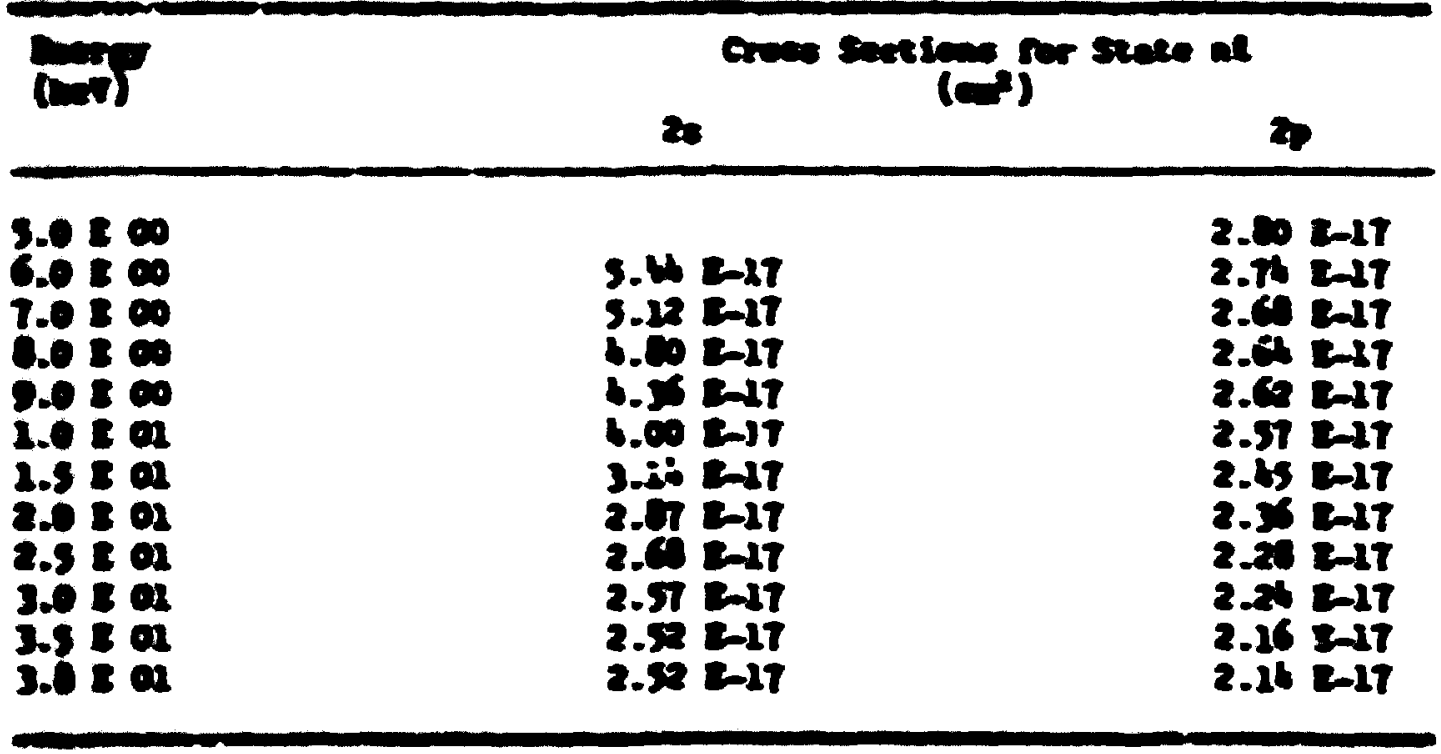

$1+1.28$

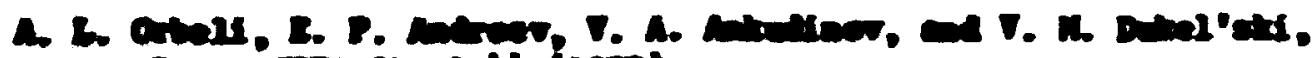

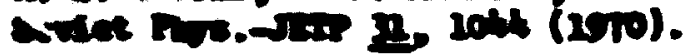

aring

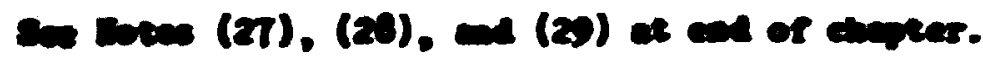

2.1.1.:

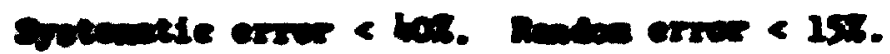




\section{a.2.28}

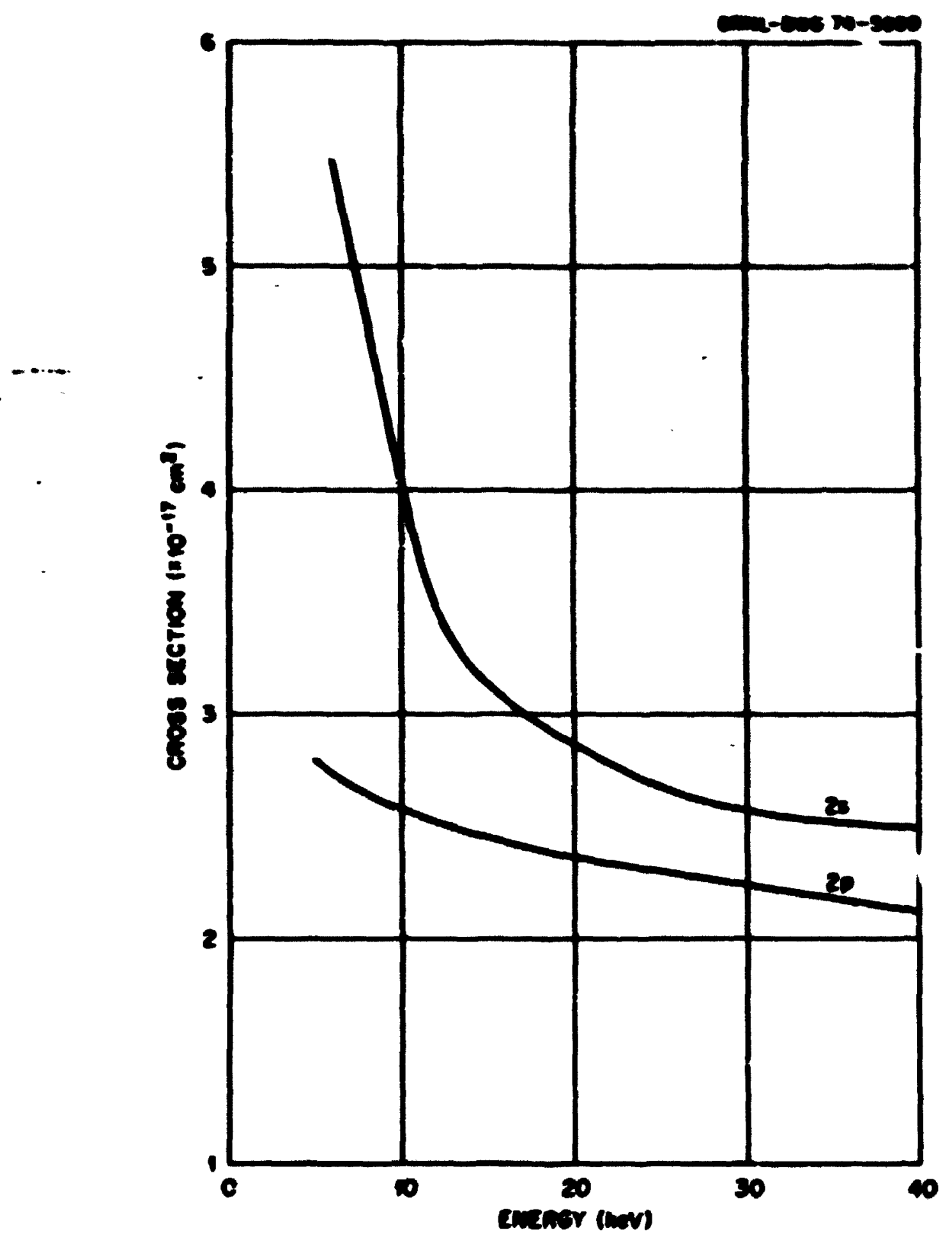


A.2.26

Breitatica Cross Sections for the Denctions

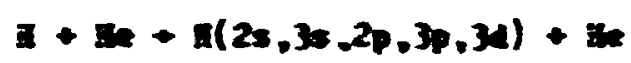

\begin{tabular}{|c|c|c|c|}
\hline & 35 & 32 & 31 \\
\hline \multirow[t]{2}{*}{ 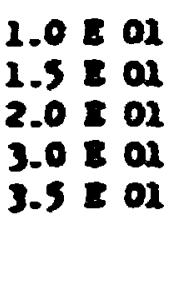 } & $\begin{array}{l}1.3 \leq-18 \\
1.6 \geq-18 \\
9.2 \geq-19 \\
7.8 \geq-19 \\
8.2 \geq-19\end{array}$ & $\begin{array}{ll}1.0 & 8-18 \\
9.7 & L-19 \\
1.8 & E-19 \\
8.0 & L-19\end{array}$ & 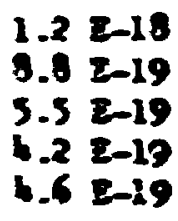 \\
\hline & 28 & 20 & \\
\hline 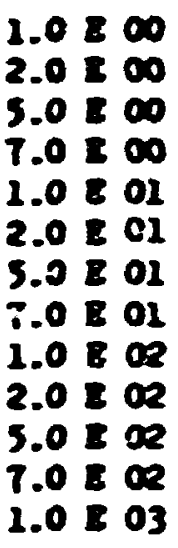 & 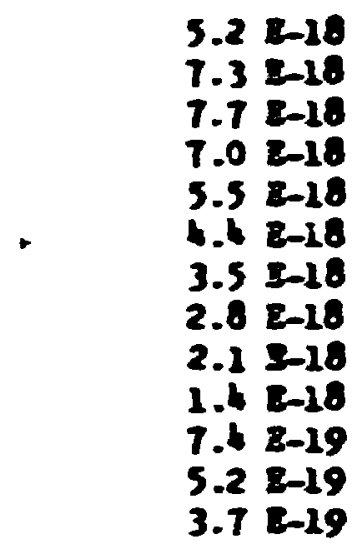 & $\begin{array}{ll}5.4 & E-17 \\
4.6 & E-17 \\
3.3 & \varepsilon-17 \\
2.6 & \varepsilon-17 \\
2.0 & E-17 \\
1.0 & E-17 \\
4.8 & E-18 \\
4.3 & \varepsilon-18 \\
4.3 & E-18 \\
3.2 & E-18 \\
2.1 & 8-18 \\
1.7 & E-18 \\
1.3 & 8-18\end{array}$ & \\
\hline
\end{tabular}

\section{Entereaces:}

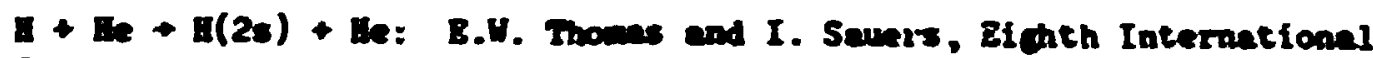
Confertace on the Puysies of Electronic atcoile Collisions: Abstract of Papers, P.166, Deogrede Instituie of Pussics Publishing Co. Beogred (1973). R.A. Buthes and Soas-sik Choe Phrs. Mev. A 5. 1758 (1972). A. Lev, Pave. Nev. 185, 7 (1969).

A + He - M(30,3p,34) + Be: R.K. Huches, H.M. Peterish, ad H. Kisaer, Puss. Rev. A 2, 2103 (1972).

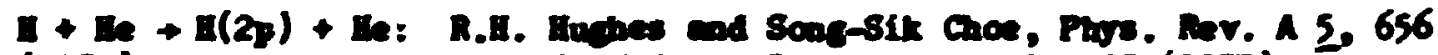
(1972). J.H. Brely ad R.J. Nefieal, Murs. Nev. A E, 257 (1972).

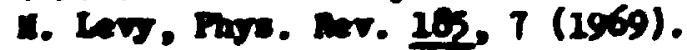

Dote:

sea lotes (25) an (26) at and of chepter.

Aceures:

8jatenatic error < 50\%. Randon error $<10 \%$. 


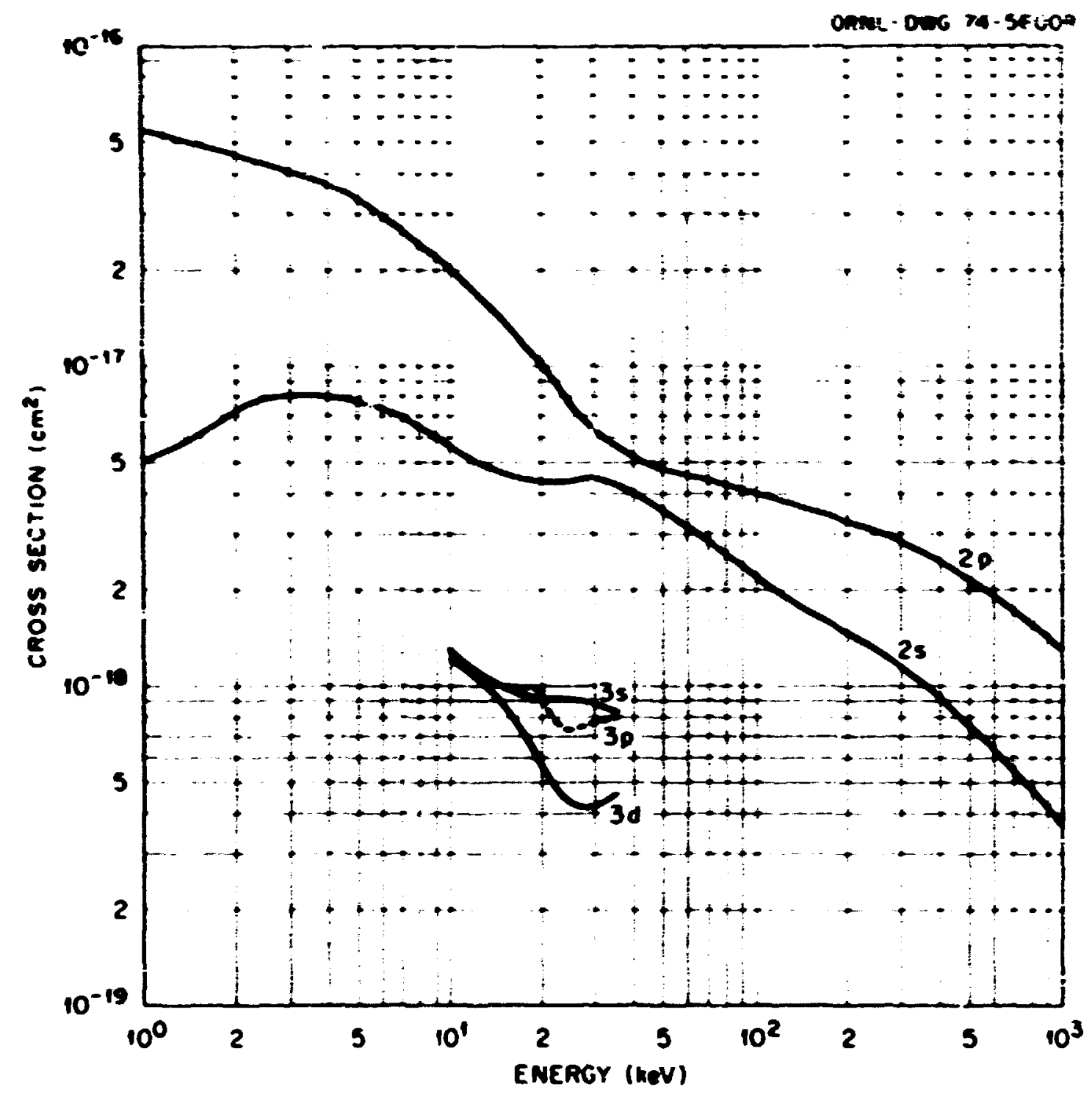




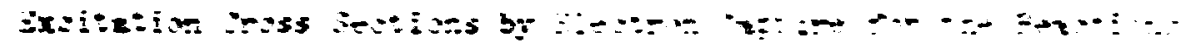

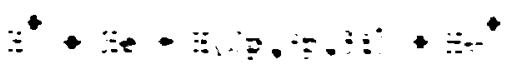

经

$\dddot{z}$

$2.5=3$

3. $3-3$

2. 3 ב-1:

:- $5 \equiv-5$

-.0 z 32

¿. 5323

$2.5 \equiv 20$

$3.5 \equiv$

$-3 \equiv 2$

$5.3 \geq 0$

$6.0 \pm 09$

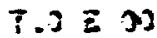

3.) 20

$9.3 \geq 0$

$1.0 \equiv 21$

¿.5 $\mathrm{E} 31$

$2.0 \geq 91$

3. $2 \mathrm{JL}$

$-2 \equiv 9 i$

$5.3 \geq 0$

6. $3 \equiv 01$

7. 501

9.0 $\Xi 01$

9. $0 \equiv \sigma_{i}$

$1.0 \equiv 02$

i. $5 \mathrm{E}, 02$

2.) E 22

!.

i

$+55-2$

$5.25-5$

-. $3 \quad \overline{-}-2$

$7.9 \pm-\therefore$

४. $3=-3$

$\therefore \ldots=-5$

$\therefore i=-\div$

i. - $=-i 9$

1. $.7 \equiv-: 9$

-. $3-\div$

$2.2 \leq-19$

$2.2 \quad 3-i 2$

$2 . \vdots \vdots-1$ ?

$3.2 \equiv-1$ ?

$3.6 \vdots-: 2$

$3.3=-5$

$2.5 \mathrm{E}-\mathrm{E}$

1. 7 E-

$1.3 \because-3$

i. 5 E-i.

$7.9 \geq-5$

$6.4 \equiv-29$

$5.4=-29$

$2.9=-19$

3.

.

$\therefore$ : 3 ?

ㄱ)

-

$\therefore E \equiv-i$ ?

¿.

i. 5 E-?

T. E-?

5.93

3.

$3 . \div$ E.

3.)

$3 . \therefore=$
Z. $\quad \therefore-$

$\because 2 \quad-2$

$\therefore$.

$\therefore \quad \because-\therefore$

;..

$\therefore$ 政?

= $5-3$

n.

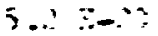

$\ldots$...

?

$3.0 \geq 32$

\section{Referen:es:}

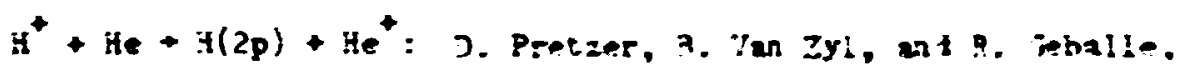

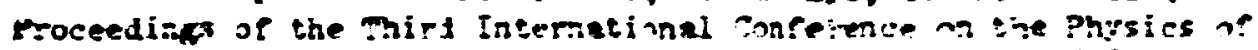

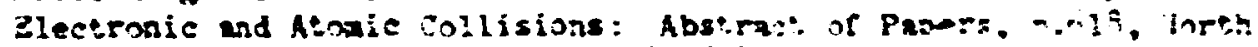
folland Plblishing co., Arstertan $(1963)$.

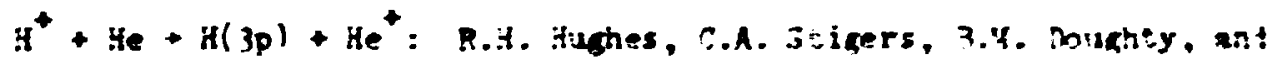

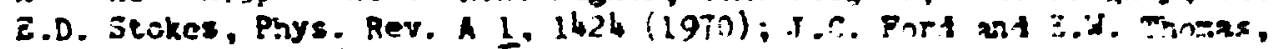
Phys. Rev. A 5.1694 (1972) (normalizet to work ne suchex et. s.1.).

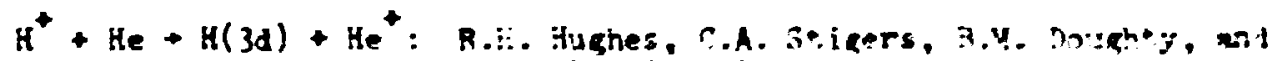
E.D. Stokes, Phys. Rer. $\ldots$ - 1424 (ig7ai).

Notes:

see Xotes (5), (13), ant (14) zt ent of chapier.

Accuracy:

Systemetic error $<50 \%$. Rendom error $<15 \%$. 


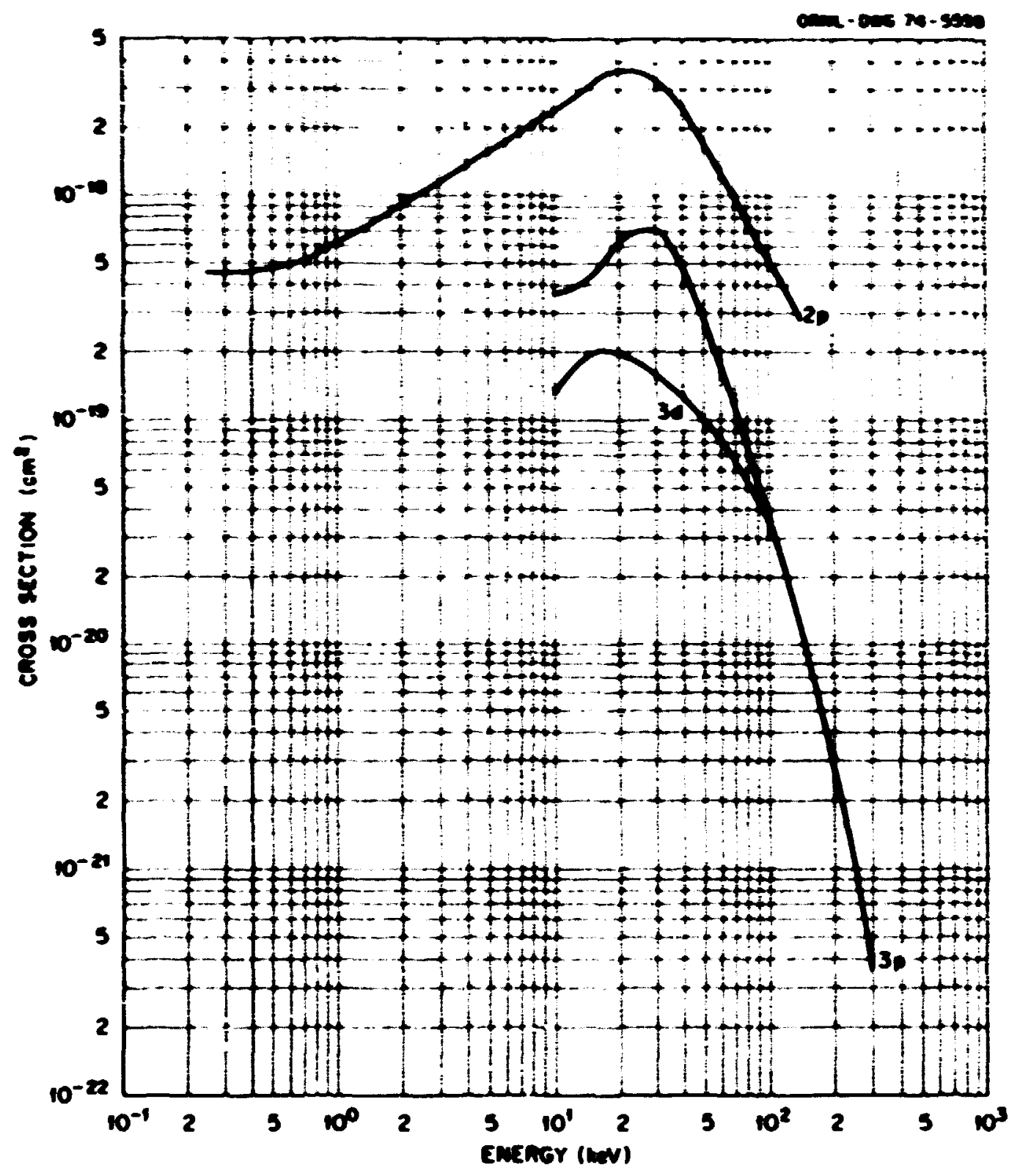




\section{A.2.30}

Breltation Crose Sections by aleetron Capture for the Rreetions

$$
a^{*}+m e+n\left(28,3=, 4=1+4 e^{*}\right.
$$

\begin{tabular}{|c|c|c|c|}
\hline$(\operatorname{lng} x)$ & \multicolumn{3}{|c|}{$\begin{array}{l}\text { Cress Section for Preited State al } \\
\left(e^{2}\right)\end{array}$} \\
\hline & 2 & 32 & ts \\
\hline 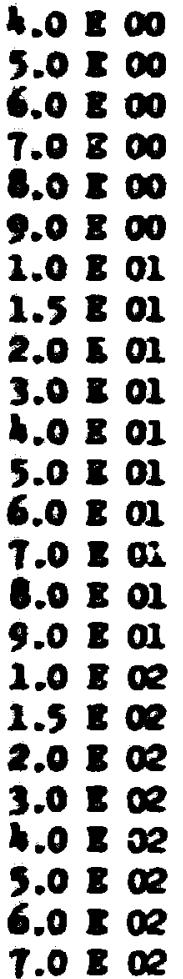 & 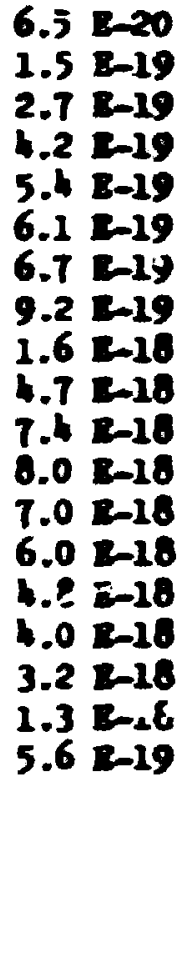 & $\begin{array}{ll}1.6 & \mathrm{E}-19 \\
2.3 & \mathrm{E}-19 \\
2.8 & \mathrm{E}-19 \\
3.3 & \mathrm{E}-19 \\
3.7 & \mathrm{E}-19 \\
4.0 & \mathrm{E}-19 \\
6.2 & \mathrm{E}-19 \\
8.0 & \mathrm{E}-19 \\
1.4 & \mathrm{E}-18 \\
1.0 & \mathrm{E}-18 \\
1.7 & \mathrm{E}-18 \\
1.4 & \mathrm{E}-18 \\
1.2 & \mathrm{E}-18 \\
1.1 & \mathrm{E}-18 \\
9.0 & \mathrm{E}-19 \\
8.0 & \mathrm{E}-19 \\
3.8 & \mathrm{E}-19 \\
1.6 & \mathrm{E}-19 \\
3.5 & \mathrm{E}-20 \\
1.0 & \mathrm{E}-20 \\
5.4 & \mathrm{E}-21 \\
3.0 & \mathrm{E}-21 \\
1.9 \mathrm{E}-21\end{array}$ & $\begin{array}{ll}4.1 & E-20 \\
5.0 & \mathrm{E}-20 \\
6.1 & \mathrm{E}-20 \\
7.2 & \mathrm{E}-20 \\
8.3 & \mathrm{~L}-20 \\
9.3 & \mathrm{E}-20 \\
1.4 & \mathrm{E}-19 \\
2.0 & \mathrm{~L}-19 \\
3.4 & \mathrm{E}-19 \\
5.6 & \mathrm{E}-19 \\
7.0 & \mathrm{E}-19 \\
6.8 & \mathrm{E}-19 \\
6.1 & \mathrm{E}-19 \\
5.0 & \mathrm{E}-19 \\
3.9 & \mathrm{E}-19 \\
3.0 & \mathrm{E}-19\end{array}$ \\
\hline
\end{tabular}

merences:

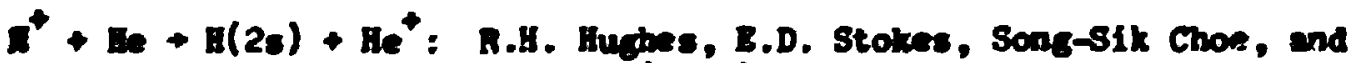
T.J. King, Phys. Rov. A k, 1453 (1971), R.L. Fitzwlison and B.H. Thomas,

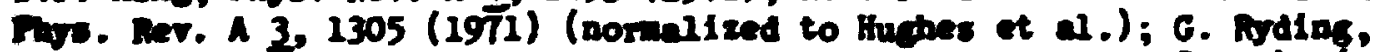
A.D. Wtthomer, and H.B. Gllbow, Proe. Phys. Soc., London 89, 547 (1966).

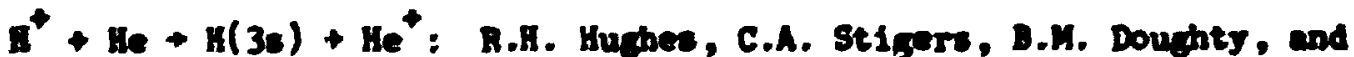
B.D. Stokes, Phys. Rer. A I, Ih2h (ig7o); R.J. Conreds, T.H. Michols, J.C. Pord, end E.W. Thonas, Phys. Rev. 1, 1928 (1973).

$\mathrm{B}^{+}+\mathrm{He}+\mathrm{H}(4 \mathrm{~S})+\mathrm{He}^{+}$: R.H. Hughes, H.R. Darreon, and B.M. Doughty, Prys. Rev. 164, 166 (1967).

\section{Dotes:}

Bee Wotes (13), (14), (15), and (16) at end of chapter.

Acouresy:

Gystematic srror < 50\%, Random error $<15 \%$. 
A.2. 31

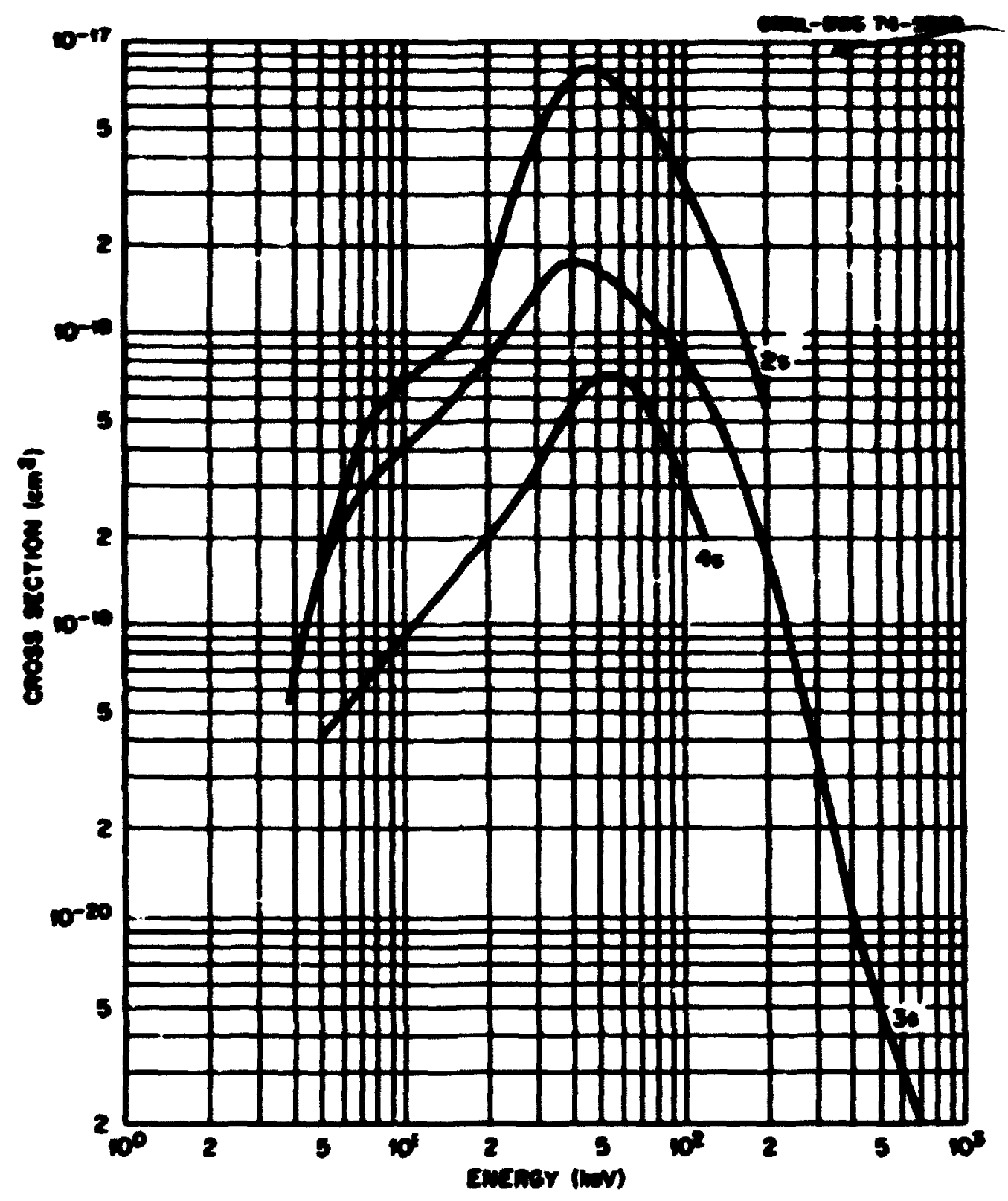




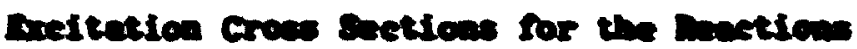

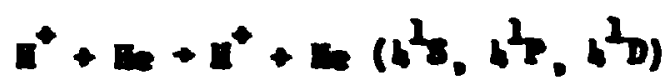

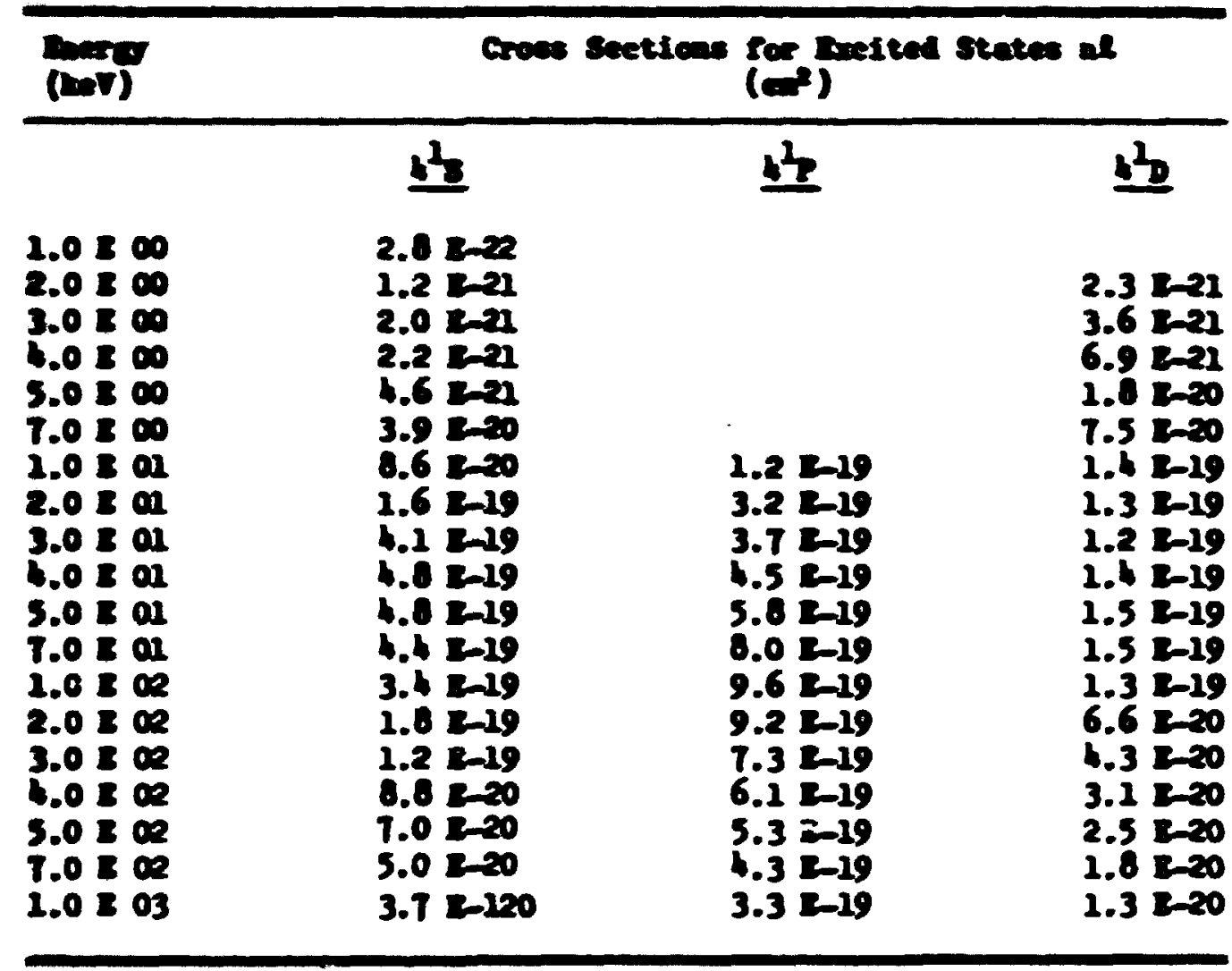

\section{Diconanes:}

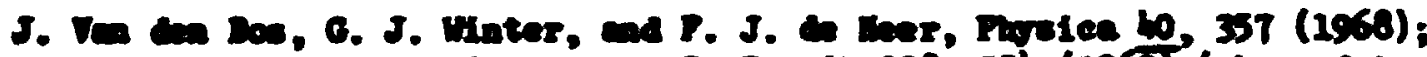

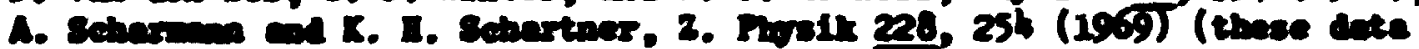

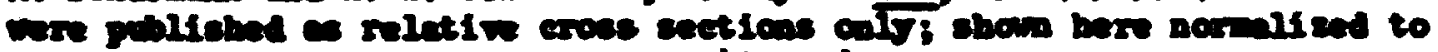

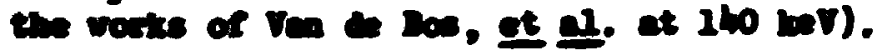

\section{Etes:}

toe Eotes (41), (42), and (43) at and of chopter.

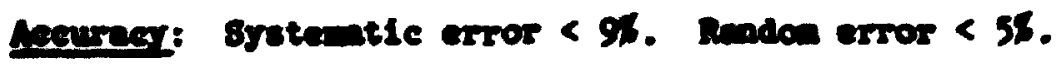




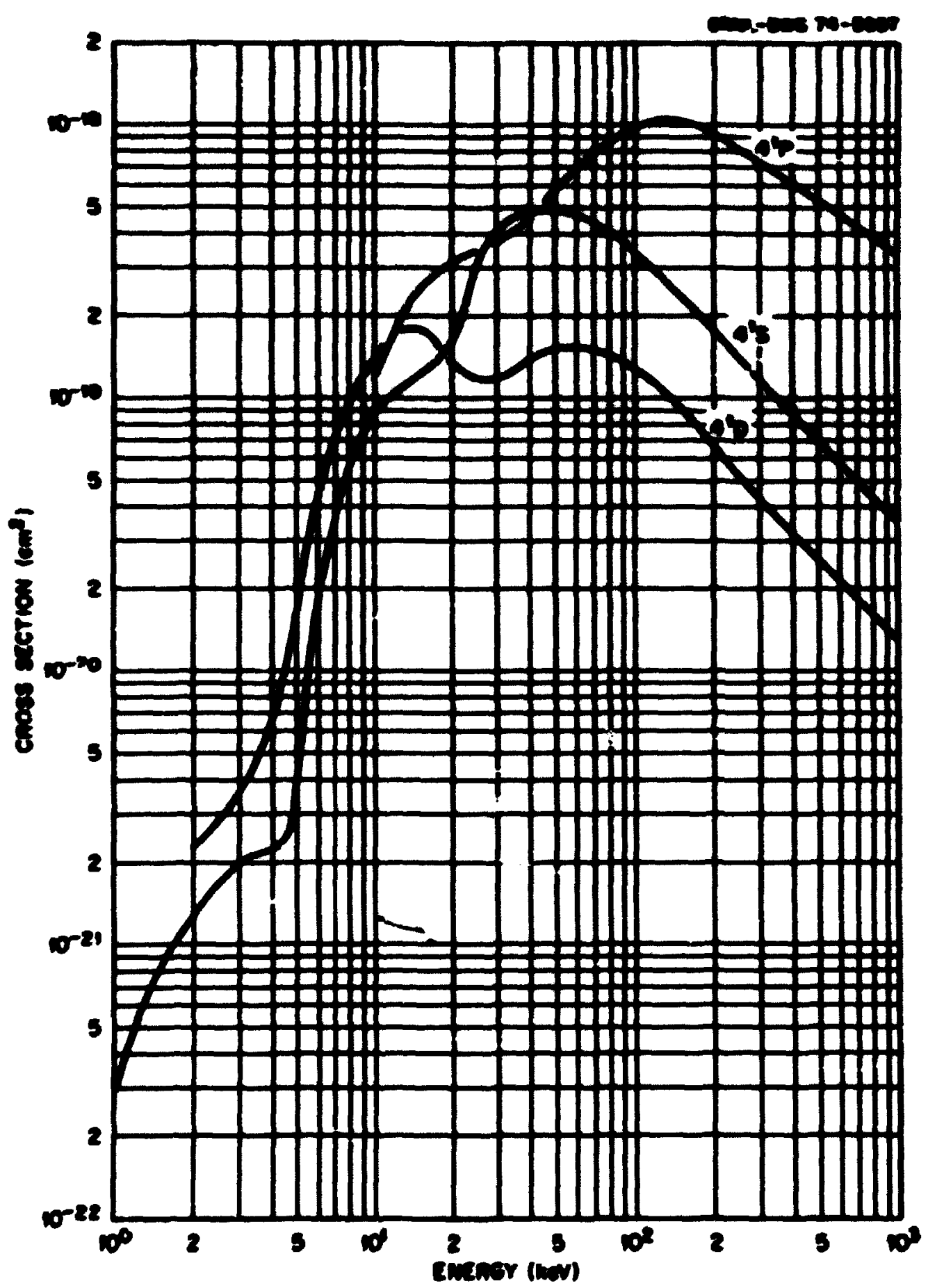




\section{A.2.3h}

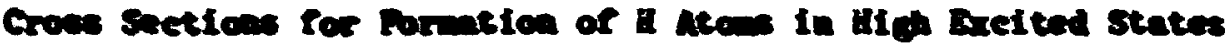

$$
\text { of } \mathrm{B}^{*} \text { Inper on } \mathrm{K}_{2}, \mathrm{~B}_{1}, \mathrm{~m}_{2}, \mathrm{O}_{2}
$$

\begin{tabular}{|c|c|c|c|c|}
\hline 9 & \multicolumn{4}{|c|}{ 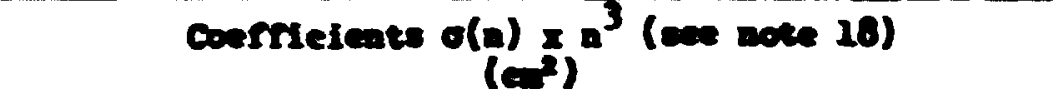 } \\
\hline & $\mathbf{E}$ & $m$ & & $\underline{\mathbf{O}_{2}}$ \\
\hline 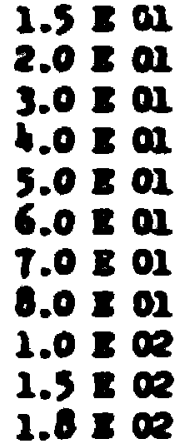 & $\begin{array}{ll}2.4 & \sum-16 \\
3.3 & =-16 \\
3.0 & =-16 \\
2.1 & \sum-16 \\
1.1 & \sum-16 \\
9.3 & =-17 \\
6.7 & =-17 \\
3.5 & \Sigma-17 \\
9.3 & \sum-18 \\
4.0 & \sum-18\end{array}$ & 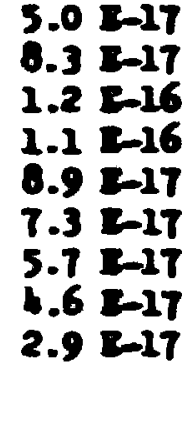 & $\begin{array}{l}3.2 \\
3.9-16 \\
3.9-16 \\
3.9 \leq-16 \\
3.6 \leq-16 \\
3.1 \leq-16 \\
2.1 \leq-16 \\
2.0 \leq-16 \\
1.3 \leq-16 \\
6.0 \leq-17\end{array}$ & $\begin{array}{ll}1.2 & \sum-16 \\
5.0 & \sum-16 \\
1.5 & \sum-16 \\
3.7 & \sum-16 \\
3.1 & \sum-16 \\
2.6 & \sum-16 \\
2.3 & E-16 \\
1.7 & E-16 \\
9.7 & \sum-17\end{array}$ \\
\hline
\end{tabular}

Derrease:

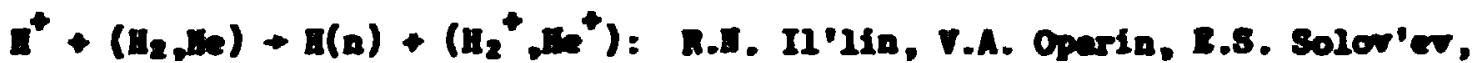

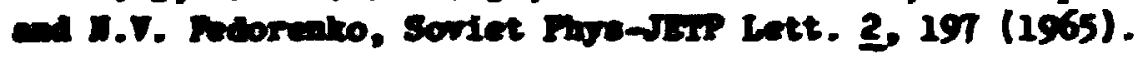

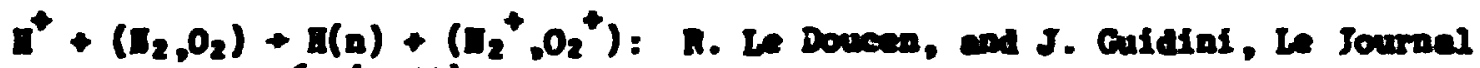
c Puss. 31,965 (1970).

\section{Estes:}

ses lotes (18) and (19) at and of chapter.

\section{Ascureg:}

Spetentic error < 10\%. Radon error < $10 \%$. 


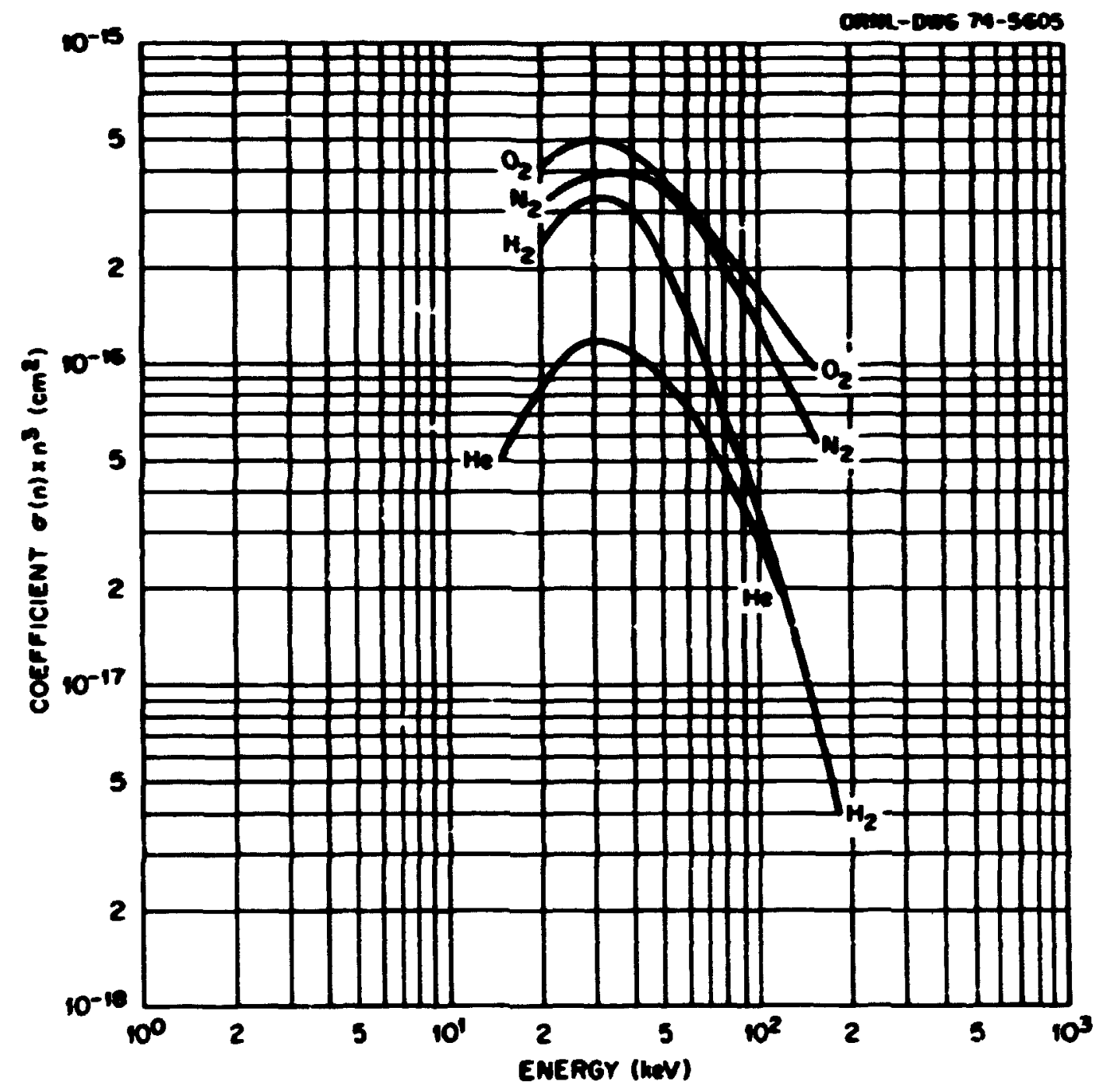




\section{A.2.36}

Bxcitetion Cross Sections for the Rections

$$
\mathrm{u}^{+}+\left(\mathrm{u}_{2}, \mathrm{O}_{2}, \mathrm{CO}_{2}\right) \rightarrow \mathrm{u}(2 \mathrm{~s})+\left(\mathrm{u}_{2}^{+}, \mathrm{O}_{2}^{+}, \infty_{2}^{+}\right)
$$

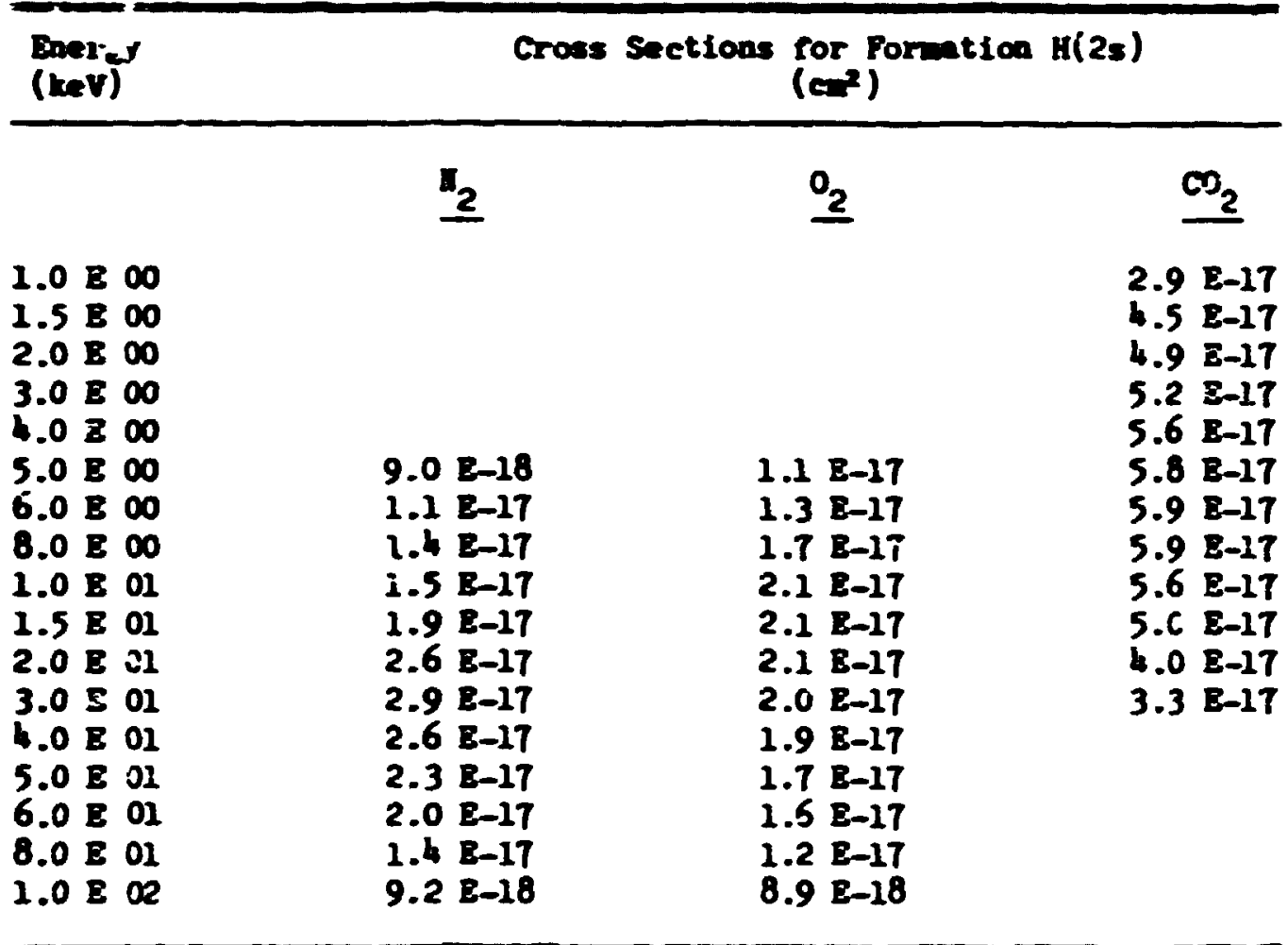

\section{Beferences:}

$H^{+}+\left(H_{2}, O_{2}\right)+H(2 s)+\left(H_{2}^{+}, \mathrm{O}_{2}^{+}\right):$R.H. Hughes, E.D. Stokes, Song-Sik Choe, and T.J. Kine, Phys. Ret. A L, 1453 (1971); R.L. Fitarilson, and E.H. Thomes, Phys. Rev. A 3, 1305 (1971) (nornelized to lughes et al.).

$\mathrm{H}^{+}+\left(\mathrm{CO}_{2}\right)+\mathrm{H}(2 \mathrm{~s})+\mathrm{CO}_{2}^{+}=$J.H. Birely and R.J. Melleal, J. Chem. Phys. 56, 2189 (1972).

\section{Notes:}

See Note (17) et end of chapter.

Accuracy:

Systematic error < 50\%. Randon error $<10 \%$. 


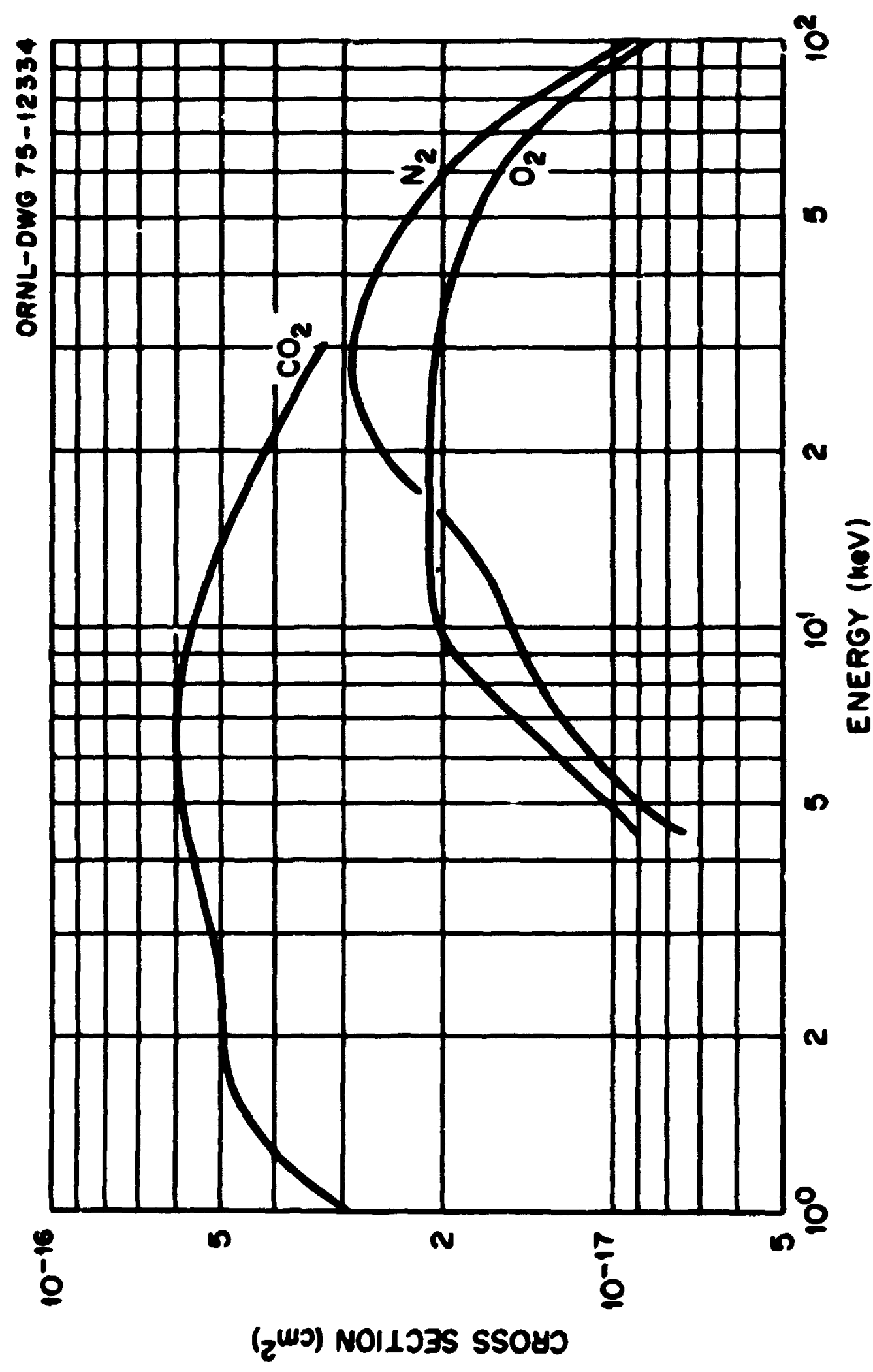




\section{A. 2.38}

Exeitation cross sections for the Reactions

$$
\mathrm{H}^{+}+\left(\mathrm{x}_{2}, \mathrm{O}_{2}, \mathrm{CO}_{2}\right) \rightarrow \mathrm{B}(3 \mathrm{~s})+\left(\mathrm{x}_{2}{ }^{+}, \mathrm{n}_{2}{ }^{+}, \mathrm{CO}_{2}{ }^{+}\right)
$$

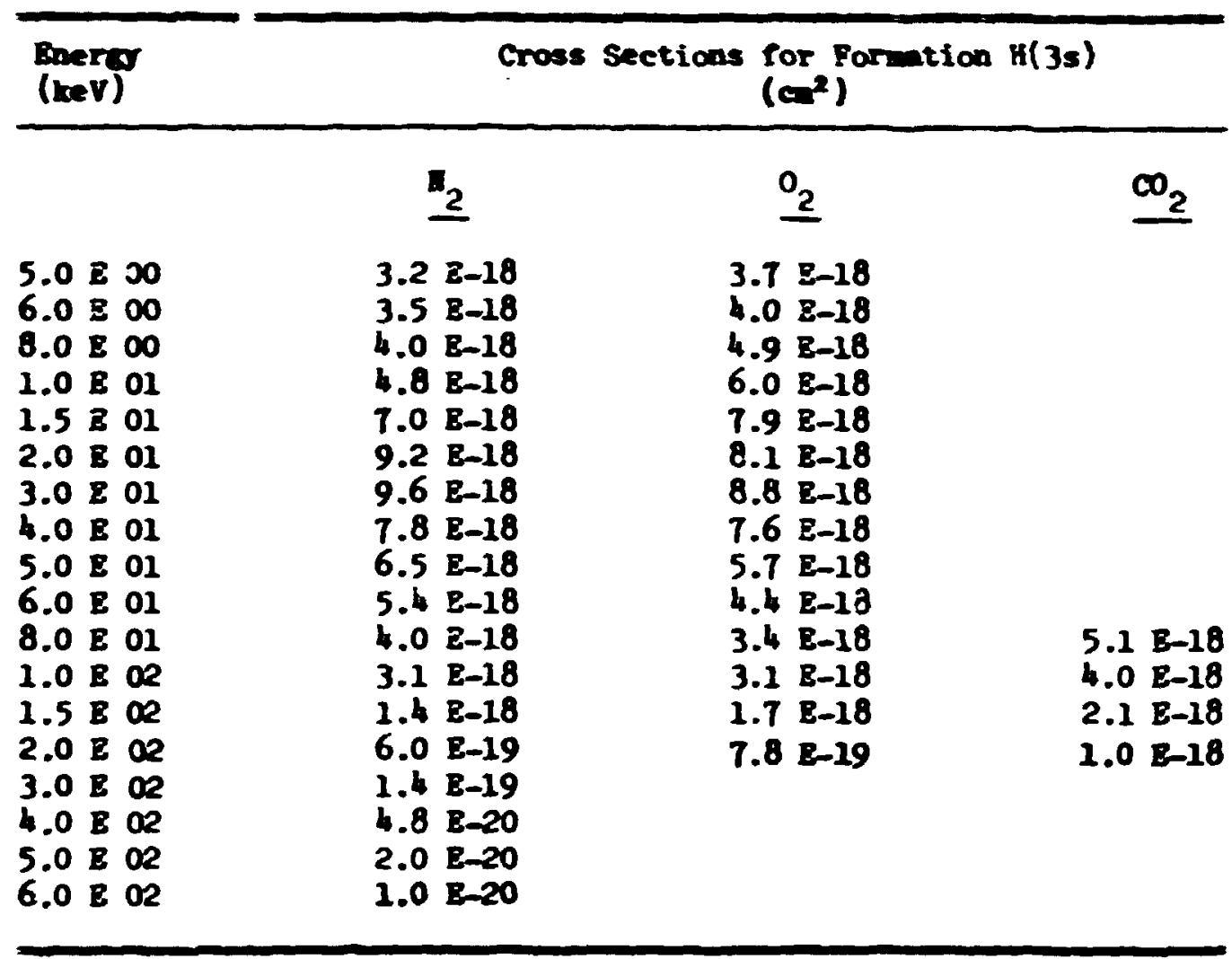

\section{Bererences:}

$\mathrm{H}^{+}+\left(\mathrm{H}_{2}, \mathrm{O}_{2}\right) \rightarrow \mathrm{H}\left(3 \mathrm{~s} ;+\left(\mathrm{N}_{2}^{+}, \mathrm{O}_{2}^{+}\right):\right.$R.H. Hughes, H.R. Dewson, B.M. Doughty, D.B. Kay, and C.A. Stigers, Phys. Rev. 146,53 (1966); J.C. Ford and E.N. Thomas, Phys. Ret. A 5, 1701 (1972).

$\mathrm{H}^{+}+\left(\mathrm{CO}_{2}\right)+\mathrm{H}(3 \mathrm{~s})+\mathrm{CO}_{2}^{+}$: J.C. Pord and E.H. Thomes, Phys. Rer. A $\underline{5}$, 1701 (1972).

\section{Dotes:}

See Note (17) at end of chapter.

\section{Accurecy:}

Systematic error < 50\%. Random error < $10 \%$. 
A.2.39

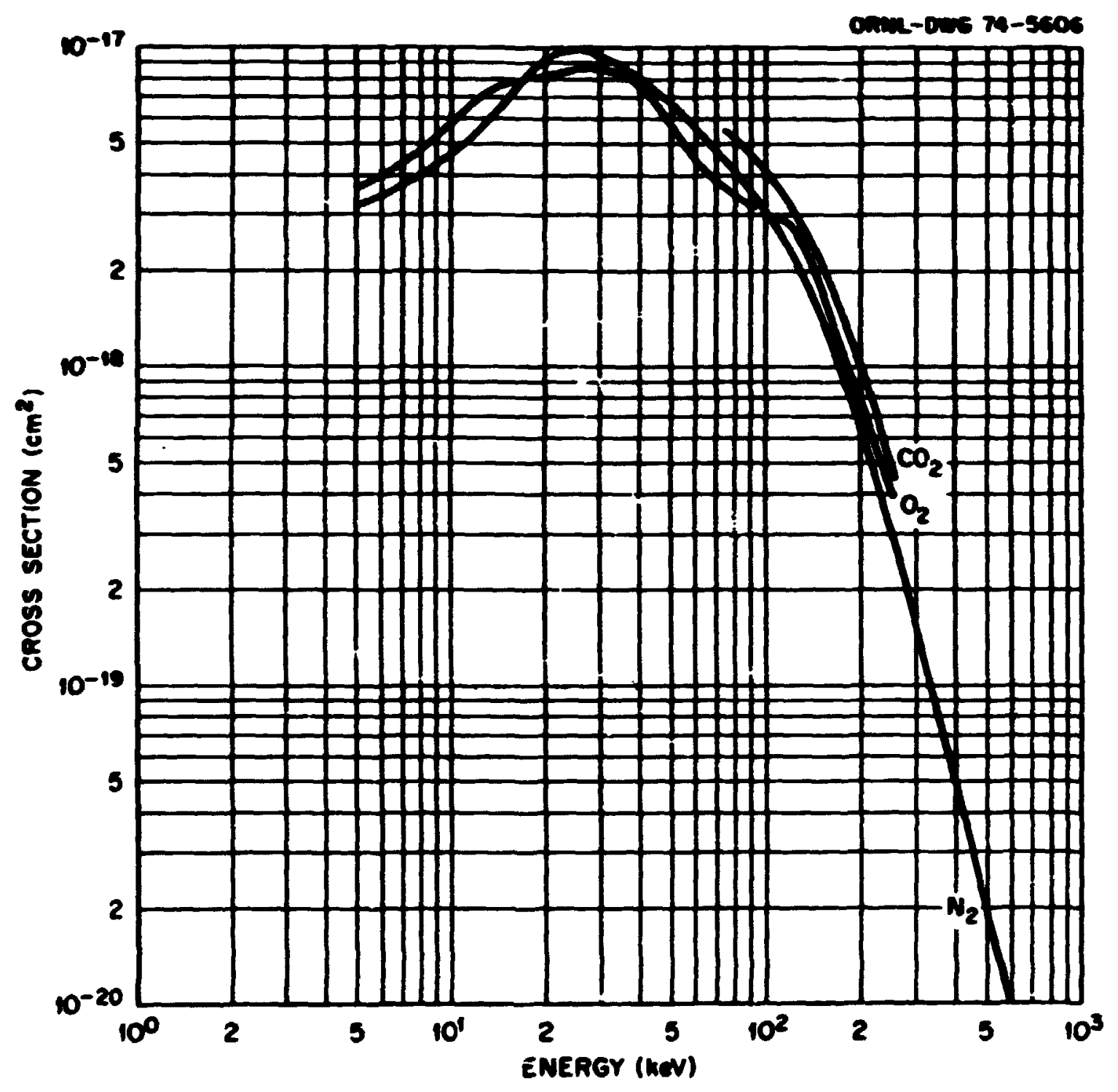


Breitation Tross Sections for the Reections

$$
H^{+}+A r+H(2 s, 3 s)+A r^{+}
$$

\begin{tabular}{|c|c|c|}
\hline $\begin{array}{l}\text { Bergy } \\
\text { (ker) }\end{array}$ & Cr & $\mathrm{nl}$ \\
\hline 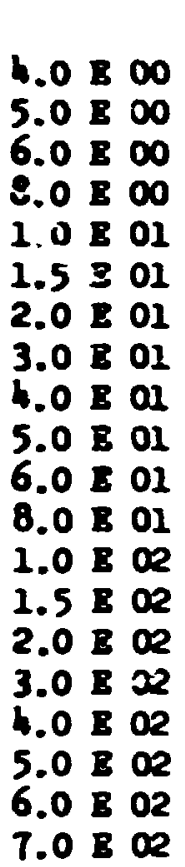 & $\begin{array}{ll}\frac{25}{18} \\
5.8 \mathrm{E}-18 \\
3.7 \mathrm{E}-18 \\
4.3 \mathrm{E}-18 \\
6.9 \mathrm{E}-18 \\
1.0 \mathrm{E}-17 \\
2.0 \mathrm{E}-17 \\
2.3 \mathrm{E}-17 \\
2.3 \mathrm{E}-17 \\
2.3 \mathrm{E}-17 \\
2.1 \mathrm{E}-17 \\
1.9 & \mathrm{E}-17 \\
1.4 & \mathrm{E}-17 \\
9.6 & \mathrm{E}-18\end{array}$ & 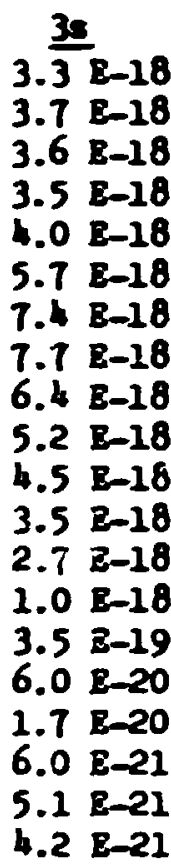 \\
\hline
\end{tabular}

Referances:

$\mathrm{B}^{+}+\mathrm{Ar}+\mathrm{H}(38)+\mathrm{Ar}^{+}$: R.H. Hughes, B.D. Stokes, Sonc-Sik Choe, and T.J. Kins Phys. Rev. A 4, 1453 (1971); R.L. Fitzwilson and E.W. Thomes, Phys. Rev. A 3, 1305 (1971) [normalized to Hughes et al.].

$H^{+}+A r \rightarrow B(33)+A r^{+}$: R.H. Hughes, H.R. Darson, B.M. Doughty, D.B. Kay, and C.A. Stigers, Phys. Rev, 146, 53 (1966); R.J. Conreds, T.H. Michols, J.C. Pord, and B.H. Thane, Phys. Rev. A I, 1928 (1973).

\section{Foter:}

See wotes (45) and (46) at end of chapter. 


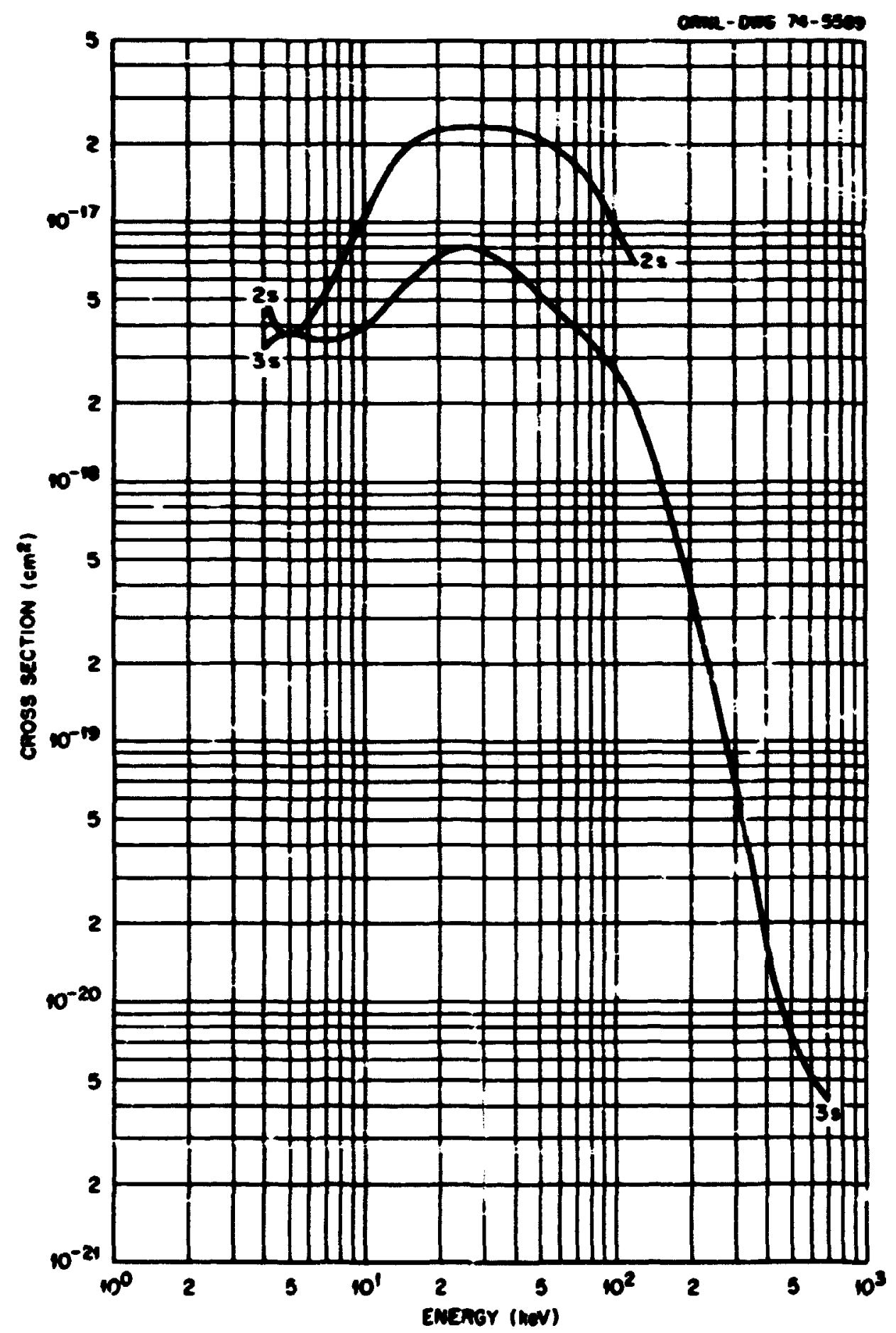




\section{Bxcitation Cross Sections for the Reactions}

$$
\mathrm{H}^{+}+(\mathrm{Cs}, \mathrm{K})+\mathrm{H}(2 \mathrm{~s})+\left(\mathrm{Cs}^{+}, \mathrm{K}^{+}\right)
$$

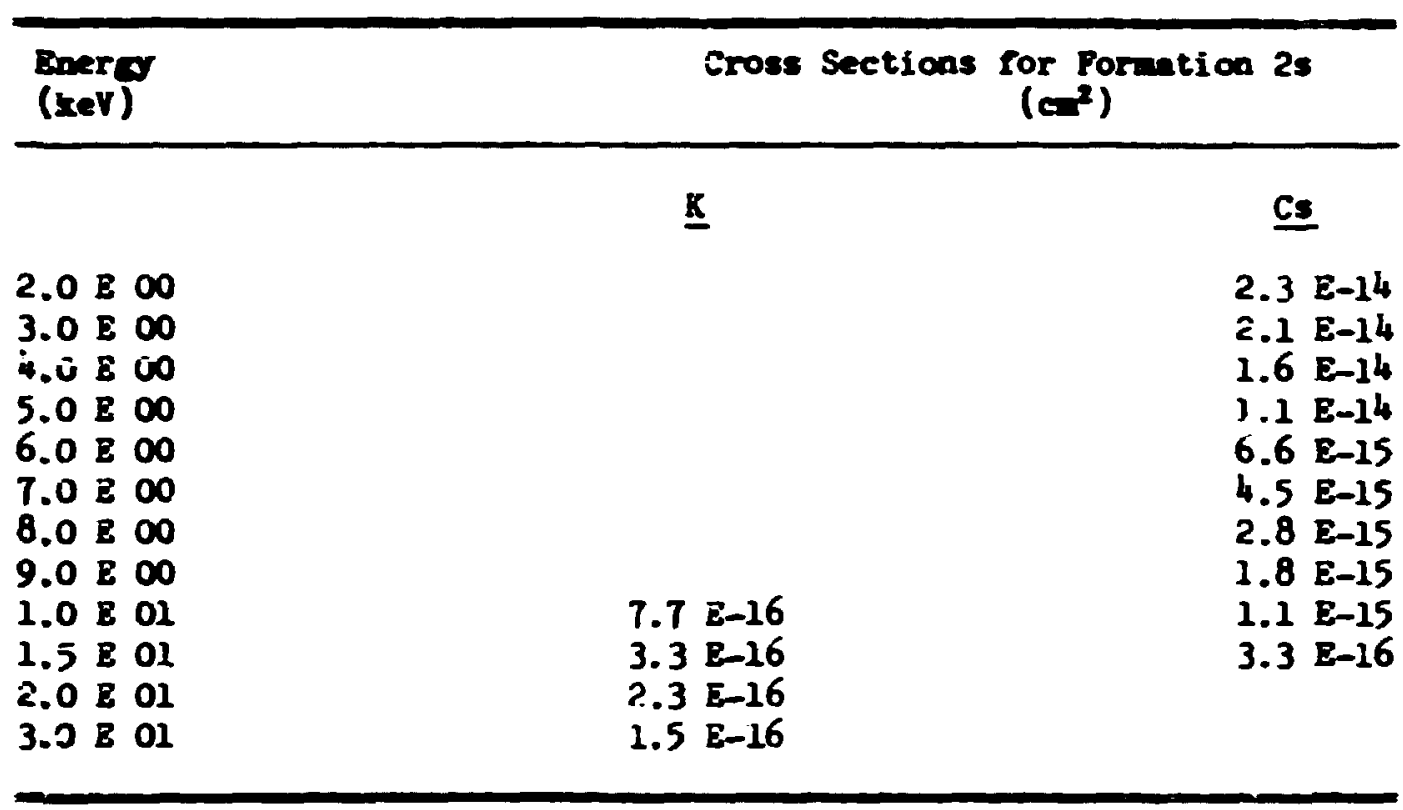

\section{Refererces:}

$H^{+}+K \rightarrow H(2 s)+K^{+}:$I.A. Sellin and L. Granoff. Phys, Letters 25A, $484(1967)$.

$\mathrm{H}^{+}+\mathrm{Cs} \rightarrow \mathrm{H}(2 \mathrm{~s})+\mathrm{Cs}^{+}$: B.L. Donnelly, T. Clapp, W. Saryer, and

M. Schultz, Phys. Rev. Letters 12, 502 (1964); I.A. Selin and L. Granoff, Phys. Letters 25A, 484 (1967).

\section{Motes:}

See Notes (45), (46), and (47) at end of chapter. 


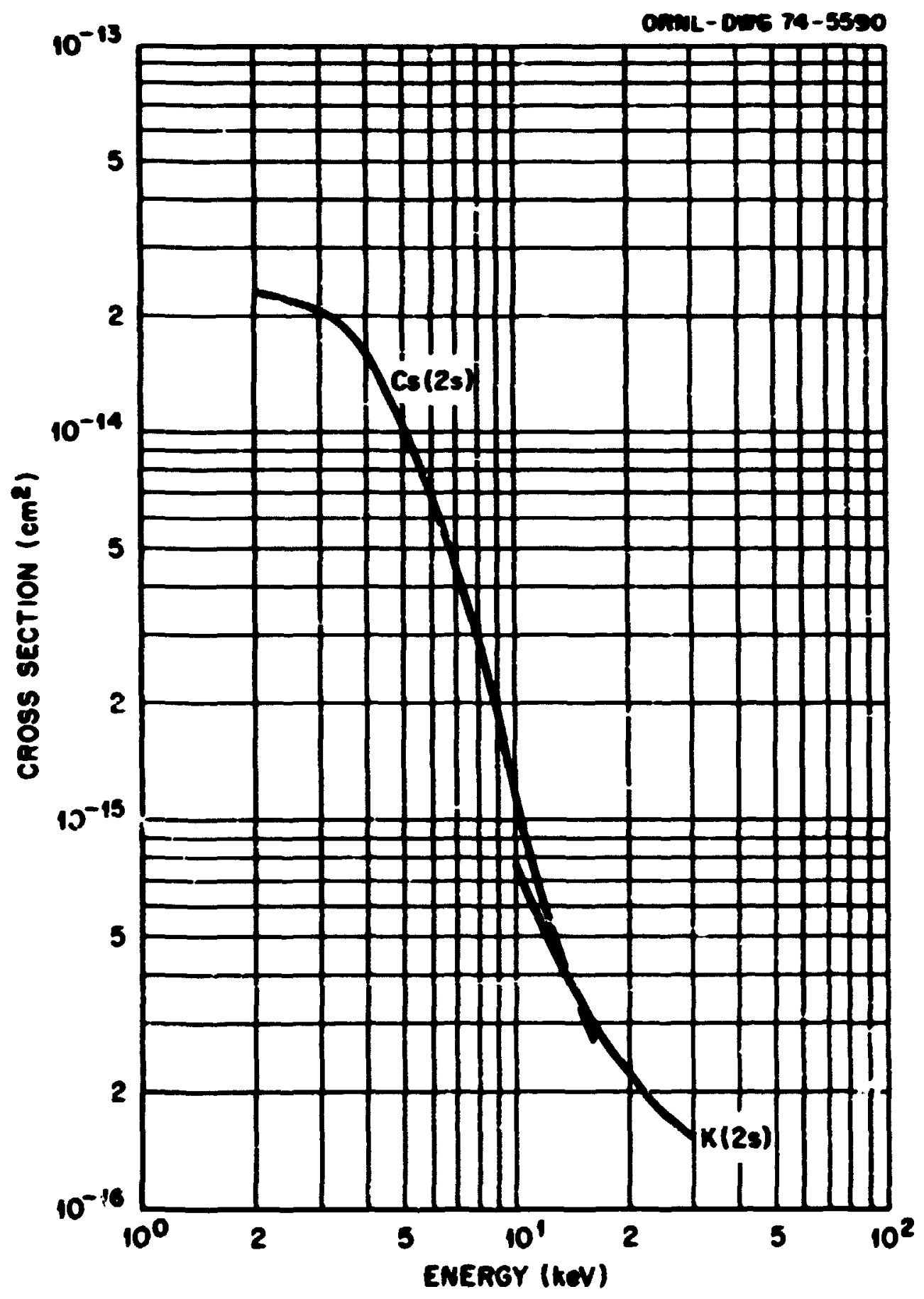




\section{A.2.4h}

Cross sections for Fornation cf H Atons in High Excited States by $\mathrm{H}^{+}$Inpact sa Na, M, $\mathrm{K}$, eid Cs

\begin{tabular}{|c|c|c|c|c|}
\hline \multirow[t]{2}{*}{$\begin{array}{l}\text { Bnerw } \\
\text { (keV) }\end{array}$} & \multicolumn{4}{|c|}{$\begin{array}{c}\left.\text { coefficients } \sigma(n) \times n^{3} \text { (see note } 20\right) \\
\left(c m^{2}\right)\end{array}$} \\
\hline & 프 & $\underline{M x}$ & $\underline{\mathbf{K}}$ & Cs \\
\hline $\begin{array}{lll}1.0 & \mathrm{E} & 01 \\
1.5 & \mathrm{E} & 01 \\
2.0 \mathrm{E} & 01 \\
3.0 \mathrm{E} & 01 \\
4.0 \mathrm{E} & 01 \\
5.0 \mathrm{E} & 01 \\
6.0 \mathrm{E} & 01 \\
\mathrm{~B} .0 \mathrm{E} & 01 \\
1.0 \mathrm{E} & 02 \\
1.5 \mathrm{E} & 02 \\
1.8 & \mathrm{E} & 02\end{array}$ & $\begin{array}{ll}7.3 & E-15 \\
4.0 & E-15 \\
2.0 & E-15 \\
4.3 & E-16 \\
7.7 & E-17 \\
4.0 & E-17 \\
3.2 & E-17 \\
2.9 & E-17 \\
2.8 & E-17 \\
2.1 & E-17 \\
1.7 & E-17\end{array}$ & $\begin{array}{ll}2.4 & E-1: \\
3.3 & E-15 \\
2.4 & E-15 \\
8.5 & E-1 G \\
2.6 & E-16 \\
1.0 & E-16 \\
3.6 & E-17 \\
1.8 & E-17 \\
1.3 & E-E-7 \\
9.8 & E-18 \\
8.4 & E-18\end{array}$ & $\begin{array}{ll}1.0 & E-14 \\
5.1 & E-15 \\
1.5 & E-15 \\
3.2 & E-16 \\
2.8 & E-16 \\
2.4 & E-10 \\
2.0 & E-16 \\
1.4 & E-16 \\
1.0 & E-16 \\
5.0 & E-17 \\
3.2 & E-17\end{array}$ & $\begin{array}{ll}1.5 & E-14 \\
6.4 & E-15 \\
2.2 & E-15 \\
7.0 & E-16 \\
5.1 & E-16 \\
4.8 & E-16 \\
4.5 & E-16 \\
3.4 & E-16 \\
2.2 & E-16 \\
7.9 & E-17 \\
4.0 & E-17\end{array}$ \\
\hline
\end{tabular}

\section{References:}

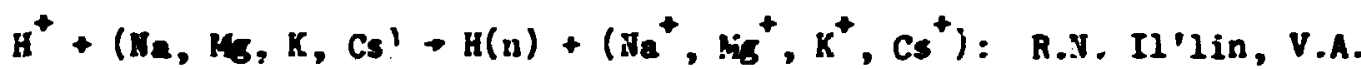
Oparin, R.S. Solor'ev, anì N.V. Fedorenko, Soriet Phys-JEPR Lett. $\underline{2}$, 197 (1965).

\section{Sotes:}

See Notes (19), (20), and (21) at end of chapter.

Acruracy:

Systematic error $<10 \%$. Sandom error $<10 \%$. 


\section{A.2.45}

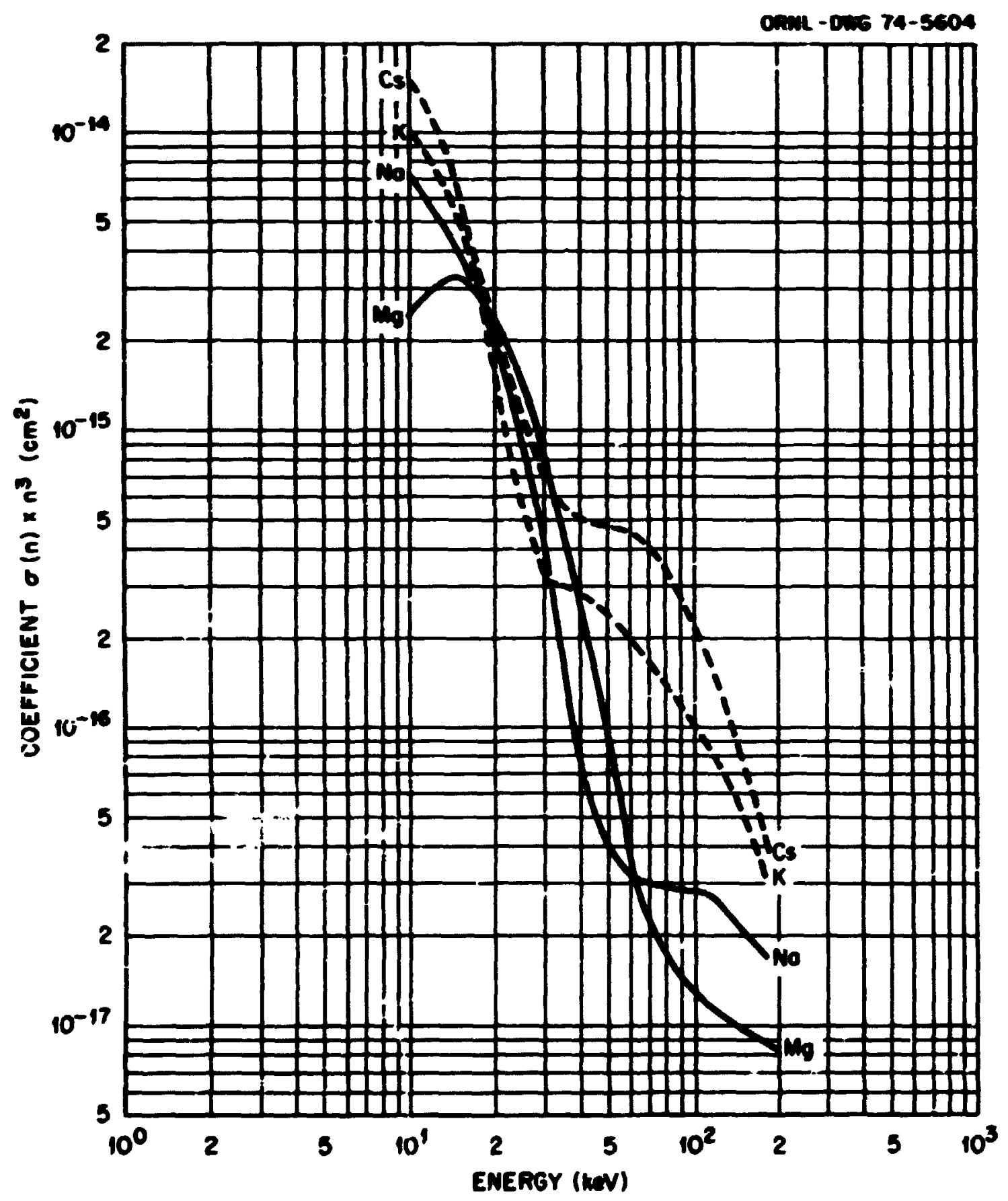




\section{A.2. 46}

Excitation Cross Sections for the Reactions

$$
\mathrm{B}_{2}^{+}+\mathrm{H}_{2}+\mathrm{H}(2 \mathrm{~s}, 3 \mathrm{~s}, 2 \mathrm{p}, 3 \mathrm{p}, 3 \mathrm{~d})+\mathrm{i}^{+}+\mathrm{H}_{2}
$$

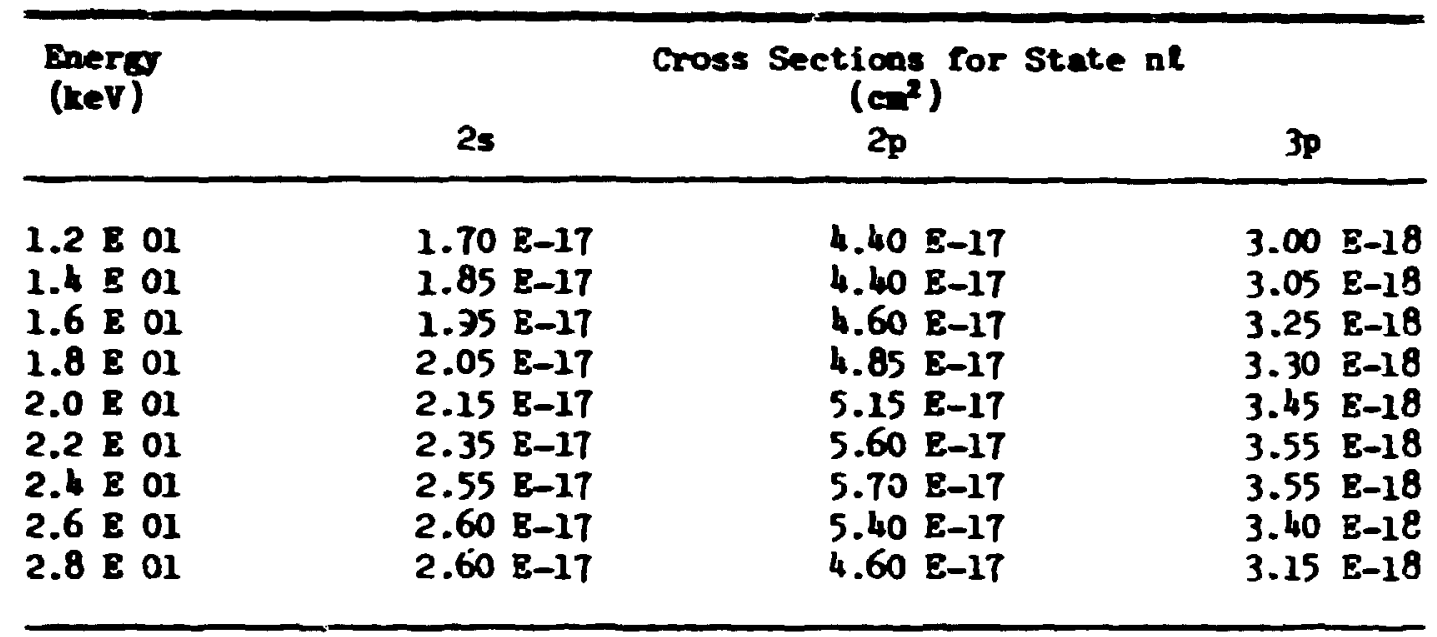

\begin{tabular}{|c|c|c|}
\hline \multirow[t]{2}{*}{$\begin{array}{l}\text { Energo } \\
\text { (kev) }\end{array}$} & \multicolumn{2}{|c|}{$\begin{array}{l}\text { Cross Sections for State nl } \\
\left(\mathrm{cm}^{2}\right)\end{array}$} \\
\hline & $3 s$ & $3 d$ \\
\hline $\begin{array}{lll}1.0 & E & 02 \\
1.5 & E & 02 \\
2.0 & E & 02 \\
3.0 & E & 02 \\
4.0 & E & 02 \\
5.0 & E & 02 \\
6.0 & E & 02 \\
1.0 & E & 02\end{array}$ & $\begin{array}{ll}3.60 & \mathrm{E}-18 \\
2.85 & \mathrm{E}-18 \\
2.43 & \mathrm{E}-18 \\
2.10 & \mathrm{E}-18 \\
2.00 & \mathrm{E}-18 \\
1.97 & \mathrm{E}-18 \\
1.70 & \mathrm{E}-18 \\
1.40 & \mathrm{E}-18\end{array}$ & $\begin{array}{ll}5.60 & \mathrm{E}-18 \\
3.60 & \mathrm{E}-18 \\
2.85 & \mathrm{E}-18 \\
2.50 & \mathrm{E}-18 \\
2.50 & \mathrm{E}-18 \\
2.50 & \mathrm{E}-18 \\
2.23 & \mathrm{E}-18 \\
2.00 & \mathrm{E}-18\end{array}$ \\
\hline
\end{tabular}

\section{Pererences:}

$\mathrm{B}_{2}^{+}+\mathrm{H}_{2}+\mathrm{H}(2 \mathrm{~s}, 2 \mathrm{p}, 3 \mathrm{p})+\mathrm{H}^{+}+\mathrm{H}_{2}$. E.P. Andreer, V.A. Anicudinor, and 8.v. Bobnober, Fifth International Conference on the Physics of Electronic atcic Collisioas: Abstract of Papers, P.309, Publishing House Mauka, Leningred, USSR (1967).

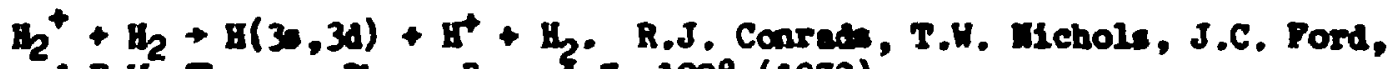
cad E.W. Thome, Phose. Rev. K I, 1928 (1973).

Sotes:

soe notes (30) and (32) at end of chepter. Aaqurnax:

Systentic error < 50\%. Randon error < $10 \%$. 


\section{A.2.47}

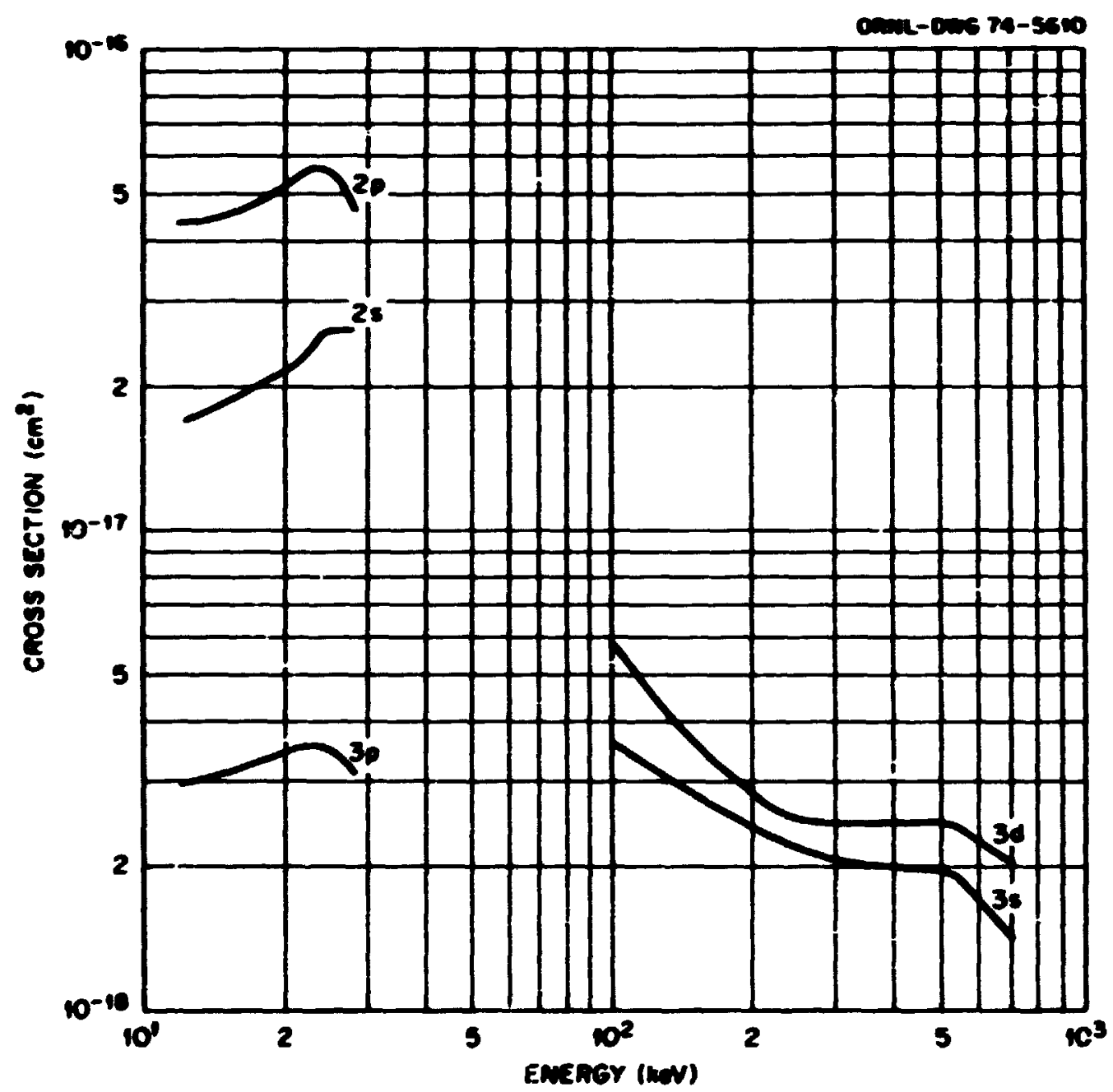




\section{A.2. 18}

Exeitation Cross Sections for the Rections

$$
\begin{aligned}
& \mathrm{H}_{2}^{+}+\mathrm{H}_{2}+\mathrm{H}_{2}^{+}+\mathrm{H}+\mathrm{H}(2 \mathrm{~s}, 2 \mathrm{p}, 3 \mathrm{p}) \\
& \mathrm{H}_{2}^{+}+\mathrm{H}_{2}+\mathrm{H}_{2}^{+}+\mathrm{H}+\mathrm{H}(\mathrm{n}=3+2)
\end{aligned}
$$

\begin{tabular}{|c|c|c|c|c|}
\hline \multirow[t]{2}{*}{$\begin{array}{l}\text { Berery } \\
\text { (keV) }\end{array}$} & \multicolumn{4}{|c|}{$\begin{array}{c}\text { Cross Sections for state nl } \\
\left(\mathrm{cm}^{2}\right)\end{array}$} \\
\hline & $\underline{2 s}$ & 22 & 32 & $n=3 \rightarrow 2$ \\
\hline $\begin{array}{lll}1.2 & \mathrm{E} & 01 \\
1.5 & \mathrm{E} & 01 \\
2.0 \mathrm{E} & 01 \\
2.5 & \mathrm{E} & 01 \\
3.0 \mathrm{E} & 01 \\
4.0 & \mathrm{E} & 01 \\
5.0 \mathrm{E} & 01 \\
6.0 \mathrm{E} & 01 \\
8.0 \mathrm{E} & 01 \\
1.0 \mathrm{E} & 02 \\
1.3 & \mathrm{E} & 02\end{array}$ & $\begin{array}{ll}4.0 & \mathrm{E}-18 \\
7.6 & \mathrm{E}-18 \\
6.8 & \mathrm{E}-18 \\
7.4 & \mathrm{E}-18 \\
7.7 & \mathrm{~B}-18\end{array}$ & $\begin{array}{ll}1.4 & \mathrm{E}-17 \\
2.3 & \mathrm{E}-17 \\
3.0 & \mathrm{E}-17 \\
3.9 & \mathrm{~B}-17 \\
3.6 & \mathrm{E}-17\end{array}$ & $\begin{array}{ll}1.5 & \text { E-18 } \\
1.9 & \text { E-18 } \\
2.6 & \text { E-18 } \\
2.4 & \text { E-18 } \\
2.1 & \text { E-18 }\end{array}$ & $\begin{array}{ll}3.8 & \mathrm{E}-18 \\
5.0 & \mathrm{E}-18 \\
5.9 & \mathrm{E}-18 \\
6.3 & \mathrm{E}-18 \\
6.4 & \mathrm{E}-18 \\
6.0 & \mathrm{E}-18 \\
5.5 & \mathrm{E}-18 \\
5.6 & \mathrm{E}-18 \\
3.8 & \mathrm{E}-18 \\
3.2 & \mathrm{E}-18\end{array}$ \\
\hline
\end{tabular}

\section{References:}

$\mathrm{H}_{2}^{+}+\mathrm{H}_{2}+\mathrm{H}_{2}^{+}+\mathrm{H}+\mathrm{H}(2 \mathrm{~s}, 2 \mathrm{p}, 3 \mathrm{P}):$ E.P. Andreer, V.A. Ankudinor, and S.V. Bobasher, Pifth International Conference on the Physics of Electroric 4tonic Collisions: Abstzect of Papers, p.309, Publisning House Maure, Leningrad, USSR (1967).

$\mathrm{H}_{2}^{+}+\mathrm{H}_{2} \rightarrow \mathrm{H}_{2}^{+}+\mathrm{H}+\mathrm{H}(\mathrm{n}=3 \rightarrow 2):$ L.L. Hatfield and R.H. Hughes, Phrs. Rer. 131, 2556 (1963).

Notes:

see lotes (32), (33), (34), and (35) at end of chapter.

Accurecy:

Systematic error $<50 \%$ for $\mathrm{H}(2 \mathrm{~s}, 2 \mathrm{p}, 3 \mathrm{~F})$; systematic error $<20 \%$ for $H(n=3+2)$. Random error $<10 \%$. 


\section{A.2.49}

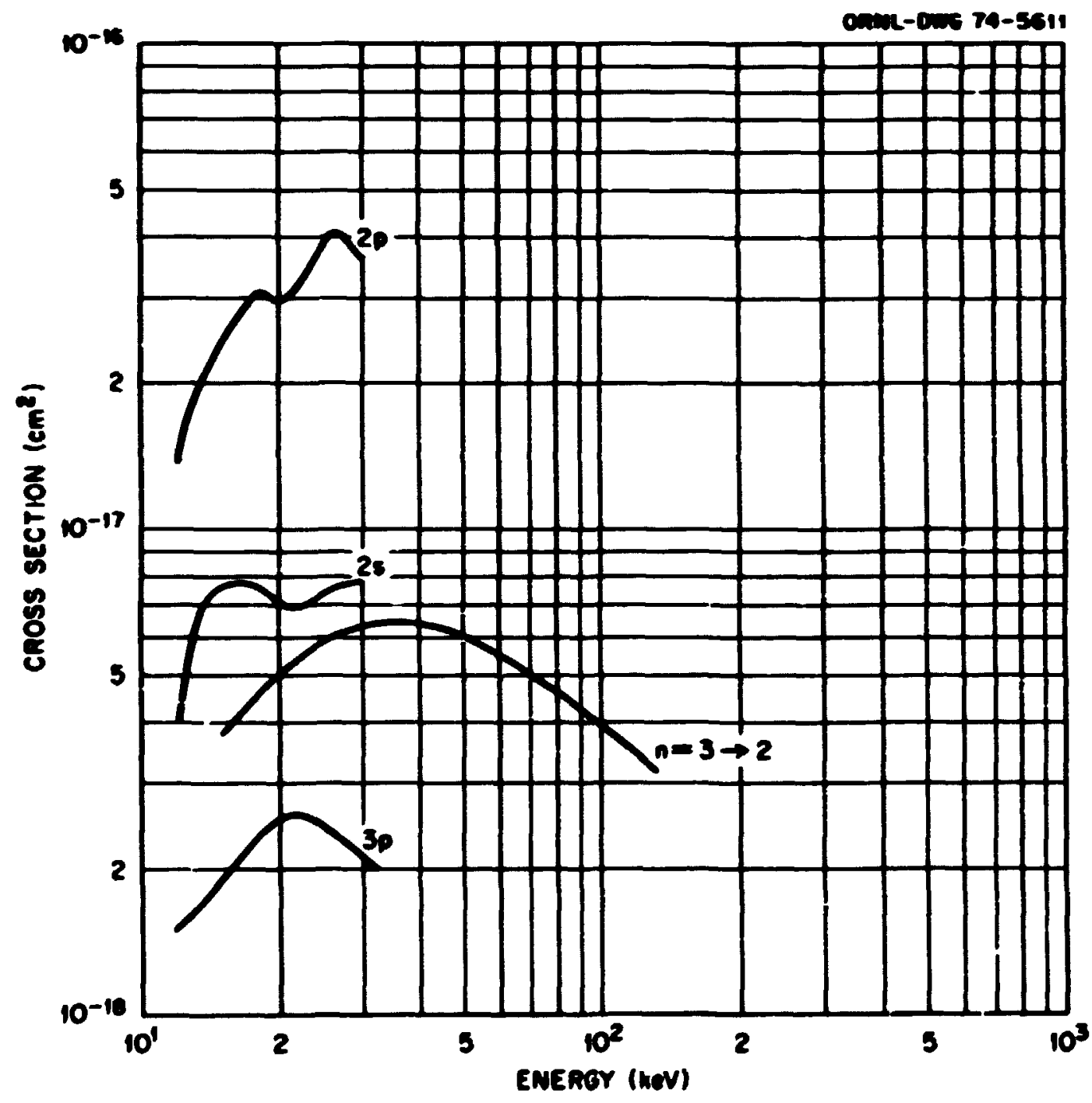




\section{A.2.50}

Excitation Cross Sections for the Rections

$$
\mathrm{H}_{2}^{+}+\mathrm{He} \rightarrow \mathrm{H}(2 \mathrm{~s}, 3 \mathrm{~s}, 3 \mathrm{p}, 3 \mathrm{~d})
$$

\begin{tabular}{|c|c|c|c|c|}
\hline \multirow[t]{2}{*}{$\begin{array}{l}\text { Bneros } \\
\text { (kev) }\end{array}$} & \multicolumn{4}{|c|}{$\begin{array}{l}\text { Cross Sections for State al } \\
\qquad\left(\omega^{2}\right)\end{array}$} \\
\hline & 28 & 3s & $2 p$ & 3d \\
\hline 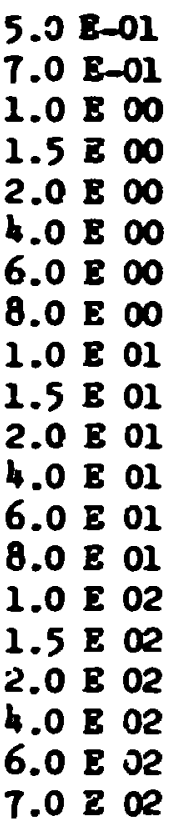 & $\begin{array}{ll}6.0 & \mathrm{E}-18 \\
6.0 & \mathrm{E}-18 \\
5.9 & \mathrm{E}-18 \\
3.8 & \mathrm{E}-18 \\
5.8 & \mathrm{~B}-18 \\
5.5 & \mathrm{~B}-18 \\
5.2 & \mathrm{~B}-18\end{array}$ & $\begin{array}{ll}2.1 & \mathrm{E}-18 \\
2.2 & \mathrm{E}-18 \\
2.1 & \mathrm{E}-18 \\
2.0 & \mathrm{E}-18 \\
1.8 & \mathrm{E}-18 \\
1.6 & \mathrm{E}-18 \\
1.1 & \mathrm{E}-18 \\
9.0 & \mathrm{E}-19 \\
8.2 & \mathrm{E}-19\end{array}$ & $\begin{array}{ll}2.6 & \mathrm{E}-17 \\
3.0 & \mathrm{E}-17 \\
3.4 & \mathrm{E}-17 \\
4.0 & \mathrm{E}-17 \\
4.3 & \mathrm{E}-17 \\
4.3 & \mathrm{E}-17 \\
4.0 & \mathrm{E}-\mathrm{i1} \\
3.6 \mathrm{E}-17 \\
3.6 & \mathrm{E}-17 \\
3.2 & \mathrm{E}-17 \\
2.9 & \mathrm{E}-17\end{array}$ & $\begin{array}{ll}2.6 & \mathrm{E}-18 \\
2.4 & \mathrm{E}-18 \\
2.3 & \mathrm{E}-18 \\
2.0 & \mathrm{E}-18 \\
1.4 & \mathrm{E}-18 \\
1.1 & \mathrm{E}-18 \\
1.0 & \mathrm{E}-18\end{array}$ \\
\hline
\end{tabular}

\section{References:}

$\mathrm{H}_{2}^{+}+\mathrm{He} \rightarrow \mathrm{H}(28)+\mathrm{He}+\mathrm{H}^{+}:$D. Jaecks and $\mathrm{E}$. Tynan, Pourth International Conference on the Physics of Electronic Atonic Collistons: Abstract of Papers, p.315, Science Bookcrafters INew York (1965).

$\mathrm{H}_{2}^{+}+\mathrm{He}+\mathrm{H}(38)+\mathrm{He}+\mathrm{H}^{+}:$R.H. Hughes, D.B. Kay, C.A. Stigers, and E.D. Stokes, Phys. Rev. 167, 26 (1968); J.C. Pord, F.M. McCoy, R. Conrads, and E.H. Thomas, Phys. Rev. A 5, 1705 (1972).

$\mathrm{H}_{2}^{+}+\mathrm{He} \rightarrow \mathrm{H}(3 \mathrm{p})+\mathrm{He}^{+}+\mathrm{H}^{+}$: B. Van $\mathrm{Zyl}$, D. Jaecks, D. Pretzer, and R. Gebslle, Pays. Rev. A 136, 1561 (1969).

$\mathrm{H}^{+}+\mathrm{He} \rightarrow \mathrm{H}(3 \mathrm{~d})+\mathrm{He}+\mathrm{H}^{+}$: J.C. Pord, R. Conrads, F.M. McCoy, and B.H. Thomas, Phys. Rer. A 2, 1705 (1972).

Notes:

see fictes (31), (36), and (37) at end of chapter. 


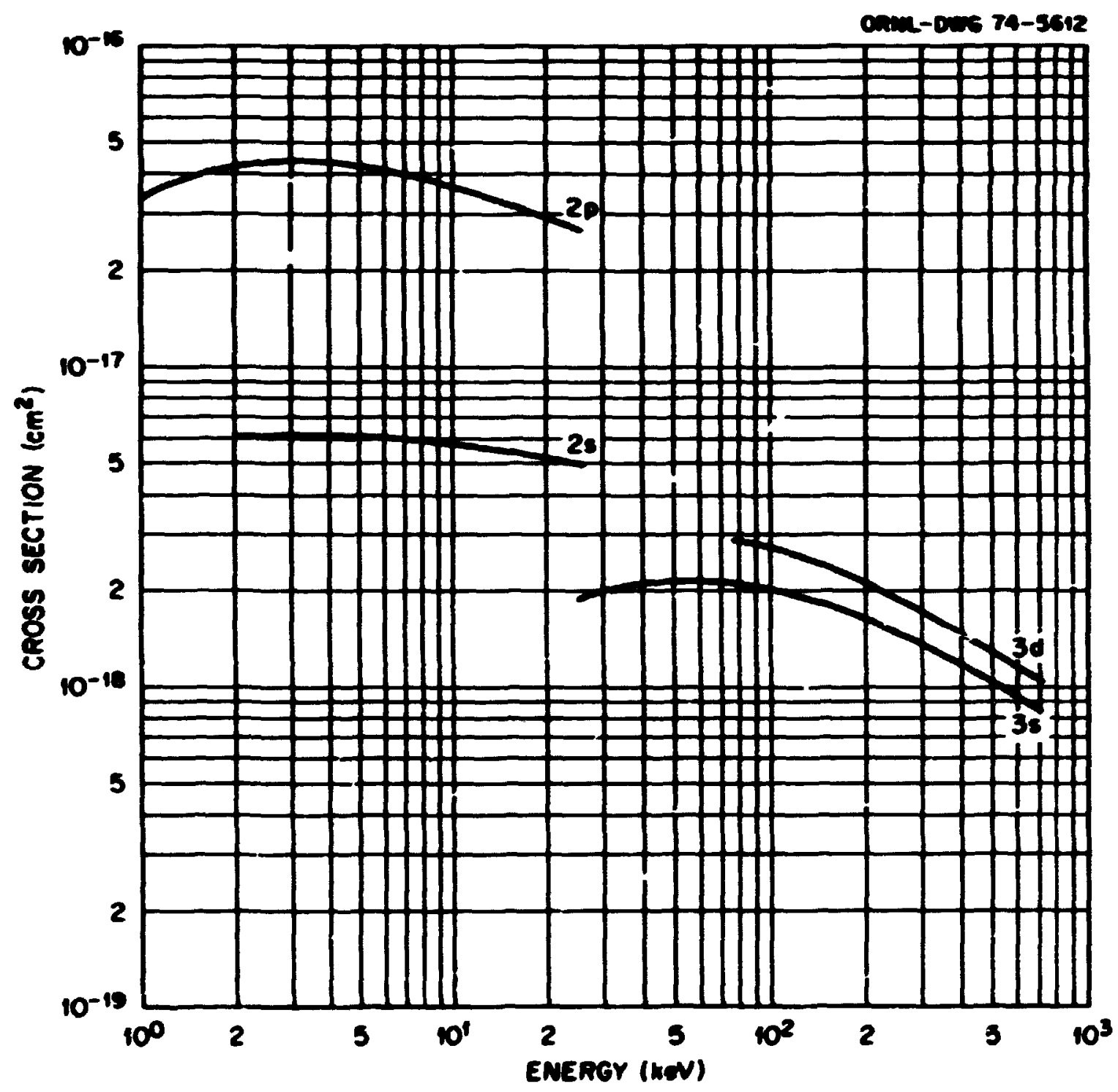




\section{A.2.52}

Bxeitation Cross Section for the Reaction

$$
\mathrm{H}_{2}^{+}+\mathrm{H}=+\mathrm{H}_{2}^{+}+\mathrm{He}^{-}(\mathrm{nl})
$$

\begin{tabular}{|c|c|c|c|c|}
\hline $\begin{array}{l}\text { Ereery } \\
\text { (keV) }\end{array}$ & & Cross See & States nl & \\
\hline & $4^{1} 5$ & $4^{1} \mathrm{P}$ & $4^{3} s$ & $4^{3} \mathrm{P}$ \\
\hline 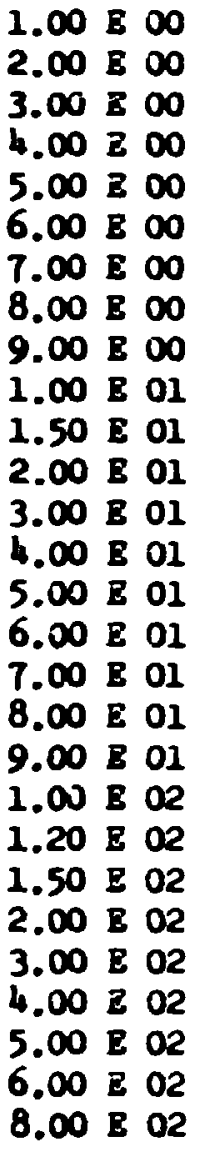 & $\begin{array}{ll}1.60 & E-21 \\
4.60 & E-21 \\
6.80 & E-21 \\
6.10 & E-21 \\
9.20 & E-21 \\
1.20 & E-20 \\
1.35 & E-20 \\
1.53 & E-20 \\
1.80 & E-20 \\
2.32 & E-20 \\
8.80 & E-20 \\
1.40 & E-19 \\
1.60 & E-19 \\
2.01 & E-19 \\
2.78 & E-19 \\
3.35 & E-19 \\
4.22 & E-19 \\
4.85 & E-19 \\
5.10 & E-19 \\
5.31 & E-19 \\
5.68 & E-19 \\
4.84 & E-19 \\
3.00 & E-19 \\
2.03 & E-19 \\
1.41 & E-19 \\
1.21 & E-19 \\
1.02 & E-19 \\
8.10 & E-20\end{array}$ & $\begin{array}{ll}1.70 & E-20 \\
2.60 & E-20 \\
3.70 & E-20 \\
6.00 & E-20 \\
7.70 & E-20 \\
9.30 & E-20 \\
1.10 & E-19 \\
1.59 & E-19 \\
2.29 & E-19 \\
3.66 & E-19 \\
4.61 & E-19 \\
4.92 & E-19 \\
5.12 & E-19 \\
5.70 & E-19 \\
6.35 & E-19 \\
6.38 & E-19 \\
6.20 & E-19 \\
7.60 & E-19 \\
8.02 & E-19\end{array}$ & $\begin{array}{ll}5.00 & \mathrm{E}-22 \\
5.90 & \mathrm{E}-21 \\
1.21 & \mathrm{E}-20 \\
9.60 & \mathrm{E}-21 \\
2.33 & \mathrm{E}-20 \\
2.96 & \mathrm{E}-20 \\
3.11 & \mathrm{E}-20 \\
3.19 & \mathrm{E}-20 \\
4.29 & \mathrm{E}-20 \\
5.38 & \mathrm{E}-20 \\
1.71 & \mathrm{E}-19 \\
2.35 & \mathrm{E}-19 \\
4.31 & \mathrm{E}-19 \\
6.32 & \mathrm{E}-19 \\
7.10 & \mathrm{E}-19 \\
6.66 & \mathrm{E}-19 \\
5.95 & \mathrm{E}-19 \\
5.04 & \mathrm{E}-19 \\
4.66 & \mathrm{E}-19 \\
4.19 & \mathrm{E}-19 \\
2.93 & \mathrm{E}-19 \\
1.72 & \mathrm{E}-19\end{array}$ & $\begin{array}{ll}6.60 & \mathrm{E}-21 \\
1.07 & \mathrm{E}-20 \\
5.03 & \mathrm{E}-20 \\
4.96 & \mathrm{E}-20 \\
5.05 & \mathrm{E}-20 \\
6.47 & \mathrm{E}-20 \\
9.48 & \mathrm{E}-20 \\
1.17 & \mathrm{E}-19 \\
1.44 & \mathrm{E}-19 \\
1.41 & \mathrm{E}-19 \\
1.34 & \mathrm{E}-19 \\
4.22 & \mathrm{E}-19 \\
5.81 & \mathrm{E}-19 \\
6.19 & \mathrm{E}-19 \\
5.86 & \mathrm{E}-19 \\
5.34 & \mathrm{E}-19 \\
4.31 & \mathrm{E}-19 \\
3.49 & \mathrm{E}-19 \\
3.06 & \mathrm{E}-19 \\
1.78 & \mathrm{E}-19 \\
1.09 & \mathrm{E}-19\end{array}$ \\
\hline
\end{tabular}

\section{Peferences:}

J. Van den Bos, G. Winter, and P. J. De Heer, Physica 44, 143 (1969). R. W. Thowas and G. D. Bent, J. Ojt. Soc. Am. 58, 138 (1968).

\section{Dotes:}

See Note (44) at end of chapter.

Accuracy: Systematic error $<10 \%$. Random error $<5 \%$. 


\section{A.2 53}

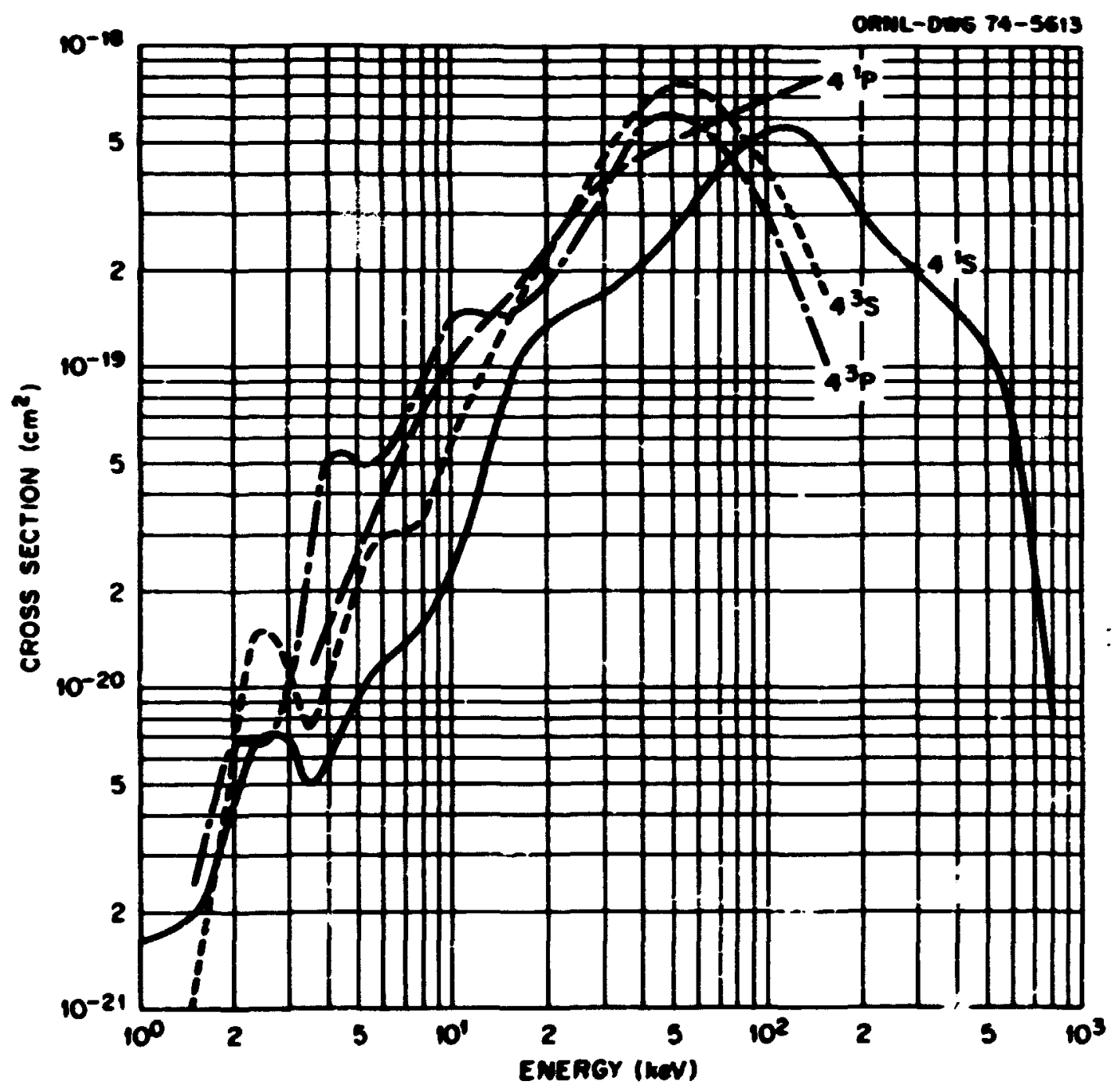




\section{A.2.54}

Exitation Cross Sections for the Reections

$$
\mathrm{H}_{3}^{+}+\mathrm{He}+\mathrm{H}(3 s, 3 d)+\mathrm{H}_{2}^{+}+\mathrm{He} \text { and } \mathrm{H}_{3}^{+}+\mathrm{H}_{2}+\mathrm{H}(3 s)+\mathrm{H}_{2}^{+}+\mathrm{H}_{2}
$$

\section{Berey}

\section{Cross Sections for States al \\ $\left(\mathrm{cos}^{2}\right)$}

38

$2.4 \mathrm{E}-18$

$2.6 \mathrm{E}-18$

$2.8 \mathrm{E}-18$

$2.9 \mathrm{E}-18$

$3.0 \mathrm{E}-18$

3.0 E-18

$3.0 \mathrm{E}-18$

$3.0 \mathrm{E}-18$

2.9 E-18

$2.8 \mathrm{E}-18$

2.7 E-18

$2.4 \mathrm{E}-18$

2.1 E-18

$1.8 \mathrm{E}-18$

$1.6 E-18$

$1.4 \mathrm{E}-18$
$3 \mathbf{3}$

$\mathrm{H}_{3}^{+}+\mathrm{He} \quad \mathrm{H}_{3}^{+}+\mathrm{H}_{2}$

3 s.

$4.0 \mathrm{~B} 00$

$5.0 \mathrm{E} 02$

$6.0 \mathrm{~B} 02$

$7.0 \mathrm{E} 02$

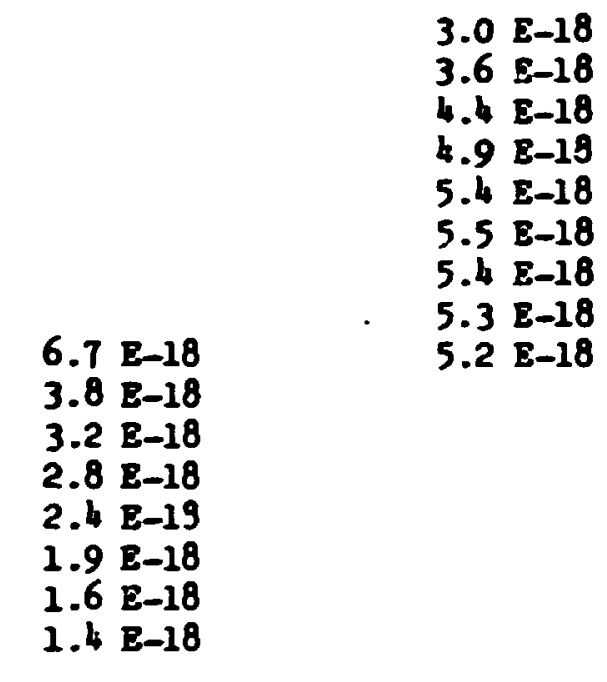

$3.0 \mathrm{E}-18$

$3.6 \mathrm{E}-18$

4.4 E-18

5.4 E-18

5.5 E-18

5.4 E-18

5.3 E-18

6.7 E-18

$3.8=18$

2.8 E-18

$2.4 \mathrm{E}-19$

$1.9 \mathrm{E}-18$

1.4 E-18

\section{Beferences:}

$\mathrm{H}_{3}^{+}+\mathrm{He} \rightarrow \mathrm{H}(3 \mathrm{~s})+\mathrm{H}_{2}^{+}+\mathrm{He}:$ R.H. Hughes, D.B. Kay, C.A. Stigers, and E.D. Stokes, Phys. Rev. 167, 26 (196:); J.C. Pord, F.M. McCoy, R. Conrads, and E.W. Thomes, Phys. Rev. A 2, 1705 (1972).

$\mathrm{H}_{3}^{+}+\mathrm{He} \rightarrow \mathrm{H}(3 \mathrm{~d})+\mathrm{H}_{2}^{+}+\mathrm{He}$ : J.C. Pord, P.M. McCoy, R. Conrads, and B.W. Thomas, Phys. Rer. A 5, 1705 (1972).

$\mathrm{H}_{3}^{+}+\mathrm{H}_{2}+\mathrm{H}(35)+\mathrm{H}_{2}^{+}+\mathrm{He}$ : R.H. Hughes, D.B. Kay, C.A. Stigers, and E.D. Stokes, Phys. Rev. 167, 26 (1968).

\section{Dotes:}

\section{Accuracy:}

Systematic error < 50\%. Randon error < $10 \%$. 


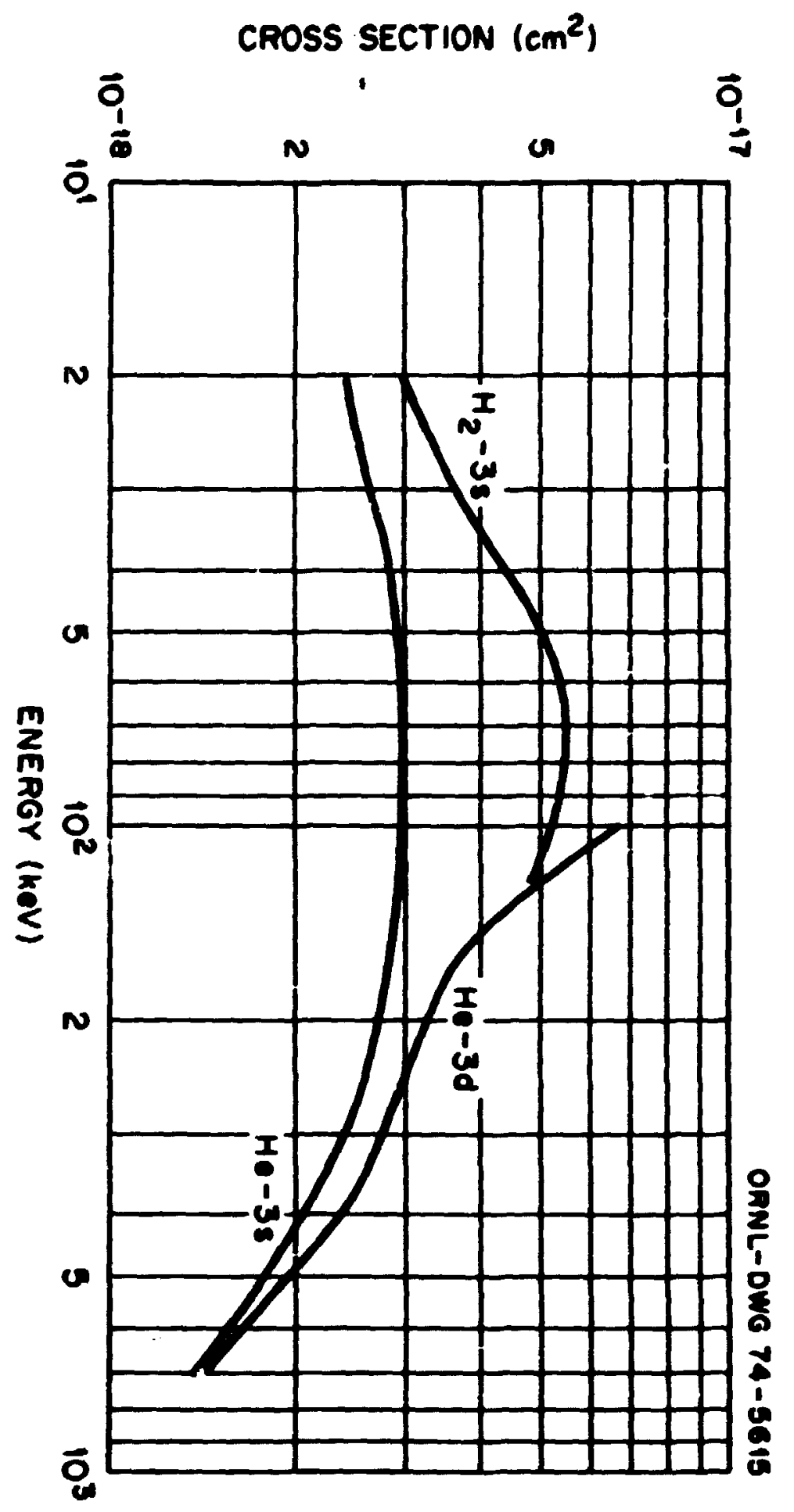




\section{A.2.56}

Excitetion Cross Sections for the Reaction

$$
\mathrm{H}_{3}^{+}+\mathrm{He}+\mathrm{H}_{3}^{+}+\mathrm{He}(\mathrm{nl})
$$

\begin{tabular}{|c|c|c|c|c|}
\hline \multirow[t]{2}{*}{$\begin{array}{l}\text { Deery } \\
\text { (kev) }\end{array}$} & \multicolumn{4}{|c|}{$\begin{array}{l}\text { Cross Sections for States al } \\
\qquad(\omega, 2)\end{array}$} \\
\hline & $4^{2} s$ & & $4^{3} s$ & $i^{3} p$ \\
\hline 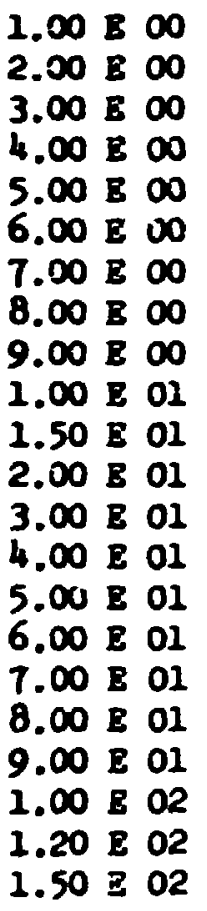 & 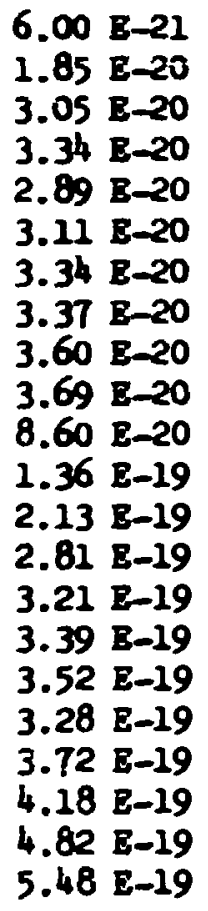 & $\begin{array}{ll}1.06 & E-19 \\
1.14 & E-19 \\
9.00 & E-20 \\
8.80 & E-20 \\
9.70 & E-20 \\
1.08 & E-19 \\
1.33 & E-19 \\
1.49 & E-19 \\
1.61 & E-19 \\
1.85 & E-19 \\
1.88 & E-19 \\
2.62 & E-19 \\
3.82 & E-19 \\
4.51 & E-19 \\
5.40 & E-19 \\
5.82 & E-19 \\
6.41 & E-19 \\
6.36 & E-19 \\
7.33 & E-19 \\
8.78 & E-19 \\
9.01 & E-19\end{array}$ & $\begin{array}{ll}2.00 & \mathrm{E}-21 \\
5.80 & \mathrm{E}-21 \\
1.71 & \mathrm{E}-20 \\
3.52 & \mathrm{E}-20 \\
2.82 & \mathrm{E}-20 \\
2.78 & \mathrm{E}-20 \\
3.71 & \mathrm{E}-20 \\
4.63 & \mathrm{E}-20 \\
4.83 & \mathrm{E}-20 \\
4.88 & \mathrm{E}-20 \\
1.10 & \mathrm{E}-19 \\
2.25 & \mathrm{E}-19 \\
3.65 & \mathrm{E}-19 \\
4.31 & \mathrm{E}-19 \\
4.88 & \mathrm{E}-19 \\
5.84 & \mathrm{E}-19 \\
6.27 & \mathrm{E}-19 \\
7.69 & \mathrm{E}-19 \\
7.30 & \mathrm{E}-19 \\
7.11 & \mathrm{E}-19 \\
6.50 & \mathrm{E}-19 \\
5.42 & \mathrm{E}-19\end{array}$ & $\begin{array}{ll}8.60 & E-21 \\
2.37 & E-20 \\
3.96 & E-20 \\
3.77 & E-20 \\
6.17 & E-20 \\
9.95 & E-20 \\
9.60 & E-20 \\
9.26 & E-20 \\
9.23 & E-20 \\
9.65 & E-20 \\
1.23 & E-19 \\
1.45 & E-19 \\
1.58 & E-19 \\
3.73 & E-19 \\
4.81 & E-19 \\
5.67 & E-19 \\
6.78 & E-13 \\
7.08 & E-19 \\
7.86 & E-19 \\
7.76 & E-19 \\
6.23 & E-19 \\
4.56 & E-19\end{array}$ \\
\hline
\end{tabular}

\section{Peferences:}

J. Ven den Bos, G. Hinter, and F. J. Defieer, Physice 44,143 (190í9).

3. H. Thomes end G. D. Bent, J. Opt. Soc. An. 53, 138 (1968).

\section{Notes:}

See lote (44) at end of chapter.

Accurecy: Systeantic error $<10 \%$. Random error $<5 \%$. 
A.2.57

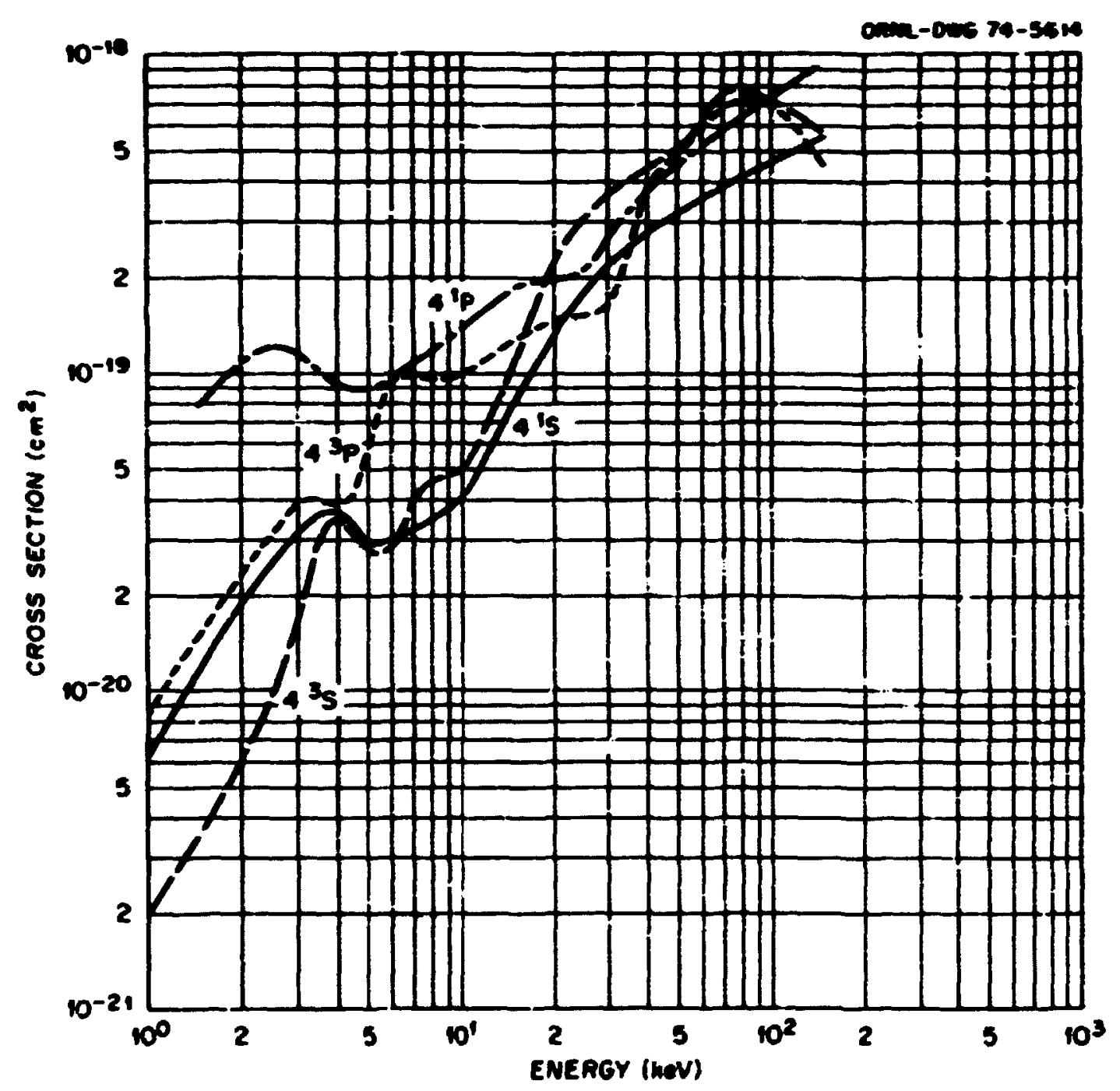


A. 2.58

Excitstion Cro is Sections for the Reaction

$$
\mathrm{He}^{+}+\mathrm{Z}^{+} \rightarrow \mathrm{He}^{+}+\mathrm{H}(2 \mathrm{O})
$$

\begin{tabular}{|c|c|}
\hline $\begin{array}{l}\text { Energy } \\
\text { (nev) }\end{array}$ & $\begin{array}{c}\text { Cross Section } \\
\left(\mathrm{cm}^{2}\right)\end{array}$ \\
\hline $\begin{array}{l}5.0 \mathrm{E}-01 \\
1.0 \mathrm{E} \quad \mathrm{Mb} \\
2.0 \mathrm{E} 00 \\
3.0 \mathrm{E} 00 \\
4.0 \mathrm{E} 00 \\
5.0 \mathrm{E} 00 \\
6.0 \mathrm{E} 00 \\
7.0 \mathrm{E} 00 \\
8.0 \mathrm{E} 00 \\
9.0 \mathrm{E} 00 \\
1.0 \mathrm{E} \mathrm{Cl} \\
1.5 \mathrm{E} 01 \\
2.0 \mathrm{E} 01 \\
2.5 \mathrm{E} 01 \\
3.0 \mathrm{E} 01\end{array}$ & $\begin{array}{l}9.0 \equiv-19 \\
2.0 \equiv-17 \\
3.0 \mathrm{E}-17 \\
3.3 \mathrm{E}-17 \\
3.5 \mathrm{E}-\mathrm{i} 7 \\
3.6 \mathrm{E}-17 \\
3.7 \mathrm{E}-17 \\
3.8 \mathrm{E}-17 \\
3.8 \mathrm{E}-17 \\
3.9 \mathrm{E}-17 \\
4.0 \mathrm{E}-17 \\
4.3 \mathrm{E}-17 \\
4.7 \mathrm{E}-17 \\
4.8 \mathrm{E}-17 \\
4.0 \mathrm{E}-17\end{array}$ \\
\hline
\end{tabular}

References:

S.J. Youne, J.S. Murray, and J.R. Sheriden, Phys. Rev. 178, 40 (1969).

\section{Eotes:}

See lote (38) at end of chapter.

Accuracy:

Systematic error $-<50 \%$. Raniom error $<10 \%$. 
A.2. 59

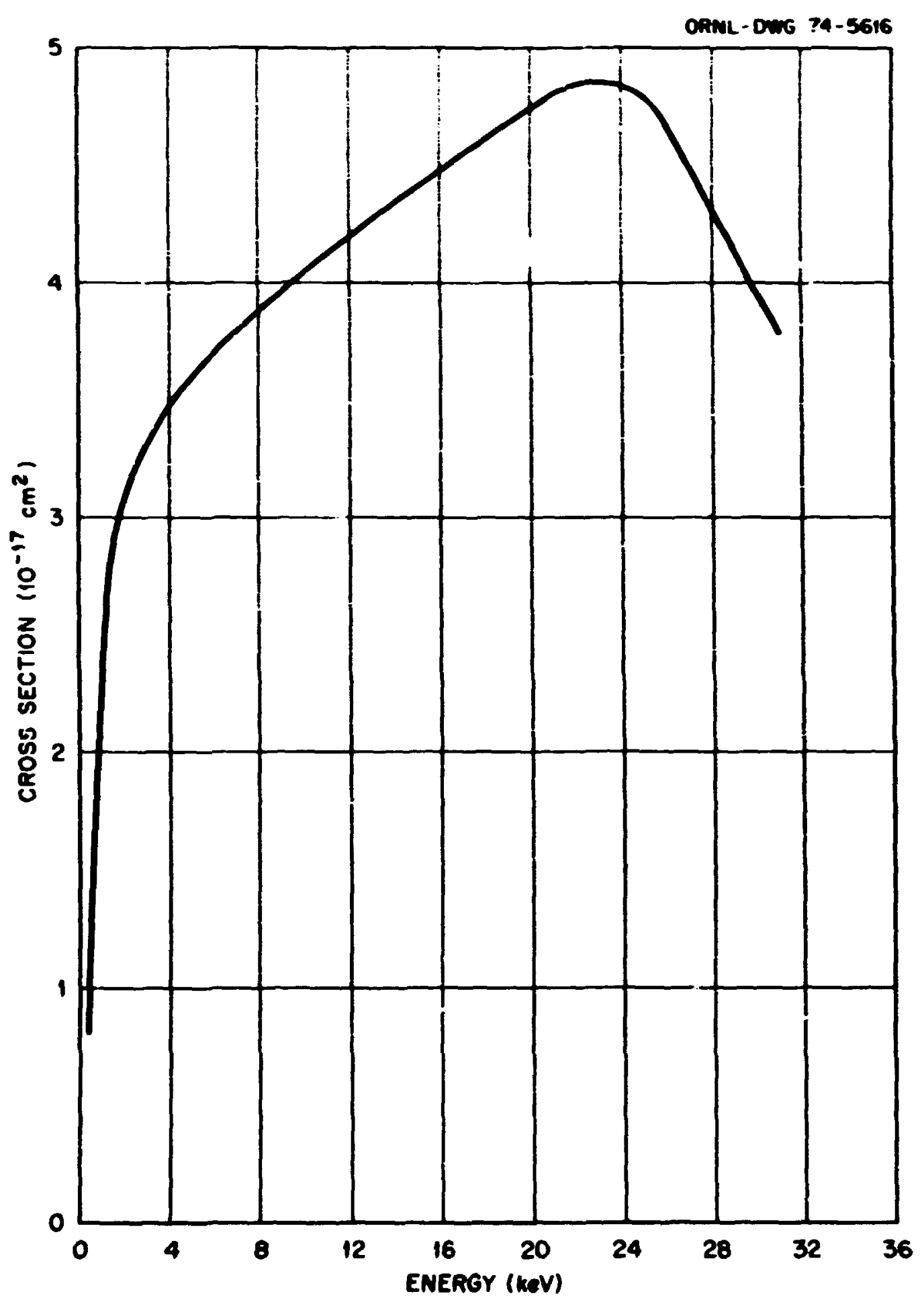




\section{A.2.60}

Cross Sections for Belmer-Mipha Jaission from the Impect of

$$
\mathrm{He}^{+} \text {and } \mathrm{He}^{\circ} \text { on } \ddot{z}_{2} \text { Gas }
$$

\begin{tabular}{|c|c|c|}
\hline \multirow[t]{2}{*}{$\begin{array}{l}\text { Ineror } \\
\text { (keV) }\end{array}$} & \multicolumn{2}{|c|}{$\begin{array}{c}\text { Cross Section } \\
\left(\mathrm{cm}^{2}\right)\end{array}$} \\
\hline & $\mathrm{He}^{+}+\mathrm{H}_{2}$ & $\mathrm{He}^{\circ}+\mathrm{I}_{2}$ \\
\hline $\begin{array}{l}1.0 \mathrm{E}-01 \\
1.5 \mathrm{E}-01 \\
2.0 \mathrm{E}-01 \\
3.0 \mathrm{E}-01 \\
4.0 \mathrm{E}-01 \\
6.0 \mathrm{E}-01 \\
8.0 \mathrm{E}-01 \\
1.0 \mathrm{E} 00 \\
1.5 \mathrm{E} 00 \\
2.0 \mathrm{E} 00 \\
3.0 \mathrm{E} 00 \\
4.0 \mathrm{E} 00 \\
6.0 \mathrm{E} 00 \\
8.0 \mathrm{E} 00 \\
1.0 \mathrm{E} 01 \\
1.5 \mathrm{E} \quad 01 \\
2.0 \mathrm{E} 01 \\
3.0 \mathrm{E} 01\end{array}$ & $\begin{array}{ll}4.6 & E-18 \\
5.4 & \mathrm{E}-18 \\
7.4 & \mathrm{E}-18 \\
6.0 & \mathrm{E}-18 \\
5.0 & \mathrm{E}-18 \\
3.8 & \mathrm{E}-18 \\
3.1 & \mathrm{E}-18 \\
2.7 & \mathrm{E}-18 \\
2.2 & \mathrm{E}-18 \\
2.1 & \mathrm{E}-18 \\
2.0 & \mathrm{E}-18 \\
1.9 & \mathrm{E}-18 \\
2.1 & \mathrm{E}-18 \\
2.2 & \mathrm{E}-18 \\
2.3 & \mathrm{E}-18 \\
2.7 & \mathrm{E}-18 \\
3.1 & \mathrm{E}-18 \\
3.5 & \mathrm{E}-18\end{array}$ & $\begin{array}{ll}6.4 & \mathrm{E}-19 \\
7.8 & \mathrm{E}-19 \\
8.7 & \mathrm{E}-19 \\
1.0 & \mathrm{E}-18 \\
1.2 & \mathrm{E}-18 \\
1.4 & \mathrm{E}-18 \\
1.5 & \mathrm{E}-18 \\
1.7 & \mathrm{E}-18 \\
1.8 & \mathrm{E}-18 \\
1.9 & \mathrm{E}-18 \\
1.9 & \mathrm{E}-18 \\
1.8 & \mathrm{E}-18 \\
1.6 & \mathrm{E}-18\end{array}$ \\
\hline
\end{tabular}

\section{Reference:}

V.A. Guser, G.X. Poljakov, V.P. Brko, Ya. M. Ponel, and A.V. Zats, Sixth International Conference on the Physics of Electronic and Atonic Collisions: Abstract of Papers, Cambridge, Mass. p. 809, MIT Press, 1969.

\section{Motes:}

See lote $(40)$ at end of chapter. 
R.2.6i

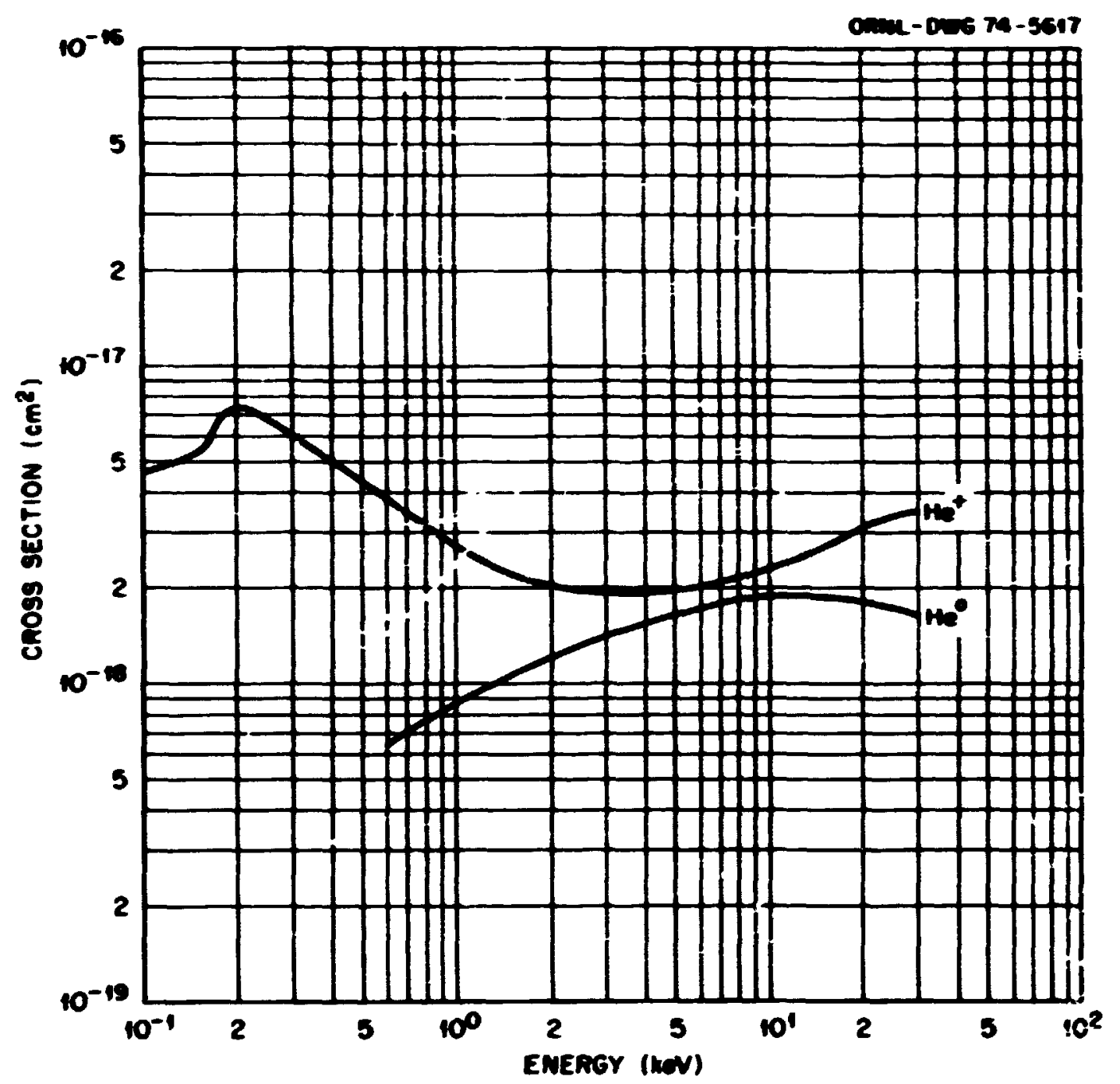




$$
\text { A. } 2.62
$$

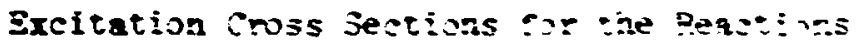

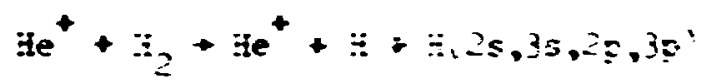

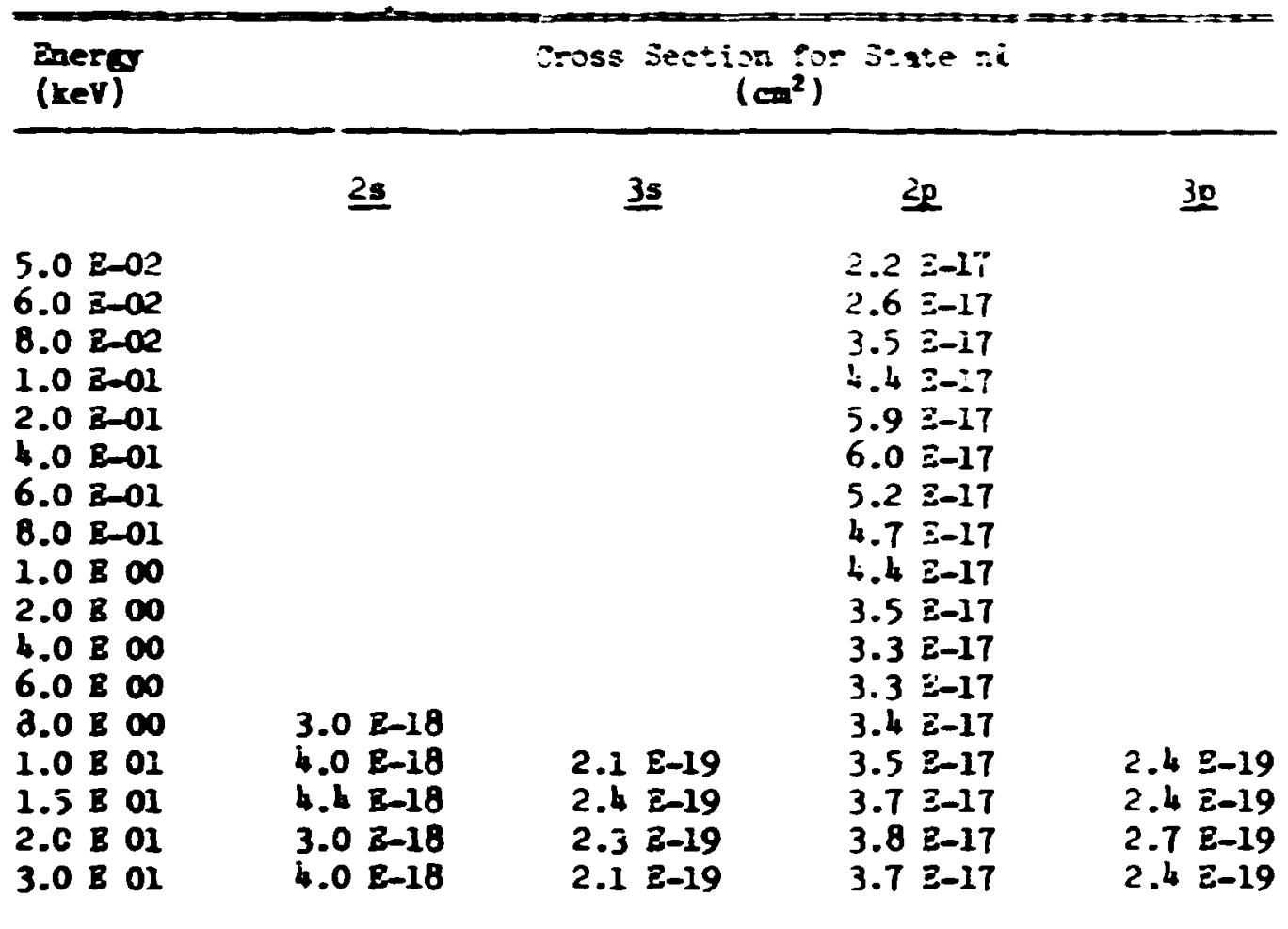

\section{Pererenses:}

$\mathrm{He}^{+}+\mathrm{H}_{2} \rightarrow \mathrm{He}^{+}+\mathrm{H}+\mathrm{H}(2 \mathrm{~s}):$ E.P. Andreev, V.A. :-2udinor, and S.V. Bobasher, Pifth Intermational Conference on the Physics of Elertronic - Atcalc Collistons: Abstract of Papers, p.3\%, Publishing House Nauka, Lenirgred, USSR (1967).

$\mathrm{Be}^{+}+\mathrm{B}_{2}+\mathrm{He}^{+}+\mathrm{H}+\mathrm{B}(3 \mathrm{~s}):$ V.A. Ankudinor, S.V. Bobasher, and E.P. Aadreer, Sorlet PhJs-JEYP 25, 236 (1967).

$\mathrm{He}^{+}+\mathrm{H}_{2} \rightarrow \mathrm{He}^{+}+\mathrm{H}+\mathrm{H}(2 \mathrm{p}):$ B. Van $\mathrm{zyl}, \mathrm{D}$. Jeecks, D. Pretzer, and R. Coballe, Phrs. Rer. 158, 29 (1967).

$\mathrm{He}^{+}+\mathrm{B}_{2}+\mathrm{He} e^{+}+\mathrm{H}+\mathrm{B}(3 \mathrm{p}):$ E.P. Andreer, V.A. Ankudinor, and S.V. Bobanher, Fifth Interntionsl Conference on the Phrsics of Elestronic - Atonle Collisions: Abstrect of Papers, p.309, Publishing House Nauke, Leningred, UBSR (1967); V.A. Ankudinov, S.V. Bobasher, and R.P. Andreev, 8oviet Plys:-JEPP 25, 236 (1967).

Iotes:

see llotes (32), (33), (35), and (39) at end of chepter. secureer:

Systemat1c error < 508. Radion error $<102$. 


\section{A.2.63}

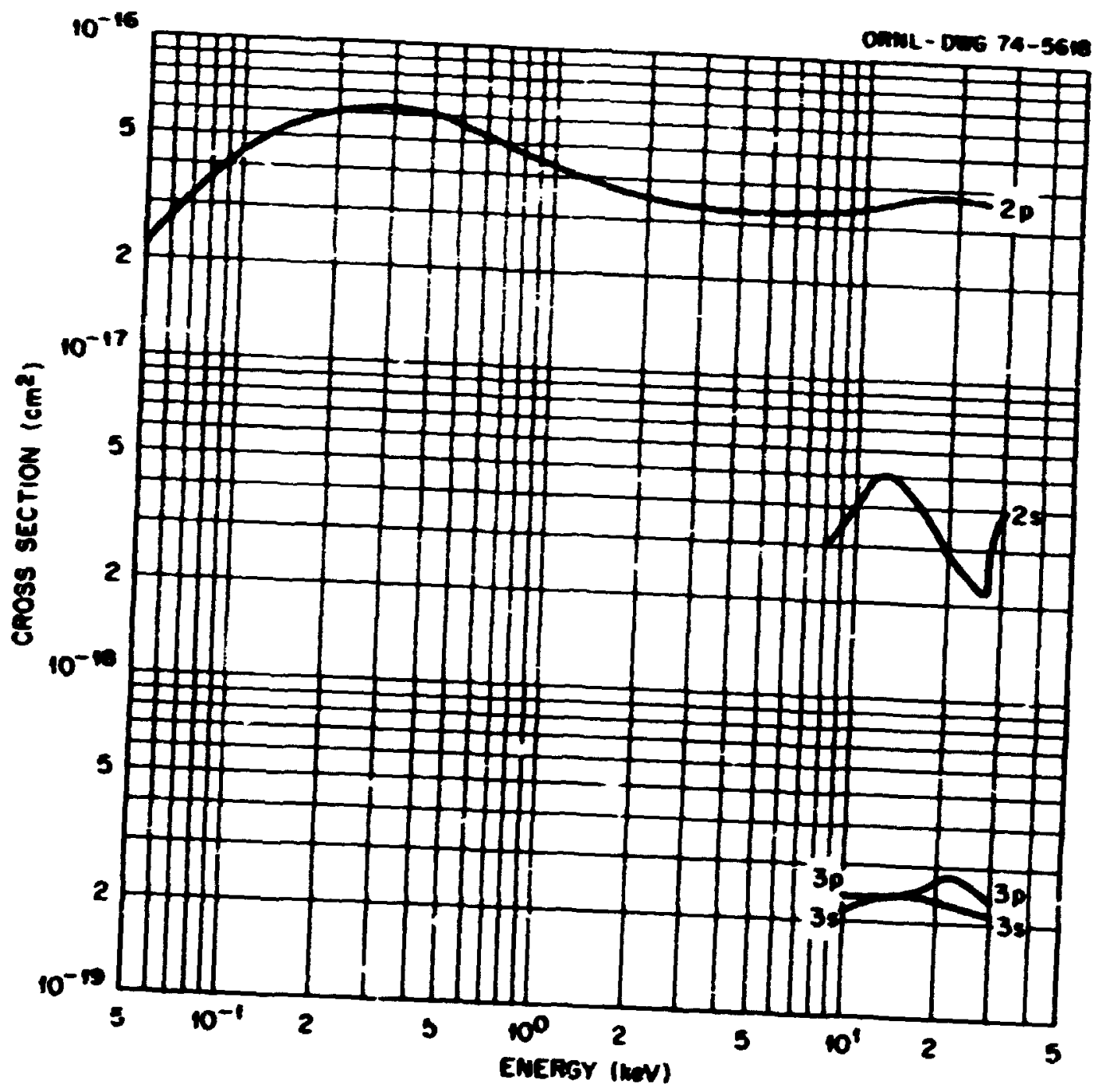




$$
\text { A.2.64 }
$$

Bxcitation Cross Sections for the Reactions

$$
\mathrm{He}^{+}+\mathrm{He} \rightarrow \mathrm{He}^{+}+\mathrm{He}^{+}\left(4^{1} \mathrm{~S}, 4^{2}=4^{1} \mathrm{D}\right)
$$

\begin{tabular}{|c|c|c|c|}
\hline $\begin{array}{l}\text { Berers } \\
\text { (kev) }\end{array}$ & Cross Sections & $\begin{array}{l}5 \text { for Excited State } \\
\left(c^{2}\right)\end{array}$ & ne \\
\hline & $4^{2} s$ & $4^{1} \mathbf{P}$ & $4^{1} D$ \\
\hline 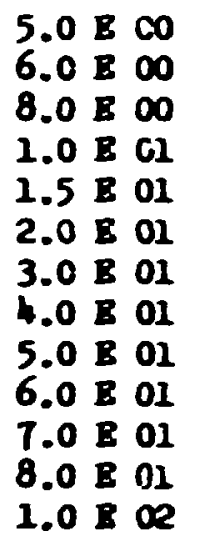 & $\begin{array}{ll}2.2 & \mathrm{E} 20 \\
2.6 & \mathrm{E}-20 \\
3.7 & \mathrm{E}-20 \\
4.9 & \mathrm{E}-20 \\
3.5 & \mathrm{E}-20 \\
4.0 & \mathrm{E}-20 \\
4.9 & \mathrm{E}-20 \\
7.8 & \mathrm{E}-20 \\
9.9 & \mathrm{E}-20 \\
8.7 & \mathrm{E}-20 \\
7.2 & \mathrm{E}-20 \\
6.8 & \mathrm{E}-20 \\
6.6 & \mathrm{E}-20\end{array}$ & $\begin{array}{ll}3.0 & E-19 \\
3.0 & E-19 \\
2.6 & R-19 \\
3.3 & E-19 \\
3.0 & E-19 \\
2.6 & E-19 \\
2.4 & E-19 \\
2.2 & E-19\end{array}$ & \begin{tabular}{ll}
5.3 & $E-20$ \\
5.6 & $E-20$ \\
6.5 & $E-20$ \\
$T . T$ & $E-20$ \\
\hdashline .9 & $E-20$ \\
1.1 & $E-19$ \\
2.8 & $E-19$ \\
2.9 & $E-19$ \\
2.0 & $E-19$ \\
1.2 & $E-19$ \\
8.1 & $E-20$ \\
7.3 & $E-20$ \\
6.7 & $E-20$
\end{tabular} \\
\hline
\end{tabular}

\section{Beference:}

F. J. de Beer and J. Vea den Bos, Pursica 31, 365 (1965).

\section{Dotes:}

See Dote (Wh) at ead of chryter.

sceureg:: Systentic error < 10\%. Randon error < $5 \%$. 


\section{2 .65}

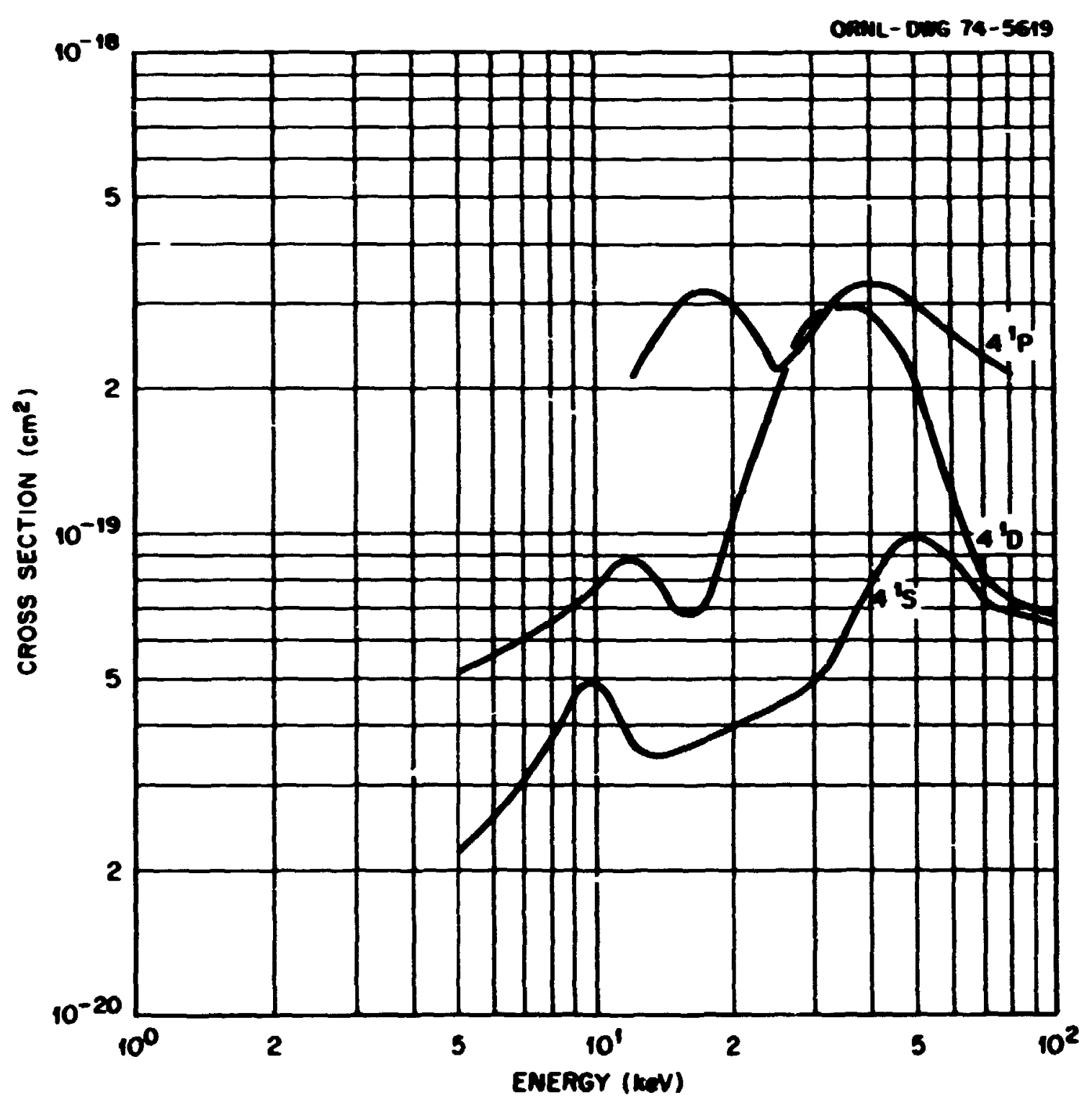




\section{A. 2.66}

Excitation Cruss jections :ur the Reartions

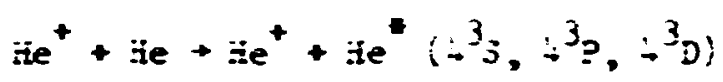

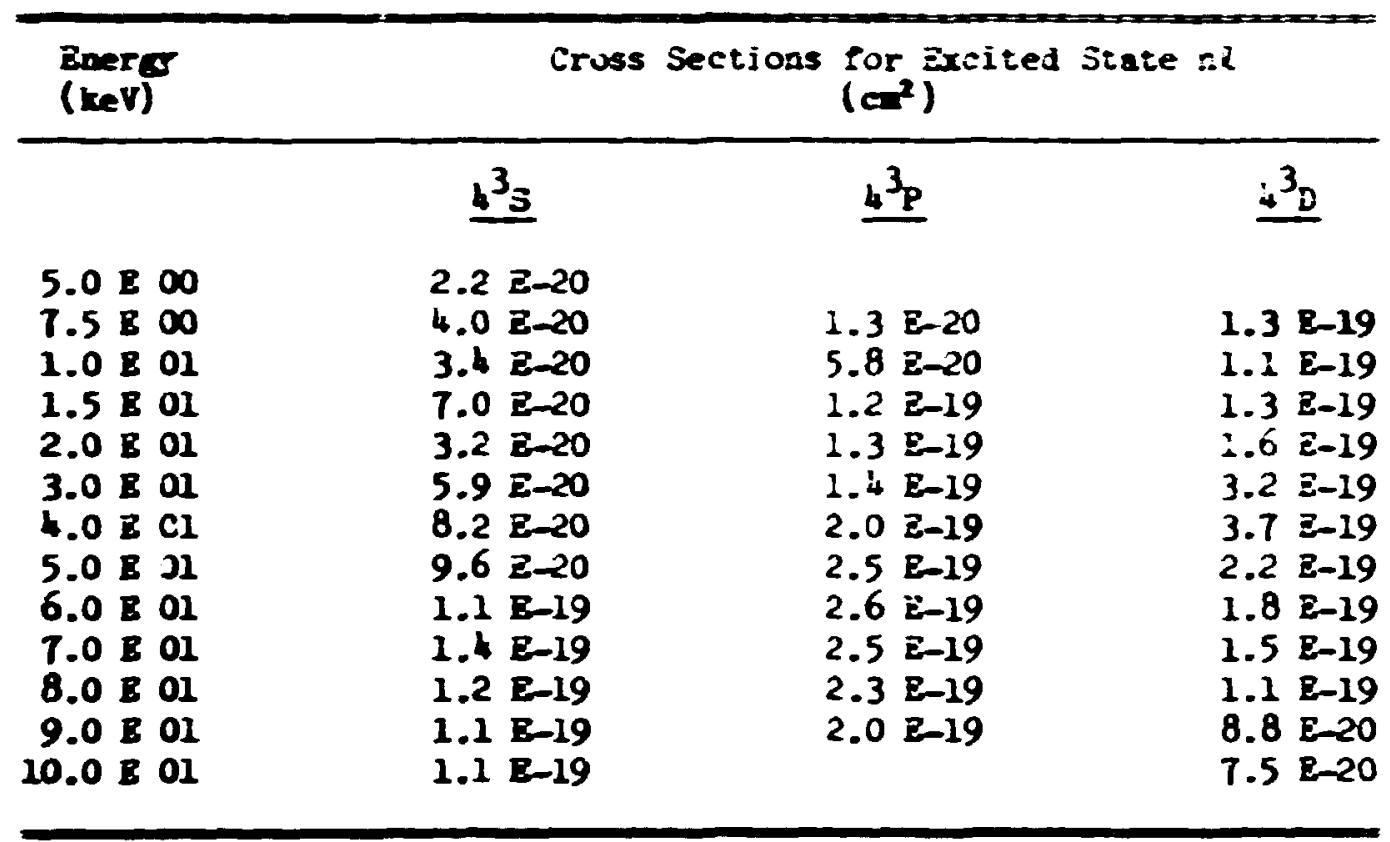

\section{Beferences:}

P. J. de lleer and J. Van ten Bos, Phusice 31, 365 (1965).

\section{Dotes:}

see tote (44) at end of chapter.

Accurag: : Systeantic error $<10 \%$. Randon error $<5 \%$. 


\section{A.2.67}

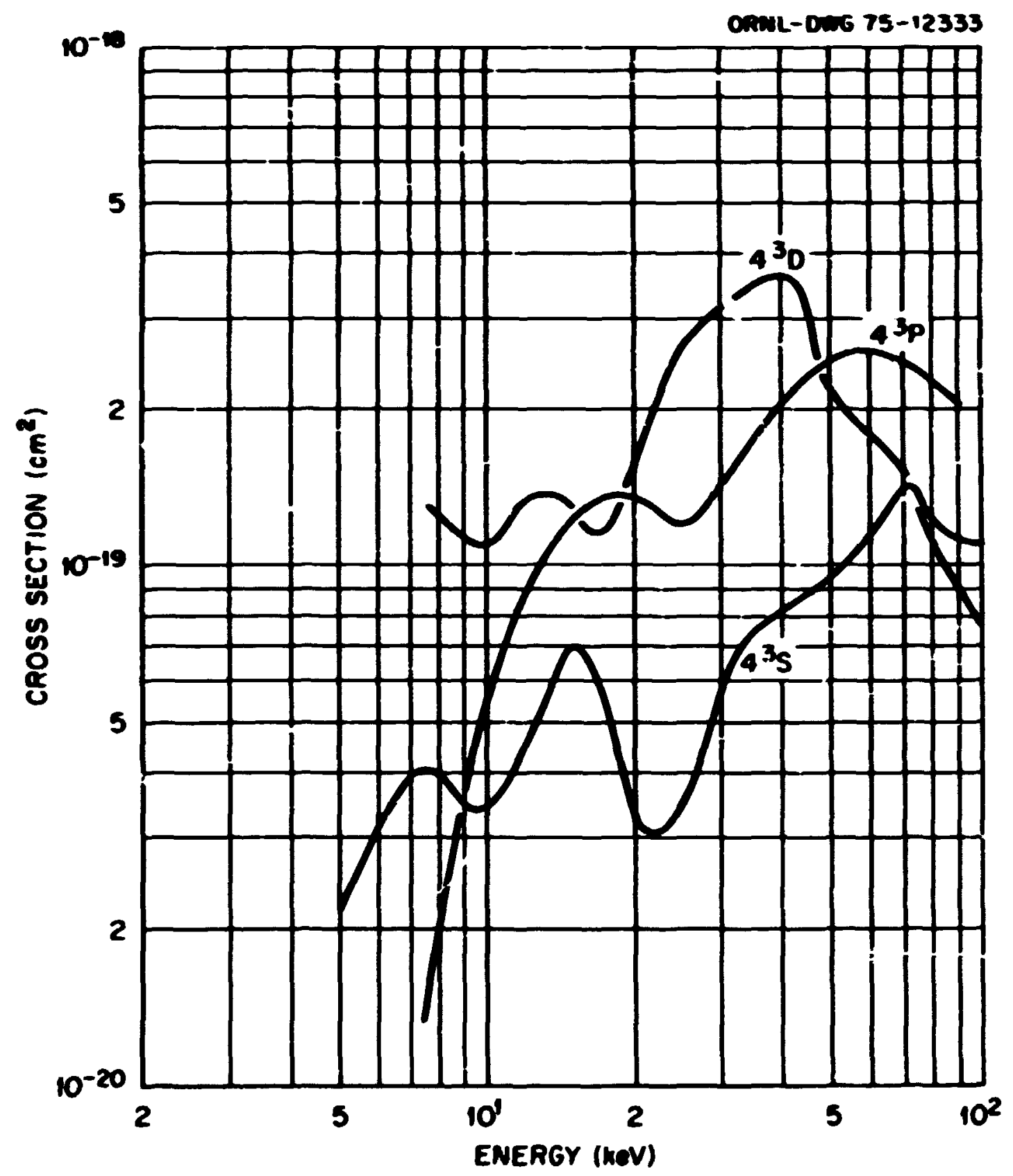




\section{A.2.69}

iotes

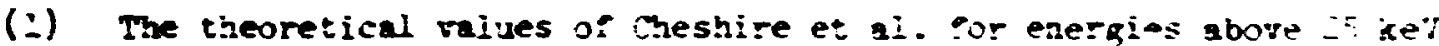
are presented sefarateiy. Althog.: not yet son ingei by experises. at high energies, they to provite a useri getiol for esting: ina eross sections at high energies.

(2) The experisental data represent cross sections for emission 0 : Irman-elphe radiation. They are equal to the sposs seat:on or formatica of the level only if cascele can be negiected; for the 2s level this is probably true bu: for the 20 ievel it any je in error.

(3) The theoretical ralues of Cheshire sre oreserted for energies greater than $25 \mathrm{keV}$. Consuit this paper for theoretical values of eross sections above $100 \mathrm{keV}$. Although not yet confirmed by experiment at these higher energies. they do provide a userul wetbod for estimating cross sections at bigh energies.

(4) The experimentel date represent cross sections for the formtion of the 2s and $2 p$ levels coly if cascade into these levels can be neclected. Mron, et 1. ., have estinated that cascade contribution to the aeasured cross section is less than 17 orer their exeres renge.

(5) In 2 il cases these cross sections have been deduced on the (unsubstantiated assuaption that cascade into the relevat levels can be neglected. With the exception of the 2p level this assumption is justified; there is insufficient date to permit a sorrection for the $2 p$ level.

(6) There is good evidence [R. H. Hughes et al. Phys. Rev. A, 1 1 24 (1970)] that cross sections for ns states decreace as $n^{-3}$ at energies above $60 \mathrm{ker}$; this fact may be utilized to predict cross sections for higher a states.

(7) In all cases these cross sections have been deduced on the (unsutstantiated) assurption that cascade into relevant states can be neglected. For the ns states this assumption is justified.

(8) The 2 s notation represents the cross sections for field induced emissior of Lyman-alpha emission $(2 s \rightarrow 2 p \rightarrow 18)$. If cascade can be neglected this is equal to the cross section for H(2s) formation.

(9) The 2p notation represents the cross sections for Lywan-alpia emission. If cascade can be neglected then this is equal to the cross section for $H(2 p)$ formetion.

(10) The 3p cross sections are for $\mathrm{H}(3 \mathrm{p})$ formation deduced from emission measurements on the (unsubstantiated) assumption that cascade can be neglected.

(11) The $1606 R$ band is the Lywan band of $H_{2}\left[B^{\prime} \varepsilon_{u}^{+} \rightarrow x^{\prime} \Sigma_{q}^{+},(4,11),(5,12)\right.$, and $(6,13) \mathrm{J}$. 
(12) The $4180 R$ band of $H_{2}$ is for the transitions $\left[3 d l_{g}+2 p I_{\Sigma_{u}^{+}}^{+}(1,0)\right]$.

(13) Date on polerization of emissions can be found in R. H. Hughes et al., Phys. Rer. A I, 1424 (1970) and in T. D. Baily et al., Phys. Rer. 167, 31 (1968).

(14) Some of these measurements have been done with $\mathrm{D}^{+}$projectiles. In these cases the cross section for $D^{+}$impact appears to be the same as for $\mathrm{H}^{+}$projectiles of the same velocity.

(15) There is goot evidence [R. H. Hughes et al., Phys. Rev. 164, 166 (1967)] that cross sections for ns states deerease as $\mathrm{n}^{-\frac{1}{a}}$ at energies above $\partial 0 \mathrm{keV}$. This fact way be utilized to predict cross sections for higher n states.

(16) In all cases the cross sections have been deduced on the (unsubstantiated) assumption that cascade into relerant levels can be neglected.

(17) It is generally found that the cross section for formation of H(ns) at energies graater than $80 \mathrm{keV}$ decreases as $\mathrm{n}^{-3}$; this ruie may be used to extrapolate to other ns levels.

(18) These data are for formation of all states having a given principal quantum nuber $a$. It is known that the cross section $\sigma(n)$ for formation of such a state is proportional to $\mathrm{n}^{-3}$. It is conventional to determine the coefricient $\sigma(n) \times n^{3}$, and it is this coefficient that is given here.

(19) The data presented here have been measured ior states ranging between 10 and 15 .

(20) These data are for formation of all states having a princlpal quantion number $n$. It is known that the cross section $\sigma(n)$ for for formation of such a state is proportional to $n^{-3}$. It is conventional to determine the coefficient $\sigma(n) \times n^{-3}$; it is that coefficient thet is given here.

(21) Il'in et al. also present data for a Li vapor target over the same energy range.

(22) These data are theoretical predictions. For experimental data obtained since the graph was made see T. J. Morgan, J. Geddes, and H. B. Gilbody, J. Phys. B I, 142 (J.974). 
(23) These data are derived from enissior measurements on the (wasubstantiated) assuaption that cascede into the excited state ay be neglected. This assu ption probably does not involve an error of $10 \%$ in wost cases.

(24) These jate ars derived rron enission measurenents on the (unsubstantiated) assuption that cascade into the excited state ma be neglected. There is no evidence vith which the validity of the assumption can be tested.

(25) For $\mathrm{H}(2 \mathrm{~s})$ and $\mathrm{H}(2 \mathrm{p})$ foration, we have used a theoretical calculation by Levy at energies of $100 \mathrm{keV}$ and greater. These salculations have nct been confirned experimertally above 100 keV but ars expected to be reliable.

(26) These date are derived from enission measurenents on the assurption that cascade into the excited state nay be neglected. With the exception of the 2p level, this is justified and in tbis case the resulting error does zot exceed $10 \%$.

(27) These data are experimental values from a single publication; no confirming data from other sources are available.

(28) There are no theoretical data available to perait extension of the energy range or to include other states.

(29) These cross sections are for enission of Lyman-alpha radiation ind were quoted as equal to the level excitation cross sections on the (unsubstantiated) assumption that cascade could be neglected.

(30) According to Ford et al. [Phys. Rev. A I, 1928 (1973)], the cross sections for $H(3 p)$ formation are approximately the same as for $H(3 s)$ and $H(3 d)$ fornation above $75 \mathrm{keV}$.

(31) These cross sections were derived from enission mesurements on the (unsubstantisted) assumption that cascade nay be neglected. In all cases this is probably a good assumption.

(32) The excitation cross sections for H(2s) are determined frcm the field induced entssion of Lyman-alpha $(2 s \rightarrow 2 p \rightarrow 1 s)$. If cascade can be neglested these are equal to the cross section for $H(2 s)$ formation.

(33) The excitation cross sections for $\mathrm{H}(2 \mathrm{p})$ are the cross sections for Lyman-alpha $(2 p \rightarrow 18)$ emission. If cascade can be neglected then these are equal to the cross sections for $H(2 p)$ formation.

(34) The excitation cross sections for $\mathrm{H}(3 \mathrm{p})$ are emission cross sections, and if cascade can be neglected these are equal to the cross sections for $H(3 p)$ formation.

(35) The $n=3+2$ transition is the $6563 \&$ Belmer-alphe radiation. 
(36) There are date for $\mathrm{D}_{2}^{+}$inpact by Van $\mathrm{zyl}$ et al. Phys. Rev. A 136, 1561 (1969) that are in good agreement with these data for $H(2 p)$ formation when plotted on the seme relocity scale.

(37) Accordiug to Ford et $\varepsilon$. [Phys. Rev. A S, 1705 (1972)] the cross section for $\mathbf{I}(3 p)$ foration is approxizately the same as that for $\mathrm{H}(3 \mathrm{~s})$ and $\mathrm{H}(3 \mathrm{~d})$ formation at energies above $75 \mathrm{keV}$.

(38) This cross section is deterwined from enission meesurements on the (unsubstantiated) assuription that cascade way be neglected.

(39) The $H(3 s)$ and $H(3 p)$ cross sections are deduced from emission measurements on the (unsubstantiated) assumption that cascade may be neglected.

(40) The Balmer-alpha enission is the $n=3 \rightarrow 2$ transition (6563 \&).

(41) Data for other excited states are available in the reference cited and are also tabulated by Thomas (Excitation in Heavy Particle Collisions; Wiley-Interscience, Hew York, 1972, Ghapter 6). Also Given by Thosas are otiner data for these same states that are in general agreesent with the values adopted here.

(42) Sone date abure $75 \mathrm{keV}$ impact energy have been taken for $\mathrm{D}^{+}$impact. These indicate that $\mathrm{D}^{+}$behaves the same as $\mathrm{H}^{+}$ions of the same energy.

(43) Excitation by triplet states by proton impact is formally forbidden because electron spin is not conserved. There are no reliable measurenents that indicate this rule to be violated.

(44) Data for other excited states are arailable in the reference cited and are also tebulated by Thomas (Excitation in Heevy Particle Collisions, Wiley-Interscience, iew York, 1972, Chapter 6).

(45) It is generally found that the cross section for formation of Híns) at energies above 80 kej decreases as $n^{-3}$; this rule may be used to extrapolate to other ns levels.

(46) Some measurements with Incident $\mathrm{D}^{+}$are available. In general these cross sections are the same as for $\mathrm{H}^{+}$projectiles of the same velocity.

(47) In our ofinion the date for $C s$ and $K$ may be unreliable in magnitude; other estimates of the cross section for cs targess are up to two orders of magnitude less than those shown here. The relative magnitudes should be reliable. 


\section{A.3 Heavy Particle Diseociation}




\section{A.3.2}

Cross Sections for the Coaversion of Fust $\mathrm{H}_{2}^{+}$Ions Into Protons in Passage Through $\mathrm{H}_{2}$ and $\mathrm{H}$

\begin{tabular}{|c|c|c|}
\hline \multirow[t]{2}{*}{$\frac{a x_{1}}{0+n}$} & \multicolumn{2}{|c|}{$\begin{array}{c}\text { Croes Section } \\
\left(c^{2}\right)\end{array}$} \\
\hline & $\underline{\mathbf{B}}$ & $\mathrm{B}_{2}$ \\
\hline 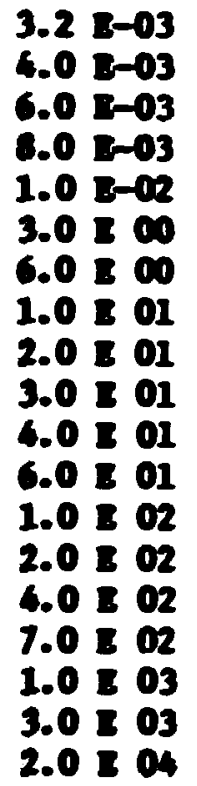 & $\begin{array}{ll}1.6 & \mathrm{E}-16 \\
2.1 & \mathrm{E}-16 \\
2.4 & \mathrm{E}-16 \\
2.3 & \mathrm{E}-16 \\
2.0 & \mathrm{E}-16 \\
1.9 & \mathrm{E}-16 \\
1.8 & \mathrm{E}-16 \\
1.6 & \mathrm{E}-16\end{array}$ & $\begin{array}{ll}3.3 & \mathrm{E}-17 \\
6.6 & \mathrm{E}-17 \\
8.8 & \mathrm{E}-17 \\
8.6 & \mathrm{E}-17 \\
8.0 & \mathrm{E}-17 \\
2.1 & \mathrm{E}-16 \\
2.4 & \mathrm{E}-16 \\
2.4 & \mathrm{E}-16 \\
2.1 & \mathrm{E}-16 \\
1.9 & \mathrm{E}-16 \\
1.9 & \mathrm{E}-16 \\
2.2 & \mathrm{E}-16 \\
2.3 & \mathrm{E}-16 \\
1.8 & \mathrm{E}-16 \\
1.2 & \mathrm{E}-16 \\
8.0 & \mathrm{E}-17 \\
6.3 & \mathrm{E}-17 \\
1.4 & \mathrm{E}-17 \\
4.9 \mathrm{E}-18\end{array}$ \\
\hline
\end{tabular}

Diterenes:

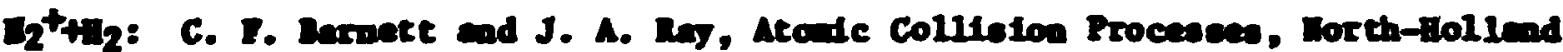
Iro1. Co., Amererdm, p. 743 (1964); K. R. Bertwar, S. H. Toplen, R. V. Pyle, ad J. W. Stemen, Fhys. Rev. 146, 91 (1966); J. Gaddint, C. R. Acad. Set.

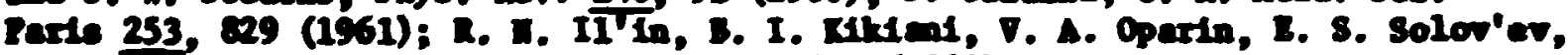

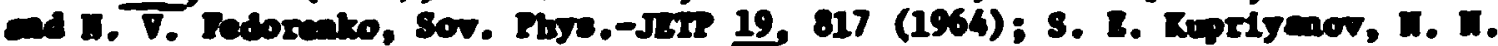

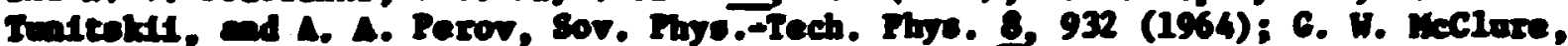
The. Rov. 130, 1832 (1963); T. T. Horen ad J. R. Roberte, J. Chen. Physe. 49,

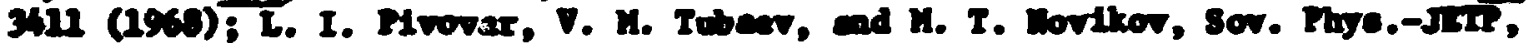
13. 23 (1961); A. Schald, Z. Ftys. 161,550 (1961); L. 8. Solor'ev, R, I. II'In,

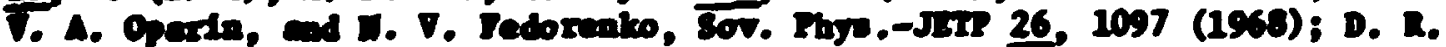

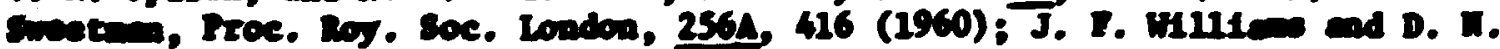
J. Duabr. Thys. Rev. 149, 62 (1965).

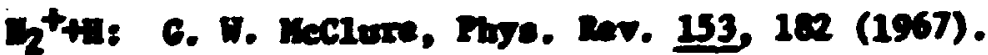

\section{searres:8}

$\$ 253$. 
A. 3.3

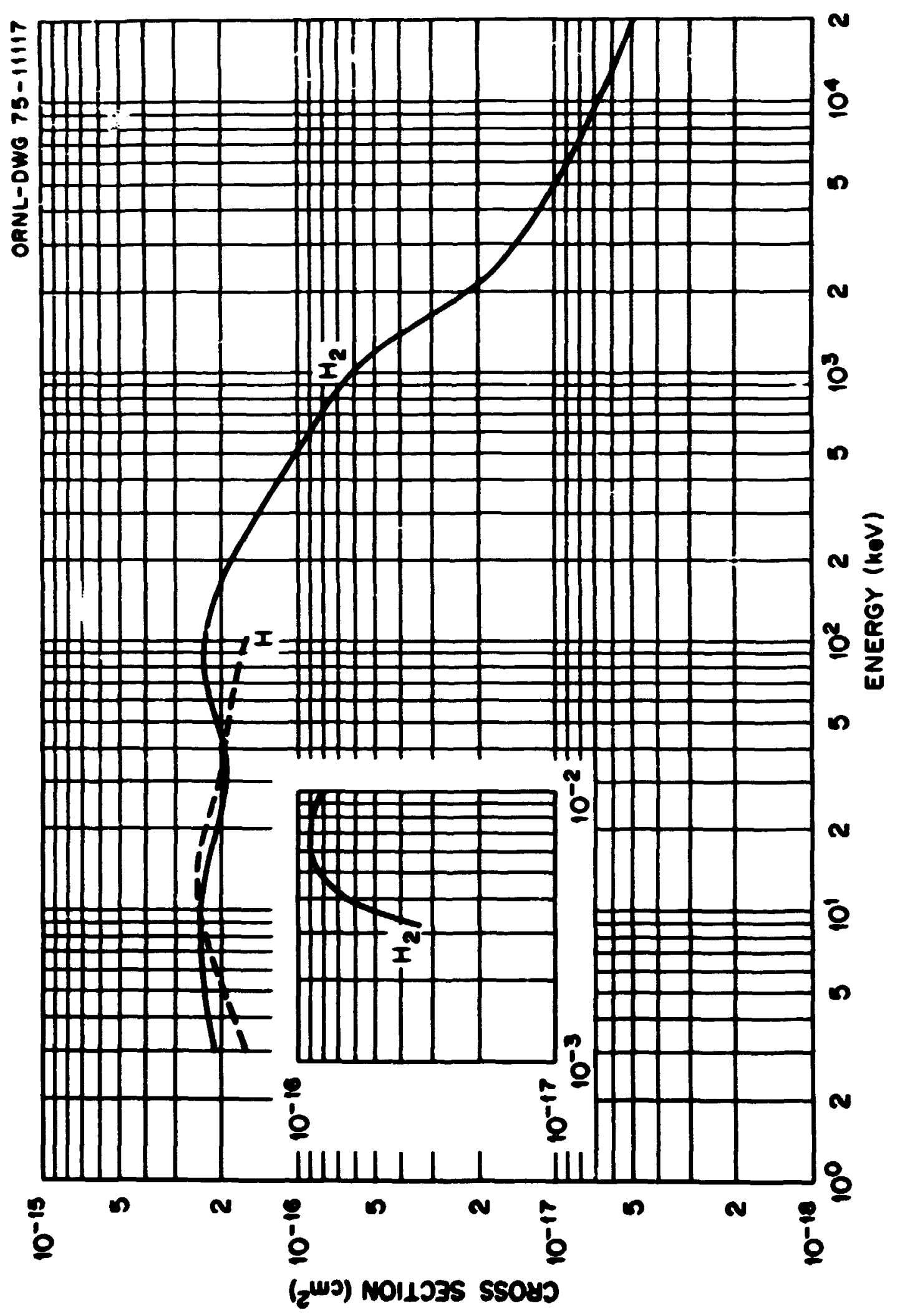




\section{A.3.4}

Diseociation Cross Sections for the Pormation of Past

$\mathrm{H}^{+}$and $\mathrm{B}^{\circ}$ for $\mathrm{H}_{2}$ Nolecules in $\mathrm{H}_{2}$

\begin{tabular}{|c|c|c|}
\hline \multirow[t]{2}{*}{$\begin{array}{l}\text { merss } \\
\text { (teen) }\end{array}$} & \multicolumn{2}{|c|}{$\begin{array}{c}\text { Croas Section } \\
\left(\mathrm{cm}^{2}\right)\end{array}$} \\
\hline & $\sigma\left(t^{+}\right)$ & $\sigma\left(\mathbf{H}^{\bullet}\right)$ \\
\hline $\begin{array}{l}6.0 \text { E } 00 \\
1.0 \text { E } 01 \\
1.5 \\
2.0 \\
2.0 \\
4.0 \\
\text { E E } 01 \\
6.0 \text { E } 01 \\
9.0 \text { E } 01 \\
3.0 \text { E } 02 \\
6.0 \text { E } 02 \\
9.0 \text { E } 02 \\
1.2 \text { E } 03\end{array}$ & $\begin{array}{ll}2.4 & \mathrm{E}-17 \\
3.8 & \mathrm{E}-17 \\
3.6 & \mathrm{E}-17 \\
4.3 & \mathrm{E}-17 \\
6.7 & \mathrm{E}-17 \\
6.7 & \mathrm{E}-17 \\
3.5 & \mathrm{E}-17 \\
6.3 & \mathrm{E}-17 \\
3.1 & \mathrm{E}-17 \\
2.4 & \mathrm{E}-17 \\
2.2 & \mathrm{E}-17\end{array}$ & $2.4 \mathrm{E}-16$ \\
\hline
\end{tabular}

\section{Bafereaces:}

C. W. Feclure, Ptyse. Rev. 134, 11226 (1964); R. H. Berloner, T. J. Horgan,

R. V. Fyle, and J. H. Stearns, Phys. Rev. A 8, 2870 (1973).

\section{Accureg:}

Denoroun.

\section{Eotes:}

The 10 w consy dete is fron KeClure's paper, whlle the high energy data is taken fron Berkner et al.

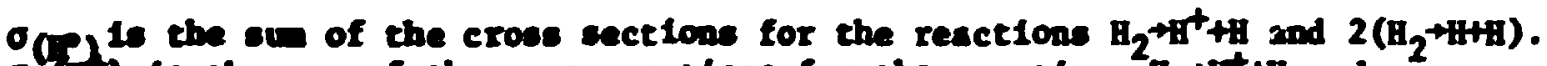
of(i) 18 the oun of the cross section for the reactions $\mathrm{H}_{2}+\mathrm{H}^{+}+\mathrm{H}$ and $2(12+2+1+2)$. 
A.3.5

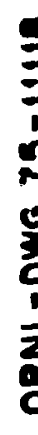

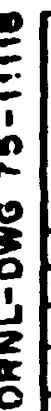
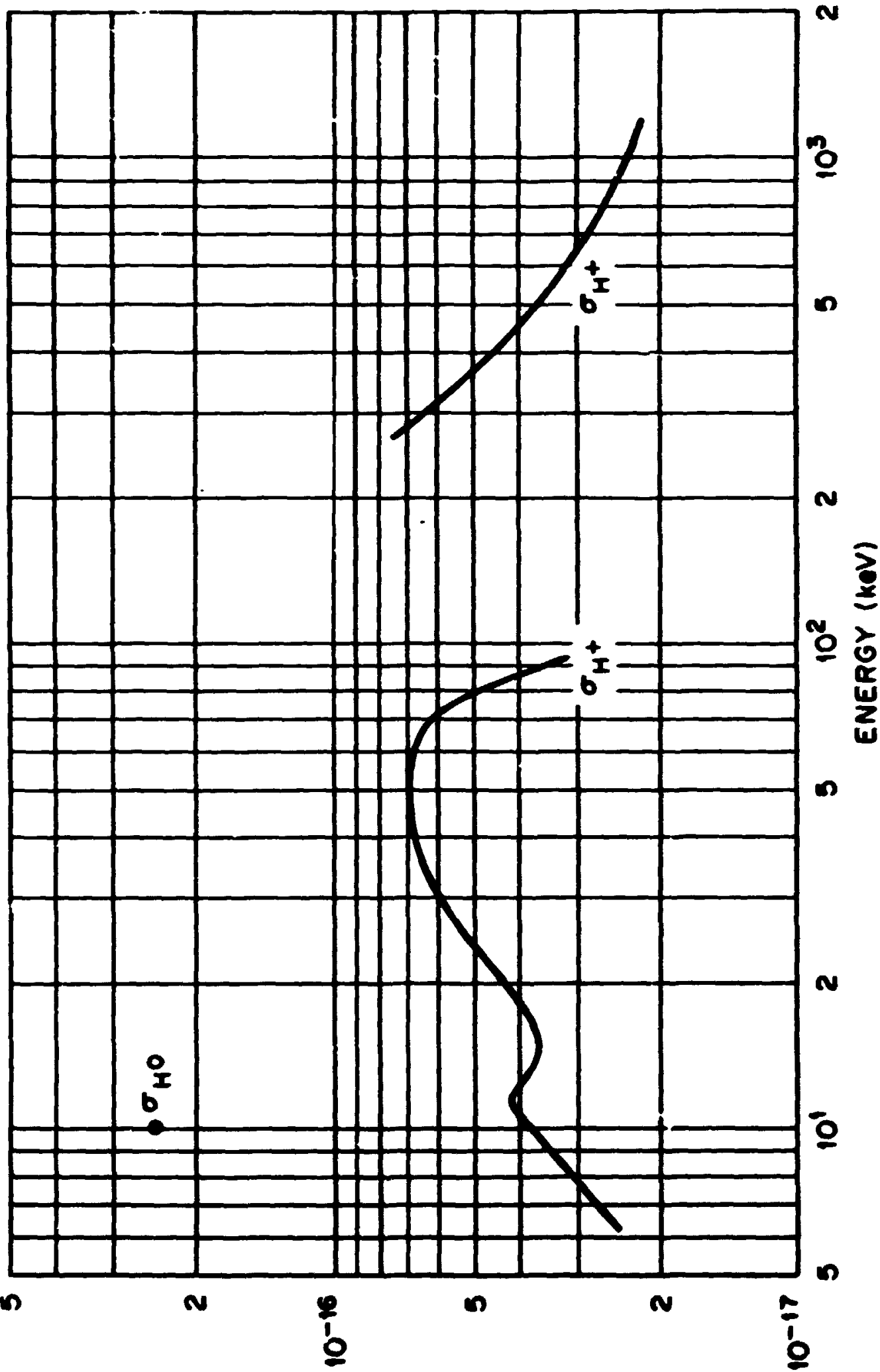

(2w) Nol123s SSOYD 


\section{A.3.6}

Partial Cross Section for Dissociation of Past $\mathrm{H}_{2}^{+}$Ions in

Passage through Hydrogen and Helium. $\mathrm{B}_{2}^{+}+\mathrm{H}^{+}+\mathrm{I}^{\circ}$

\begin{tabular}{|c|c|}
\hline $\begin{array}{l}\text { Derro } \\
\text { (atev) }\end{array}$ & $\begin{array}{c}\text { Croos Sections } \\
\left(c^{2}\right)\end{array}$ \\
\hline
\end{tabular}

$\mathbf{1 2}$

Be

1.7 E 00

$1.6 \mathrm{E}-16$

$3.0=00$

$1.6 \mathrm{E}-16$

$6.0 \mathrm{E} 00$

$1.7 \mathrm{E}-16$

$1.0 \mathrm{E} 01$

$1.7 \mathrm{E}-16$

2.0 E 01

1.6 E-16

5.0 E 01

1.4 E-16

6.7 E-17

$1.2 \mathrm{E}-16$

$6.6 \mathrm{E}-17$

7.0 E 01

1.0 E-16

6.2 E-17

$2.0=02$

6.8 E-16

4.5 E-17

5.0 E 02

3.7 E-16

2.2 E-17

$2.9 g-16$

1.7 E-17

7.0 E 02

2.2 E-16

1.2 E-17

$1.0 \mathrm{E} 03$

1.7 E-16

8.2 E-18

$1.5=03$

1.4 E-16

6.2 E-18

\section{Deferences:}

$\mathrm{B}_{2}^{+}+\mathrm{I}_{2}$ : J. Coldint, C. R. Acad. Se1. Paris, 253, 829 (1961); I. I. P1wovar,

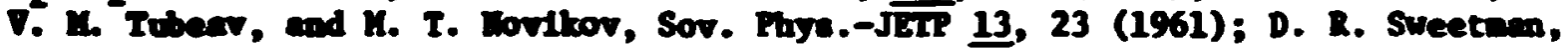
uapubliched; M. I. Tunitakt, E. S. Zhurkti, and M. V. Tikhondrov, Sov. Phys.Jir Letere, 12, 210 (1970).

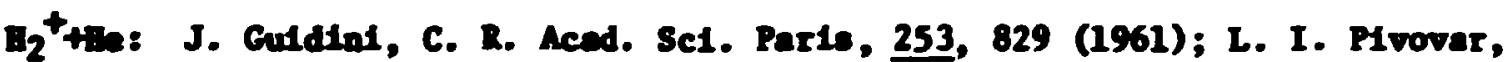
V. M. Tubeav, and R. T. Hovikov, Sov. Phys.-JETP 13, 23 (1961); D. R. Sweetman, apoblished.

\section{Acorrag:}

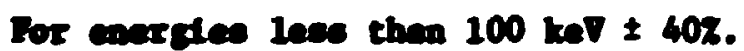

Dor carriden greater then 100 keV $\pm 20 \%$.

\section{Exese:}

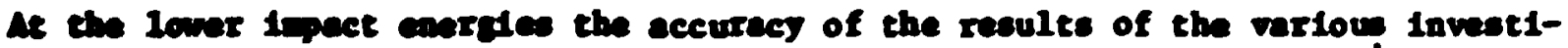

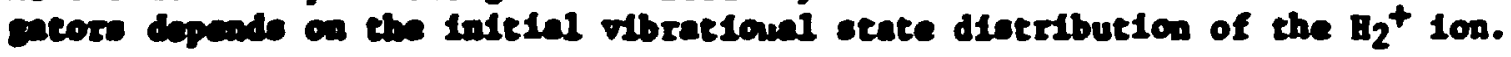




\section{A.3.7}

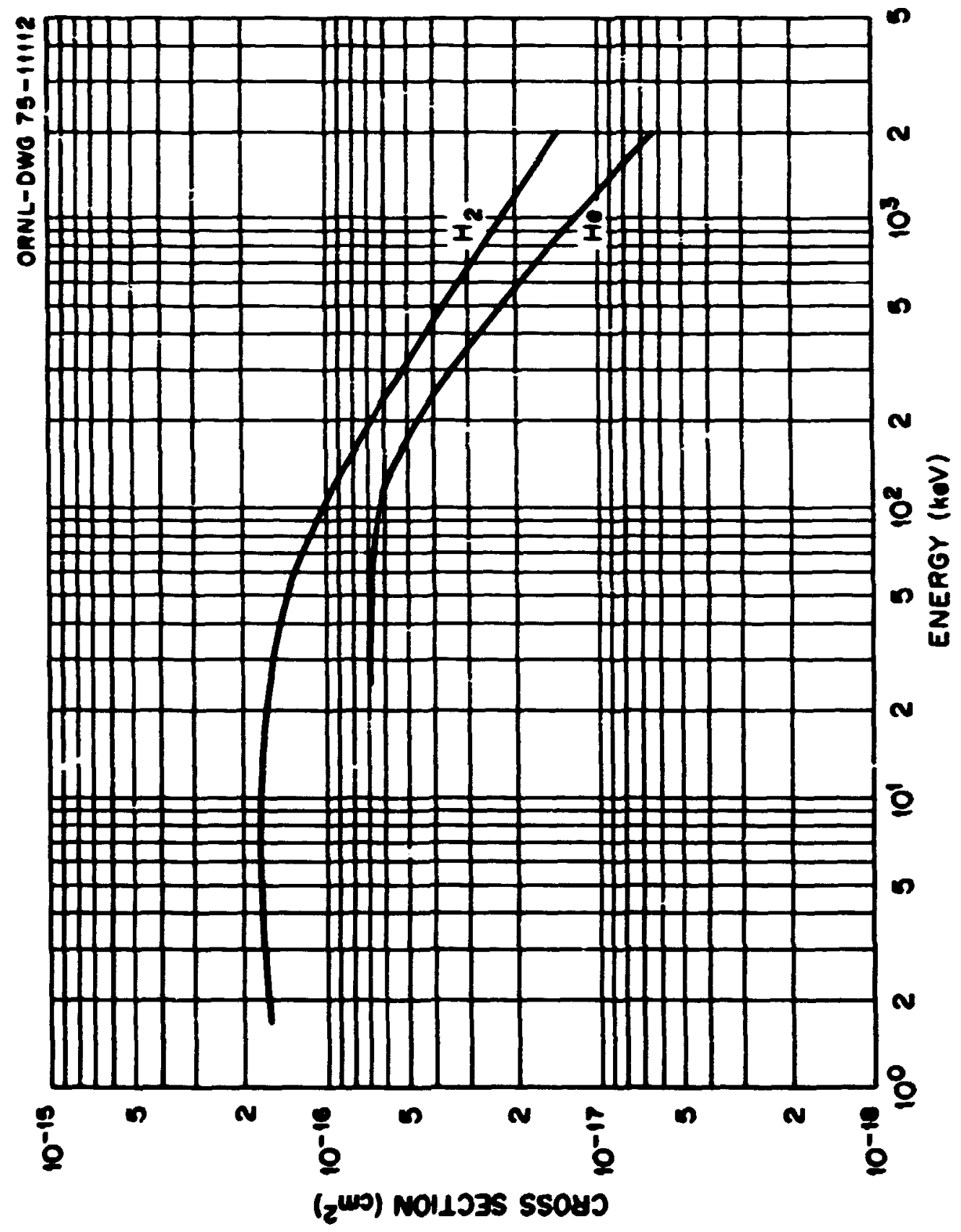


Dissociation Cross Sections for the Pornation of Protons for $\mathrm{H}_{2}^{+}$in lie and the Fornation of Fast $\mathrm{H}^{\circ}$ Atoms for $\mathrm{H}_{2}^{+}$in $\mathrm{H}_{2}$

\begin{tabular}{|c|c|c|}
\hline \multirow[t]{2}{*}{$\begin{array}{l}\text { Enery } \\
\text { (tevt) }\end{array}$} & \multicolumn{2}{|c|}{$\begin{array}{c}\text { Croes Sections } \\
\left(c^{2}\right)\end{array}$} \\
\hline & $\sigma_{B}{ }^{+}(B e)$ & $\sigma_{\mathrm{n}} \cdot\left(\mathrm{A}_{2}\right)$ \\
\hline $\begin{array}{l}2.0 \text { E } 00 \\
4.0 \text { E } 00 \\
6.0 \text { E } 00 \\
8.0 \text { E } 00 \\
1.0 \text { E } 01 \\
3.0 \text { E } 01 \\
5.0 \text { E } 01 \\
7.0 \text { E } 01 \\
1.0 \text { E } 02 \\
2.0 \text { E } 02 \\
3.0 \text { E } 02 \\
7.0 \text { E } 02 \\
1.0 \text { E } 03\end{array}$ & $\begin{array}{ll}3.7 & E-17 \\
7.6 & E-17 \\
1.0 & E-16 \\
1.2 & E-16 \\
1.3 & E-16 \\
1.8 & E-16 \\
2.0 & E-16 \\
2.0 & E-16 \\
2.0 & E-16 \\
1.7 & E-16 \\
1.2 & E-16 \\
5.9 & E-16 \\
4.2 & E-16\end{array}$ & $\begin{array}{ll}5.1 & \mathrm{E}-16 \\
6.3 & \mathrm{E}-16 \\
7.1 & \mathrm{E}-16 \\
7.7 & \mathrm{E}-16 \\
8.3 & \mathrm{E}-16 \\
6.9 & \mathrm{E}-16 \\
5.3 & \mathrm{E}-16 \\
3.6 & \mathrm{E}-16 \\
1.2 & \mathrm{E}-16\end{array}$ \\
\hline
\end{tabular}

\section{References:}

Battik: R. H. Il'In, B. I. KAk1ani, V. A. Oparin, E. S. Solov'ev, and u. V. Pedorenko, Sov. Phys. JETP 19, 817 (1964); J. Guidint, C. R. Acad. Se1. Parls 253, 829 (1961); I. I. PIwovar, V. M. Tubeev, M. T. Novkkov, Sow. Puyse.-JLIP 13, 23 (1961); J. P. Hillias and D. M. P. Dunber, Phys. Dev. 149, 62 (1966).

$\mathrm{B}_{2}^{+}+\mathrm{H}_{2}$ : G. W. Mcclure, Thye. Rev. 130, 1852 (1963); A. Schidd, 2. Phys. 161. 350 (1961); R. S. Solov'ev, R. H. I1'In, V. A. Oparin, and N. V. Fadorenko, Sov. Physe.-JETP 26, 1097 (1968); D. R. Siveetran, Proc. Roy. Soc. London 2564, 416 (1960).

\section{Accureg:}

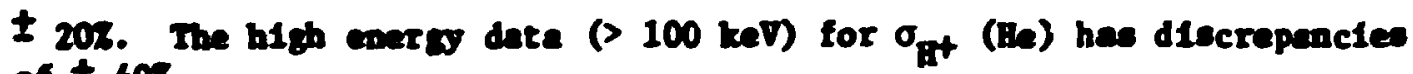
of \pm 408 .

\section{Dores:}

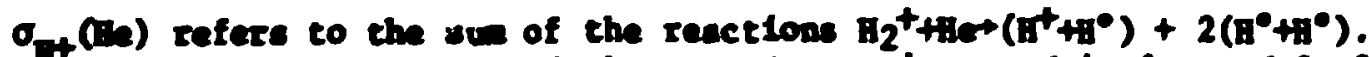

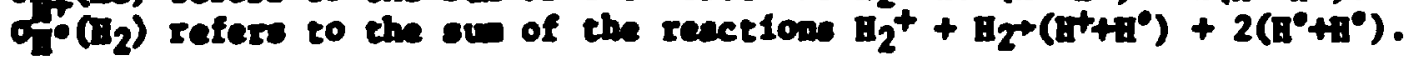


A.3.9

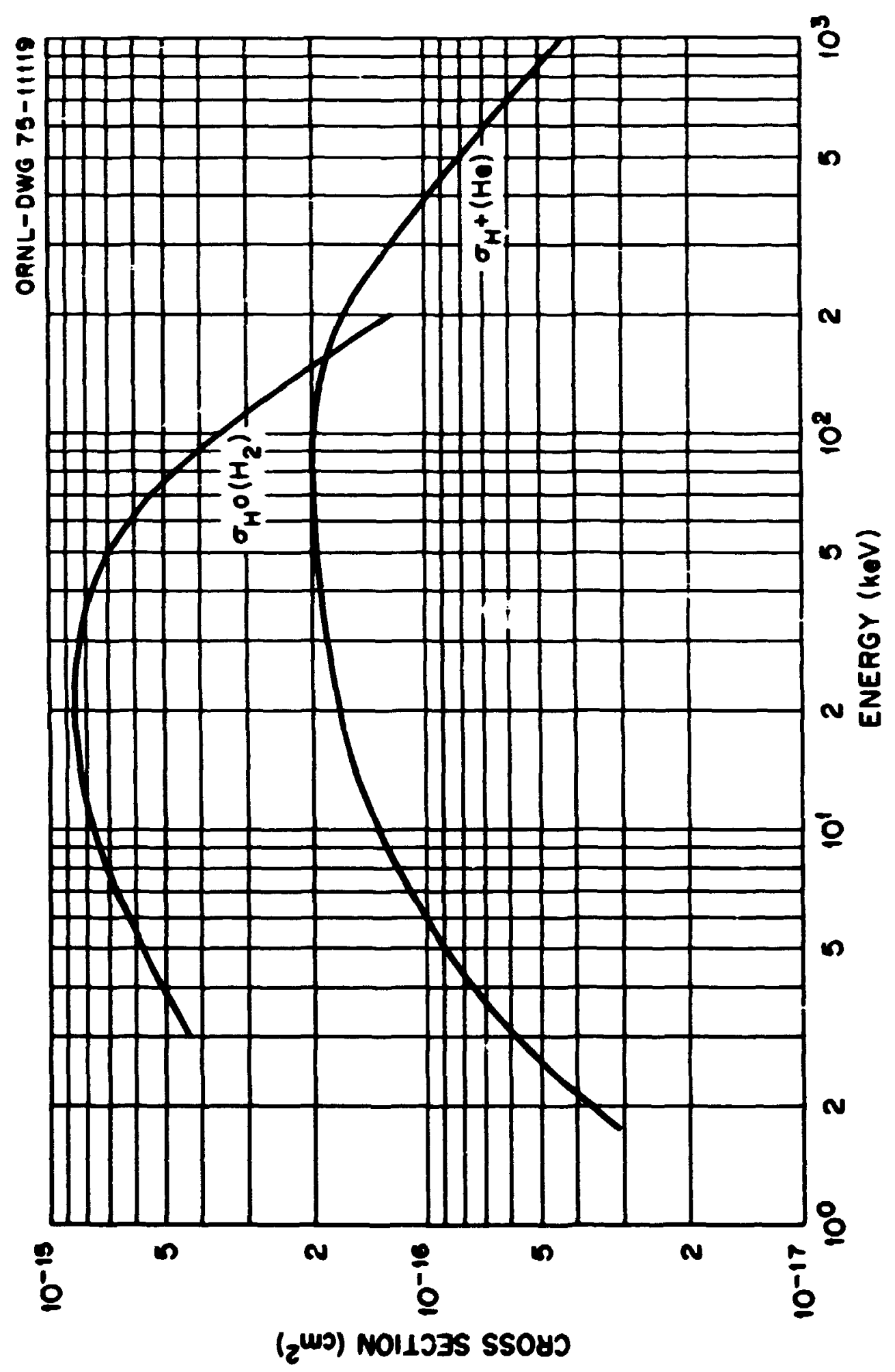




\section{A. 3.10}

Dissociation Cross Sections for the Formetion: of Protons for $\mathrm{H}_{2}{ }^{+}$in $\mathrm{H}_{2}$ and $\mathrm{Ie}$

\begin{tabular}{|c|c|c|}
\hline \multirow[t]{2}{*}{$\begin{array}{l}\text { Deness } \\
(t \in V)\end{array}$} & \multicolumn{2}{|c|}{$\begin{array}{c}\text { Cross Sectlous } \\
\text { (ca) }\end{array}$} \\
\hline & $\sigma_{1}+\left(a_{2}\right)$ & $\sigma_{\mathrm{B}}$ (ne) \\
\hline $\begin{array}{l}2.0 \text { E } 00 \\
4.0 \text { E } 00 \\
6.0 \text { E } 00 \\
1.0 \text { E } 01 \\
2.0 \text { E } 01 \\
4.0 \text { E } 01 \\
6.0 \text { E } 01 \\
1.0 \text { E } 02 \\
1.5 \text { E } 02 \\
3.0 \text { E } 02 \\
7.0 \text { E } 02 \\
1.0 \text { E } 03 \\
2.0 \text { E } 03\end{array}$ & $\begin{array}{ll} & \\
2.0 & \mathrm{E}-16 \\
2.5 & \mathrm{E}-16 \\
3.3 & \mathrm{E}-16 \\
4.6 & \mathrm{E}-16 \\
5.9 & \mathrm{E}-16 \\
6.5 & \mathrm{E}-16 \\
7.0 & \mathrm{E}-16 \\
7.0 & \mathrm{E}-16 \\
6.5 & \mathrm{E}-16 \\
5.4 & \mathrm{E}-16 \\
4.8 & \mathrm{E}-16 \\
3.0 & \mathrm{E}-16\end{array}$ & $\begin{array}{ll}6.5 & E-17 \\
9.5 & E-17 \\
1.2 & E-16 \\
1.5 & E-16 \\
2.1 & E-16 \\
2.6 & E-16 \\
3.0 & E-16 \\
3.3 & E-16 \\
3.2 & E-16\end{array}$ \\
\hline
\end{tabular}

\section{Deferences:}

$B_{2}++u_{2}$ : J. Gutdint, C. R. Aced. Sc1., Par1s, 253, 829 (1961); R. I. Il'In, B. I. Kiktan1, V. A. Oparin, E. S. Solov'ev, and M. V. Fedorenko, Sov. Phys.JETP 19, 817 (1964); L. I. P1vovar, V. M. Tuberv, and H. T. Movikov, Sov. Fiss.-JEIP 13, 23 (1961); D. R. Sreetma, upubliohed; J. F. William and D. I. F. Dusbar, Fuyse. Dev. 149, 62 (1966).

B, ${ }^{+}$the: R. S. Solov'ev, R. I. II'In, V. A. Oparin, and H. P. Pedorenko, Sor. Pbye.-JETP 26, 1097 (1968); J. P. Willians and D. M. P. Duabar, Phye. 2av. 149, 62 (1966).

\section{Accuras:}

$\pm 258$.

Deter

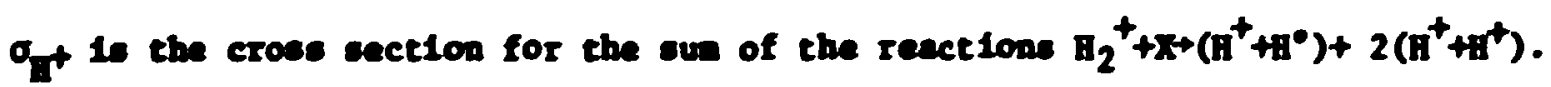


A.3.11

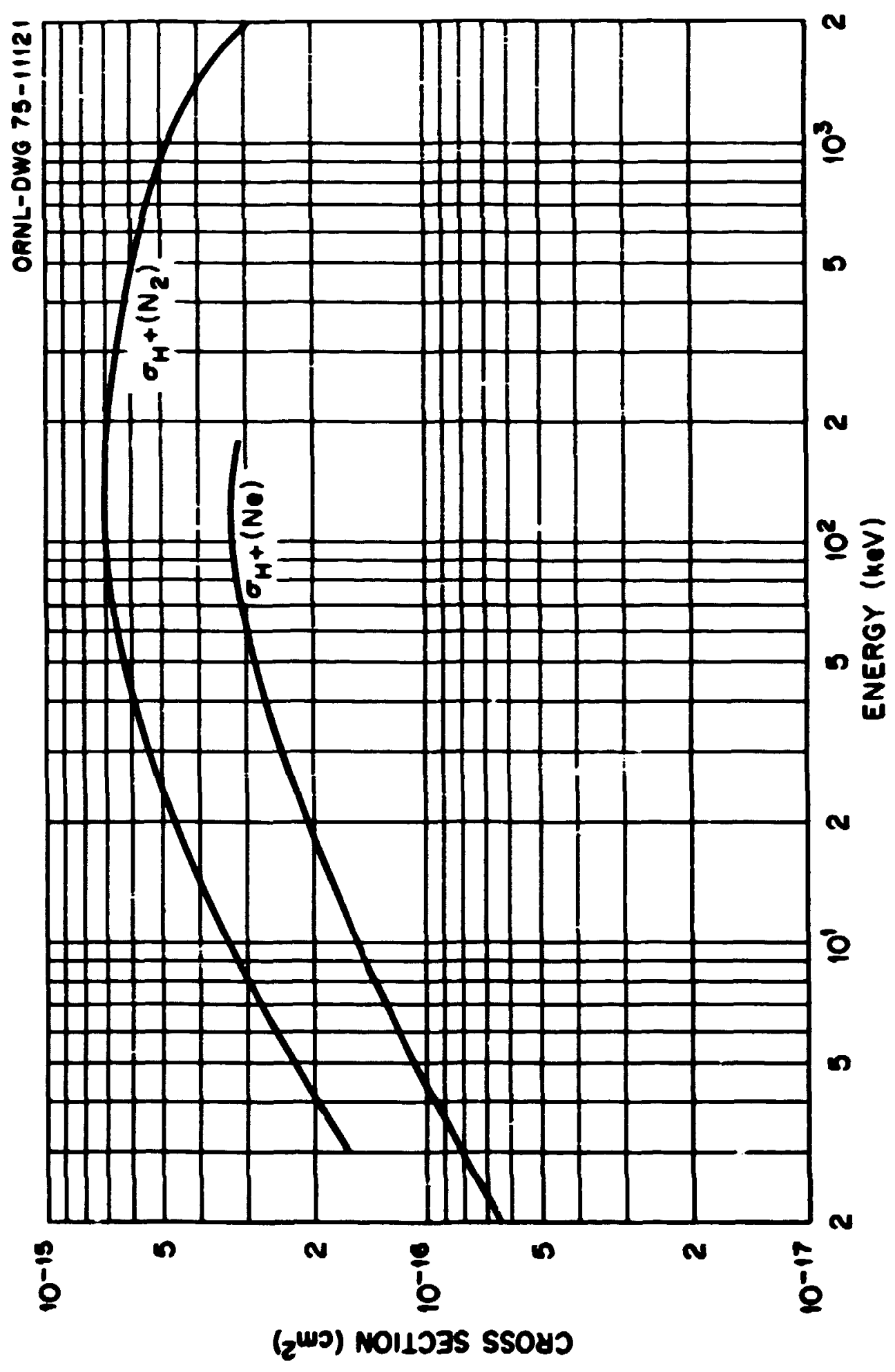




\section{A.3.12}

Cross Sections for the Production Protons and $\mathrm{H}$ Atcas for $\mathrm{H}_{2}{ }^{+}$in $\mathrm{Mg}$ and tha

\begin{tabular}{|c|c|c|c|}
\hline \multirow[t]{2}{*}{$\begin{array}{l}\text { Deres } \\
\text { (ter) }\end{array}$} & \multicolumn{3}{|c|}{$\begin{array}{c}\text { Crose Section } \\
\left(\mathrm{c}^{2}\right)\end{array}$} \\
\hline & $\sigma_{\mathrm{n}^{+}}\left(\mathrm{O}_{3}\right)$ & $\sigma_{\mathrm{B}^{+}}\left(\mathrm{k}_{\mathrm{n}}\right)$ & $\sigma_{B^{-}}\left(\mathrm{H}_{8}\right)$ \\
\hline $\begin{array}{l}2.0 \text { \& } 01 \\
3.0 \text { E } 01 \\
4.0 \text { E } 01 \\
5.0 \text { E } 01 \\
6.0 \text { E } 01 \\
8.0 \text { E } 01 \\
1.0 \text { E } 02 \\
1.5 \text { E } 02 \\
1.8 \\
\text { E } 02\end{array}$ & $\begin{array}{ll}6.8 & \mathrm{E}-17 \\
9.7 & \mathrm{E}-17 \\
6.5 & \mathrm{E}-17 \\
8.9 & \mathrm{E}-17 \\
1.4 & \mathrm{E}-16 \\
1.6 & \mathrm{E}-16 \\
1.6 & \mathrm{E}-16 \\
1.8 & \mathrm{E}-16 \\
1.9 & \mathrm{E}-16\end{array}$ & $\begin{array}{ll}6.3 & E-17 \\
7.7 & E-17 \\
9.0 & E-17 \\
1.1 & E-16 \\
1.6 & E-16 \\
1.7 & E-16 \\
1.8 & E-16 \\
2.0 & E-16 \\
2.2 & E-16\end{array}$ & $\begin{array}{ll}4.9 & E-16 \\
3.6 & E-16 \\
2.9 & E-16 \\
2.4 & E-16 \\
2.0 & E-16\end{array}$ \\
\hline
\end{tabular}

\section{References:}

E. S. Solov'ev, R. M. Il'in, V. A. Oparin, and N. V. Fedorenko, Sov. Phys.JET 26, 1097 (1968).

\section{Accuracs:}

$\pm 30 \%$. 


\section{A. 3.13}

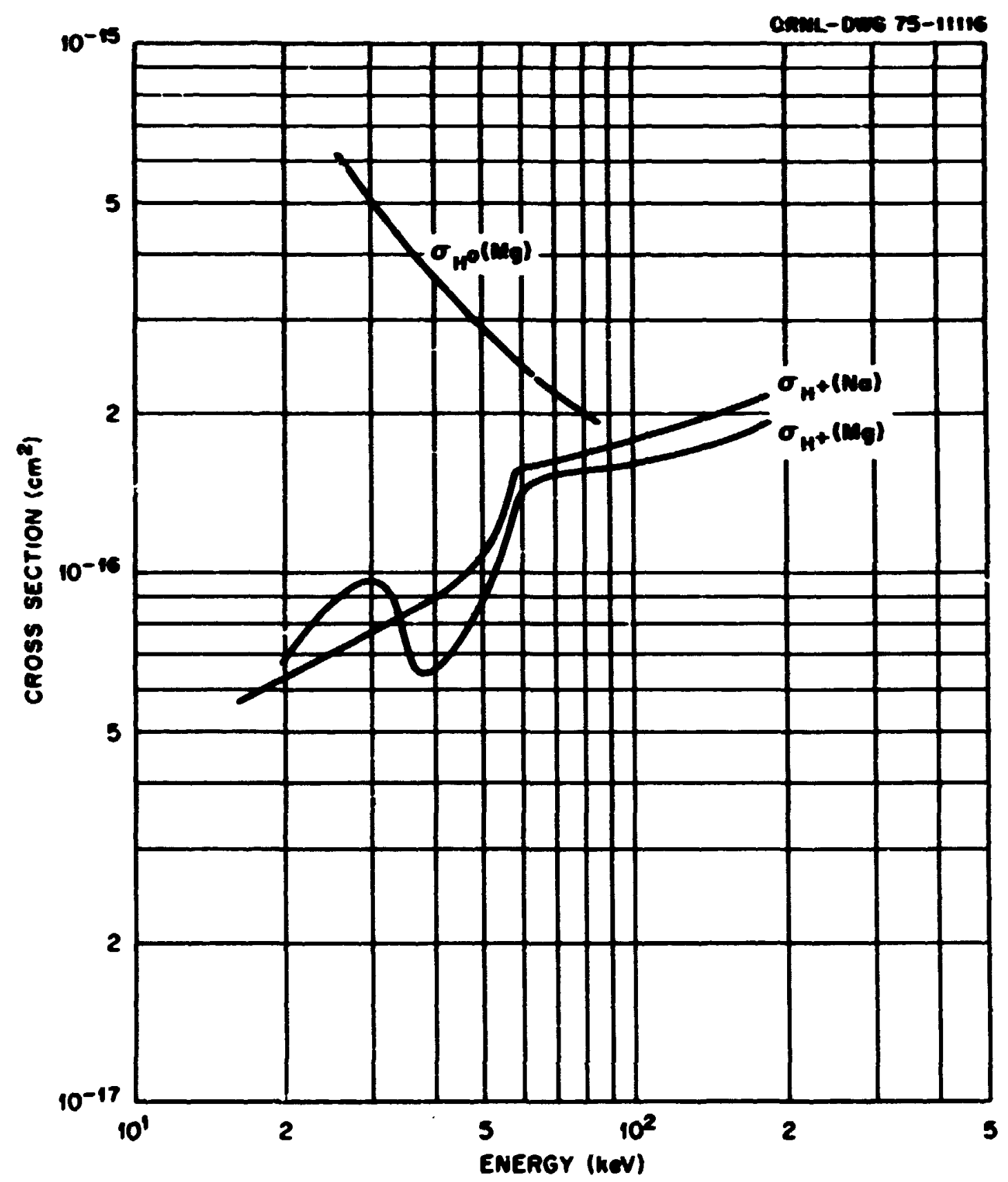




\section{A.3.14}

Dissociation Cross Sections for the Production of Protons for the Reaction Products $\left(\mathrm{H}^{+}+\mathrm{H}^{\circ}\right)$ for $\mathrm{H}_{2}^{+}$in $A x$

\begin{tabular}{|c|c|c|}
\hline \multirow[t]{2}{*}{$\begin{array}{l}\text { Dares } \\
(m+V)\end{array}$} & \multicolumn{2}{|c|}{$\begin{array}{c}\text { Crose Section } \\
\left(\mathrm{cm}^{2}\right)\end{array}$} \\
\hline & $\sigma_{\text {Et }}$ & $\sigma_{E^{+}+H^{\circ}}$ \\
\hline $\begin{array}{l}2.0 \text { E } 00 \\
4.0 \text { E } 00 \\
7.0 \text { E } 00 \\
1.0 \text { E } 01 \\
2.0 \text { E } 01 \\
4.0 \text { E } 01 \\
7.0 \text { E } 01 \\
1.0 \text { E } 02 \\
2.0 \text { E } 02 \\
4.0 \text { E } 02 \\
7.0 \text { E } 02 \\
1.0 \text { E } 03 \\
2.0 \text { E } 03\end{array}$ & $\begin{array}{ll}8.7 & E-17 \\
1.3 & E-16 \\
1.8 & E-16 \\
2.2 & E-16 \\
3.3 & E-16 \\
4.6 & E-16 \\
5.7 & E-16 \\
6.4 & E-16 \\
6.6 & E-16 \\
5.6 & E-16 \\
4.7 & E-16 \\
4.1 & E-16 \\
3.1 & E-16\end{array}$ & 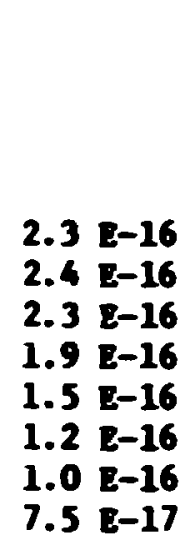 \\
\hline
\end{tabular}

\section{Deference:}

$\mathrm{B}_{2}^{+}+\mathrm{Ar} \rightarrow \sigma_{\mathrm{Z}}:$ J. Guid1n1, C. R. Acad. Sc1. Par1s, 253, 829 (1961); R. M. II'In, B. Y. Rikiani, V. A. Oparin, E. S. Solov'ev, and R. V. Pedorenko, Sov. Phys.-JETP 19, 817 (1964); L. I. P1 vover, V. M. Tubeev, and M. T. Wovikov, Sov. Frys.-JETP 13, 23 (1961); D. R. Sweetmen, unpublished; J. F. W1111.me and D. M. F. Dunbar, Thys. Rev. 169, 62 (1966).

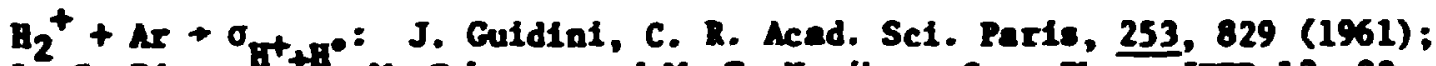

L. I. P1vovar, H. M. Tubeev, and M. T. Novikov, Sov. Phys.-JETP 13, 23

(1961); D. R. Sreetenn, unpublished.

\section{Accuracy:}

$\pm 25 \%$.

\section{Motes:}

$\sigma_{\mathrm{H}^{+}}$refers to the sun of the reactions $\mathrm{H}_{2}^{+}+\mathrm{Ar}+\left(\mathrm{H}^{+}+\mathrm{H}^{\circ}\right)+2\left(\mathrm{H}^{+}+\mathrm{H}^{+}\right)$.

$\sigma_{\mathrm{H}^{+}+\mathrm{H}^{\bullet}}$ refers to the reaction $\mathrm{H}_{2}^{+}+\mathrm{Ar}^{+}+\mathrm{H}^{+}+\mathrm{H}^{\bullet}$. 
A.3.15

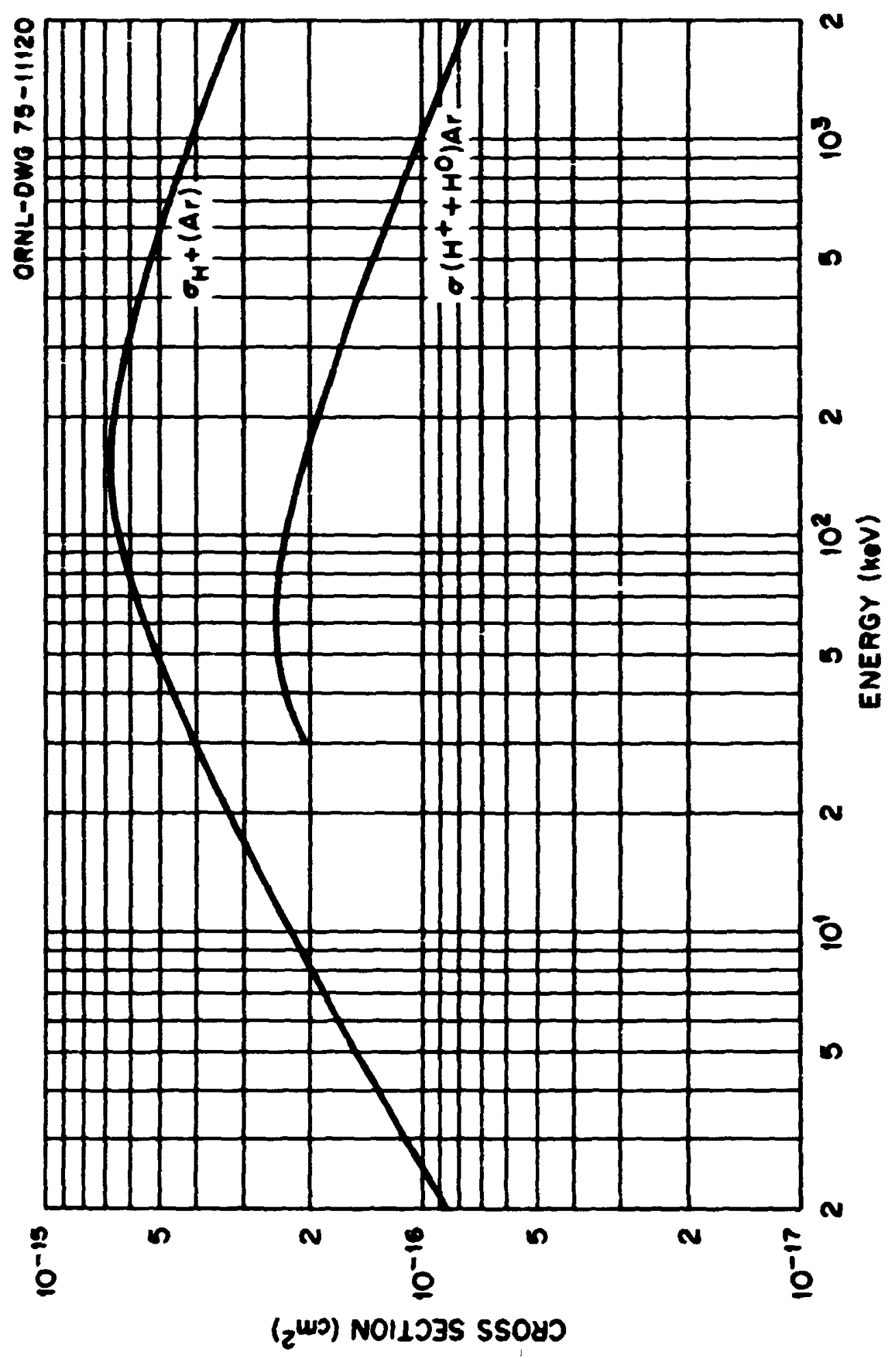




\section{A.3.16}

Cross Sections for the Pormtion of Blectronically Beited H Atoms fron the Dissociation of $\mathrm{H}_{2}^{+}$and $\mathrm{H}_{3}^{+}$in $\mathrm{H}_{2}$, Me, $\mathrm{M}_{3}$, and Ma

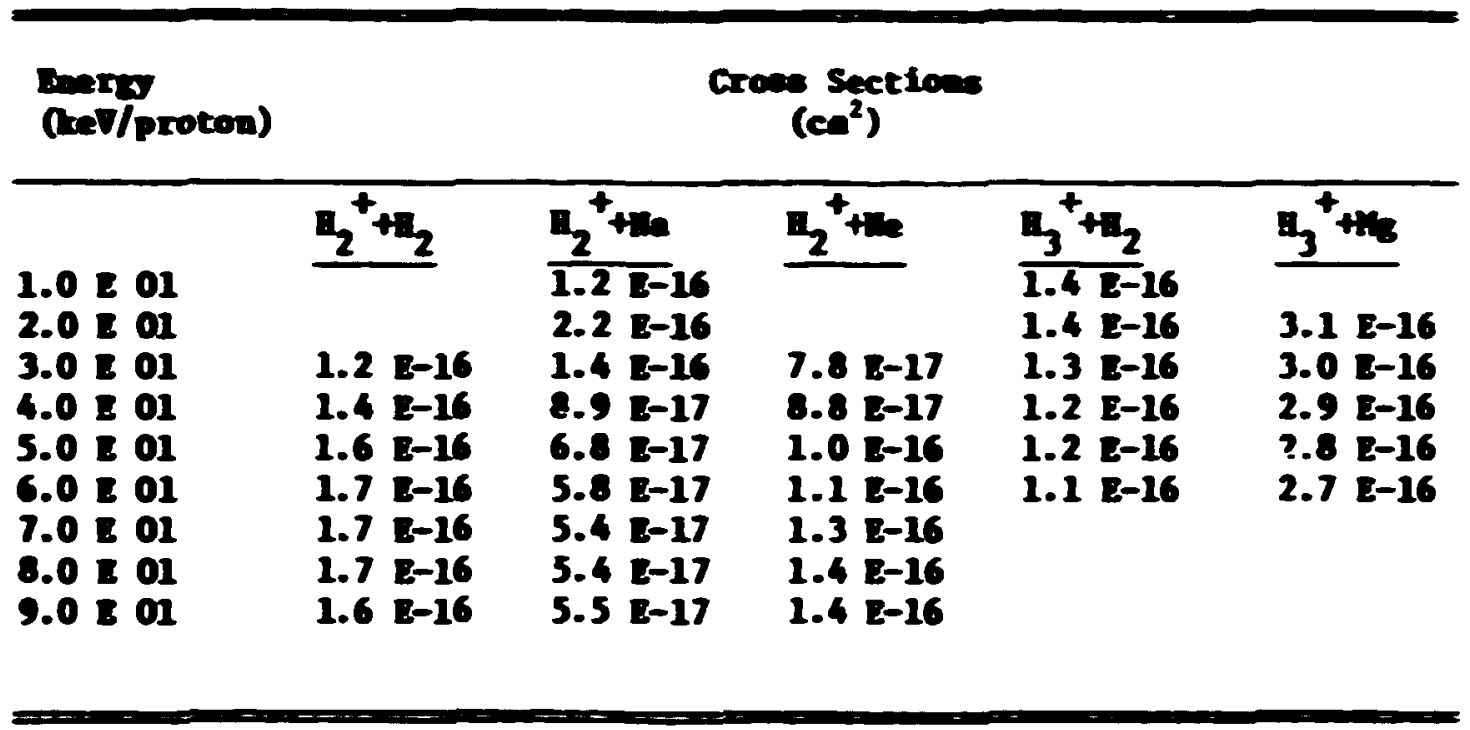

\section{Deferences:}

E. S. Solor'ev, R. II. II'In, V. A. Oparia ad II. V. Pedorenko, Sov. Pugre.JLI 26, 1097 (1S68).

\section{Aceares:}

\pm 302 .

Eoce:

To obtatn the croag section for fornetion of level $a$, divide the tabulated croves eection by $\mathrm{n}^{3}$. 
4.3.17

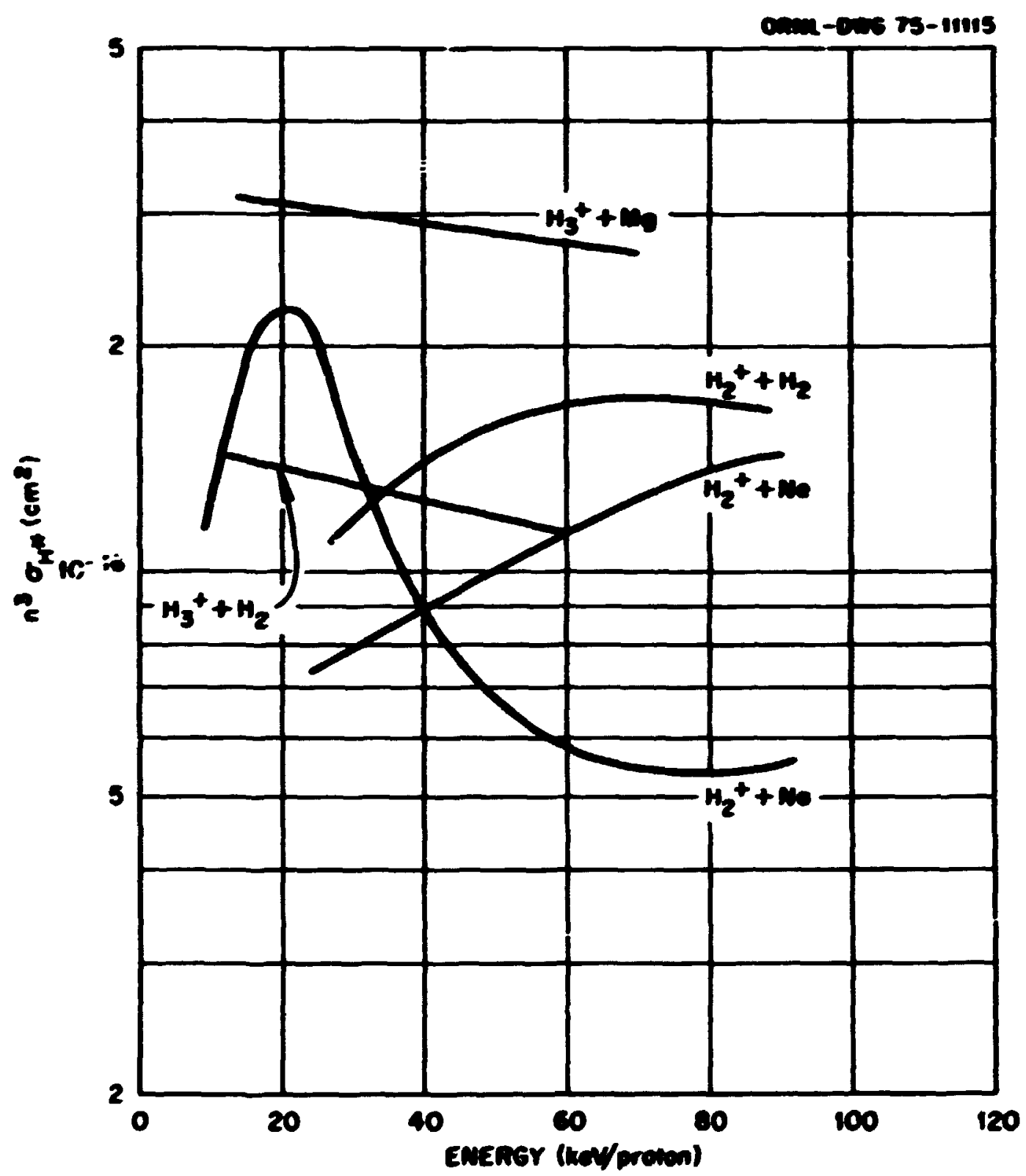




\section{A.3.18}

Disecietion Cross Section for $\mathrm{HD}^{+}$Ions in $B$ e and $E$

\begin{tabular}{|c|c|c|c|c|}
\hline \multirow[t]{2}{*}{$(a)$} & \multicolumn{4}{|c|}{$\begin{array}{l}\text { Crose Sections } \\
\left(c^{2}\right)\end{array}$} \\
\hline & $\sigma_{1}\left(\sigma_{2}\right)$ & $\sigma_{1}\left(a_{2}\right)$ & $\sigma_{2}\left(\sigma_{2}\right)$ & $\sigma_{2}\left(\sigma_{2}\right)$ \\
\hline $\begin{array}{l}5.0=1 \\
1.0=01 \\
1.5=01 \\
2.0=01 \\
2.5=01 \\
3.0=01 \\
3.5=01 \\
4.0=01 \\
4.5=01\end{array}$ & $\begin{array}{ll}1.6 & E-17 \\
1.9 & E-17 \\
2.4 & E-17 \\
2.9 & E-17 \\
2.2 & E-17 \\
2.1 & E-17 \\
2.1 & E-17 \\
2.3 & E-17 \\
2.1 & E-17\end{array}$ & $\begin{array}{ll}4.4 & E-17 \\
5.0 & E-17 \\
4.7 & {[-17} \\
8.0 & {[-17} \\
8.2 & {[-17} \\
5.0 & E-17 \\
5.0 & {[-17} \\
5.1 & E-17 \\
4.8 & E-17\end{array}$ & $\begin{array}{ll}2.3 & E-17 \\
3.0 & E-17 \\
4.0 & E-17 \\
4.9 & E-17 \\
3.6 & E-17 \\
3.3 & E-17 \\
3.8 & E-17 \\
3.9 & E-17 \\
3.5 & E-17\end{array}$ & $\begin{array}{ll}7.2 & {[-17} \\
7.9 & {[-17} \\
7.2 & E-17 \\
1.1 & {[-16} \\
1.1 & E-16 \\
9.3 & {[-17} \\
9.0 & E-17 \\
8.9 & E-17 \\
8.6 & E-17\end{array}$ \\
\hline
\end{tabular}

Defore:

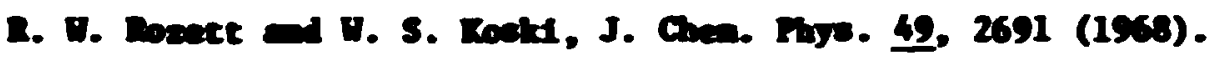

secters:

Unowown

Dotes:

$\sigma_{1}$ is for reaction products $B^{+}+D$ and $\sigma_{2}$ is for $H+D^{+}$. 


\section{A.3.19}

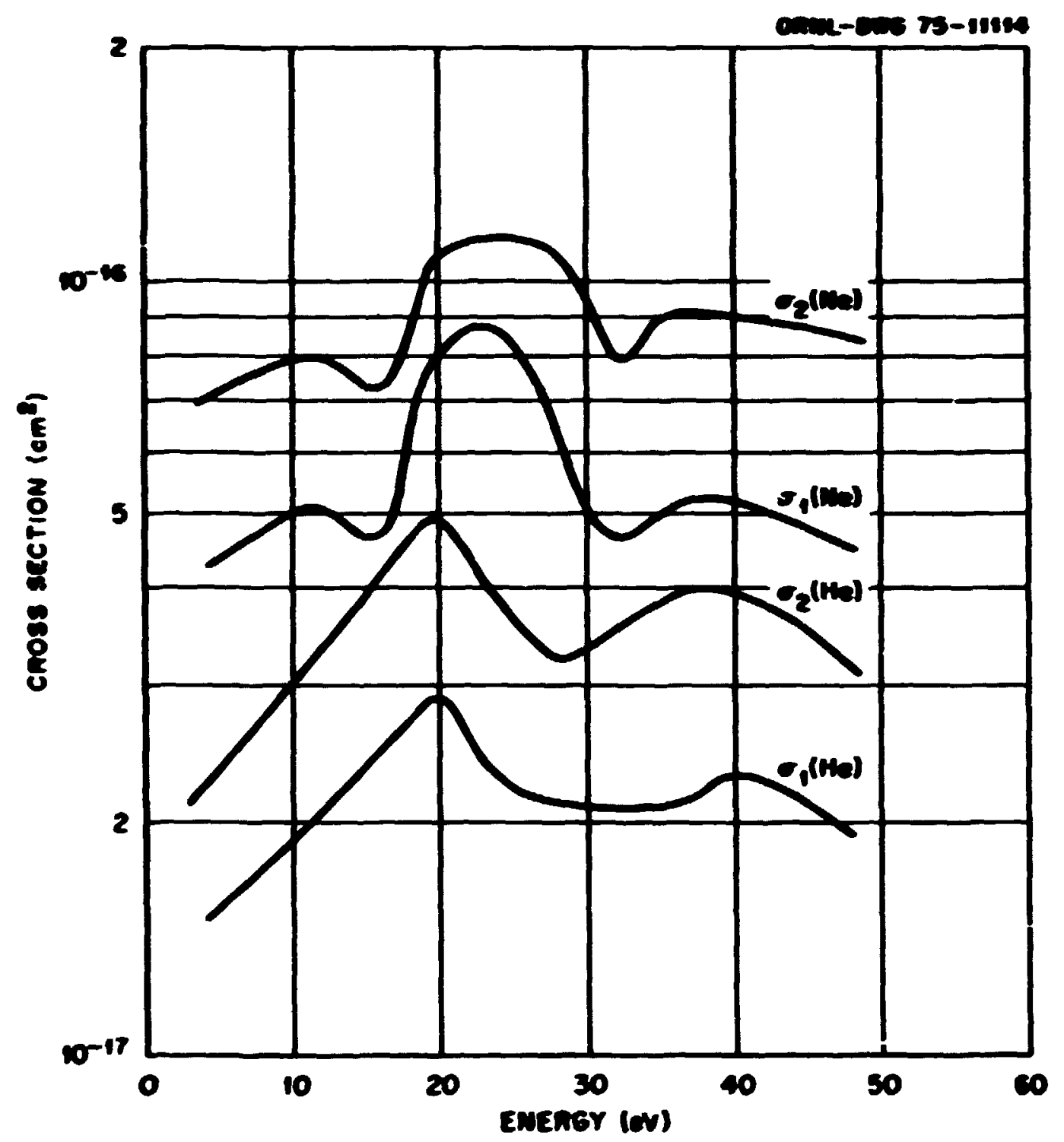




\section{A.3.20}

Disediation Crose Sections for the Production of Past $\mathrm{H}^{+}$and $\mathrm{E}_{2}^{+}$from $\mathrm{H}_{3}^{+}$in $\mathrm{H}_{2}$

\begin{tabular}{|c|c|c|}
\hline & \multicolumn{2}{|c|}{$\begin{array}{c}\text { Croves Sections } \\
\left(\mathrm{com}^{2}\right)\end{array}$} \\
\hline & oan & $\mathrm{OO}_{2}+3$ \\
\hline 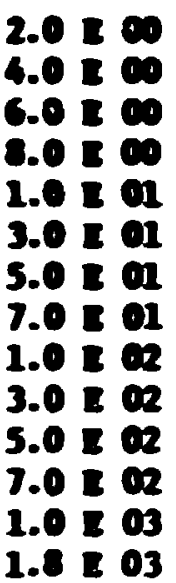 & $\begin{array}{ll}1.2 & {[-17} \\
3.4 & {[-17} \\
5.5 & {[-17} \\
7.2 & {[-17} \\
2.6 & {[-17} \\
1.8 & {[-16} \\
2.1 & {[-16} \\
2.2 & {[-16} \\
2.3 & {[-16} \\
2.3 & {[-16} \\
2.0 & {[-16} \\
1.6 & {[-16} \\
1.3 & E-16 \\
7.3 & E-17\end{array}$ & $\begin{array}{ll}3.1 & {[-17} \\
6.1 & {[-17} \\
6.0 & {[-17} \\
9.6 & {[-17} \\
1.1 & {[-16} \\
1.3 & E-16 \\
1.2 & E-16 \\
1.1 & E-16 \\
9.9 & E-17 \\
6.6 & E-17 \\
5.3 & {[-17} \\
4.2 & E-17 \\
3.7 & E-17 \\
2.0 & E-17\end{array}$ \\
\hline
\end{tabular}

\section{Deferees:}

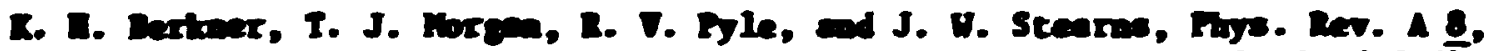
2670 (1973); J. T. Bllles ad D. H. T. Debar, The. Rev. 149, 62 (1966); G. D. HeClure, Fuse. Rev. 130, 1852 (1563).

\section{Aering:}

$\pm 30$.

\section{Extes:}

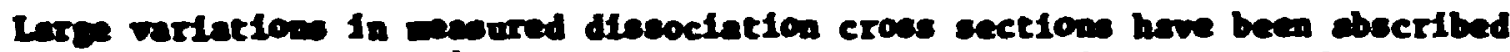

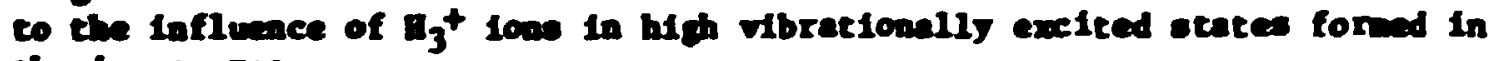
the loa sorres. 


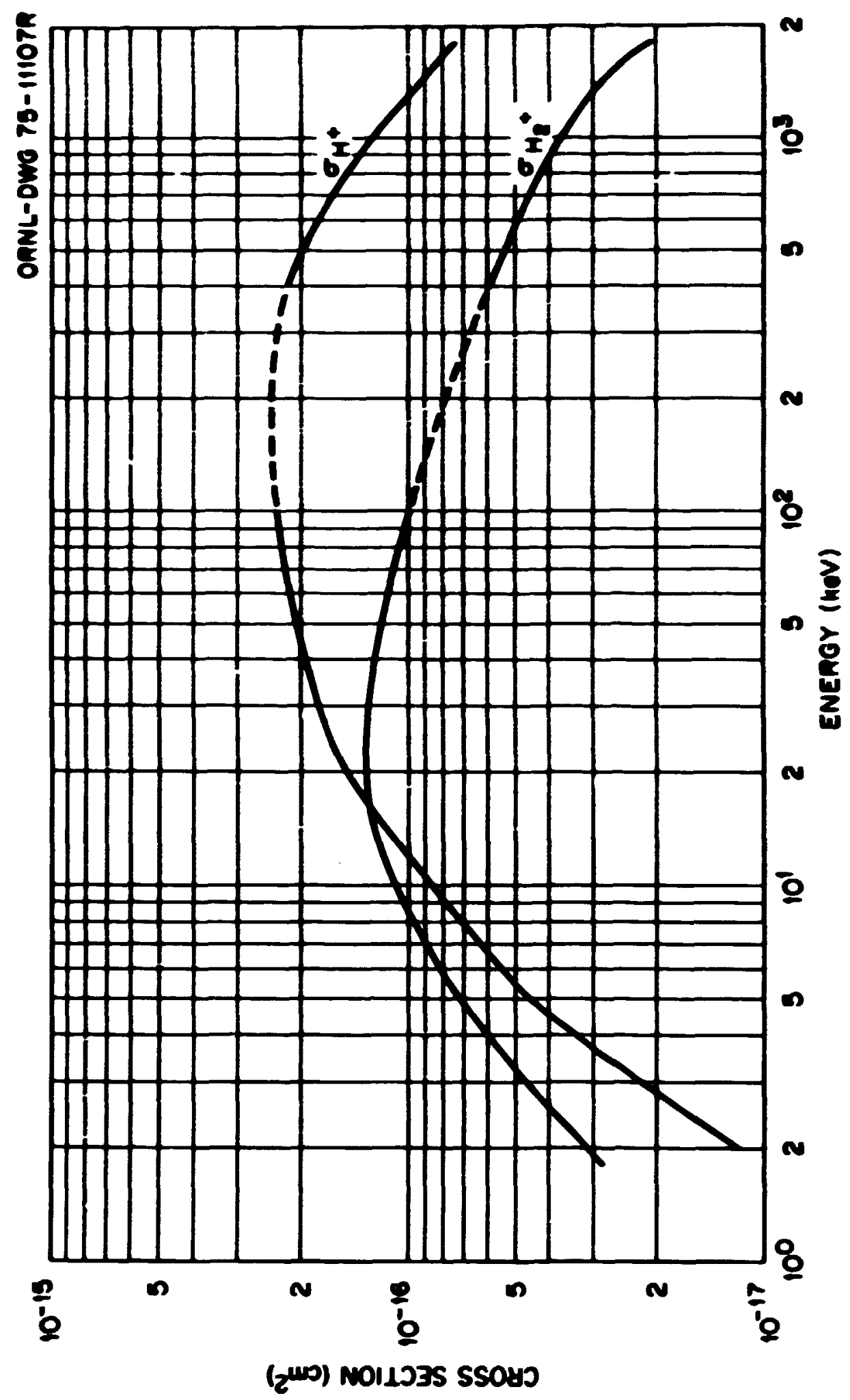




\section{A.3.22}

Discocietion Crose Seetions for the Production of Lend $\mathrm{B}_{2}{ }^{\circ}$ from $\mathrm{H}_{3}{ }^{+}$in $\mathrm{H}_{2}$

\begin{tabular}{|c|c|c|}
\hline $\lim$ & \multicolumn{2}{|c|}{$\begin{array}{c}\text { Orow Sections } \\
\left(\mathrm{cm}^{2}\right)\end{array}$} \\
\hline & o(a) & $\sigma\left(z_{2}{ }^{\circ}\right)$ \\
\hline 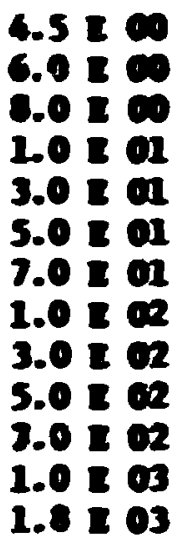 & 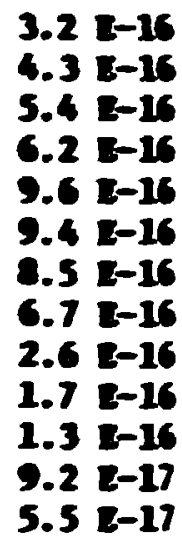 & 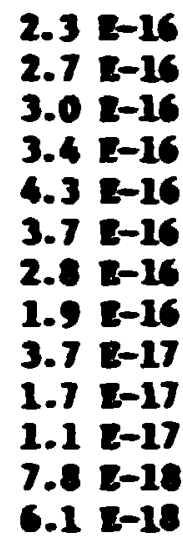 \\
\hline
\end{tabular}

\section{Eferenea:}

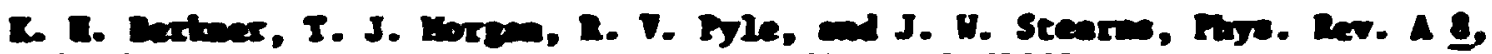
2370 (1973); G. D. Veclere, Fyse. Rev. 130; 1652 (1563).

\section{Seeres:}

$\pm 302$.

\section{Eece:}

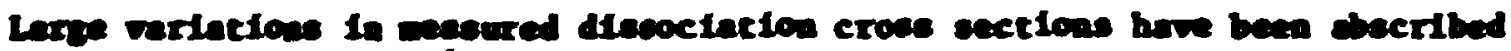

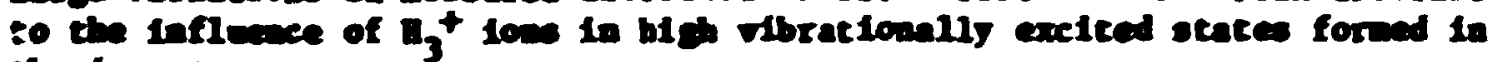
the 100 couree. 


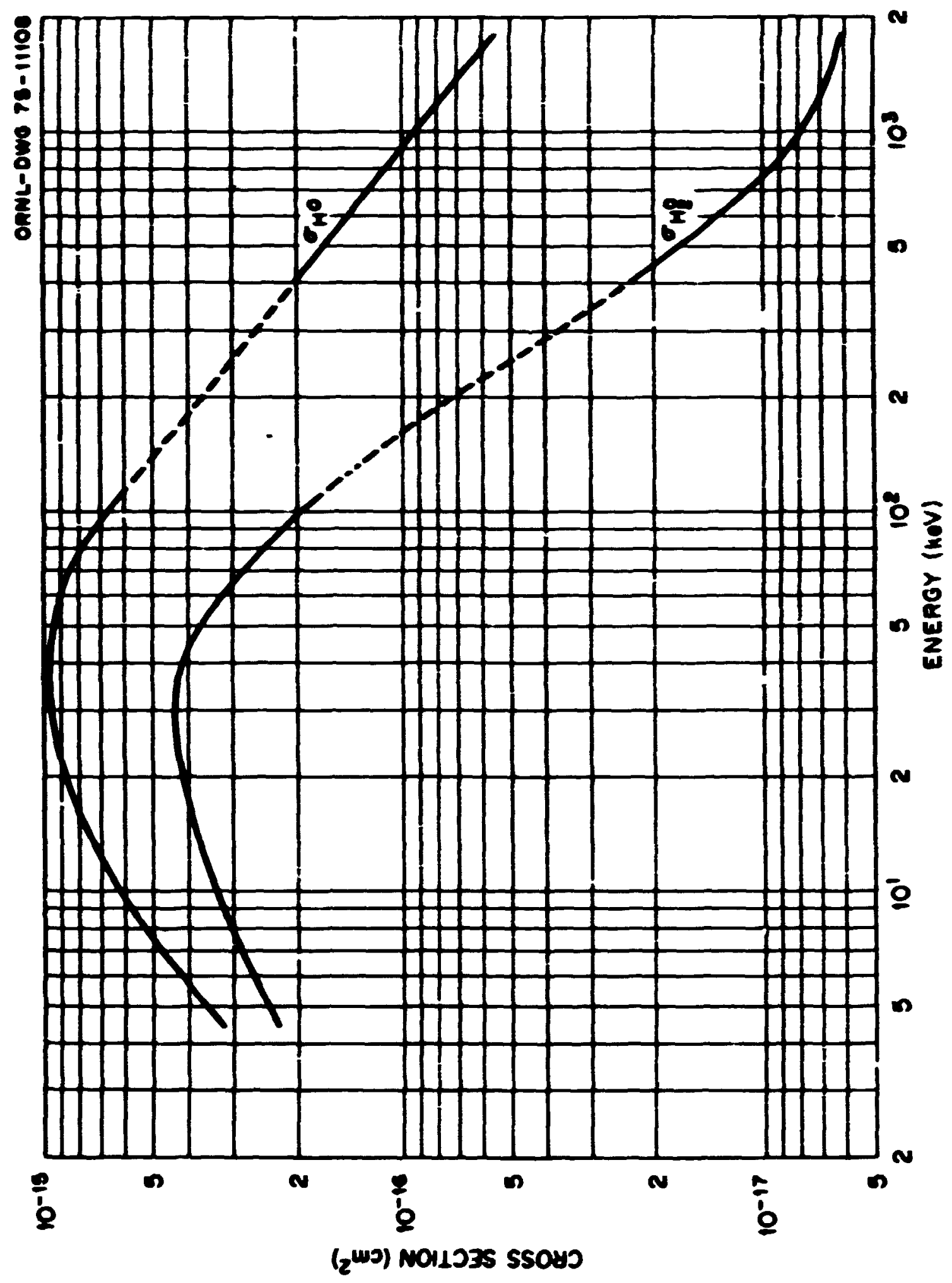


Cross Sections for the Porntion of Past $\mathrm{A}^{+}$ad $\mathrm{B}_{2}{ }^{+}$ rron the Disscociation of $\mathrm{H}_{3}{ }^{*}$ in $\mathrm{Be}$ and the

\begin{tabular}{|c|c|c|c|c|}
\hline (nes) & & $\begin{array}{r}\text { Croes sex } \\
\left(\mathrm{cm}^{2}\right)\end{array}$ & & \\
\hline & $0_{1}\left(\theta_{2}\right)$ & $\sigma_{x}+(t=)$ & $\sigma_{n_{2}}+\left(\sigma_{2}\right)$ & $\sigma_{n_{2}+\left(\sigma_{2}\right)}$ \\
\hline $\begin{array}{l}3.0=0 \\
5.0=00 \\
7.0=00 \\
1.0=01 \\
2.0 \text { घ } 01 \\
3.0 \geq 01 \\
5.0=0\end{array}$ & $\begin{array}{ll}2.2 & E-17 \\
3.6 & E-17 \\
4-8 & E-17 \\
0.4 & E-17 \\
9.8 & E-17 \\
1.2 & E-16 \\
1.4 & E-16\end{array}$ & $\begin{array}{ll}2.6 & E-17 \\
4.2 & E-17 \\
5.6 & E-17 \\
7.4 & E-17 \\
1.2 & E-16 \\
1.4 & E-16 \\
1.8 & E-16\end{array}$ & $\begin{array}{ll}1.7 & {[-17} \\
2.8 & {[-17} \\
3.7 & E-17 \\
4.8 & {[-17} \\
7.3 & {[-17} \\
0.8 & {[-17} \\
1.1 & {[-16}\end{array}$ & $\begin{array}{ll}2.2 & {[-17} \\
3.5 & {[-17} \\
4.5 & {[-17} \\
5.8 & {[-1]} \\
0.7 & {[-1]} \\
1.0 & {[-16} \\
1.2 & {[-16}\end{array}$ \\
\hline
\end{tabular}

\section{Daferes:}

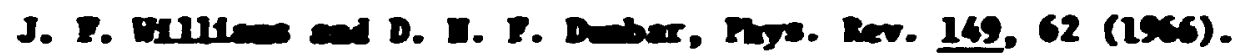

\section{Peeses:}

$\pm 20$. 


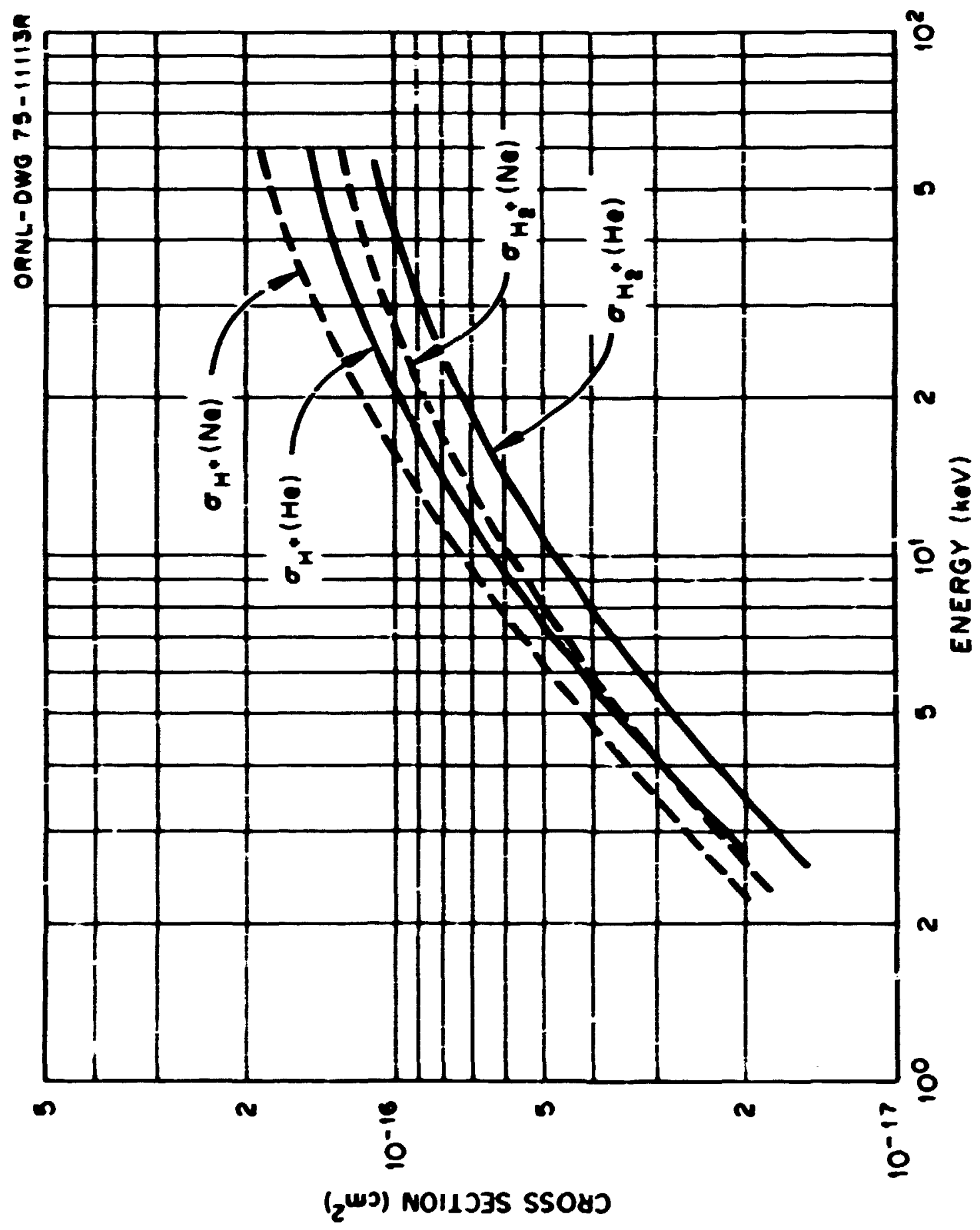


Cross Sections for the Formation of $\mathrm{H}^{+}, \mathrm{H}_{2}^{+}$, and $\left(\mathrm{H}_{2}{ }^{+}+\mathrm{H}^{\circ}\right)$ fron the Dissociation of $\mathrm{H}_{3}^{+}$in Hater Vapor

\begin{tabular}{|c|c|c|c|}
\hline \multirow[t]{2}{*}{$\begin{array}{l}\text { Rners } \\
\text { (tev) }\end{array}$} & \multicolumn{3}{|c|}{$\begin{array}{c}\text { Cross Sections } \\
\left(\mathrm{ce}^{2}\right)\end{array}$} \\
\hline & $\sigma\left(\mathrm{H}^{\circ}+\mathrm{H}_{2}^{\circ}\right)$ & $\sigma\left(\mathrm{B}^{+}\right)$ & $\sigma\left(\mathrm{B}_{2}^{+}\right)$ \\
\hline $\begin{array}{lll}4.5 & E & 00 \\
6.0 & E & 00 \\
8.0 & E & 00 \\
1.0 & E & 01 \\
2.0 & E & 01 \\
4.0 & E & 01 \\
5.0 & E & 01 \\
6.0 & E & 01\end{array}$ & 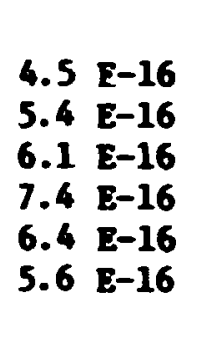 & $\begin{array}{ll}1.3 & E-16 \\
1.4 & E-16 \\
1.7 & E-16 \\
2.0 & E-16 \\
3.2 & E-16 \\
6.2 & E-16 \\
8.2 & E-16\end{array}$ & $\begin{array}{ll}1.1 & E-16 \\
1.2 & E-16 \\
1.4 & E-16 \\
1.5 & E-16 \\
1.9 & E-16 \\
2.3 & E-16 \\
2.4 & E-16 \\
2.5 & E-16\end{array}$ \\
\hline
\end{tabular}

\section{Deference:}

R. Dagnac, J. Angles, D. Blanc, D. Molina and R. Pradal, C. R. Acad. Sct. Paris B 273, 460 (1971).

\section{Accuracy:}

$\pm 30 \%$. 


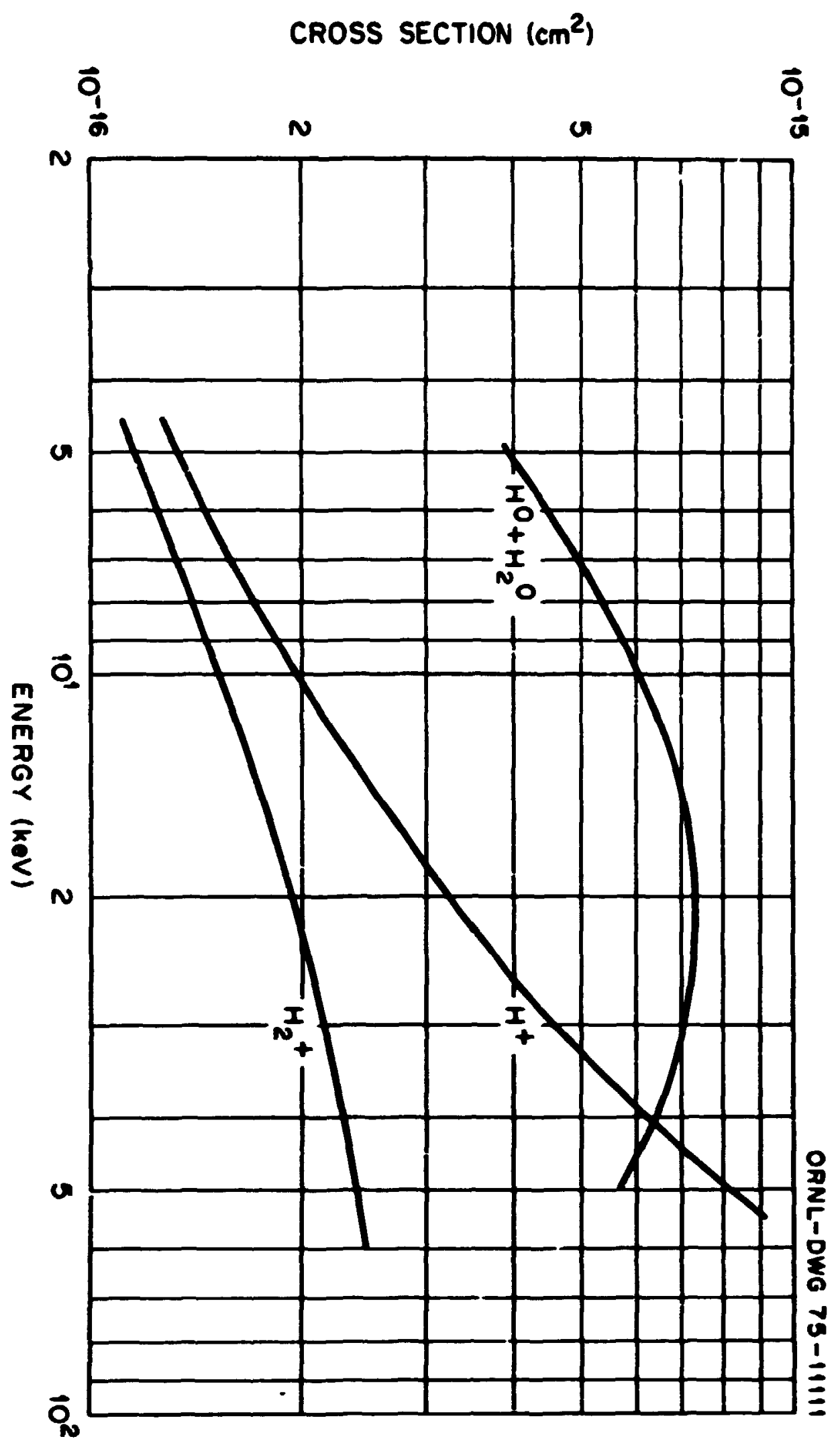

$L Z^{\cdot} \cdot V$ 


\section{A.3.28}

Totel and Partial Cross Sections for the Dissociation of $\mathrm{HeH}^{+}$in He

\begin{tabular}{|c|c|c|c|c|}
\hline \multirow[t]{2}{*}{$\begin{array}{l}\text { Buerw } \\
\text { (tev) }\end{array}$} & \multicolumn{4}{|c|}{$\begin{array}{c}\text { Croes Sections } \\
\left(\mathrm{cm}^{2}\right)\end{array}$} \\
\hline & $\sigma_{\mathbf{I}}$ & $0\left(\operatorname{lic}^{+}+\mathrm{H}^{+}\right)$ & $a\left(\mathrm{Bi}^{+}+\mathrm{H}^{\circ}\right)$ & $\alpha\left(E_{e}^{0}+B^{+}\right)$ \\
\hline $\begin{array}{lll}5.0 & \text { E } & 01 \\
1.0 & \text { E } & 02 \\
3.0 & \text { E } & 02 \\
5.0 & \text { E } & 02 \\
7.0 & \text { E } & 02 \\
9.0 & \text { E } & 02 \\
1.0 & \text { E } & 03\end{array}$ & $\begin{array}{ll}2.1 & \mathrm{E}-16 \\
3.2 & \mathrm{E}-16 \\
2.2 & \mathrm{E}-16 \\
1.4 & \mathrm{E}-16 \\
1.1 & \mathrm{E}-16 \\
9.6 & \mathrm{E}-17 \\
9.2 & \mathrm{E}-17\end{array}$ & $\begin{array}{ll}6.4 & E-17 \\
5.8 & E-17 \\
5.3 & E-17 \\
5.0 & E-17\end{array}$ & $\begin{array}{ll}3.4 & \mathrm{E}-17 \\
2.9 & \mathrm{E}-17 \\
2.6 & \mathrm{E}-17 \\
2.4 & \mathrm{E}-17\end{array}$ & $\begin{array}{ll}1.9 & E-17 \\
1.5 & E-17 \\
1.2 & E-17 \\
1.1 & E-17\end{array}$ \\
\hline Beerry & & $\left.+\mathrm{H}^{\circ}\right)$ & $\sigma\left(\mathrm{Be}^{++}+\mathrm{H}^{+}\right)$ & $\sigma\left(\mathrm{Be}^{++}+\mathrm{H}^{0}\right)$ \\
\hline $\begin{array}{lll}5.0 & \text { E } & 02 \\
7.0 & \text { E } & 02 \\
9.0 & \text { E } & 02 \\
1.0 & \text { E } & 03\end{array}$ & & $\begin{array}{l}-17 \\
-17 \\
-18 \\
-18\end{array}$ & $\begin{array}{ll}1.5 & E-18 \\
1.6 & E-18 \\
1.6 & E-18 \\
1.6 & E-18\end{array}$ & $\begin{array}{ll}1.3 & E-18 \\
1.4 & E-18 \\
1.3 & E-18 \\
1.3 & E-18\end{array}$ \\
\hline
\end{tabular}

\section{Refereaces:}

J. W. Stearn, R. B. Berkne:, R. V. Pyle, B. P. Briegleb and M. L. Warren, Phye. Rev. A \&. 1960 (1971); W. D. H1lson, Larrence Radiation Laboratory, Report No. Ucrit-16308.

\section{Accuracy:}

Betinated to be $\pm 20 \%$.

\section{Mote:}

$\sigma_{\text {I }}$ 1e the cotal dissociation cross section obtained from the sur of the partial crose sections. 


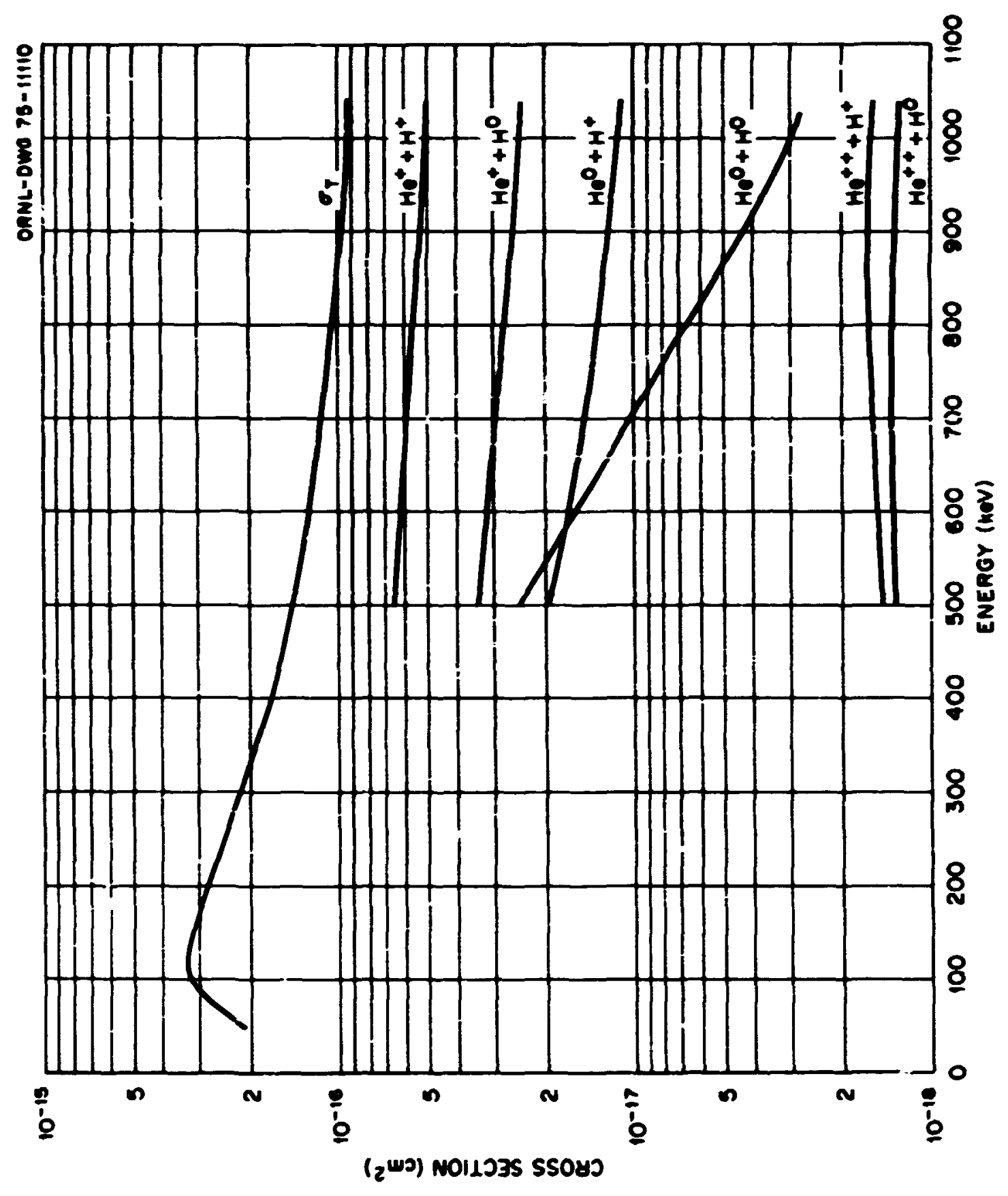




\section{A.3.30}

Total and Partial Cross Sections for the Dissociation of $\mathrm{HeH}^{+}$in $\mathrm{H}_{2}$

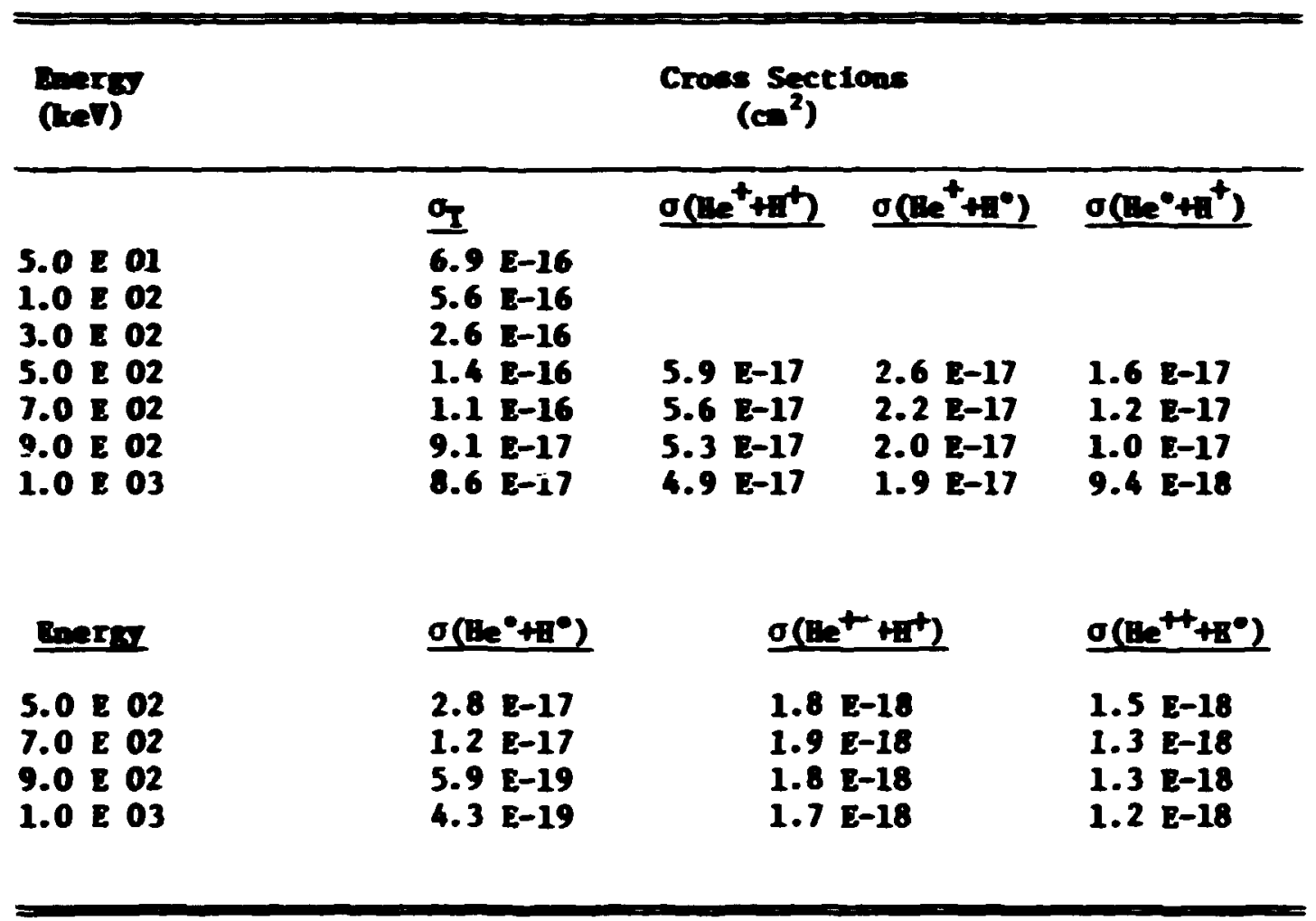

\section{Deferences:}

J. W. Steart, K. H. Berkner, R. V. Pyle, B. P. Briegleb and M. L. Harten, Phys. Rev. A 4, 1960 (1971); W. D. WHIson, Lawreace Radiation Laboratory, Report No. OCDI-16308.

\section{Accuracz:}

Estiented to be $\pm 20 z$.

Dote:

$\sigma_{\mathrm{T}}$ Is the total dissoctation cross section obtained fron the sun of the partial crose sections. 


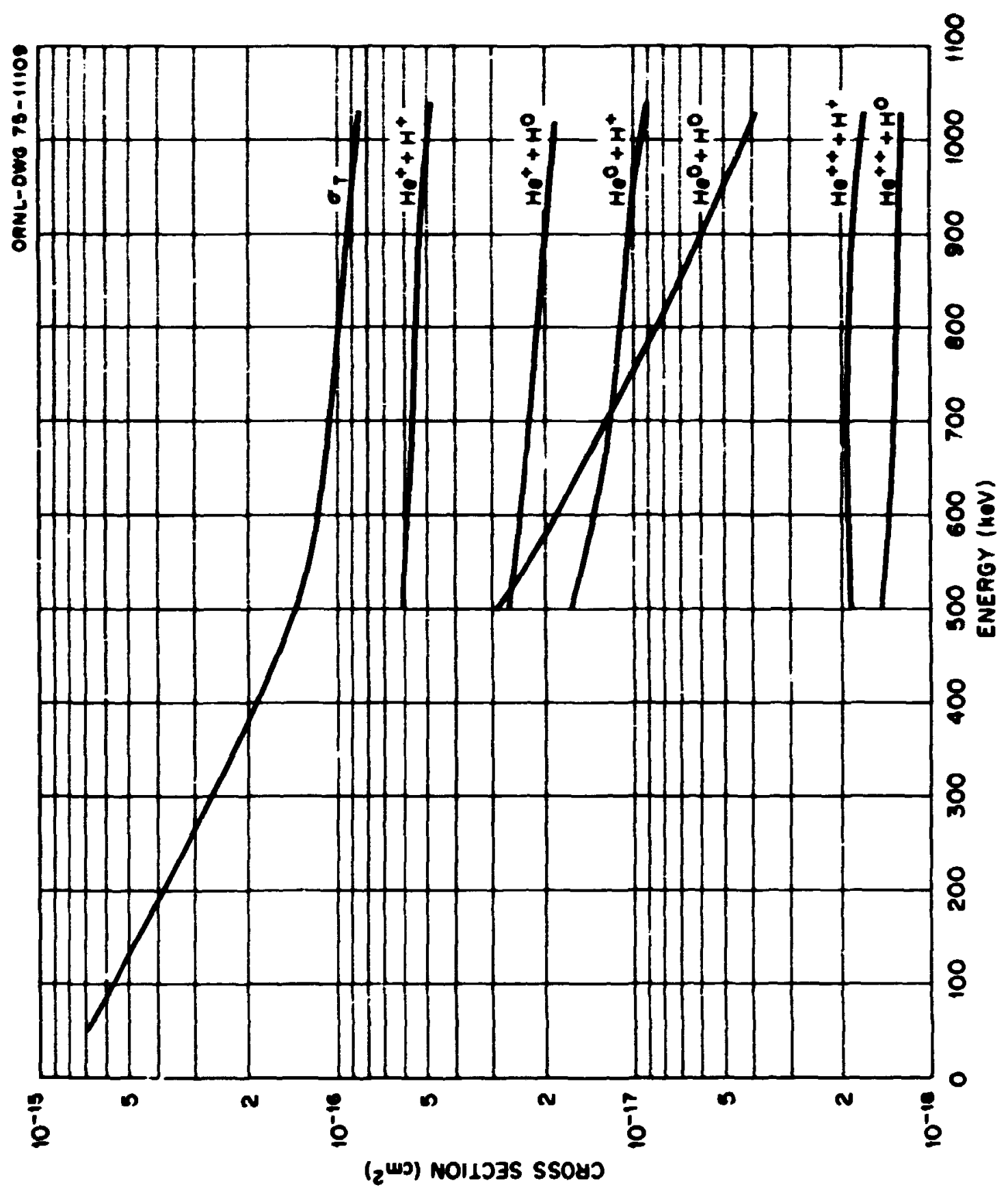




\section{A.3.33}

(Continationes)

$$
\begin{aligned}
& \text { Croas Sections for the Coaveraion of Fast } \mathrm{k}_{2}^{+} \text {Ioss into } \\
& \text { Protone is Pasarese Through } \mathrm{B}_{2} \text { and } \mathrm{B}
\end{aligned}
$$

\section{Doces:}

(1) The proton probetion crons gections is the su of the crose sections

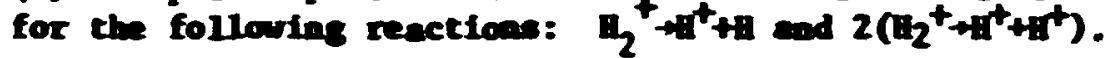

(2) The discrepencies in the cross sections mesured by different observers, particulaply at the lower lepact corries, probubly arise minly through the we of $\mathrm{B}_{2}{ }^{+}$been having different vibrational aneries.

(3) Por $\mathrm{B}_{2}$ target ouly two easuremeats have been ade above $1 \mathrm{Hel}$ - at 3 and 20 Hin. 
4.6.2

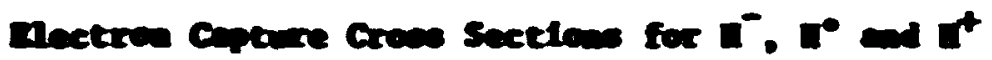

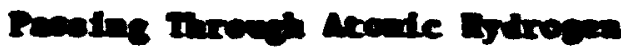

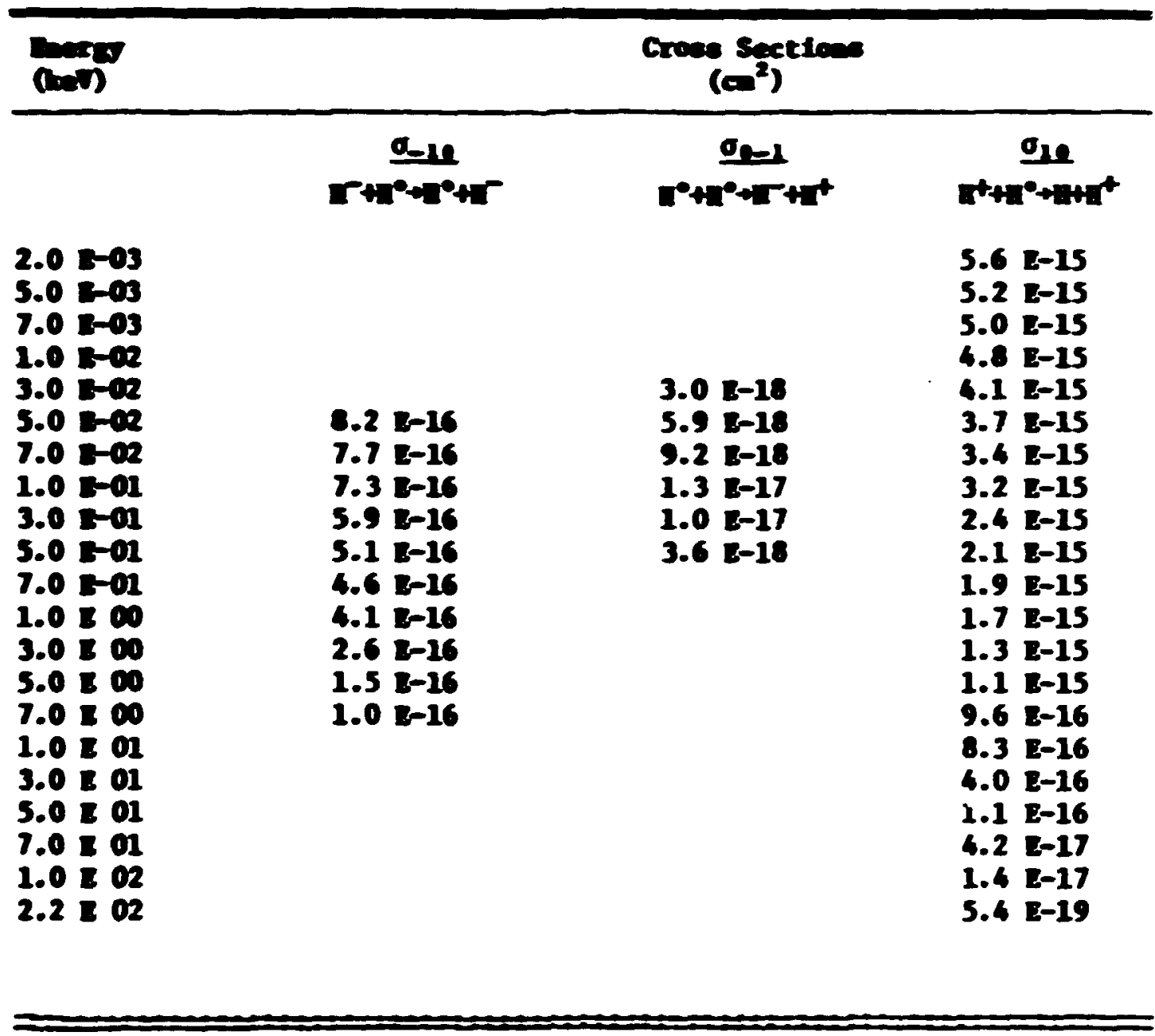

\section{Deference:}

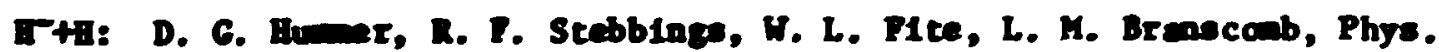
ov. 119, 668 (1960).

$\mathrm{n}^{\circ}+\mathrm{H}^{\bullet}$ : G. W. HeClure, Physe. Rev. 166, 22 (1968).

$\mathrm{H}^{+}+\mathrm{H}^{\bullet}$ : V. A. Belyaev, B. D. Breshnev, and E. M. Eraetov, Sov. Phys.JE1 25, 777 (1967)); G. W. HeClure, Phye. Rev. 148, 47 (1966); H. L. Fite, A. C. R. Srith and R. T. Stebbing, Proc. Roy. Soc. (London) 1268 , 527 (1962); W. L. Ftee, R. P. Stebbinge, D. G. Hurer, and R. T. Brackien, Phys. Rev. 119, 663 (1960); A. B. Mnitekover, G. Ryding, and H. B. Gilbody, Proc. Physe. Soc. 89, 541 (1966).

\section{Aceures:}

t207. 


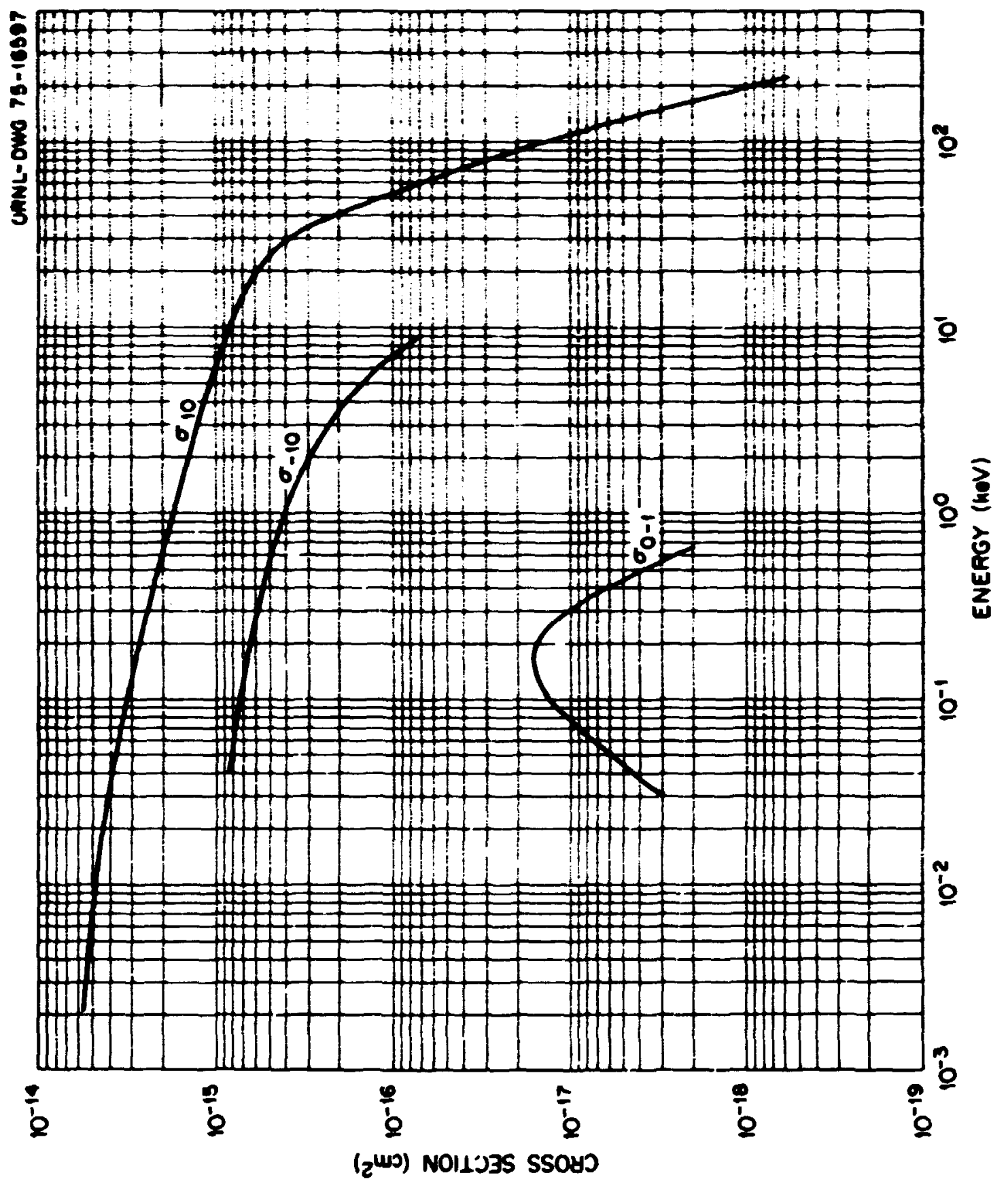


Wlectron Coptwre Croas Section for $\mathrm{a}^{+}$and $\mathrm{a}^{\circ}$

Peasing Irrough $\mathrm{m}_{2}$

\begin{tabular}{|c|c|c|c|}
\hline Dery & \multicolumn{3}{|c|}{$\begin{array}{c}\text { Croas Sections } \\
\left(c^{2}\right)\end{array}$} \\
\hline & $\sigma_{10}$ & $\sigma_{1-1}$ & $\sigma_{0-}$ \\
\hline
\end{tabular}

$5.0 \mathrm{E}-02$

$1.0 \mathrm{E}-01$

2.0 E-OI

$5.0 \mathrm{E}-01$

1.0 E 00

$2.0 \geq 00$

5.0 E 00

1.0 E 01

2.0 E 01

4.0 E 01

6.0 E 01

1.0 E 02

$2.0 \geq 02$

4.0 E 02

6.0 E 02

1.0 E 03

$2.0 \mathrm{E} 03$

$3.0 \geq 03$
$2.7[-17$

$4.1 \quad \mathrm{E}-17$

7.3 E-17

$2.3=-16$

$5.2=-16$

8.5 E-16

8.6 E-16

8.5 E-16

$6.0 \mathrm{E}-16$

2.6 E-16

$1.1 \mathrm{E}-16$

2.8 E-17

$1.8 \mathrm{E}-18$

$5.7 E-20$

7.2 E-21

4.6 E-22

1.2 E-23

2.4 E-24 $\begin{array}{llll}1.2 & E-20 & & \\ 4.4 & E-20 & & \\ 1.2 & E-19 & & \\ 3.8 & E-19 & 4.5 & E-18 \\ 1.6 & E-18 & 2.1 & E-17 \\ 4.7 & E-18 & 2.2 & E-17 \\ 1.0 & E-17 & 1.9 & E-17 \\ 2.5 & E-18 & 9.5 & E-18 \\ 2.2 & E-19 & 5.3 E-18 \\ 1.0 & E-20 & 2.1 & E-18 \\ 1.2 & E-22 & 1.4 & E-19 \\ 1.3 & E-24 & 6.0 \mathrm{E}-21\end{array}$

2.4 E-26

2.6 E-28

\section{Beferences:}

$\mathrm{B}^{+}+\mathrm{B}_{2}+\mathrm{H}^{\bullet}$ : V. V. Afrosinov, G. A. Lelko, Yu. A. Manaev, and H. N. Panov, Sov. Phys.-JETP 29, 648 (1969); J. Abbe and J. Adloff, Bull. Soc. Chim. Frence 6, 1212 (1966); C. F. Barnett and H. K. Reynolds, Phys. Rev. 109. 335 (1958); R. H. Berkner, R. V. Pyle, and J. H. Stearns, Nuc. Pusion 10, 145 (1970); W. H. Craser, J. Chen. Phys. 35, 836 (1961); R. Curran, T. M. Donahue, and H. H. Kasner, Phys. Rev. 114, 490 (1959); F. J. de Beer, J. Schoteen and H. Moustafa, Physica 32,1766 (1966); J. Desesquelles, G. D. Cao, and M. Dufay Compt. Rend. 262B, 1329 (1966); Ya. M. Pogel, L. I. Krupnik, and B. G. Safronov, Sov. Phys.-JETP 1, 415 (1955); Yu. S. Gordeev and M. N. Peaov, Sov. Phys.-Tech. Phys. 2, 656 (1964); 0. Hollricher, 2. Physik 187, 41 (1965); D. W. Koopman, Phys. Rev. 154, 79 (1967); P. L. Ribe, Phys. Rev. 83, 1217 (1951); P. M. St1er and C. F. Barnett, Phys. Rev. 103, 896 (1956); U. Schryber, Hel. Phy. Act. 140, 1023 (1967); P. Schwirzke, 2. Phys1k 157, 510 (1960); L. H. Welsh, R. H. Berkner, S. N. Kaplan, and R. V. Pyle, Phys. Rev. 158, 85 (1967); J. B. H. SEedeford and J. B. Hasted, Proc. Roy Soc. (London) 1227,466 (1955); J. P. W1111am, Phys. Rev. 179, 240 (1969); L. H. Tobuten, M. Y. Raka1, and R. A. Langley, Phys. Rev. 171, 114 (1968). 


\section{A.4.5}

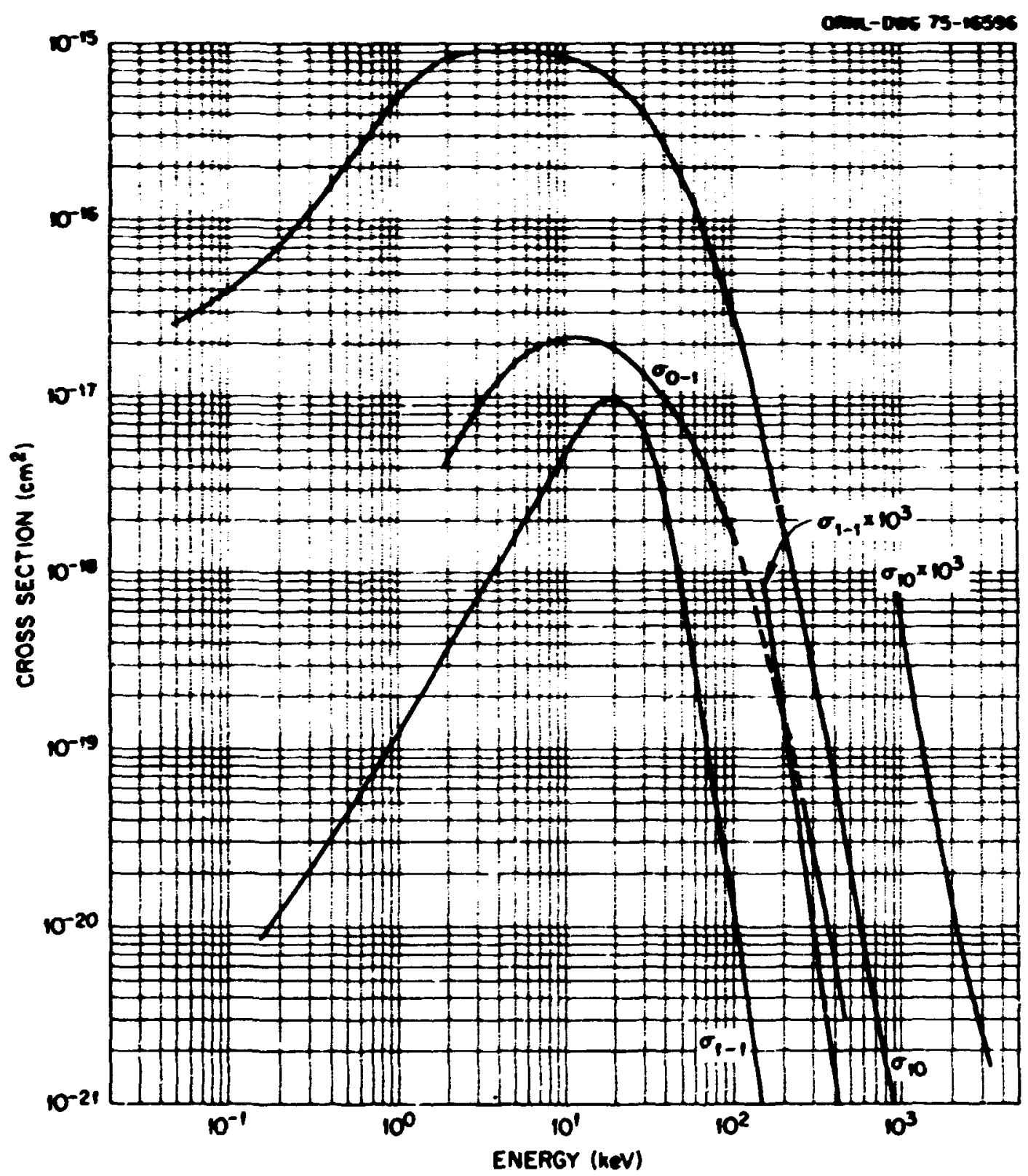


Electron Copture Crose Section for $\mathbf{u}^{+}$ad $\mathbf{u}^{*}$

Paseing Trrouth Eelio

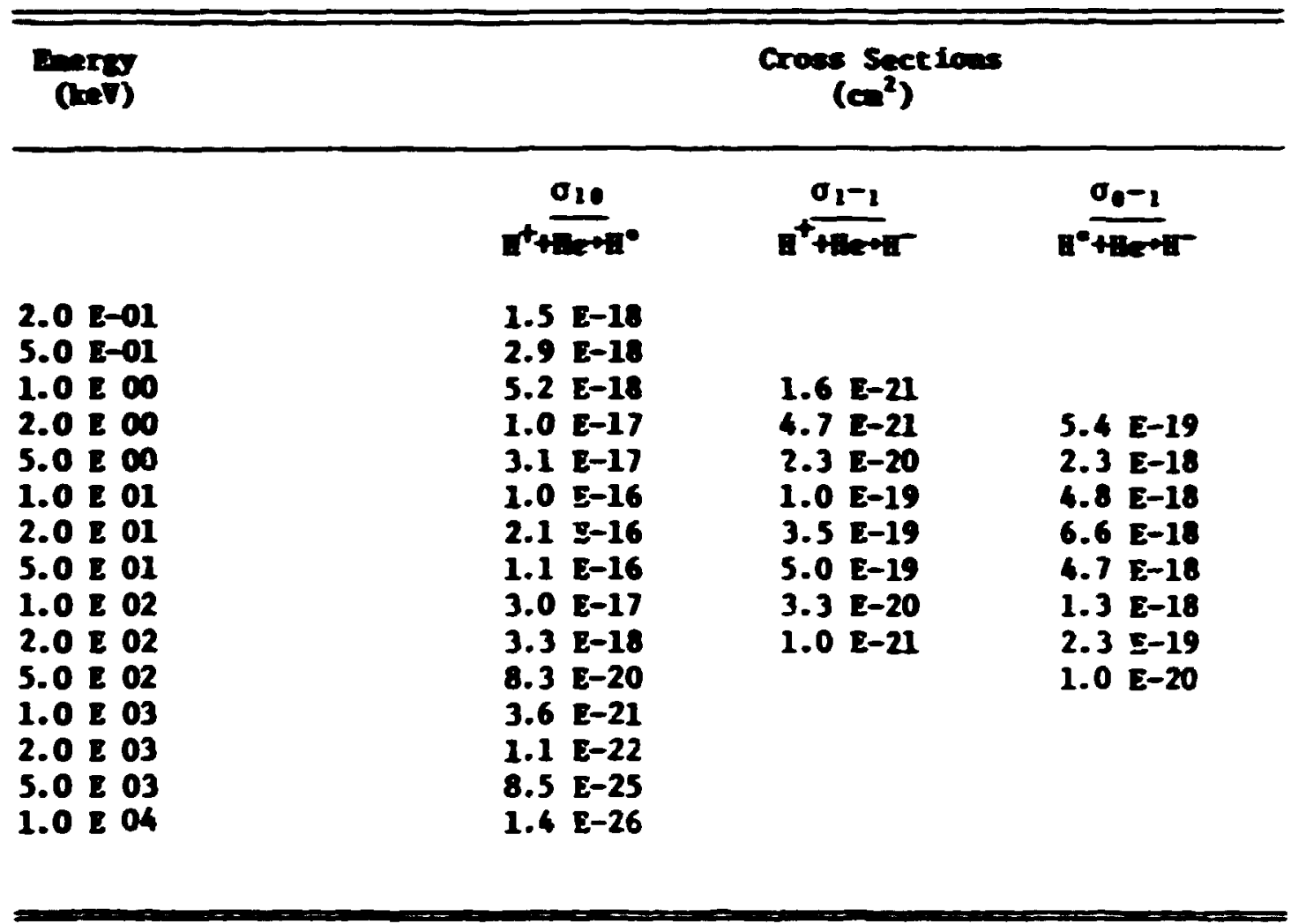

\section{Deferences:}

H+Hie+H*: V. V. Afrosiov, Yu. A. Manev, M. R. Panov, and M. V. Pedorenko, Sov. Phys.-Tech. Phys. 14, 109 (1969); S. K. Allison, Rev. Mod. Phys. 30, 1137 (1958); C. F. Barnett and H. R. Reynolds, Phys, Rev. 109, 355 (1958); K. H. Berkner, S. I. Kaplan, G. A. Paulikas, and R. V. Pyle, Phys. Rev. 140, A729 (1965); L. Coll1, F. Cristofor1, C. E. Prigerio, and P. G. Stone, Phys. Letters 3, 62 (1962); R. J. de Heer, J. Schutten, and H. Moustafa, Physica 32, 1766 (1966); J. B. H. Stedeford and J. B. Hasted, Proc. Roy. Soc. (London) 227A, 466 (1954); J. Desesquelles, G. D. Ceo, and M. Dufay, Compt. Rend. 262B, 1329 (1966); U. Schryber, Hel. Phy. Act. 140, 1023 (1962); P. M. Stier and C. T. Barnete, Phys. Rev. 103, 896 (1956); L. H. Toburen and M. Y. Makat, Phys. Rev. 177, 191 (1969); I. M. Welsh, K. H. Berkner, S. K. Kaplan, and R. V. Pyle, Phys. Rev. 158, 85 (1967); J. F. Willian, Phys. Rev. 157, 97 (1967).

$\mathrm{A}^{+}+\mathrm{He} \mathrm{H}^{-}$: V. F. Rozlov and S. A. Bonder, Sov. Phys,-JETP 23, 195 (1966); Ya. H. Pogel, Sov. Phys.-Usp. 3, 390 (1960); U. Schryber, Hel. Phy. Act. A40, 1023 (1967); L. H. Toburen and $\bar{M}$. Y. Nakal, Phys. Rev. 177, 191 (1969); J. F. W111ian, Phys. Rev. 150, 7 (1966). 


\section{A.6.7}

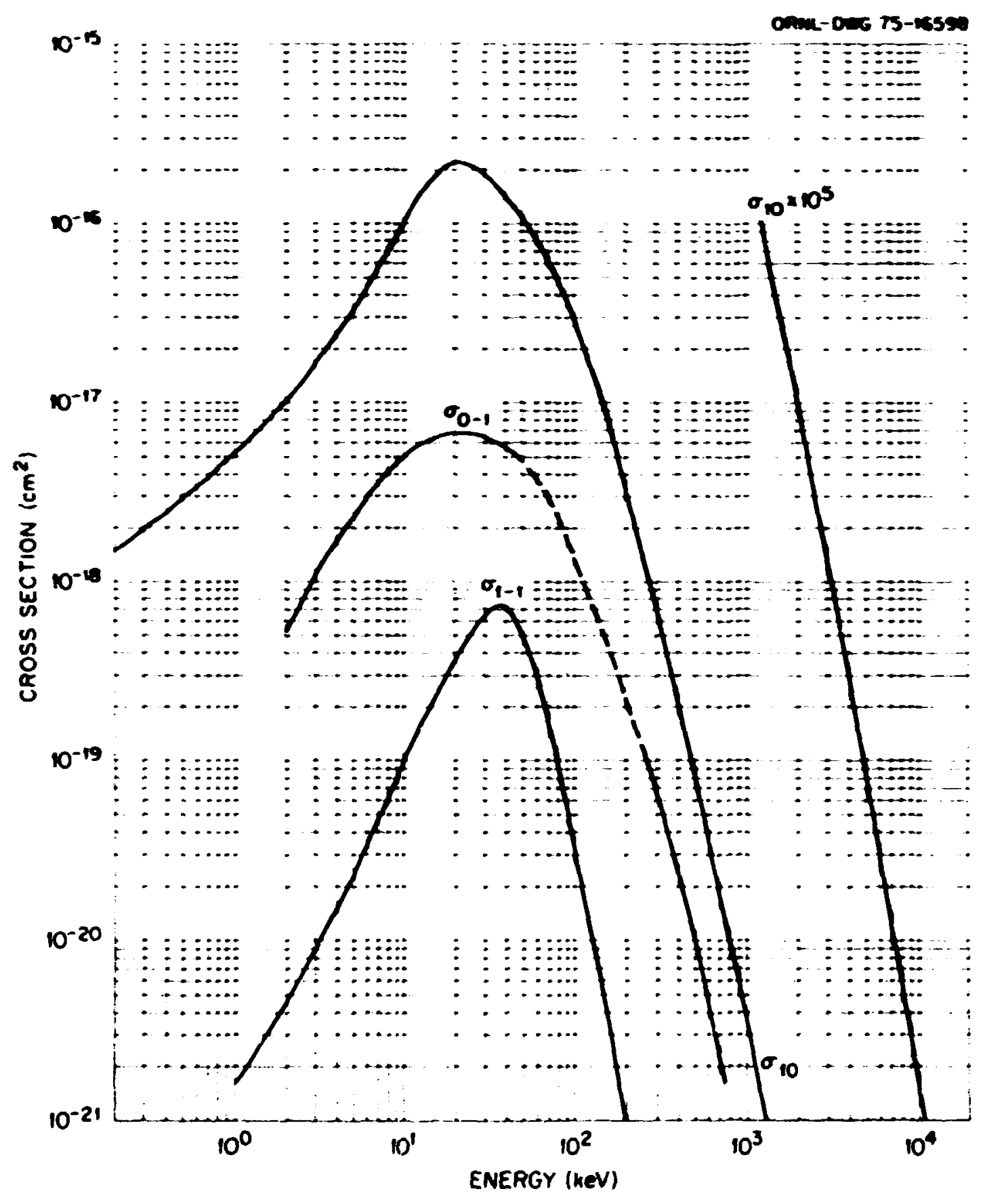


Electron Capture Cross Sections for $\mathrm{H}^{+}$and $\mathrm{H}^{\bullet}$

Passing Through $N_{2}$

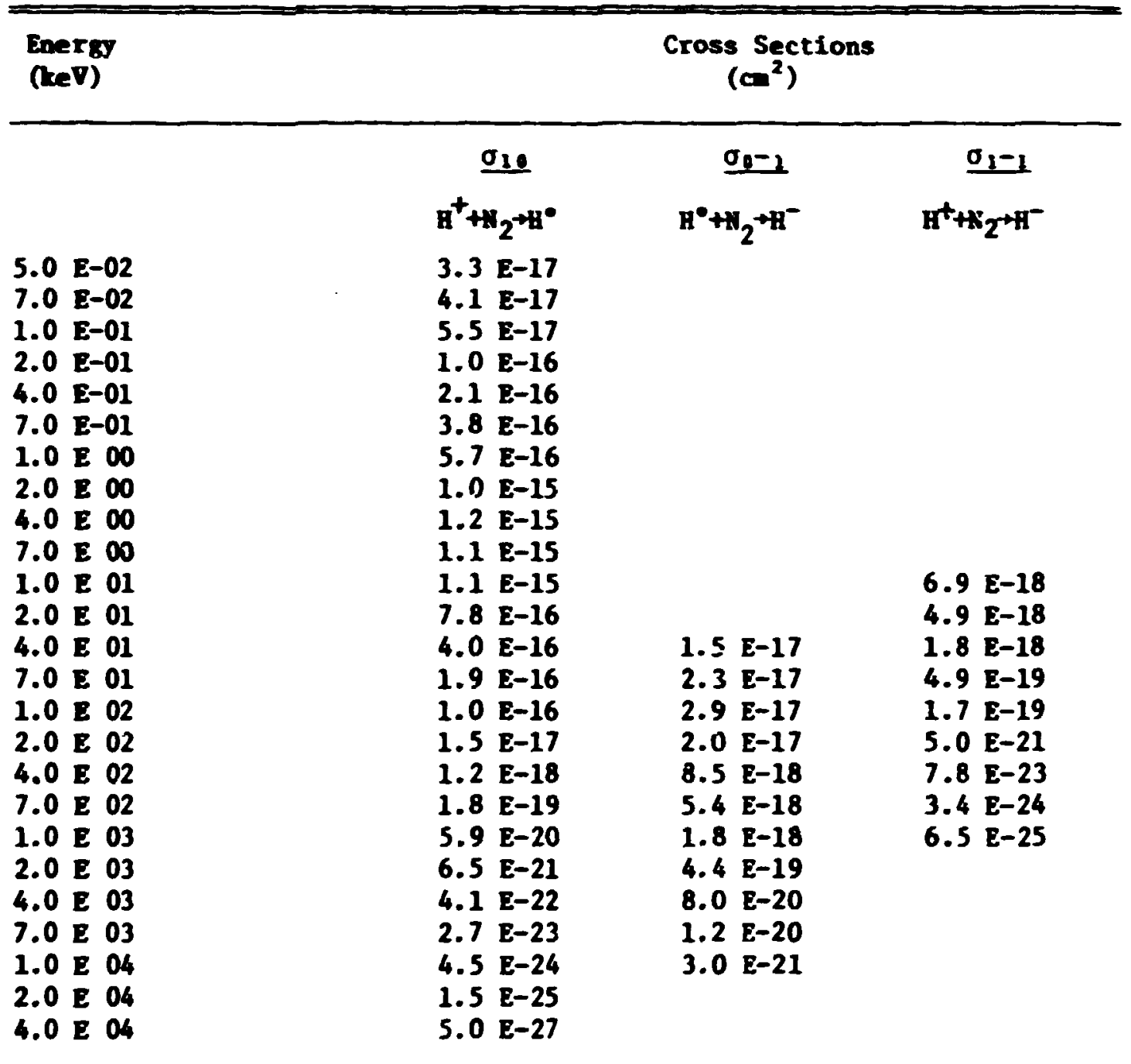

References:

$H^{+}+H_{2}+H^{\circ}$ : E. Acerbi, M. Castiglione, G. Dutto, F. Resmini, G. Succ1, and G. Taglloferri, Nuovo Cimento SOB, 176 (1967); S. K. Allison, Rev. Mod. Phys, 30, 1137 (1958); C. F. Barnete and H. K. Reynolds, Phys. Rev. 109, 355 (1958); F. J. de Heer, J. Schutten, and H. Moustafa, Physica 32, 1766 (1966); M. Dufay, J. Desquelles, M. Druet ta, and M. Eldeloberg, Ann. Geophys. 22, 614 (1966); H. B. Gilbody and J. B. Hasted, Proc. Roy. Soc. 238, 334 (1956); D. W. Koopman, Phys. Rev. 166, 57 (1968); R. J. McNeal and D. C. Clark, J. Geophys. Res. 74, 5065 (1969);U. Schryber, Helv. Phys. Acta 40, 1023 (1967); P. M. Stier and C. P. Barnete, Phys. Rev. 103, 896 (1956); L. H. Toburen, M. Y. Nakat, and R. A. Langley, Phys. Rev. 171, $1 \overline{14}$ (1968); M. Welsh, K. H. Berkner, S. N. Kaplan, and R. V. Pyle, Phys. Rev. 158, 85 (1967). 


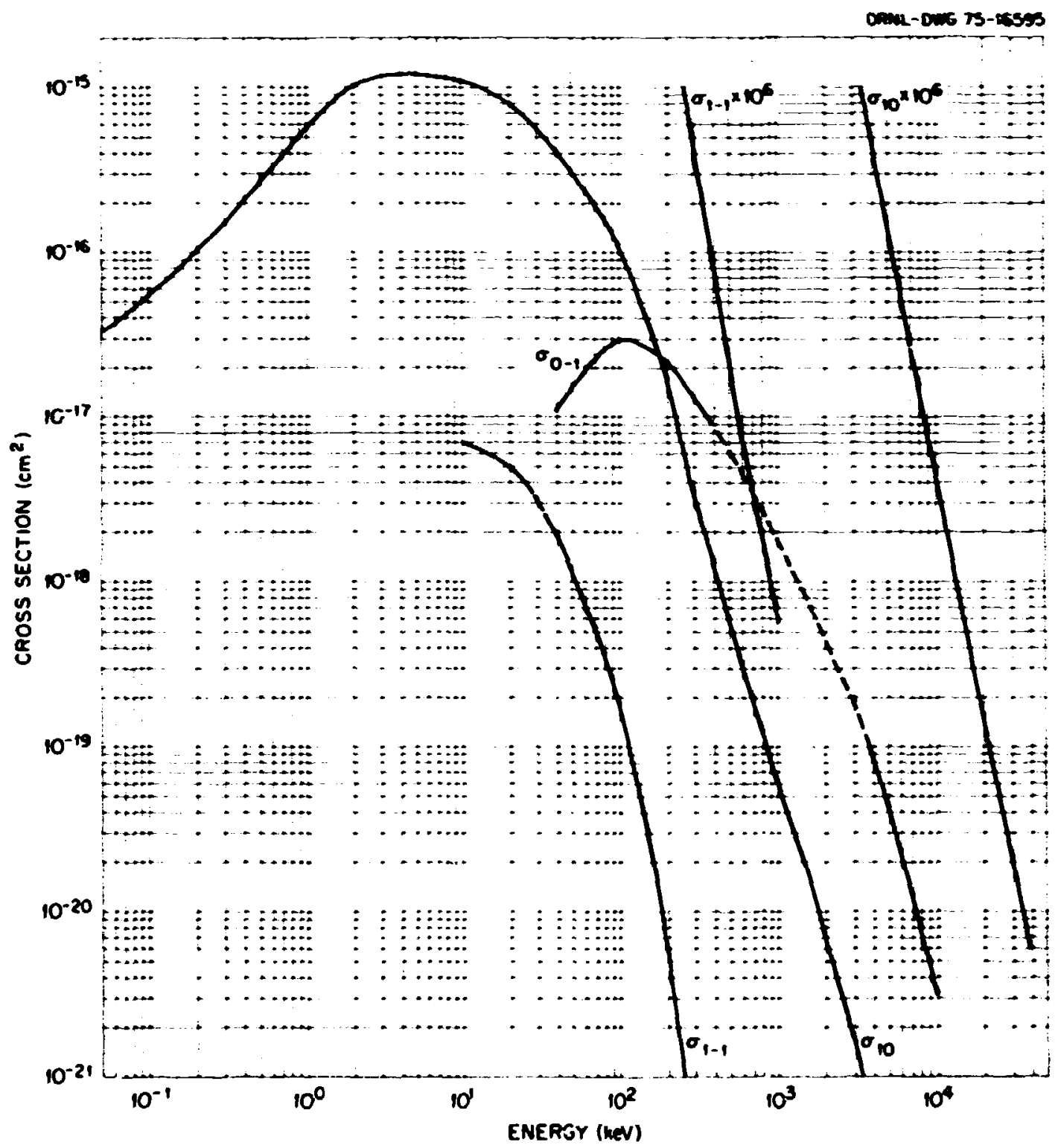




\section{A.4.10}

Electron Capture Cross Sections for $\mathrm{H}^{+}$and

$\mathrm{H}^{\bullet}$ Passing Through $\mathrm{O}$ and $\mathrm{O}_{2}$

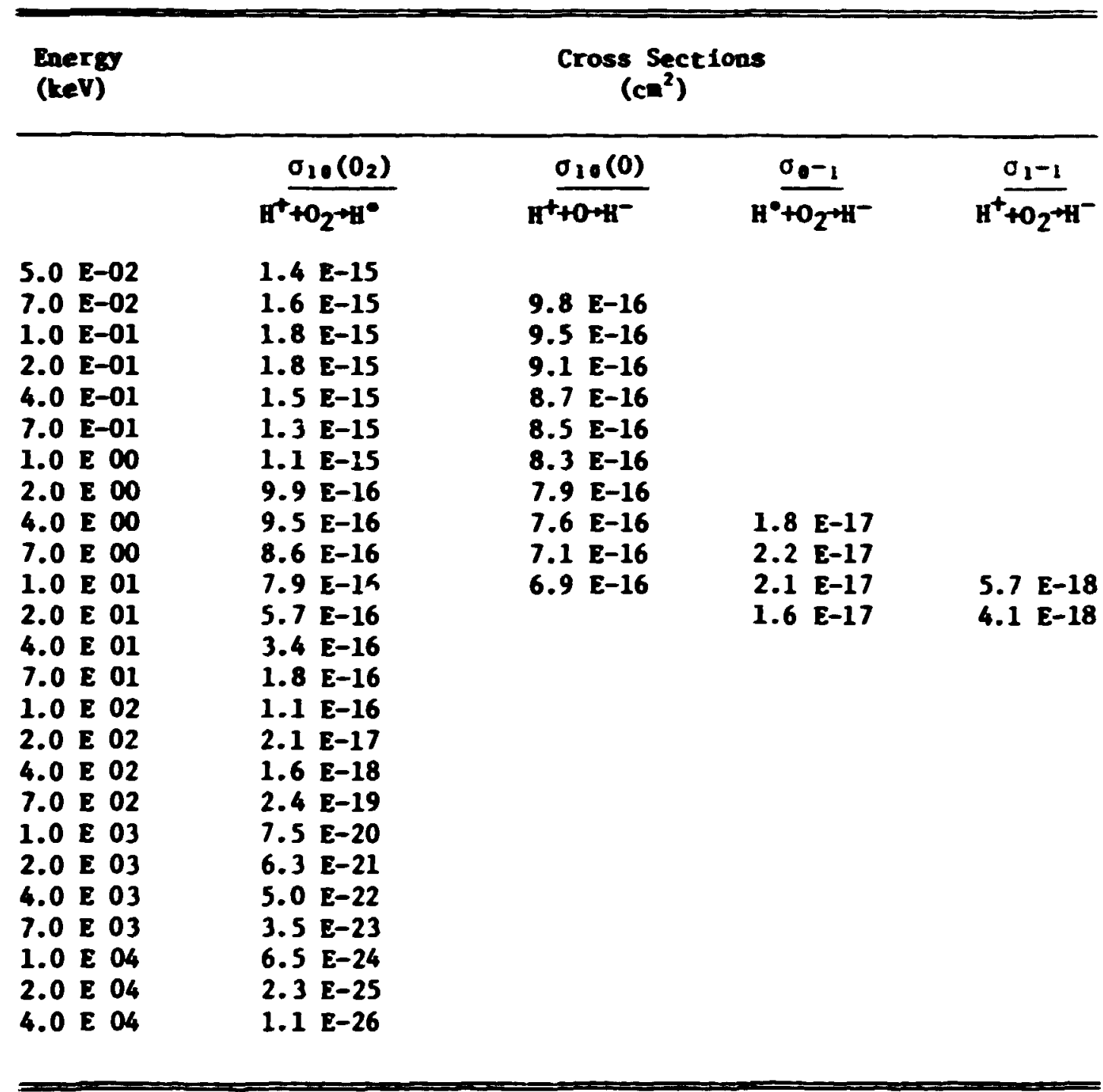

\section{References:}

$\mathrm{H}^{+}+\mathrm{O}_{2}+\mathrm{H}^{\circ}$ : E. Acerbi, M. Castiglioni, G. Dutto, F. Resmini, G. Succi, and G. Taglioferri, Nuovo Cimento 50B, 176 (1967); S. K. Allison, Rev. Mod. Phys. 30, 1137 (1958); F. J. de Heer, J. Schutten, and H. Moustafa, Phystca 32, 1766 (1966); J. Desquelles, G. D. Cao, and M. Dufay Compt. Rend. 262B, 1329 (1966); M. Dufay, Ann. Geophys. 22, 641 (1966); D. H. Kocpann, Phys. Rev. 166, 57 (1968); L. H. Toburen M. Y. Nakal, and R. A. Langley, Phys. Rev. 171,114 (1968); U. Schryber, Helv. Phys. Acta 40, 1023 (1967);

R. F. Stebbings, A. C. H. Saith, and H. Ehrhardt, J. Geophys. Res. 69, 2349 (1964); P. M. Stier and C. F. Barnett, Phys. Rev. 103, 896 (1956). 


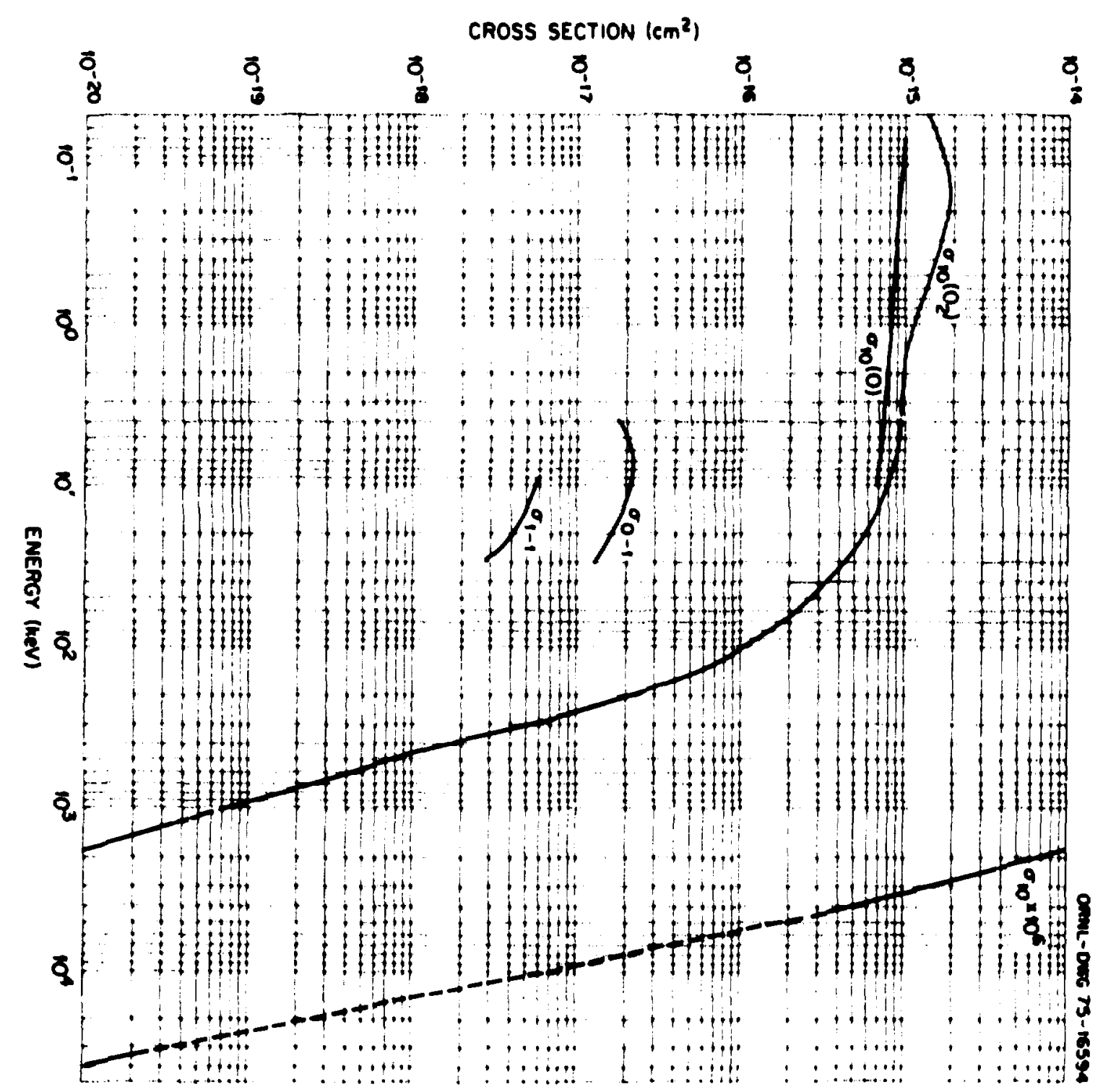


Electron Capture Cross Sections for $\mathrm{H}^{+}$and $\mathrm{H}^{\bullet}$

Passing Through ste Gas

\begin{tabular}{|c|c|c|c|}
\hline \multirow[t]{2}{*}{$\begin{array}{l}\text { Enerso } \\
\text { (Rev) }\end{array}$} & \multicolumn{3}{|c|}{$\begin{array}{c}\text { Cross Sections } \\
\left(\mathrm{cm}^{2}\right)\end{array}$} \\
\hline & $\sigma_{11}$ & $\underline{\sigma_{0}-1}$ & $\underline{\sigma_{1}-1}$ \\
\hline & $n^{+}+1$ we-He & $\mathrm{H}^{\circ}+\mathrm{lll}+\mathrm{K}$ & $\mathrm{H}^{+}+$Hie-HT \\
\hline $\begin{array}{l}4.0 \\
4.0 \\
7.0\end{array}$ & $\begin{array}{ll}8.6 & E-18 \\
2.1 & E-17 \\
3.5 & E-17 \\
8.8 & E-17 \\
1.8 & E-16 \\
2.8 & E-16 \\
3.0 & E-16 \\
2.3 & E-16 \\
1.3 & E-16 \\
7.6 & E-17 \\
4.7 & E-17 \\
1.4 & E-17 \\
2.1 & E-18 \\
2.6 & E-19 \\
5.0 & E-20 \\
4.2 & E-21 \\
3.2 & E-22\end{array}$ & $\begin{array}{ll}1.7 & E-18 \\
6.0 & E-18 \\
1.3 & E-17 \\
1.5 & E-17 \\
1.1 & E-17 \\
4.5 & E-18\end{array}$ & 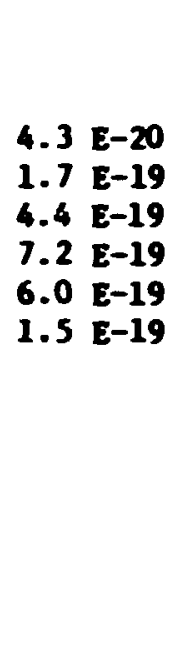 \\
\hline
\end{tabular}

References:

It+hera': V. V. Afrosimov, R. M. Il'in, and E. S. Solov'ev, Sov. Phys.Tech. Phys. 5, 661 (1960); S. K. Allison, Rev. Mod. Phys. 30, 1137 (1958); P. J. de Heer, J. Schutcea, and H. Houstafa, Physica 32, $17 \overline{766}$ (1966); J. B. H. Stedeford and J. B. Hasted, Proc. Roy. Soc. (London) 1227, 466 (1955); P. M. Stier and C. F. Bamett, Phys. Rev. 103, 896 (1956); U. Schryber, Helv. Phys. Acte 40, 1023 (1967); J. P. Wilileas and D. K. F. Dubar, Phys. Rev. 149, 62 (1966).

$H^{\circ}+$ the+H': S. K. Allison, Rev. Mod. Phys. 30, 1137 (1958); P. M. Stier and C. P. Barnett, Phys. Rev. 103, 896 (1956); J. F. Milliam, Phys. Rev. 153, 117 (1967).

Ht+lle+fi: S. K. Allieon, Rev. Hod. Phys. 30, 1137 (1958); V. F. Rozlow and S. A. Bondar, Sov. Phys.-JETP 23, 195 (1966); Ya. M. Pogel, R. V. Mitin, V. P. Rozlow, and M. D. Ronachko, Sov. Phys.-JETP ㅇ, 390 (1959); J. F. Hill1es, Phys. Rev. 150, 7 (1966).

Accuracy:

$\sigma_{10}- \pm 202 \quad \sigma_{0-1}- \pm 252 \quad \sigma_{1-1}=$ unreliable data.

Motes:

O1. has not been wasured between $200 \mathrm{keV}$ and $1 \mathrm{MeV}$. 


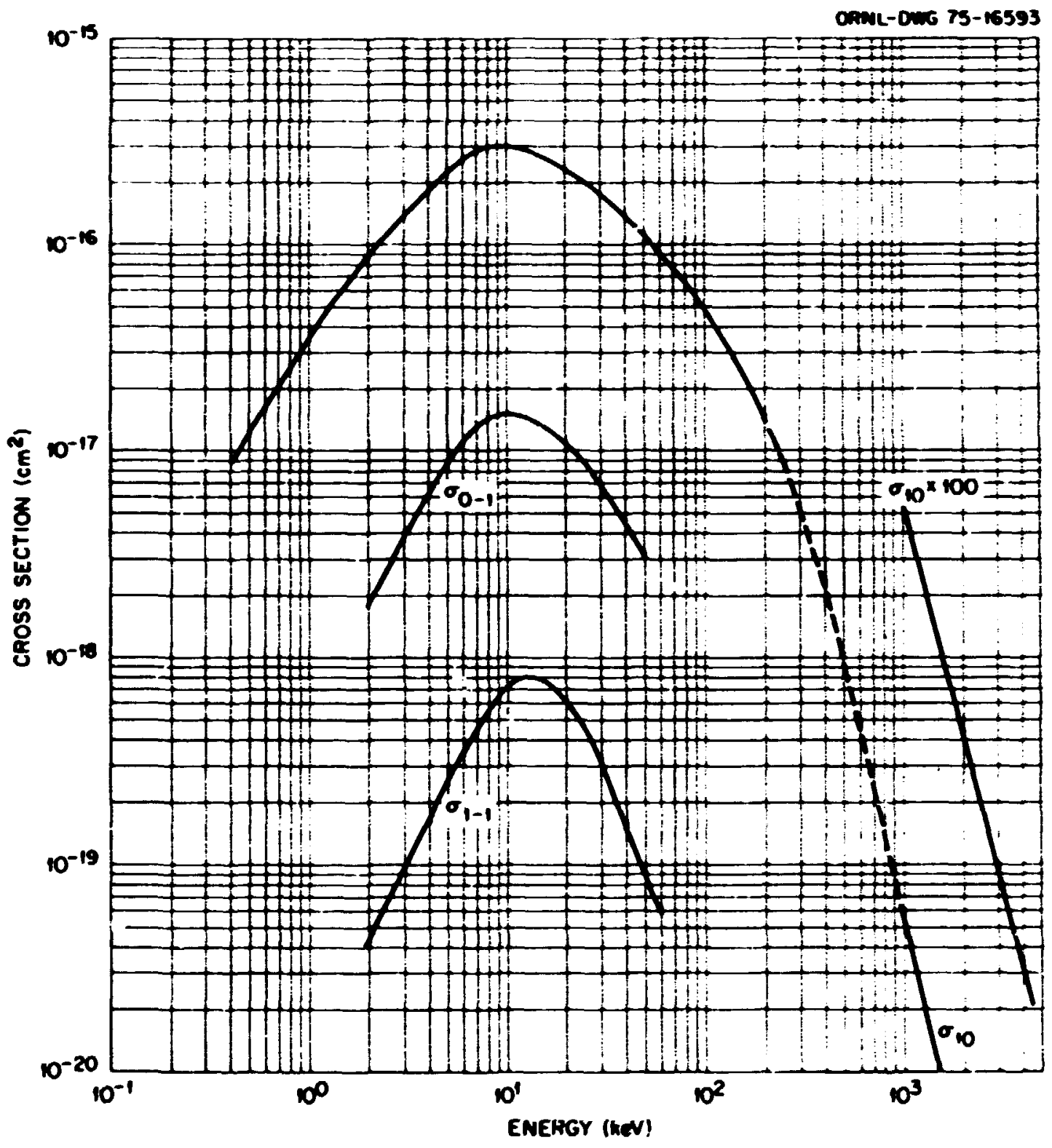


Electron Capture Cross Sections for $\mathrm{H}^{+}$and $\mathrm{H}^{*}$

Passing Through Ar

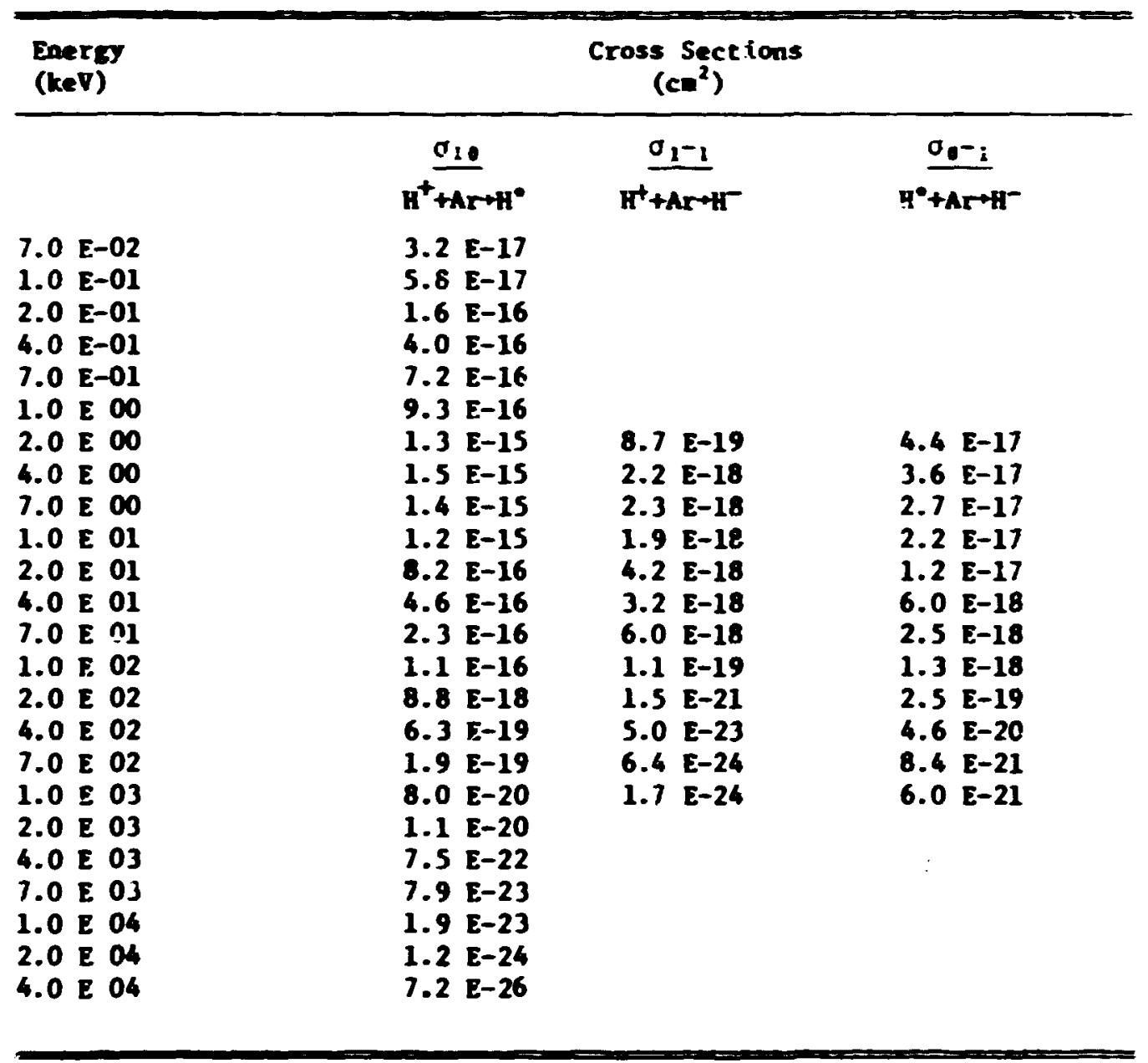

\section{References:}

$\mathrm{H}^{+}+\mathrm{Ar}+\mathrm{H}^{*}$ : V. V. Afrosilov, Yu. A. Mamaev, M. A. Panov, V. Uroskevich, Sov. Phys.-Tech. Phys. 12, 512 (1967); V. V. Afrosimov, R. N. Iliǹ, E. S. Solovév, Sov. Phys.-Tecin. Phys. 5, 661 (1960); E. Acerb1, M. Caseigliont, G. Dutto, F. Reseini, G. Succi, and G. Tagliaferti, Nuovo Cimento, SOB, 176 (1967); C. F. Barnete and H. R. Reynolds, Phys. Rev. 109, 355 (1958); K. H. Berkner, S. N. Kaplan, G. A. Paullkes, and R. V. Pyle, Phys. Rev. 140, A729 (1965); L. M. Welsh, K. H. Berkner, S. M. Raplan, H. Selig, and R. V. Pyle, Phys. Rev. 158, 85 (1967); $x^{\prime}$. M. Stier and C. F. Barnett, Phys. Rev. 103, 896 (1956); YU. S. Cordeev and M. N. Panov, Sov. Phys.-Tech. Phys. 9, 656 (1964); F. J. de Heer, 3. Schutten, and H. Houstafa, Physica 32. 1766 (1966); D. H. Roopman, Phys. Rev. 154, 79 (1967); G. Monnom, Report IEUR-CEA-FC-762 (1975); l. Schryber, He1v. Phys. Acta 40, 1023 (1967); L. H. Toburen, M. Y. Maka1, and R. A. Langley, Phys. Rev. 17i, 114 (1968); J. F. Hillias and D. N. F. Durbar, Phys. Rev. 149, 62 (1966); P. M. Stier and C. F. Bamete, Phys. Rev. 103, 896 (1956). 


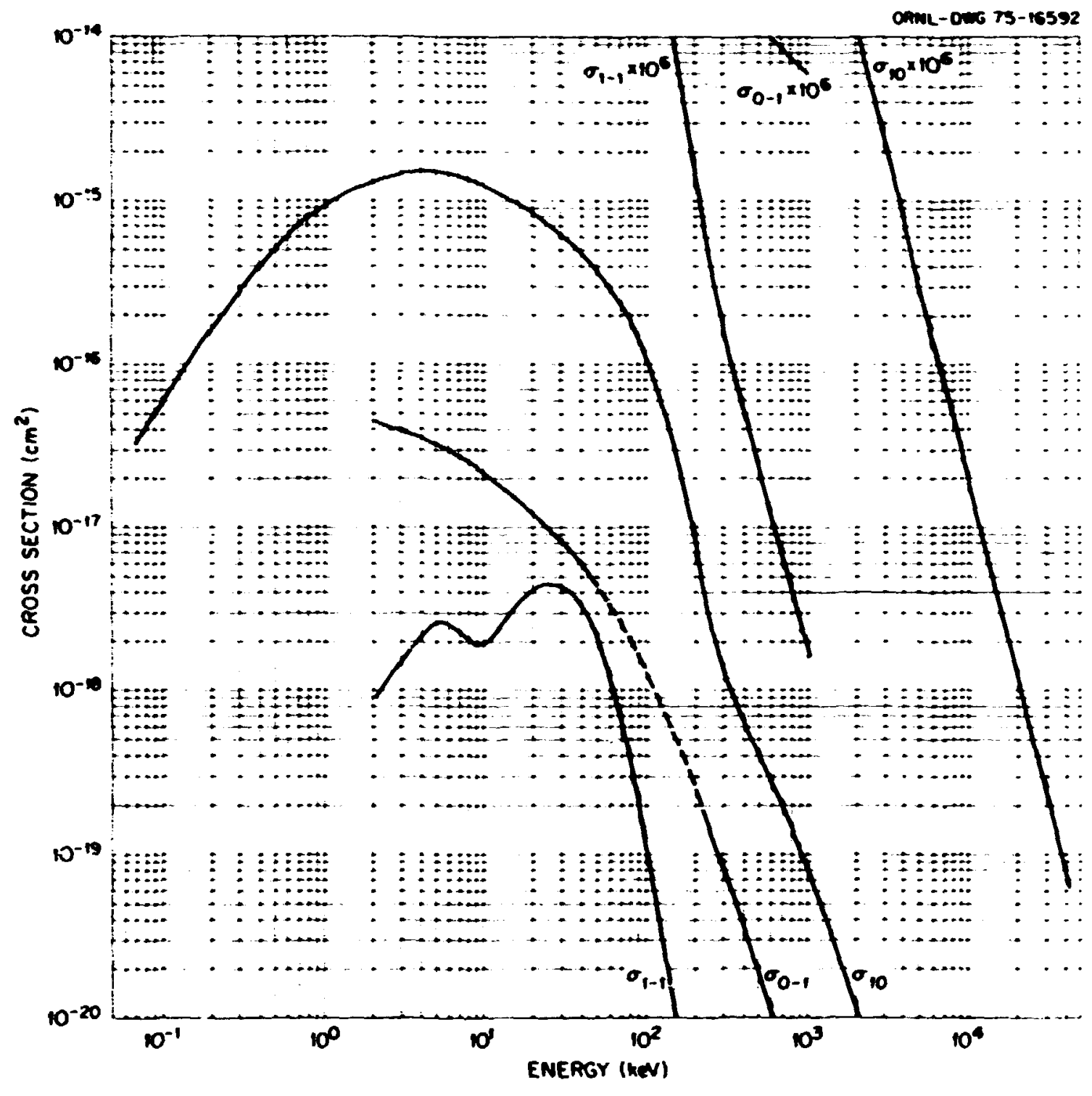


Electron Copture Cross Sectlons for $\mathbf{u}^{+}$

$$
\text { In } \mathrm{B}_{2} \mathrm{O} \text { and } \mathrm{CH}_{6}
$$

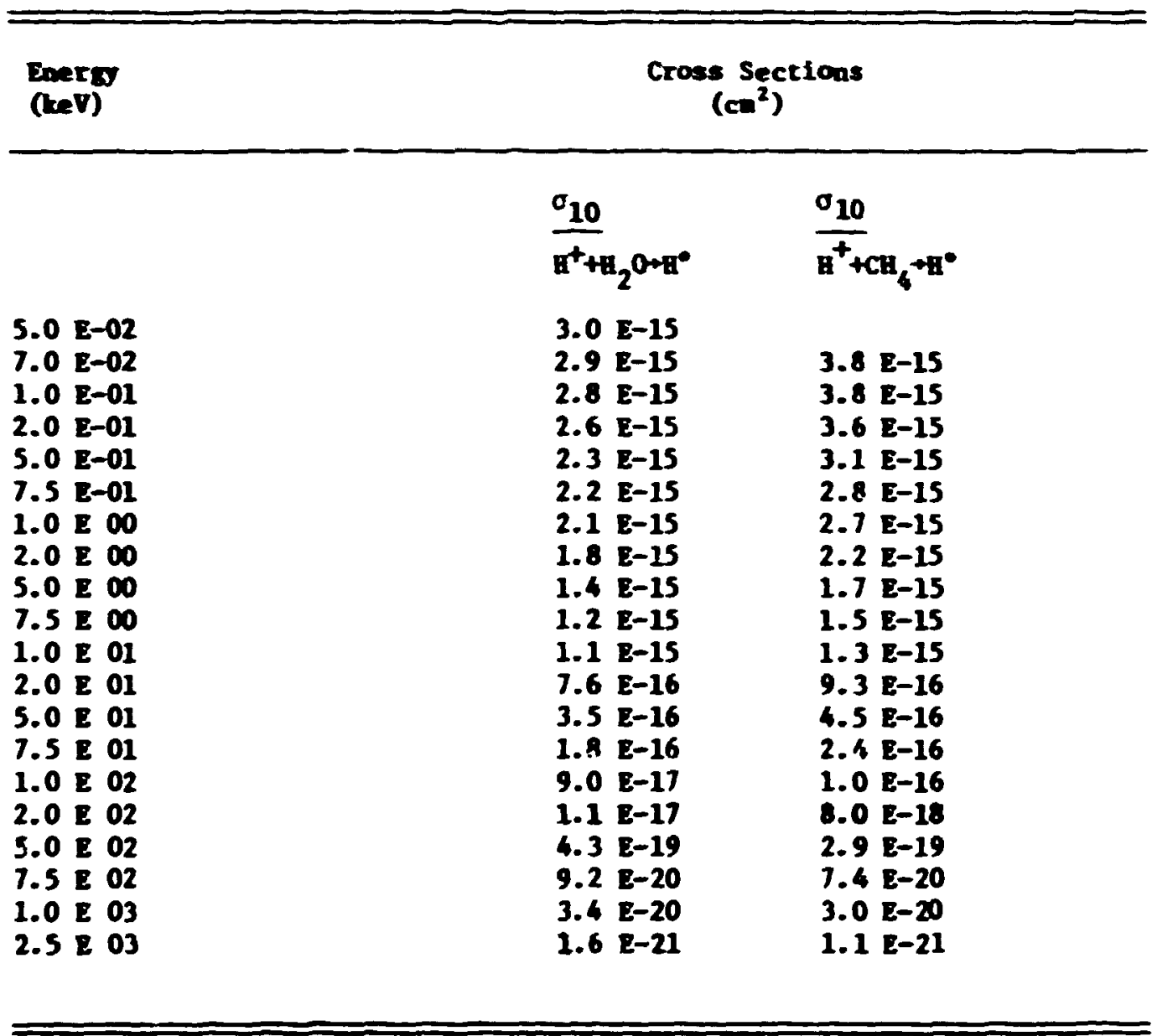

\section{Deferences:}

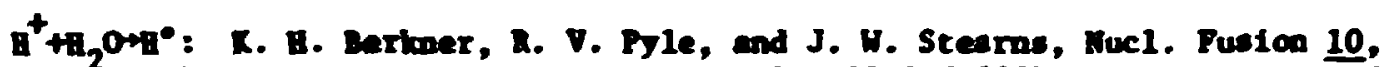
145 (1970); Z. S. Chembers, Report I0. UCL-14214 (1965); M. A. Coplan and R. W. Odive, J. Chen. Phye. 52, 4is4 (1970); R. Dagnac, D. Binac, and D. Hollac, J. Frye. B. 3. 1239 (1970); D. U. Roopmen, Phys. Rev. 166, 57 (1968); L. B. Toburea, H. T. Rakat, and R. A. Langley, Phys. Rev. 171, 114 (1968).

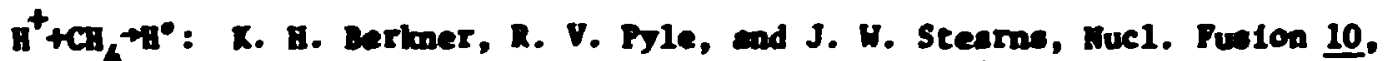
145 (1970); R. S. Chabers, Report no. UChL-14214 (1965); J. G. Collin and P. Rerbarle, J. Chea. Phys. 46, 1087 (1967); J. Desesquelles, G. D. Cao, and H. Duf 3, Come. Read. 2621, I329 (1966); D. W. Koopman, J. Chea. Phys. 49. 5203 (1968); R. J. Fellent, J. Che. Phys. 53, 4308 (1970); L. H. Toburen, M. T. Make1, and R. A. Lengley, Thre. Rev. 171, 114 (1968).

Accures:

$a^{+}+\mathrm{B}_{2} \mathrm{O}:=258$
$\mathrm{~B}^{+}+\mathrm{a}_{4}= \pm 25 \%$ 


\section{A. 4.17}

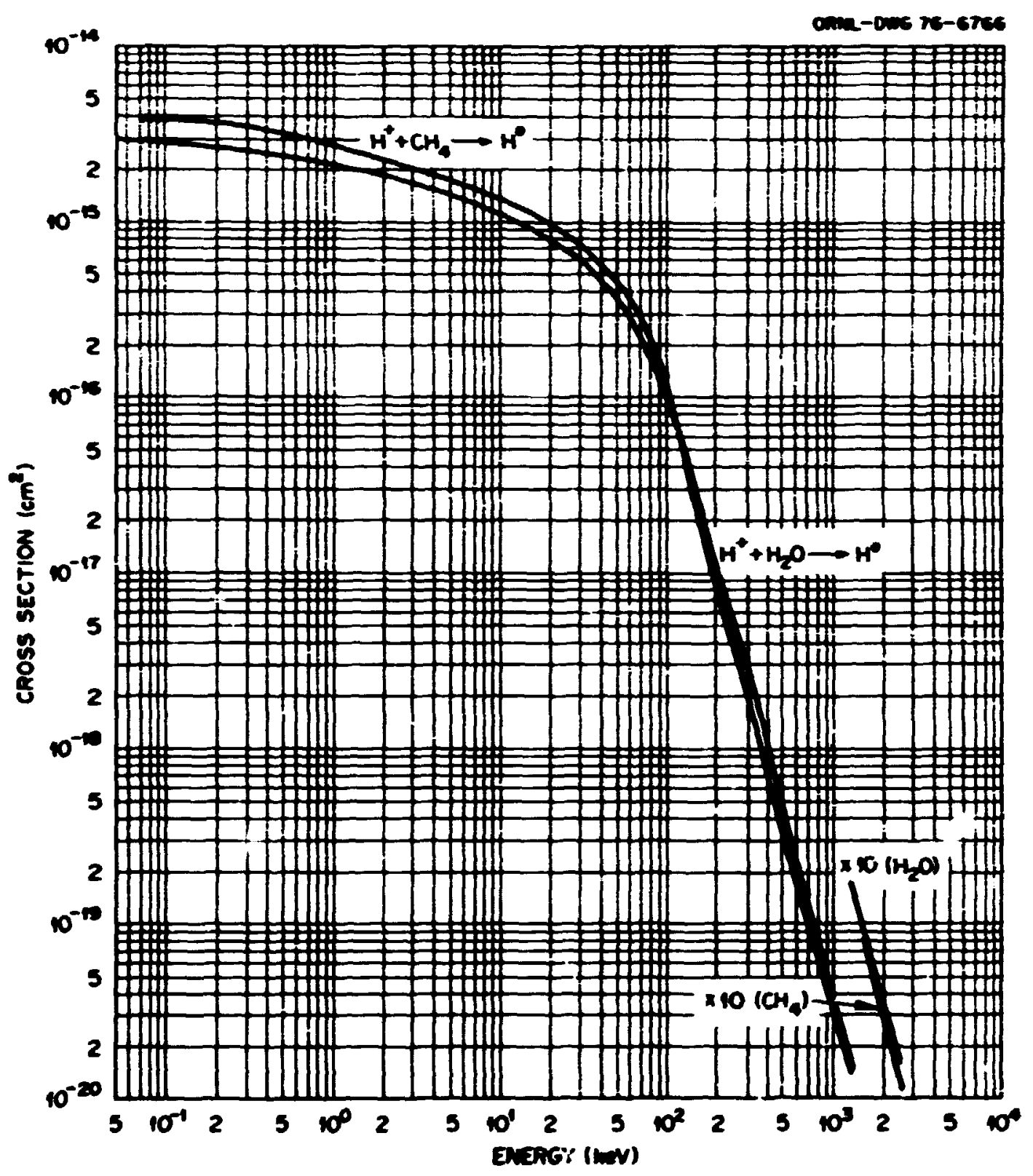




\section{A.4.18}

Electron Capture Cross Sections for

$$
\mathrm{H}^{+} \text {in } \mathrm{CO} \text { and } \mathrm{CO}_{2}
$$

\begin{tabular}{|c|c|c|}
\hline \multirow[t]{2}{*}{$\begin{array}{l}\text { Enersy } \\
\text { (keV) }\end{array}$} & \multicolumn{2}{|c|}{$\begin{array}{c}\text { Cross Sections } \\
\left(\cos ^{2}\right)\end{array}$} \\
\hline & $\frac{\sigma_{1 e}}{\mathrm{~A}^{+}+\cos ^{\circ}}$ & $\frac{\sigma_{10}}{n^{+}+\infty_{2}+H^{*}}$ \\
\hline 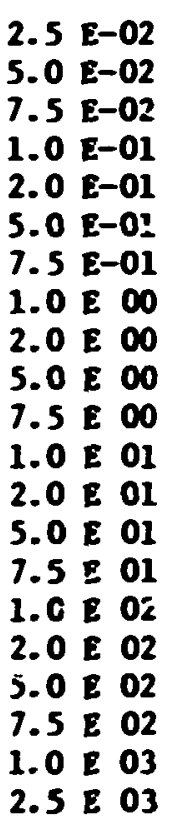 & $\begin{array}{ll}4.7 & E-16 \\
7.7 & E-16 \\
1.0 & E-15 \\
1.3 & E-15 \\
1.7 & E-15 \\
1.9 & E-15 \\
1.8 & E-15 \\
1.8 & E-15 \\
1.7 & E-15 \\
1.3 & E-15 \\
1.1 & E-15 \\
1.0 & E-15 \\
6.8 & E-16 \\
2.5 & E-16 \\
1.3 & E-16 \\
7.9 & E-17 \\
1.5 & E-17 \\
6.5 & E-19 \\
1.8 & E-19 \\
6.7 & E-20 \\
3.0 & E-21\end{array}$ & $\begin{array}{ll}2.9 & E-15 \\
2.8 & E-15 \\
2.7 & E-15 \\
2.4 & E-15 \\
1.9 & E-15 \\
1.7 & E-15 \\
1.5 & E-15 \\
1.3 & E-15 \\
1.1 & E-15 \\
1.0 & E-15 \\
9.4 & E-16 \\
7.5 & E-16 \\
3.8 & E-16 \\
2.3 & E-16 \\
1.6 & E-16 \\
2.5 & E-17 \\
1.1 & E-18 \\
2.6 & E-19 \\
9.0 & E-20 \\
3.6 & E-21\end{array}$ \\
\hline
\end{tabular}

\section{References:}

A $^{+}+\mathrm{CO}+\mathrm{H}^{\bullet}:$ R. H. Berkner, R. V. Pyle, and J. W. Stearns, Nucl. Fusion 10, 145 (1970); E. S. Chambers, Report No. UCRL-14214 (1965); J. Desesquelles, G. D. CaO, and K. Dufay, Comp. Rend. 262B, 1329 (1966); H. B. Gilbody and J. B. Hasted, Proc. Roy. Soc. London 239A, 336 (1956); E. Gustafssch and and E. Lindroln, Ark. Pysik, 18, 219 (1960); R. J. Helieal, J. Chen. Phys. 53, 4308 (1970); M. C. Poulizac, J. Desesquelles, M. Dufay, Annls. Astrophys. 30,301 (1967); L. H. Toburen, M. Y. Makal, and R. A. Langley, Phys. Rev. 171, 114 (1968).

$\mathrm{H}^{+}+\mathrm{CO}_{2}+\mathrm{H}^{\circ}$ : M. A. Coplan and R. W. Ogilvie, J. Chen. Phys. 52, 4154 (1970); J. Defesquelles, G. D. CaO, M. Dufay, Compt. Rend. 262B, 1329 (1966); D. W. Koopman, Phys. Rev. 166, 57 (1968); R. J. Melleal, J. Chea. Phys. 53, 4308 (1970); L. H. Toburen, M. Y. Nakal, and R. A. Langley, Phys. Rev. 171, 114 (1968).

Hote: $R$. H. Berkner, et al. results are for incident $D^{\circ}$. Data 1s plotted at $1 / 2 \mathrm{E}$. Accuracz:

$H^{+}+\cos H^{*}: \pm 20 \%$

$\mathrm{H}^{+}+\mathrm{CO}_{2} \rightarrow \mathrm{H}^{\circ}: \pm 20 \%$. Below 1 keV uncertainty is large. 


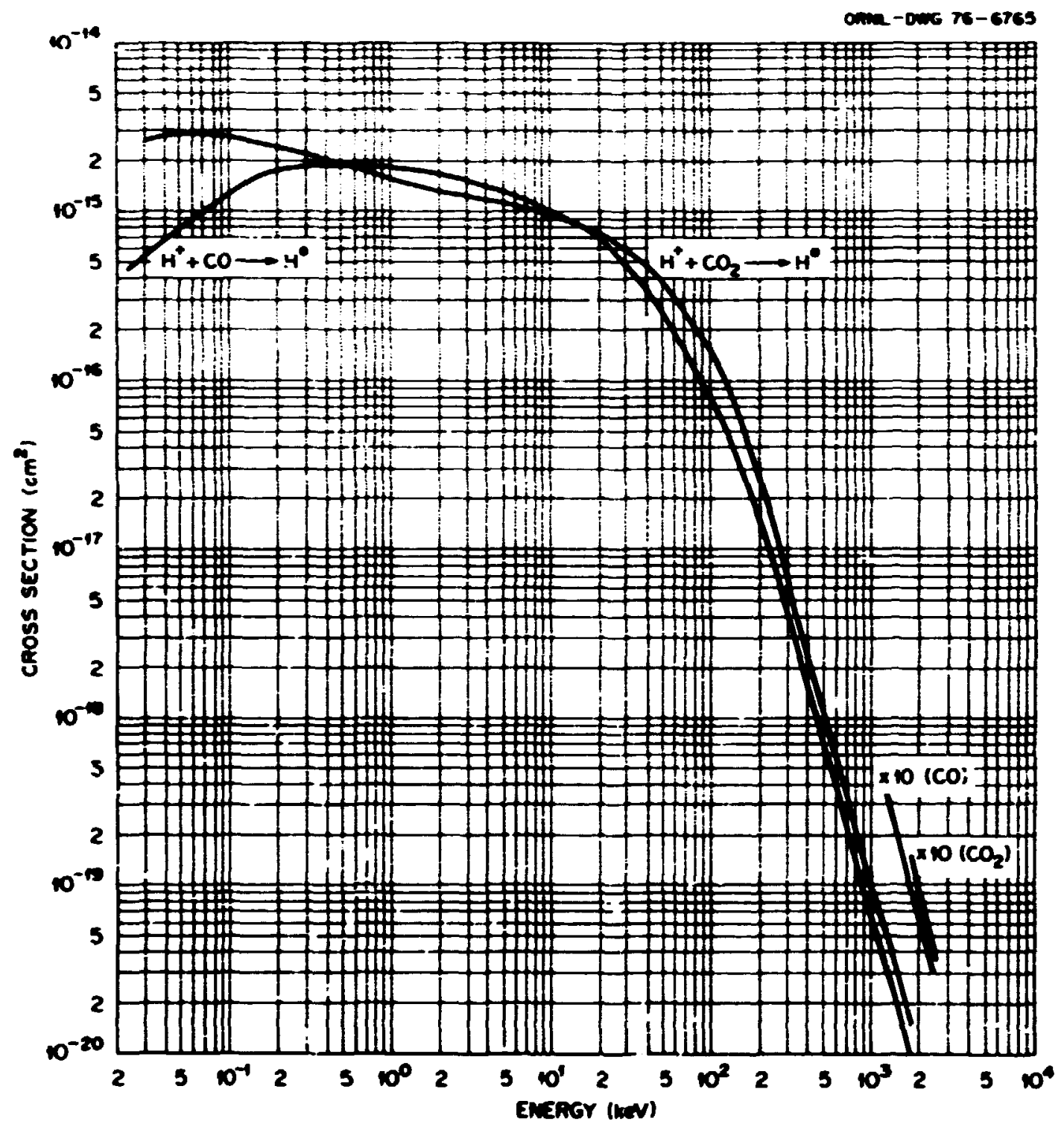


A. 4.20

Electron Capture Cross Sections of $\mathrm{H}^{+}$in $\mathrm{Li}$ Vapor

\begin{tabular}{|c|c|c|}
\hline \multirow[t]{2}{*}{$\begin{array}{l}\text { Energy } \\
\text { (tev) }\end{array}$} & \multicolumn{2}{|c|}{$\begin{array}{l}\text { Cross Sections } \\
\left(\mathrm{ca}^{2}\right)\end{array}$} \\
\hline & $\frac{\sigma_{10}}{\mathrm{H}^{2}+L_{L}+H^{\circ}}$ & $\frac{\sigma_{1-1}}{B^{+}+E^{\prime}+H^{-}}$ \\
\hline $\begin{array}{lll}1.0 & E & 00 \\
2.0 & E & 00 \\
4.0 & E & 00 \\
7.0 & E & 00 \\
1.0 & E & 01 \\
2.0 & E & 01 \\
4.0 & E & 01 \\
7.0 & E & 01 \\
1.0 & E & 02 \\
2.0 & E & 02 \\
4.0 & E & 02\end{array}$ & $\begin{array}{ll}2.3 & E-15 \\
4.2 & E-15 \\
8.2 & E-15 \\
5.3 & E-15 \\
3.0 & E-15 \\
2.9 & E-16 \\
4.1 & E-17 \\
2.2 & E-17 \\
1.1 & E-17 \\
2.6 & E-18 \\
6.0 & E-19\end{array}$ & $\begin{array}{ll}9.5 & E-18 \\
2.4 & E-17 \\
5.6 & E-17 \\
5.8 & E-17 \\
2.8 & E-17 \\
2.0 & E-18\end{array}$ \\
\hline
\end{tabular}

\section{References:}

$\mathrm{H}^{+}+\mathrm{L}, 1+\mathrm{H}^{\circ}$ : B. A. D'yachkov, Sov. Atom. Energy 27, 958 (1969); W. Grüebler, P. A. Schnelzbach, V. Könls and P. Marnder, HeIv. Phys. Actz. 43, 254 (1970); R. M. II'In, V. A. Oparin, E. S. Solov'ev, and K. V. Fedorenko, Sov. Phys.Tech. Phys. 11, 92 (1967).

$\mathrm{A}^{+}+L_{1+\mathrm{E}^{-}:}$: . Griebler, P. A. Schnelzbach, V. König, and P. Marnier, Helv. Phys. Acta. 43, 254 (1970).

Accuracy:

Unknown. 


\section{A.4. 21}

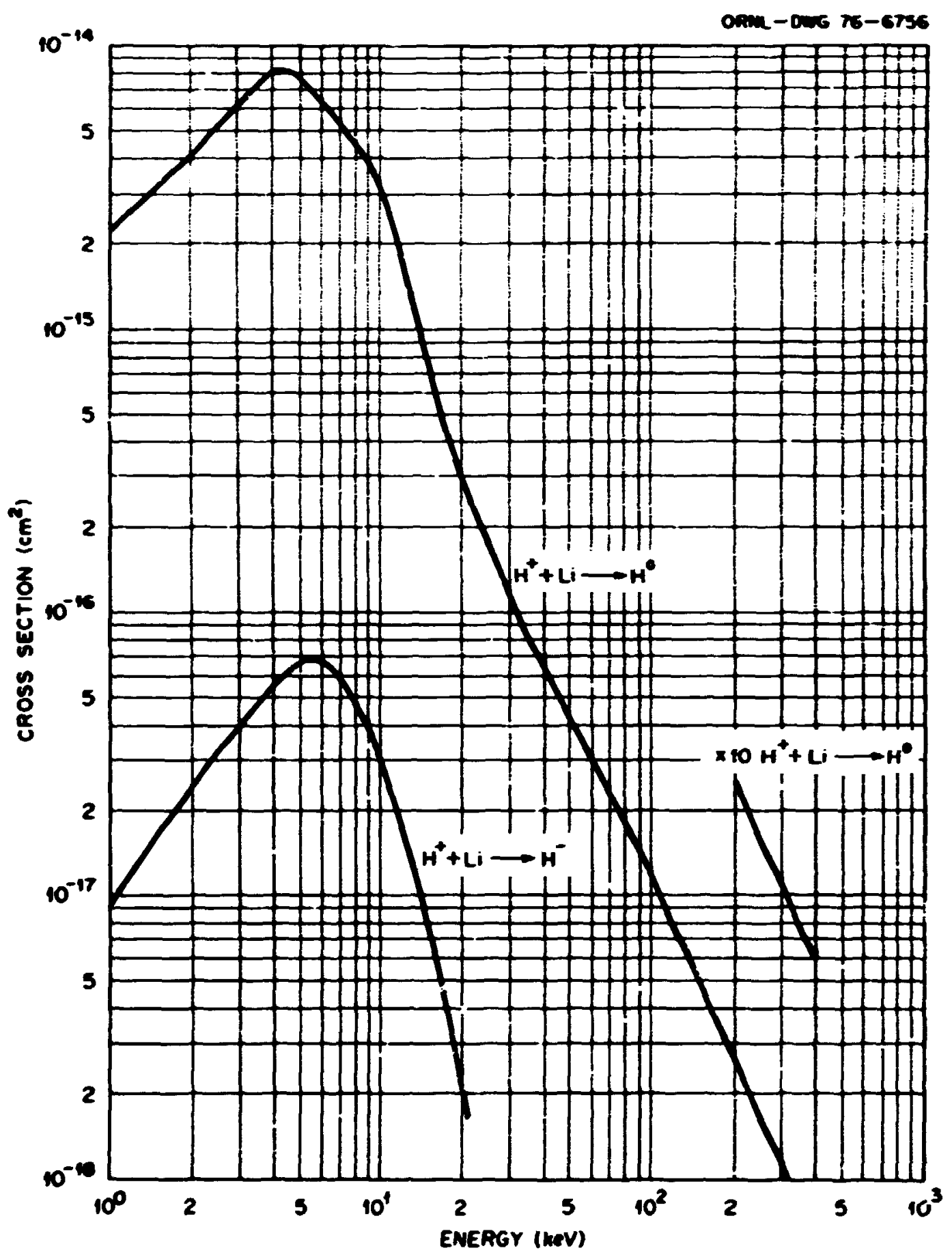




$$
\text { A.4. } 22
$$

Electron Capture Cross Sections for

$$
\mathrm{H}^{+} \text {in Na Vapor }
$$

\begin{tabular}{|c|c|c|}
\hline \multirow[t]{2}{*}{$\begin{array}{l}\text { Energy } \\
\text { (keV) }\end{array}$} & \multicolumn{2}{|c|}{$\begin{array}{c}\text { Cruss Sertions } \\
\left(\mathrm{cm}^{2}\right)\end{array}$} \\
\hline & $\frac{\sigma_{10}}{\mathrm{H}^{+}+\mathrm{Na}+\mathrm{H}^{\circ}}$ & $\frac{\mathrm{C}_{1}-1}{\mathrm{H}^{+}+\mathrm{Na}+\mathrm{H}^{-}}$ \\
\hline $\begin{array}{l}5.0 \text { E- } 01 \\
7.0 \text { E- } 01 \\
1.0 \text { E } 00 \\
2.0 \text { E } 00 \\
4.0 \text { E } 00 \\
6.0 \text { E } 00 \\
8.0 \text { E } 00 \\
1.0 \text { E } 01 \\
2.0 \text { E } 01 \\
4.0 \text { E } 01 \\
6.0 \text { E } 01 \\
1.0 \text { E } 02 \\
1.8 \text { E } 02\end{array}$ & $\begin{array}{ll}2.7 & E-15 \\
3.3 & E-15 \\
4.4 & E-15 \\
8.3 & E-15 \\
1.2 & E-14 \\
3.0 & E-15 \\
4.2 & E-15 \\
2.2 & E-15 \\
3.2 & E-16 \\
7.5 & E-17 \\
5.0 & E-17 \\
3.7 & E-17 \\
2.1 & E-17\end{array}$ & $\begin{array}{ll}3.8 & E-17 \\
6.5 & E-17 \\
1.9 & E-16 \\
5.0 & E-16 \\
4.2 & E-16 \\
2.3 & E-16 \\
1.4 & E-16\end{array}$ \\
\hline
\end{tabular}

\section{References:}

$H^{+}+\mathrm{Na}+H^{\bullet}:$ W. Grüebler, P. A. Schmelzbach, V. König and P. Marmier, Helv. Phys. Acta. 43, 254 (1970); R. N. Il'In, V. A. Oparin, E. S. Solov'ev, and N. V. Fedorenko, Sov. Phys.-Tech. Phys. 11, 921 (1967).

$H^{+}+\mathrm{Na} \rightarrow H^{-}:$W. Grüebler, P. A. Schmelzbach, v. Könfg, and P. Marnter, Helv. Phys. Acta. 43, 254 (1970).

\section{Accuracy:}

Unknown. 


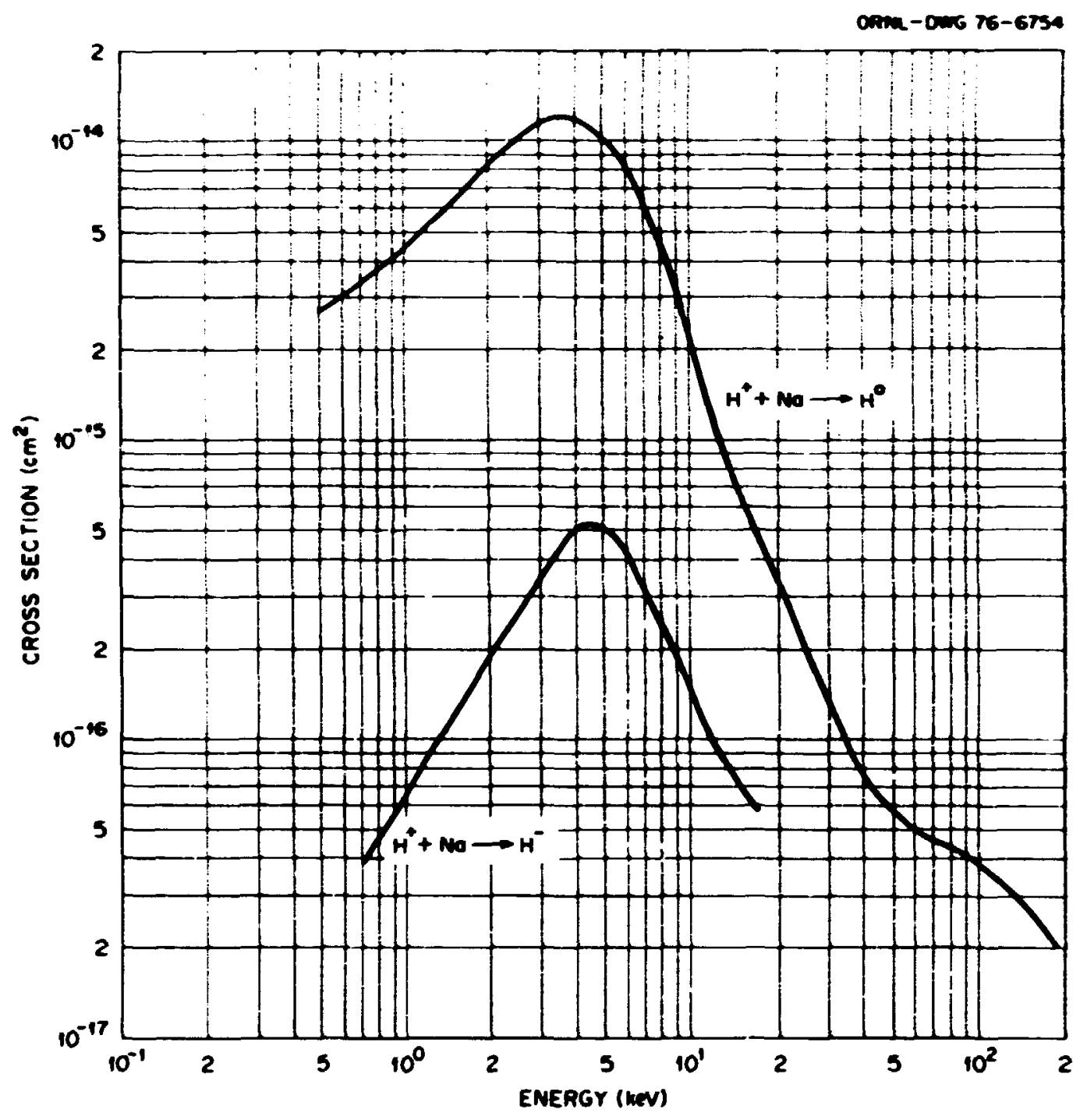




\section{A.4.24}

Electron Capture Cross Sections of $\mathrm{H}^{+}$in $\mathrm{X}$ Vapor

\begin{tabular}{|c|c|c|}
\hline \multirow[t]{2}{*}{$\begin{array}{l}\text { Energy } \\
\text { (keV) }\end{array}$} & \multicolumn{2}{|c|}{$\begin{array}{c}\text { Cross Sections } \\
\left(c^{2}\right)\end{array}$} \\
\hline & $\frac{\sigma_{u}}{u^{+}+x+n^{0}}$ & 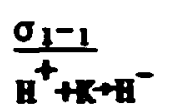 \\
\hline 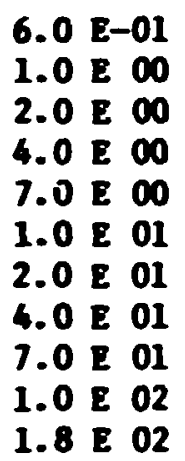 & $\begin{array}{ll}5.2 & E-15 \\
7.4 & E-15 \\
9.5 & E-15 \\
7.8 & E-15 \\
4.5 & E-15 \\
2.5 & E-15 \\
6.9 & E-16 \\
3.7 & E-16 \\
2.4 & E-16 \\
1.2 & E-16 \\
2.6 & E-17\end{array}$ & $\begin{array}{ll}2.2 & E-17 \\
3.2 & E-17 \\
4.0 & E-17 \\
3.4 & E-17 \\
2.1 & E-17 \\
1.3 & E-17 \\
3.4 & E-18\end{array}$ \\
\hline
\end{tabular}

\section{References:}

$B^{+}+K+B^{\bullet}:$ W. Grüebler, P. A. Schwelzbach, V. König, and P. Mander, Helv. Phys. Acta. 43, 254 (1970); R. M. Il'In, V. A. Oparin, E. S. Solov'ev, and M. V. Pedorentio, Sov. Phys.-Tech. Phys. 11, 921 (1967).

$a^{+}+K+B^{-}:$W. Grüebler, P. A. Schmelzbach, V. König, and P. Marnier. Helv. Phys. Acta. 43, 254 (1970).

Accuracy:

Unknown. 


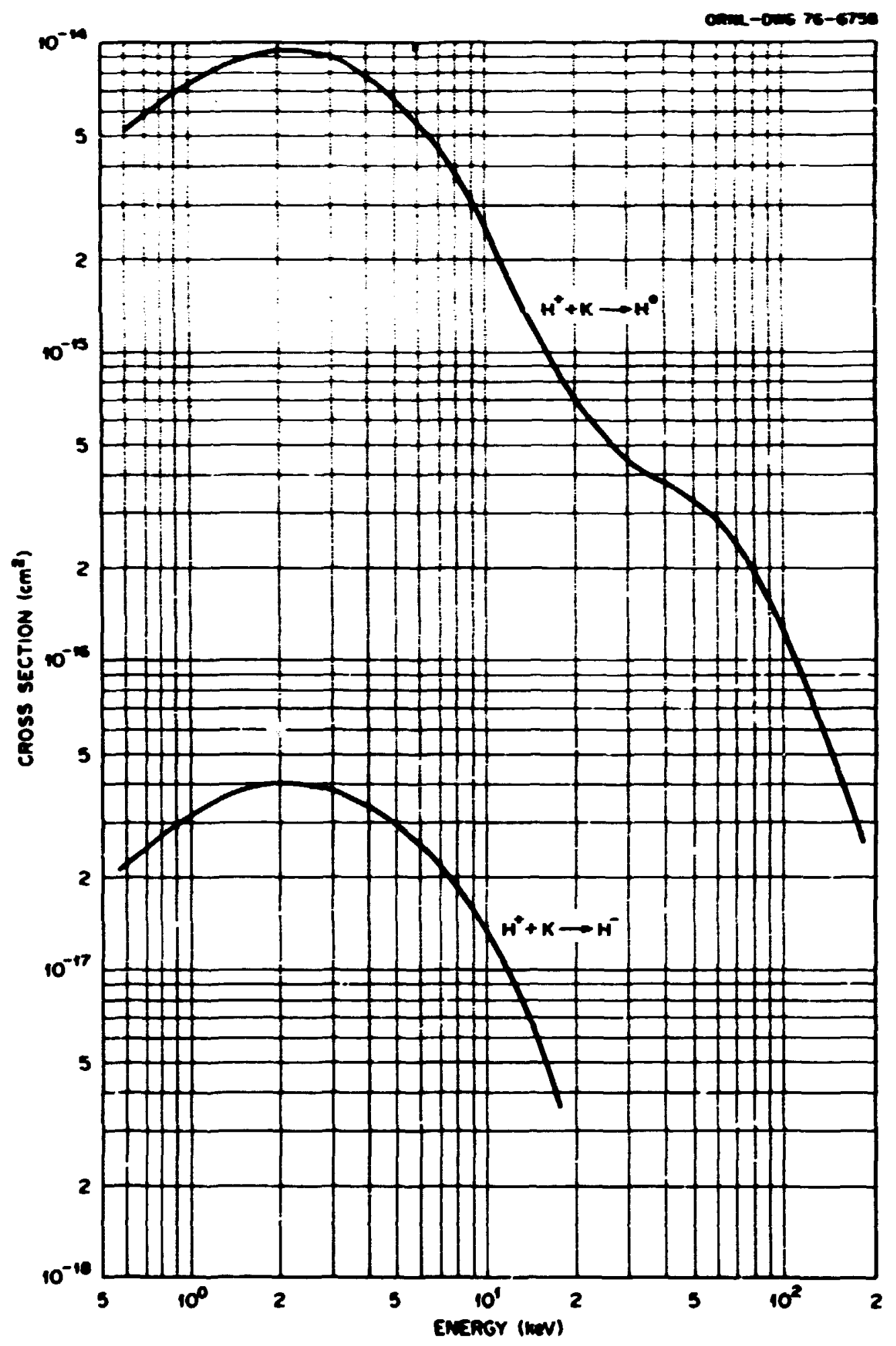




$$
\text { A. } 4.26
$$

Electron Capture Cross Sections for

$$
\mathrm{R}^{+} \text {In M8 Vapor }
$$

\begin{tabular}{|c|c|c|}
\hline \multirow[t]{2}{*}{$\begin{array}{l}\text { Derers } \\
\text { (keV) }\end{array}$} & \multicolumn{2}{|c|}{$\begin{array}{c}\text { Crous Sections } \\
\left(\mathrm{cm}^{2}\right)\end{array}$} \\
\hline & $\frac{0_{10}}{n^{t}+x+n^{\circ}}$ & $\frac{0_{1}-1}{a^{+}+x_{8}+a^{-}}$ \\
\hline 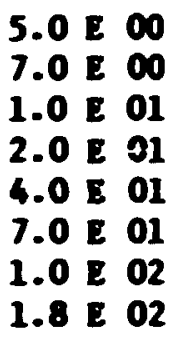 & $\begin{array}{ll}1.5 & E-15 \\
2.1 & E-15 \\
1.8 & E-15 \\
6.3 & E-16 \\
7.2 & E-17 \\
1.6 & E-17 \\
1.2 & E-17 \\
9.6 & E-18\end{array}$ & $\begin{array}{ll}1.0 & \mathrm{E}-16 \\
8.6 & \mathrm{E}-17 \\
6.3 & \mathrm{E}-17 \\
2.0 & \mathrm{E}-17 \\
3.4 \mathrm{E}-18 \\
1.1 \mathrm{E}-18\end{array}$ \\
\hline
\end{tabular}

\section{Defereaces:}

A $^{+}+1+\mathrm{H}^{\circ}$ : R. B. Berkner, R. V. Pyle, and J. W. Stearns, Phys. Rev. 178 , 248 (1969); A. H. Futch and K. G. Hoses, Sth Int. Coaf. Elect. Atom.

Coll., p. 12, Leaingred, USSR (1967); R. M. I1'In, V. A. Operí, E. S. Solov'ev, and 1. V. Pedorenko, Sov. Phys.-Teeh. Phys. 11, 921 (1967).

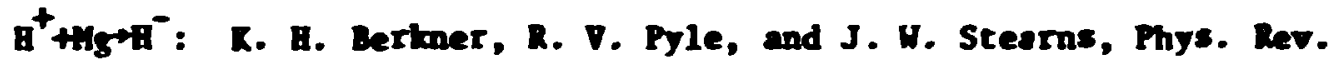
178,248 (1969).

\section{Accuracy:}

Onknown. 


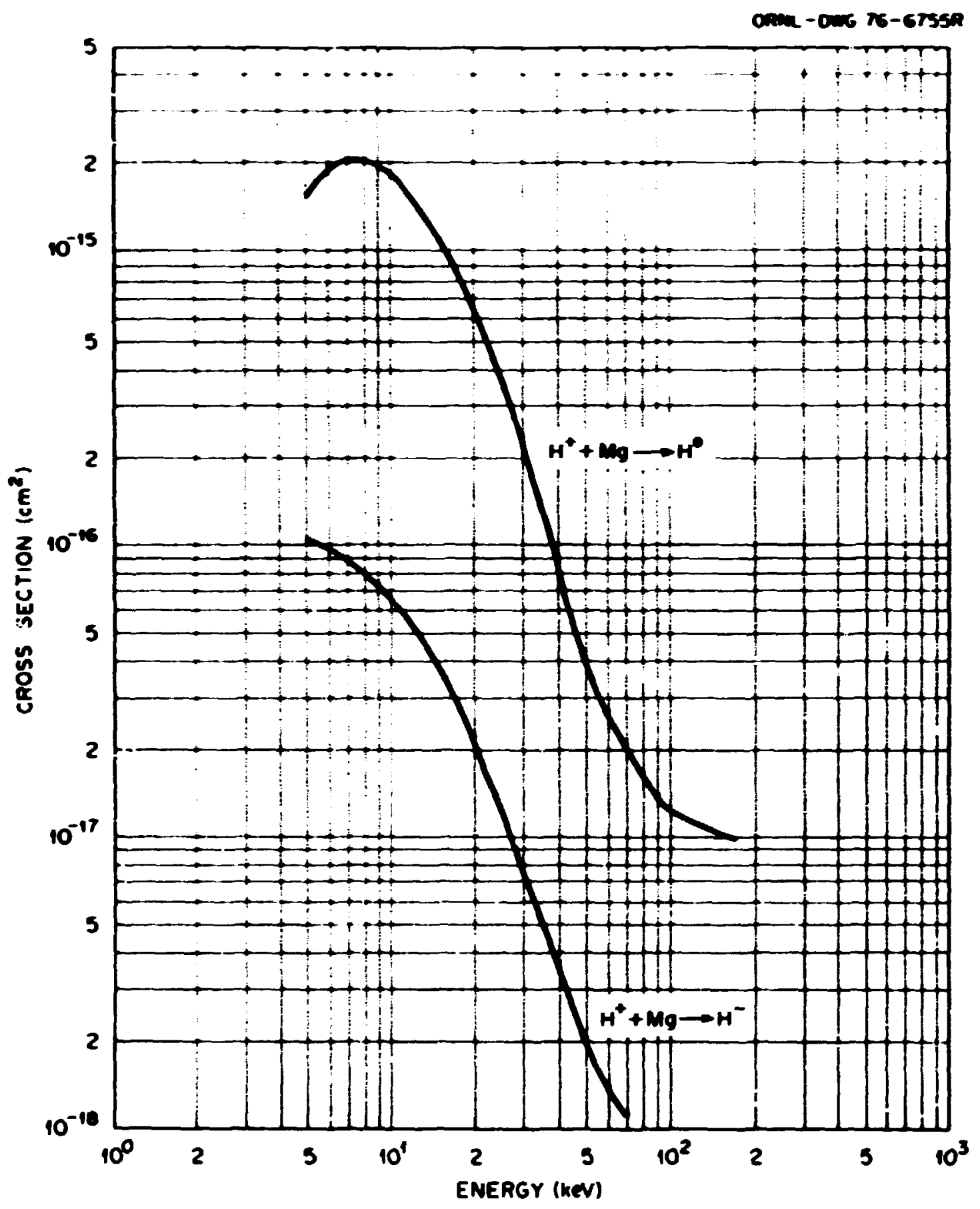


Electron Capture Cross Sections of in Cs Vapor

\begin{tabular}{|c|c|c|c|}
\hline \multirow[t]{2}{*}{$\begin{array}{l}\text { Rerns } \\
\text { (nev) }\end{array}$} & \multicolumn{3}{|c|}{$\begin{array}{c}\text { Croes Sections } \\
\left(\mathrm{ce}^{2}\right)\end{array}$} \\
\hline & $\frac{0_{10}}{a^{t}+c^{2}+a^{0}}$ & $\frac{\sigma_{0}-1}{x^{0}+c_{0}+x^{2}}$ & $\frac{\sigma_{1}-1}{x^{+}+a_{n+1}}$ \\
\hline $\begin{array}{l}2.5 E-01 \\
5.0 \mathrm{E}-01 \\
7.0 \mathrm{E}-01 \\
1.0 \mathrm{E} 00 \\
2.0 \mathrm{E} 00 \\
4.0 \mathrm{E} 00 \\
7.0 \mathrm{E} 00 \\
1.0 \mathrm{E} 01 \\
2.0 \mathrm{E} 01 \\
4.0 \mathrm{E} 01 \\
7.0 \mathrm{E} 01 \\
1.0 \mathrm{E} 02 \\
1.8 \mathrm{E} 02\end{array}$ & 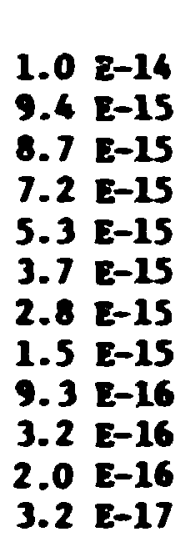 & $\begin{array}{ll}3.8 & E-16 \\
3.4 & E-16 \\
2.9 & E-16 \\
2.3 & E-16 \\
1.4 & E-16 \\
7.2 & E-17 \\
3.7 & E-17 \\
2.4 & E-17\end{array}$ & $\begin{array}{ll}1.0 & E-16 \\
7.4 & E-17 \\
6.0 & E-17 \\
4.7 & E-17 \\
2.8 & E-17 \\
1.3 & E-17 \\
4.2 & E-18 \\
1.5 & E-18 \\
1.4 & E-19\end{array}$ \\
\hline
\end{tabular}

\section{Eeferences:}

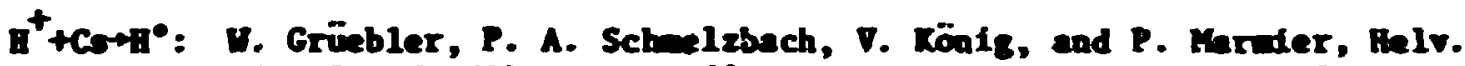
Fuys. Acea. 43, 254 (1970); R. W. I1'In, V. A. Oparia, E. S. So:ov'ev, and \#. V. Fedorenko, Sov. Fuys.-Tech. Fuys. 11, 92 (1967); A. S. Sehlacheer, P. J. Bjortholn, D. H. Loyd, L. W. Aaderson, and H. Haeber11, Mhya. Rev. 177, 184 (1965); G. Splese, A. Valance, and P. Pradel, Phys. Rev. A 6, $\overline{746}$ (1972); V. H. Tuen, G. Gautherin, and A. S. Schlachter, Phys. Rev. A 9, 1242 (1972).

H'+Cort: C. Cisneros, I. Mlvarez, C. P. Rarnett and J. A. Ray, Phys. Rev. A (1976); A. S. Schlechter, P. J. Bjorkhola, D. H. Loyd, L. H. Anderson and W. Baeber11, Phys. Nev. 177, 184 (1969).

$\mathrm{I}^{+}+\mathrm{Cor} \mathrm{H}^{-}$: C. Cisneros, I. Aivarez, C. F. Bamett and J. A. Ray, Mhs. Rev. A (1976); W. Grǘble, , P. A. Srhmelzbech, V. Xönis, and P. Marnter, Helv. Thys. Acta. 43, 254 (1970); A. S. Schlacheer, P. J. Bjorkholn, D. B. Loyd, L. W. Anderson, and W. Hacber11, Phys. Rev. 177, 184 (1969).

\section{Acsureas:}

Onknown. 


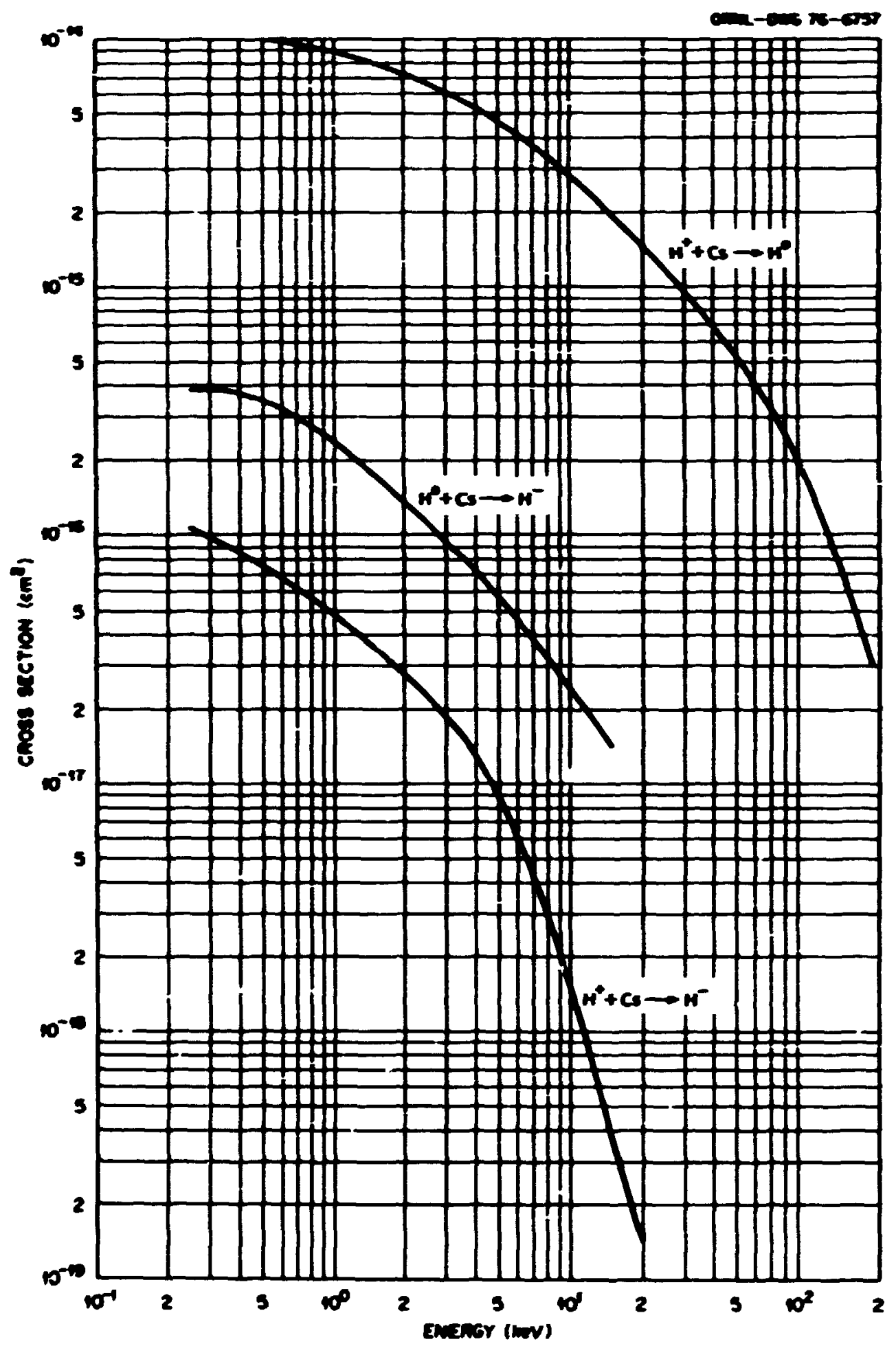


A.6. 30

Electron Capture Cross Sections for che Reactions

$$
\mathrm{n}_{2}^{+}+\mathrm{n}_{2} \operatorname{and} \mathrm{n}_{2}^{+}+\mathrm{n}
$$

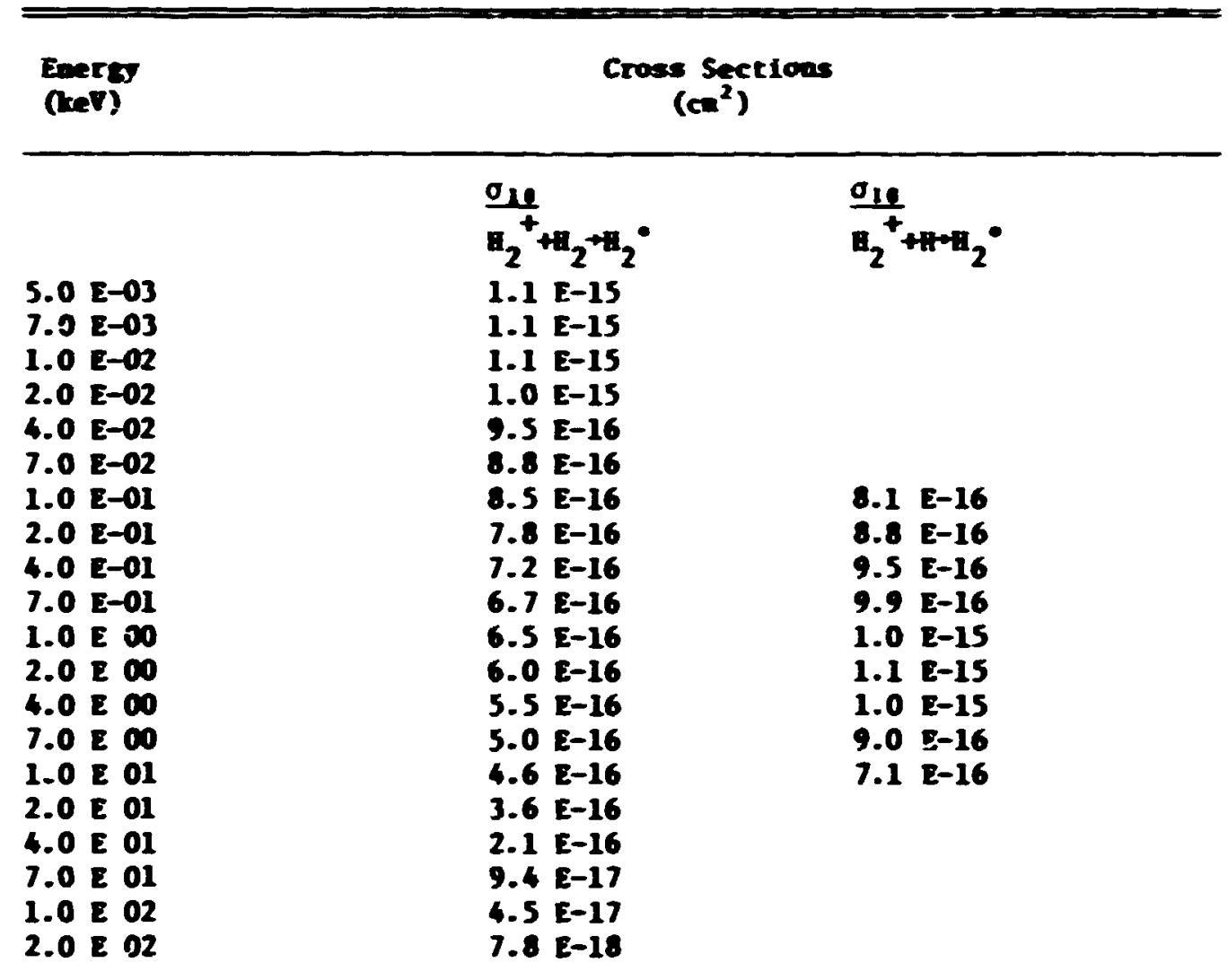

Deferences:

$H_{2}{ }^{+}+H_{2}$ : W. H. Craeer, J. Chea. Phys. 35, 836 (1961); H. B. Gilbody and J. B. 2hasted, Proc. Roy. Soc. A 238, 334 (1956); Yu. S. Cordeev and M. M. Penov, Sov. Phys.-Tech. Phys. 9, 656 (1964); H. C. Haydea and R. C. Aleme, Phys. Rev. 172, 104 (1968); D. W. Roopan, Phys. Rev. 154, 79 (1967); J. J. Leventhal, T. P. Horan, L. Priedan, J. Chen. Phys - 46, 4666 (1967); C. W. Heclure, Phys. Rev. 130, 1852 (1963); A. Schadd, 2. Thys. 161, 550 (1961); J. B. H. Stedeford and J. B. Hested, Proc. Roy. Soc. A227, 466 (1955); D. R. Seretwan, Proc. Roy. Soc. (London) A256, 416 (1960); D. V. Vance and T. $L$. Bailey, 2. Chen. Phys. 44, 486 (1966).

H, ${ }^{+}+\mathrm{HI}$ : U. L. P1te, R. T. Brackan, and W. R. Snow, Mhys. Rev. 112, 1161 (1958).

Accurac:

$\mathrm{H}_{2}^{+}+\mathrm{H}_{2}: \pm 40 \%$.

$\mathrm{H}_{2}^{+}+\mathrm{H}: \pm 20 \%$.

Lote:

Some Investigators have found that the cross sectlons vere dependent on the vibrational state of the $\mathrm{H}_{2}^{+}$ion. 


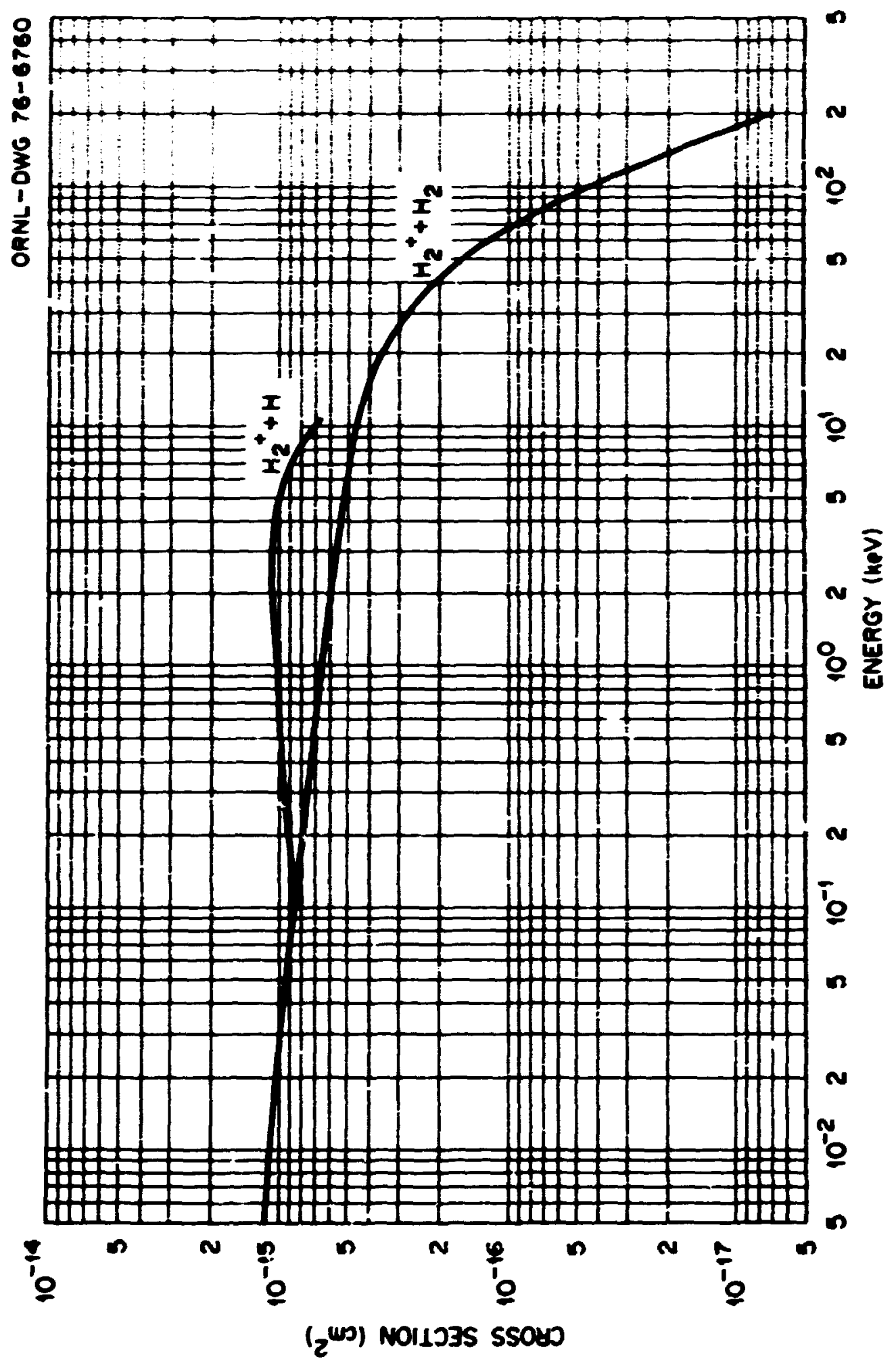




\section{A.4.32}

Electron Copture Cross Sections for

$$
\mathrm{He}^{+} \text {in } \mathrm{H}_{2} \text { and } \mathrm{He}
$$

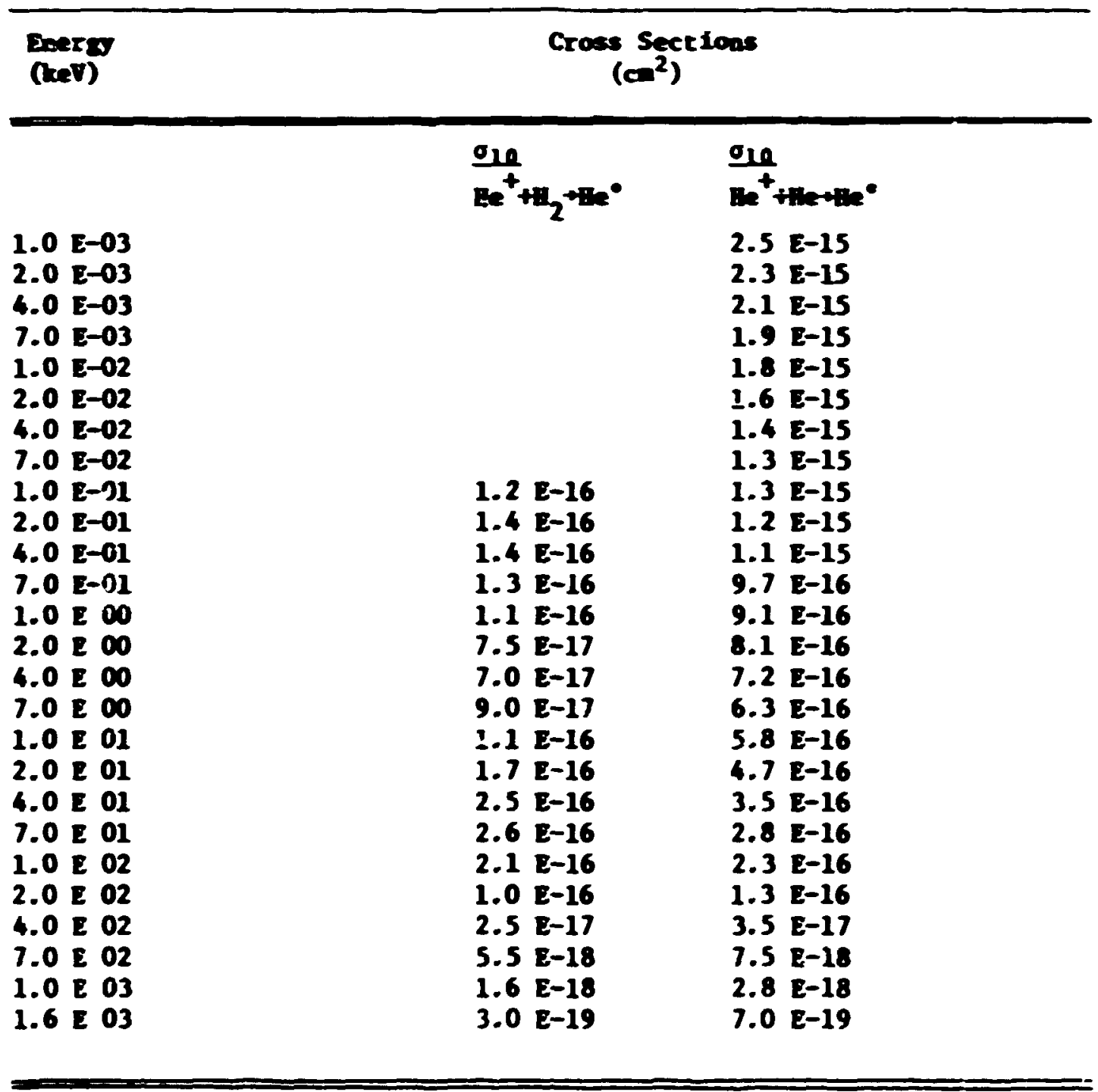

\section{References:}

Het $+H_{2}$ : S. K. Allison, J. Cuevas, P. G. Murphy, Phys. Rev. 102, 1041 (1956): C. F. Barnett and P. M. St1er, Phys. Rev. 109, 385 (1938); H. B. Gilbody, J. B. Hasted, J. V. Irelend, A. R. Lee, E. W. Thomes and A. S. Whiteman, Proc. Roy. Soc. London, A274, 40 (1963); F. J. Defleer, J. Schutcen and H. Moustafa, Physica 32, $1793(\overline{1966})$; L. I. PIvovar, V. M. Tubsev, and M. T. Novikov, Sov. Phys.-JETP 14, 20 (1962); J. B. H. Stedeford and J. B. Hasted, Proc. Roy. Soc. London 1227, 466 (1955); A. B. Wittkower, G. Levy, and H. B. Gilbody. Proc. Phys. Soc. London 91, 862 (1967). 


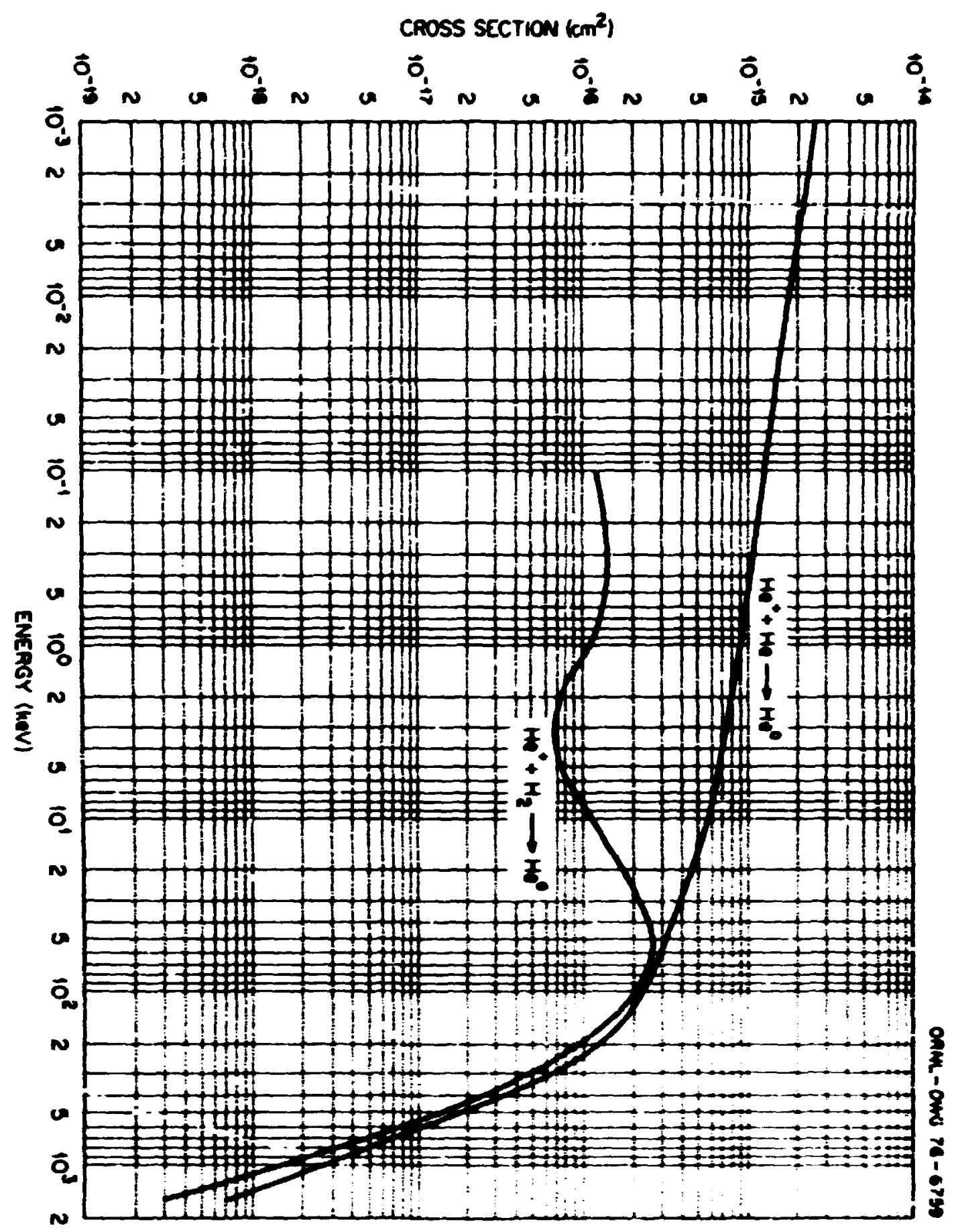


Electron Capture Crcise Sections for

$$
\mathrm{He}^{+} \text {in } \mathrm{O}_{2} \text { and } \mathrm{x}_{2}
$$

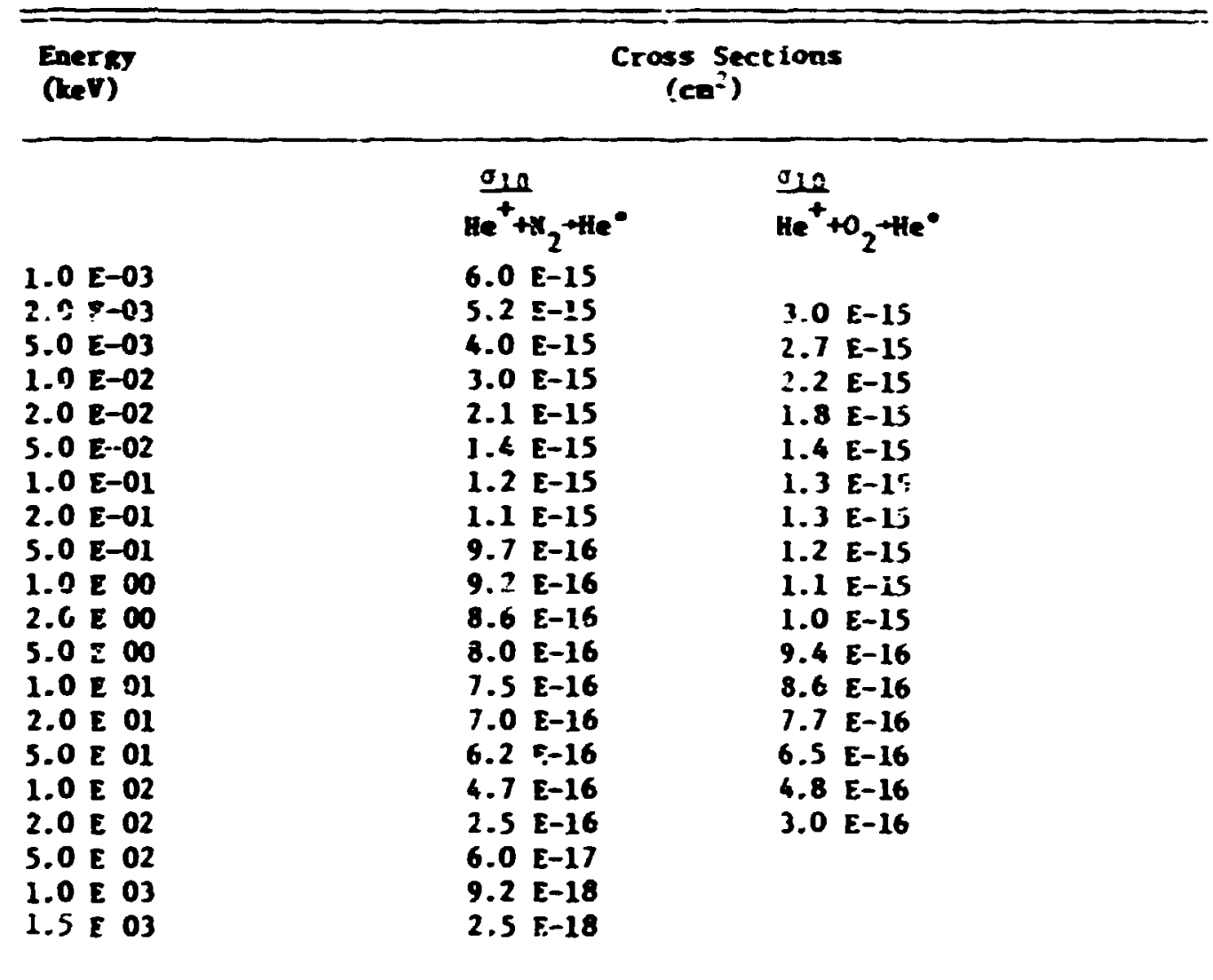

\section{Deferences:}

Re $+H_{2}:$ C. F. Barnete and P. M. Stler, Fhys. Rev. 109, 385 (1958); F. J. De Hefr, J. Schuten, and H. Houstafa, Physica 32, 1793 (i966); D. H. Koopean, Phys. Rev. 166, 57 (1968): R. C. C. Lac, R. Rozete, and H. S. Rosk1, J. Chen. Phys. 49, 4202 (1968); P. Mahadevan and C. D. Marnuson, Fifth Int. Conf - on Electron and Aconic Collistons, p. 405, Leningrad (1967); V. S. Mikolaev, I. S. Daferfev, L. M. Fateeva, and Ya. A. Teplova, Sov. Phys.-JETP 13, 695 (1961); L. I. Pivovar, V. M. Tubaev, and M. T. Novikov. Sov. Phys.-JET $\overline{16}, 20$ (1962); R. F. Stebbinge, J. A. Ruthertiord, and D. R. Turner, Planet. Space Sc1. 13, 1125 (1965); R. F. Stebbings, A. C. H. Salth, and E. Ehrhardt, J. Chen. Fhys. 39, 968 (1963).

Het+0 ${ }_{2}$ : C. P. Barnete and P. M. Scier, Phys. Rev. 109, 38; (1958); F. J. De Heer, J. Schutten, and H. Mostafa, Physica 32, 1793 :I966): H. L. Fite, A. C. A. Selth, R. F. Stebbings, and J. L. Rutherford, J. Geophys. Res. 68, 3225 (1963); D. W. Koopean, Phys. Rev. 166, 57 (1968); P. Mahadevan and C. D. Magnusoil, Fifth Int. Conf. on Electron an Atomic Collisions, p. 405, Lenfngrad (1967): P. Mahadevan and C. D. Magnusm, Phys. Rev. 171, 103 (1968); R. F. Stebbings, A. C. H. Saith and E. Ehrharde, J. Chen. Phys. 39, 968 (1963).

\section{Accuracy:}

$\mathrm{He}^{+}+\mathrm{H}_{2}: \pm 25 \%$.
$\mathrm{He}^{+}+\mathrm{O}_{2}: \pm 25 \%$. 
A.4.35

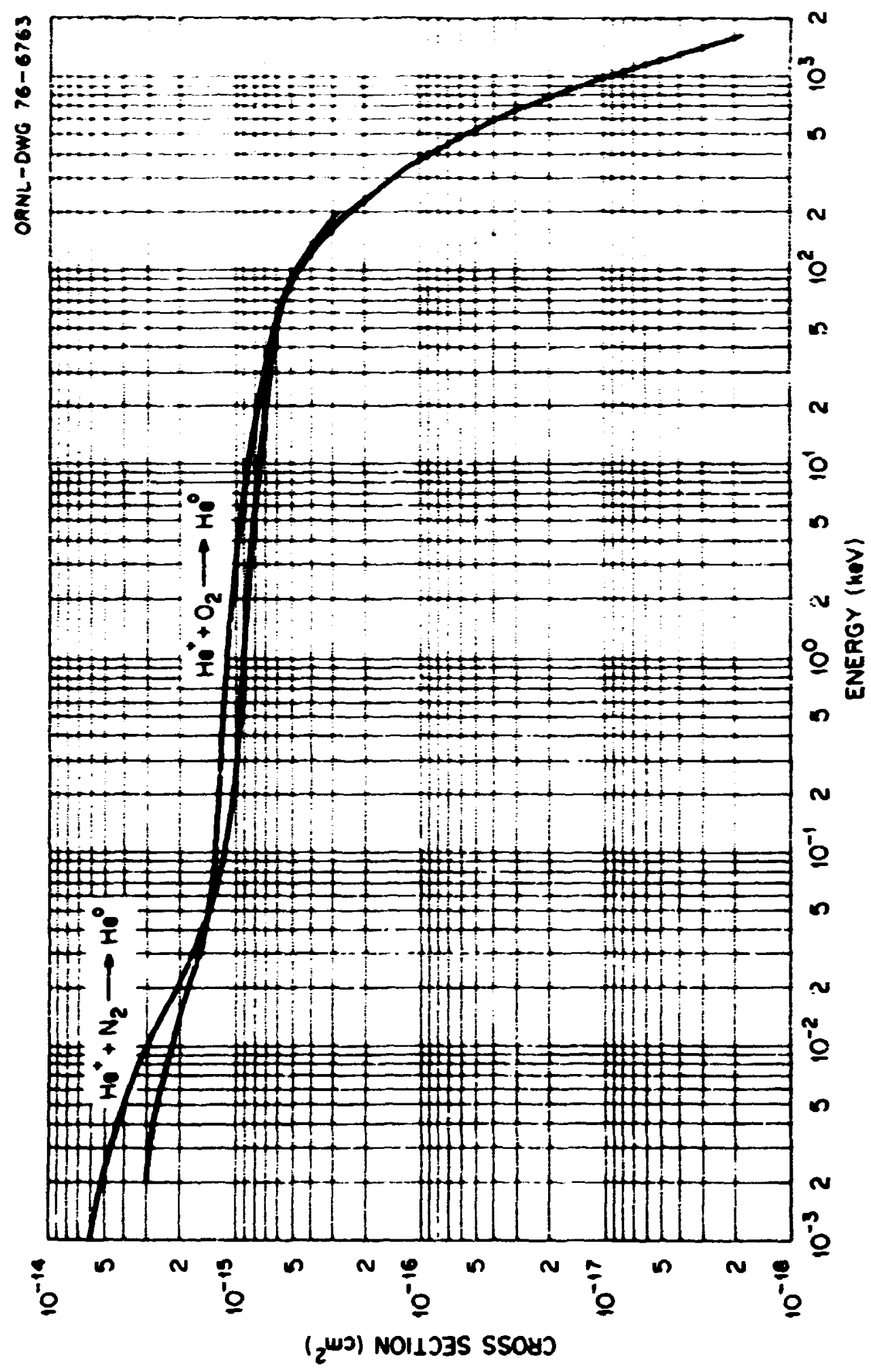


Electron Capture Cross Sections for

$$
H e^{H} \text { in } H \text { and } H_{2}
$$

\begin{tabular}{|c|c|c|c|}
\hline \multirow[t]{2}{*}{$\begin{array}{l}\text { Energy } \\
\text { (keV) }\end{array}$} & \multicolumn{3}{|c|}{$\begin{array}{c}\text { Cross Sections } \\
\left(\mathrm{cm}^{2}\right)\end{array}$} \\
\hline & $\frac{0_{21}}{\mathrm{He}^{++}+\mathrm{H}+\mathrm{He}}$ & $\frac{\sigma_{2 l}}{\mathrm{He}^{++}+\mathrm{H}_{2}+\mathrm{He}^{+}}$ & $\frac{\mathrm{O}_{2 \mathrm{O}}}{\mathrm{He} \mathrm{e}^{++}+\mathrm{H}_{2}+\mathrm{He} \mathrm{e}^{\circ}}$ \\
\hline $\begin{array}{lll}6.0 & \text { E } & 00 \\
8.0 & \text { E } & 00 \\
1.0 & \text { E } & 01 \\
2.0 & \text { E } & 01 \\
5.0 & \text { E } & 01 \\
7.5 & \text { E } & 01 \\
1.0 & \text { E } & 02 \\
2.0 & \text { E } & 02 \\
5.0 & E & 02 \\
7.5 & E & 02 \\
1.0 & \text { E } & 03 \\
1.4 & \text { E } & 03 \\
2.0 & \text { E } & 03 \\
3.0 & \text { E } & 03 \\
3.8 & E & 03\end{array}$ & $\begin{array}{ll}3.7 & E-16 \\
5.4 & E-16 \\
7.2 & E-16 \\
1.4 & E-15 \\
1.6 & E-15 \\
1.4 & E-15\end{array}$ & $\begin{array}{ll}1.7 & E-16 \\
5.2 & E-16 \\
1.0 & E-15 \\
1.0 & E-15 \\
8.3 & E-16 \\
3.7 & E-16 \\
5.5 & E-17 \\
1.5 & E-17 \\
5.2 & E-18 \\
1.3 & E-18 \\
3.0 & E-19 \\
4.4 & E-20 \\
1.8 & E-20\end{array}$ & $\begin{array}{ll}1.0 & E-17 \\
1.8 & E-17 \\
5.7 & E-17 \\
6.9 & E-17 \\
6.8 & E-17 \\
3.0 & E-17 \\
2.2 & E-18 \\
4.8 & E-19 \\
1.6 & E-19 \\
4.7 & E-20\end{array}$ \\
\hline
\end{tabular}

References:

Het+the ${ }^{+}$: W. L. Fice, A. C. H. Smith, and R. F. Stebbings, Proc. Roy. Soc. London A268, 527 (1962); J. E. Bayfield and G. A. Mhayrallah, Phys.

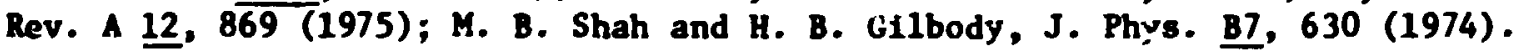

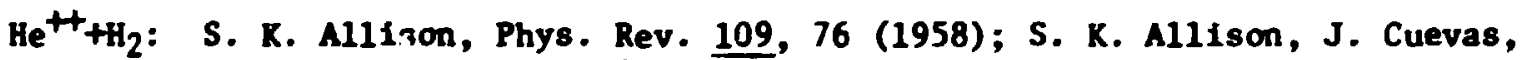
and P. G. Murphy, Phys. Rev. 102, 1041 (1956); R. A. Baragiola and I. B. Nemirovsky, Nucl. Insc. and Meth. 110, 511 (1973); J. E. Bayfield and G. A. Khayrallah, Phys. Rev. A 11, 920 (1975); P. Hvelplund, J. Heinemeier, E. H. Pedersen and F. R. Simpson, 9th Int. Conf. Atomc. Elect. Coll. P. 185, Seattle, Wash. (1975); L. I. PIvovar, M. T. Novikov and V. M. Tubaev, Sov. Phys.-JETP 15, 1035 (1.962); L. I. Plvovar, V. M. Tubaev, and M. T. Hovikov, Sov. Phys.-JETP 14, 20 (1962); M. B. Shah and H. B. Gilbody, J. Phys. B I, 256 (1974).

Accuracy:

Het+H: $\pm 40 \%$.

$\mathrm{He}^{++}+\mathrm{H}_{2}: \pm 40 \%$. 


\section{A.4.37}

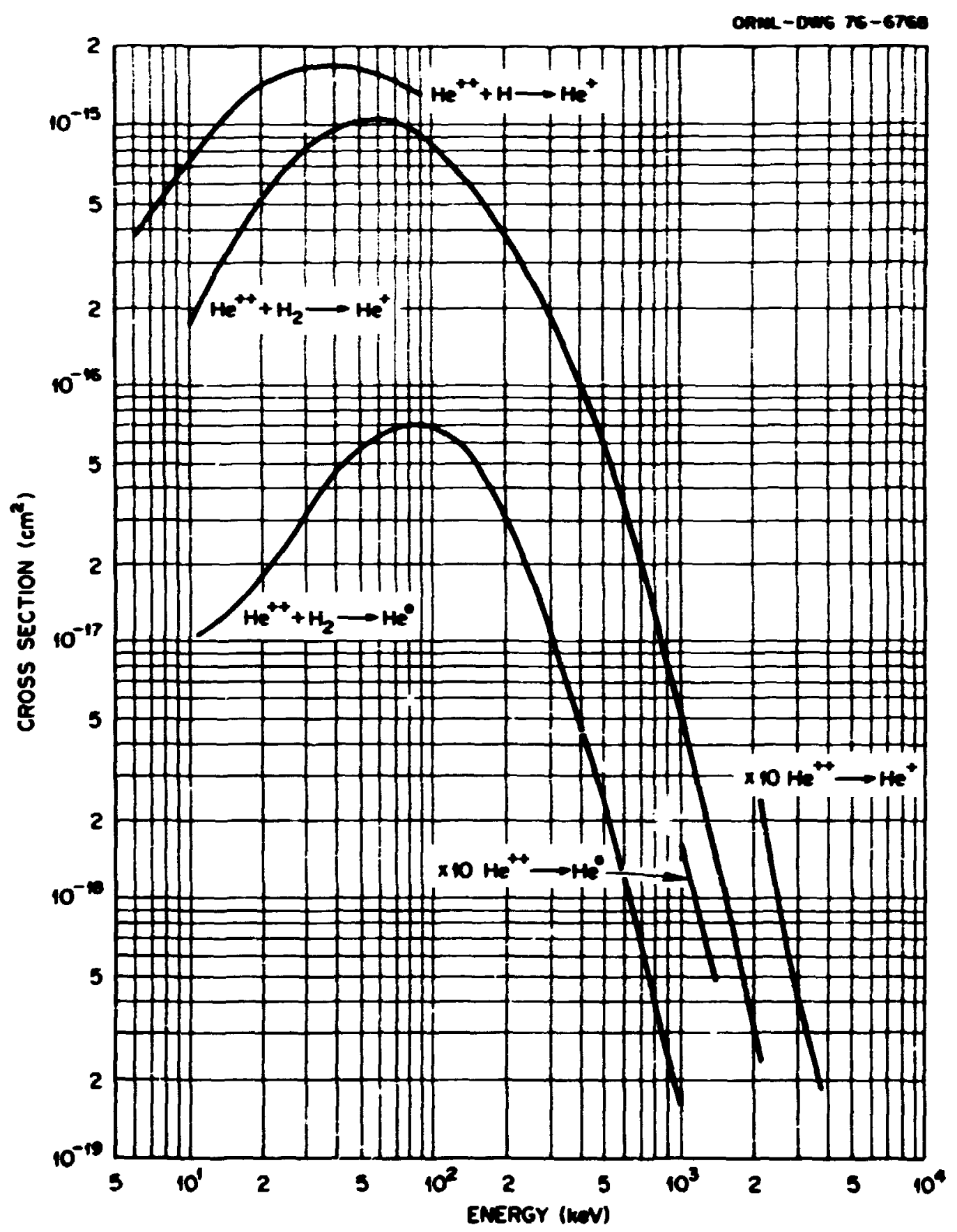


Electron Capture Cross Sections for $\mathrm{He}^{++}$in He

\begin{tabular}{|c|c|c|}
\hline \multirow[t]{2}{*}{$\begin{array}{l}\text { Energy } \\
\text { (keV) }\end{array}$} & \multicolumn{2}{|c|}{$\begin{array}{c}\text { Cross Sections } \\
\left(\mathrm{cm}^{2}\right)\end{array}$} \\
\hline & $\frac{\mathrm{J}_{20}}{\mathrm{He}^{++}+\mathrm{He} \rightarrow \mathrm{He}}$ & $\frac{\mathrm{J}_{21}}{\mathrm{He}}+\mathrm{He}+\mathrm{He}+$ \\
\hline $\begin{array}{l}6.0 \text { E-02 } \\
1.0 \text { E-01 } \\
2.0 \text { E-01 } \\
4.0 \text { E-01 } \\
7.0 \text { E-01 } \\
1.0 \text { E } 00 \\
2.0 \text { E } \mathrm{CO} \\
5.0 \text { E } 00 \\
7.5 \text { E } 00 \\
1.0 \text { E } 01 \\
2.0 \text { E } 01 \\
5.0 \text { E } 01 \\
7.5 \text { E } 01 \\
1.0 \text { E } 02 \\
2.0 \text { E } 02 \\
5.0 \text { E } 02 \\
7.5 \text { E } 02 \\
1.0 \text { E } 03 \\
1.4 \text { E } 03 \\
3.0 \text { E } 03 \\
6.8 \text { E } 03\end{array}$ & $\begin{array}{ll}1.6 & E-16 \\
1.5 & E-16 \\
1.2 & E-16 \\
1.1 & E-16 \\
9.0 & E-17 \\
4.0 & E-17 \\
3.1 & E-18 \\
9.5 & E-19 \\
2.6 & E-19 \\
3.6 & E-20\end{array}$ & $\begin{array}{ll}1.3 & E-17 \\
1.6 & E-17 \\
2.1 & E-17 \\
3.2 & E-17 \\
3.9 & E-17 \\
4.5 & E-17 \\
7.1 & E-17 \\
1.8 & E-16 \\
2.7 & E-16 \\
3.3 & E-16 \\
2.4 & E-16 \\
7.0 & E-17 \\
3.3 & E-17 \\
2.0 & E-17 \\
6.0 & E-18 \\
3.0 & E-19 \\
7.3 & E-20\end{array}$ \\
\hline
\end{tabular}

\section{References:}

$\mathrm{He}^{++}+\mathrm{He} \rightarrow \mathrm{Hl}{ }^{+}$: V.V. Afrosimov, G.A. LeǏko, Yu. A. Mamaev, and M.N. Panov, Sov. Phys.-JETP 40, 661 (1975); S. K. Allison, Phys. Rev. 109, 76 (1958); J. E. Bay field and G. A. Khayrallah, Phys. Rev. A 11, $920 \overline{(1975) ; ~ K . ~ H . ~}$ Berkner, R. V. Pyle, J. W. Steams, and J. C. Harren, Phys. Rev. 166, 44 (1968); G. R. Hertel and W. S. Kosk1, J. Chem. Phys. 40, 3452 (1964); P. Hvelplund, J. Heinemeler, E. H. Pedersen, and F. R. Simpson, 9th Int. Conf. Elect. and Atom. Coll., p. 185, Seattle, Wash. (1975); V. S. Nikolaev, I. S. Dmitriev, L. N. Pateeva, and Ya. A. Teplova, Sov. Phys.-JETP 13, 695 (1961); L. I. Pivovar, V. M. Tubaev, and M. T. Novikov, Sov. Phys.-JETP 14, 20 (1962); L. I. Pivovar, M. R. Novikov, and V. M. Tubaev, Soc. Phys.-JEPT 15, 1035 (1962); M. B. Shah and H. B. Gilbody, J. Phys. B 7, 256 (1974).

$H^{t+}+$ tlettle $e^{\circ}$ S. K. Allison, Phys. Rev. 109, 76 (1958); J. E. Bayfield and C. A. Khayrallah, Phys. Rev, A 11, 920 (1975); K. H. Berkner, R. V. Pyle, J. W. Stearns, and J. C. Harren, Phy's. Rev. 166, 44 (1968); V. S. Nikolaev, L. N. Pateeva, I. S. Dmitriev, and Ya. A. Teplova, Snv. Phys.-JETP 14, 67 (1962); L. I. Pivovar, M. T. Novikov, and V. M. Tubaev, Soc. Phys.-JETP 15, 1035 (1962); H. Schrey and B. Huber, Z. Phy. A 273, 401 (1975); M. B. Shah and H. B. Gilbody, J. Phys, B I, 256 (1974).

Accuracy:

$\mathrm{He}^{++}+\mathrm{He}+\mathrm{He}^{+}: \pm 25 \%$ He $\mathrm{He}^{++}+\mathrm{He}+\mathrm{He} e^{\circ}: \pm 20 \%$. 


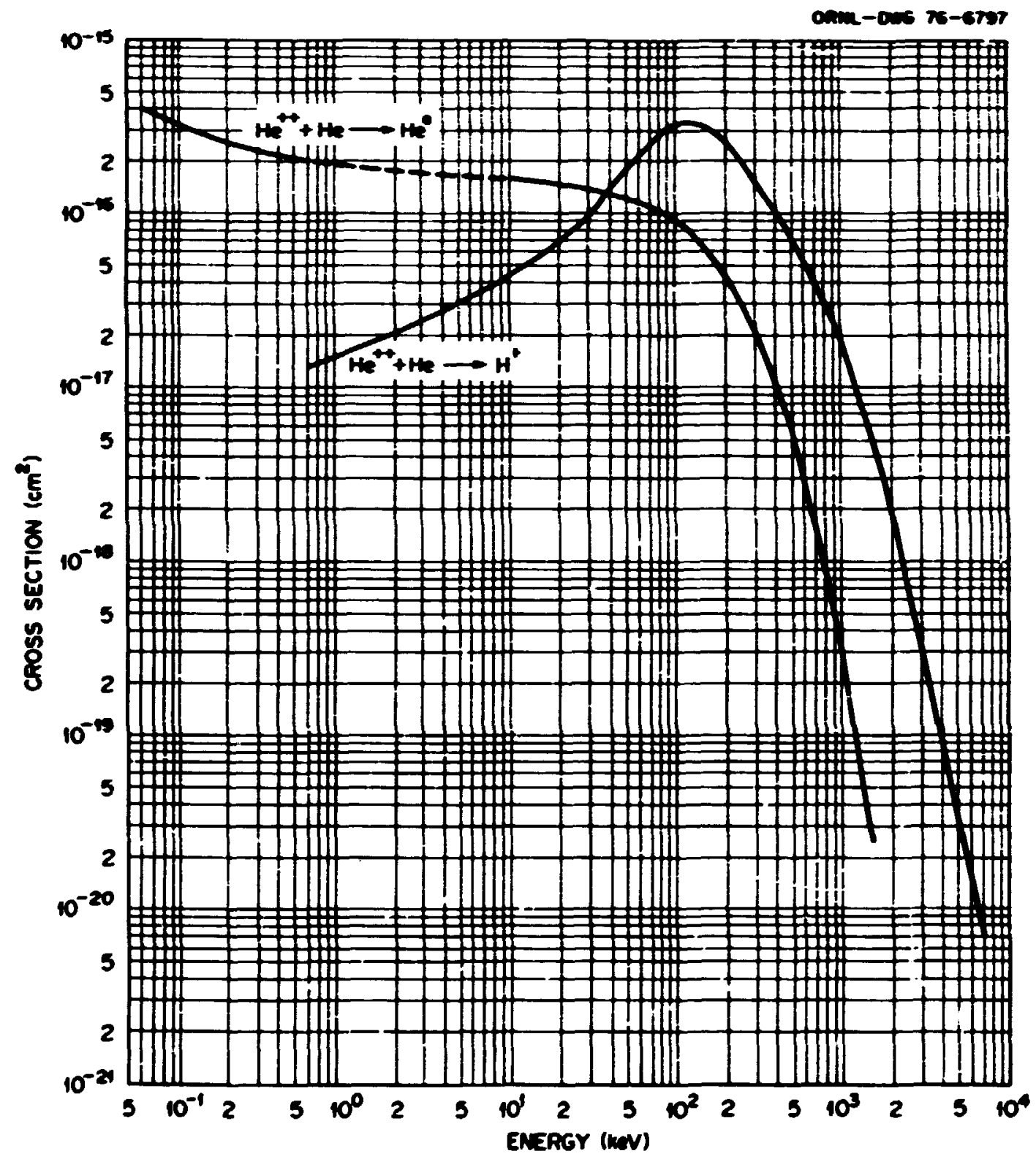


Electron Capture Cross Sections for Hett in $\mathrm{N}_{2}$ and $\mathrm{O}_{2}$

\begin{tabular}{|c|c|c|c|c|}
\hline \multirow[t]{2}{*}{$\begin{array}{l}\text { Eneres } \\
\text { (keV) }\end{array}$} & \multicolumn{4}{|c|}{$\begin{array}{l}\text { Cross Sections } \\
\left(\cos ^{2}\right)\end{array}$} \\
\hline & $\begin{array}{l}\sigma_{21} \\
\mathrm{Be}^{++}+\mathrm{H}_{2}+\mathrm{He}^{+}\end{array}$ & $\begin{array}{l}\sigma_{20} \\
B e^{++}+H_{2}+H_{e}\end{array}$ & $\mathrm{He}^{++}+\mathrm{O}_{2+\mathrm{He}} \mathrm{O}^{+}$ & $\mathrm{He}_{2 \mathrm{O}}+\mathrm{O}_{2}+\mathrm{He}$ \\
\hline $\begin{array}{lll}1.0 & E & 01 \\
2.0 & E & 01 \\
3.0 & E & 01 \\
5.0 & E & 01 \\
7.0 & E & 01 \\
1.0 & E & 02 \\
2.0 & E & 02 \\
4.0 & E & 02 \\
7.0 & E & 02 \\
1.0 & E & 03 \\
1.5 & E & 03\end{array}$ & $\begin{array}{ll}5.6 & E-16 \\
8.9 & E-16 \\
1.1 & E-15 \\
1.2 & E-15 \\
1.2 & E-15 \\
1.1 & E-15 \\
6.1 & E-16 \\
2.0 & E-16 \\
7.3 & E-17 \\
3.4 & E-17 \\
7.7 & E-18\end{array}$ & $\begin{array}{ll}2.5 & E-16 \\
3.1 & E-16 \\
3.3 & E-16 \\
3.3 & E-16 \\
3.1 & E-16 \\
2.6 & E-16 \\
1.0 & E-16 \\
1.2 & E-17 \\
2.0 & E-18 \\
6.0 & E-19 \\
1.4 & E-19\end{array}$ & $\begin{array}{ll}5.6 & E-16 \\
8.1 & E-16 \\
8.4 & E-16 \\
8.5 & E-16\end{array}$ & $\begin{array}{ll}3.6 & E-16 \\
3.8 & E-16 \\
3.7 & E-16 \\
3.2 & E-16\end{array}$ \\
\hline
\end{tabular}

\section{References:}

He ${ }^{++}+\mathrm{H}_{2}$ : J. E. Bayfield and G. A. Thayrallah, Phys. Rev. A 11, 920 (1975); V. S. Hikolaev, I. S. Daitriev, L. N. Pateeva, and Ya. A. Teplova, Sov. Phys.-JETP 13, 695 (1961); V. S. Mikolaev, L. N. Paceeva, I. S. Datriev, and Ya. A. Teplova, Sov. Phys.-JETP 14, 67 (1962); L. I. Pivovar, M. T. Movikov, and F. M. Tubaev, Sov. Phys.-JETP 15, 1035 (1962); L. I. Pivovar, V. M. Tubeev, and H. T. Novikov, Sov. Phys.-JETP 14, 20 (1962); M. B. Shah and H. B. Gilbody, J. Phys. B I, 256 (1974).

$H e^{t+}+O_{2}$ : M. B. Shah and H. B. Gilbody, J. Phys. B I, 256 (1974).

Accuracy:

$H e^{++H_{2}}- \pm 20 \%$.

$$
\mathrm{He}^{++}+\mathrm{O}_{2}- \pm 20 \%
$$




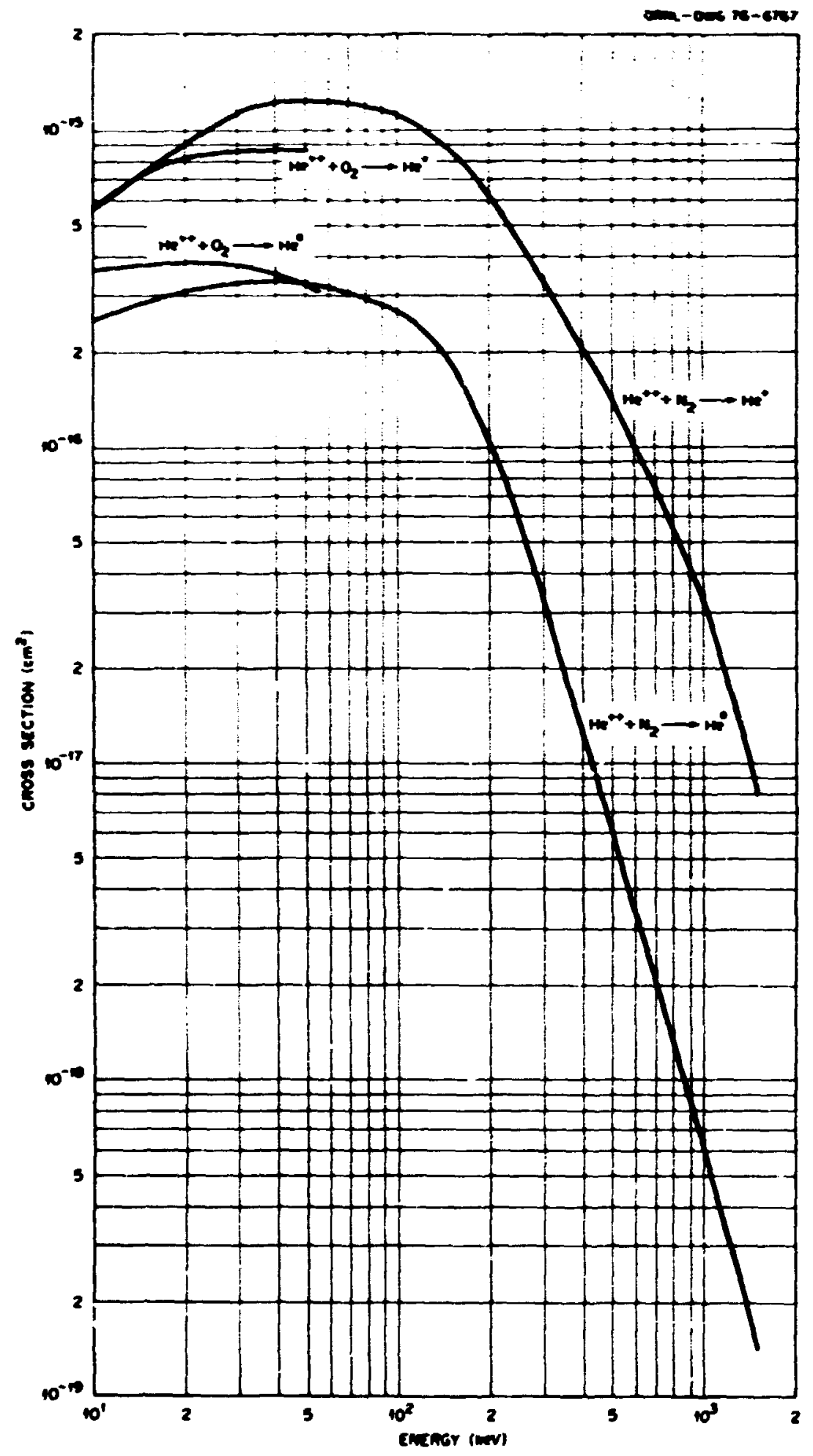


Electron Capture Cross Sections for $c^{+}$and $c^{\bullet}$ in $\mathrm{H}_{2}$

\begin{tabular}{|c|c|c|c|}
\hline \multirow[t]{2}{*}{$\begin{array}{l}\text { Derergs } \\
\text { (keV) }\end{array}$} & \multicolumn{3}{|c|}{$\begin{array}{c}\text { Croas Sections } \\
\left(\mathrm{c}^{2}\right)\end{array}$} \\
\hline & $\frac{\sigma_{10}}{C^{+}+H_{2}+C^{\circ}}$ & $\frac{\sigma_{1}-1}{c^{+}+n_{2}+C^{-}}$ & $\frac{\sigma_{0-1}}{c^{*}+1_{2}+c}$ \\
\hline 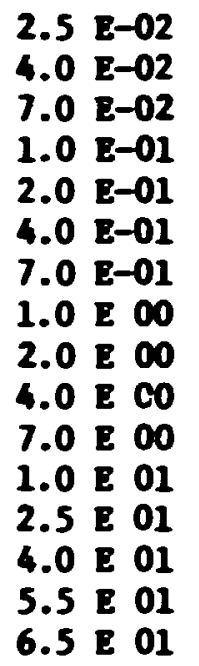 & $\begin{array}{ll}1.0 & \mathrm{E}-16 \\
1.1 & \mathrm{E}-16 \\
1.2 & \mathrm{E}-16 \\
1.3 & \mathrm{E}-16 \\
1.5 & \mathrm{E}-16 \\
1.6 & \mathrm{E}-16 \\
1.9 & \mathrm{E}-16 \\
1.9 & \mathrm{E}-16 \\
1.8 & \mathrm{E}-16 \\
1.7 & \mathrm{E}-16 \\
1.7 & \mathrm{E}-16 \\
2.0 & \mathrm{E}-16 \\
4.3 & \mathrm{E}-16 \\
5.0 & \mathrm{E}-16\end{array}$ & $\begin{array}{ll}3.1 & E-19 \\
1.0 & E-18 \\
2.2 & E-18 \\
4.0 & E-18\end{array}$ & $\begin{array}{ll}4.5 & E-17 \\
4.2 & E-17 \\
5.1 & E-17 \\
5.5 & E-17\end{array}$ \\
\hline
\end{tabular}

\section{Deferences:}

$C^{+}+H_{2}+C^{\bullet}$ : H. B. Gilbody and J. B. Hasted, Proc. Roy. Soc. A238, 334 (1956): E. Gustafsson and E. Lindholm, Ark. Fysik, 18, 219 (1960).

$C^{+}+H_{2}+C^{-}:$Ya. M. Fogel, R. V. Mitin, and A. G. Koval, Sov. Phys.-JETP 4 . 359 (1957).

$\mathrm{CH} \mathrm{H}_{2} \mathrm{C}^{-}$: Ya. M. Pogel, V. A. Ankudinov, and D. V. Pilipenko, Sov. Phys.JETP, $\underline{8}, 601$ (1959).

Accuracy:

Unknown. 


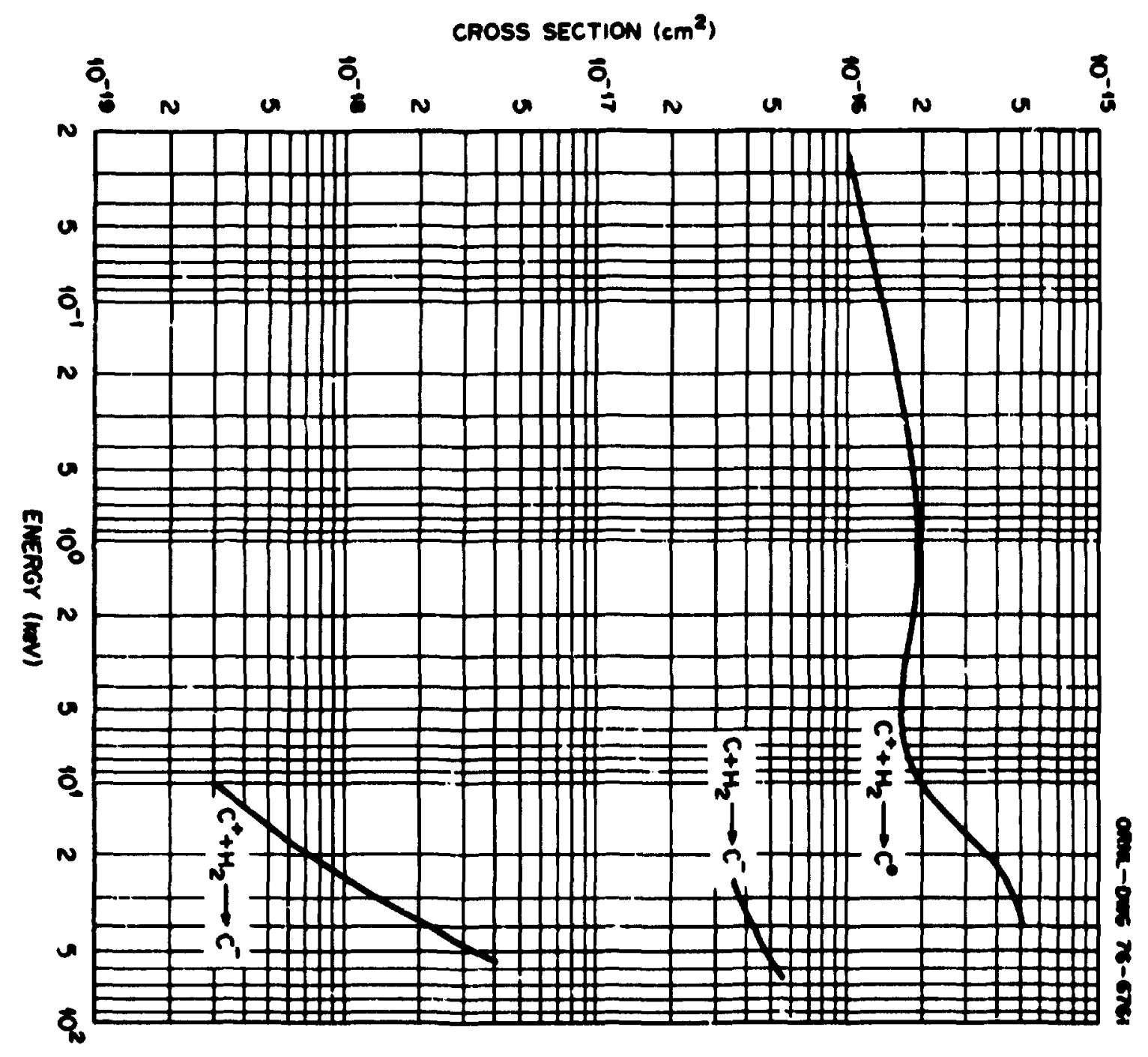




\section{A.4. 44}

Electron Capture Cross Sections for the Reactions

$$
\mathrm{A}^{+}+\mathrm{B}_{2} \text { and } \mathrm{x}^{+}+\mathrm{H}
$$

\begin{tabular}{|c|c|c|}
\hline \multirow[t]{2}{*}{$\begin{array}{l}\text { Energs } \\
\text { (keV) }\end{array}$} & \multicolumn{2}{|c|}{$\begin{array}{c}\text { Cross Sections } \\
\left(\mathrm{cm}^{2}\right)\end{array}$} \\
\hline & $\frac{\sigma_{10}}{x^{t}+n_{2}+x^{\circ}}$ & $\frac{\sigma_{10}}{n^{+}+1+\pi^{0}}$ \\
\hline 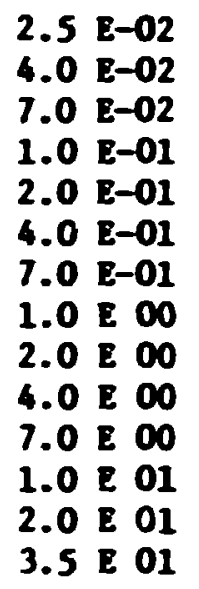 & $\begin{array}{ll}1.7 & E-16 \\
2.2 & E-16 \\
3.1 & E-16 \\
3.4 & E-16 \\
4.6 & E-16 \\
5.5 & E-16 \\
6.0 & E-16 \\
6.0 & E-16 \\
6.0 & E-16 \\
6.2 & E-16 \\
6.8 & E-16 \\
8.5 & E-16 \\
1.1 & E-15 \\
1.2 & E-15\end{array}$ & 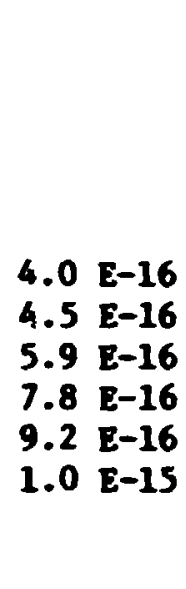 \\
\hline
\end{tabular}

\section{References:}

$H^{+}+H_{3}:$ P. J. De Heer, W. Huizenga, and J. Kistenaker, Physisa 23, 181

(1957); H. B. Gilbody and J. B. Hasted, Proc. Roy. Soc. 1238, 334 (1956);

E. Gustafeson and E. Lindholn, Ark. Fysik 18, 219 (1960).

$A^{+}+$H: R. Y. Stebb1ngs, H. L. Fite and D. G. Humer, J. Chee. Phys. 33, $1226(1960)$.

Accuracy:

$n^{+}+\mathrm{B}_{2}= \pm 50 \%$.

$n^{+}+1: \pm 20 \%$. 


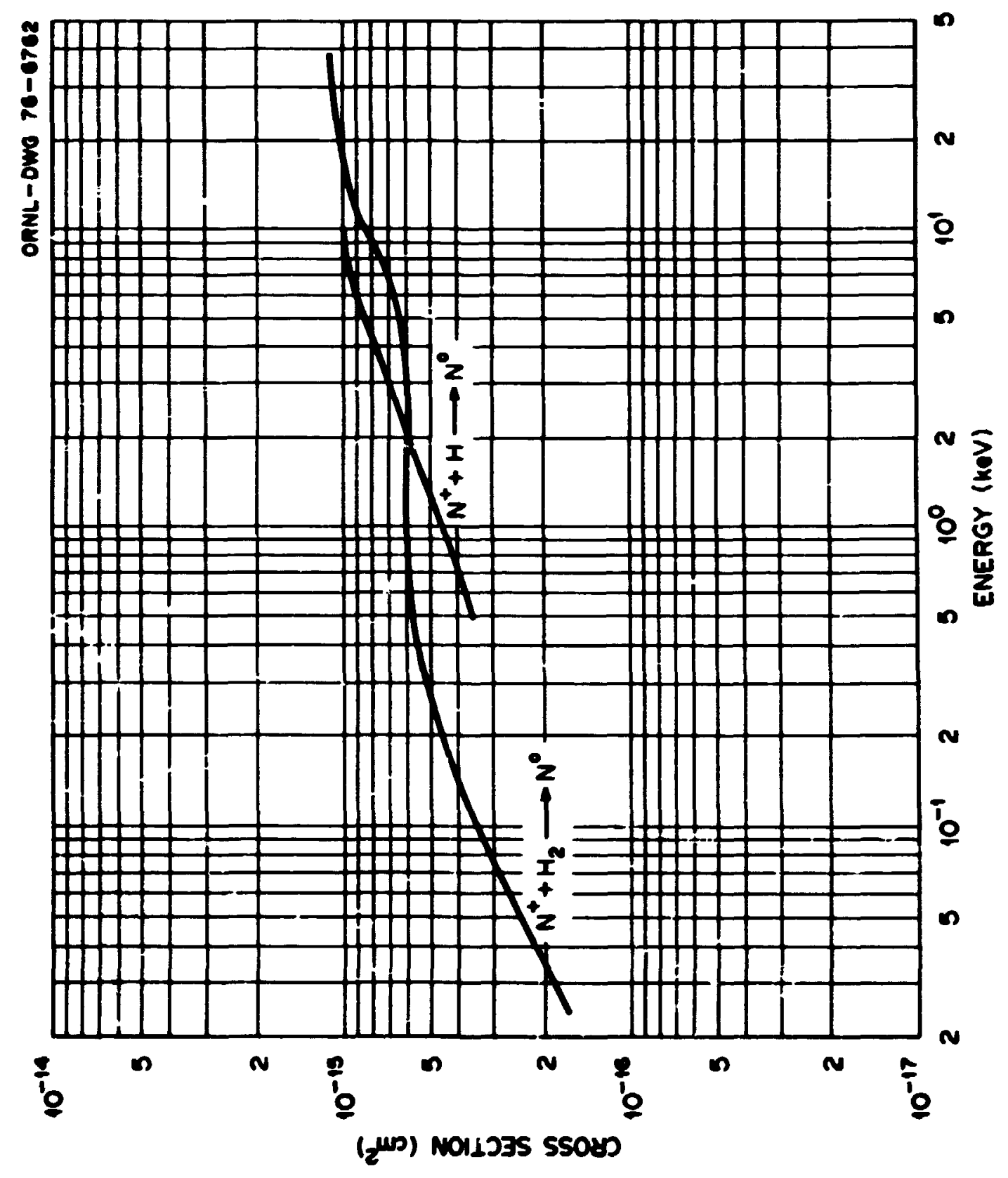


Electron Capture Cress Sections for the Reactions

$$
\mathrm{O}^{+}+\mathrm{B}+0^{\bullet}, \mathrm{O}^{+}+\mathrm{B}_{2}+0^{\bullet} \text {, and } \mathrm{O}^{+}+\mathrm{H}_{2}+\mathrm{O}^{-}
$$

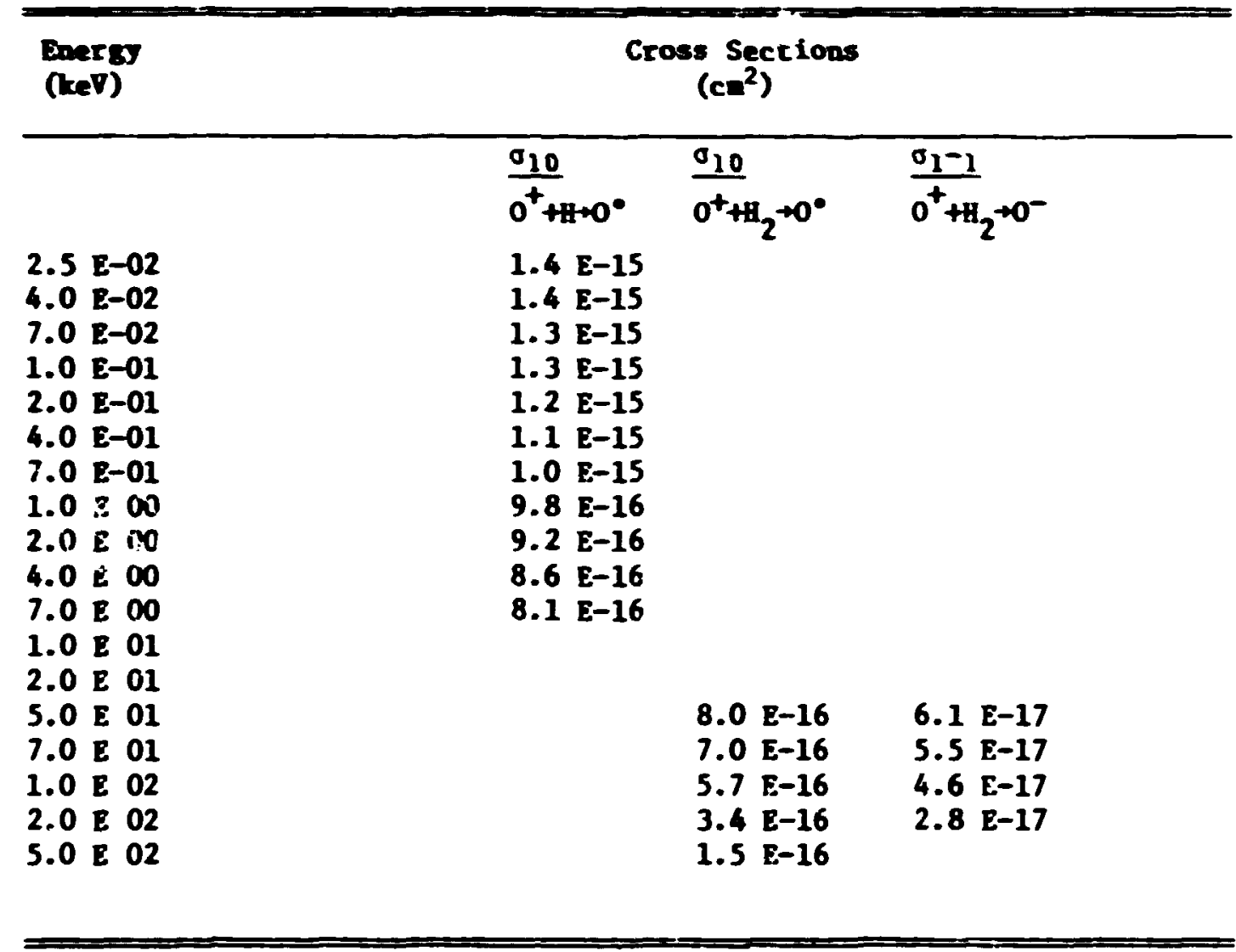

\section{References:}

$0^{+}+\mathbb{0 ^ { \circ }}$ : W. L. Fite, A. C. H. Smith, and R. F. Stebbings, Proc. Roy. Soc. A268, 527 (1962): R. F. Stebbings, H. L. Fite, and D. G. Hummer, J. Chem. Phys. 33, 1226 (1960).

$0^{+}+\mathrm{H}_{2} \rightarrow 0^{-}$: T. Jorgensen, Jr., G. E. Ruyate, W. W. Lang, D. C. Lorentz, and C. A. Sautzer, Phys. Rev. 140, A 1481 (1965).

$0^{+}+H_{2}+0^{\circ}:$ T. Jorgensen, Jr., G. E. Kuyate, W. W. Lang, D. C. Lorentr, and C. A. Sautter, Phys. Rev. 140. A1481 (1965); J. P. Oisen and P. Hvelplund, J. Phys. B I, 1331 (1974).

\section{Accuracy:}

$\mathrm{O}^{+}+\mathrm{H}_{2}+\mathrm{O}^{\circ}: \pm 25 \%$.

$0^{+}+\ldots+0^{\circ}: \pm 20 \%$.

$0^{+}+\mathrm{H}_{2}+\mathrm{O}^{-}: \pm 25 \%$. 


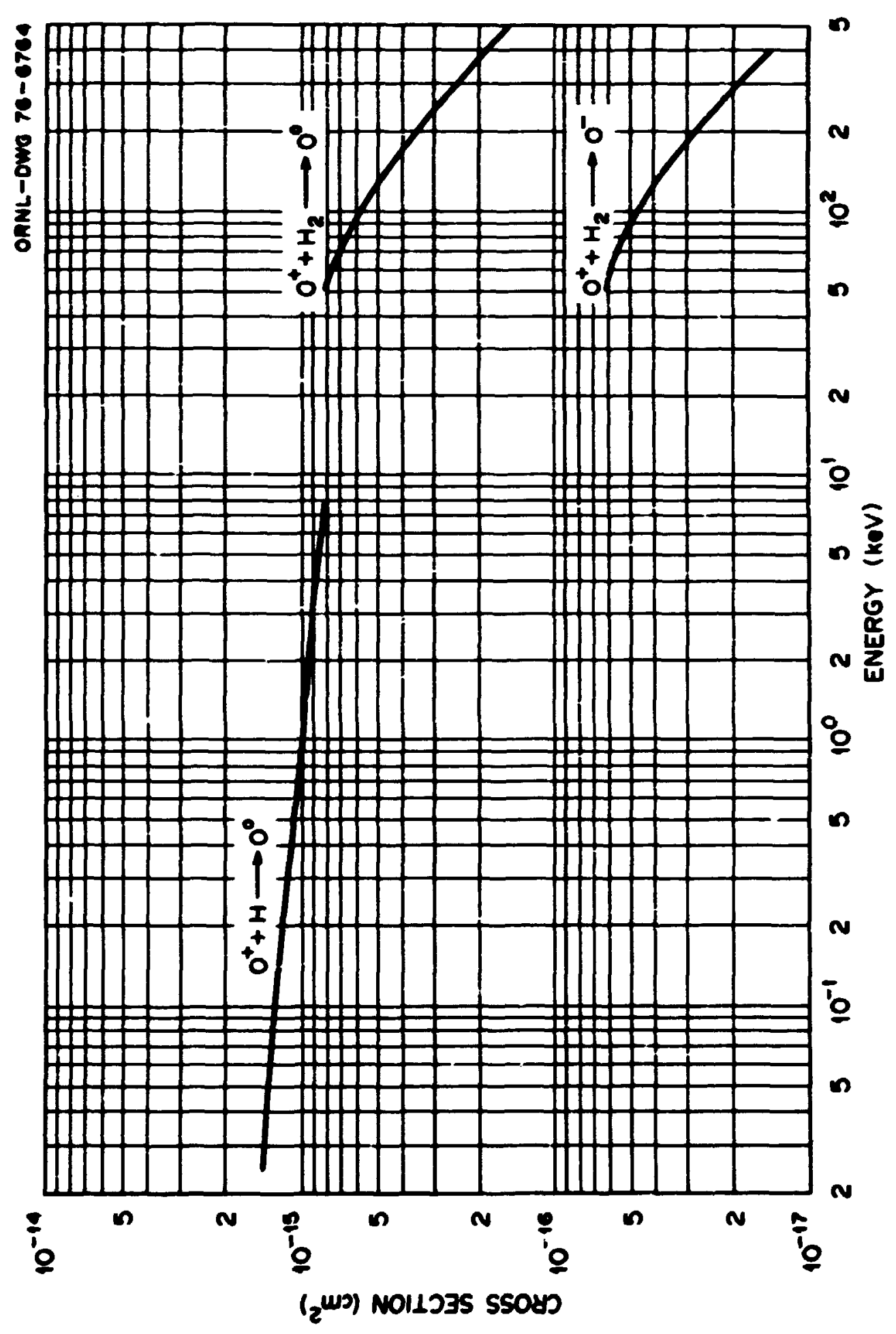




\section{A. $\$ .48$}

Electron Capture Cross Sections for

$$
0^{\circ} \text { and } \mathrm{O}^{+\mathrm{H}} \text { in } \mathrm{H}_{2}
$$

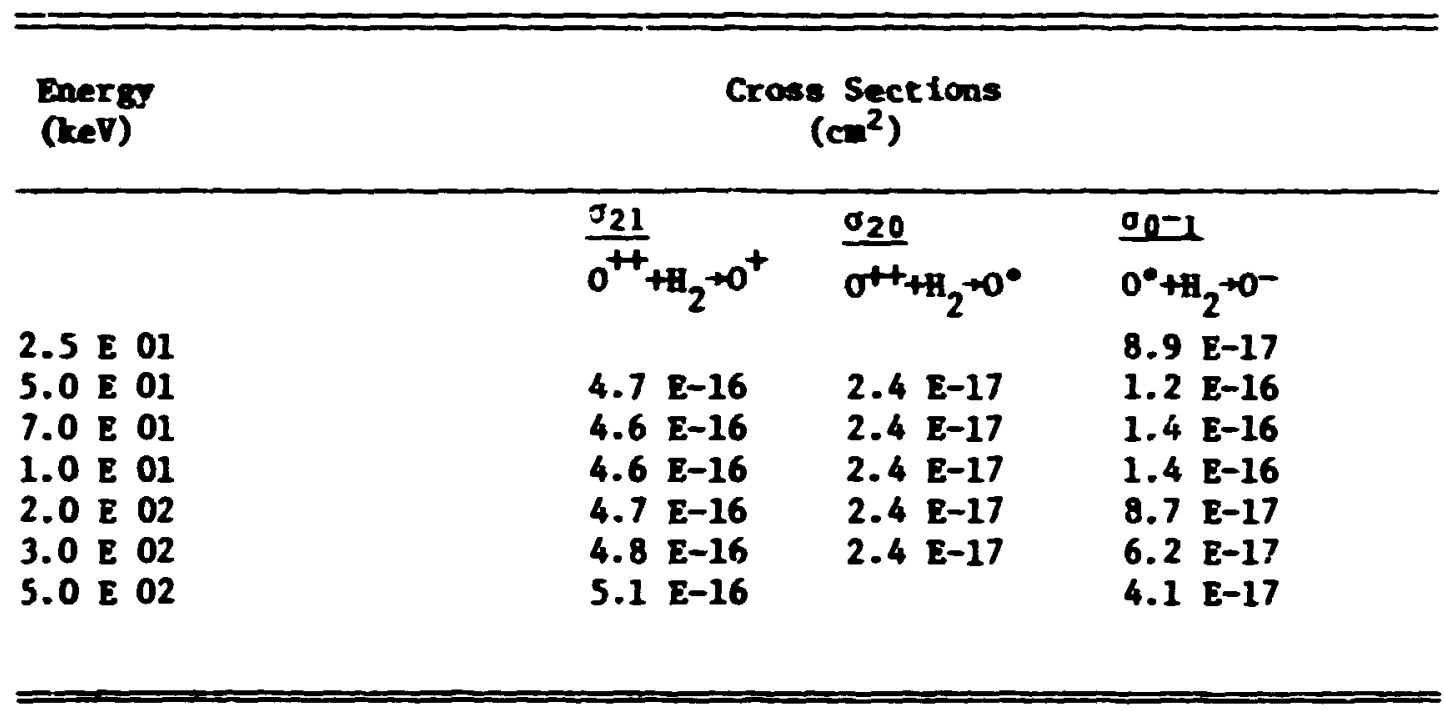

\section{References:}

$0^{++}+\mathrm{H}_{2} \rightarrow 0^{+}:$T. Jorgensen, Jr., G. E. Ruyatr, W. W. Lang, D. C. Lorentz, and $C^{2}$. A. Sauter, Phys. Rev. 140, Al481 (1965); J. O. O1sen and P. Hvelplund, J. Phys. B I, 1331 (1974).

$0^{++}+\mathrm{H}_{2}+0^{\circ}:$ T. Jorgensen, Jr., G. E. Kuyate, W. H. Lang, D. C. Lorentz, and C. A. Sauter, Phys. Rev. 140, Al481 (1965); J. O. O1sen and P. Hvelplund, J. Phys. B I, 1331 (1974).

$0^{\circ}+H_{2}+0^{-}$: Ya. M. Pogel, V. A. Ankudinov, D. V. P1lipenko, Sov. Phys .-JETP 8, 601 (1959); T. Jorgensen, Jr., G. E. Kuyatt, W. W. Lang, D. C. Lorentz, and C. A. Sautter, Phys. Rev. 140, A1481 (1965); J. O. Olsen and P. Hvelplund, J. Phys. B I, 1331 (1974).

Accuracy:

$\mathrm{O}^{++}+\mathrm{H}_{2}+\mathrm{O}^{+}: \pm 20 \%$.

$\mathrm{O}^{++}+\mathrm{H}_{2} \rightarrow \mathrm{O}^{\circ}$ : Unknown.

$0^{\circ}+\mathrm{H}_{2}+0^{-}: \pm 40 \%$. 


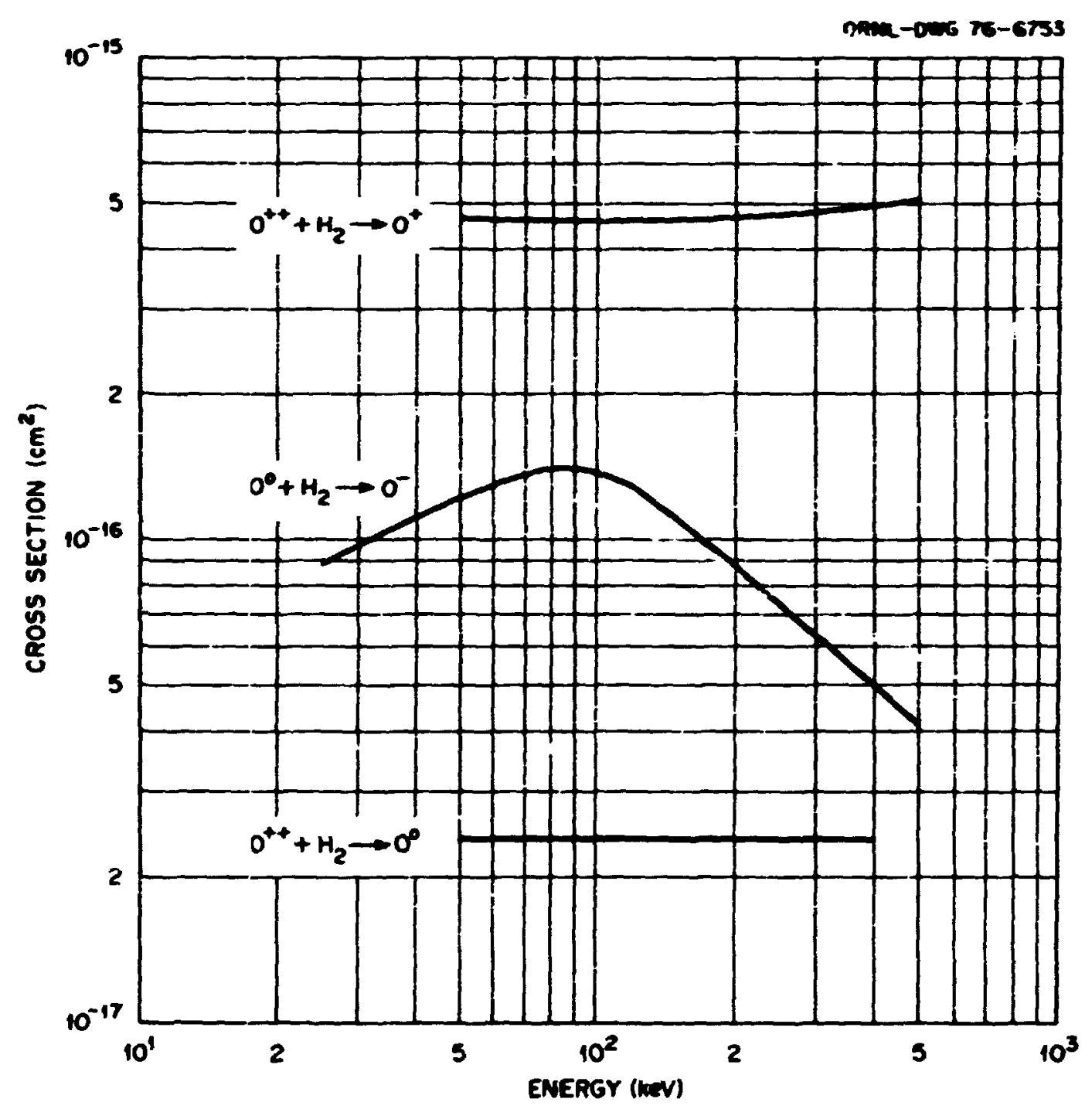




\section{A.4.51}

(Continuations)

$$
\begin{aligned}
& \text { ilectron Capture Cross Sections for } \mathrm{H}^{+} \text {and } \mathrm{a}^{*} \\
& \text { Pacsing Through } \mathrm{B}_{2}
\end{aligned}
$$

\section{Beferences: (contd.)}

$\mathrm{H}^{+}+\mathrm{H}_{2}+\mathrm{H}^{-}$: V. V. Afrosinov, G. A. Leiko, Yu. A. Manev, M. M. Panor, and A. V. Fedorenko, Sov. Phys.-JETP 35, 1070 (1972); V. F. Tozlor and S. A. Bondar, Sov. Thys.-JETP 23, 195 (1966); G. H. Ecclure, Thys. Rev. 132, 1636 (1963); Te. H. FopeI, R. V. Mitin, V. F. Toxlov, and A. D. Ronaskico, Sov. Pbys.-JETR 35, 390 (1959); D. Schryber, Rel. Phy. Act. 140, 1023 (1967); L. A. Toburen ad n. Y. Dakat, Phys. Rev. 177, 191 (1969); J. F. H111en, Phys. Rev. 150, 7 (1966); J. F. iflllev, Thys. Rev. 157, 97 (1967).

$\mathrm{H}^{\circ}+\mathrm{H}_{2}+\mathrm{H}^{-}$: V. V. Afroeinov, G. A. Leiko, Tu. A. H-aev, M. M. I eor, and H. V. Fedorenko, Sov. Phys.-JETP 35, 1070 (1972); G. H. HeClure, Phys. Dev. 134, 11226 (1964); T. Jorecensen, Jr., C. E. Tuyete, H. H. Lang, D. C. Lorentz, and C. A. Sautter, Phys. Rev. 140, A1481 (1965); U. Schryber, Bel. Phy. Act. 140, 1023 (1967); P. M. Stier ad C. P. Barmett, Phye. Dev. 103, 896 (1956); J. P. Williem, Phys. Rev. 153, 116 (1967).

\section{Accuracs:}

$0_{10} \pm 302$

$\sigma_{1-1} \pm 507$

$\sigma_{0-1} \pm 408$

\section{Notes:}

0 1-1 has not been plotted for energies greater then $400 \mathrm{keV}$. For cross sections between 400-1000 kev see J. F. H1111.m, Phys. Rev. 157, 97 (1967).

\section{Electron Capture Crose Sections for $\mathrm{B}^{+}$and $\mathrm{B}^{\bullet}$ \\ Paesing Through Hellu}

\section{Beferences: (contd.)}

H the+H: Y. M. Pogel, V. A. Ankudinov, D. V. PLlipenko, and M. V. Topolia, Sov. Phys.-JLIP 34, 400 (1958); P. M. Stier ad C. P. Bermete, Phys. Rev. 103, 896 (1956); U. Schryber, Hel. Phy. Act. A60, 1023 (1967).

Accuracy:

$010 \pm 25 \%$

$0_{1-1} \mathrm{E}>10 \mathrm{keV} \pm 25 \% ; \mathrm{E}>10 \mathrm{keV} \pm 60 \%$

$00-1 \pm 257$

Notes:

Barkner, et al., reeulte for $D^{+}$in be hes been plotted at $E / 2$. 
(continuatisas)

Electron Capture Cross Sections for $\mathrm{u}^{+}$and $\mathrm{H}^{*}$

Passiag Through $x_{2}$

Deferences: (contd.)

$\mathrm{B}^{\circ}+\mathrm{l}_{2}+\mathrm{A}^{-}$: S. K. Allison, Rev. Hod. Phys. 30, 1137 (1958); Ya. H. Fogel, V. A. Antudinov, D. V. Pilipeoto, ad X. V. Topolle, Sov. Ptys.-JETP 7, 400 (1958); D. V. Pllipenko ad Ys. H. Fogel, Sov. Phys.-Tech, Phys. I5, 646 (1962); P. M. Stier and C. F. Banett, Phys. Dev. 103, 896 (1956).

At+1 $_{2}+\mathrm{H}^{-}$: S. K. Allison, Lev. Hod. Ftys. 30, 1137 (1958); Ta. H. Pogel and R. V. Hicen, Sov. Phys.-JETP 3, 334 (1956); D. Schryber, Eelv. Phys. Acte. 140,1023 (1967); L. H. Toburen, M. Y. Makne, and R. A. Lagley, Thyse. Rev, 171, 114 (1968).

Accurac:

$\sigma_{10} \pm 20 \%$

$\sigma_{1-1} \pm 20 \%$

$\sigma_{0-1} \pm 252$

Electron Capture Crose Sections for $\mathrm{H}^{+}$and $\mathrm{H}^{*}$

Paasing Through $\mathrm{O}$ and $\mathrm{O}_{2}$

\section{Eeference: (contd.)}

H $^{+}+0+\mathrm{B}^{-}:$R. P. Stebbinga, A. C. H. Sadth, and H. Ehrhardt, J. Ceophys. las. 69, 2349 (1964).

H'+02+1: Ya. M. Pogel, V. A. Aukudinov, D. V. Pilipenko, and I. V. Topolis, Sov. Phys.-Tech. Phys. 7, 400 (1958); D. V. Ptlipenko and Ya. H. Poegl, sov. Phys.-JLIP 15, $64 \overline{6}$ (1962); P. H. Stier and C. F. Bamet, Fiys. Rev. 103, 896 (1956).

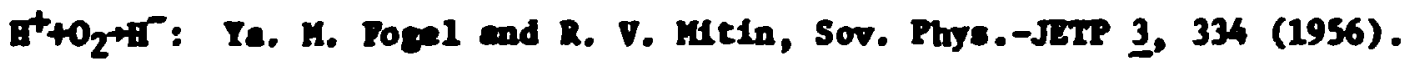
Accurac:
$\sigma_{10}\left(O_{2}\right)= \pm 308$
$\sigma_{10}(0)= \pm 30 \%$
$\sigma_{0-1}\left(O_{2}\right)= \pm 25 \%$
$\sigma_{1-1}=$ minown 
(Continuations)

Electron Capture Cross Sections for $\mathrm{H}^{+}$and $\mathrm{u}^{\bullet}$

Passing Through Ar

References: (contd.)

Ht+Ar+H: V. V. Afrosinov, Yu. A. Mneve, M. P. Penow, and V. Oroskevich, Sov. Phys. -Tech. Phys. 12, 512 (1967); Ya. M. Posel, Sov. Phys. Usp. 3, 390 (1960); V. V. Afrosinov, R. H. II'in, and E. S. Solov'ev, Sow. Phys.Tech. Phys. 5, 661 (1960); U. Schryber, Delv. Phys. Acte 40. 1023 (1967); L. H. Tobureă, H. Y. Nakal, and R. A. Lngley, Phys. Rev. 177, 191 (1969); J. F. Millie, Phys. Dev. 150, 7 (1966).

$H^{\circ}+A r+H^{-}$: T. H. Donchue and F. Hushfar, Phys. Rev. 124, 138 (1961); Ya. H. Pogel, V. A. Ankudinov, D. V. Phipento, ad A. V. Topolis, Sov. Phys.-JETP I, 400 (1958); U. Schryber, Helv. Phys. Acte 40, 1023 (1967); P. H. Seier and C. F. Ramet, Thys. Rev. 103, 896 (1956); J. P. Millies, Phys. Rev. 153, 117 (1967).

Accuras:

$\sigma_{10}- \pm 252$

$\sigma_{0-1} \pm \mathbf{4 0 2}$

$\sigma_{1-1} \pm 402$

Electror. Capture Croos Sections for

$\mathrm{He}^{+}$in $\mathrm{H}_{2}$ and $\mathrm{Be}$

\section{Deferences: (contd.)}

Bet+lie: S. R. Allison, J. Cueves, P. G. Murphy, Phys. Rev. 102, 1041 (1956);

C. P. Barmett and P. M. Stier, Phys. Rev. 109, 385 (1958); 1. V. Pedorenko, L. G. P1Lppenko, and I. P. Fieke, Sov. Physe-Tech. Phys. 5, 45 (1960); A. Gall1, A. Gierdini-Guddont, G. G. Volp1, Nuovo C1mento 26, 845 (1962); I. J. Delber, J. Schutten ad H. Moustafa, Physica 32, 1793 (1966); H. B. Gilbod, J. B. Hested, J. V. Ireland, A. R. Lee, E. W. Thone, and A. S. Mhitenen, Proc. Ros. Soc. London 1274, 10 (1963); H. B. Gtlbods and J. B. Hasted, Proc. Boy. Sxc. London 1238, 334 (1956); H. C. Basden and H. G. Veterback, Phys.

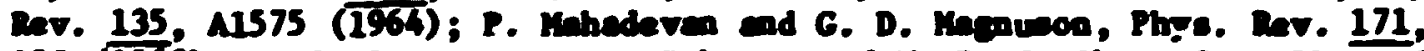
103 (1968); L. I. P1wovar, V. M. Tubeev, and M. T. Movikow, Sov. Phys.-Jit? 14. 20 (1962); R. F. Potter, J. Chem. Phye. 22, 974 (1954); W. W. Sheltea and P. A. Stoycheff, Thye. Rev. A 3, 613 (1971); J. B. H. Stedetiord and J. B. Hested, Proc. Roy. Soc. Londor 1227,446 (1954).

Accurag:

$B^{+}+B_{2}: \pm 158$

int+ik: \pm 158 
A.5 Ionization and Stripping by Heary Particles

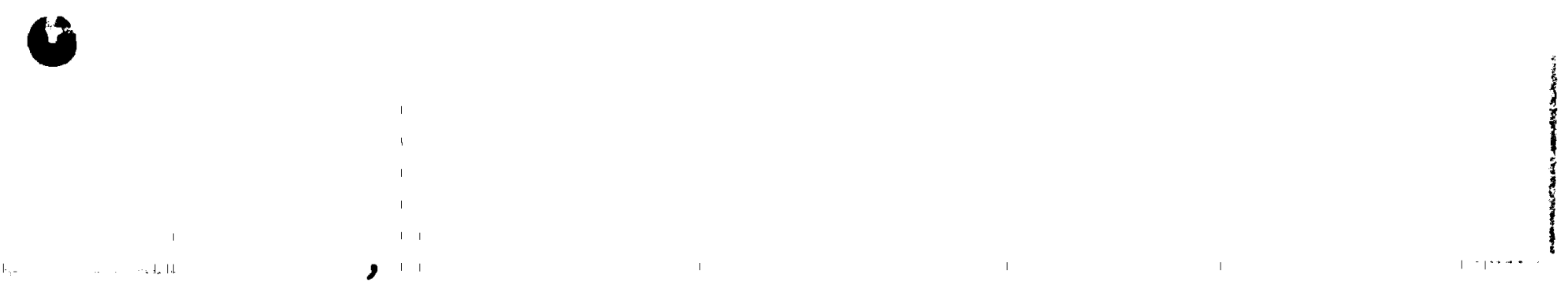




\section{A.5.2}

Cross Sections for Ionization of Atonic it bo $\mathrm{H}^{+}$

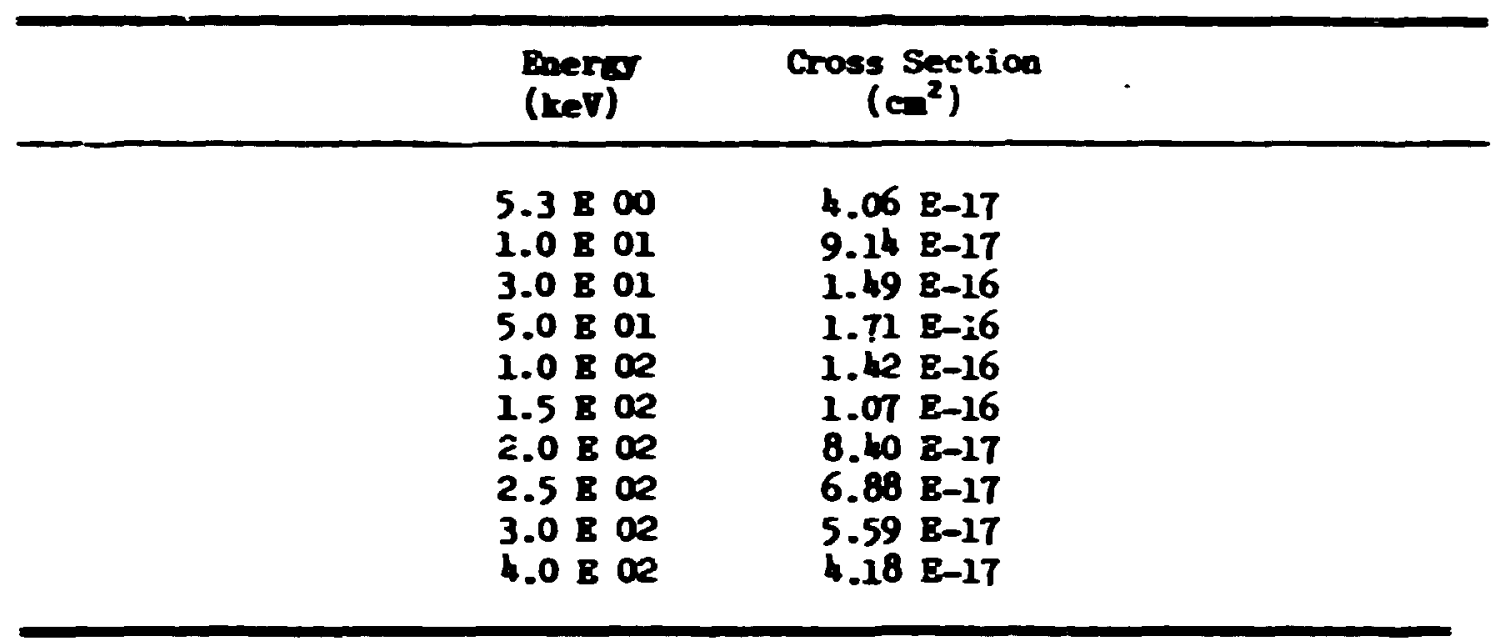

\section{Deferences:}

W.I. Fte, R.I. Stebbine, D.G. Humer, ad R.T. Bracken, Puys. Rer. 119, 663 (1960); H.B. Gillod ad J.V. Irelna, Proc. Ros. Soc. $1-277,137$ (1964).

\section{secures:}

$\pm 20: 3$. 


\section{‥5.3}

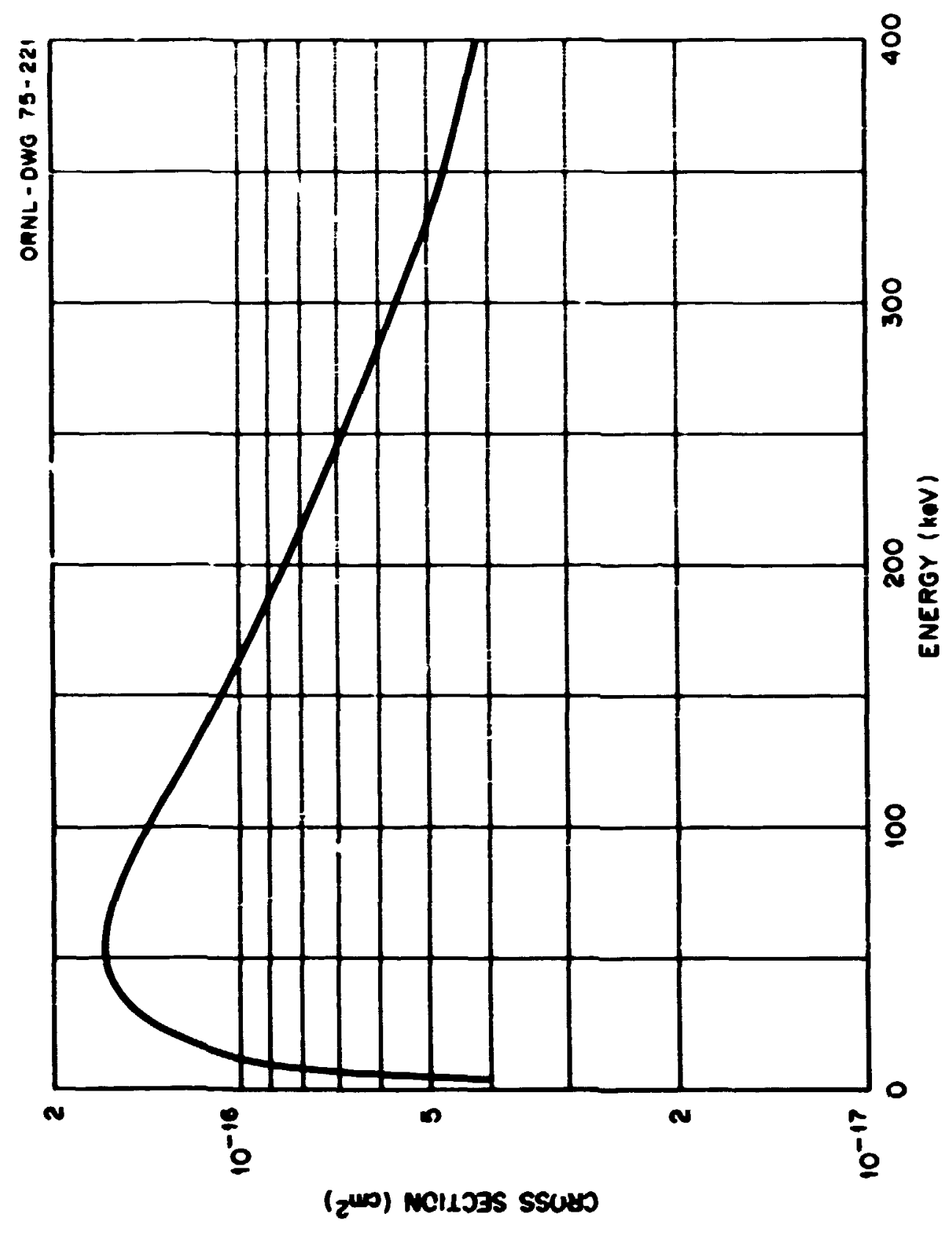




$$
\text { A. } 5.4
$$

Cross sections for the Production of positire vharzes in $\mathrm{x}_{2}$ and ife by $\mathrm{I}^{\star}$

\begin{tabular}{|c|c|c|}
\hline \multirow[t]{2}{*}{$\begin{array}{l}\text { Inergd } \\
\text { (kev) }\end{array}$} & \multicolumn{2}{|c|}{$\begin{array}{c}\text { Cross jections } \\
\left(\mathrm{cm}^{2}\right)\end{array}$} \\
\hline & $\mathbf{H}_{2}$ & ت̈e \\
\hline 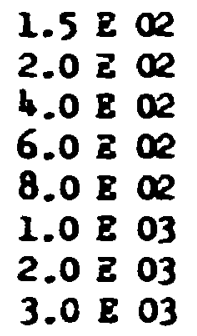 & $\begin{array}{rr}1.77 & E-16 \\
1.40 & \Xi-16 \\
7.59 & =-17 \\
5.37 & =7 \\
4.18 & E-17 \\
3.44 & \Xi-17 \\
1.91 & E-17 \\
1.35 & \Xi-17\end{array}$ & $\begin{array}{ll}8.66 & \Xi-17 \\
0.99 & \Xi-17 \\
4.1 \Xi & \Xi-17 \\
3.08 & \Xi-17 \\
2.43 & \Xi-17 \\
2.09 & \Xi-17 \\
1.24 & \Xi-17 \\
9.29 & \Xi-18\end{array}$ \\
\hline
\end{tabular}

\section{References:}

B.H. MeDaniel, J.H. Hooper, D.d. Martin, and D.S. jarmer, Proc. Pifth Edt. Coaf. on Ionizntion Pbenonem in Geses (Muich, 1961) Vorth-Holland Publisking Co. (Asterdea) Vol. 1, 60 (1962); L.I. Pivover and Yu. Z. Eerchenko, Sor. PWVs._JETP 25, $2 \overline{7}$ (1967). 


\section{A. 5.5}

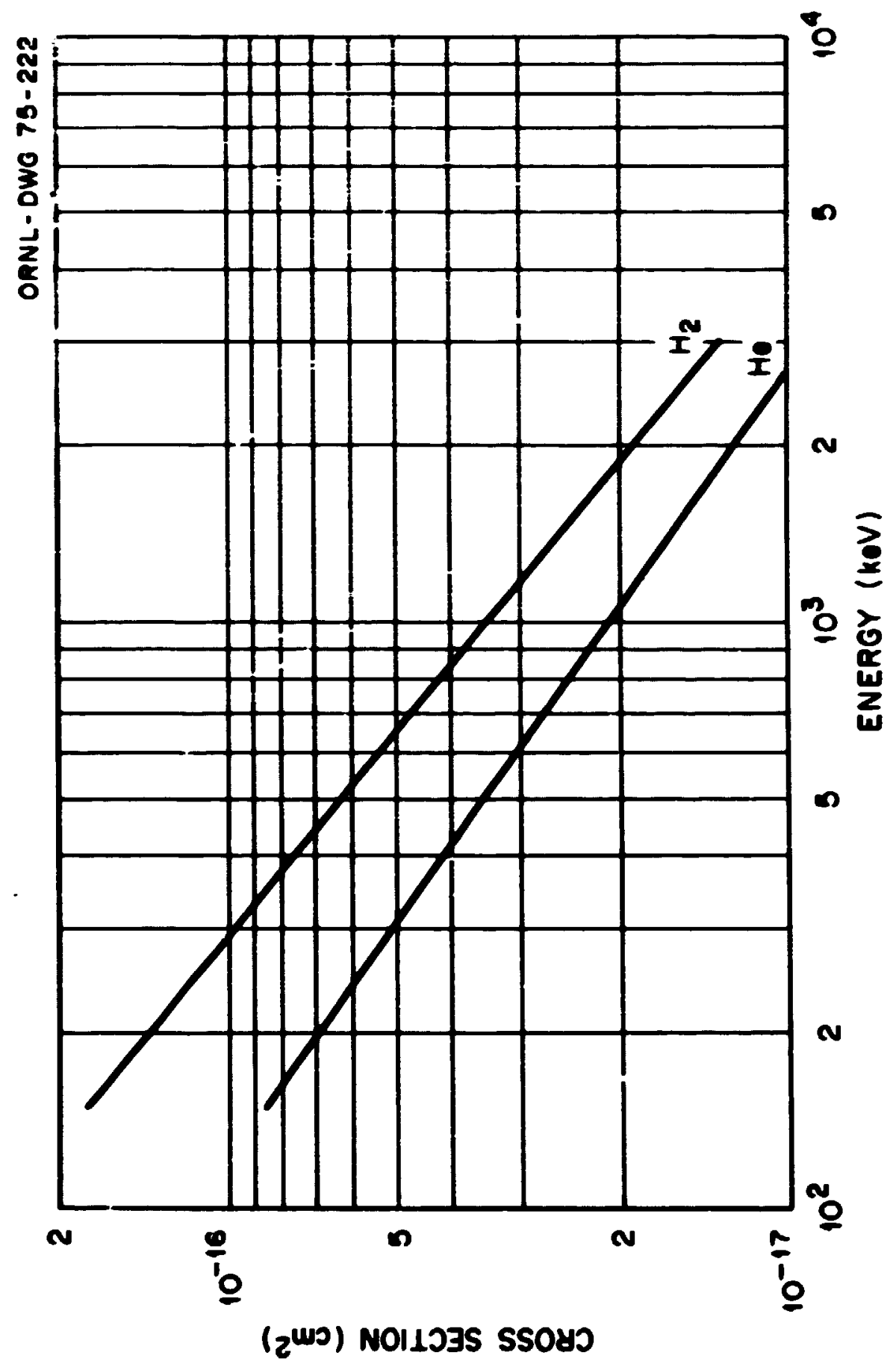




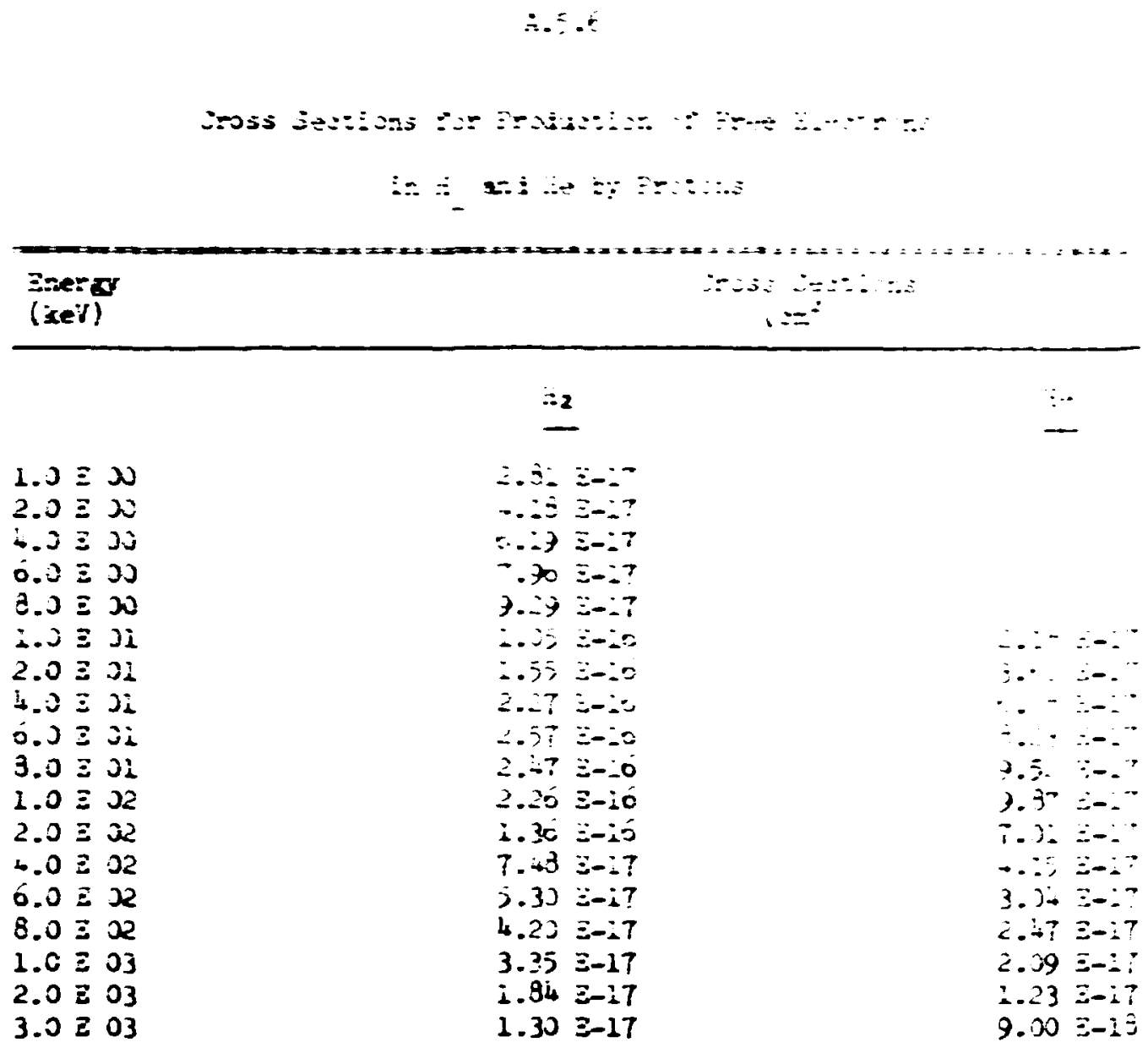

\section{References:}

$4^{+}+\mathrm{H}_{2}$ : J.H. Hooper, Z.H. MeDaniel, D.H. Mrtin, and J.3. Harmer, Phys. Rev. 121, 1123 (1961); L.I. Pivovar and Yu. Z. Levchenko, Soviet Phys.-JETP 25, 27 (1967); Yu. S. Gordeer and M.X. Panov, Soriet Pins.Tech. Phys. 9,656 (1964); Z.S. Soior'ev, R.J. IL'in, V.A. Ops:in, and M.V. Pedorenko, Soviet Phys . JETP 15, 459 (1962); F.J. de Jieer, J. Jehutter, and H. Mustafe, Physica 32, $1766(1966)$; V.V. Afrosianov, R.X. II'in, and X.V. Pedorenko, Soviet Phys .-JETP $I, 968$ (1958); P. Jehwirzice, 2. Piys. 15T, 510 (1960); Ye. M. Fogel, L.I. Krupnik, and B.G. Safronor, Soriet Phys.-JEIP 1, 415 (1955); K.3. Gilbody and A.R. iee, Proc. Phys. Joc. A-274, 365 (1963); M.E. Rudd, C.A. Sautter, and C.L. Bailley, Phys. Rer. 151, 20 (1966).

$\mathrm{H}^{+}+$He: E.W. McDariel, J.W. Hooper, D.W. Martín, and D.3. Harmer, Proc. Fifth Int. Cont. on Ionization Pt.?nomena in Gases (Munich, 1961) Worth-Holland Publishing Co. (Aasterdam) Vol. 1, 60 (1962); L.I. Pi vovar and Yu. Z. Levchenko, Soriet Phys.-JETP 25, 27 (1967); 8.J. de Heer, $\therefore$ Schutten, and H. Moustafa, Physica 32, 1766 (1966); H.B. Gilbody and A.R. Lee, Proc. Roy. Soc. A-274, $365\left(\frac{1963) ;}{196 . V . ~ P e d o r e n k o, ~ V . V . ~ A l r o s i m o v, ~}\right.$ R.N. Il'in, and Z.S. Solor'ev, Proc. Pourth Int. Conf. on Ionization Phenomene in Gases (Uppsala, 1959), Horth Holland Publishing Co. (Amsterdan) Vol. I, IA-47 (2960).

\section{Accuracy:}

$\pm 25 \%$. 


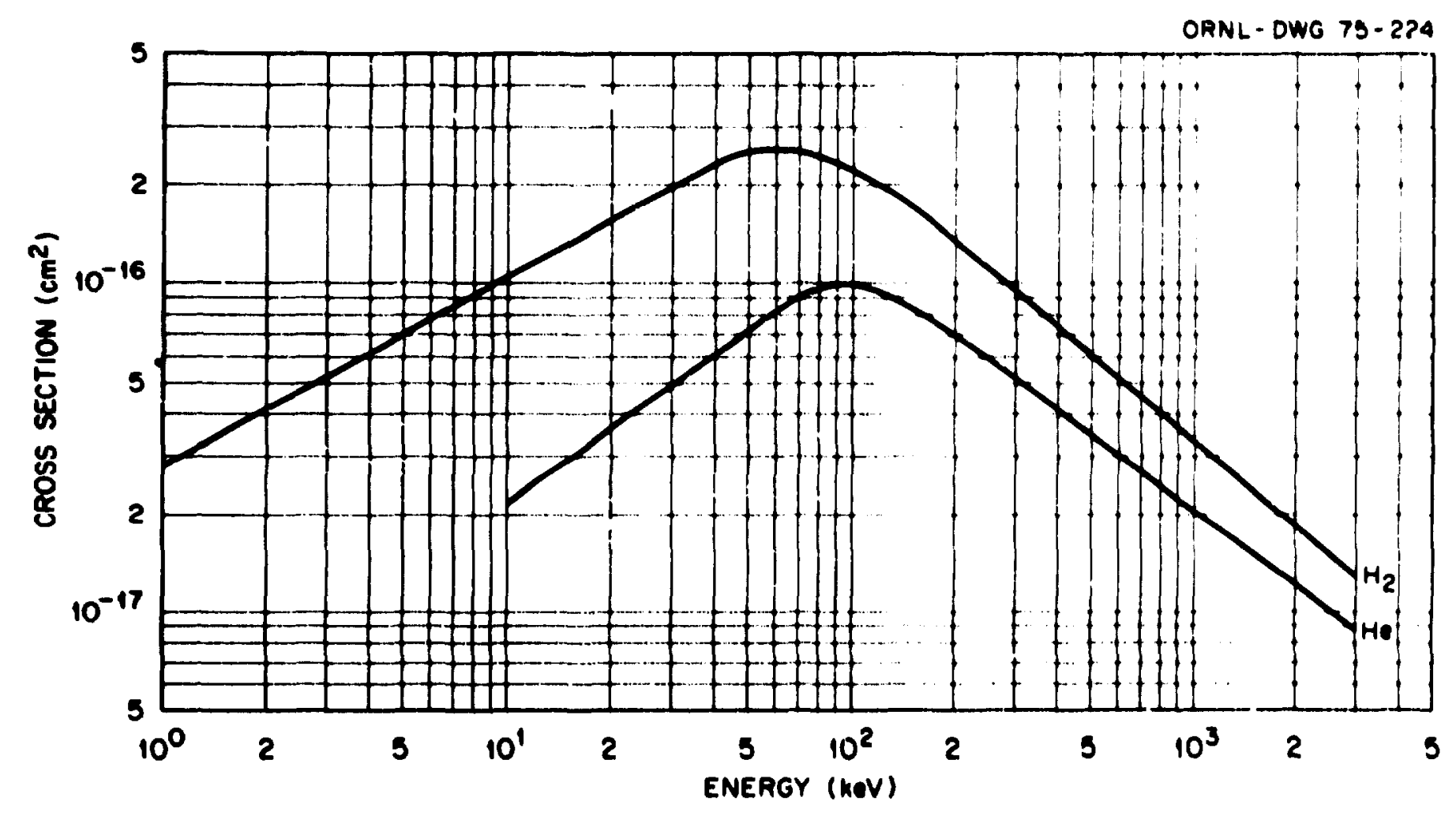




\section{A.5.8}

Cross Sections for the Production of Slor

$$
\mathrm{H}_{2}^{+} \text {and } \mathrm{H}^{+} \text {Tron } \mathrm{H}^{+} \text {and } \mathrm{H}^{\circ} \text { Imfact on } \mathrm{u}_{2}
$$

\begin{tabular}{|c|c|c|c|c|}
\hline \multirow[t]{2}{*}{$\begin{array}{l}\text { Bnergy } \\
\text { (heV) }\end{array}$} & \multicolumn{4}{|c|}{$\begin{array}{c}\text { Cross Sections } \\
\left(\mathrm{cm}^{2}\right)\end{array}$} \\
\hline & $\mathrm{H}^{+}+\mathrm{Hi}_{2}+\mathrm{H}_{2}{ }^{+}$ & $\mathrm{Z}^{+}+\mathrm{H}_{2}+\mathrm{i}^{+}$ & $\mathrm{H}^{\mathrm{O}}+\mathrm{H}_{2}+\mathrm{H}_{2}^{+}$ & $\mathrm{Hi}^{\mathrm{O}}+\mathrm{i}_{2}+\mathrm{HH}^{+}$ \\
\hline $\begin{array}{lll}5.0 & \mathrm{E} & 00 \\
1.0 \mathrm{E} & 01 \\
2.0 \mathrm{E} & 01 \\
3.0 \mathrm{E} & 01 \\
4.0 \mathrm{E} & 01 \\
5.0 \mathrm{E} & 01\end{array}$ & $\begin{array}{ll}8.1 & z-16 \\
8.0 & z-16 \\
5.2 & z-15 \\
4.8 & E-16 \\
3.8 & E-16 \\
3.4 & E-16\end{array}$ & $\begin{array}{ll}4.0 & 2-17 \\
9.3 & E-17 \\
1.2 & E-16 \\
8.7 & E-17 \\
6.3 & E-17 \\
4.4 & E-17\end{array}$ & $\begin{array}{ll}4.0 & \mathrm{E}-17 \\
8.2 & \mathrm{E}-17 \\
1.3 & \mathrm{E}-16 \\
1.3 & \mathrm{E}-16 \\
1.2 & \mathrm{E}-16 \\
1.2 & \mathrm{E}-16\end{array}$ & $\begin{array}{ll}2.6 & \mathrm{E}-\mathrm{i} 8 \\
1.1 & \mathrm{E}-17 \\
1.7 & \mathrm{E}-17 \\
1.5 & \mathrm{E}-17 \\
1.5 & \mathrm{E}-\mathrm{i} 7 \\
1.2 & \mathrm{E}-17\end{array}$ \\
\hline
\end{tabular}

\section{References:}

$\mathrm{H}^{\dagger}+\mathrm{H}_{2}$ : V.V. Afrosimov, G.A. LeǏko, Yu. A. Yamaev, M.M. Panov, and I.V. Fedorenko, Sov. Phys.-JETP 35, 1070 (1972); R. Browning and H.B. Gilbody, J. Phys. E 1, 1149 (1968).

$H^{\circ}+H_{2}$ : V.V. Afrosimov, G.A. Leǐko, Yu. A. Mamaer, M.M. Panov, and I.V. Pedarenko, Sov. Phys.-JEIP 35, 1070 (1972).

\section{Accuracy:}

$\pm 20 \%$ 
A.5.9

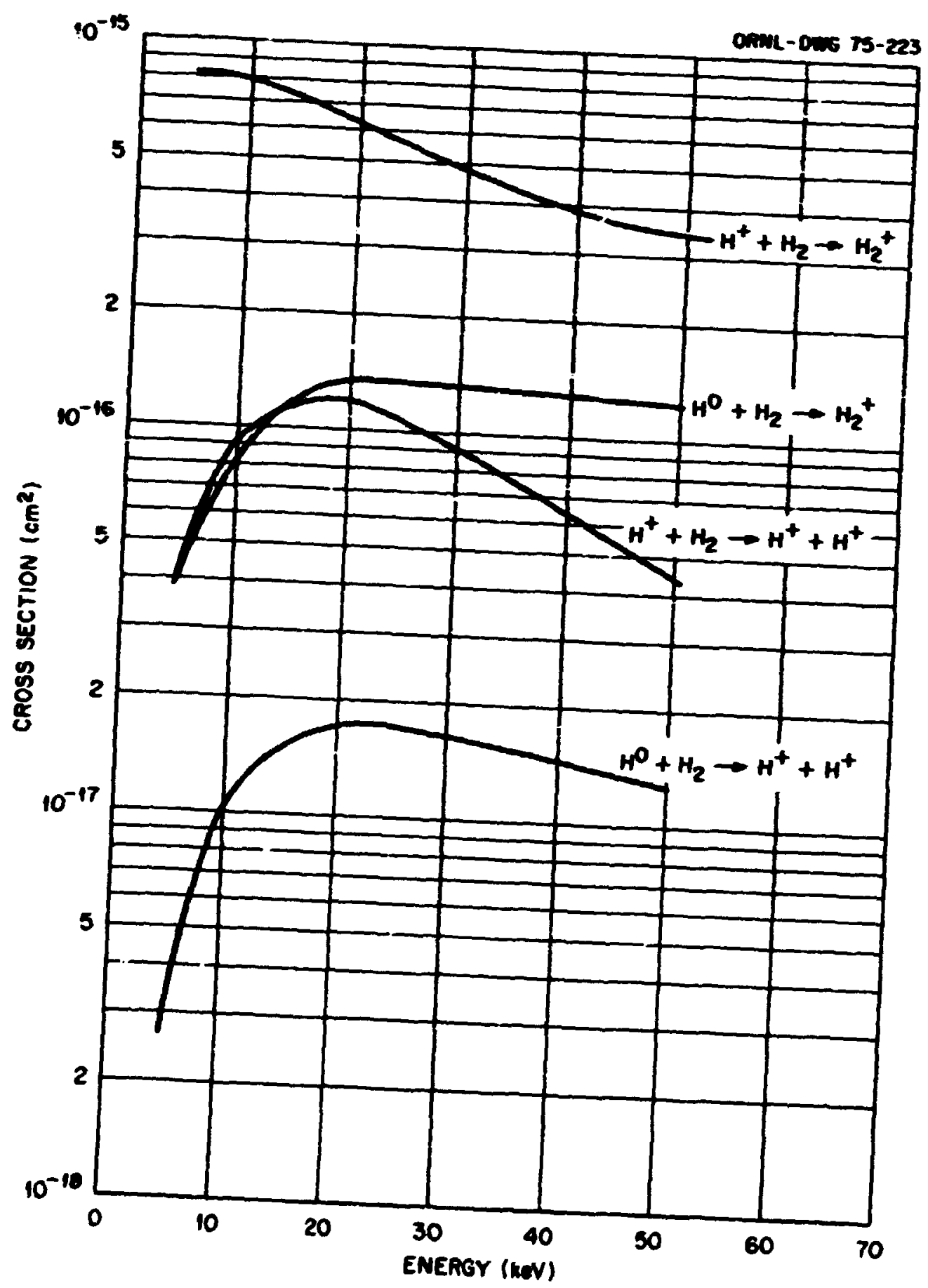


Cross Sections for Positive Ion Production

$$
\text { in } \mathrm{x}_{2} \text { and } \mathrm{O}_{2} \text { by } \mathrm{H}^{+}
$$

\begin{tabular}{|c|c|c|}
\hline \multirow[t]{2}{*}{$\begin{array}{l}\text { Zneroy } \\
\text { (keV) }\end{array}$} & \multicolumn{2}{|c|}{$\begin{array}{l}\text { Cross sections } \\
\left(\mathrm{cm}^{2}\right)\end{array}$} \\
\hline & $\mathbb{N}_{2}$ & $o_{2}$ \\
\hline $\begin{array}{lll}5.0 & \mathrm{E} & 00 \\
8.0 & \mathrm{E} & 00 \\
1.0 & \mathrm{E} & 01 \\
3.0 & \mathrm{E} & 01 \\
6.0 & \mathrm{E} & 01 \\
1.0 & \mathrm{E} & 02 \\
3.0 & \mathrm{E} & 02 \\
6.0 & \mathrm{E} & 02 \\
1.0 & \mathrm{E} & 03 \\
3.0 & \mathrm{E} & 03\end{array}$ & $\begin{array}{ll}1.2 & E-15 \\
1.2 & E-15 \\
1.2 & E-15 \\
9.7 & E-16 \\
8.0 & \Xi-16 \\
6.7 & z-16 \\
3.4 & \Xi-16 \\
2.1 & E-16 \\
1.4 & E-16 \\
6.4 & E-17\end{array}$ & $\begin{array}{ll}1.1 & \mathrm{E}-15 \\
1.0 & \mathrm{E}-15 \\
9.2 & \mathrm{E}-16 \\
9.0 & \mathrm{E}-16 \\
7.7 & \mathrm{E}-16 \\
7.0 & \mathrm{E}-16 \\
4.1 & \mathrm{E}-16 \\
2.2 & \mathrm{E}-16 \\
1.5 & \mathrm{E}-16\end{array}$ \\
\hline
\end{tabular}

\section{References:}

E.H. MeDaniel, J.W. Hooper, D.W. Martin, and D.S. Harner, Proc. Fifth Int. Conf. on Ionization Phenorene in Gases (Munich, 1961) Jorth-ilollend Publishing Co. (Amsteriam) Vol. 1, 60 (1952); L.I. Pivovar, and Yu. Z. Levchenko, Sov. Phys.-JEMP 25, $2 \overline{7}$ (1967); R. Browning and H.B. Gilbedy, J. Phys. I, il49 (1968).

Accuracy:

$\pm 20 \%$. 


\section{A.5.21}

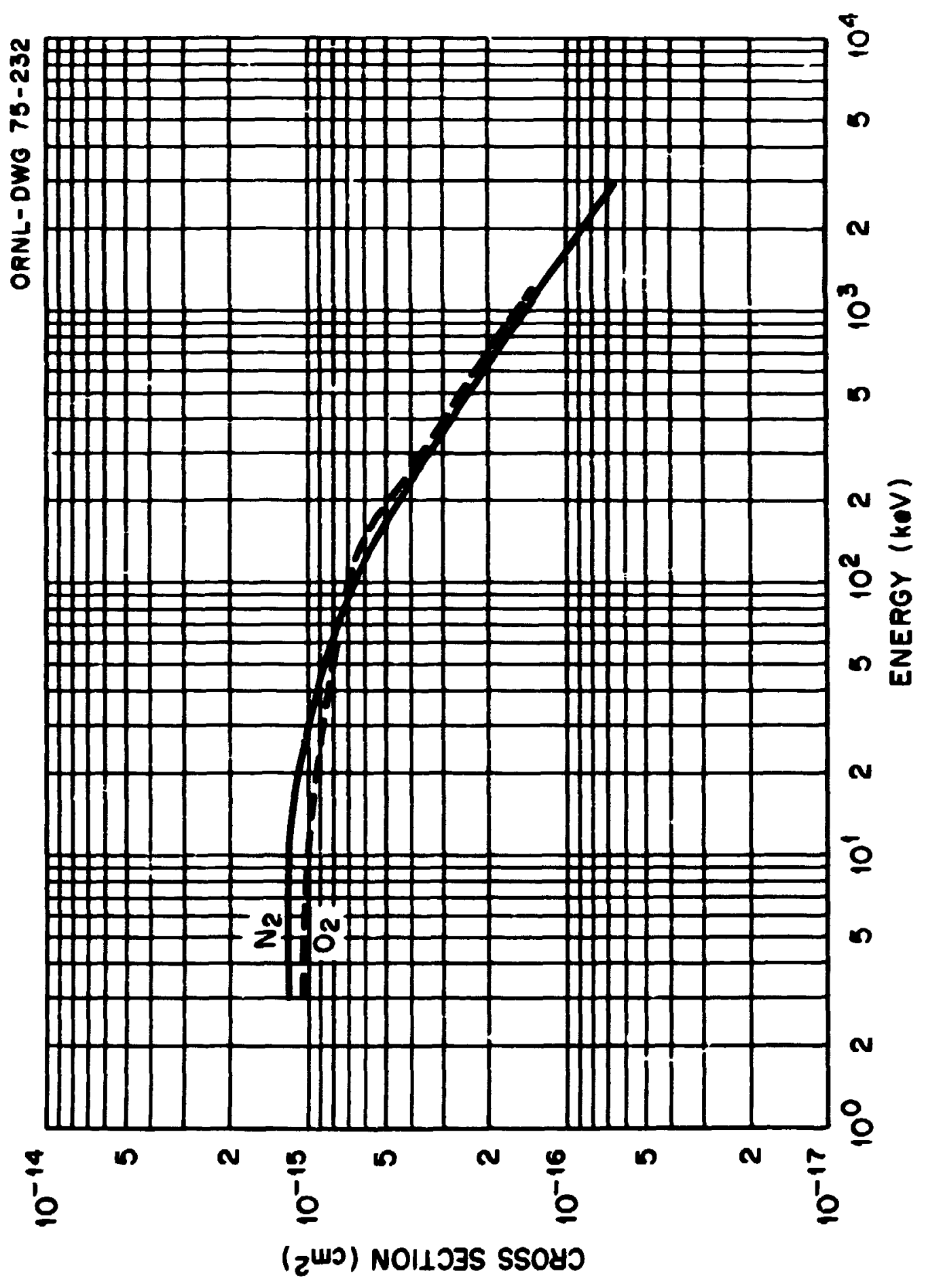




\section{A.5.12}

Cross Sections for Electron Production

in $\mathrm{x}_{2}$ and $\mathrm{O}_{2}$ by $\mathrm{H}^{+}$

\begin{tabular}{|c|c|c|}
\hline \multirow[t]{2}{*}{$\begin{array}{l}\text { Bner: } \\
\text { (keV) }\end{array}$} & \multicolumn{2}{|c|}{$\begin{array}{c}\text { Cross Sections } \\
\left(\mathrm{cm}^{2}\right)\end{array}$} \\
\hline & $\boldsymbol{I}_{2}$ & $\mathrm{O}_{2}$ \\
\hline $\begin{array}{l}1.0 \mathrm{~B} 00 \\
2.0 \mathrm{~B} 00 \\
4.0 \mathrm{E} 00 \\
6.0 \mathrm{E} \quad 00 \\
1.0 \mathrm{~B} 01 \\
2.0 \mathrm{E} 01 \\
.0 \mathrm{~B} 01 \\
6.0 \mathrm{E} 01 \\
1.0 \mathrm{E} 02 \\
2.0 \mathrm{E} 02 \\
4.0 \mathrm{E} 02 \\
6.0 \mathrm{~B} 02 \\
1.0 \mathrm{~B} 03 \\
2.0 \mathrm{E} 03 \\
3.0 \mathrm{E} 03\end{array}$ & 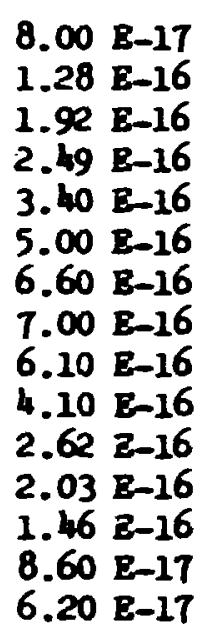 & $\begin{array}{ll}5.50 & E-17 \\
9.90 & E-17 \\
1.40 & E-16 \\
2.10 & E-16 \\
3.35 & E-16 \\
5.10 & E-16 \\
6.00 & E-16 \\
6.00 & E-16 \\
4.40 & E-16 \\
2.95 & E-16 \\
2.22 & E-16 \\
1.58 & E-16\end{array}$ \\
\hline
\end{tabular}

\section{References:}

$\mathrm{H}^{+}+\mathrm{H}_{2}$ : E.H. MeDaniel, J.H. Hooper, D.W. Martin, and D.S. Harner, Proc. Pifth Int. Conf. on Ioni zation Pinenomena in Cases (Munich, 1961), Wortb-Holland Publishing Co. (Asterden) Vol. 1, 60 (1962); P.J. de Heer, J. Schutten, and H. Moustafa, Physica 32, 1766 (1966); J.B. Crooks ard M.E. Rudd, Phus. Rev. A 3, 1628 (1971); L.I. PI vovar and Yu. Z. Levehenko, Soviet Physics-JETP 25, 27 (1967); E.S. Solov'ev, R.I. Il'in, V.A. Oparin, and I.V. Pedorenko, Sov. Phrs.-JEIP 15, 459 (1962); Yu. S. Cordeev and M.L. Panov, Sov. Phys.-Tech. Phys. 9, 656 (1964); R.J. Meileal and D.C. Clark, J. Ceophys. Res. 74, 5065 (1969); M.J. Dufay, M.D. Desesquelles, and M. Bidelebers, Ans. Ceophys. 22, 614 (1966); J.G. Collins and P. Kebarle, J. Chen. Phys. 46, 1082 (1967).

$\mathrm{H}^{+}+\mathrm{O}_{2}$ : Z.H. McDaniel, J.H. Hooper, D.H. Mrtin, and D.S. Harmer, Proc. Fifth Int. Conf. on Ionization Phesomens in Gases (Munich 1961), Morth-Holland Publishing Co. (Amsterdan) Vol. 1, 60 (1962); P.J. de Heer, J. Schutten, and H. Moustafa, Physice 32, 1766 (1966); J.B. Crooks and M.E. Rudd, Phys. Rev. A 3, 1628 (1971); M.J. Dufay, M.D. Desesquelles, ad K. E1delsberg, Ann. Ceophys. 22, 614 (1966); R.J. Mcileal and J.H. Birely, Rev. Ceophys. and Space Phys. 11, 633 (1973).

\section{Accurecy:}

$\pm 50 \%$. 


\section{A.5.13}

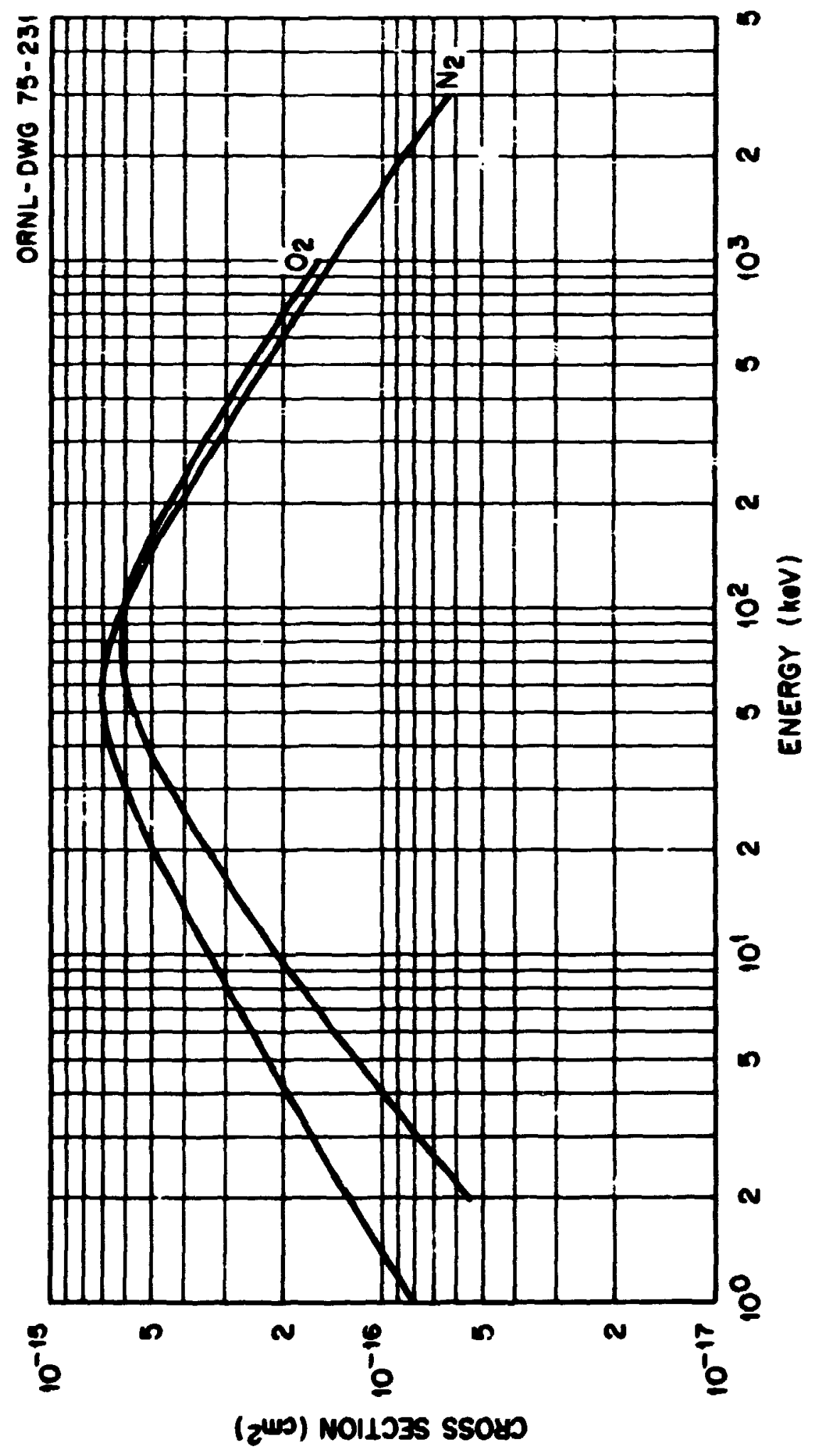




$$
\text { A. } 5.14
$$

Cross Sections for Production of

$$
x_{2}^{+}, x^{+} \text {, and } x^{2+} \text { Ions by Protons in } x_{2} \text { Ges }
$$

\begin{tabular}{|c|c|c|c|}
\hline \multirow[t]{2}{*}{$\begin{array}{l}\text { Dneros } \\
\text { (tev) }\end{array}$} & \multicolumn{3}{|c|}{$\begin{array}{c}\text { Cross Sections } \\
\left(c^{2}\right)\end{array}$} \\
\hline & $\mathbf{x}^{+}$ & $x_{2}^{+}$ & $x^{2^{+}}$ \\
\hline $\begin{array}{lll}3.0 & \mathrm{E} & 00 \\
1.0 & \mathrm{E} & 01 \\
2.0 & \mathrm{E} & 01 \\
3.0 \mathrm{E} & 01 \\
4.0 \mathrm{E} & 01 \\
4.6 \mathrm{E} & 01\end{array}$ & $\begin{array}{ll}1.05 & E-16 \\
2.64 & E-16 \\
3.23 & E-16 \\
3.27 & E-16 \\
3.07 & E-16 \\
2.86 & E-16\end{array}$ & $\begin{array}{ll}1.00 & E-15 \\
9.20 & E-16 \\
7.10 & E-16 \\
6.21 & E-16 \\
5.60 & E-16 \\
5.00 & E-16\end{array}$ & $\begin{array}{ll}3.37 & \Xi-18 \\
7.58 & E-18 \\
1.23 & E-17 \\
1.45 & \Xi-17 \\
1.49 & E-17\end{array}$ \\
\hline
\end{tabular}

\section{Refereaces:}

R. Browning and H.B. Gilbody, J. Phys. B 1, 1149 (1968).

\section{Accuracy:}

$\pm 20 \%$. 


\section{A. 5.15}

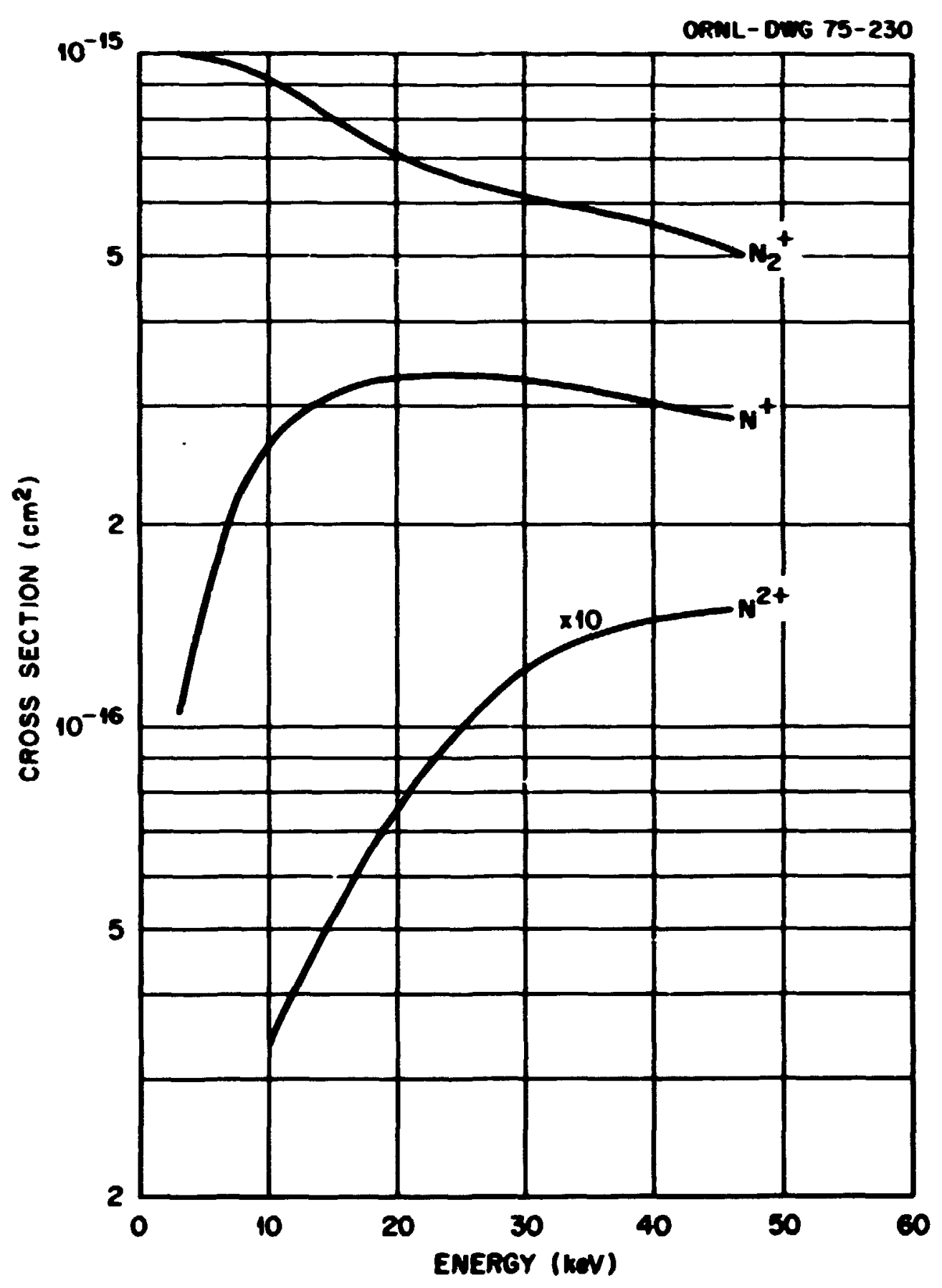

1 


\section{A.5.16}

Cross Sections for Proluction of $\mathrm{O}_{2}^{+}, 0^{+}$, and $\mathrm{O}^{2+}$ Ions by Protons in $\mathrm{O}_{2}$

\begin{tabular}{|c|c|c|c|}
\hline \multirow[t]{2}{*}{$\begin{array}{l}\text { Boergy } \\
\text { (keV) }\end{array}$} & \multicolumn{3}{|c|}{$\begin{array}{c}\text { Cross Sections } \\
\left(\mathrm{cm}^{2}\right)\end{array}$} \\
\hline & $0^{+}$ & $\mathrm{O}_{2}^{+}$ & $0^{2+}$ \\
\hline $\begin{array}{lll}6.0 & \mathrm{E} & 00 \\
1.0 & \mathrm{~B} & 01 \\
2.0 & \mathrm{~B} & 01 \\
3.0 & \mathrm{~B} & 01 \\
4.0 & \mathrm{~B} & 01 \\
4.6 & \mathrm{~B} & 01\end{array}$ & $\begin{array}{ll}2.40 & E-16 \\
3.08 & E-16 \\
3.76 & E-16 \\
3.87 & E-16 \\
3.81 & E-16 \\
3.75 & E-16\end{array}$ & $\begin{array}{ll}8.12 & 2-16 \\
7.00 & \Xi-16 \\
5.10 & E-16 \\
4.47 & E-16 \\
4.12 & E-16 \\
4.00 & E-16\end{array}$ & $\begin{array}{ll}2.34 & \Xi-18 \\
4.26 & \mathrm{E}-18 \\
9.64 & \mathrm{E}-18 \\
1.47 & \mathrm{E}-17 \\
1.77 & \mathrm{E}-17 \\
1.84 & \mathrm{E}-17\end{array}$ \\
\hline
\end{tabular}

\section{References:}

R. Brownins and H.B. Gilbod, J. Phys. B 1, 1149 (1968).

\section{Aceuregr:}

$\pm 20 \%$. 


\section{A. 5.17}

j)

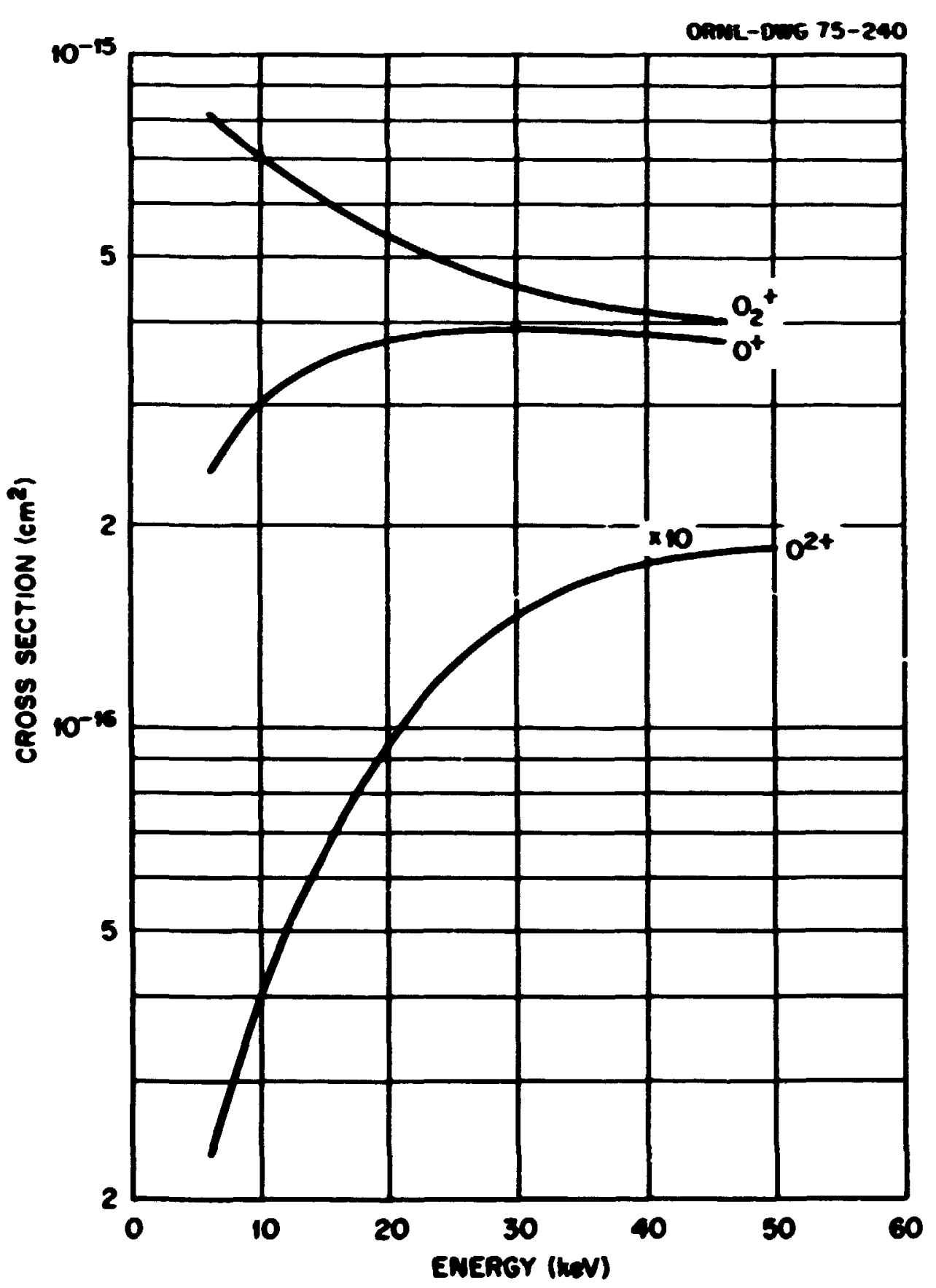

u 
A. 5.18

Angular Distributions of Electrons from 300-keV Proton Inpact in $\mathrm{H}_{2}$

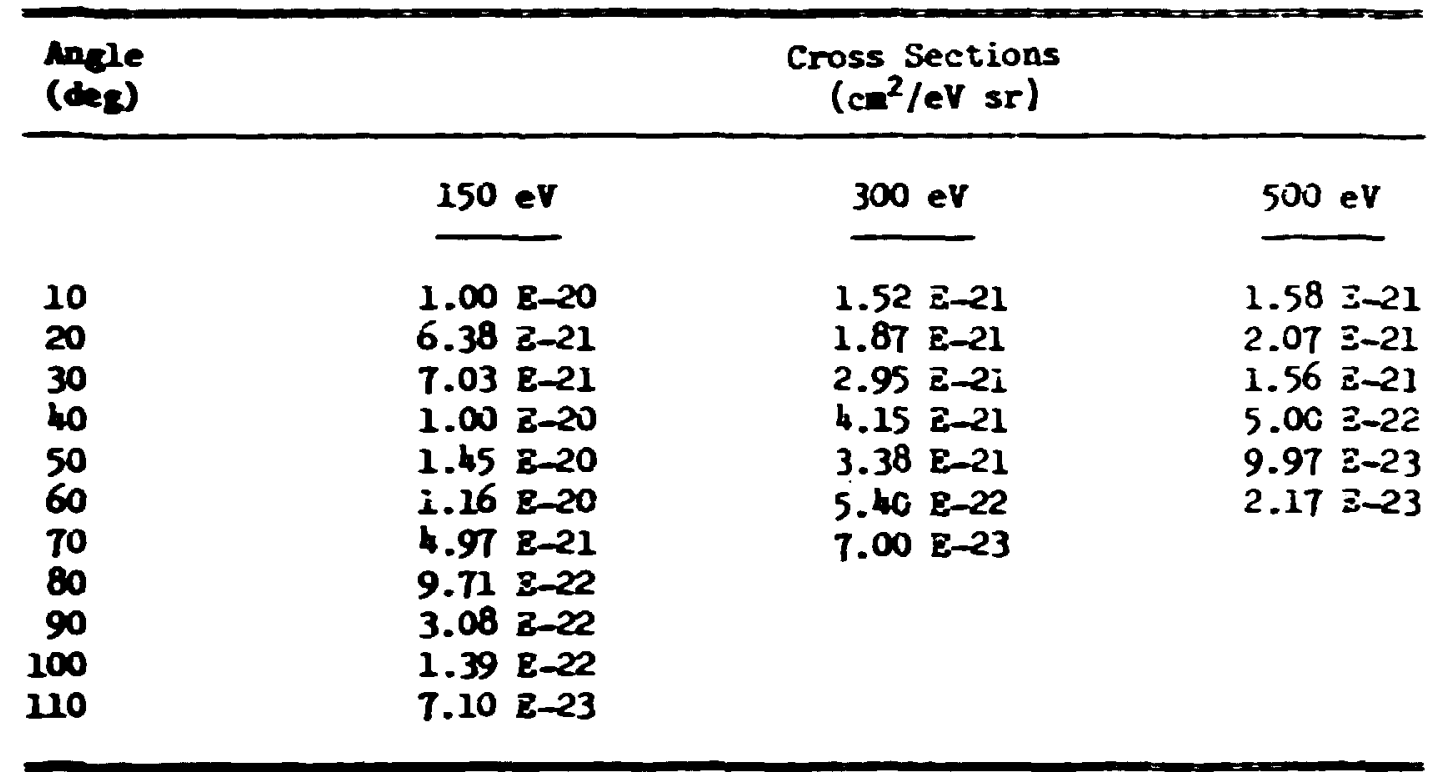

References:

M.B. Fudd and J.i. Macek, Case Studies in Atwric Physics 3, 73 (1972).

M.Z. Rudd, C.A. Sautter, and C.L. Bailey, Phrs. Rer. 151, 20 (1966).

iccurecr:

$\pm 20 \%$ 
A. 5.19

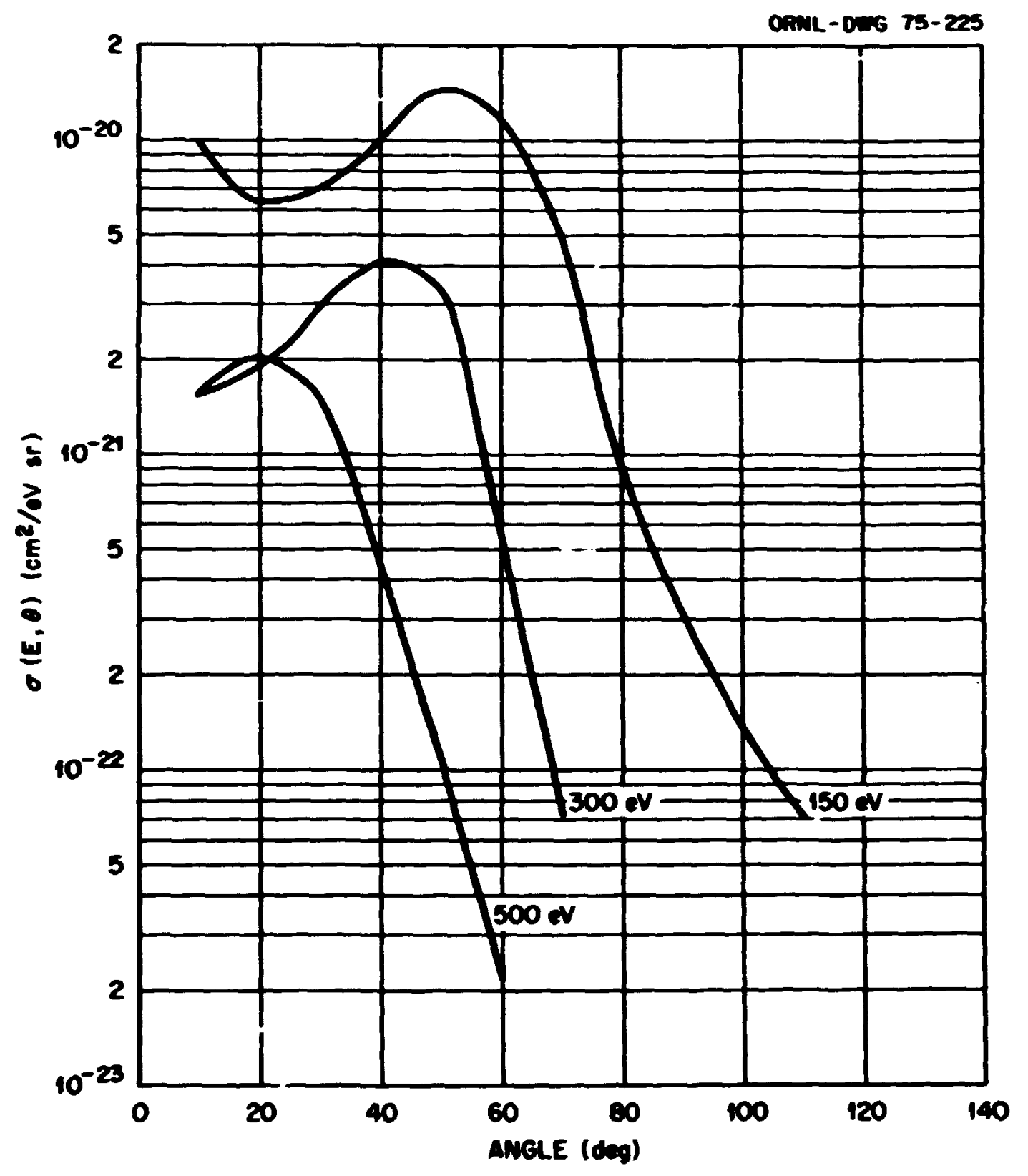




$$
\text { A. } 5.20
$$

Angular Distribution of Electrons of Various Bnergies

fron 300-keV Proton Impacts in $\mathrm{d}_{2}$

\begin{tabular}{|c|c|c|c|}
\hline \multirow[t]{2}{*}{$\begin{array}{l}\text { Angle } \\
\text { (deg) }\end{array}$} & \multicolumn{3}{|c|}{$\begin{array}{c}\text { Cross Sections } \\
\left(\mathrm{cm}^{2} / \mathrm{eV} \text { sr) }\right.\end{array}$} \\
\hline & $10 \mathrm{ev}$ & $20 \mathrm{eV}$ & $60 \mathrm{eV}$ \\
\hline $\begin{array}{l}10 \\
20 \\
30 \\
40 \\
50 \\
60 \\
70 \\
80 \\
90 \\
100 \\
110 \\
120 \\
130 \\
140 \\
150 \\
160\end{array}$ & $\begin{array}{ll}5.31 & E-19 \\
4.79 & E-19 \\
5.11 & E-19 \\
5.21 & E-19 \\
5.11 & E-19 \\
4.79 & E-19 \\
4.32 & E-19 \\
3.51 & E-19 \\
2.48 & E-19 \\
1.62 & E-19 \\
1.23 & E-19 \\
9.65 & E-20 \\
8.76 & E-20 \\
8.11 & E-20 \\
7.70 & E-20 \\
7.31 & E-20\end{array}$ & $\begin{array}{ll}2.34 & E-19 \\
2.16 & E-19 \\
2.40 & E-19 \\
2.56 & E-19 \\
2.59 & E-19 \\
2.43 & E-i 9 \\
2.08 & E-19 \\
1.61 & E-19 \\
9.94 & E-20 \\
5.60 & E-20 \\
3.71 & E-20 \\
2.73 & E-20 \\
2.25 & E-20 \\
1.95 & E-20 \\
1.74 & E-20 \\
1.62 & \mathrm{E}-20\end{array}$ & $\begin{array}{ll}4.89 & E-20 \\
3.42 & E-20 \\
3.84 & E-20 \\
4.48 & E-20 \\
5.49 & E-20 \\
5.99 & E-20 \\
5.08 & E-20 \\
2.71 & E-20 \\
9.81 & E-21 \\
3.52 & E-21 \\
2.01 & E-21 \\
1.31 & E-21 \\
1.10 & E-21 \\
9.16 & E-22 \\
8.24 & E-22 \\
7.20 & E-22\end{array}$ \\
\hline
\end{tabular}

\section{Peferences:}

M.B. Rudd and J.H. Macek, Case Studies in Atonic Physics 3, 73 (1972).

M.E. Rudd, C.A. Sautter, and C.L. Bafley, Phys. Rev. 151, 20 (1966).

\section{Accurecy:}

$\pm 20 x$. 
A. 5.21

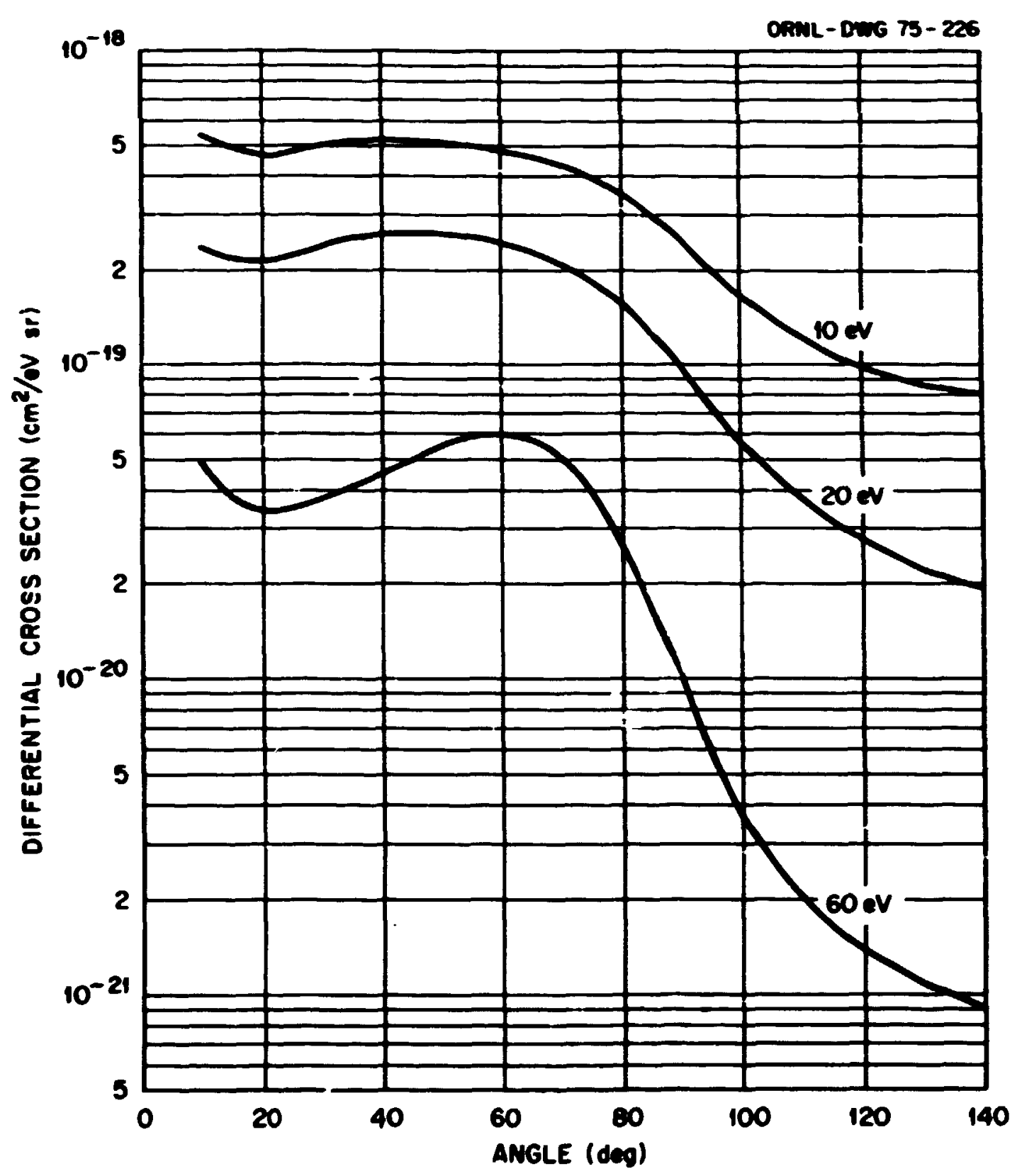

J 


\section{A. 5.22}

Differential Cross Sections for Ejection of Electrons iron $\mathrm{H}_{2}$ molecules by 50-keV $\mathrm{H}^{+}$Projectiles

(The electron energy distributions were measured at various angles, shoum elongside the curres, from the incident bean direction.)

\begin{tabular}{|c|c|c|c|c|}
\hline \multirow[t]{2}{*}{$\begin{array}{c}\text { Baeray } \\
\text { (ev) }\end{array}$} & \multicolumn{4}{|c|}{$\begin{array}{l}\text { Differential Cross Sections } \\
\left(\mathrm{cm}^{2} / \mathrm{eV}^{-} \mathrm{s} \rightarrow \text { wlecule }\right)\end{array}$} \\
\hline & $90^{\circ}$ & $67.5^{\circ}$ & $45^{\circ}$ & $23^{\circ}$ \\
\hline $\begin{array}{lll}5.0 & E & 00 \\
1.0 & E & 01 \\
1.5 & E & 01 \\
2.0 & E & 01 \\
3.0 & E & 01 \\
4.0 & E & 01 \\
5.0 & E & 01 \\
6.0 & E & 01 \\
1.0 & E & 01 \\
8.0 & E & 01 \\
9.0 & E & 01 \\
1.0 & E & 02 \\
1.1 & E & 02 \\
1.2 & E & 02 \\
1.3 & E & 02 \\
1.4 & E & 02 \\
1.5 & E & 02\end{array}$ & $\begin{array}{ll}3.80 & \mathrm{E}-19 \\
2.69 & \mathrm{E}-19 \\
1.45 & \mathrm{E}-19 \\
8.70 & \mathrm{E}-20 \\
3.34 & \mathrm{E}-20 \\
1.41 & \mathrm{E}-20 \\
6.40 & \mathrm{E}-21 \\
3.00 & \mathrm{E}-21\end{array}$ & $\begin{array}{ll}5.70 & E-19 \\
5.39 & E-19 \\
3.59 & E-19 \\
2.42 & E-19 \\
1.13 & E-19 \\
5.58 & E-20 \\
2.79 & E-20 \\
1.41 & E-20 \\
7 . & \mathrm{E}-21 \\
3.90 & E-21 \\
2.08 & E-21 \\
1.13 & E-21\end{array}$ & $\begin{array}{ll}9.30 & \mathrm{E}-19 \\
1.40 & \mathrm{E}-18 \\
1.20 & \mathrm{E}-18 \\
9.80 & \mathrm{E}-19 \\
6.19 & \mathrm{E}-19 \\
3.70 & \mathrm{E}-19 \\
2.10 & \mathrm{E}-19 \\
1.14 & \mathrm{E}-19 \\
6.20 & \mathrm{E}-20 \\
3.32 & \mathrm{E}-20 \\
1.73 & \mathrm{E}-20\end{array}$ & $\begin{array}{ll}2.40 & E-18 \\
4.10 & E-18 \\
3.38 & E-18 \\
2.70 & E-18 \\
1.80 & E-18 \\
1.18 & E-18 \\
7.59 & E-19 \\
4.83 & E-19 \\
3.00 & E-19 \\
1.81 & E-19 \\
1.06 & E-19 \\
6.10 & E-20 \\
3.47 & E-20 \\
1.92 & E-20 \\
1.02 & E-20 \\
5.01 & E-21 \\
2.02 & E-21\end{array}$ \\
\hline
\end{tabular}

Reference:

C.E. Kuysatt and T. Jorgensen, Phys. Rer. 130, 1444 (1963).

Accuracy:

Eptimates of total error are indicated by the vertical bars on the draving. 


\section{A. 5.23}

i)

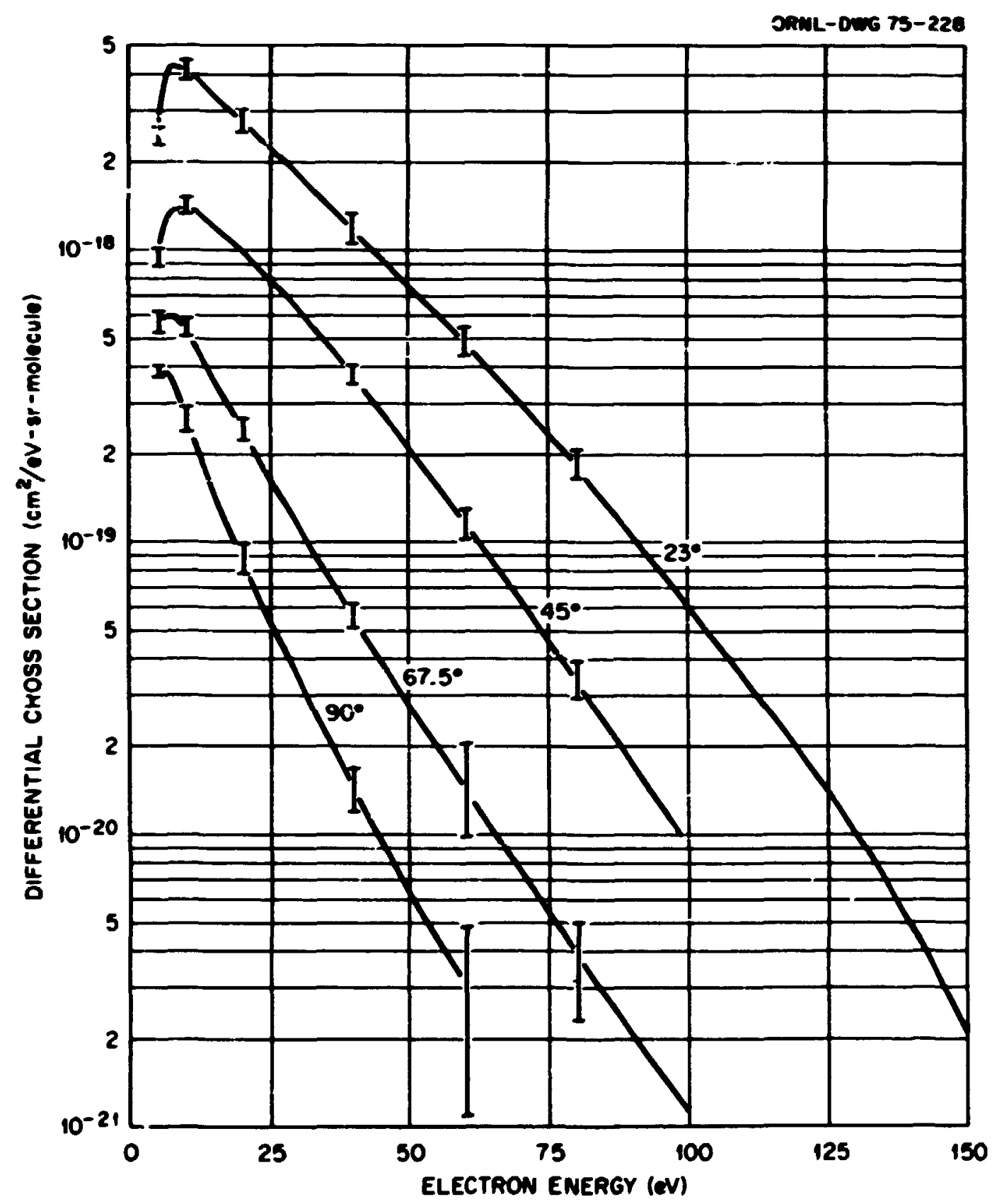




\section{A. 5.24}

Difrerential Cross Sections for Ejection of Elections from He Atoms by 50-keV H ${ }^{+}$Projectiles

(The electron energy distributions were measured at rarious angles, shom angside the curves, from the incident bean direction.)

\begin{tabular}{|c|c|c|c|c|c|}
\hline \multirow[t]{2}{*}{$\begin{array}{l}\text { Bnereg } \\
(\mathrm{eV})\end{array}$} & \multicolumn{5}{|c|}{ 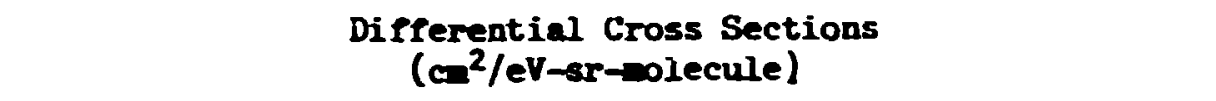 } \\
\hline & $160^{\circ}$ & $70^{\circ}$ & $\underline{50^{\circ}}$ & $30^{\circ}$ & $10^{\circ}$ \\
\hline $\begin{array}{ccc}0 & \mathrm{E} & 01 \\
2.0 & \mathrm{~B} & 01 \\
4.0 & \mathrm{E} & 01 \\
6.0 & \mathrm{E} & 01 \\
8.0 & \mathrm{E} & 01 \\
1.0 & \mathrm{E} & 02 \\
1.2 & \mathrm{E} & 02 \\
1.4 & \mathrm{E} & 02 \\
1.6 & \mathrm{E} & 02 \\
1.8 & \mathrm{E} & 02 \\
2.0 & \mathrm{E} & 02 \\
2.2 & \mathrm{E} & 02 \\
2.4 & \mathrm{E} & 02 \\
2.6 & \mathrm{E} & 02 \\
2.8 & \mathrm{E} & 02 \\
3.0 & \mathrm{E} & 02\end{array}$ & $\begin{array}{ll}1.20 & \mathrm{E}-19 \\
4.00 & \mathrm{E}-20 \\
1.03 & \mathrm{E}-20 \\
2.29 & \mathrm{E}-21 \\
7.60 & \mathrm{E}-22 \\
3.10 & \mathrm{E}-22 \\
1.40 & \mathrm{E}-22 \\
6.80 & \mathrm{E}-23 \\
3.40 & \mathrm{E}-23 \\
1.80 & \mathrm{E}-23\end{array}$ & $\begin{array}{ll}2.00 & \mathrm{E}-19 \\
7.79 & \mathrm{E}-20 \\
2.97 & \mathrm{E}-20 \\
1.01 & \mathrm{E}-20 \\
3.68 & \mathrm{E}-21 \\
1.33 & \mathrm{E}-21 \\
5.15 & \mathrm{E}-22 \\
2.12 & \mathrm{E}-22 \\
8.97 & \mathrm{E}-23 \\
4.30 & \mathrm{E}-23 \\
2.25 & \mathrm{E}-23 \\
1.29 & \mathrm{E}-23\end{array}$ & $\begin{array}{ll}1.46 & \mathrm{E}-19 \\
5.49 & \mathrm{E}-20 \\
2.08 & \mathrm{E}-20 \\
8.00 & \mathrm{E}-21 \\
3.14 & \mathrm{E}-21 \\
1.30 & \mathrm{E}-21 \\
5.44 & \mathrm{E}-22 \\
2.40 & \mathrm{E}-22 \\
1.09 & \mathrm{E}-22 \\
5.02 & \mathrm{E}-23 \\
2.45 & \mathrm{E}-23\end{array}$ & $\begin{array}{ll}3.46 & \mathrm{E}-19 \\
1.63 & \mathrm{E}-19 \\
7.72 & \mathrm{E}-20 \\
3.14 & \mathrm{E}-20 \\
1.31 & \mathrm{E}-20 \\
5.40 & \mathrm{E}-21 \\
2.14 & \mathrm{E}-21 \\
8.90 & \mathrm{E}-22 \\
3.77 & \mathrm{E}-22 \\
1.63 & \mathrm{E}-22 \\
7.20 & \mathrm{E}-23 \\
3.36 & \mathrm{E}-23 \\
1.69 & \mathrm{E}-23\end{array}$ & $\begin{array}{ll}1.51 & E-18 \\
2.00 & E-18 \\
4.75 & E-19 \\
2.04 & E-19 \\
9.40 & E-20 \\
4.00 & E-20 \\
1.60 & E-20 \\
6.22 & E-21 \\
2.50 & E-2 \\
1.01 & E-21 \\
4.39 & E-22 \\
1.89 & E-22 \\
8.33 & E-23 \\
3.93 & E-23 \\
1.92 & E-23 \\
1.06 & E-23\end{array}$ \\
\hline
\end{tabular}

Reference:

M.E. Rudd and T. Jorgensen, Phys. Rev. 131, 666 (1963).

\section{Accuracy:}

Estimates of total error are indicated by the vertical bers on the drawing. 


\section{A.5.25}

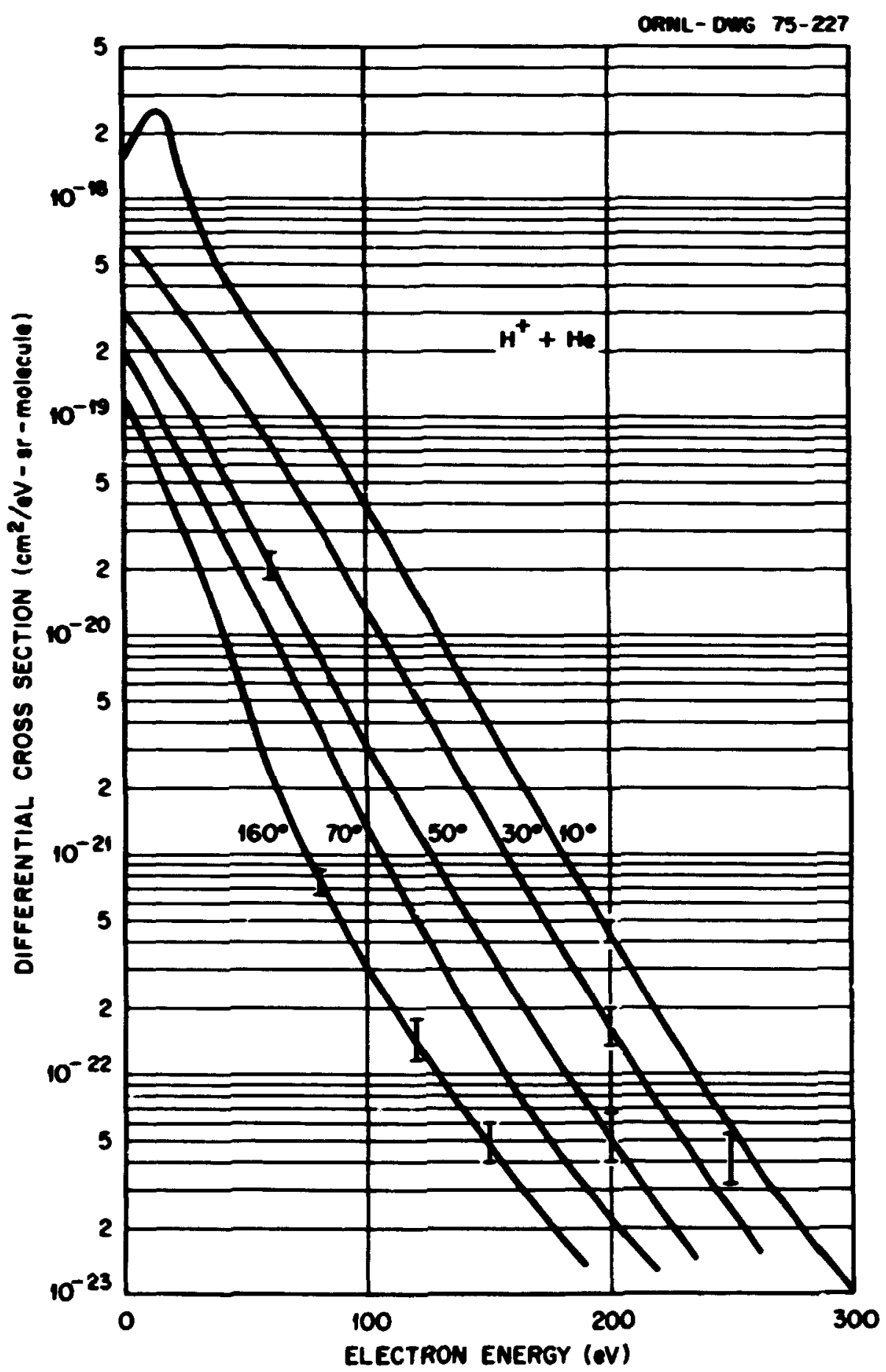




$$
\begin{gathered}
\text { A.5.26 } \\
\text { Cross Sections for Prociuction of Slor } \\
\text { Electrons in Collisions of } \mathrm{H}_{2}^{+} \text {and } \mathrm{H}_{3}^{+} \text {in } \mathrm{H}_{2}
\end{gathered}
$$

\begin{tabular}{|c|c|c|}
\hline \multirow[t]{2}{*}{$\begin{array}{l}\text { Baergy } \\
\text { (keV) }\end{array}$} & \multicolumn{2}{|c|}{$\begin{array}{c}\text { Cross Sections } \\
\left(\mathrm{cm}^{2}\right)\end{array}$} \\
\hline & $\mathrm{H}_{2}^{+}$ & $\mathrm{H}_{3}^{+}$ \\
\hline $\begin{array}{lll}1.0 & \mathrm{E} & 00 \\
3.0 & \mathrm{E} & 00 \\
6.0 & \mathrm{E} & 00 \\
1.0 & \mathrm{~B} & 01 \\
3.0 & \mathrm{E} & 01 \\
6.0 & \mathrm{E} & 01 \\
1.0 & \mathrm{~B} & 02\end{array}$ & $\begin{array}{ll}2.9 & \mathrm{E}-17 \\
5.7 & \mathrm{E}-17 \\
1.0 & \mathrm{E}-16 \\
1.6 & \mathrm{E}-16 \\
3.2 & \mathrm{E}-16 \\
4.0 & \mathrm{E}-16 \\
4.0 \mathrm{E}-16\end{array}$ & $\begin{array}{ll}1.3 & \mathrm{E}-17 \\
3.6 & \mathrm{~B}-17 \\
7.2 & \mathrm{~B}-17 \\
1.1 & \mathrm{E}-16 \\
2.6 & \mathrm{~B}-16 \\
3.6 & \mathrm{~B}-16 \\
4.3 & \mathrm{E}-16\end{array}$ \\
\hline
\end{tabular}

\section{References:}

Iu. S. Cordeer and M.I. Panor, Sov. Phys.-Tech. Phys. 9, 656 (1964); V.V. Arrosinor, R.X. II'in, and X.V. Pedorenko, Sor. Phys.-Tech. Phys. 3. 2080 (1958).

\section{Accuracy:}

$\pm 25 \%$. 
A.5.27

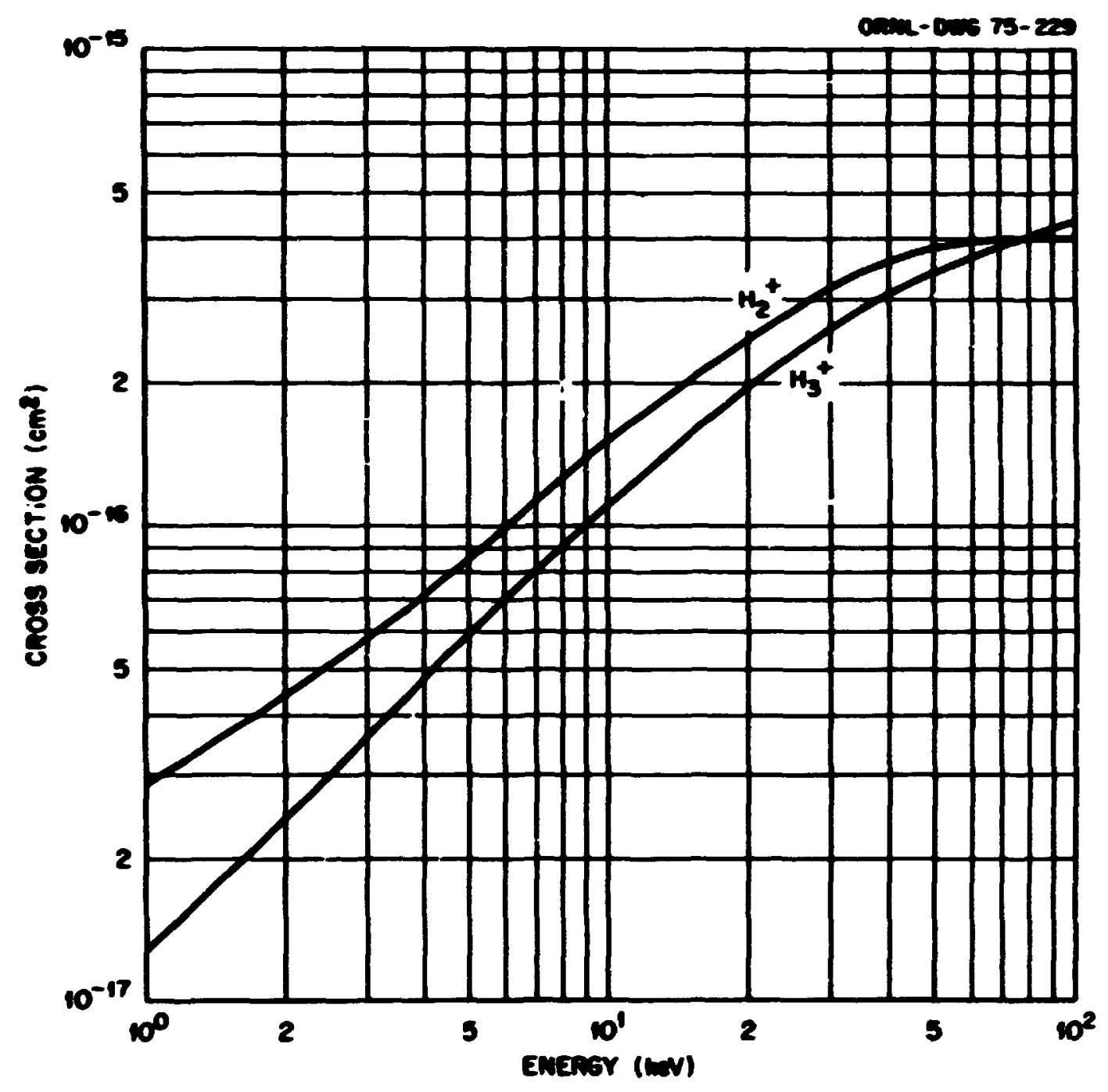


Cross Sections for Blectron Loss or Strippint of $\mathrm{H}$ Atoms in $\mathrm{H}$ and $\mathrm{H}_{2}$

\begin{tabular}{|c|c|c|}
\hline \multirow[t]{2}{*}{$\begin{array}{l}\text { Bnered } \\
\text { (keV) }\end{array}$} & \multicolumn{2}{|c|}{$\begin{array}{l}\text { Cross Sections } \\
\left(\mathrm{cm}^{2} / \text { molecule }\right)\end{array}$} \\
\hline & H & $\underline{\mathbf{H}_{2}}$ \\
\hline $\begin{array}{l}1.2 \mathrm{E} 00 \\
2.0 \mathrm{E} 00 \\
5.0 \mathrm{~B} 00 \\
7.0 \mathrm{E} 00 \\
1.0 \mathrm{~B} 01 \\
2.0 \mathrm{~B} 01 \\
5.0 \mathrm{~B} 01 \\
7.0 \mathrm{E} 01 \\
1.0 \mathrm{~B} 02 \\
2.0 \mathrm{~B} 02 \\
5.0 \mathrm{~B} 02 \\
7.0 \mathrm{E} 02 \\
1.0 \mathrm{~B} 03 \\
2.0 \mathrm{~B} 03 \\
5.0 \mathrm{E} 03 \\
7.0 \mathrm{~B} 03 \\
1.0 \mathrm{E} 04\end{array}$ & $\begin{array}{ll}3.0 & \mathrm{E}-18 \\
2.0 & \mathrm{~B}-17 \\
4.2 & \mathrm{E}-17 \\
5.6 & \mathrm{E}-17 \\
7.7 & \mathrm{E}-17 \\
1.2 & \mathrm{E}-16 \\
8.8 & \mathrm{E}-17 \\
7.5 & \mathrm{E}-17 \\
6.2 & \mathrm{E}-17 \\
4.3 & \mathrm{~B}-17\end{array}$ & $\begin{array}{ll}6.0 & \mathrm{E}-17 \\
7.3 & \mathrm{E}-17 \\
7.4 & \mathrm{E}-17 \\
9.2 & \mathrm{E}-17 \\
1.3 & \mathrm{E}-16 \\
1.5 & \mathrm{E}-16 \\
1.3 & \mathrm{E}-16 \\
1.1 & \mathrm{E}-16 \\
7.0 & \mathrm{E}-17 \\
3.2 & \mathrm{E}-17 \\
2.3 & \mathrm{E}-17 \\
1.7 & \mathrm{E}-17 \\
9.2 & \mathrm{E}-18 \\
4.1 & \mathrm{E}-18 \\
3.1 & \mathrm{E}-18 \\
2.3 & \mathrm{E}-18\end{array}$ \\
\hline
\end{tabular}

\section{Deforence:}

B + I: G.W. NeClure, Phys. Rev. 166, 22 (1968); A.B. Hittkower, G. Levg, and H.B. Gllsod, Proc. Plos. Soc. 91,306 (1967); L.M. Welsh, K.H. Bertwer, S.I. Teplen, and R.V. PJle, Phys. Rev. 158, 85 (1967).

A + H2: C.P. Barnett and H.K. Resnolds, Phors. Rer. 109, 355 (1958); P.M. Stler and C.P. Baruett, Phys. Rev. 103, 896 (1956); G.H. Mcclure, Prov. Rev. 134, A-1226 (1964); L.H. Toburen, M.Y. Iakad, and R.A. Iangley, Plars. Rev. 172, 124 (2968); J.P. N1llies, Plus. Dev. 157, 97 (1967);

C.H. Bertener, S.I. Kroplen, ad R.V. PYle, Phys. Rev. 137, Al461 (1964); L.M. Veloh, K.H. Bertener, S.I. Keplen, and R.V. PJle, Phrs. Rev. 158, 85 (1967).

\section{Aceures:}

$\pm 258$ 


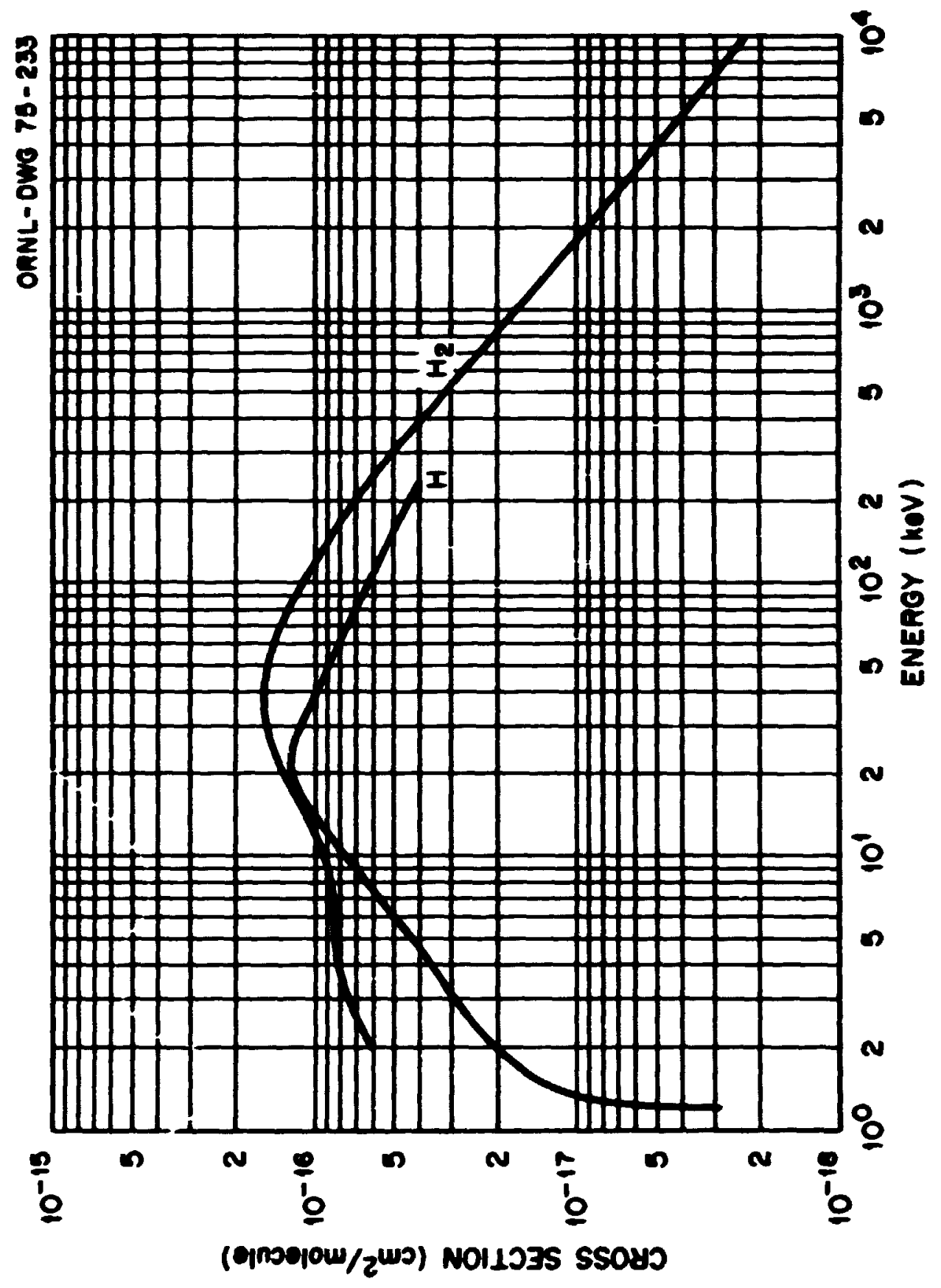




\begin{tabular}{|c|c|c|}
\hline \multicolumn{3}{|c|}{ A.5.30 } \\
\hline \multicolumn{3}{|c|}{ 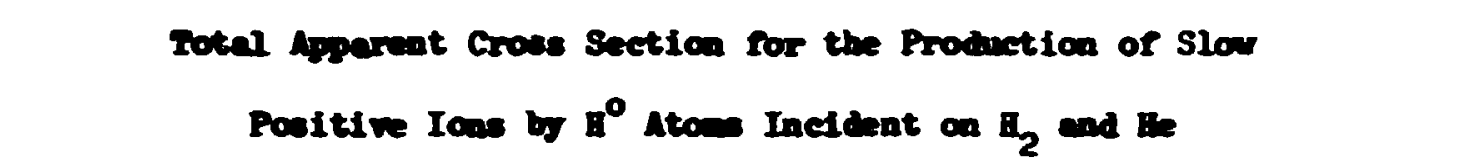 } \\
\hline (In) & $\begin{array}{l}\mathrm{H}_{2} \\
\text { Croses Seetion } \\
\left(\mathrm{e}^{2}\right)\end{array}$ & $\begin{array}{l}\text { He } \\
\text { Cross Section } \\
\left(\omega^{2}\right)\end{array}$ \\
\hline $\begin{array}{l}1.5=\infty \\
2.0 \geq \infty \\
3.0 \geq \infty 2 \\
4.0=\infty\end{array}$ & $\begin{array}{ll}9.15 & E-17 \\
8.01 & E-17 \\
0.20 & E-17 \\
4.99 & E-17\end{array}$ & $\begin{array}{ll}1.00 & \mathrm{E}-17 \\
3.80 & \mathrm{E}-17 \\
3.15 & \mathrm{E}-17 \\
2.61 & \mathrm{E}-17\end{array}$ \\
\hline
\end{tabular}

\section{Beforences:}

$\mathrm{H}_{2}$ : L.J. Pucirett, G.0. Tavlor, and D.W. Mrtin, PhJs. Rer. 178, 271 (1969).

He: L.J. Puckett, G.0. Tarlor, and D.J. Mrtin, Phrs. Rer. 178, 271 (1969).

scenregr:

$\pm 20$. 


\section{A.5.31}

$\ldots$

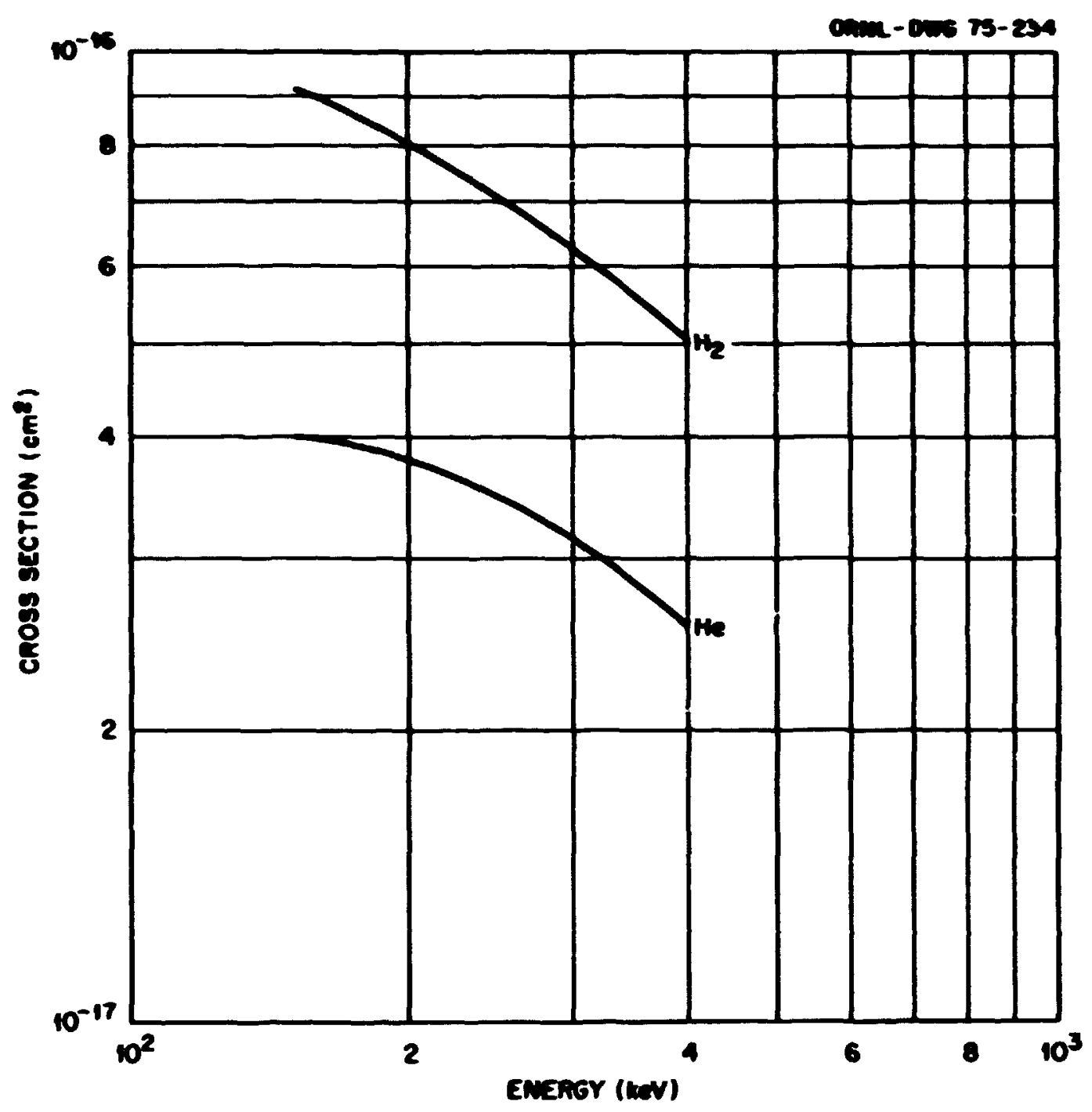

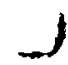




\section{A.5.32}

Totel Crose Section for the Production of

Pree Blectrons bs $\mathrm{H}^{\circ}$ Atom in $\mathrm{H}_{2}$ and $\mathrm{Be}$

\begin{tabular}{|c|c|c|}
\hline \multirow[t]{2}{*}{$(\operatorname{mer})$} & \multicolumn{2}{|c|}{$\begin{array}{c}\text { Croas Sections } \\
\left(c^{2}\right)\end{array}$} \\
\hline & $\mathrm{B}_{2}$ & ide \\
\hline $\begin{array}{llll}1.5 & \mathrm{~B} & C 2 \\
2.0 & \mathrm{~B} & 02 \\
2.5 & \mathrm{~B} & \infty 2 \\
3.0 & \mathrm{~B} & \alpha 2 \\
3.3 & \mathrm{~B} & C 2\end{array}$ & $\begin{array}{ll}1.91 & \mathrm{Z}-16 \\
1.67 & \mathrm{E}-16 \\
1.35 & \mathrm{E}-16 \\
1.12 & \mathrm{E}-16 \\
1.00 & \mathrm{E}-16\end{array}$ & $\begin{array}{ll}1.08 & \mathrm{E}-16 \\
1.04 & \mathrm{E}-16 \\
9.00 \mathrm{E}-17 \\
8.00 \mathrm{E}-17 \\
7.50 \mathrm{E}-17\end{array}$ \\
\hline
\end{tabular}

\section{Peforeace:}

$H_{2}$ : L.J. Puckett, G.0. Tarlor, ad D.W. Martin, Phos. Rev. 178, 271 (1969).

He: L.J. Puckett, G.0. Torlor, and D.K. Martin, Phys. Rev. 178, 271 (1969). seours:

$\pm 208$. 


\section{A.5.33}

j

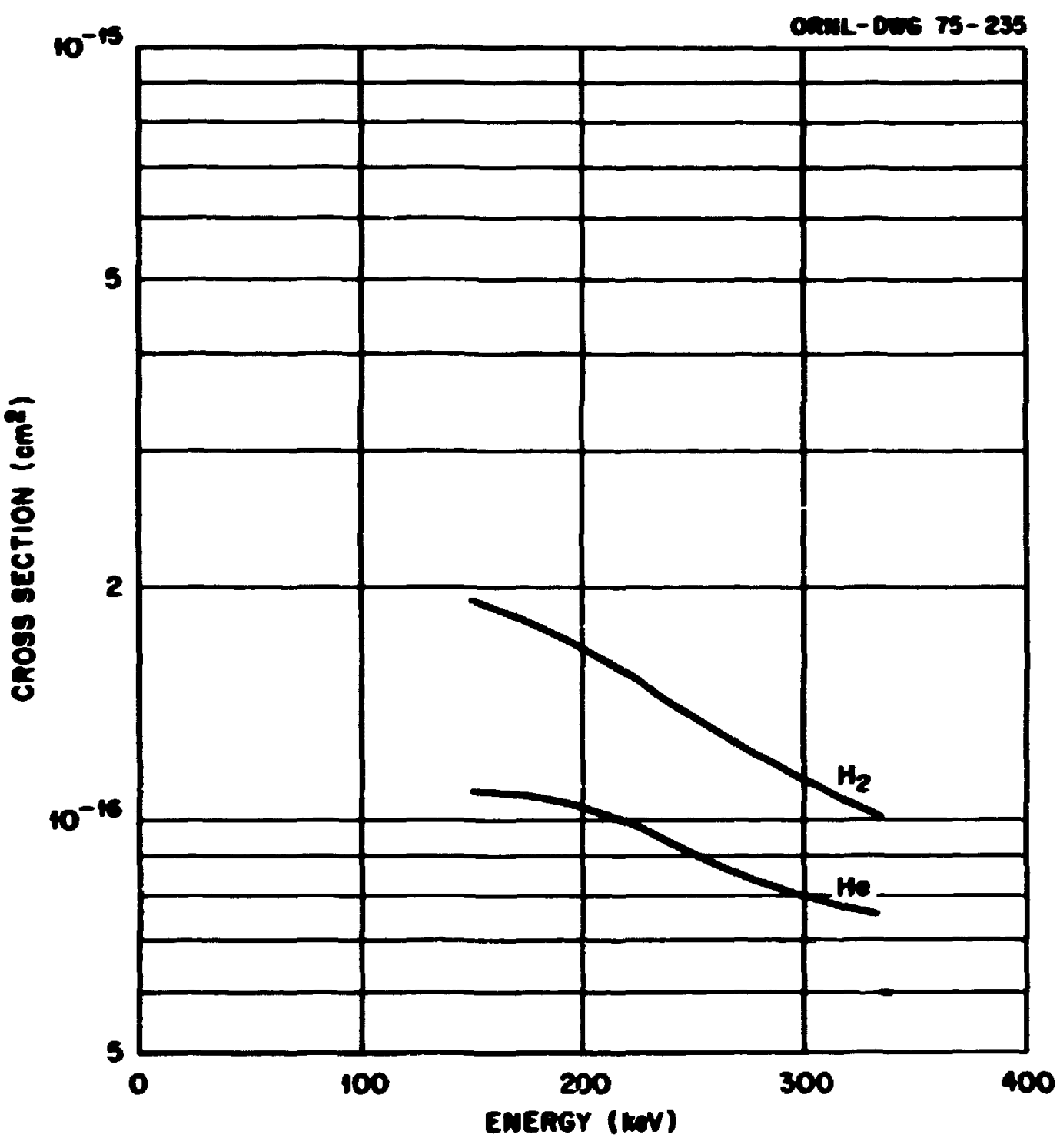

$J$ 


$$
\text { A. } 5.34
$$

Cross Sections for Electron Loss or Stripping

for Netastable $\mathrm{H}(2 s)$ Atom in $\mathrm{H}_{2}$ and He

\begin{tabular}{|c|c|c|}
\hline \multirow[t]{2}{*}{$\begin{array}{l}\text { Bueras } \\
\text { (tev) }\end{array}$} & \multicolumn{2}{|c|}{$\begin{array}{l}\text { Cross Sections } \\
\left(c^{2}\right)\end{array}$} \\
\hline & $\mathrm{H}_{2}$ & ile \\
\hline $\begin{array}{l}5.0 \mathrm{E} 00 \\
1.0 \mathrm{E} 01 \\
1.5 \mathrm{E} 01 \\
2.0 \mathrm{E} 01 \\
4.0 \mathrm{E} 01 \\
5.0 \mathrm{E} 01 \\
1.0 \mathrm{E} 02 \\
2.0 \mathrm{E} 02 \\
4.0 \mathrm{E} 02 \\
5.0 \mathrm{E} 02\end{array}$ & $\begin{array}{ll}2.5 & \mathrm{E}-16 \\
2.9 & \mathrm{E}-10 \\
3.5 & \mathrm{E}-16 \\
3.9 & \mathrm{E}-16 \\
4.5 & \mathrm{E}-16 \\
4.5 & \mathrm{E}-16 \\
3.2 & \mathrm{E}-16 \\
2.0 & \mathrm{E}-16 \\
1.0 & \mathrm{E}-16 \\
8.0 & \mathrm{E}-17\end{array}$ & $\begin{array}{ll}2.9 & \mathrm{E}-16 \\
3.4 & \mathrm{E}-16 \\
3.2 & \mathrm{E}-16 \\
3.1 & \mathrm{E}-16 \\
2.3 & \mathrm{E}-16 \\
2.0 & \mathrm{E}-16 \\
1.3 & \mathrm{E}-16 \\
7.7 & \mathrm{E}-17 \\
5.5 & \mathrm{E}-17 \\
5.0 & \mathrm{E}-17\end{array}$ \\
\hline
\end{tabular}

\section{References:}

H(2s) + $\mathrm{H}_{2}$ : H.B. Gilbody, R.M. Reynolds, and G.I. Riddell, J. Phys. B 4, S4 (1971); H.B. Gilbody and J.L. CorT, J. Phys. B I, 1953 (1974).

H(2s) + Be: H.B. Gilbod, R. Broming, R.M. Regnolds, and G.I. Riddell, J. Ptors. B 4, 94 (1971); R.H. Huches and S.S. Choe, Phos. Rev. A 6, 1413 (1972); H.B. Gllbod and J.L. Corr, J. Plys. B I. 1953 (1974).

\section{Accuracy:}

$\pm 20 \%$ 
A.5.35

.)

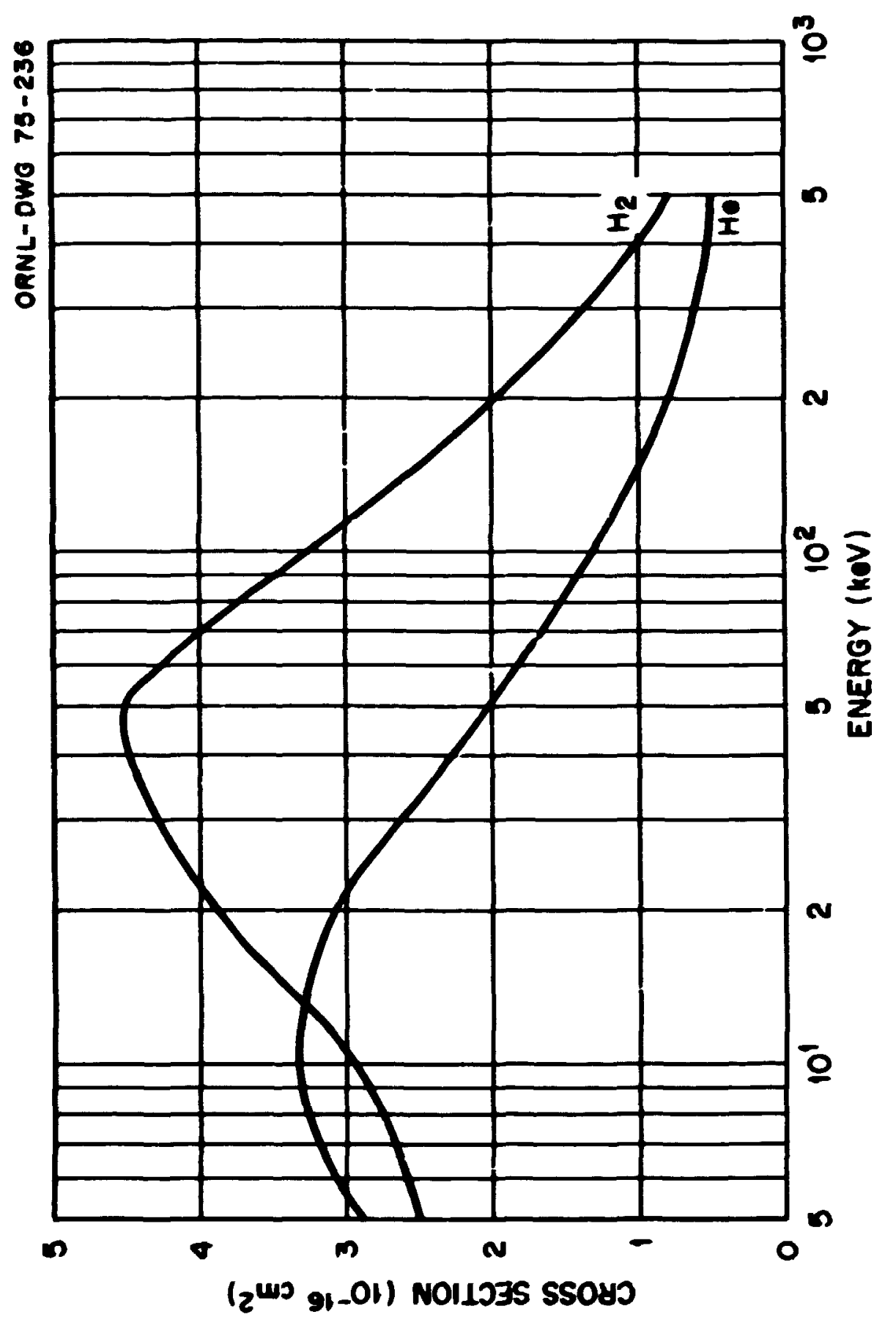


A. 5.36

Cross Sections for Electron Stripping

H Atoms in He and re

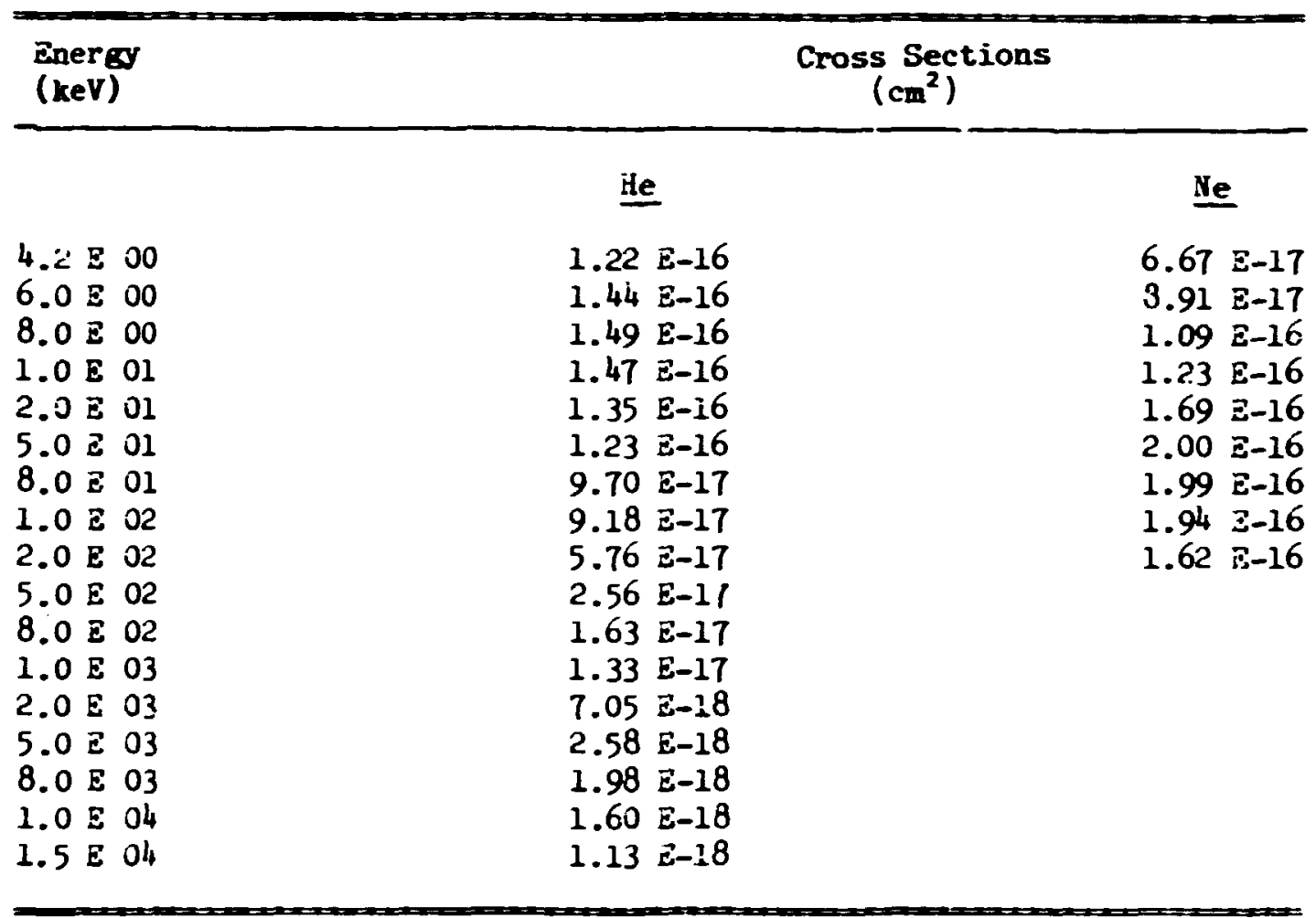

References:

it + lie: C.F. Barnett and H.K. Reynolds, Phys. Rev. 109, 355 (1958); P.M. St,ier and C.F. Barnett, Phys. Rev. 103, 896 (1958); G.W. McClure, Phys. Rev. 134, A-1226 (1964); L.H. Toburen, M.Y. Nakai, and R.A. Langley, Phys. Rev. 171, 114 (1968); J.F. Williams, Phys. Rev. 157, 97 (1967);

K.H. Berkner, S.N. Kaplan, and R.V. Pyle, Phys. Rev. 134, A-1461 (1964); L.M. Welsh, K.H. Berkner, S.N. Kaplan, and R.V. Pyle, Phys. Rev. 158, 85 (1967).

II + Ne: P.M. Stier and C.F. Barnett, Phys. Rev. 103, 896 (1956).

Accuracy:

$\pm 20 \%$ 
A. 5.37

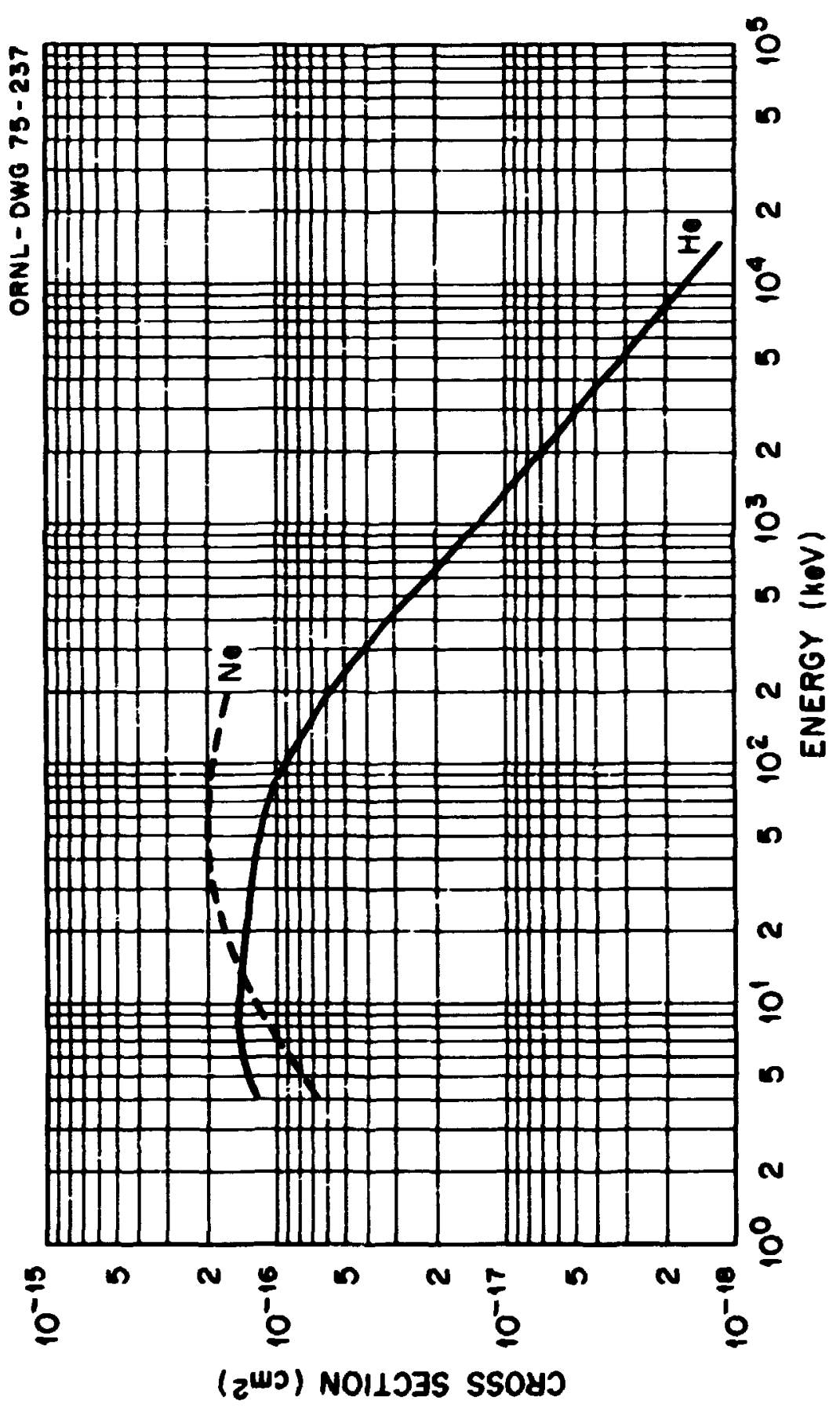




\section{A. 5.38}

Cross Sections for Electron Loss of H Atoms

$$
\text { in } x_{2}, \mathrm{O}_{2}, \text { and } \mathrm{x}_{2} \mathrm{O}
$$

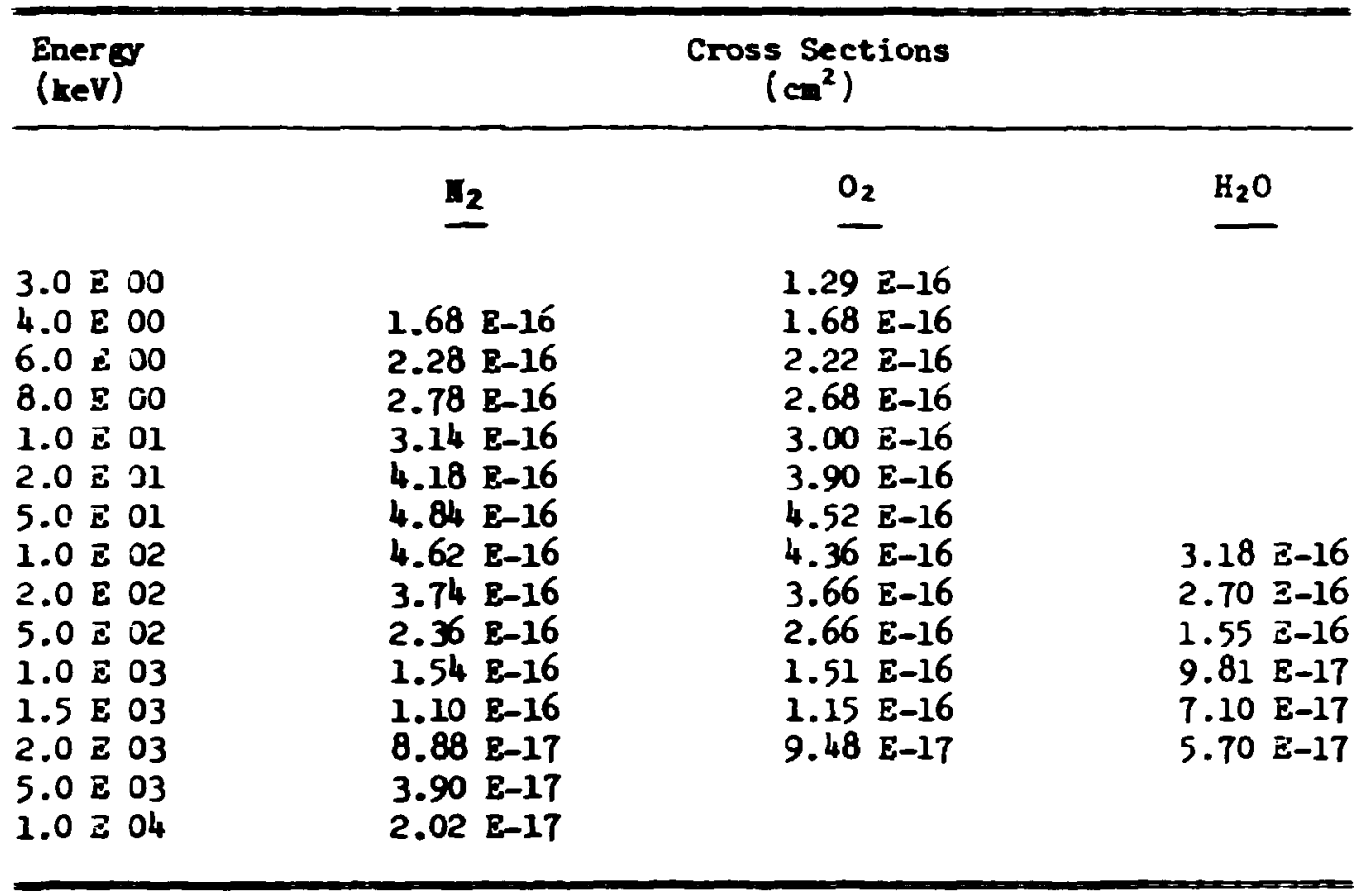

References:

$H+N_{2}$ : C.F. Barnett and i.K. Reynolds, Phys. Rev. 109, 355 (1958);

P.M. Stier and C.F. Barnett, Phys. Rev. 103, 896 (1956); L.H. Toburen, M.Y. Nakai, and R.A. Lmazley, Phys. Rev. 171 , 114 (1968); K.it. Berkner, S.N. Kaplan, and R.V. Pyl.z, Phys. Rev. 134, A-1461 (1964); L.M. Welsh, K.H. Berkner, S.N. Kaplar, and R.V. Pyle, Phys. Rev. 158, 85 (196?); U. Schryber, Hel. Phys. Acta 40, 1023 (1967); H. Tarara and A. Russek, Rev. Mod. Phys. 45, $178(1973)$.

H $+0_{2}$ : P.M. Stier and C.F. Barnett, Phys. Rev. 103, 896 (1956); L.H. Toburen, M.Y. Hekai, and R.A. Langley, Phys. Rev. 171, 114 (1968); H. Tavara and A. Russek, Rev. Mod. Phys. 45, 178 (1973).

$\mathrm{H}+\mathrm{H}_{2} \mathrm{O}$ : L.H. Toburen, M.Y. Nakai, and R.A. Langley, Phys. Rev. 171, $114(1968)$.

Accuracy:

$\pm 25 \%$ 


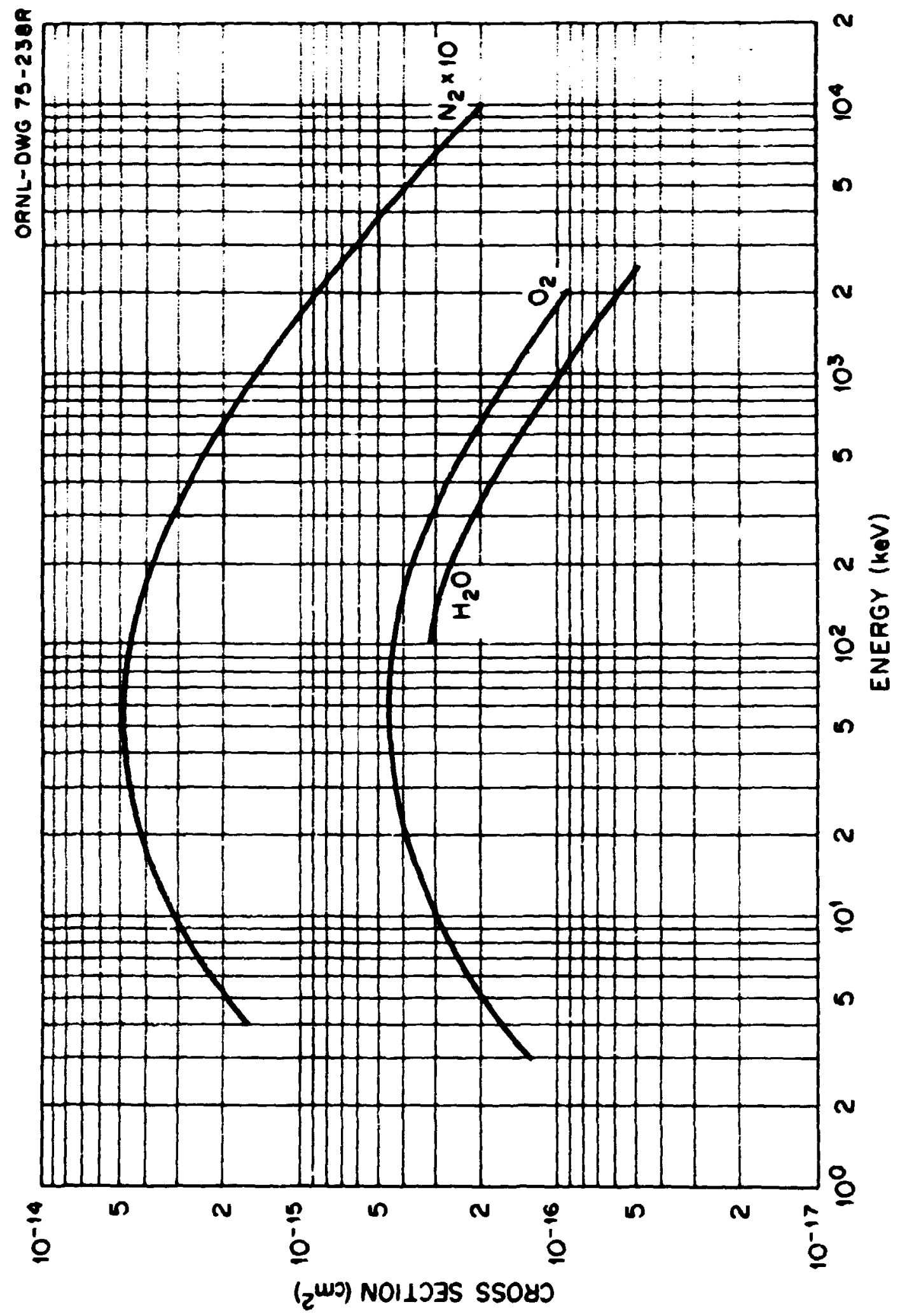




$$
\text { A. } 5.40
$$

Cross Sections for Electron Production in

$$
\mathrm{d}_{2} \text { and } \mathrm{O}_{2} \text { by if Atom Impact }
$$

\begin{tabular}{|c|c|c|}
\hline \multirow[t]{2}{*}{$\begin{array}{l}\text { Zanergy } \\
\text { (keV) }\end{array}$} & \multicolumn{2}{|c|}{$\begin{array}{c}\text { Cross Jections } \\
\left(\mathrm{cm}^{2}\right)\end{array}$} \\
\hline & $\underline{N_{2}}$ & $\mathrm{O}_{2}$ \\
\hline $\begin{array}{l}1.0 \mathrm{E}-01 \\
2.0 \mathrm{E}-01 \\
4.0 \mathrm{E}-01 \\
6.0 \mathrm{E}-01 \\
1.0 \mathrm{E} 00 \\
2.0 \mathrm{E} 00 \\
4.0 \mathrm{E} 00 \\
6.0 \mathrm{E} 00 \\
1.0 \mathrm{E} 01 \\
2.0 \mathrm{E} 01 \\
4.0 \mathrm{E} 01 \\
6.0 \mathrm{E} 01 \\
1.0 \mathrm{E} 02 \\
2.0 \mathrm{E} 02 \\
4.0 \mathrm{E} 02\end{array}$ & $\begin{array}{ll}8.0 & \mathrm{E}-18 \\
2.4 & \mathrm{E}-17 \\
5.9 & \mathrm{E}-17 \\
9.0 & \mathrm{E}-17 \\
1.5 & \mathrm{E}-16 \\
2.6 & \mathrm{E}-16 \\
4.2 & \mathrm{E}-16 \\
5.0 & \mathrm{E}-16 \\
7.4 & \mathrm{E}-16 \\
1.0 & \mathrm{E}-15 \\
1.1 & \mathrm{E}-15 \\
1.0 & \mathrm{E}-15 \\
8.0 & \mathrm{E}-16 \\
5.8 & \mathrm{E}-16 \\
4.2 & \mathrm{E}-16\end{array}$ & $\begin{array}{ll}1.5 & \mathrm{E}-17 \\
3.7 & \mathrm{E}-17 \\
8.3 & \mathrm{E}-17 \\
1.2 & \mathrm{E}-16 \\
1.7 & \mathrm{E}-16 \\
2.5 & \mathrm{E}-16 \\
3.7 & \mathrm{E}-16 \\
4.3 & \mathrm{E}-16 \\
5.0 & \mathrm{E}-16\end{array}$ \\
\hline
\end{tabular}

\section{References:}

H $+\mathbb{N}_{2}$ : H.H. Fleischmann and R.A. Young, Phys. Lett. 29A, 287 (1969); L.J. Puckett, G.0. Taylor, and D.W. Martin, Phys. Rev. 178,271 (1969); E.S. Solov'ev, R.N. II'in, V.A. Ofarin, and N.V. Pedorenko, Sov. Phys.JETP 15, 459 (1962); R.J. MeNeal and D.C. Clark, J. Geophys. Res. T4, $5065(1969)$.

$\mathrm{H}+\mathrm{O}_{2}$ : H.H. Fleischmann and R.A. Young, Phys. Lett. 29A, 287 (1969); R.J. McNeal, D.C. Clark, and R.A. Klingbery, Phys. Rev. A 2, 131 (2970). Accuracy:

$\pm 50 \%$. 


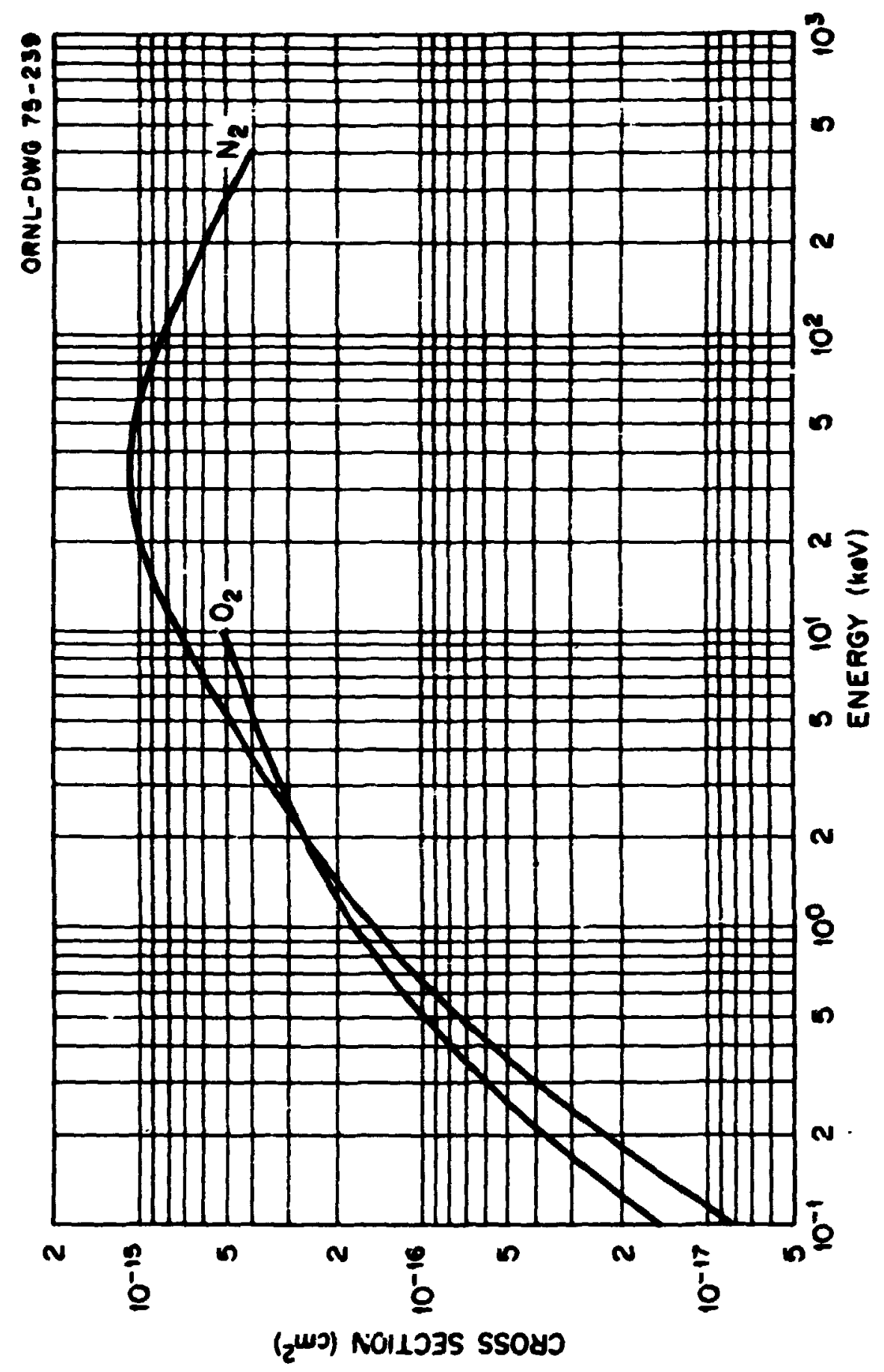




\section{A.5. 42}

Cross Sections for Ose Electron Loss

of H Atoms in $\mathrm{Xa}, \mathrm{K}$, and $\mathrm{is}$

\begin{tabular}{|c|c|c|c|c|c|}
\hline \multirow[t]{2}{*}{$\begin{array}{l}\text { Bneres } \\
\text { (keV) }\end{array}$} & \multicolumn{5}{|c|}{$\begin{array}{c}\text { Cross Sections } \\
\left(\mathrm{c}^{2}\right)\end{array}$} \\
\hline & $\mathbf{K}$ & $I n_{1}$ & $\mathrm{Hez}$ & $\mathbf{1 8 1}$ & $\mathbf{4 8 2}$ \\
\hline $\begin{array}{lll}4.0 & E & 00 \\
6.0 & E & 00 \\
8.0 & E & 00 \\
1.0 & E & 01 \\
1.5 & E & 01 \\
2.0 & E & 01 \\
2.5 & E & 01 \\
3.0 & E & 01 \\
4.0 & E & 01 \\
6.0 & E & 01\end{array}$ & $\begin{array}{ll}2.6 & z-16 \\
3.6 & z-16 \\
4.6 & z-16 \\
5.9 & z-16 \\
5.3 & z-16 \\
4.5 & z-16\end{array}$ & $\begin{array}{ll}4.8 & E-16 \\
4.0 & E-16\end{array}$ & $\begin{array}{ll}2.4 & \mathrm{E}-16 \\
3.1 & \mathrm{E}-16 \\
3.5 & \mathrm{E}-16 \\
3.8 & \mathrm{E}-16 \\
3.9 & \mathrm{E}-16 \\
3.7 & \mathrm{E}-16\end{array}$ & $\begin{array}{ll}1.9 & \mathrm{E}-17 \\
3.3 & \mathrm{E}-17 \\
5.2 & \mathrm{E}-17 \\
7.6 & \mathrm{E}-17 \\
1.8 & \mathrm{E}-16 \\
3.3 & \mathrm{E}-16 \\
3.3 & \mathrm{E}-16 \\
3.1 & \mathrm{E}-16 \\
1.5 & \mathrm{E}-16\end{array}$ & $\begin{array}{ll}2.7 & z-16 \\
2.5 & z-16 \\
3.0 & z-16 \\
3.3 & \mathrm{z}-16 \\
3.1 & \mathrm{z}-16 \\
2.8 & \mathrm{z}-16\end{array}$ \\
\hline
\end{tabular}

\section{References:}

i + K: R.A. Mieman, Ph.D. Thesis, University of Pittsburch, Pittsburch, PA.

H + We: R.A. Ifeman, Ph.D. Thesis, University of Pittsburgh, Pittsburgh, PA.

H + Maz: V.A. Oparin, R.J. Il'in, and E.S. Solor'er, Sov. Phys.-JETP 25, 240 (1967).

H + Ms2: V.A. Oparin, R.X. Il'in, and E.S. Solov'er, Sov. Phys.-JETP 25, $240(1967)$.

H + MG1: A.H. Putch and K.G. Moses, Fifth Intl. Conf. on Electronic \& Atomic Collisions (Leningrad, 1967) Publishing House Nauka (Leningrad) p. 12 (1967).

\section{Accurecy:}

The accuracy is no better then a factor of 2 to 3.

Note:

Non and $M_{\delta_{2}}$ refer to a different set of measurements and not a molecule. 


\section{A.5.43}

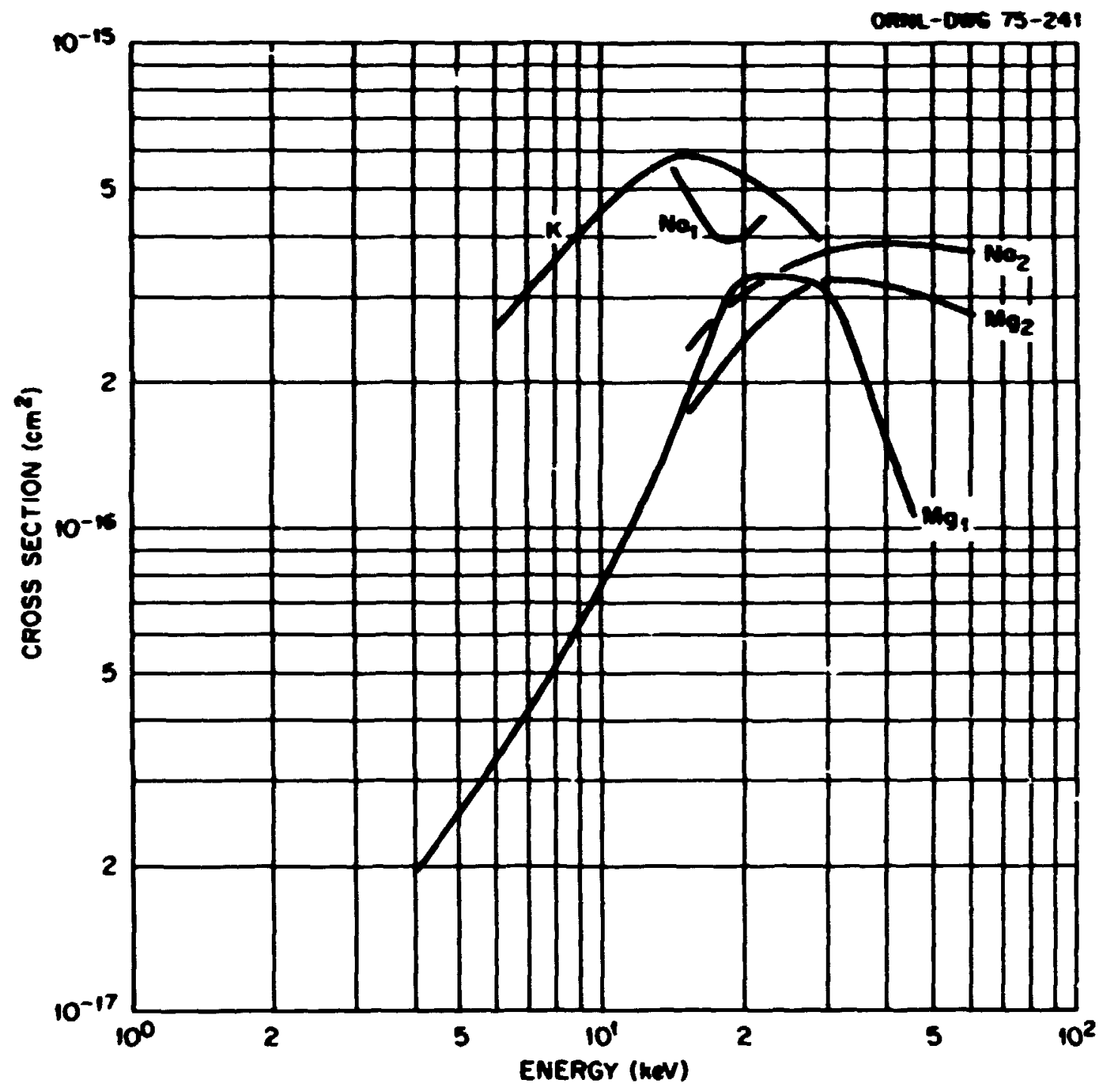




\section{A.5.4.}

Cross jections for One Electron Loss

$$
\text { of } \mathrm{H}_{2} \text { and } \mathrm{J}_{2} \text { in } \mathrm{H}_{2} \text { and } \mathrm{J}_{2} \text { ies }
$$

\begin{tabular}{|c|c|c|}
\hline \multirow[t]{2}{*}{$\begin{array}{l}\text { Bherg } \\
\text { (keV) }\end{array}$} & \multicolumn{2}{|c|}{$\begin{array}{c}\text { Cross Sections } \\
\left(\mathrm{cm}^{2}\right)\end{array}$} \\
\hline & $\mathrm{H}_{2}+\mathrm{H}_{2}$ & $D_{2}+D_{2}$ \\
\hline 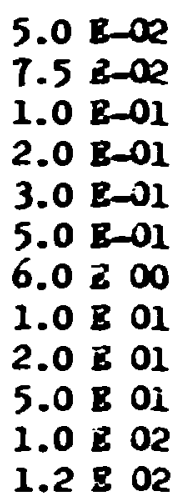 & $\begin{array}{ll}5.0 & \mathrm{z}-19 \\
2.8 & \mathrm{z}-18 \\
5.7 & \mathrm{E}-18 \\
1.4 & \mathrm{E}-17 \\
1.9 & \mathrm{E}-17 \\
2.2 & \mathrm{E}-17 \\
5.2 & \mathrm{E}-17 \\
6.7 & \mathrm{z}-17 \\
1.2 & \mathrm{E}-16 \\
1.8 & \mathrm{E}-16 \\
2.0 & \mathrm{E}-16 \\
2.0 & \mathrm{E}-16\end{array}$ & $\begin{array}{ll}3.8 & \Xi-19 \\
1.3 & \Xi-18 \\
2.7 & \Xi-18 \\
8.7 & \Xi-18 \\
1.3 & E-17 \\
1.7 & \Xi-17\end{array}$ \\
\hline
\end{tabular}

\section{References:}

$\mathrm{d}_{2}+\mathrm{H}_{2}$ : R.X. Peterson and M. Eisner, Phys. Rev. A 8,1289 (1973); G.W. Meclure, Phys. Rev. 134, A 1226 (1964); T.J. Morgan, K. H. Berkner, and R.V. Pyle, Phys. Rev. A 5, 1591 (1972).

$D_{2}+D_{2}$ : R.K. Peterson and M. Eisner, Phys. Rev. A 8,1289 (1973).

\section{Accuracy:}

$\pm 20 \%$. 


\section{A.5.45}

,

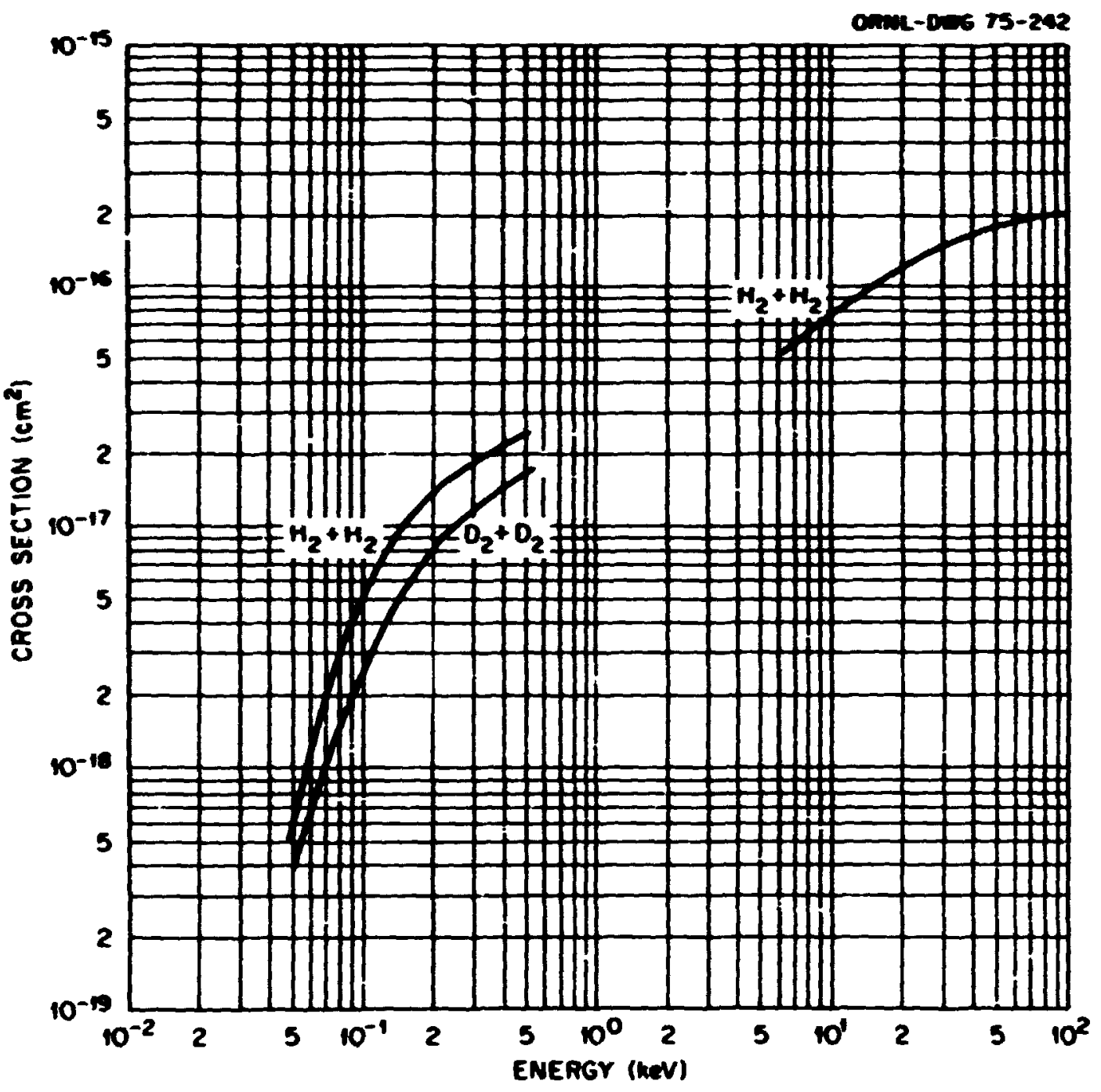




\section{A.5.46}

Cross Sections for Electron Production

in Coilisions of $y^{-}$vith it atoms

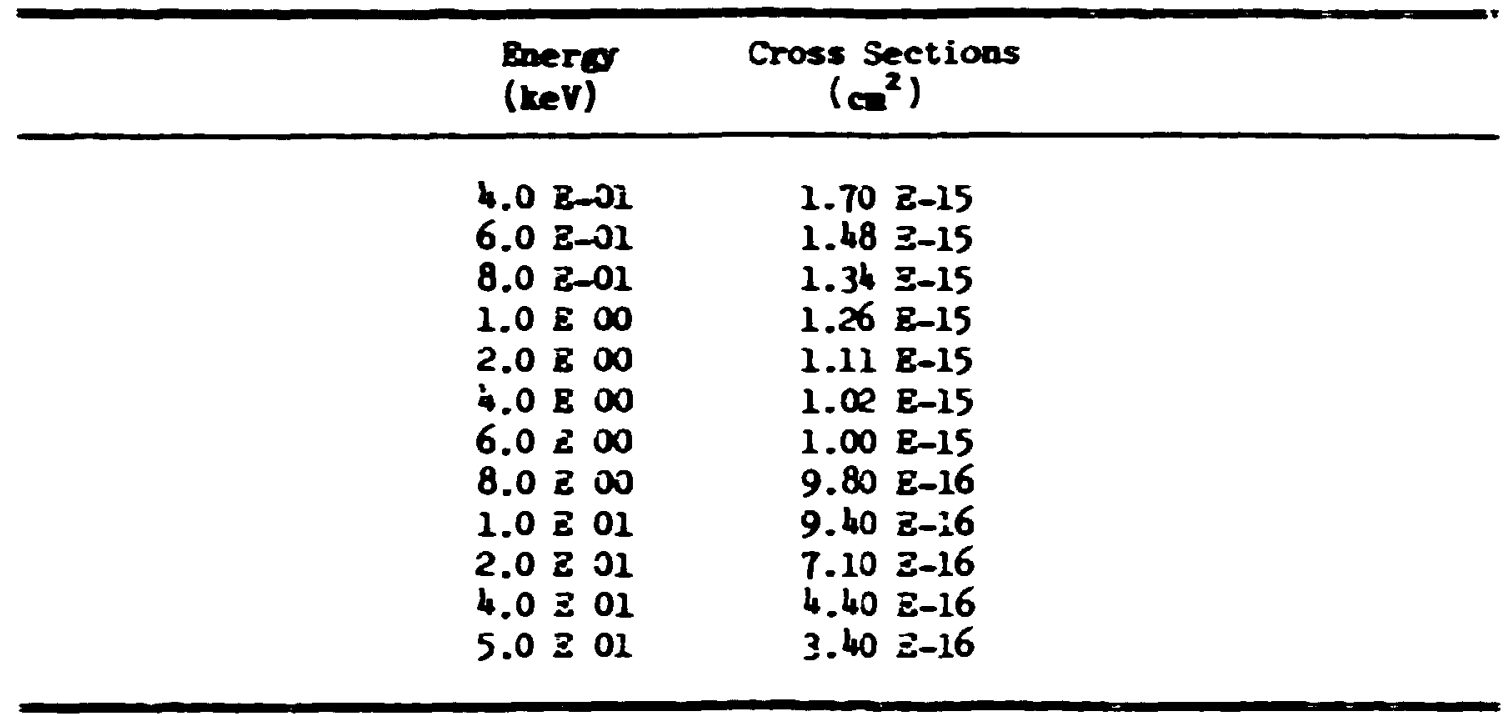

\section{References:}

D.G. Ylumer, R.F. Stebbings, H.L. Fite, and L.M. Branscaab, Phys. Rer. 119, $668(1960)$.

\section{Accurecy:}

$\pm 30 \%$. 


\section{A. 5.67}

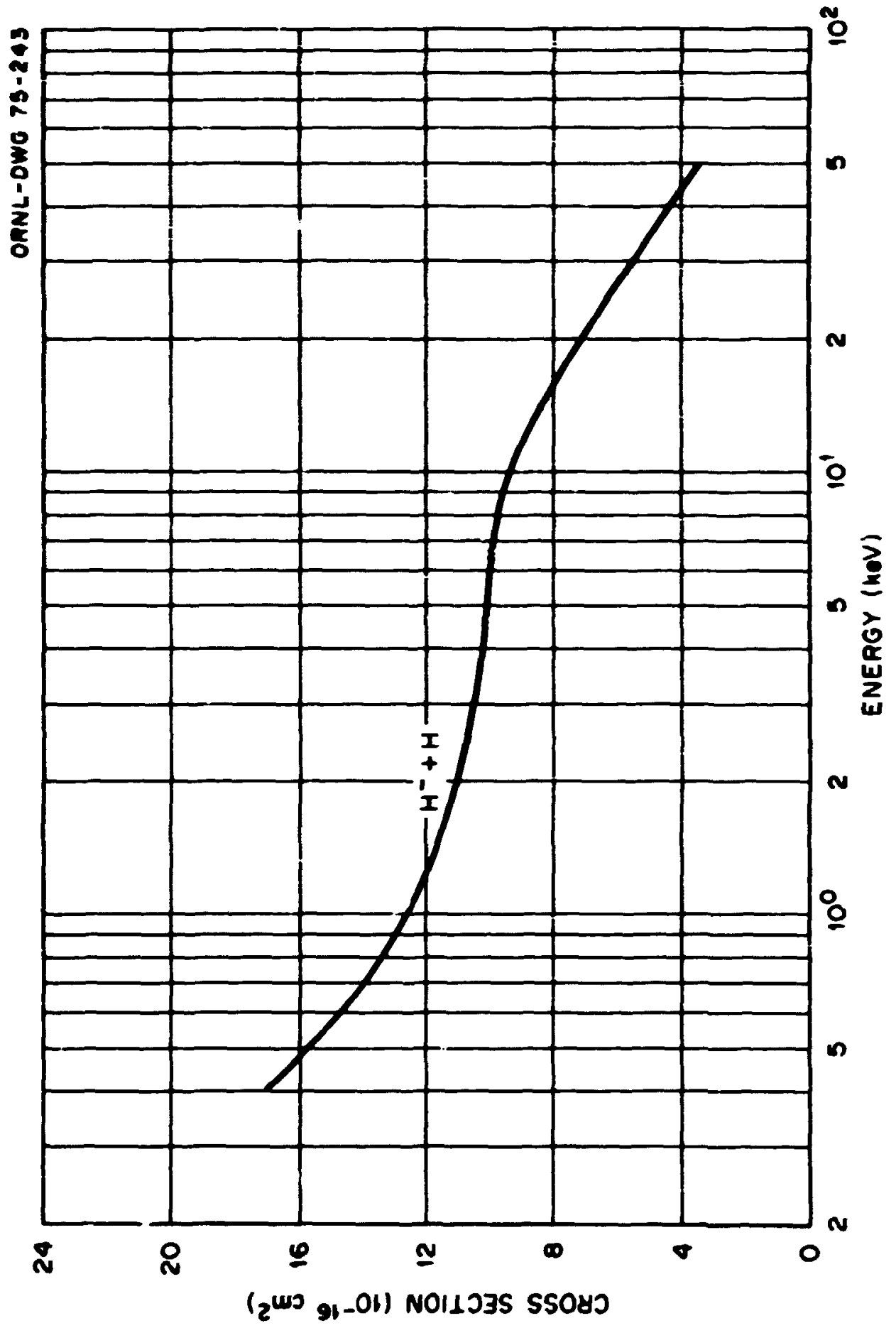




\section{A. 5.48}

Cross Jections for One Bieitron Loss

or stripping for $\mathrm{I}^{-}$in $\ddot{i}_{2}$ and $\mathrm{ie}$

\begin{tabular}{|c|c|c|}
\hline \multirow[t]{2}{*}{$\begin{array}{l}\text { Deergo } \\
\text { (keV) }\end{array}$} & \multicolumn{2}{|c|}{$\begin{array}{c}\text { Cress Sections } \\
\left(\mathrm{sm}^{2}\right)\end{array}$} \\
\hline & $\ddot{z}_{2}$ & :Ie \\
\hline $2.0 \mathrm{E}-01$ & $\tau-5 \equiv-16$ & $4.6=-10$ \\
\hline $5.0 \equiv-01$ & $1.0=-15$ & $5.2 \equiv-i 6$ \\
\hline $1.0 \equiv 00$ & i.1 E-15 & $5.3 \equiv-16$ \\
\hline $2.0 \equiv 00$ & $1.23-15$ & 6.1 E-16́ \\
\hline $5.0 \mathrm{z} 00$ & $1.2 \mathrm{z}-15$ & $6.0 \equiv-16$ \\
\hline $1.0=01$ & $1.0 \equiv-15$ & $5.4 \equiv-16$ \\
\hline $2.0 \mathrm{E} \mathrm{OI}$ & $9.7 \equiv-16$ & $4.4 \equiv-i 6$ \\
\hline $5.0 \mathrm{E} 01$ & $0.0 \Sigma-16$ & $3.2 \mathrm{E}-$ \\
\hline $1.0 \mathrm{E} 02$ & $4.0 \mathrm{E}-16$ & $2.2 \equiv-: 6$ \\
\hline $2.0 \mathrm{E} 02$ & $2.6 \pm-16$ & $1.5 \mathrm{E}-\mathrm{io}$ \\
\hline $5.0 \mathrm{E} 02$ & $1.32-17$ & $7.3 \Xi-i 7$ \\
\hline $1.0=03$ & $7.2 \equiv-17$ & $4.0 \Xi-17$ \\
\hline $2.0 \mathrm{E} 03$ & $3.3 \Sigma-17$ & $2.2 \quad \ddot{z}-17$ \\
\hline $5.0=03$ & $1.2 \sum-17$ & $9.0 \mathrm{z}-19$ \\
\hline $1.0 \geq 04$ & $5.5 \mathrm{E}-18$ & $4.7 \equiv-18$ \\
\hline $1.7 \mathrm{E} 0_{4}$ & $2.7 \equiv-18$ & \\
\hline
\end{tabular}

\section{References:}

$H^{-}+\mathrm{H}_{2}$ : J.F. Willians, Phys. Pev. 154, 9 (1967); P.M. Stier and C.E.

Barnett, Phys. Rev. 103, 996 (1956); ?.it. Rose, R.J. Connor, and R.P. Sastide, Bull. Am. Phys. Soc. II-3, 40 (1958); G.I. Dimov and V.S. Dudnikov, Sov. Phys.-Tenh. Phys. 11, 919 (1967); K.ii. Berkner, S.J. Kaplan, and R.V. Pyle, Phys. Rev. 134, A1461 (1964); R. Smythe and J.H. Toevs, Phys. Rev. 139, A-15 (1965); H. Tawara and A. Risseik, Rev. :Hod. Phys. 45, 178 (1973); J.S. Risley and R. Geidalle, Phys. Rev. A 9, 2485 (1974); F.R. Jimpson and H.B. Gilbody, J. Phys. B $\underline{5}, 1959$ (1972).

$\mathrm{H}^{-}+$ite: J.F. Willians, Phys. Rev. 154, 9 (1967); G.I. Dimor and V.G. Dudnikov, Sov. Phys.-Te=h. Phys. I1, $9 i 9$ (1967); P.M. Stier and C.F. Barnett, Phys. Rev. 103, 896 (:956); K.H. Berkner, S.N. Kaplan, and R.V. Pyle, Phys. Rev. 134, A-1461 (1964); F.R. Simpson and i. B. Gilbody, J. Phys. B S, 1959 (1972); J.J. Risley and R. Geballe, Phys. Rev. A 9, 2485 (1974); H. Tawara and A. Russek, Rev. Mod. Phjs. 45, 178 (1973).

Accuracy: $\pm 25 \%$.

\section{Note:}

For total detachment cross sections $\left(\sigma_{-10}+2 \sigma_{-11}\right)$ see $J . S$. Risley and R. Geballe, Phys. Rev. A 2, 2485 (1974); J.S. Risley, Phys. Rev. A 10, 731 (1974); J.B. Hasted and R.A. Smith, Proc. Roy. Soc. (Lond.) A235, 349 (1956); J.B.H. Stedeford and J.B. Hasted, Proc. Roy. Joc. (Lond.) A227, 466 (1955); E.E. Muschlitz, Jr., T.L. Bailey, anci J.H. Simons, J. Chem. Phys. 24, 1202 (1956) and J. Chem. Phys. 26, 711 (1957). 


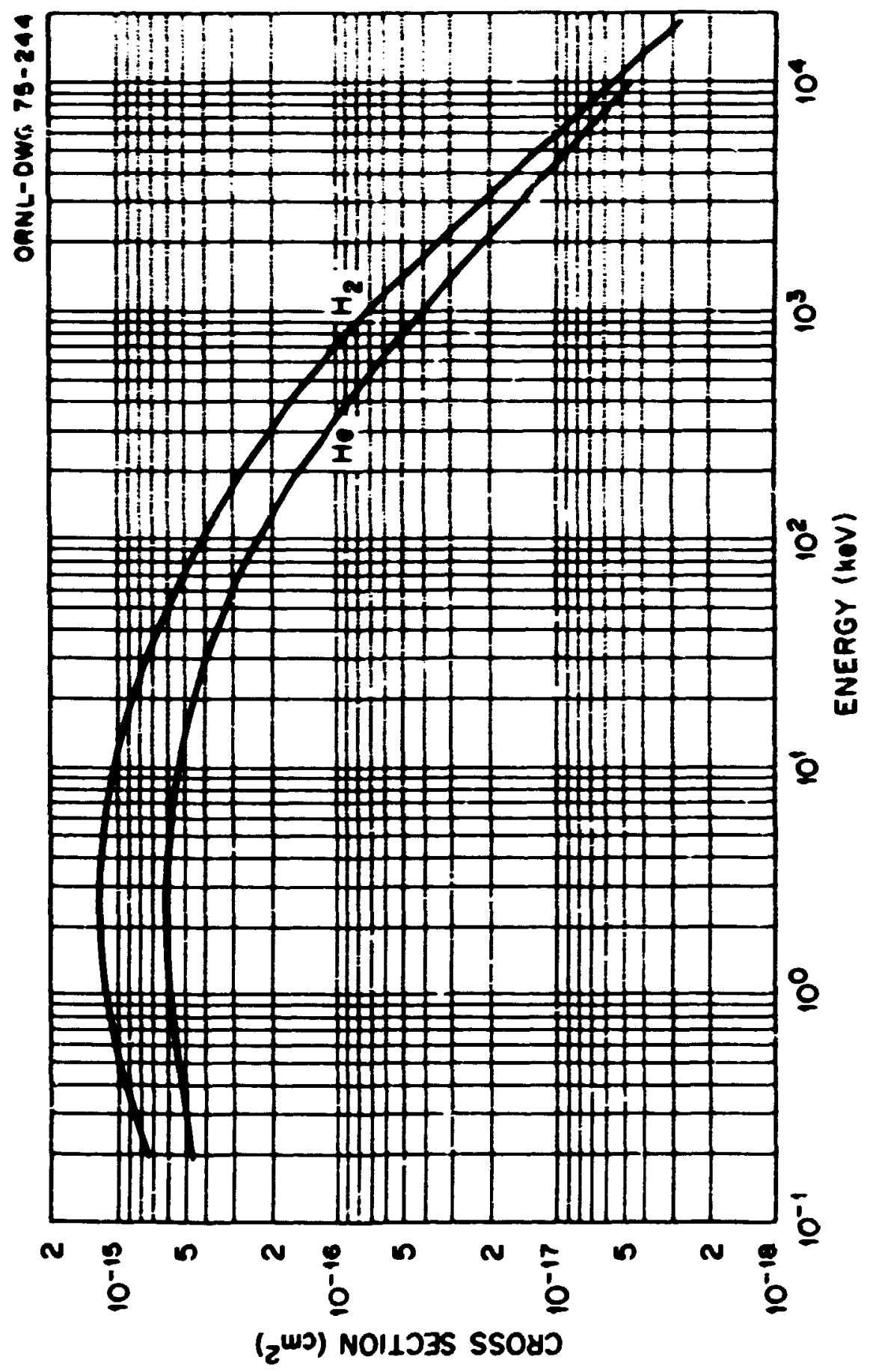


A. 5.50

Cross Sections for Loss of Two Electrons

in Collisions of $\mathrm{H}^{-}$with $\mathrm{H}_{2}$ and He

\begin{tabular}{|c|c|c|}
\hline \multirow[t]{2}{*}{$\begin{array}{l}\text { Energy } \\
(\mathrm{keV})\end{array}$} & \multicolumn{2}{|c|}{$\begin{array}{l}\text { Cross Sections } \\
\left(\mathrm{cm}^{2}\right)\end{array}$} \\
\hline & $\underline{\mathrm{H}_{2}}$ & He \\
\hline 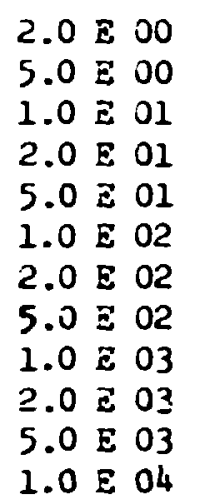 & $\begin{array}{ll}2.1 & \mathrm{E}-17 \\
3.1 & \mathrm{E}-17 \\
4.0 & \mathrm{E}-17 \\
4.4 & \mathrm{E}-17 \\
4.2 & \mathrm{E}-17 \\
3.0 & \mathrm{E}-17 \\
1.7 & \mathrm{E}-17 \\
7.0 & \mathrm{E}-18 \\
3.5 & \mathrm{E}-18 \\
1.7 & \mathrm{E}-18 \\
6.5 & \mathrm{E}-19 \\
3.0 & \mathrm{E}-19\end{array}$ & $\begin{array}{ll}2.7 & \mathrm{E}-17 \\
4.7 & \mathrm{E}-17 \\
5.1 & \mathrm{E}-17 \\
4.3 & \mathrm{E}-17 \\
2.3 & \mathrm{E}-17 \\
1.2 & \mathrm{E}-17 \\
6.0 & \mathrm{E}-18 \\
2.5 & \mathrm{E}-18 \\
1.3 & \mathrm{E}-18 \\
6.5 & \mathrm{E}-19 \\
2.7 & \mathrm{E}-19 \\
1.4 & \mathrm{E}-19\end{array}$ \\
\hline
\end{tabular}

\section{References:}

$\mathrm{H}^{-}+\mathrm{H}_{2}$ : H. Tawara and A. Russek, Rev. Mod. Phys. 45, 178 (1973);

J.F. Wiliiams, Phys. Rev. 154, 9 (1967); K.H. Berkner, S.N. Kaplan, and

R.V. Pyle, Phys. Rev. 134, A-1461 (1964); R. Smythe and J.W. Toevs, Phys. Rev. 139, A-15 (1965); G. Tisone and L.M. Branscomb, Bull. Am. Phys. Soc. 9, 535(1964); Ya. M. Fogel, V.A. Ankudinov, and R.E. Slabospitski, Sov. Phys.-JETP 5, 382 (1957).

$\mathrm{H}^{-}+\mathrm{iHe}$ J.F. Willians, Phys. Rev. 154, 9 (1967); G.I. Dimov, and V.G. Dudnikov, Sov. Phys.-Tech. Fhys. 11, 919 (1967); K.H. Berkner, S.V. Kaplan, and R.V. Pyle, Phys. Rev. 134, A-1461 (1964); Ya. M. Fogel, V.A. Ankudinov, and R.E. Slabospitski, Sov. Phys.-JETP 5, 382 (1957).

\section{Accura:y:}

$\pm 25 \%$ 


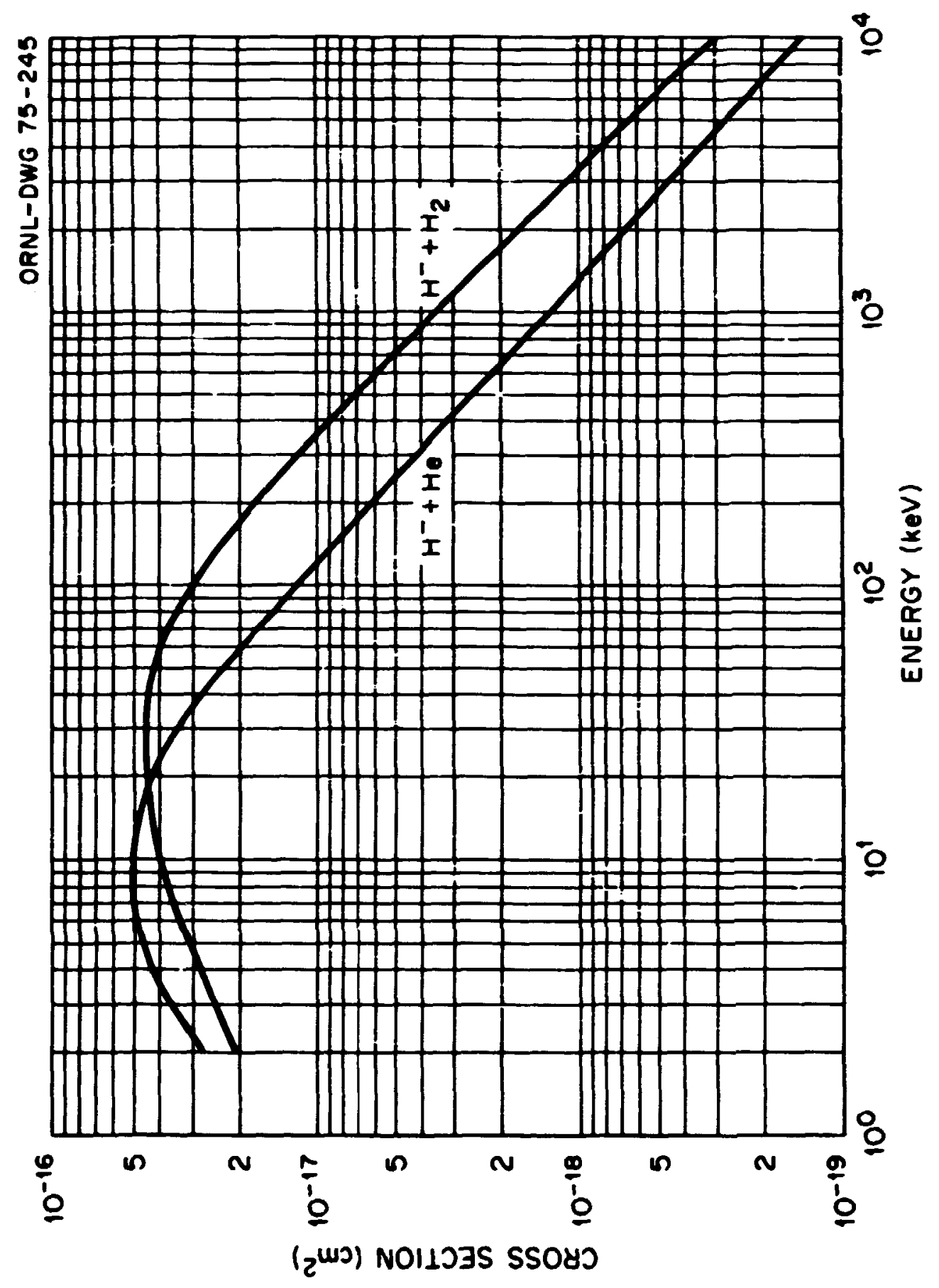


Cross Sections for One Electron Loss or Stripping for $\mathrm{H}^{-}$in $\mathrm{O}_{2}$ and $\mathrm{I}_{2}$

\begin{tabular}{|c|c|c|}
\hline \multirow[t]{2}{*}{$\begin{array}{l}\text { Energy } \\
\text { (keV) }\end{array}$} & \multicolumn{2}{|c|}{$\begin{array}{c}\text { Cross Sections } \\
\left(\mathrm{cm}^{2}\right)\end{array}$} \\
\hline & $\underline{x_{2}}$ & $\underline{\mathrm{O}_{2}}$ \\
\hline 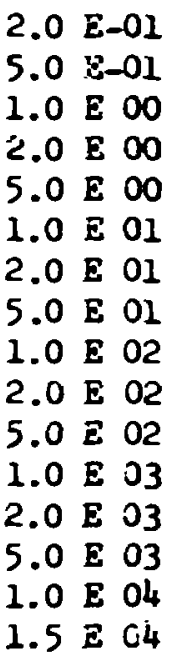 & $\begin{array}{ll}1.1 & E-15 \\
1.6 & E-15 \\
1.9 & E-15 \\
2.1 & E-15 \\
1.9 & E-15 \\
1.7 & E-15 \\
1.5 & E-15 \\
1.2 & E-15 \\
9.8 & E-16 \\
7.5 & E-16 \\
4.6 & E-16 \\
3.0 & E-16 \\
1.9 & E-16 \\
1.0 & E-16 \\
5.5 & E-17 \\
3.8 & E-17\end{array}$ & $\begin{array}{ll}1.5 & \mathrm{E}-15 \\
1.4 & \mathrm{E}-15 \\
1.3 & \mathrm{E}-15 \\
1.1 & \mathrm{E}-15 \\
1.1 & \mathrm{E}-15 \\
1.1 & \mathrm{E}-15 \\
1.2 & \mathrm{E}-15 \\
1.1 & \mathrm{E}-15 \\
1.0 & \mathrm{E}-15 \\
9.0 & \mathrm{E}-15 \\
5.8 & \mathrm{E}-15 \\
3.6 & \mathrm{E}-15 \\
2.1 & \mathrm{E}-15 \\
9.8 & \mathrm{E}-15 \\
5.3 & \mathrm{E}-15 \\
4.0 & \mathrm{E}-15\end{array}$ \\
\hline
\end{tabular}

\section{References:}

$\mathrm{H}^{-}+\mathrm{N}_{2}$ : H. Tawara and A. Russek, Rev. Mod. Phys. 45, 178 (1973); G.I. Dimov and V.G. Dudnikov, Sov. Phys.-Tech. Phys. 11, 919 (1967); K.H. Berkner, S.N. Kaplan, and R.V. Pyle, Phys. Rev. 134, A-1461 (1964); I. Kovacs, Nucl. Instr. Meth. 51, 224 (1967); J.S. Risley and R. Geballe, Phys. Rev. A 9, 2485 (1974); R. Smythe and J.W. Toevs, Phys. Rev. 139, A-15 (1965); P.M. Stier and C.F. Barnett, Phys. Hev. 103, 896 (1956).

$\mathrm{if}^{-}+\mathrm{O}_{2}$ : P.M. Stier and C.F. Barnett, Phys. Rev. 103, 896 (1956); P.M. Rose, R.J. Connor, and R.P. Bastide, Bull. Am. Phys. Soc. II-3, 40 (1958); J.S. Risley and R. Geballe, Phys. Rev. A 9, 2485 (1974); R. Smythe and J.W. Toevs, Phys. Rev. 139, A-15 (1965); D.V. Phipenko, V.A. Gusev, and Ya. M. Fogel, Sov. Phys.-JETP 22, 965 (1966).

Accuracy: $\pm 25 \%$.

Note:

For total detachment cross sections $\left(\sigma_{-10}+2 \sigma_{-11}\right)$ see:

J.S. Risley and R. Geballe, Phys. Rev. A 9, 2485 (1974); R. Sruthe and J.H. Toevs, Phys. Fev. 139, A-15 (1965); J.S. Risley, Phys. Rev. A 10 , 731 (1974); T.L. Bailey and P. Mahadevan, J. Chem. Phys. 52, 179 (1970). 


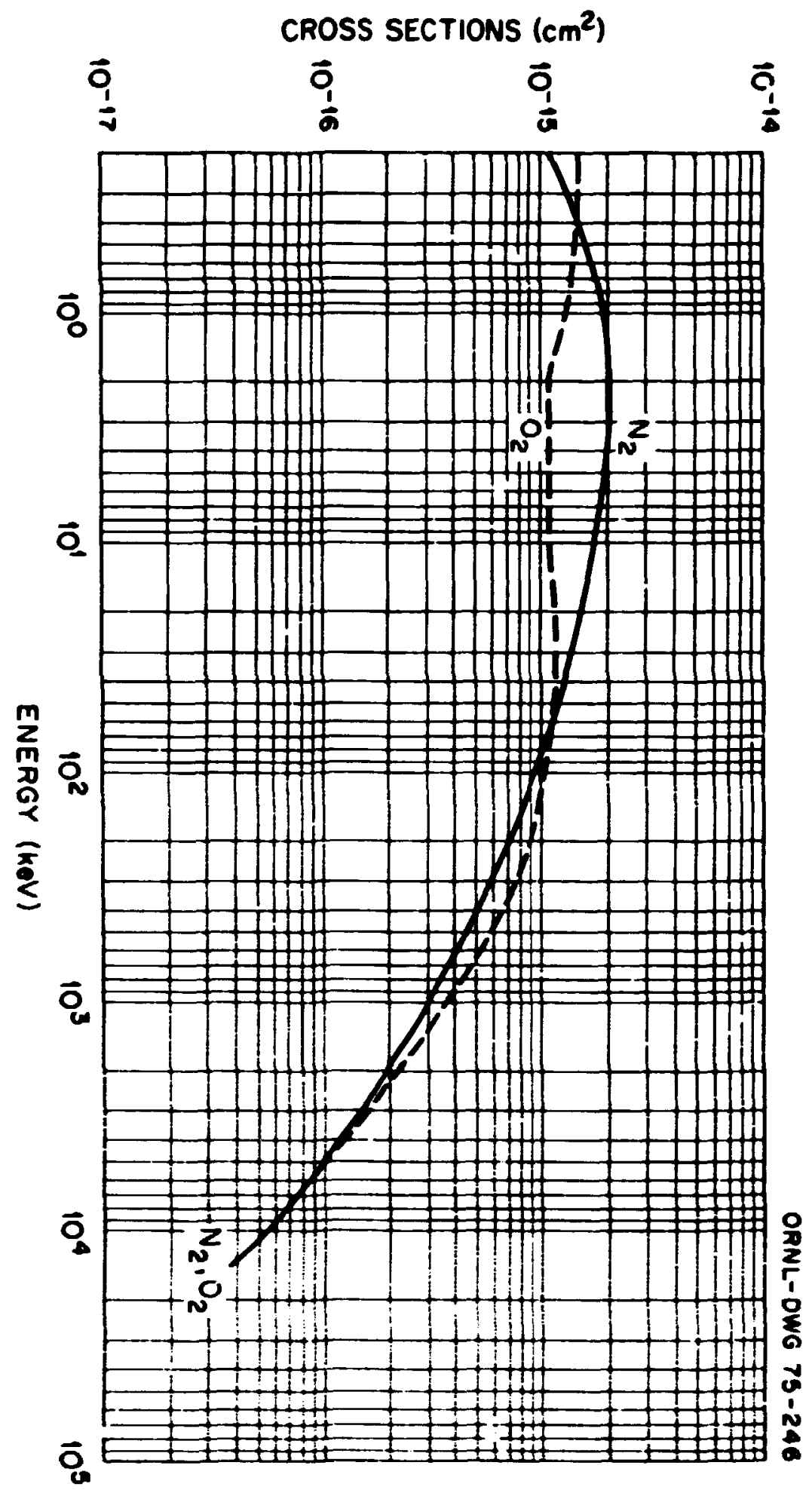


Cross Sections for Loss of Two Electrons by in $^{-}$in $\mathrm{x}_{2}$ and $\mathrm{C}_{2}$

\begin{tabular}{|c|c|c|}
\hline \multirow[t]{2}{*}{$\begin{array}{l}\text { Znergy } \\
\text { (keV) }\end{array}$} & \multicolumn{2}{|c|}{$\begin{array}{c}\text { Cross Sections } \\
\left(\mathrm{cm}^{2}\right)\end{array}$} \\
\hline & $\underline{y_{2}}$ & $\underline{O_{2}}$ \\
\hline $\begin{array}{lll}5.0 & E & 00 \\
1.0 & E & 01 \\
2.0 & E & 01 \\
4.0 & E & 01 \\
5.0 & E & 01 \\
1.0 & E & 02 \\
2.0 & E & 02 \\
5.0 & E & 02 \\
1.0 & E & 03 \\
2.0 & E & 03 \\
5.0 & E & 03 \\
1.0 & E & 04\end{array}$ & $\begin{array}{ll}1.1 & E-16 \\
1.1 & E-16 \\
1.3 & E-16 \\
1.8 & E-16 \\
2.0 & E-16 \\
3.0 & E-16 \\
2.8 & E-16 \\
1.1 & E-16 \\
4.7 & E-17 \\
2.0 & \mathrm{E}-17 \\
6.5 & \mathrm{E}-18 \\
2.7 & \mathrm{E}-18\end{array}$ & $\begin{array}{ll}8.4 & \mathrm{~J}-17 \\
9.2 & \mathrm{E}-17 \\
1.2 & \mathrm{~J}-16 \\
1.6 & \mathrm{E}-26\end{array}$ \\
\hline
\end{tabular}

\section{References:}

$\mathrm{H}^{-}+\mathrm{H}_{2}$ : Ya. M. Pogel, V.A. Ankudinov, and R.E. Slabospitski, Sov. Phys.JETP 5, 382 (1957); K.H. Berkner, S.N. Kaplan, and R.V. Pyle, Phys. Rev. 134, $\bar{A}-1461$ (1964); I. Kovacs, Nucl. Instr. Meth. 51, 224 (1967); H. Tarara and A. Russek, Rev. Mod. Phys. 45, 178 (1973).

$\mathrm{H}^{-}+\mathrm{O}_{2}=$ Ya. M. Fogel, V.A. Ankudinov, and R.E. Slajospitski, Sor. Phys.JEPT $\underline{5}, 382$ (1957).

Accurecy:

$\pm 25 \%$ 


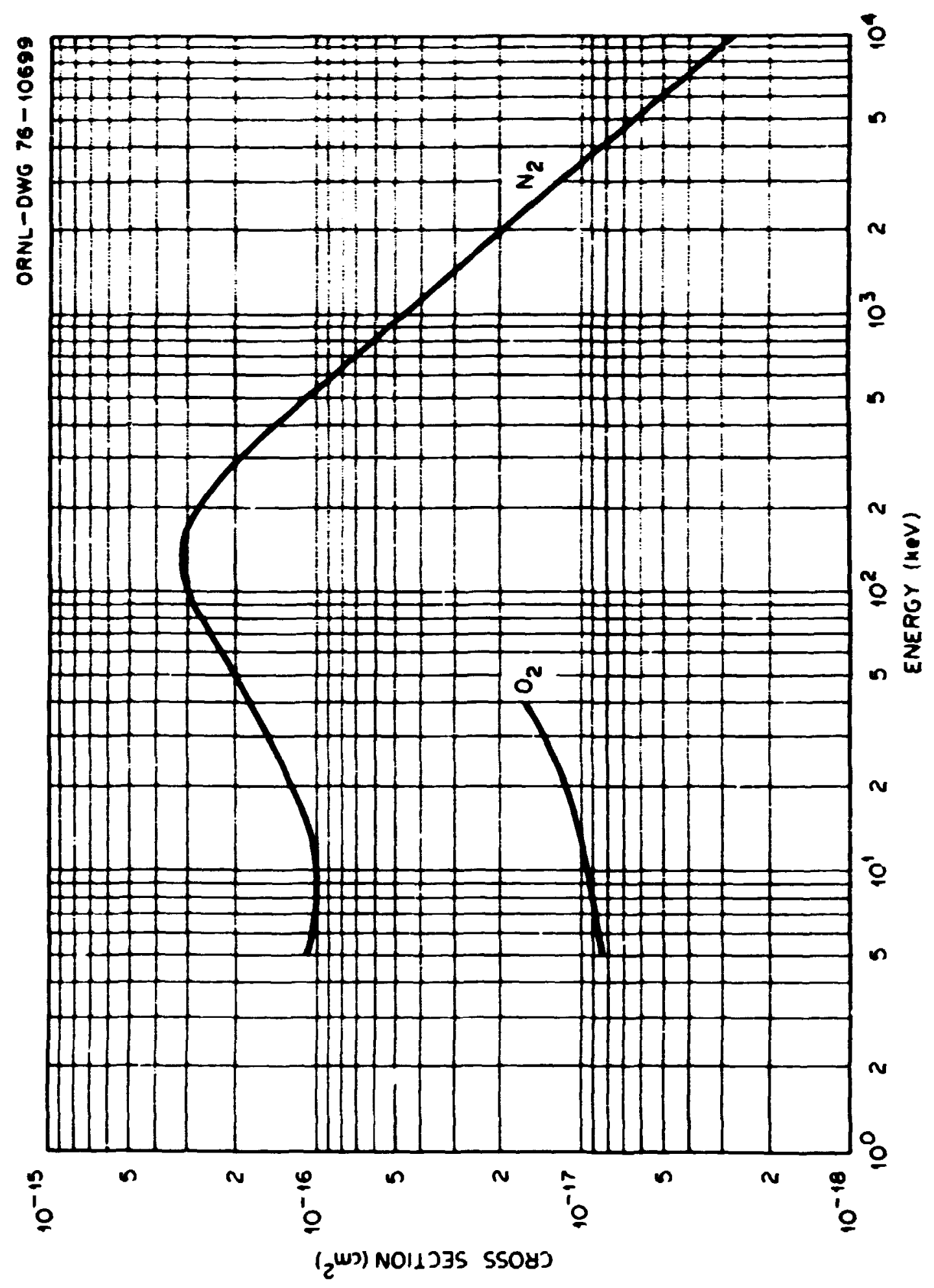


A.5.56

Cross Sections for Single Electron Los3 or

Stripping for $\mathrm{at}^{-}$in ar and ie

\begin{tabular}{|c|c|c|}
\hline \multirow[t]{2}{*}{$\begin{array}{l}\text { Bnergs } \\
\text { (keV) }\end{array}$} & \multicolumn{2}{|c|}{$\begin{array}{c}\text { Cross Sections } \\
\text { (ct) }\end{array}$} \\
\hline & $A r$ & Ye \\
\hline $2.0 \mathrm{E} 00$ & $6.5 \bar{E}-16$ & $3.0 \equiv-16$ \\
\hline $4.0 \mathrm{E} 00$ & $9.3 \bar{z}-16$ & $3.3 z-16$ \\
\hline $6.0 \mathrm{E} 00$ & $9.7 \bar{E}-16$ & $3.5 z-16$ \\
\hline $1.0 \mathrm{E} 01$ & $1.2=-15$ & $3.7 \mathrm{E}-16$ \\
\hline $2.0 \mathrm{E} 01$ & $1.6 z-15$ & $4.2 \mathrm{z}-16$ \\
\hline $5.0 \geq 01$ & $1.5 \approx-15$ & $4.9 \equiv-16$ \\
\hline $7.0 \leq 01$ & $1.3 \bar{E}-15$ & \\
\hline $1.0 E 02$ & $1.2 \mathrm{E}-15$ & \\
\hline $2.0 \& 02$ & $9.0 \mathrm{z}-16$ & \\
\hline $5.0 \mathrm{E} 02$ & $6.0 \Sigma-16$ & \\
\hline $7.0 E 02$ & $5.0 \mathrm{E}-16$ & \\
\hline $1.0 E 03$ & $4.0=-16$ & \\
\hline $2.0 E 03$ & $2.6 \mathrm{~g}-16$ & \\
\hline $5.0 \mathrm{E} 03$ & $1.3 \mathrm{E}-16$ & \\
\hline 7.0 E 03 & $1.0 \mathrm{E}-16$ & \\
\hline $1.0 \mathrm{E} 04$ & $7.5=-17$ & \\
\hline
\end{tabular}

References:

$H^{-}+$Ar: K.H. Berkner, S.Y. Kaplan, and R.V. Pyle, Phys. ReV. 134, Al461 (1964); J.B. Hasted and J.B.H. Stedeford, Proc. Roy. Soc. (London) M227, 466 (1955); P.M. Stier and C.F. 3arnett, Phys. Rev. 103, 896 (1956); J.F. Williams, Phys. Rev. 154, 9 (1967).

$\mathrm{H}^{-}+$He: R. Surthe and J.H. Toevs, Phys. Rev. 139, Al5 (1965); P.M. Stier and C.P. Barnett, Phys. Rev. 103, 896 (1956); J.F. Williams, Phys. Rev. 154. 9 (1967).

\section{Accuracy:}

$\pm 257$

Note:

Berkner, et al., result for $\mathrm{H}^{-}+\mathrm{Ar}$ at $10 \mathrm{MeV}$ were obtained from $\mathrm{D}^{-}+\mathrm{Ar}$ at $20 \mathrm{MeV}$. 


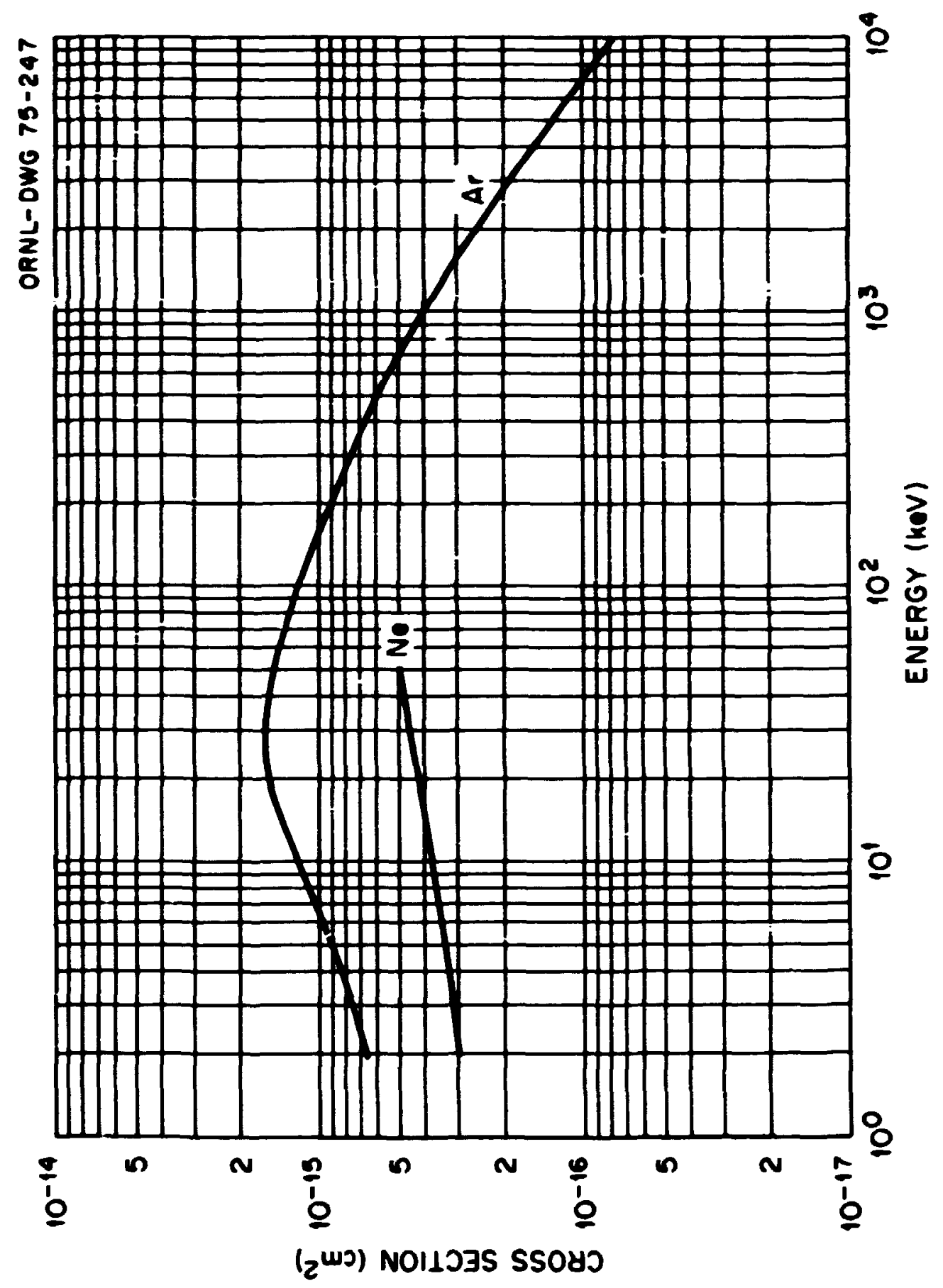




\section{A. 5.58}

Cross Sections for De Eleetron Loss

$$
\text { of } \mathrm{se}^{+} \text {Ions in } \mathrm{I}_{2} \text { and } \mathrm{He}
$$

\begin{tabular}{|c|c|c|}
\hline \multirow[t]{2}{*}{$\begin{array}{l}\text { Bneros } \\
\text { (kev) }\end{array}$} & \multicolumn{2}{|c|}{$\begin{array}{c}\text { Cross sections } \\
\left(\mathrm{cm}^{2}\right)\end{array}$} \\
\hline & $\mathrm{a}_{2}$ & He \\
\hline 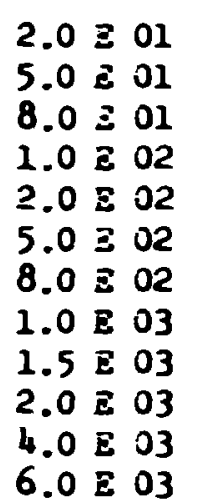 & $\begin{array}{ll}2.4 & \mathrm{~J}-18 \\
i .1 & \mathrm{E}-17 \\
2.0 & \mathrm{E}-17 \\
1.8 & \mathrm{E}-17 \\
1.5 & \mathrm{E}-17 \\
9.6 & \mathrm{E}-18\end{array}$ & $\begin{array}{ll}6.0 & \mathrm{E}-19 \\
1.9 & \mathrm{E}-18 \\
3.4 & \mathrm{z}-18 \\
4.4 & \mathrm{E}-18 \\
1.0 & \mathrm{E}-17 \\
2.2 & \mathrm{~J}-17 \\
2.3 & \mathrm{~J}-17 \\
2.2 & \mathrm{E}-17 \\
1.8 & \mathrm{E}-17 \\
1.4 & \mathrm{z}-17 \\
1.7 & \mathrm{E}-18 \\
5.2 & \mathrm{E}-18\end{array}$ \\
\hline
\end{tabular}

\section{References:}

$\mathrm{He}^{+}+\mathrm{H}_{2}$ : S.K. Allison, J. Cueves, and P.G. Murphy, Phys. Rev. 102 , 1041 (19́); L.I. Pirovar, V.M. Tubeer, and M.T. Novikor, Sov. Phys.JETP 14, 20 (1962); S.K. Allison, Phys. Rev. 109, 76 (1958); R.C. Dehmel, H.K. Chau, and H.H. Fleischmann, Atomic Data $\overline{5}, 231$ (1973).

$\mathrm{He}^{+}+\mathrm{He}$ : S.K. Allison, J. Cuevas, and P.G. Murphy, Phys. Rev. 102, 1041 (1956); P.R. Jones, F.P. Ziembe, H.A. Woses, and R. Everhart, Phys. Rev. 1:3, 182 (1959); N.V. Pedorenko, V.V. Afrosimov, and D.M. Kaminker, Sov. Phys.-Tech. Phys. 1, 1861 (1956); I. S. Dmitriev, V.S. Nikolaev, L.N. Pateeva, and Ya. A. Teplova, Suv. Phys.-JETP 15, 11 (1962); S.K. Allison, Phys. Rev. 109, 76 (1958); L.I. Pivovar, V.M. Tubaev, and X.T. Novikov, Sov. Phys.-JETP 14, 20 (1962); R.C. Dehmel, H.K. Chau, and H.H. Fleischmann, Atomic Data 5, 231 (1973); A.R. Lee and H.B. Gilbody, 3rd Int. Conf. on Phys. of Electronic Atomic Collisions (London, 15j3) North-Holland Publishing Co. (Amsterdam) p. 692 (1964). 


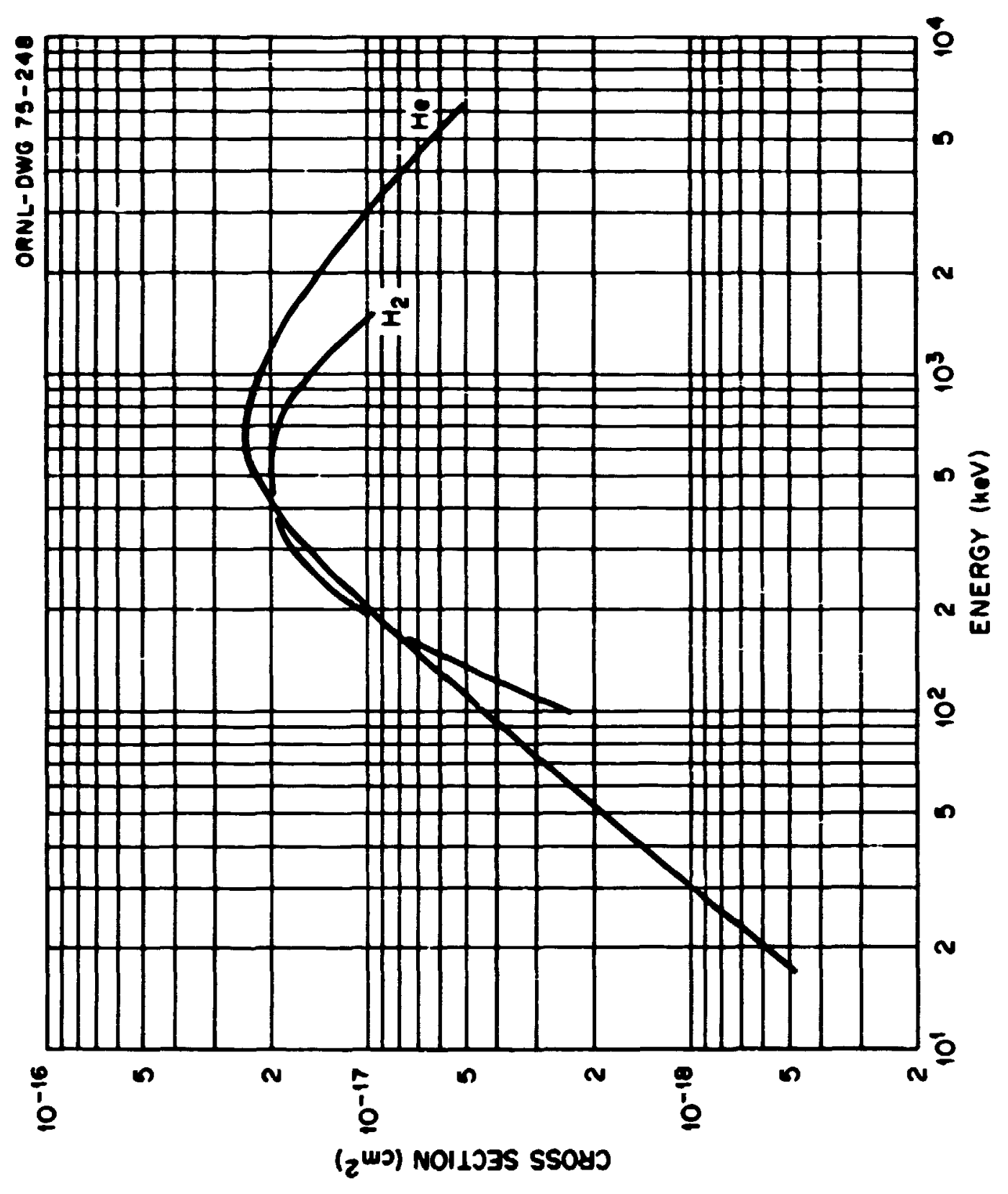


Cross Sections for the Production of Slow Electrons by ie ${ }^{+}$Ions in $i_{2}$ and ife

\begin{tabular}{|c|c|c|}
\hline \multirow[t]{2}{*}{$\begin{array}{l}\text { Energr } \\
\text { (keV) }\end{array}$} & \multicolumn{2}{|c|}{$\begin{array}{l}\text { Cross Sections } \\
\left(\mathrm{cm}^{2}\right)\end{array}$} \\
\hline & $\underline{\mathrm{H}_{2}}$ & ife \\
\hline 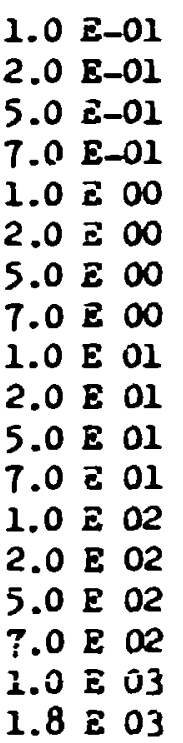 & $\begin{array}{ll}2.2 & \mathrm{E}-17 \\
3.7 & \mathrm{E}-17 \\
4.1 & \mathrm{E}-17 \\
4.0 & \mathrm{E}-17 \\
3.9 & \mathrm{E}-17 \\
3.9 & \mathrm{E}-17 \\
4.0 & \mathrm{E}-17 \\
4.0 & \mathrm{E}-17 \\
4.0 & \mathrm{E}-17 \\
5.0 & \mathrm{E}-17 \\
1.2 & \mathrm{E}-16 \\
1.7 & \mathrm{E}-16 \\
2.4 & \mathrm{E}-16 \\
3.5 & \mathrm{E}-16 \\
2.8 & \mathrm{E}-16 \\
2.4 & \mathrm{E}-16 \\
2.1 & \mathrm{E}-16 \\
1.5 & \mathrm{E}-16\end{array}$ & $\begin{array}{ll}2.4 & \mathrm{E}-17 \\
2.5 & \mathrm{E}-17 \\
2.7 & \mathrm{E}-17 \\
2.8 & \mathrm{E}-17 \\
2.9 & \mathrm{E}-17 \\
3.2 & \mathrm{E}-17 \\
3.8 & \mathrm{E}-17 \\
4.2 & \mathrm{E}-17 \\
4.4 & \mathrm{E}-17 \\
5.2 & \mathrm{E}-17 \\
7.3 & \mathrm{E}-17 \\
8.6 & \mathrm{E}-17 \\
1.1 & \mathrm{E}-16 \\
1.8 & \mathrm{E}-16 \\
1.8 & \mathrm{E}-16 \\
1.6 & \mathrm{E}-16 \\
1.3 & \mathrm{E}-16 \\
9.0 & \mathrm{E}-17\end{array}$ \\
\hline
\end{tabular}

\section{References:}

$\mathrm{He}^{+}+\mathrm{H}_{2}$ : R.A. Langley, D.W. Martin, D.S. Harmer, J.W. Hooper, and E.W. McDaniel, Phys. Rev. 136, A379 (i964); L.I. Pivovar, Yu. Z. Levchenko, and A.N. Grigor'ev, Sor. Phys.-JETP 27, 699 (190́8); i..B. Gilbody and J.B. Hasted, Proc. Roy. Soc. A240, $3 \overline{82}$ (1957); H.B. Gilbody, J.B. Hasted, J.V. Ireland, A.R. Lee, E.W. Thomas, and A.S. Whitman, Proc. Roy. Soc. A274, 40 (1963); E.S. Solov'ev, R.A. II'in, V.A. Oparin, and I.V. Fedorenko, Sor. Phys.-JETP 18, 342 (1964); F.J. de Heer, J. Schutten, and H. Moustafa, Physica 32, 1793 (i966).

$\mathrm{He}^{+}+$He: F.J. de Heer, J. Schutten, and H. Moustafa, Physica 32, 1793 (1966); E.S. Solov'ev, R.N. II'in, V.A. Oparin, and N.V. Fedorenko, Sov. Phys.-JETP 18, 342 (1964); N.V. Fedorenko, V.V. Afrosimov, and D.M. Kaminker, Sov. Phys.-JETP $\frac{1}{1}, 1861$ (1956); H.B. Gillbody, J.B. Hasted, J.V. Ireland, A.R. Lee, E.W. Thomas, and A.S. Whiteman, Proc. Roy. Soc. A274, 40 (1963); H.B. Gilbody and J.B. Hasted, Proc. Roy. Snc. A240, 382 (1957); L.I. Pivovar, Yu. Z. Levchenko, and A.I. Grigor'ev, Sov. Phys.-JETP 27, 699 (1968); R.A. Langley, D.W. Martin, D.S. Harmer, J.W. Hooper, and E.iH. McDaniel, Phys. Rev. 136, A379 (1964).

\section{Accuracy:}

$\pm 25 \%$ 
A. 5.61

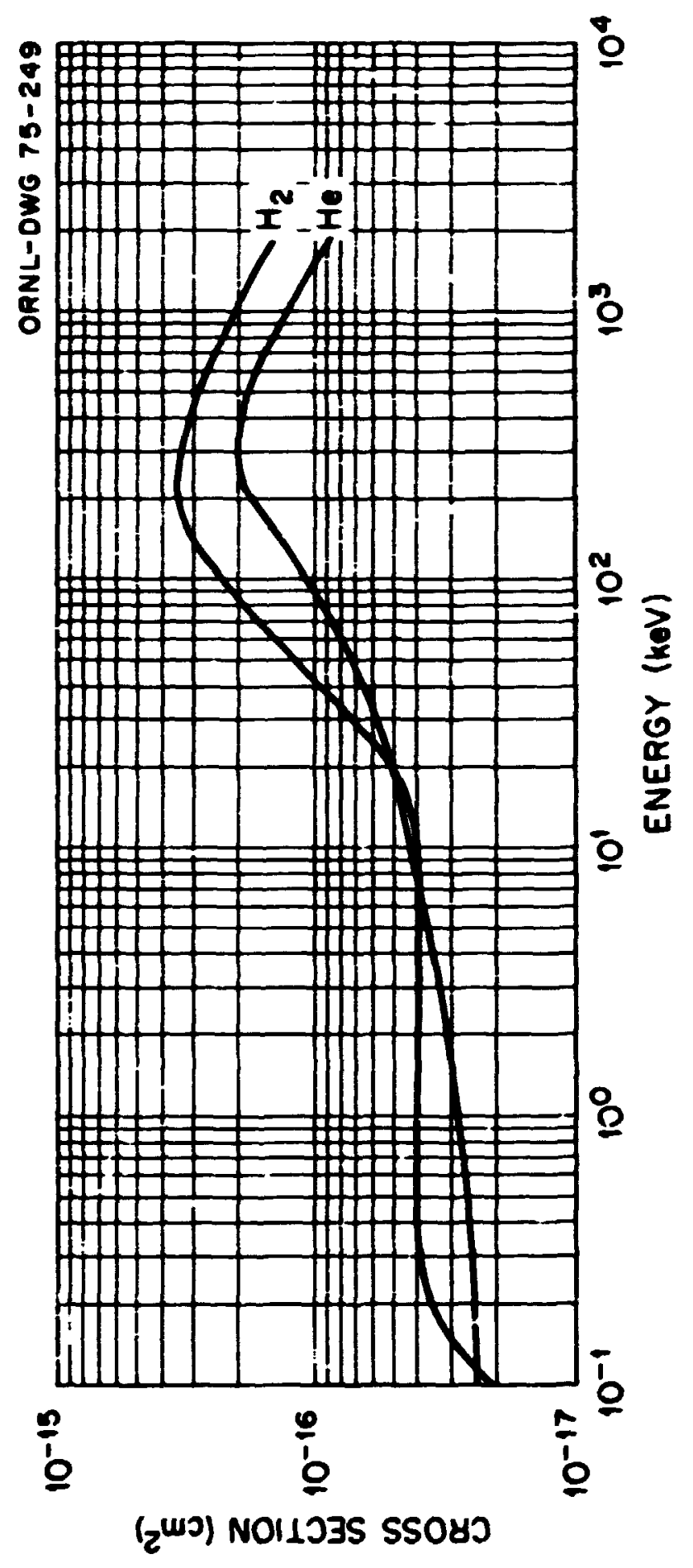




\section{A.5.62}

Cross Sections for the Production of Positive

Ians by $\mathrm{He}^{+}$Ions in $\mathrm{H}_{2}$ and ite

\begin{tabular}{|c|c|c|}
\hline \multirow[t]{2}{*}{$\begin{array}{l}\text { Energy } \\
\text { (keV) }\end{array}$} & \multicolumn{2}{|c|}{$\begin{array}{c}\text { Cross Sections } \\
\left(\mathrm{cm}^{2}\right)\end{array}$} \\
\hline & $\mathrm{H}_{2}$ & Se \\
\hline 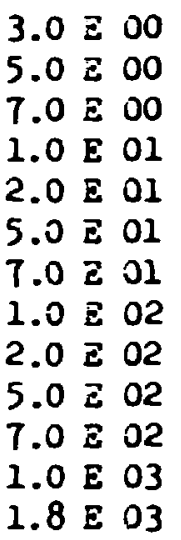 & $\begin{array}{ll}1.3 & E-16 \\
1.1 & \Xi-16 \\
1.2 & \mathrm{E}-16 \\
1.5 & \mathrm{E}-16 \\
2.2 & \mathrm{E}-16 \\
4.0 & \mathrm{E}-16 \\
4.7 & \mathrm{E}-16 \\
5.3 & \mathrm{E}-16 \\
4.5 & \mathrm{E}-16 \\
2.8 & \mathrm{E}-16 \\
2.3 & \mathrm{E}-16 \\
1.8 & \mathrm{E}-16 \\
1.3 & \mathrm{E}-16\end{array}$ & 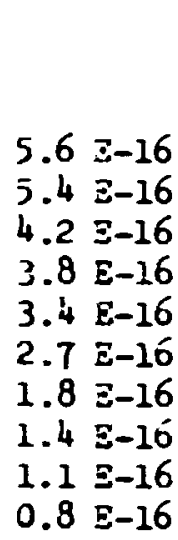 \\
\hline
\end{tabular}

\section{References:}

$\mathrm{He}^{+}+\mathrm{H}_{2}$ : K.A. Langley, D.W. Martin, D.S. Harmer, J.W. Hooper, a:Id E.H. McDaniel, Phys. Rev. 136, A379 (1964); L.I. Pivovar, Yu. Z. Levcherko, and A.N. Grigor'ev, Sov. Phys.-JETP 27, 699 (1968); H.B. Gilbciy, J.B. Hasted, J.V. Ireland, A.R. Lee, E.W. Thomas, and A.S. Whiteman, Proc. Roy. Soc. A274, 40 (1963); E.S. Solov'ev, R.N. Il'in, V.A. Oparin, and N.V. Fedorenko, Sov. Phys.-JETP 18, 342 (1964); F.J. de Heer, J. Schutten, and H. Moustafa, Physica 32, 1793 (1966); R. Browning, C.J. Latimer, and H.B. Gilbody, J. Phys. B 2, 534 (1969).

He $^{+}+$He: N.V. Fedorenko, V.V. Afrosimov, and D.M. Kaminker, Sov. Phys.JETP 1, 1861 (1956); F.J. de Heer, J. Schutten, and H. Moustafa, Physica 32, 1793 (1966); E.S. Solov'ev, R.N. Il'in, F.A. Oparin, and I.V. Fedorenko, Sov. Phys.-JETP 18, 342 (1964); H.B. Gilbody, J.B. Hasted, J.V. Irelant, A.R. Lee, E.W. Thomas, and A.S. Whiteman, Proc. Roy. Soc. A274, $40($ i 6,3$)$; L.I. Pivovar, Yu. Z. Levchenko, and A.N. Grigor'ev, Scv. Phys--JETP 27, 699 (1968); R.A. Langley, D.W. Martin, D.S. Harmer, J.H. Hooper, and E.H. McDaniei, Phys. Rev. 136, A 379 (1964).

\section{Accuracy:}

$\pm 25 \%$ 


\section{A. 5.63}

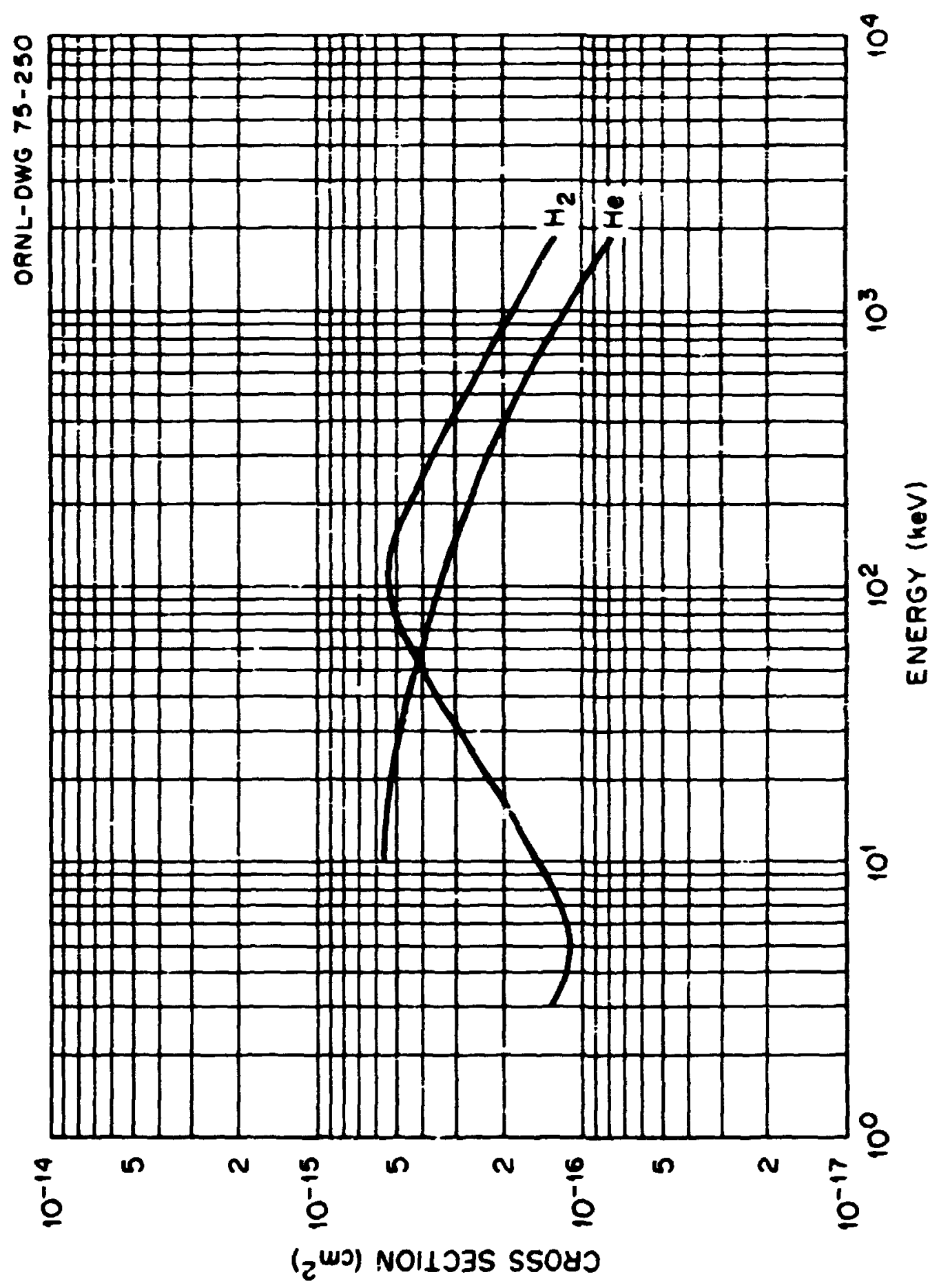




\section{A. 5.64}

Cross jections for Production of

$$
x_{2}^{+}, f^{+} \text {, and } i^{2+} \text { oy } \mathrm{He}^{+} \text {in } i_{2}
$$

\begin{tabular}{|c|c|c|c|}
\hline $\begin{array}{l}\text { Energy } \\
\text { (keV) }\end{array}$ & & $\begin{array}{l}\text { Tross Section } \\
\qquad\left(\mathrm{sm}^{2}\right)\end{array}$ & \\
\hline & $\mathrm{x}_{2}{ }^{+}$ & $x^{+}$ & $i^{2+}$ \\
\hline $\begin{array}{lll}5.5 & \mathrm{E} & 0 \\
1.0 & \mathrm{E} & 01 \\
2.0 & \mathrm{E} & 01 \\
3.0 & \mathrm{E} & 01 \\
4.0 & \mathrm{E} & \mathrm{O} \\
4.5 & \mathrm{E} & 01\end{array}$ & $\begin{array}{ll}7.91 & E-17 \\
1.67 & E-16 \\
3.47 & E-10 \\
4.34 & E-16 \\
4.70 & E-16 \\
4.98 & \Xi-16\end{array}$ & 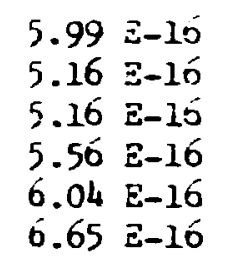 & $\begin{array}{ll}1.44 & \Xi-17 \\
2.20 & \Xi-17 \\
4.03 & \Xi-17 \\
5.99 & \Xi-17 \\
7.66 & \Xi-17 \\
8.28 & \mathrm{E}-17\end{array}$ \\
\hline
\end{tabular}

\section{References:}

R. Browning, C.J. Latimer, and H.B. Gilbody, J. Phys. B 2, 534 (2969).

\section{Accuracy:}

$\pm 20 \%$. 


\section{A. 5.65}

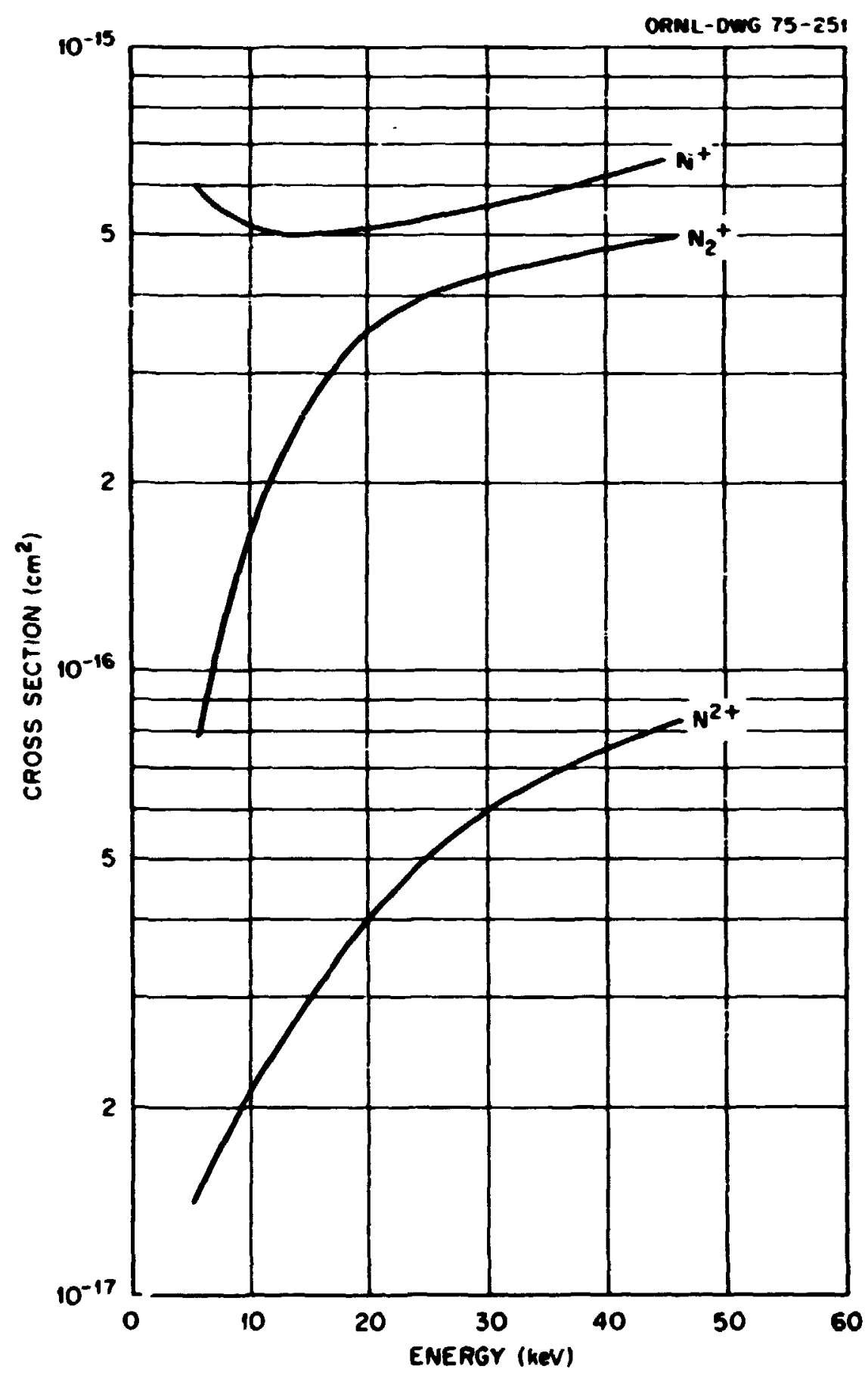




\section{A. 5.66}

Cross Sections for the Production of

$$
\mathrm{O}_{2}^{+}, \mathrm{O}^{+} \text {, and } \mathrm{O}^{2+} \text { Ions by } \mathrm{He}^{+} \text {in } \mathrm{O}_{2}
$$

\begin{tabular}{|c|c|c|c|}
\hline \multirow[t]{2}{*}{$\begin{array}{l}\text { Energy } \\
\text { (kev) }\end{array}$} & \multicolumn{3}{|c|}{$\begin{array}{c}\text { Cross Sections } \\
\left(\mathrm{cm}^{2}\right)\end{array}$} \\
\hline & $\mathrm{O}_{2}^{+}$ & $0^{+}$ & $0^{2+}$ \\
\hline 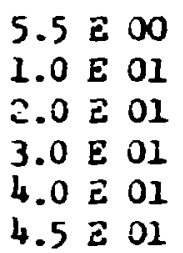 & $\begin{array}{ll}8.22 & \Xi-17 \\
1.43 & \mathrm{E}-16 \\
2.62 & \mathrm{E}-16 \\
3.34 & \mathrm{z}-16 \\
3.56 & \mathrm{z}-16 \\
3.59 & \mathrm{z}-16\end{array}$ & $\begin{array}{ll}8.20 & \mathrm{~J}-16 \\
7.64 & \mathrm{E}-16 \\
6.95 & \mathrm{E}-16 \\
7.06 & \mathrm{\Xi}-16 \\
7.22 & \mathrm{E}-16 \\
7.24 & \mathrm{E}-16\end{array}$ & $\begin{array}{ll}1.31 & \Xi-17 \\
2.17 & E-17 \\
4.18 & \ddot{E}-17 \\
5.95 & E-17 \\
6.99 & E-17 \\
7.10 & E-17\end{array}$ \\
\hline
\end{tabular}

References:

R. Browning, C.J. Latimer, and H.B. Gilbody, J. Phys. 3 2, 534 (1969). Accuracy:

$\pm 20 \%$. 


\section{A. 5.67}

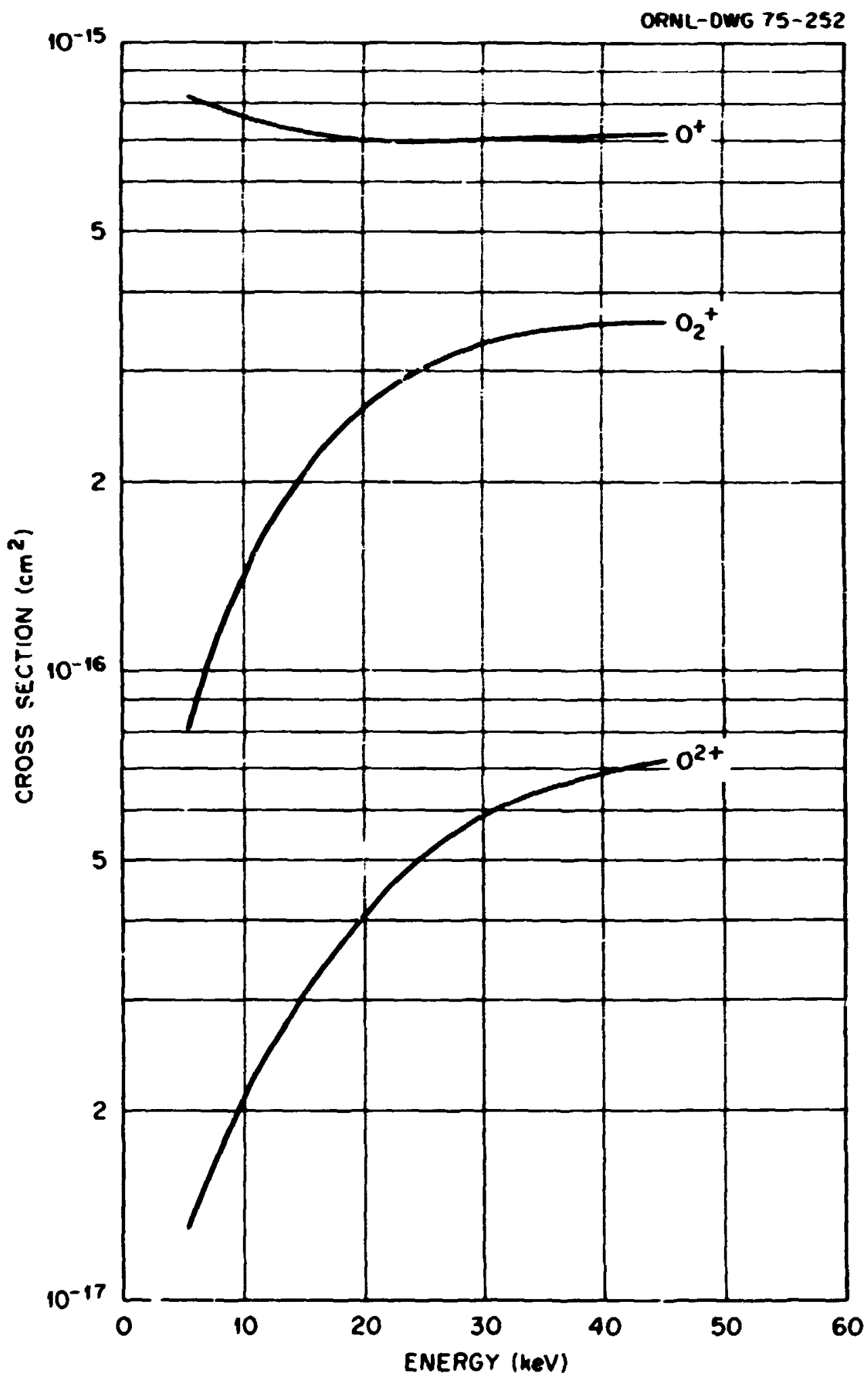




\section{A.5.68}

Cross Jections for Positive Ion Production

ty He $^{+t}$ Ions in if 2 and ie

\begin{tabular}{|c|c|c|}
\hline \multirow[t]{2}{*}{$\begin{array}{l}\text { Bneror } \\
\text { (kei) }\end{array}$} & \multicolumn{2}{|c|}{$\begin{array}{l}\text { Cross Sections } \\
\left(\mathrm{cm}^{2}\right)\end{array}$} \\
\hline & $\underline{H_{2}}$ & :e \\
\hline $\begin{array}{lll}6.8 & E & 00 \\
1.0 & \Xi & 01 \\
2.0 & E & 01 \\
4.0 & E & 01 \\
6.0 & \Xi & 01 \\
8.0 & E & 01 \\
1.0 & E & 02 \\
1.5 & E & 02 \\
2.0 & E & 02 \\
4.0 & E & 02 \\
6.0 & E & 02 \\
8.0 & E & 02 \\
1.0 & E & 02\end{array}$ & $\begin{array}{ll}3.1 & \mathrm{E}-16 \\
4.2 & \mathrm{E}-16 \\
6.8 & \mathrm{E}-16 \\
1.2 & \mathrm{E}-15 \\
1.3 & \mathrm{z}-15 \\
1.3 & \mathrm{E}-15 \\
1.3 & \mathrm{E}-15 \\
1.3 & \mathrm{E}-15 \\
1.2 & \mathrm{E}-15 \\
8.1 & \mathrm{z}-16 \\
6.3 & \mathrm{E}-16 \\
5.1 & \mathrm{z}-16 \\
4.5 & \mathrm{E}-16\end{array}$ & $\begin{array}{ll}5.7 & \mathrm{~B}-16 \\
5.4 & \mathrm{E}-16 \\
4.0 & \mathrm{E}-16 \\
3.2 & \mathrm{E}-10 \\
2.6 & \mathrm{Z}-16 \\
2.3 & \mathrm{E}-16\end{array}$ \\
\hline
\end{tabular}

\section{References:}

ite $+H_{2}$ : L.J. Puckett, G.O. Taylor, and D.J. Martin, Phys. Rev. 178, 271 (1969); R.A. Langley, D.W. Martin, D.S. Harmer, J.A. Hooper, and E.H. MeDaniel, Phys. Rev. 136́, A 379 (1964); J.G. Graham, C.J. Latimer, R. Browning, and H.B. Gilbody, J. Phys. B I, L405 (1974).

He + He: L.J. Puckett, G.0. Taylor, and D.d. Martin, Phys. Rev. 178 , 271 (1969); R.A. Langley, D.W. Mrtin, D.j. Harmer, J.W. Hooper, and E.H. McDaniei, Phys. Rev. 136, A379 (1964).

Accuracy:

$\pm 20 \%$. 
A. 5.69

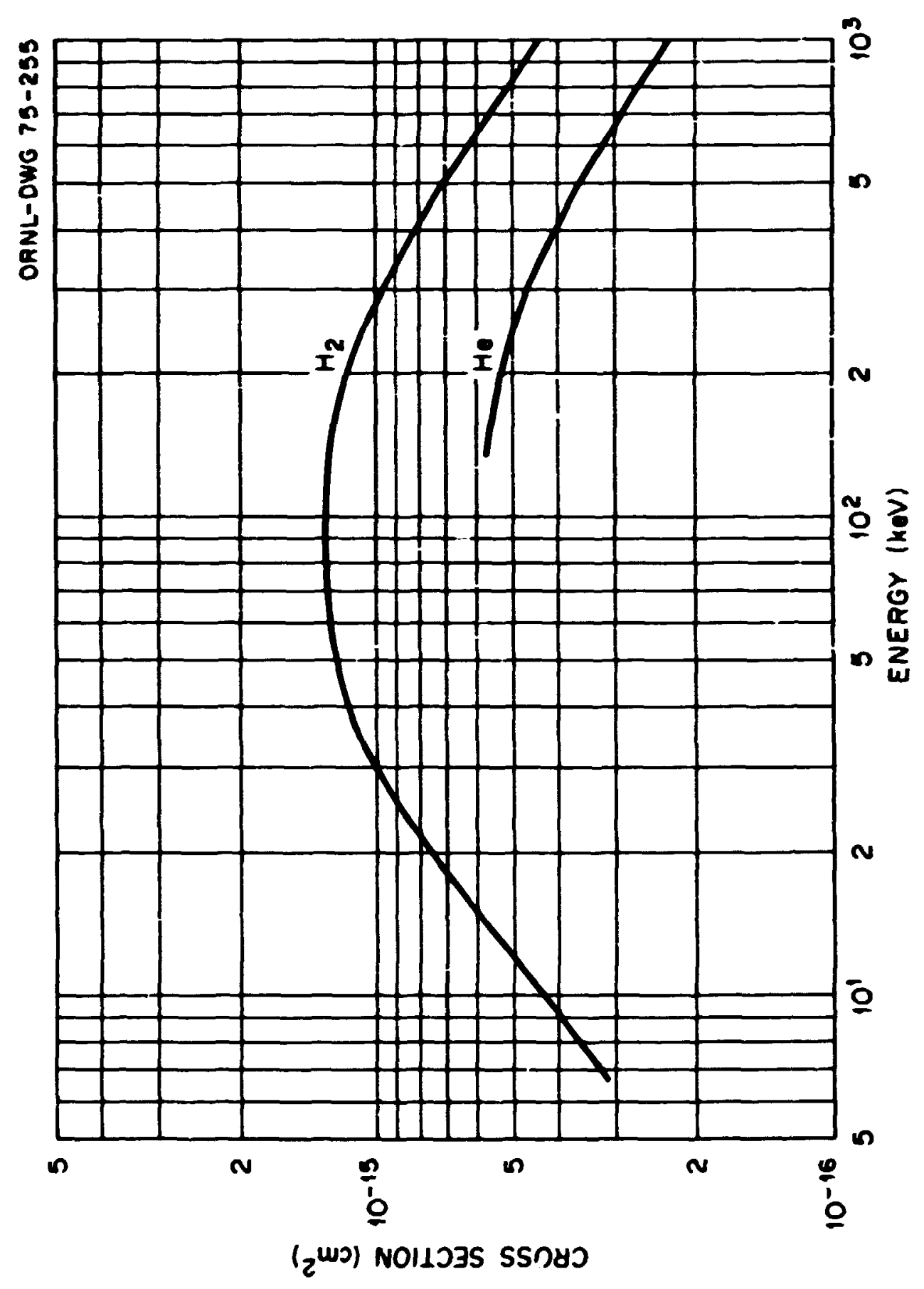




\section{A. 5.70}

Cross Sections for the Production of Free

zlectrons by $\mathrm{He}^{\mathrm{t+}}$ in $\mathrm{H}_{2}$ and ite

\begin{tabular}{|c|c|c|}
\hline \multirow[t]{2}{*}{$\begin{array}{l}\text { Derce } \\
\text { (keV) }\end{array}$} & \multicolumn{2}{|c|}{$\begin{array}{c}\text { Cross Sections } \\
\left(\mathrm{cm}^{2}\right)\end{array}$} \\
\hline & $\mathbf{H}_{2}$ & he \\
\hline 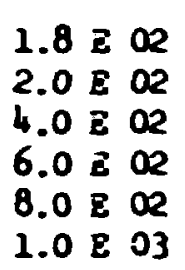 & $\begin{array}{l}6.3 \mathrm{E}-16 \\
6.9 \mathrm{E}-16 \\
5.9 \mathrm{E}-16 \\
4.9 \mathrm{E}-16 \\
4.2 \\
.2-16\end{array}$ & $\begin{array}{ll}1.3 & \mathrm{E}-16 \\
1.5 & \mathrm{E}-16 \\
2.5 & \mathrm{E}-16 \\
2.5 & \mathrm{z}-16 \\
2.3 & \mathrm{~J}-16 \\
2.1 & \mathrm{E}-16\end{array}$ \\
\hline
\end{tabular}

\section{References:}

$\mathrm{He}^{+t}+\mathrm{H}_{2}$, He: L.J. Puckett, G.O. Taylor, and D.it. Martin, Phys. Rev. 178,271 (1969).

Accuracy:

$\pm 20 \%$. 


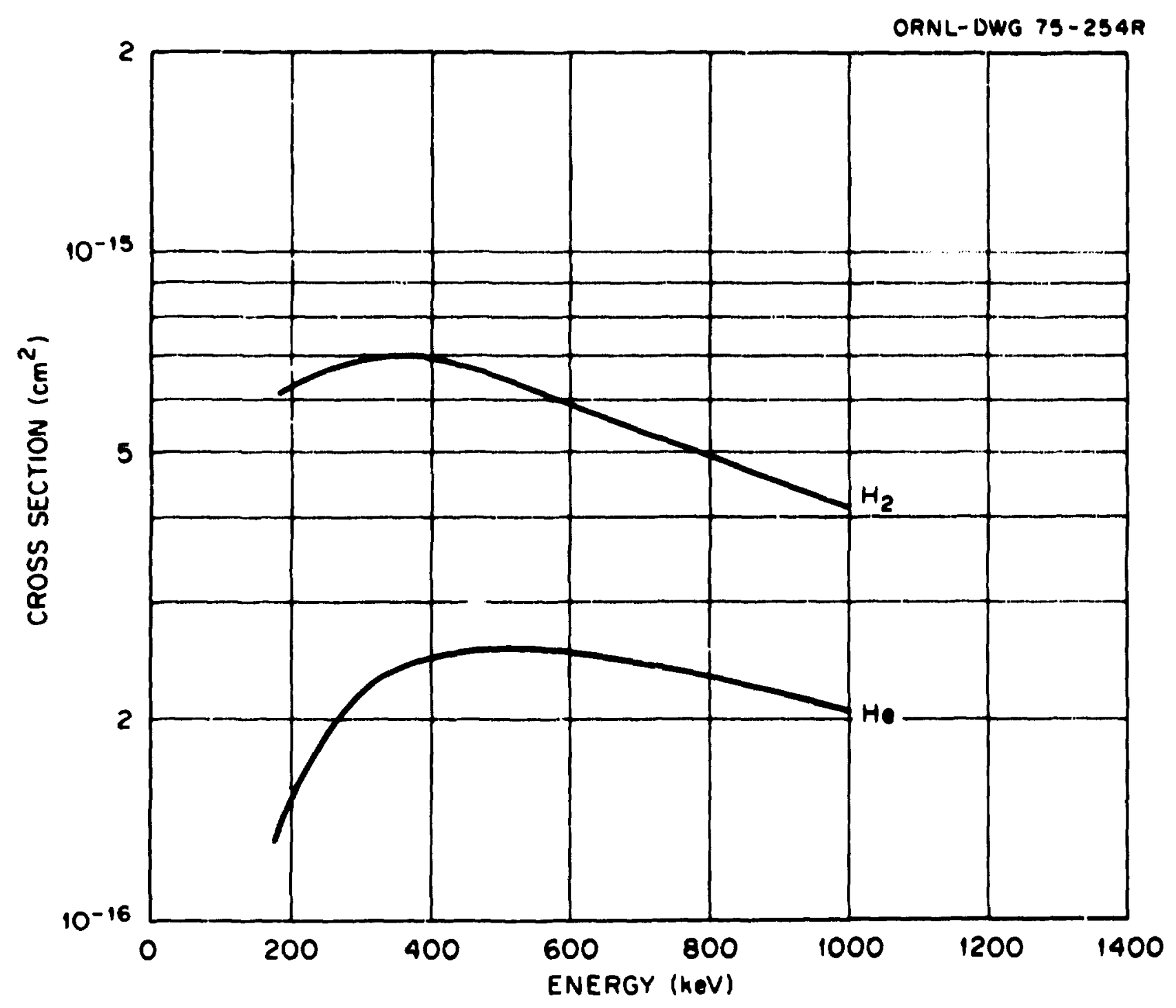


Cross jections for De and Tro Bisciron Woss

for ie Atoms in $\ddot{Z}_{2}$ and He

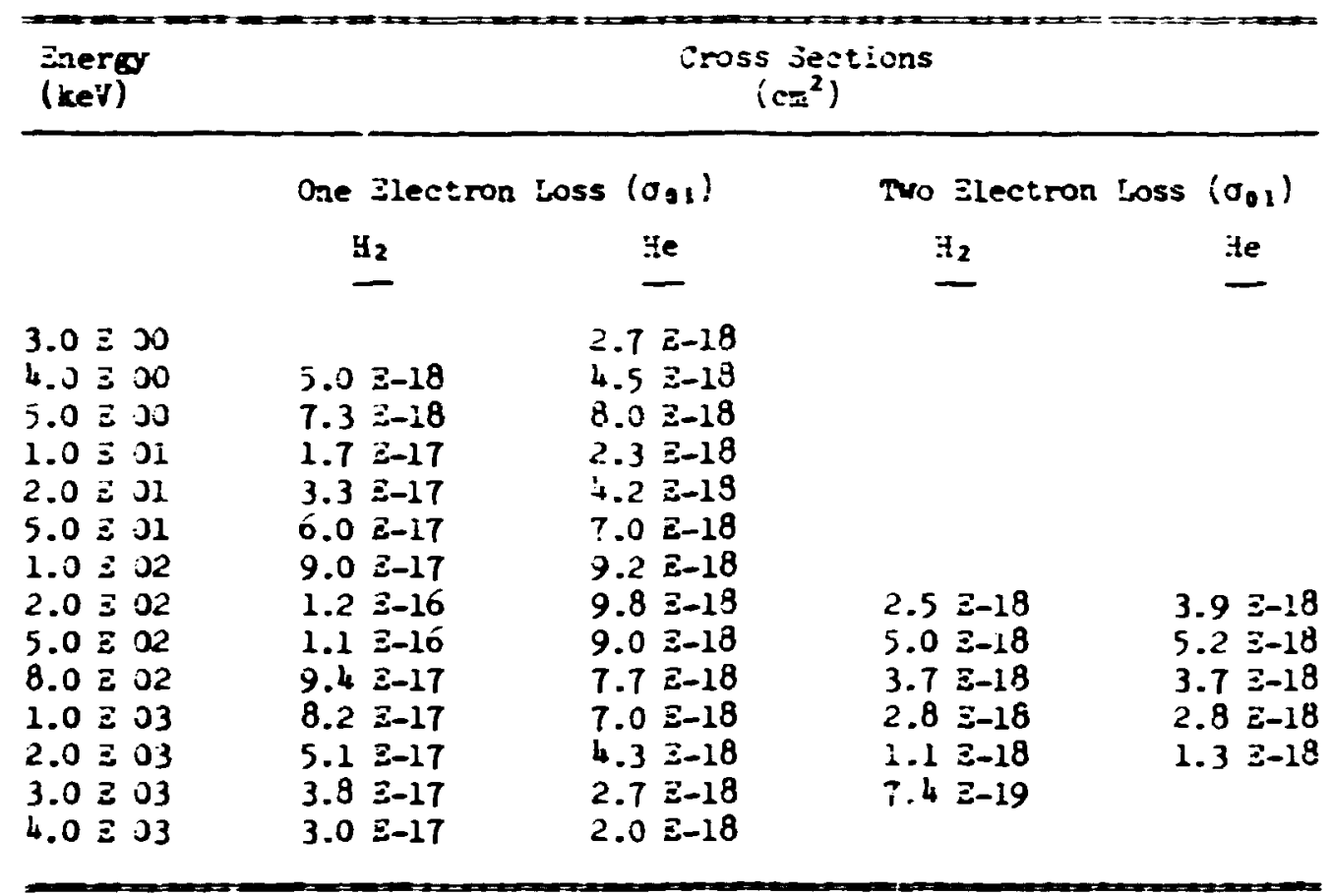

\section{References:}

He + I $_{2}:$ Z.h. Pedersen and P. Ivelplund, J. Phys. B 7, 132 (1974);

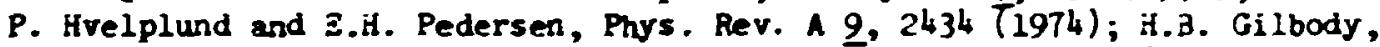
K.F. Dunn, R. 3rowning, and C.J. Latimer, J. Phys. 3 3, 1105 (1970); C.P. Barnett and P.A. Stier, Phys. Rev. 109, 385 (1958); H. Tavara, J. Phys. Sx. Jap. 31, 871 (1971); S.K. Allison, Rev. Mod. Phys. 30, 1137 (1958); Y3. M. FogeI, V.A. Anicudinov, and D.V. Pilipenko, jov. Phys.J JTP $11,18(1960)$; i. B. Gilbody, R. Browning, G. Levy, A.I. McIntosh, and K.P. Dunn, J. Phys. B 1, 863 (1968); A.3. Nittkower, J. Levy, and i.3. Gilbody, Proc. Phys. Soc. 91,862 (1967).

He t ile: Z.i. Pedersen and P. Hvelplund, J. Phys. 3 7, 132 (1974); P. Hvelplund and $\Xi . H$. Pedersen, Phys. Rev. A 2, 2434 (1974); C.F. Barnett and P.M. Stier, Phys. Rev. 109, 386 (1958); :. Tawara, J. Phys. Joc. Jap. 31, 236 (1971); 3.K. Allison, Rev. Mod. Phys. 30, 1137 (1958); Ya. M. Pogel, V.A. Ankudinov, and D.V. Pilipenko, Sov. Phys.-JETP 11, 18 ( 1960); :1.B. Jilbody, K.P. Durs, R. Broming, and C.J. Latimer, J. Phys. B 3, 1105 (1970); W.L. Williams and F.M. Goldberg, VII Int. Conf. on the Phys. of Electronic Atomic Collisions, Jorth Jolland Publishing Co. Amsterdann (1971) p. 1087; i.3. Gilbody, R. Browning, G. Levy, A.I. MeIntosh, and K.F. Dunn, J. Phys. B 1, 863 (1968); A.3. Wittkower, 6. Levy, and H.B. Gillbody, Proc. Phys. Joc. 91 , 862 (1967).

\section{Hote:}

Measurements made before 1970 did not properly take into account the presence of He metastable states.

Accuracy: $\pm 25 \%$. 


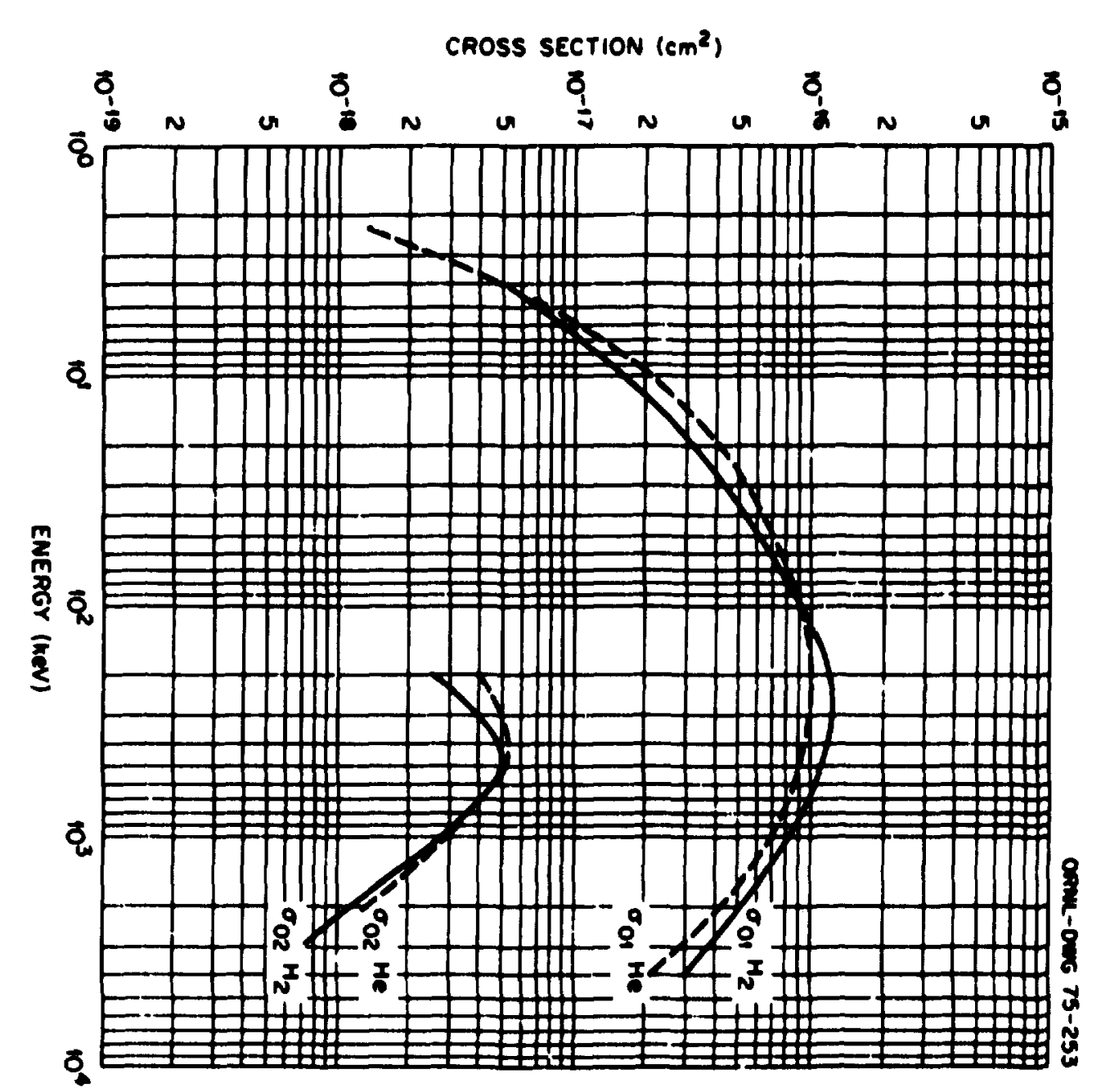


Cross Sections for Ionization of $\mathrm{H}_{2}$ and ife by He Atoms

\begin{tabular}{|c|c|c|}
\hline \multirow[t]{2}{*}{$\begin{array}{l}\text { Energy } \\
\text { (keV) }\end{array}$} & \multicolumn{2}{|c|}{$\begin{array}{c}\text { Cross Sections } \\
\left(\mathrm{cm}^{2}\right)\end{array}$} \\
\hline & $\underline{I_{2}}$ & He \\
\hline $\begin{array}{ll}4.6 & \Xi-02 \\
5.0 & \Xi-02 \\
7.0 & \Xi-02 \\
1.0 & \Xi-01 \\
2.0 & \mathrm{E}-01 \\
5.0 & \mathrm{E}-01 \\
9.0 \mathrm{E}-01 \\
3.0 \mathrm{E} 00 \\
5.0 \mathrm{E} 00 \\
1.0 \mathrm{E} 01 \\
2.0 \mathrm{E} & 01 \\
5.0 \mathrm{E} & 01 \\
1.0 \mathrm{E} & 02 \\
2.0 \mathrm{E} & 02 \\
5.0 \mathrm{E} & 02 \\
1.0 \mathrm{E} & 03\end{array}$ & $\begin{array}{ll}2.5 & E-20 \\
3.5 & \mathrm{E}-19 \\
6.0 & \mathrm{E}-19 \\
2.2 & \mathrm{E}-18 \\
1.0 & \mathrm{E}-17 \\
2.9 & \mathrm{E}-17 \\
4.3 & \mathrm{E}-17 \\
3.8 & \mathrm{E}-17 \\
5.0 & \mathrm{E}-17 \\
7.7 & \mathrm{E}-17 \\
1.1 & \mathrm{E}-16 \\
1.8 & \mathrm{E}-16 \\
2.4 & \mathrm{E}-16 \\
2.6 & \mathrm{E}-16 \\
1.9 & \mathrm{E}-16 \\
1.2 & \mathrm{E}-16\end{array}$ & $\begin{array}{ll}5.0 & \mathrm{E}-17 \\
8.4 & \mathrm{E}-17 \\
1.3 & \mathrm{E}-16 \\
1.4 & \mathrm{E}-16 \\
1.1 & \mathrm{E}-16 \\
7.3 & \mathrm{E}-17\end{array}$ \\
\hline
\end{tabular}

\section{References:}

He + H2: N.G. Itterback, Phys. Rev. Letts. 12, 295 (1964); L.J. Puckett, G.0. Taylor, and D.W. Martin, Phys. Rev. 178, 271 (1969); E.S. Solov'ev, R... Il'in, V.A. Oparin, and N.V. Fedorenko, Sov. Phys.-JETP 18, 342 (1964); E.S. Solov'ev, R.N. Il'in, V.A. Oparin, and N.V. Pedorenko, 3rd Int. Conf. on Phys. of Electronic Atomic Collisions (London, 1963) North-iolland Publishing Co. (Amsterdarm) P. 692 (1964); R. Browning, C.J. Latimer, and H.B. Gilbody, J. Phys. B $\underline{3}, 667$ (1970).

He + He: I..J. Puckett, G.0. Taylor, and D.W. Martin, Prys. Rev. 178, 271 (1969); E.S. Solov'ev, R.i. II'in, V.A. Oparin, and Y.V. Fedorenko, Sov. Phys.-JETP 18 , 342 (1964); E.S. Solov'ev, R.N. Il'in, V.A. Cparin, and N.V. Fedorenixo, $\overline{3} d$ Int. Conf. on Phys. of Electronic \& Atomic Collisions (London, 1963) North-Holland Publishing Co. (Amsterdanm) p. 672 (1964).

\section{Accuracy:}

$\pm 25 \%$. 
A. 5.75

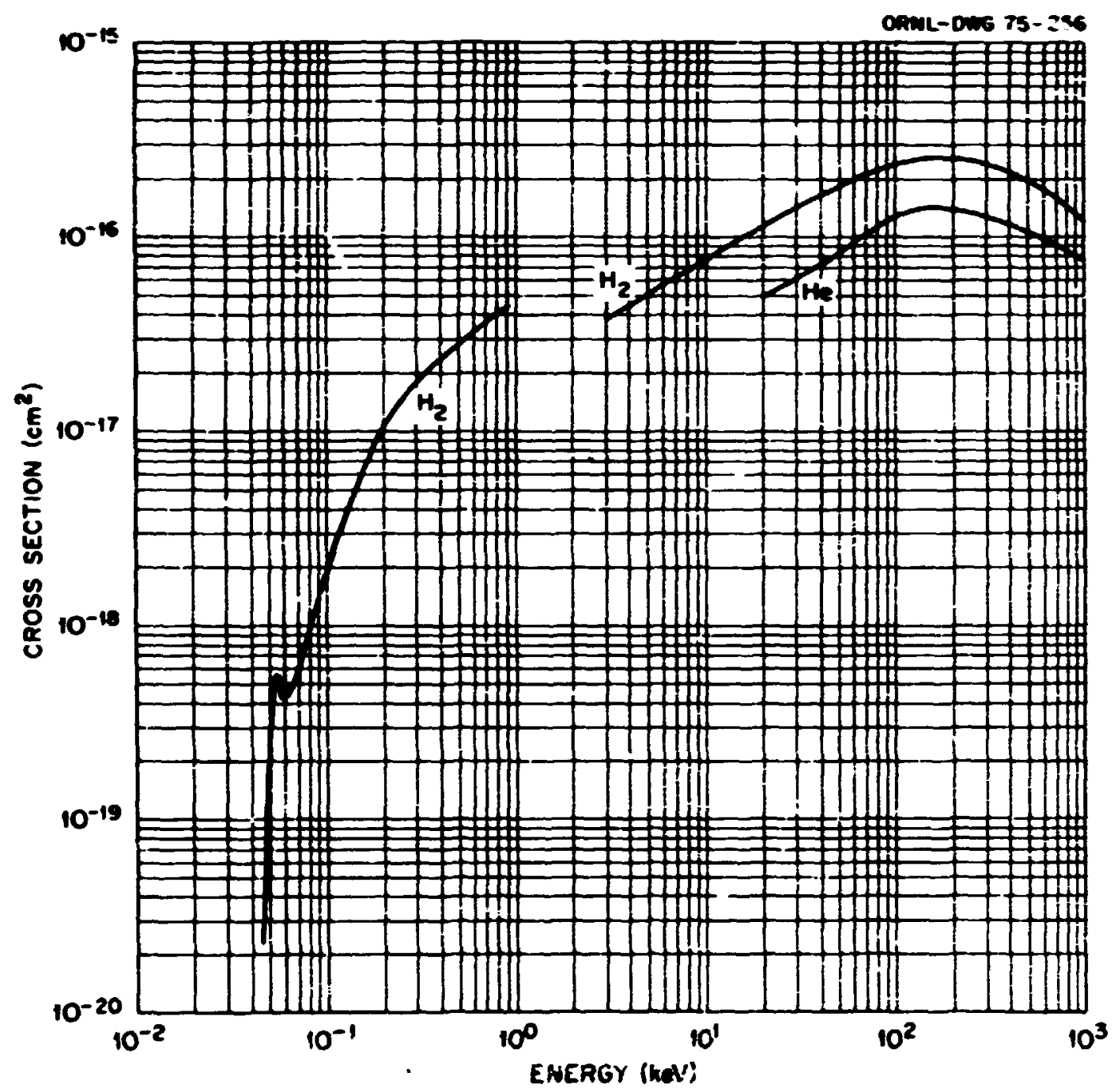




\section{A.6 Equilibrium Fractions}


Equilibrium Fractions of a Hydrogen Beam in $\mathrm{H}$ and $\mathrm{H}_{2}$

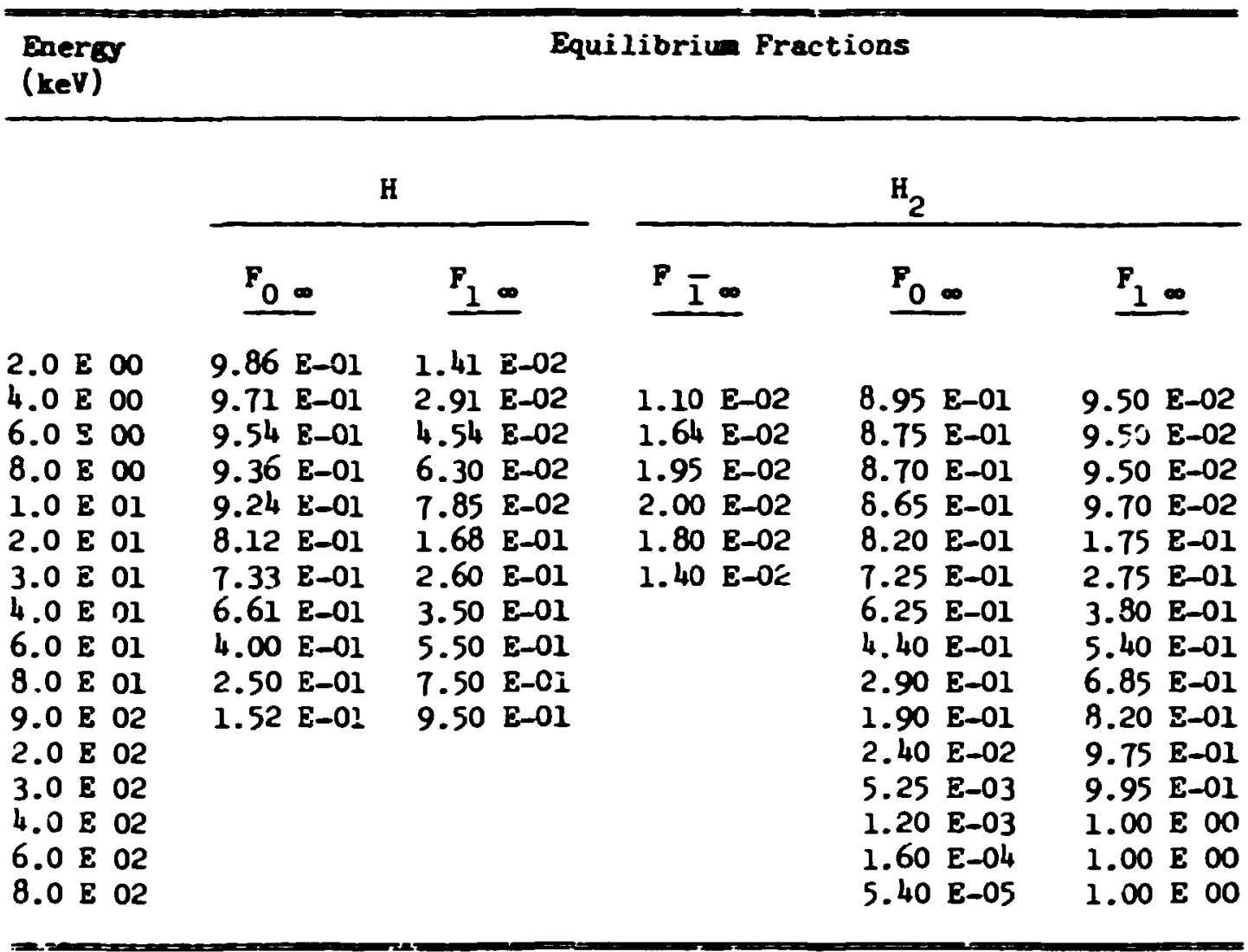

References:

$\mathrm{H}^{+}+\mathrm{H}$ : There is no direct measurement for this case. We have nere generated fractions using the formulae $F_{0 \infty}=\sigma_{10} /\left(\sigma_{10}+J_{01}\right)$ and $F_{1 \infty}=$ $\sigma_{01} /\left(\sigma_{10}+\sigma_{01}\right)$; the values of $\sigma_{01}$ and $\sigma_{10}$ are from M. Tawara and A. Russek, Fev. Mod. Phys. 45, 178 (1973). There is a slight error (perhaps as ruch as $2 \%$ ) in neglect of negative state formation and loss.

$\mathrm{H}^{+}+\mathrm{H}_{2}$, Experimental: From the review by S.K. Allison and M. GarcisKunoz, "Atomic and Molecular Processes," (ed. D.R. Bates, Academic Press, N.Y. 1962) page 721. Also derived values (see Note 1 at end of chapter) are used above $1000 \mathrm{keV}$ based on cross section values of L. Toburen et al. Phys. Rev. 171, 114 (1968) and U. Schryber, Helv. Phys. Acta 39, $562(1906)$.

Accuracy:

Systematic error is negligible. Random error $< \pm 5 \%$.

Notes:

See Note (4) at end of chapter. 


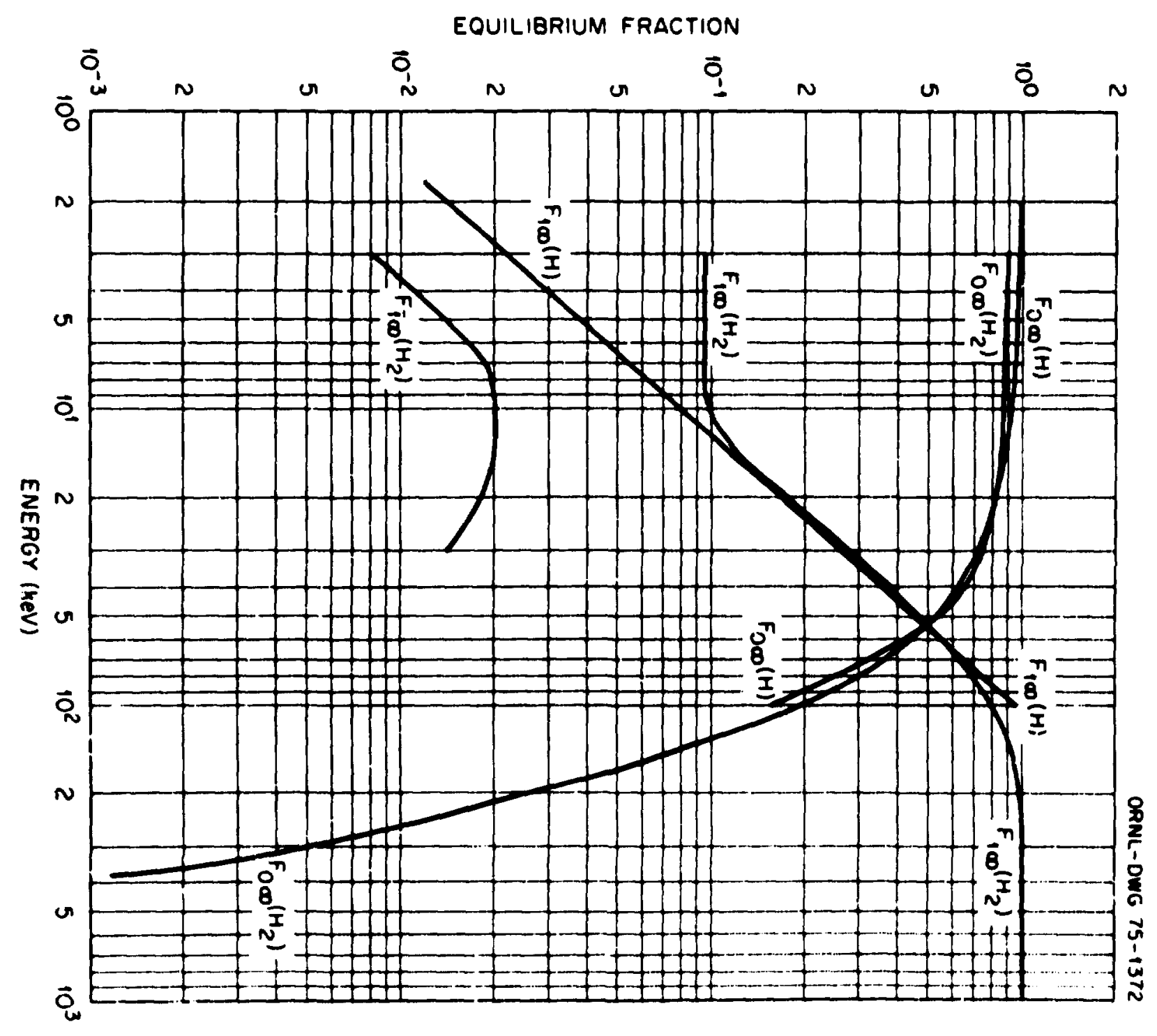




\section{A.6.4}

Equilibrium Fractions of a djdrogen Beam in He and Ar

\begin{tabular}{|c|c|c|c|c|c|c|}
\hline \multirow[t]{3}{*}{$\begin{array}{c}\text { Energy } \\
\text { (kev) }\end{array}$} & \multicolumn{6}{|c|}{ Equilibriu Fractions } \\
\hline & \multicolumn{3}{|c|}{$\mathrm{He}$} & \multicolumn{3}{|c|}{ Ar } \\
\hline & $\overline{1} \overline{1}_{\infty}$ & $F_{0 \infty}$ & $F_{1 \infty}$ & $\mathbf{F} \bar{I}_{\infty}$ & $F_{0 \infty}$ & $F_{1 \infty}$ \\
\hline $\begin{array}{lll}4.0 & E & 00 \\
6.0 & E & 00 \\
8.0 & E & 00 \\
1.0 & E & 01 \\
2.0 & E & 01 \\
3.0 & E & 01 \\
4.0 & E & 01 \\
6.0 & E & 01 \\
8.0 & E & 01 \\
1.0 & E & 02 \\
2.0 & E & 02 \\
3.0 & E & 02 \\
4.0 & E & 02 \\
6.0 & E & 02 \\
8.0 & E & 02 \\
1.0 & E & 03\end{array}$ & $\begin{array}{ll}9.20 & E-03 \\
9.55 & E-03\end{array}$ & $\begin{array}{ll}1.25 & E-01 \\
2.38 & E-01 \\
3.35 & E-01 \\
4.10 & E-01 \\
6.00 & E-01 \\
6.00 & E-01 \\
5.45 & E-01 \\
4.40 & E-01 \\
3.35 & E-01 \\
2.54 & E-01 \\
6.05 & E-02 \\
1.75 & E-02 \\
6.45 & E-03 \\
1.55 & E-03 \\
5.20 & E-04 \\
3.60 & E-04\end{array}$ & 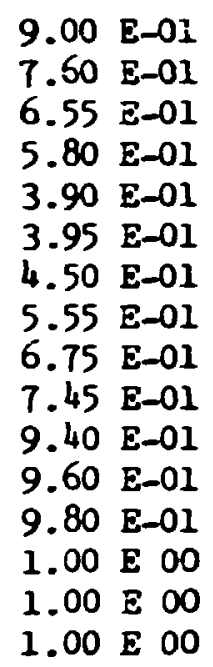 & $\begin{array}{ll}4.00 & E-02 \\
3.10 & E-03 \\
2.00 & E-02 \\
1.30 & E-02 \\
5.00 & E-03\end{array}$ & $\begin{array}{ll}9.20 & E-01 \\
8.80 & E-01 \\
8.50 & E-01 \\
8.20 & E-01 \\
6.75 & E-01 \\
5.75 & E-01 \\
4.85 & E-01 \\
3.55 & E-01 \\
2.50 & E-01 \\
1.75 & E-01 \\
3.10 & E-02 \\
8.30 & E-03 \\
3.25 & E-03 \\
1.00 & E-03 \\
8.93 & E-04 \\
1.20 & E-04\end{array}$ & 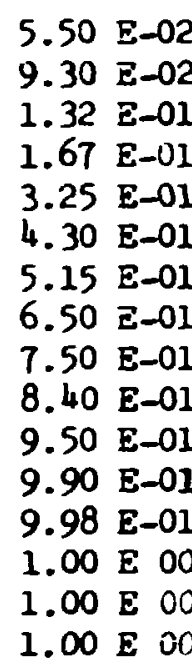 \\
\hline
\end{tabular}

\section{References:}

$\mathrm{H}^{+}+\mathrm{He}$, Experimental: From the review by S.K Allison and M. Garciamunoz,

"Atomic and Molecular Processes" (ed. D.R. Bates, Academic Press, N.Y. 1962j page 721. Also derived values (see Note 1 at end of chapter) are used above $1000 \mathrm{keV}$ based on the cross section values of L. Toburen et al., Prys. Rev. 171, 114 (1968) and U. Schryber, Helv. Phys. Acta 39, 562 (1966).

$\mathrm{H}^{+}+$Ar: P.M. Stier and C.F. Barnett, Phys. Rev. 103, 896 (1956). P.M. Stier, c. F. Barnett, and G.E. Evans, Phys. Rev. 96, 973 (1954). Also derived values (see Jote 1 at end of chapter) are used above $250 \mathrm{keV}$ based on the cross section values of L. Toburen et al., Phys. Rev. 171, 114 (1968).

\section{Accuracy:}

Systematic error is negligible. Random error $< \pm 5 \%$.

\section{Notes:}

See Note (4) at end of chapter. 


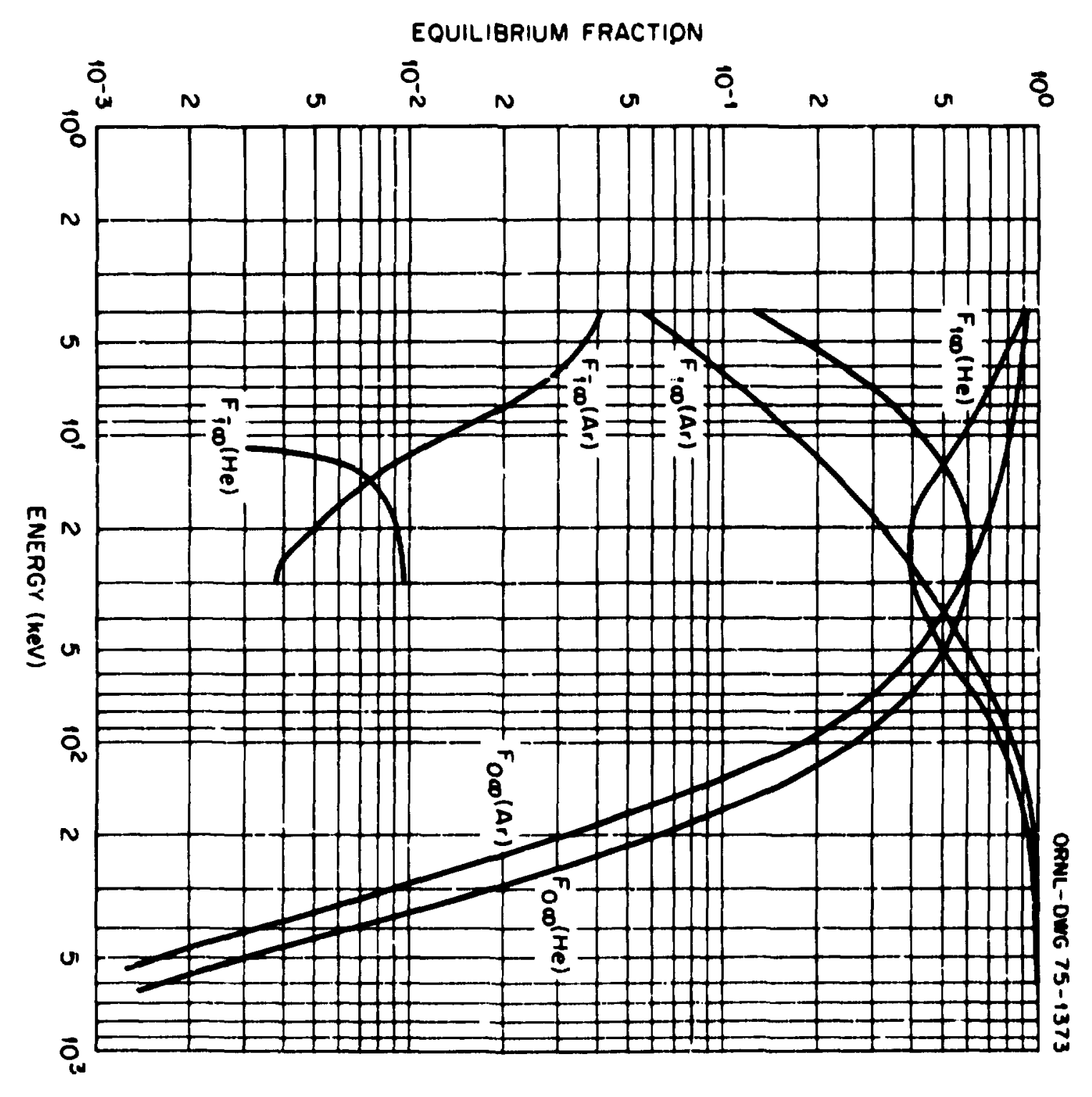


Equilibrium Fractions of aydrogen Beam in $x_{2}$ and $0_{2}$

\begin{tabular}{|c|c|c|c|c|c|c|}
\hline \multirow{3}{*}{$\begin{array}{l}\text { Energy } \\
(\text { (keV) }\end{array}$} & \multicolumn{6}{|c|}{ Equilibriu Fractions } \\
\hline & \multicolumn{3}{|c|}{$\mathbf{x}_{2}$} & \multicolumn{3}{|c|}{$\mathrm{O}_{2}$} \\
\hline & $\mathbf{F} \overline{1}$ & $F_{0 \infty}$ & $F_{1 \infty}$ & $P I \infty$ & $F_{0} \propto$ & $F_{1 \infty}$ \\
\hline $4.0 E 00$ & $3.70 \quad E-03$ & $8.9 n E-01$ & $1.25 \mathrm{E}-01$ & $1.66 \mathrm{E}-02$ & $8.60 \mathrm{E}-01$ & $1.35 \mathrm{E}-01$ \\
\hline $6.0 \equiv 00$ & 9.05 E-03 & $8.45 \mathrm{E}-01$ & $1.62 E-01$ & $1.78 E-02$ & $8.00 \mathrm{E}-01$ & $1.87 E-01$ \\
\hline $8.0 \mathrm{E} 00$ & $1.23 \mathrm{E}-02$ & 8.00 E-01 & $1.95 E-01$ & 1.70 E-02 & $7.60 \mathrm{E}-02$ & $2.28 \quad 3-01$ \\
\hline $1.0 \mathrm{E} 01$ & $1.36 \mathrm{E}-02$ & $7.60 \vec{E}-01$ & $2.24 \quad E-01$ & $1.56 \mathrm{E}-02$ & $7.25 \mathrm{E}-01$ & $2.63 \mathrm{E}-01$ \\
\hline $2.0 \mathrm{E} 01$ & $1.00 \mathrm{E}-02$ & $6.40 \mathrm{E}-01$ & $3.50 \mathrm{E}-01$ & $1.05 E-02$ & $6.05 \mathrm{E}-01$ & $3.85 \mathrm{E}-01$ \\
\hline $3.0 \mathrm{E} 01$ & $6.05 \mathrm{E}-03$ & $5.45 \mathrm{E}-01$ & $4.55 \mathrm{E}-01$ & 7.50 E-03 & $5.30 \mathrm{E}-01$ & $4.70 \quad E-01$ \\
\hline $4.0 \mathrm{EO} 01$ & & 4.80 E-01 & $5.40 E-01$ & & 4.70 E-01 & $5.38 \mathrm{E}-01$ \\
\hline $6.0 \mathrm{E} \mathrm{Ol}$ & & $3.55 \mathrm{E}-01$ & $6.55 \mathrm{E}-01$ & & $3.65 \mathrm{E}-01$ & 6.45 E-01 \\
\hline 8.0 E OI & & $2.55 \overline{\mathrm{E}}-0 \mathrm{I}$ & $7.40 \mathrm{E}-01$ & & $2.80 \mathrm{E}-01$ & $7.25 \mathrm{E}-01$ \\
\hline $1.0 \mathrm{E} 02$ & & $1.86 \cdot \mathbf{E}-01$ & $8.05 \mathrm{E}-01$ & & $2.12 \mathrm{E}-01$ & $7.75 \mathrm{E}-01$ \\
\hline $2.0 \equiv 02$ & & $3.60 \mathrm{E}-02$ & 9.60 E-01 & & $5.10 \mathrm{E}-02$ & 9.40 E-01 \\
\hline $3.0 \equiv 02$ & & $1.00 \mathrm{E}-02$ & $9.90 \mathrm{E}-01$ & & $1.54 \mathrm{E}-02$ & $9.80 \mathrm{E}-01$ \\
\hline $4.0 \mathrm{E} 02$ & & $4.05 \mathrm{E}-03$ & $1.00 \approx 00$ & & $6.50 \mathrm{E}-03$ & $9.90 \mathrm{E}-01$ \\
\hline $6.0 E 02$ & & $1.14 \mathrm{E}-03$ & $1.00 \mathrm{E} 00$ & & $1.95 \mathrm{E}-03$ & $1.00 \equiv 00$ \\
\hline $8.0 \approx 02$ & & 5.00 E-04 & $1.00 \mathrm{E} 00$ & & 7.64 E-04 & $1.00 \mathrm{E} 00$ \\
\hline $1.0 \mathrm{E} 03$ & & $2.80 \mathrm{E}-04$ & $1.00 \equiv 00$ & & $4.38 \mathrm{E}-04$ & $1.00 E 00$ \\
\hline $2.0 E 03$ & & 7.53 E-05 & $1.00 \mathrm{E} 00$ & & $6.90 \mathrm{E}-05$ & $1.00 E 00$ \\
\hline
\end{tabular}

\section{References:}

$\mathrm{H}^{+}+\mathrm{N}_{2}$, Experimental: From the review by S.K. Allison and M. Garcia-Munoz, "Atomic and Molecular Processes" (ed. D.R. Bates, Academic Press, N.Y. 1962) page 721. Also derived values (see Note 1 at end of chapter) are used above $1000 \mathrm{keV}$ based on the cross section values of L. Toburen et al., Phys. Rev. 171,114 (1968).

$\mathrm{H}^{+}+\mathrm{O}_{2}$, Experimental: From the review by S.K. Allison and $M$. Garcia-Munoz, "Atomic and Molecular Processes" (ed. D.R. Bates, Academic Press, N.Y. 1962) page 721. Also derived values (sze Note $I$ at end of chapter) are used above $1000 \mathrm{keV}$ based on the cross section values of L. Toburen et al., Phys. Rev. $171,114(1968)$.

\section{Accuracy:}

Systematic error is negligible. Random error < 5\%.

Yotes:

See rote (4) at end of chapter. 


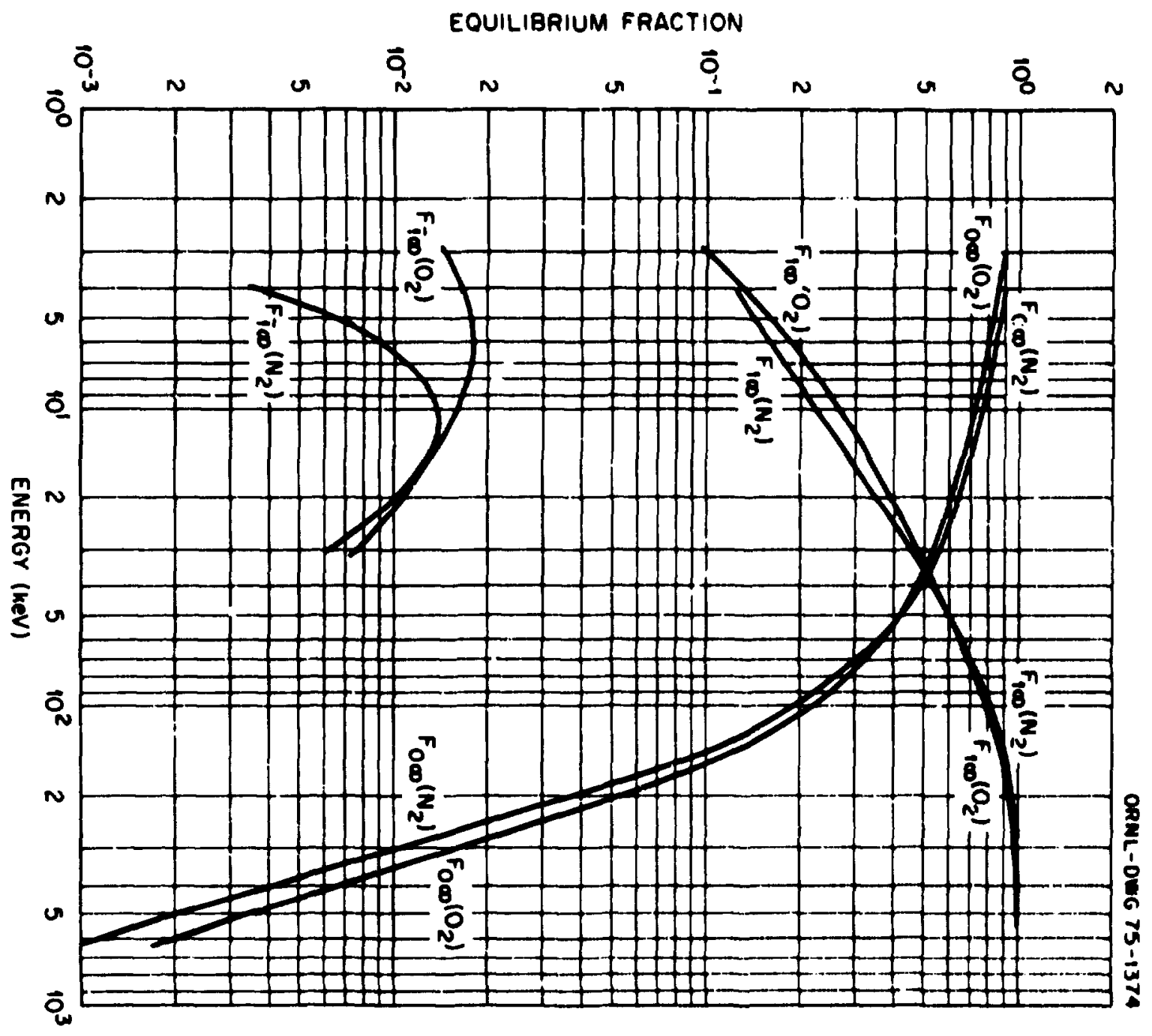


A.6.8

Equilibriu Fractions of a Hydrogen Beam in $\mathrm{H}_{2} \mathrm{O}$

\begin{tabular}{|c|c|c|c|}
\hline \multirow{3}{*}{$\begin{array}{l}\text { Energy } \\
\text { (keV) }\end{array}$} & \multicolumn{3}{|c|}{ Equilibriv Fractions } \\
\hline & \multicolumn{3}{|c|}{$\mathrm{H}_{2} \mathrm{O}$} \\
\hline & $P_{\bar{I} \infty}$ & $P_{0}=$ & $F_{1 \infty}$ \\
\hline $\begin{array}{lll}4.0 & E & 00 \\
6.0 & E & 00 \\
8.0 & E & 00 \\
1.0 & E & 01 \\
2.0 & E & 01\end{array}$ & $\begin{array}{ll}3.60 & E-02 \\
4.30 & E-02 \\
4.50 & E-02 \\
4.45 & E-02 \\
2.78 & E-02\end{array}$ & & \\
\hline $\begin{array}{lll}3.0 & \mathrm{E} & 01 \\
4.0 & \mathrm{E} & 01 \\
6.0 & \mathrm{E} & 01 \\
8.0 & \mathrm{E} & 01 \\
1.0 & \mathrm{E} & 02 \\
2.0 & \mathrm{E} & 02 \\
3.0 & \mathrm{E} & 02 \\
4.0 & \mathrm{E} & 02 \\
6.0 & \mathrm{E} & 03 \\
3.0 & \mathrm{E} & 02 \\
1.0 & \mathrm{E} & 03 \\
2.0 & \mathrm{E} & 03\end{array}$ & $1.50 \mathrm{E}-02$ & $\begin{array}{ll}5.40 & E-01 \\
4.35 & E-01 \\
3.70 & E-01 \\
2.80 & E-01 \\
2.10 & E-01 \\
3.60 & E-01 \\
1.22 & E-02 \\
5.65 & E-03 \\
1.92 & E-03 \\
6.07 & E-04 \\
2.86 & E-04 \\
5.73 & E-05\end{array}$ & $\begin{array}{ll}4.50 & \mathrm{E}-01 \\
5.20 & \mathrm{E}-01 \\
6.25 & \mathrm{E}-01 \\
7.10 & \mathrm{E}-01 \\
7.70 & \mathrm{E}-01 \\
9.60 \mathrm{E}-01 \\
9.80 \mathrm{E}-01 \\
9.90 \mathrm{E}-01 \\
1.00 \mathrm{E} 00 \\
1.00 \mathrm{E} 00 \\
1.00 \mathrm{E} 00 \\
1.00 \mathrm{E} 00\end{array}$ \\
\hline
\end{tabular}

\section{References :}

$\mathrm{H}^{+}+\mathrm{H}_{2} \mathrm{O}$ : At energies above $100 \mathrm{keV}$ we have generated fractions using the formalae $F_{0}=\sigma_{10} /\left(\sigma_{10}+\sigma_{01}\right)$ and $F_{1}=\sigma_{01} /\left(\sigma_{10}+\sigma_{01}\right)$; the values of $\sigma_{01}$ and $\sigma_{10}$ are from L. Toburen, et al., Phys. Rev. 171, 114 (1968). There is a slight error (perhaps as much as 2x) in neglect of negative state formation and loss. $I$. at low energies ( $E \leq 30 \mathrm{keV}$ ) is rrom K.B. Brooks, et al., Physics Letts. 6, 169 (1963); F $\overline{\overline{1}}, \mathrm{~F}_{0}$, and $F_{1}$ - at $30 \mathrm{keV}$ are by A.B. Wittkower, et 르., Phys. Letts. 13, 134 $(1964)$.

Accuracy:

Systematic error is negligible. Random error $< \pm 5 \%$.

\section{Notes :}

See Note (4) at end of chapter. 


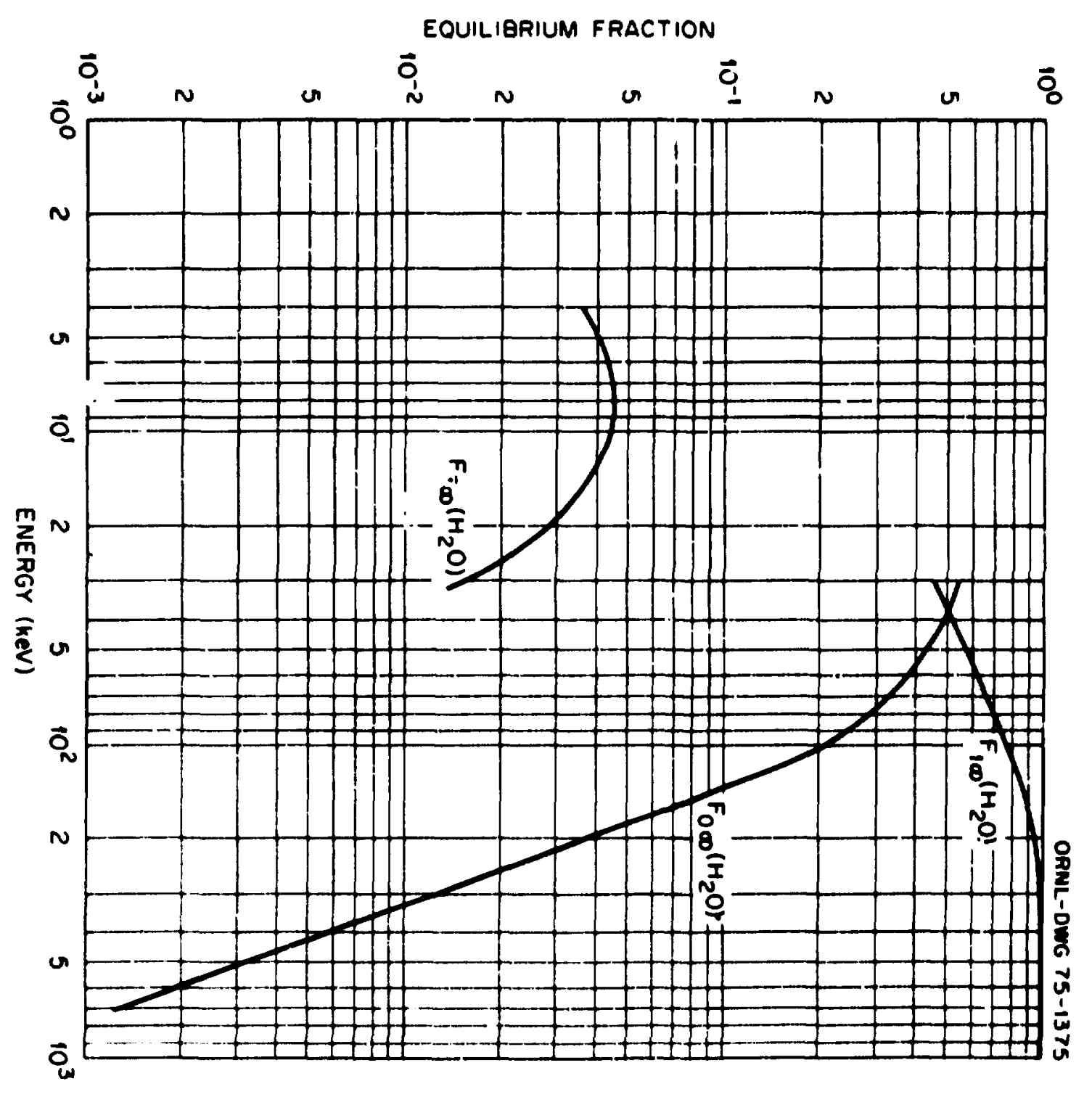




\section{$\mathbf{1} .6 .10$}

Equilibriun Fractions of a Hydrogen Beam in $\mathrm{Li}$ and $\mathrm{Ma}$

\begin{tabular}{|c|c|c|c|c|c|c|}
\hline \multirow{3}{*}{$\begin{array}{l}\text { Energs } \\
\text { (keV) }\end{array}$} & \multicolumn{6}{|c|}{ Equilibrion Fractions } \\
\hline & \multicolumn{3}{|c|}{$\mathbf{L i}$} & \multicolumn{3}{|c|}{ Xe } \\
\hline & $F \bar{I}$ & $\mathbf{P}_{0} \infty$ & $F_{1 \infty}$ & $\mathbf{F} \Sigma_{\infty}$ & $F_{0}=$ & $F_{1 \infty}$ \\
\hline 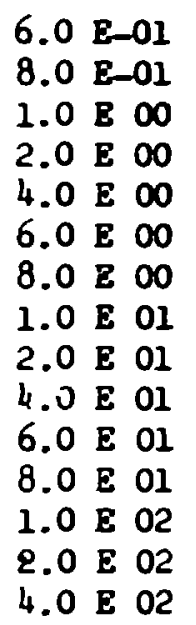 & $\begin{array}{ll}4.68 & \mathrm{E}-02 \\
4.80 & \mathrm{E}-02 \\
4.92 & \mathrm{E}-02 \\
5.64 & \mathrm{E}-02 \\
5.88 & \mathrm{E}-02 \\
4.60 & \mathrm{E}-02 \\
3.21 & \mathrm{E}-02 \\
2.40 & \mathrm{E}-02 \\
8.50 & \mathrm{E}-03\end{array}$ & 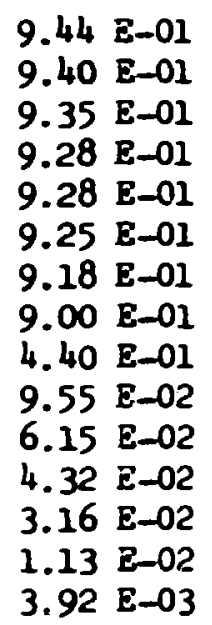 & $\begin{array}{ll}8.00 & E-03 \\
2.15 & E-02 \\
4.41 & E-02 \\
7.86 & E-02 \\
6.03 & E-01 \\
9.08 & E-01 \\
9.40 & E-01 \\
9.58 & E-01 \\
9.65 & E-01 \\
9.82 & E-01 \\
9.88 & E-01\end{array}$ & $\begin{array}{ll}8.00 & \mathrm{E}-02 \\
9.50 & \mathrm{E}-02 \\
1.04 & \mathrm{E}-01 \\
1.20 & \mathrm{E}-01 \\
1.06 & \mathrm{E}-01 \\
8.60 & \mathrm{E}-02 \\
6.50 & \mathrm{E}-02 \\
5.20 & \mathrm{E}-02\end{array}$ & $\begin{array}{l}9.25 \mathrm{E}-01 \\
9.10 \mathrm{E}-01 \\
9.00 \mathrm{E}-01 \\
8.75 \mathrm{E}-01 \\
8.75 \mathrm{E}-01 \\
8.90 \mathrm{E}-01 \\
9.20 \mathrm{E}-01 \\
9.30 \mathrm{E}-01 \\
6.40 \mathrm{E}-01 .\end{array}$ & $\begin{array}{ll}1.05 & \mathrm{E}-02 \\
1.68 & \mathrm{E}-02 \\
2.45 & \mathrm{E}-02 \\
3.50 & \mathrm{E}-02 \\
3.30 \mathrm{E}-01\end{array}$ \\
\hline
\end{tabular}

\section{References:}

$\mathrm{H}^{+}+\mathrm{WL}$, Experimental: H. Grïebler, et al., Helv. Phys. Acta 43, 254 (1970). Derived values (see Note 1 at end of chepter) are used above $2 \overline{00} \mathrm{keV}$ based on the cross sections by B.A. Dyachkor, Soviet Atomie Dnergy 27, 958 (1969).

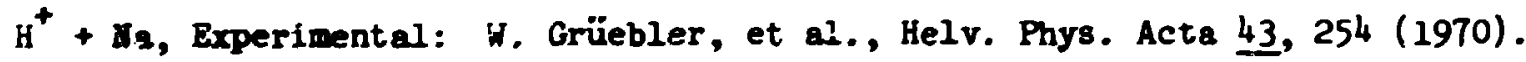

\section{Accuracy:}

Systematic error is negligiole. Random er::or $< \pm 4 \%$.

\section{Notes:}

See Note (4) at end of chapter. 


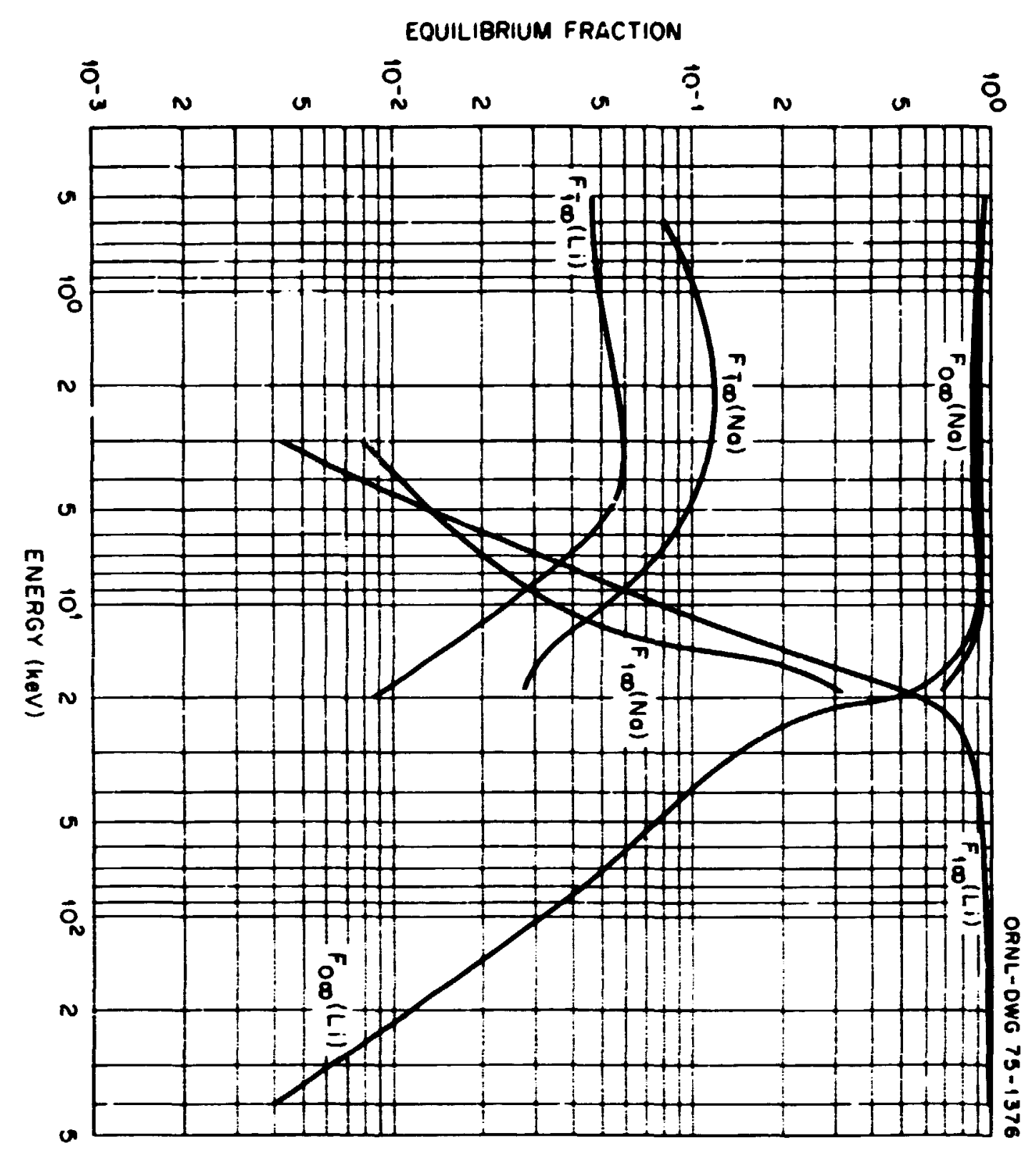


A.6.12

Equilibrium Fractions of a idydrogen Beam in $k$ and Cs

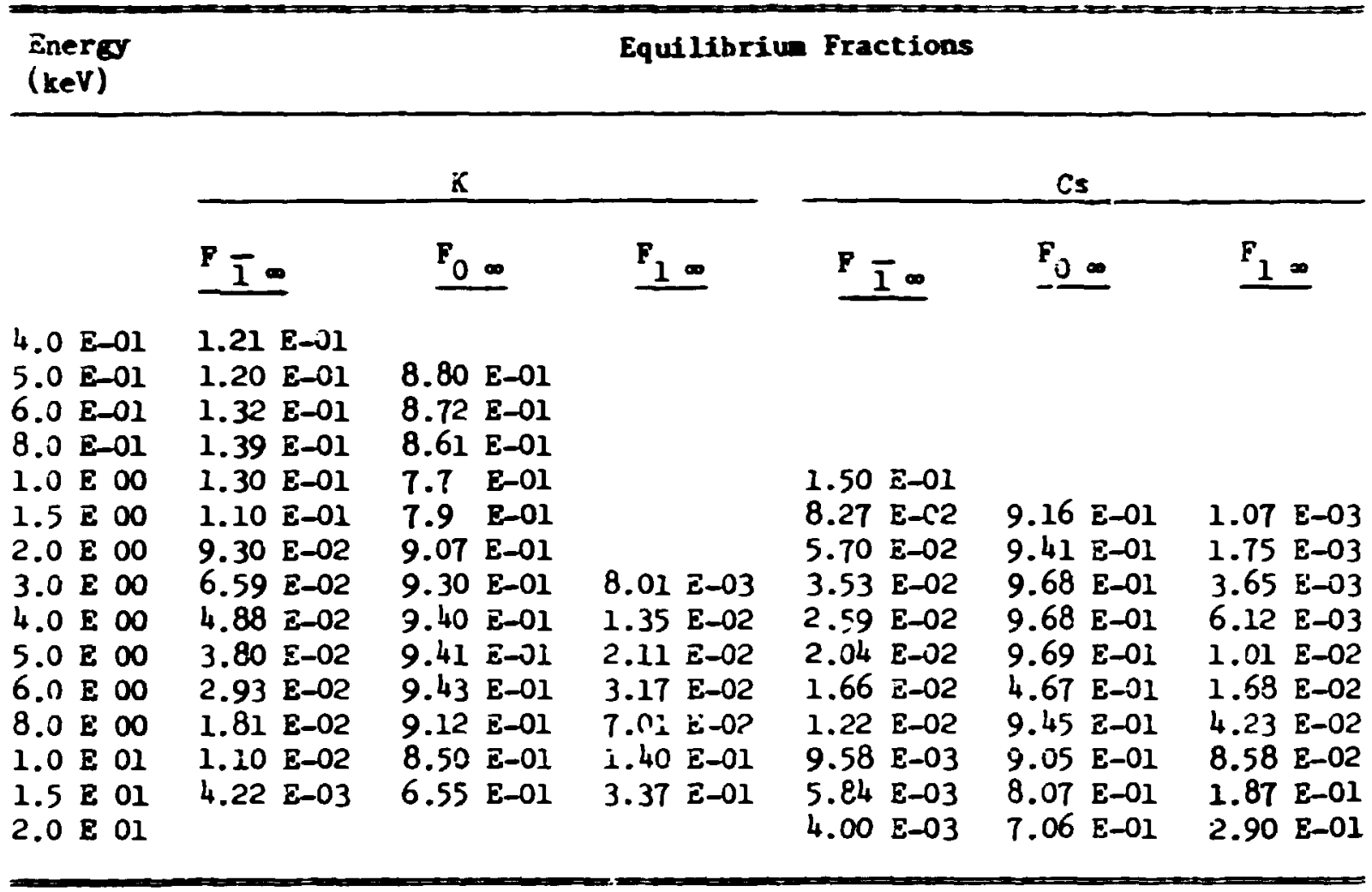

\section{References:}

$\mathrm{H}^{+}+\mathrm{K}$, Experimental: W. Grübler et al., Helv. Phys. Acta 43, 254 (1970).

$\mathrm{H}^{+}+$Cs, Experimental: A.S. Schlachter et al., Phys. Rev. 177, 184 (1969).

\section{Accuracy:}

Systematic irror is negligible. Random error $< \pm 4 \%$.

Notes:

See Note (4) at end of chapter. 


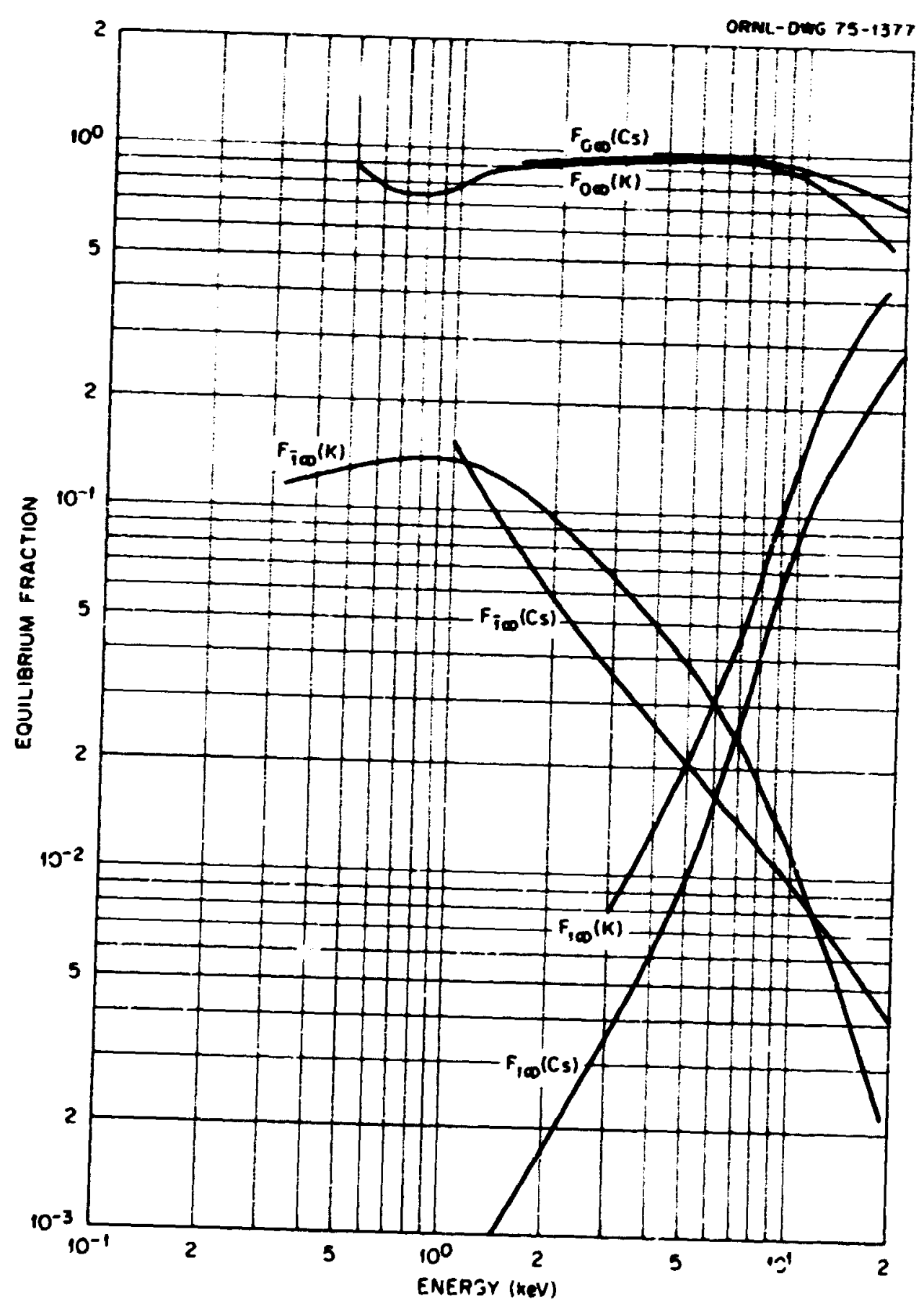


A.6.14

Equilibrium Excited State Fractions for

Hydroger: Beams Traversing Sodium and Potassium

\begin{tabular}{|c|c|c|c|}
\hline \multirow[t]{2}{*}{$\begin{array}{l}\text { Bnergy } \\
\text { (kev) }\end{array}$} & \multicolumn{3}{|c|}{ Fxcited State Fraction $\times \mathrm{n}^{3 *}$} \\
\hline & Na & $\underline{\mathbf{M}_{3}}$ & $\underline{K}$ \\
\hline $\begin{array}{lll}i .5 & E & 01 \\
2.0 & \mathrm{E} & 01 \\
2.5 & \mathrm{E} & 01 \\
3.0 & \mathrm{E} & 01 \\
4.0 & \mathrm{E} & 01 \\
6.0 & \mathrm{E} & 01 \\
8.0 & \mathrm{E} & 01 \\
1.0 & \mathrm{E} & 02\end{array}$ & 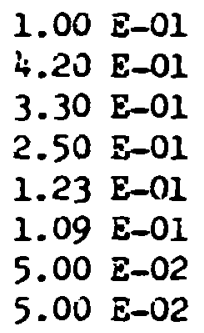 & $\begin{array}{ll}4.50 & E-02 \\
3.83 & E-01 \\
4.55 & E-01 \\
4.67 & E-01 \\
2.37 & E-01 \\
8.60 & E-02 \\
4.76 & E-02 \\
3.10 & E-02\end{array}$ & $3.1 \mathrm{I}-01$ \\
\hline
\end{tabular}

See Note (2) at end of chapter.

References:

H + Ka, Experimental: V.A. Oparin, R.N. Il'in, and E.S. Solov'ev, 2h. Eks. Teor. Fiz. 52, 369 (1967). [Soviet Phys. JETP 25, 240 (1967)].

$H+K$, Experimental: R.N. Il'in, V.A. Oparin, E.S. Solor'ev, N.V. Fedorenko, Zh. Tekh. Fiz. 36, 124? (1966). [Soviet Phys. Tech. Phys. 11, $921(1967)\}$.

H + Mg, Experimental: V.A. Oparin, R.N. Il'in, ard E.S. Solov'ev, 2h. Eks. Teor. Piz. 52, 369 (1967). [Soviet Phys. JEIP 25, 240 (1967)]. Accuracy:

Systematic error $< \pm 10 \%$.

Notes:

See Note (3) at end of chapter. 


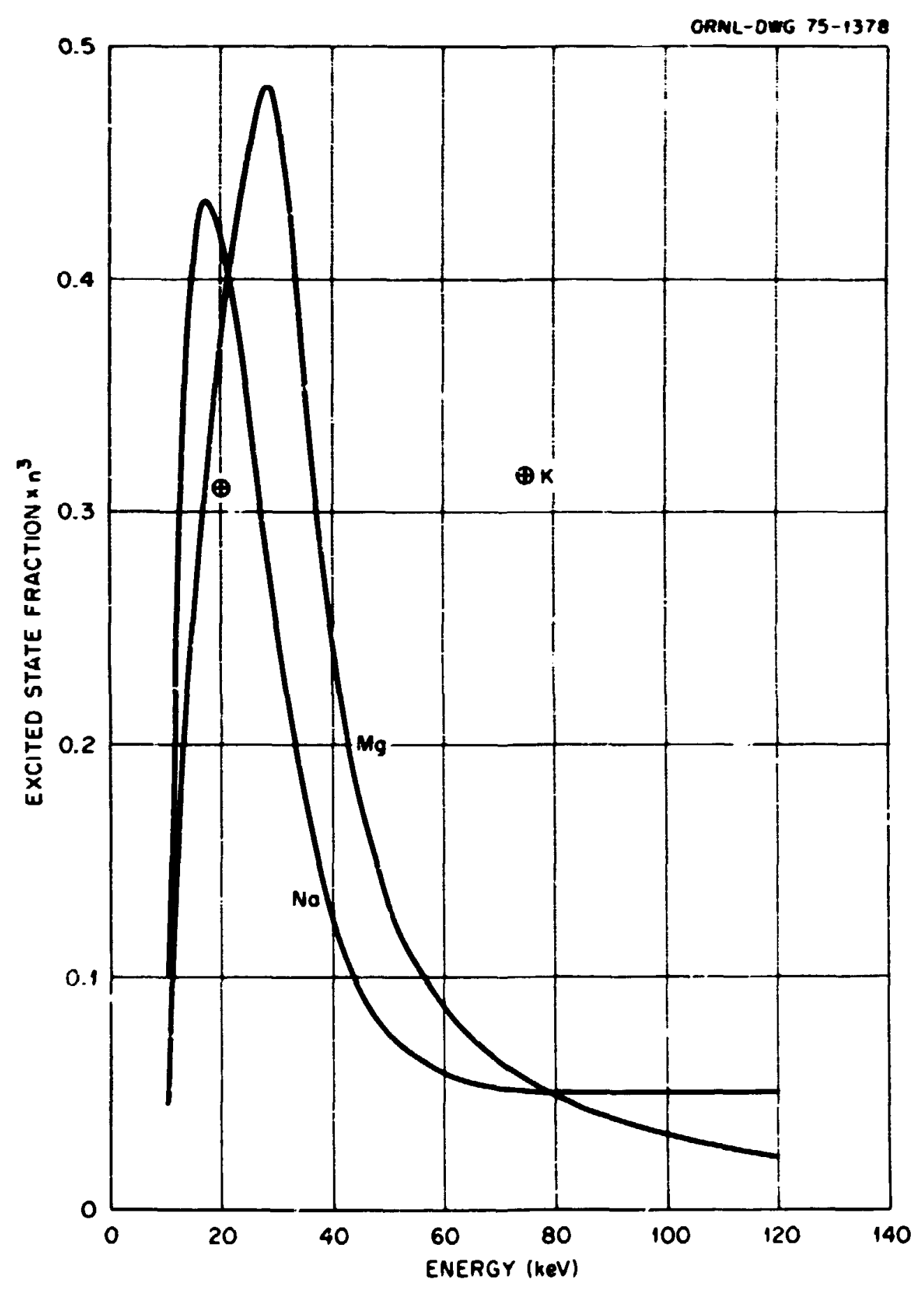




\section{A.6.16}

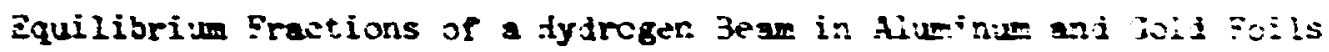

\begin{tabular}{|c|c|c|c|c|c|c|}
\hline \multirow{3}{*}{$\begin{array}{l}\text { anergy } \\
\text { (inev) }\end{array}$} & \multicolumn{6}{|c|}{ Equilibriu Fractions } \\
\hline & \multicolumn{3}{|c|}{ Al } & \multicolumn{3}{|c|}{$\mathrm{Au}$} \\
\hline & $\bar{I}$ & $F_{G \infty}$ & $F_{1}$ & $\mathbf{F}=$ & $5_{0 \infty}$ & $5_{1}$ \\
\hline 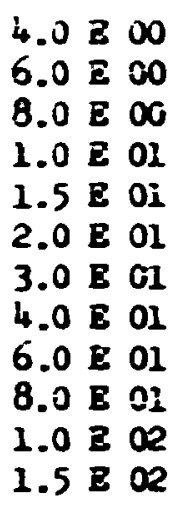 & $\begin{array}{ll}6.20 & E-02 \\
5.21 & E-02 \\
4.59 & E-02 \\
4.00 & E-02 \\
3.06 & E-02 \\
2.40 & E-02 \\
1.41 & E-02 \\
8.00 & E-03\end{array}$ & $\begin{array}{ll}8.50 & E-01 \\
8.45 & \mathrm{E}-01 \\
8.13 & \mathrm{E}-01 \\
8.18 & \mathrm{E}-0 \mathrm{I} \\
7.71 & \mathrm{E}-01 \\
7.25 & \mathrm{E}-01 \\
5.85 & \mathrm{E}-01 \\
4.58 & \mathrm{E}-01 \\
2.90 & \mathrm{E}-01 \\
1.92 & \mathrm{E}-01 \\
1.20 & \mathrm{E}-01 \\
4.50 & \mathrm{E}-02\end{array}$ & $\begin{array}{ll}8.20 & \mathrm{E}-02 \\
1.02 & \mathrm{E}-01 \\
1.99 & \mathrm{E}-01 \\
1.42 & \mathrm{E}-01 \\
1.85 & \mathrm{E}-01 \\
2.51 & \mathrm{E}-01 \\
4.01 & \mathrm{E}-1 \\
5.34 & \mathrm{E}-01 \\
7.10 & \mathrm{E}-01 \\
8.10 & \mathrm{E}-01 \\
8.80 & \mathrm{E}-01 \\
9.55 & \mathrm{E}-01\end{array}$ & $\begin{array}{ll}4.11 & \mathrm{E}-02 \\
3.93 & \mathrm{E}-02 \\
3.74 & \mathrm{E}-02 \\
3.50 & \mathrm{E}-02 \\
3.16 & \mathrm{E}-02 \\
2.69 & \mathrm{E}-02 \\
1.79 & \mathrm{E}-02 \\
9.58 & \mathrm{E}-02\end{array}$ & $\begin{array}{ll}8.59 & \Xi-01 \\
8.44 & \mathrm{E}-01 \\
9.01 & \mathrm{E}-01 \\
8.00 & \mathrm{E}-01 \\
7.50 & \mathrm{E}-01 \\
7.00 & \mathrm{E}-01 \\
5.90 & \mathrm{E}-01 \\
4.92 & \mathrm{E}-01 \\
3.58 & \mathrm{E}-0 \mathrm{I} \\
2.69 & \mathrm{E}-01 \\
2.10 & \mathrm{E}-01 \\
1.28 & \mathrm{E}-01\end{array}$ & $\begin{array}{ll}1.00 & \mathrm{E}-01 \\
1.23 & \mathrm{E}-01 \\
1.44 & \mathrm{E}-\mathrm{Ol} \\
1.65 & \mathrm{E}-01 \\
2.14 & \mathrm{E}-01 \\
2.68 \mathrm{E}-\mathrm{Cl} \\
3.80 \mathrm{E}-01 \\
4.97 \mathrm{E}-01 \\
6.55 \mathrm{E}-01 \\
7.52 \mathrm{E}-01 \\
8.09 & \mathrm{E}-01 \\
9.00 & \mathrm{E}-01\end{array}$ \\
\hline
\end{tabular}

\section{Reierence:}

H + Al, Al, Experimental: J.A. Phillips, Phys, Rev. 97, 404 (1955).

\section{Accuract:}

Randon error $< \pm 58$. 


\section{A.6.17}

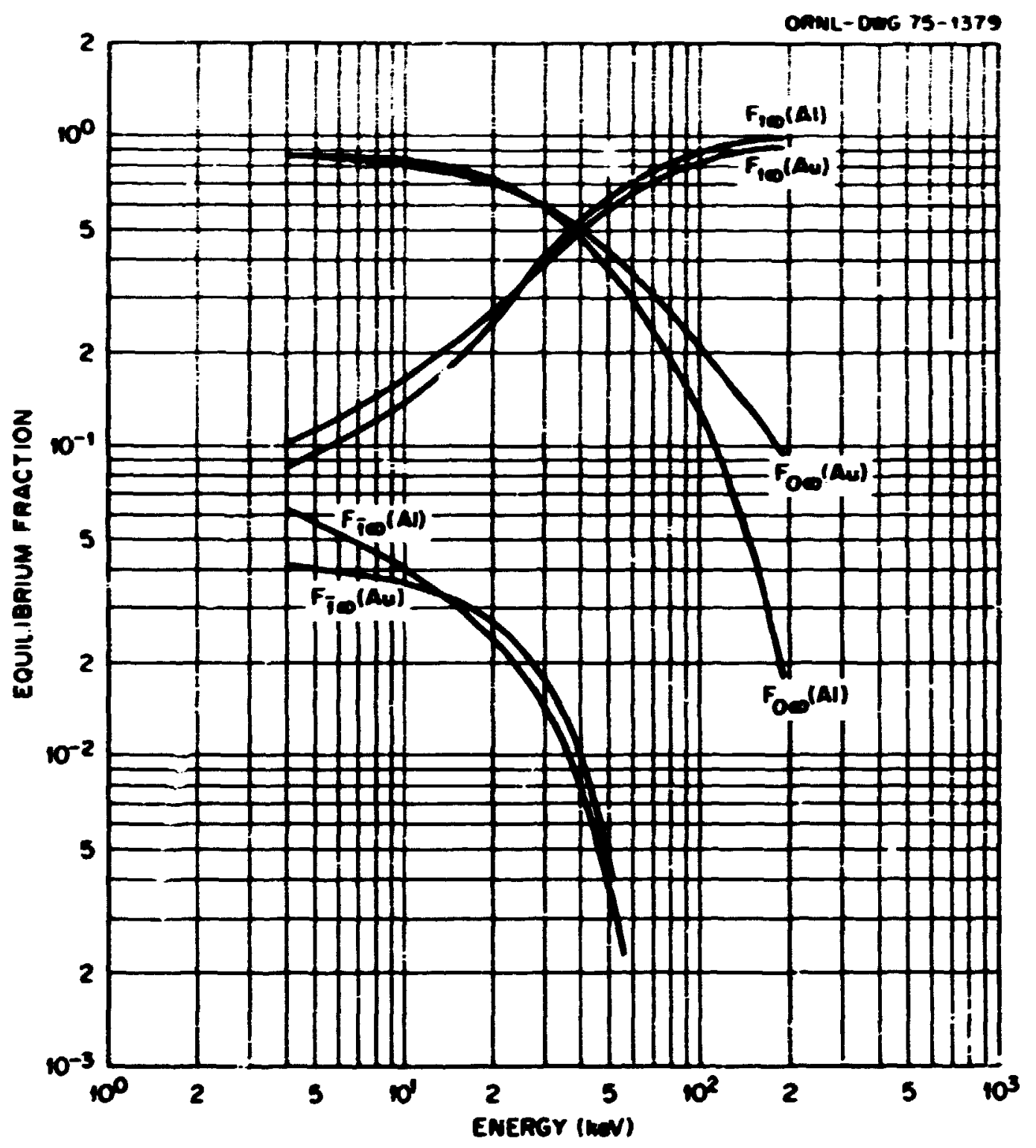




\section{A.6.18}

Yiald of $\mathrm{H}^{\circ}$ and $\mathrm{H}^{-}$Observed for an $\mathrm{H}_{2}^{+}$Bean Traversing $\mathrm{H}_{2}$ and $\mathrm{H}_{2} \mathrm{O}$

\begin{tabular}{|c|c|c|c|}
\hline $\begin{array}{l}\text { Deero } \\
(k=1)\end{array}$ & Yield & Atoms Per & $+(\infty)$ \\
\hline & $\mathrm{H}_{2}$ Target & \multicolumn{2}{|c|}{$\mathrm{H}_{2} \mathrm{O}$ Target } \\
\hline & $\mathrm{H}^{\circ}$ Yield & ${\underline{\mathrm{H}^{-}} \mathrm{rield}}$ & $\underline{H}^{\circ}$ Yield \\
\hline $\begin{array}{lll}1.0 & \mathrm{~B} & 01 \\
1.5 & \mathrm{~B} & 01 \\
2.0 & \mathrm{~B} & 01 \\
3.0 & \mathrm{~B} & 01\end{array}$ & & $\begin{array}{ll}6.15 & \mathrm{E}-02 \\
8.17 & \mathrm{E}-02 \\
7.78 & \mathrm{E}-02 \\
6.27 & \mathrm{E}-02\end{array}$ & \\
\hline $\begin{array}{llll}6.0 & \mathrm{~B} & 01 \\
8.0 & \mathrm{~B} & 01 \\
1.0 & \mathrm{~B} & 02 \\
1.5 & \mathrm{~B} & 02 \\
2.0 & \mathrm{~B} & 02 \\
3.0 & \mathrm{~B} & 02 \\
4.0 & \mathrm{~B} & 02\end{array}$ & $\begin{array}{l}1.40 \mathrm{E} \infty \\
1.12 \mathrm{E} \infty \\
9.20 \mathrm{E}-01 \\
6.06 \mathrm{E}-01 \\
1.24 \mathrm{E}-01 \\
2.35 \mathrm{E}-01 \\
1.46 \mathrm{E}-01\end{array}$ & & $\begin{array}{l}1.16 \mathrm{E} \quad 00 \\
9.40 \mathrm{E}-01 \\
7.82 \mathrm{E}-01 \\
5.47 \mathrm{E}-01 \\
3.96 \mathrm{E}-01 \\
2.35 \mathrm{E}-01 \\
1.51 \mathrm{E}-01\end{array}$ \\
\hline
\end{tabular}

\section{Rererences:}

$\mathrm{H}_{2}^{+}+\left(\mathrm{H}_{2}+\mathrm{H}_{2} \mathrm{O}\right)+\mathrm{H}^{\mathrm{O}}$, Beperinentel: C.P. Barnett, M. Rankin, J.A. Ray, Prsc. 6th Intl. Conf. on Ionization Phesonene in cases (SBRM, Paris 1963) Vol. 1 page 63.

$\mathrm{H}_{2}^{+}+\mathrm{H}_{2} \mathrm{O} \rightarrow \mathrm{H}^{-}$, Beperimental: M.B. Brooks, M. Roos, R.P. Bastide, P.I. Rose, and A.B. Hitthower, Phys. Letts. 6, 169 (1963).

Necurec:

Randon error $< \pm 20 \%$. 


\section{A.6.19}

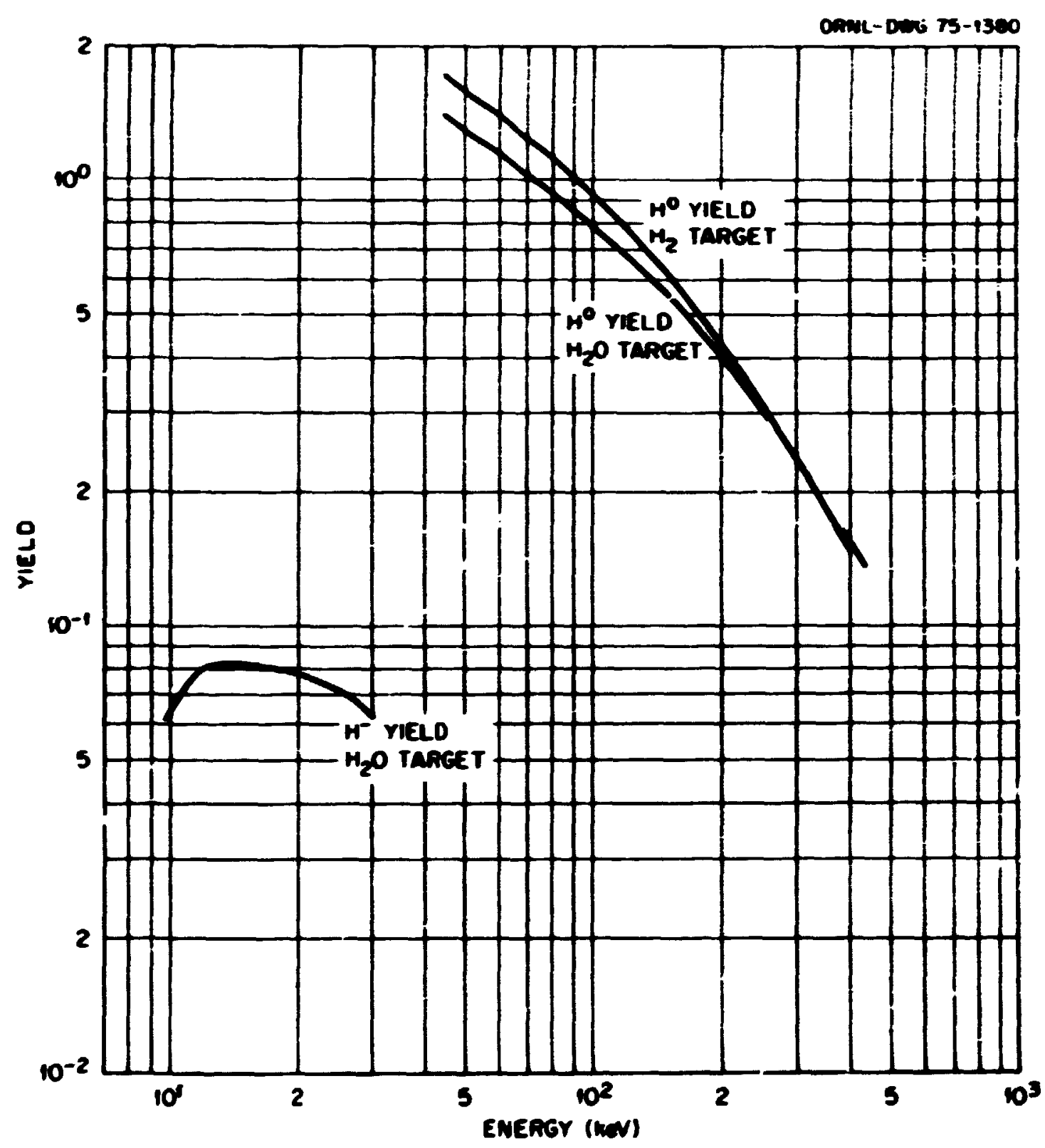




\section{A. 6.20}

Yield of $\mathrm{E}^{\circ}$ and $\mathrm{H}^{-}$Observed for an $\ddot{z}_{3}{ }^{+}$Beam Traversing $\mathrm{B}_{2}$ and $\ddot{z}_{2} \mathrm{O}$

\begin{tabular}{|c|c|c|c|}
\hline $\begin{array}{l}\text { Bnerg } \\
\text { (neV) }\end{array}$ & rield & Atoms Per & ${ }_{3}^{+}$Ion) \\
\hline & $\dot{y}_{2}$ Trret & \multicolumn{2}{|c|}{$\Xi_{2} 0$ Target } \\
\hline & $\mathrm{H}^{0}$ rield & ${\underline{y^{-}} \text {Yield }}$ & $\underline{0}^{0}$ yield \\
\hline 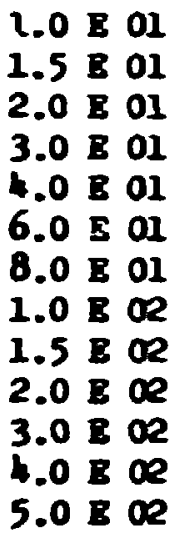 & $\begin{array}{lll}2.74 & \mathrm{E} & 00 \\
2.30 & \mathrm{E} & 00 \\
1.58 & \mathrm{~B} & \infty \\
1.16 & \mathrm{~B} & 00 \\
7.20 & 8-01\end{array}$ & $\begin{array}{ll}5.50 & E-02 \\
1.36 & \Xi-01 \\
1.66 & z-01 \\
9.30 & E-01\end{array}$ & $\begin{array}{lll}2.53 & E & 00 \\
2.24 & E & 00 \\
1.98 & E & 00 \\
1.73 & E & 00 \\
1.30 & E & 00 \\
1.01 & E & 00 \\
6.99 & E & 00 \\
5.05 & E & 00 \\
3.90 & E & 00\end{array}$ \\
\hline
\end{tabular}

\section{Deferences:}

$\mathrm{H}_{3}^{+}+\mathrm{H}_{2}+\mathrm{H}^{0}$, Eqperimentel: C.P. Barnett, H. Rankin, J.A. Ry, Proc. 6th Int 1. Conf. on Ionization Fbenonene in Cases (Stain, Paris 1953) V01. I pare 63. C.R. Midaleton, M.P. Pajpe, A.C. Riviere, J. Phos. B 4 , 180 (1971).

$\mathrm{H}_{3}^{+}+\mathrm{H}_{2} \mathrm{O}-\mathrm{H}^{-}$, Beperiweatal: H.B. Brooks, H. Roos, R.P. Bastide, P.II. Rose, ad A.B. Witthower, Phys. Letts. 6, 169 (1963).

$\mathrm{H}_{3}^{+}+\mathrm{H}_{2} \mathrm{O} \rightarrow \mathrm{H}^{\circ}$, Reperinentel: C.P. Barnett, K. Rankía, J.A. Ras, Proc. 6th Int1. Conf. a Ionization Pbencinene in Geses (SEFin, Paris 1963)

Vo1. 1 pare 63.

\section{secures:}

Irado exror $< \pm 20 \%$. 


\section{A.6.21}

3

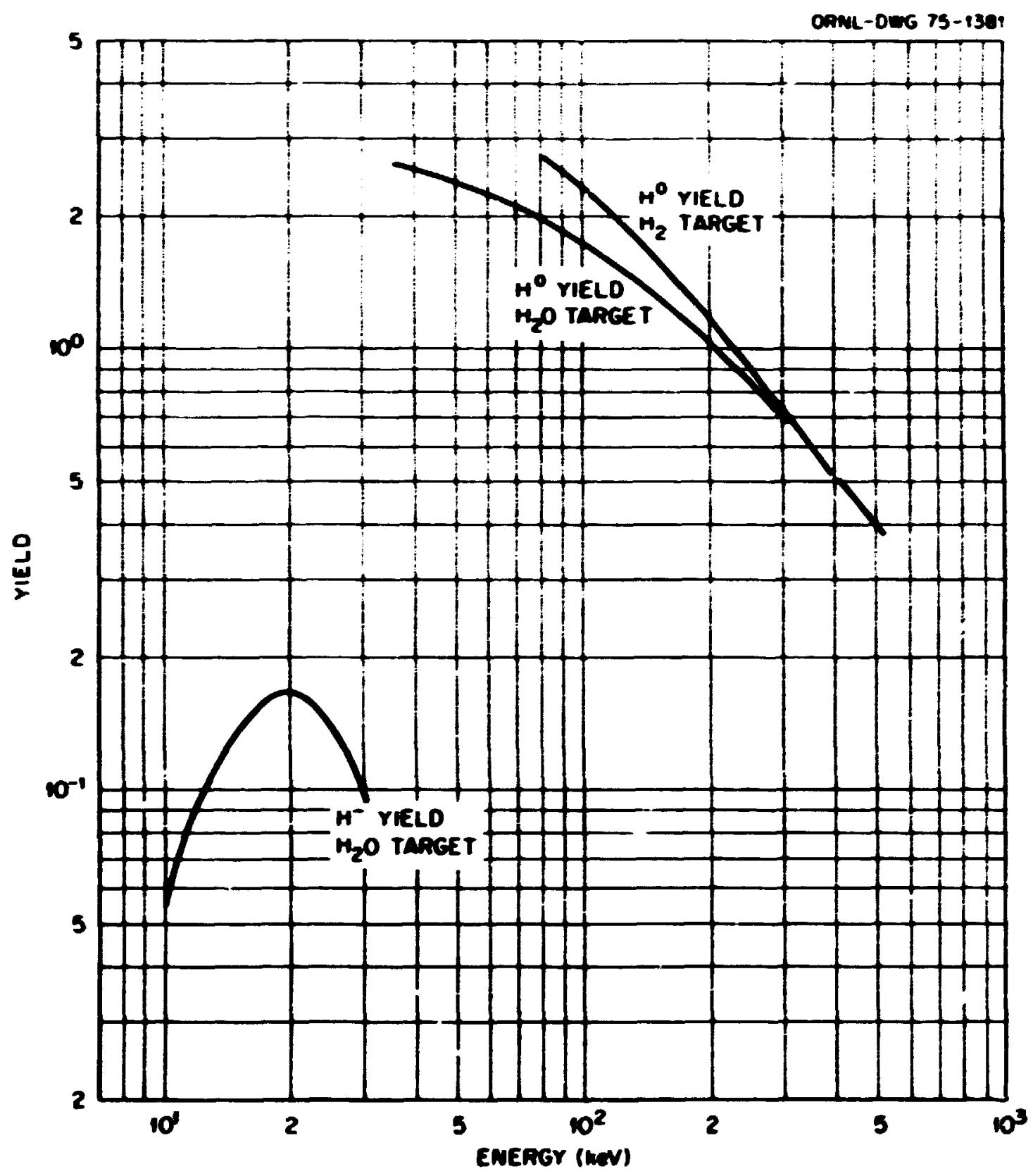




\section{A.6.22}

Fractions of $\mathrm{H}_{3}^{+}, \mathrm{H}_{2}^{+}, \mathrm{H}_{2}, \mathrm{H}^{+}$, and $\mathrm{H}$ as a Function of Parget Thickness for $100-\mathrm{keV} \mathrm{H}{ }_{3}$ t Traversing $\mathrm{H}_{2}$

\begin{tabular}{|c|c|c|c|c|c|}
\hline \multirow{2}{*}{$\begin{array}{c}\text { Terget } \\
\text { Thieleness } \\
\text { (Dicron-c) }\end{array}$} & \multicolumn{5}{|c|}{ Practions of Components Mormalized to $\mathrm{H}_{3}{ }^{+}$} \\
\hline & $\mathrm{H}_{3}^{+}$ & $\mathrm{H}_{2}^{+}$ & $\mathrm{H}_{2}^{\circ}$ & $\mathrm{H}^{+}$ & $\mathrm{a}^{\circ}$ \\
\hline 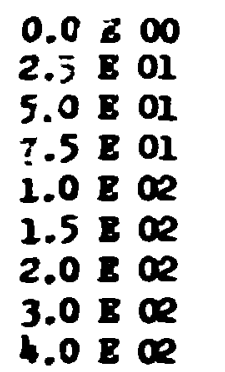 & 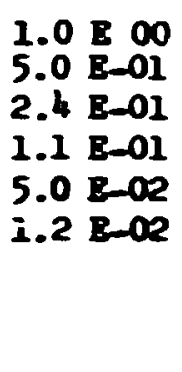 & $\begin{array}{ll}3.6 & \mathrm{E}-02 \\
3.9 & \mathrm{E}-02 \\
3.3 & \mathrm{E}-02 \\
2.4 & \mathrm{E}-02 \\
1.2 & \mathrm{E}-02\end{array}$ & $\begin{array}{ll}8.8 & \mathrm{E}-02 \\
1.0 & \mathrm{E}-01 \\
9.3 & \mathrm{E}-02 \\
8.0 & \mathrm{E}-02 \\
4.7 & \mathrm{E}-02 \\
1.0 & \mathrm{E}-02\end{array}$ & $\begin{array}{l}1.4 \mathrm{E}-01 \\
2.4 \mathrm{E}-01 \\
3.0 \mathrm{E}-01 \\
3.4 \mathrm{E}-01 \\
4.0 \mathrm{E}-01 \\
4.3 \mathrm{E}-01 \\
4.4 \mathrm{E}-01 \\
4.5 \mathrm{E}-01\end{array}$ & 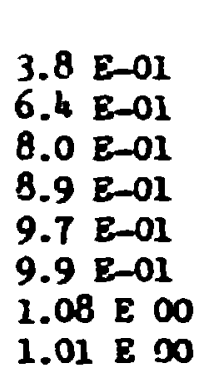 \\
\hline
\end{tabular}

\section{Erencee:}

Theoretical, besed on experfental data: C.P. Barnett, M. Dankin,

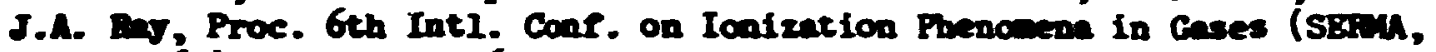
Faris 1963) Vol. 1 pace 63. 
ORNL-OWG 63-2112

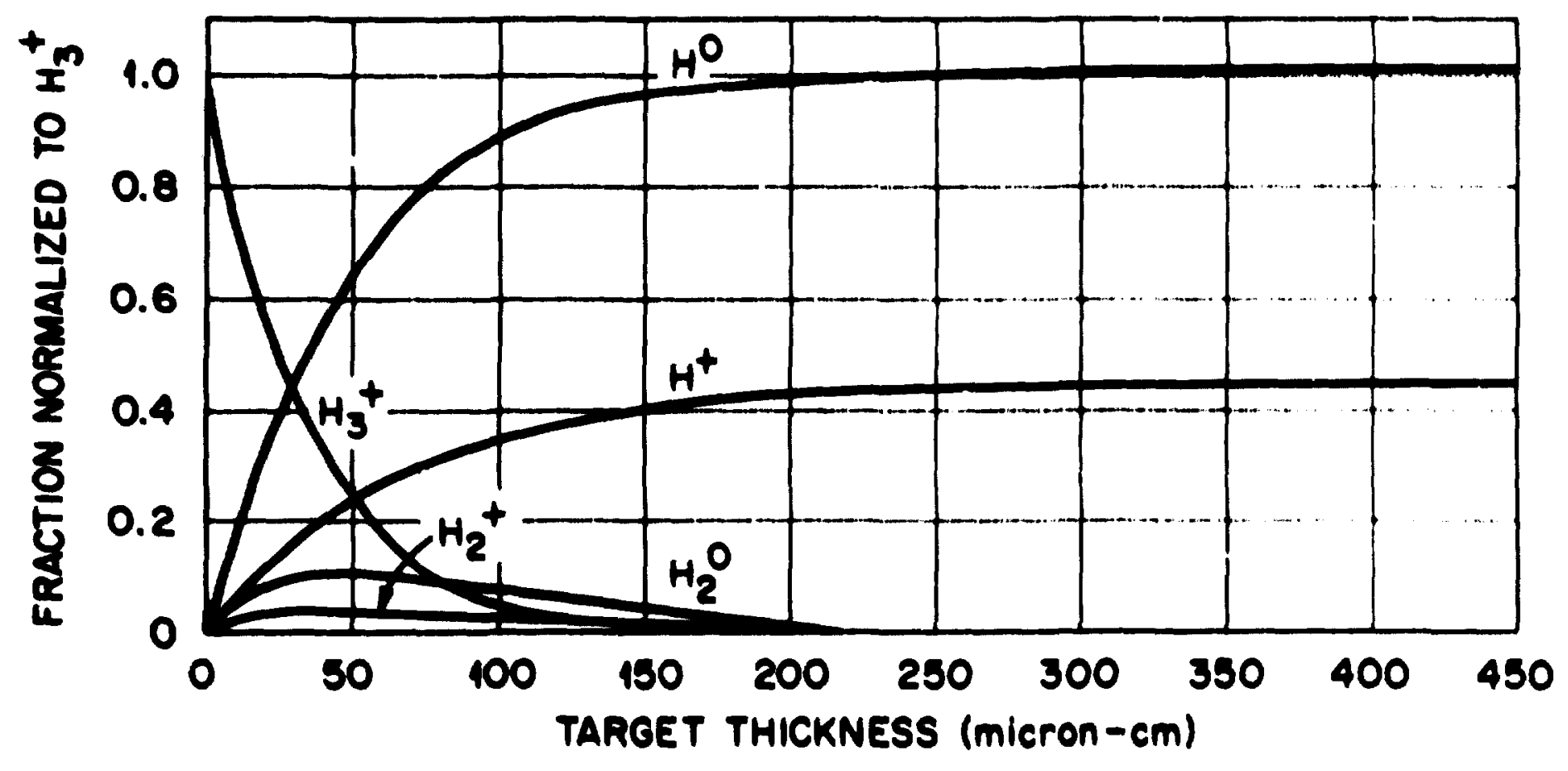




\section{A.6.2k}

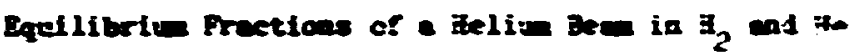

\begin{tabular}{|c|c|c|c|c|c|c|c|}
\hline \multirow{3}{*}{$\begin{array}{c}\text { aners } \\
\text { (xerl) }\end{array}$} & \multicolumn{7}{|c|}{ Ephibrib Prection } \\
\hline & \multicolumn{4}{|c|}{$E_{2}$} & \multicolumn{3}{|c|}{ Je } \\
\hline & $1 \overline{1}$ & $\mathbf{P}_{\mathbf{0}}$ & $I_{1}=$ & $P_{2}$ & $F_{0}$ & $P_{1}=$ & $\underline{2}$ \\
\hline 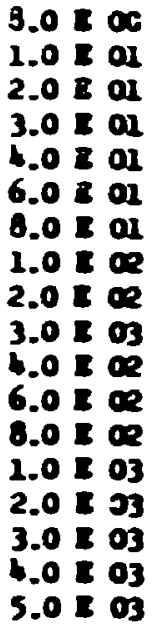 & 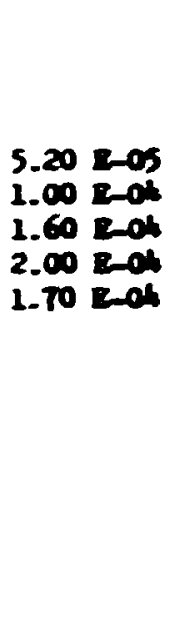 & $\begin{array}{l}8.30=-01 \\
8.10=-01 \\
7.60 E-01 \\
7.00=-01 \\
4.38=-01 \\
2.46=01 \\
1.18=01\end{array}$ & $\begin{array}{ll}1.51 & 2-01 \\
1.57 & E-01 \\
1.62 & E-01 \\
1 . & -01 \\
1.60 & E-01 \\
1.90 & E-01 \\
2.40 & 2-01 \\
2.89 & E-01 \\
5.50 & 8-01 \\
6.50 & E-01 \\
7.30 & 8-01\end{array}$ & $\begin{array}{ll}1.00 & E-03 \\
1.23 & \geq-02 \\
6.60 & z-02 \\
1.67 & z-01\end{array}$ & 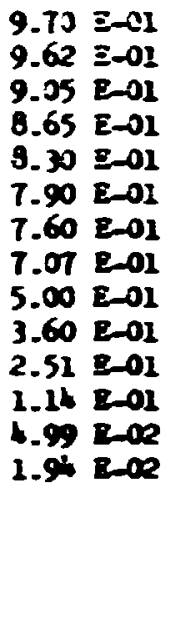 & $\begin{array}{ll}2.90 & E-02 \\
3.71 & E-02 \\
7.50 & E-02 \\
1.09 & E-01 \\
1.00 & E-0 i \\
1.99 & E-01 \\
2.50 & E-01 \\
2.94 & E-01 \\
1.68 & E-01 \\
5.90 & E-01 \\
6.69 & E-01 \\
6.23 & E-01 \\
5.20 & E-01 \\
3.60 & E-01 \\
1.01 & 2-01 \\
3.91 & 2-02 \\
1.60 & E-02 \\
9.60 & E-03\end{array}$ & 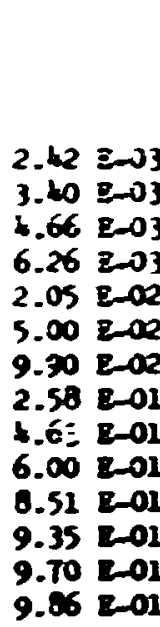 \\
\hline
\end{tabular}

\section{Derines:}

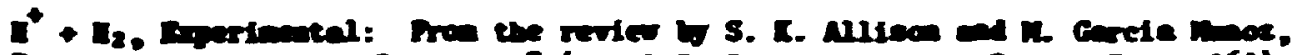

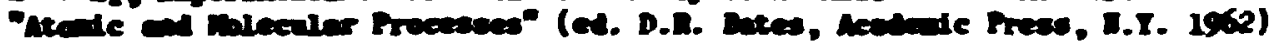
ner.

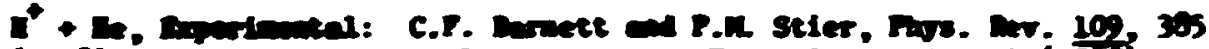

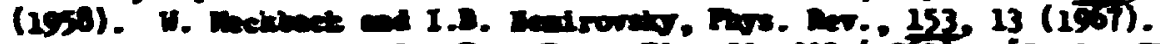

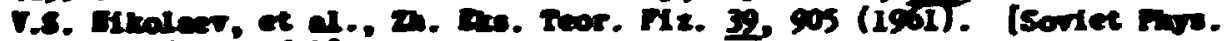
Jut 13, 61 (2051)].

\section{$4=x+2=$}

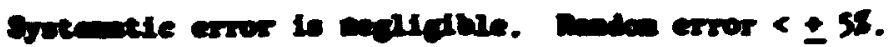


A.6.2:

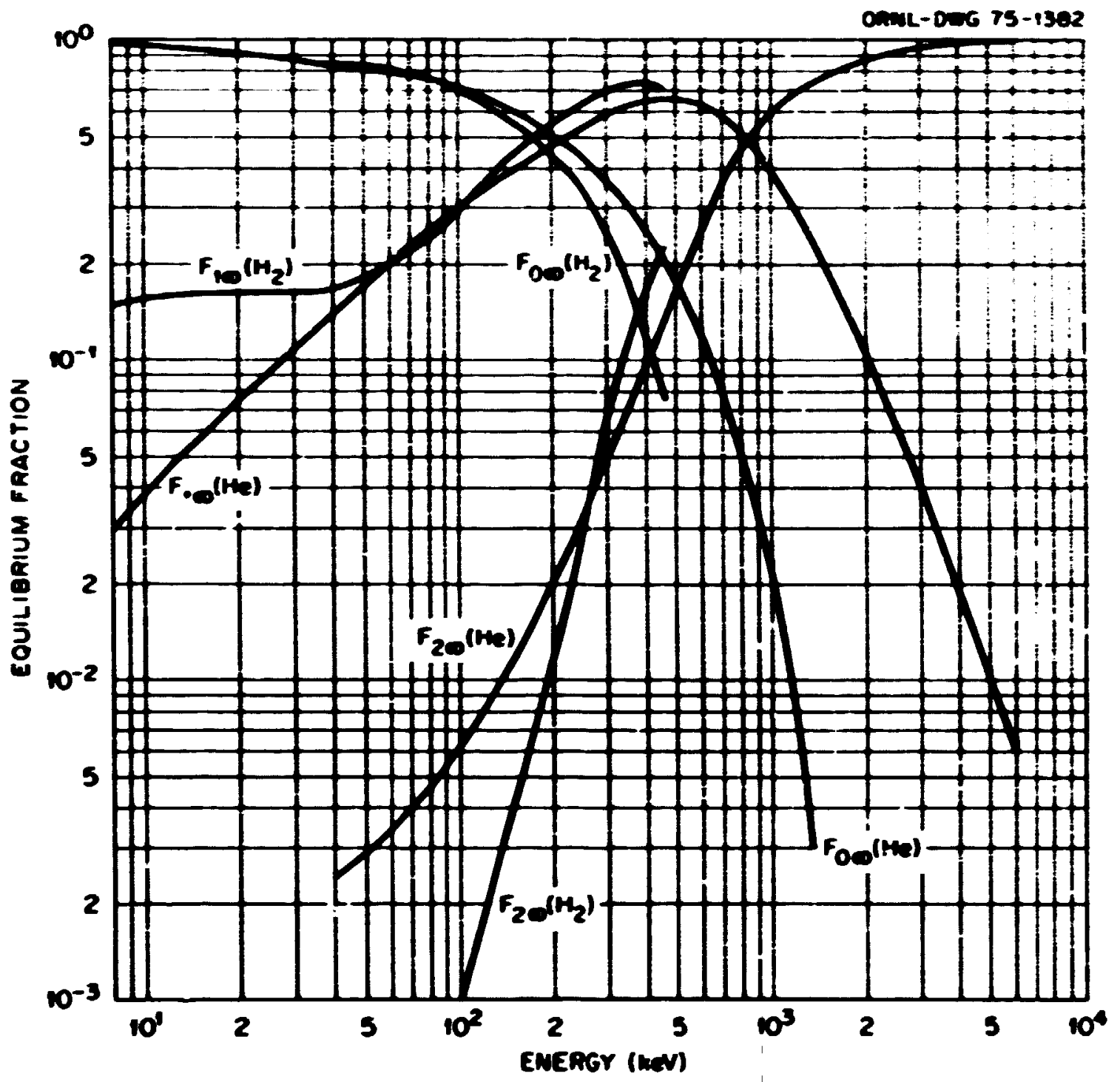




\section{A.6.26}

Equilibriun Frections of a teliue Been in $x_{2}$ eat $s_{2}$

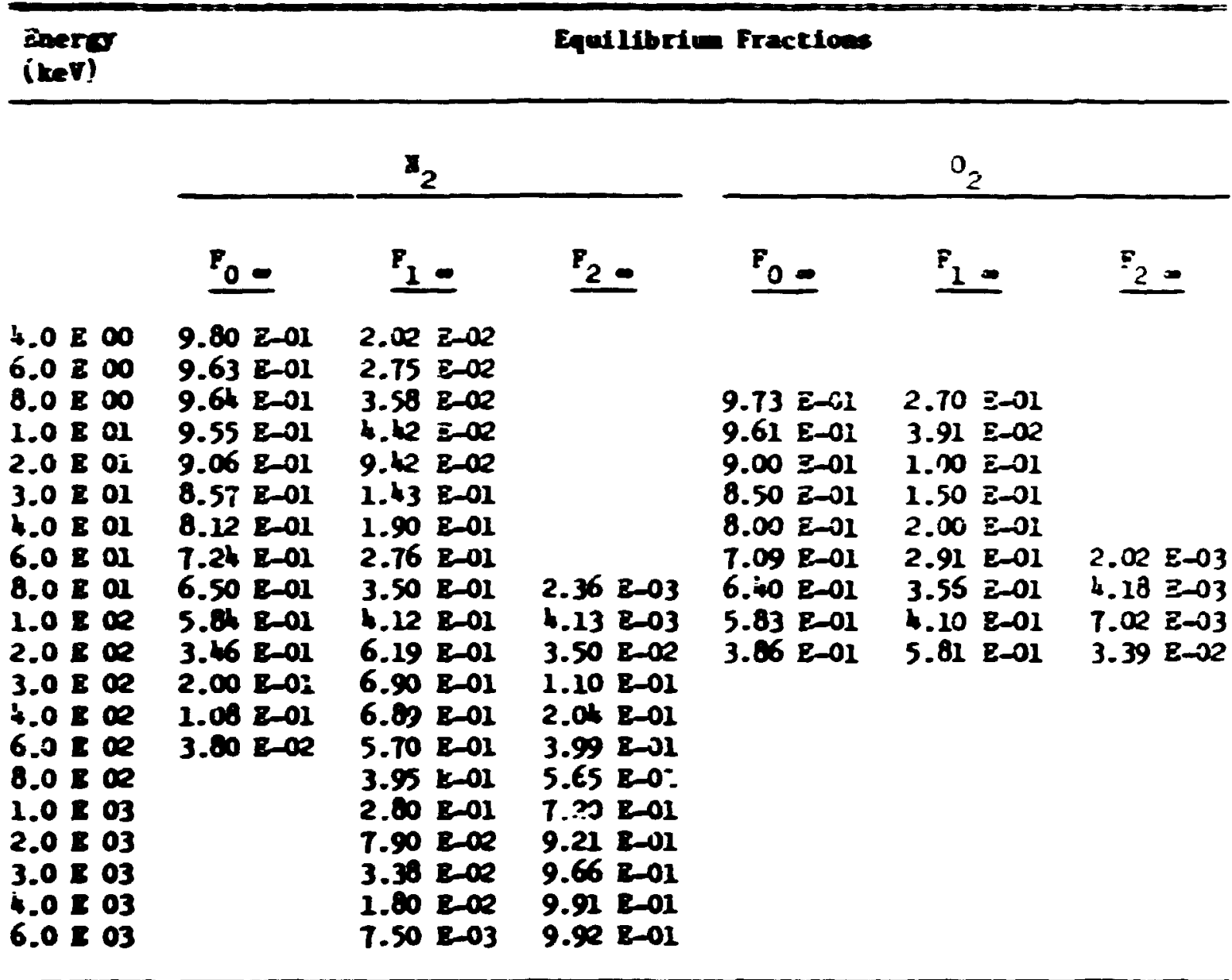

\section{Anterences:}

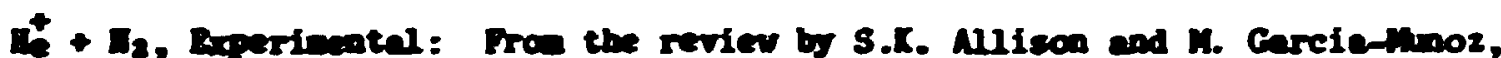
"hkonie ad Mblecular Prosesses," (ed. D.R. Bates, Mecdeaic Press I.T. 1962) pace T21. V.S. Hibioleer et al., 2h. Bus. Teor. Piz. 39, 905 (1961)

[sovet Pus. Jat 12, 627 (196i)].

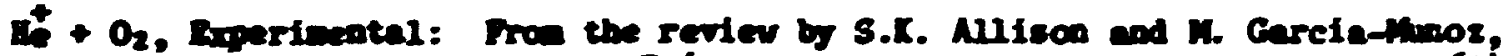
"Atodie ad holecular Proceaces," (ed. D.R. Bates, Mcodenic Preas M.I. 1962) pace Ted.

seover:

Sptenatic error is aedidble. Radon error $< \pm 58$. 


\section{A.6.2?}

,

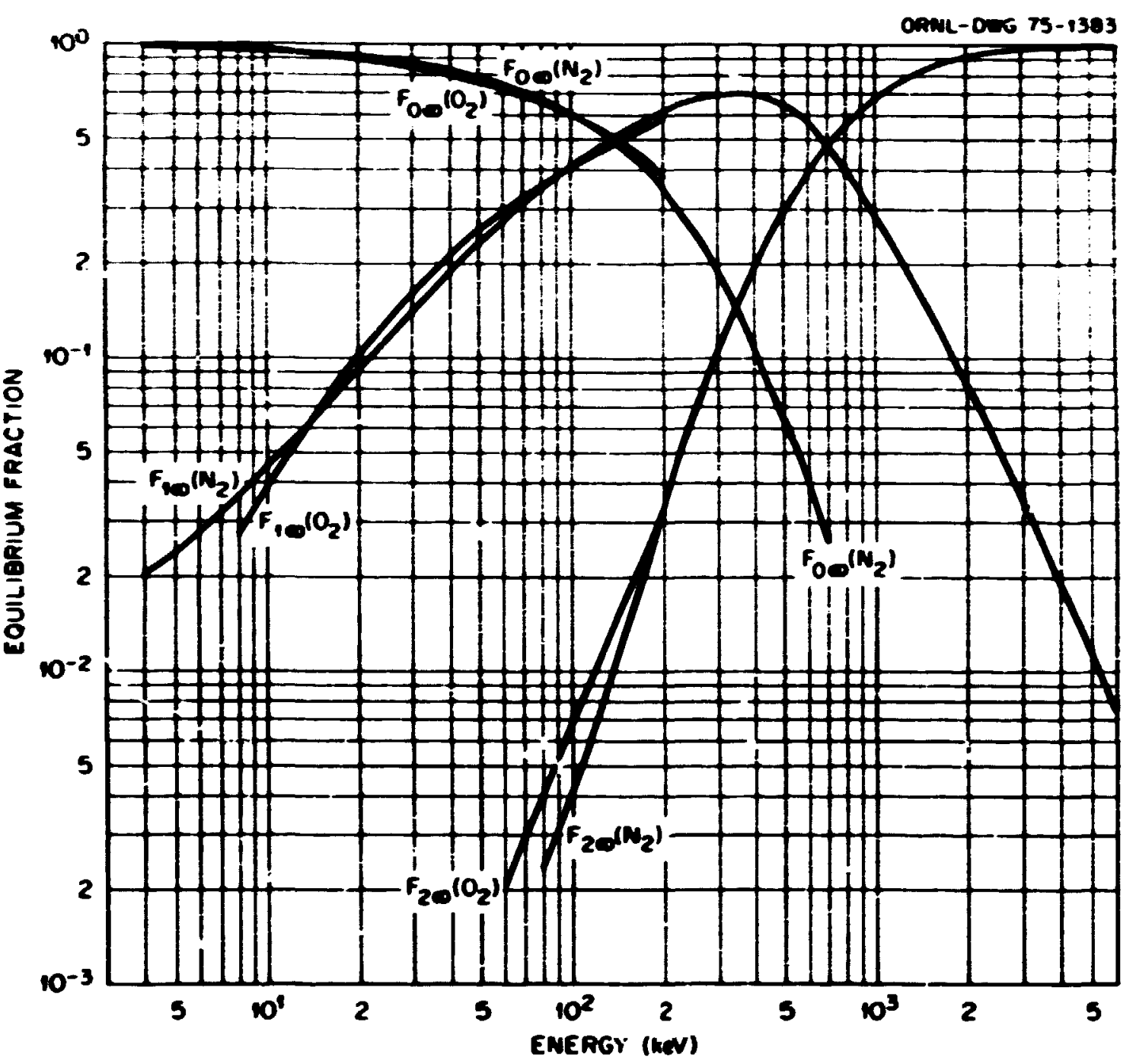

J 


\section{A.6.28}

Bquilibriun Fractions of a Heliun Beas in Ar

\begin{tabular}{|c|c|c|c|}
\hline \multirow{3}{*}{$\begin{array}{l}\text { Bneray } \\
\text { (keV) }\end{array}$} & \multicolumn{3}{|c|}{ Equilibrion Fractions } \\
\hline & \multicolumn{3}{|c|}{ Ar } \\
\hline & $P_{0}=$ & $\underline{P_{1}}$ & $\mathbf{F}_{2}$ \\
\hline 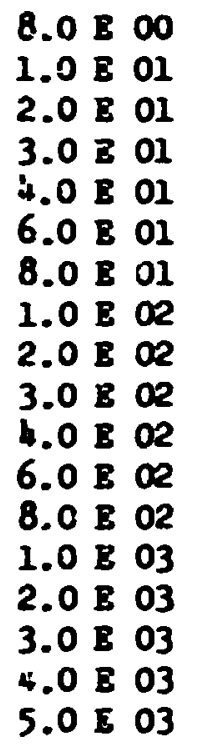 & $\begin{array}{ll}9.95 & \mathrm{E}-01 \\
9.91 & \mathrm{E}-01 \\
9.65 & \mathrm{E}-01 \\
9.30 & \mathrm{E}-01 \\
8.91 & \mathrm{E}-01 \\
8.08 & \mathrm{E}-01 \\
7.23 & \mathrm{E}-01 \\
6.53 & \mathrm{E}-01 \\
3.53 & \mathrm{E}-01 \\
1.36 & \mathrm{E}-01 \\
6.90 & \mathrm{E}-02 \\
1.90 & \mathrm{E}-02\end{array}$ & $\begin{array}{ll}5.00 & E-03 \\
8.70 & E-03 \\
3.51 & E-02 \\
6.90 & E-02 \\
1.09 & E-01 \\
1.92 & E-01 \\
2.76 & E-01 \\
3.44 & E-01 \\
6.20 & E-01 \\
7.70 & E-01 \\
7.14 & E-01 \\
4.90 & E-01 \\
3.33 & E-01 \\
2.35 & E-01 \\
8.00 & E-02 \\
4.00 & E-02 \\
2.40 & E-02 \\
1.60 & E-02\end{array}$ & $\begin{array}{ll}1.10 & \mathrm{E}-03 \\
2.53 & \mathrm{E}-03 \\
2.70 & \mathrm{E}-02 \\
9.39 & \mathrm{E}-02 \\
2.07 & \mathrm{E}-01 \\
4.90 & \mathrm{E}-01 \\
6.67 & \mathrm{E}-01 \\
\mathrm{~T} .65 & \mathrm{E}-01 \\
9.20 & \mathrm{E}-01 \\
9.60 & \mathrm{E}-01 \\
9.68 & \mathrm{E}-01 \\
9.84 & \mathrm{E}-01\end{array}$ \\
\hline
\end{tabular}

Rererences:

He + Ar. Bxperizentel: Prom the review by S.K. Allison and M. GarciaMunoz, "Atonic and Wolecular Processes," (ed. D.R. Bates, Academic

Press, M.Y. 1962) page 721. V.S. Nikolaev et al, 2h. Bks. Teor. Fix. 39, 905 (1961) [Soviet Phys. JEIP 12, 627 (1961)].

\section{Accuracy:}

Systematic error is negligible. Random error $< \pm 5 \%$. 
c

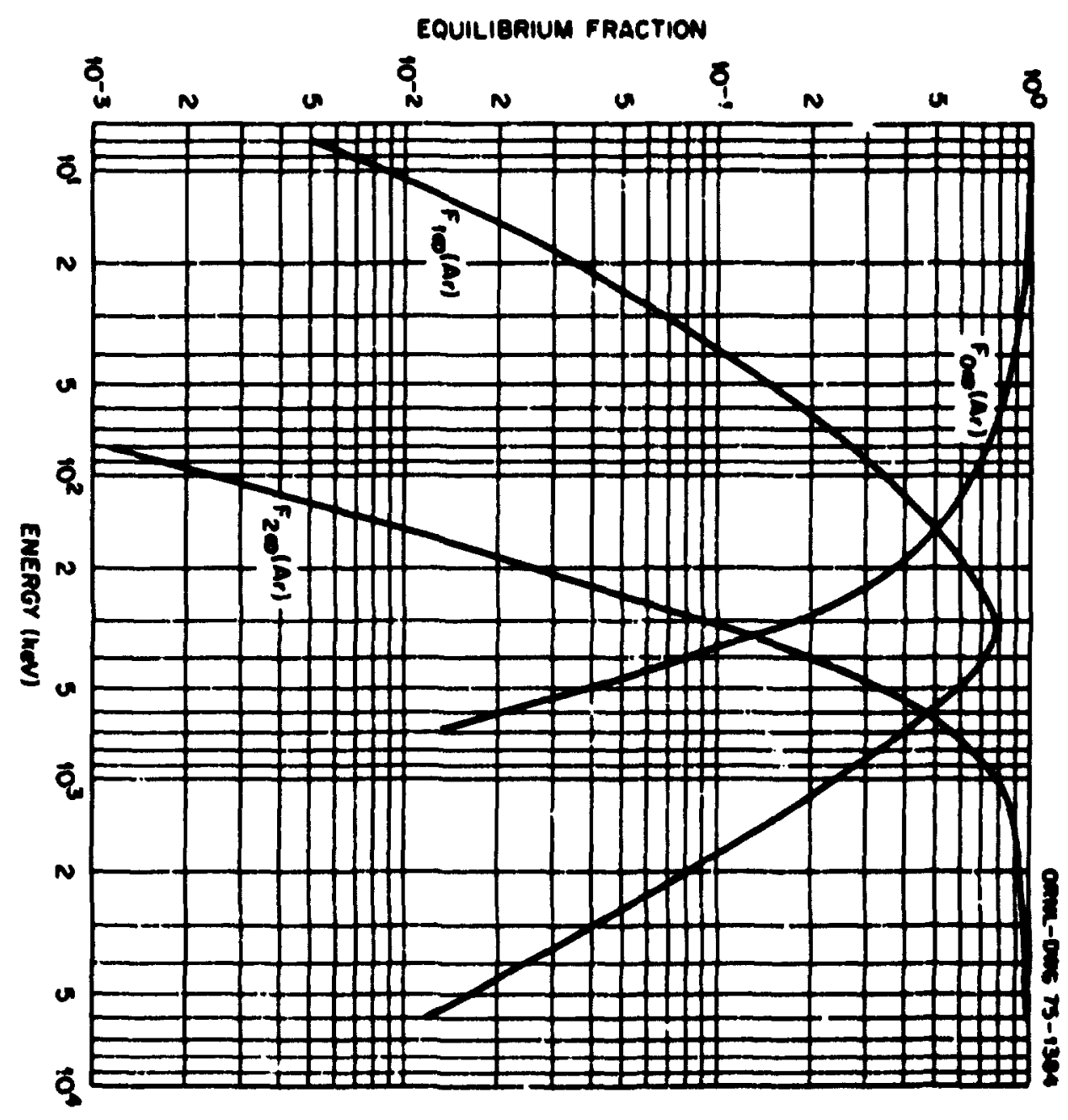




\section{A.6.31}

\section{Dotes}

(1) At very hich enerdies the equilibriu frections are expected tc be given bo $F_{0}=\sigma_{10} /\left(\sigma_{10}+\sigma_{11}\right)$ and $F_{1}=\sigma_{01} /\left(\sigma_{10}+\sigma_{11}\right)$ where $\sigma_{01}$ is the cross section for electron stripping fron and $\sigma_{10}$ is the cross section for electron cepture bs $\mathrm{H}^{+}$. We have used direct neasurenents of fractions vherever possible but where these are not arailable we have cenerated velues using cross sections frow the references cited.

(2) The equilibrium-excited state rrection referred to bere is the fraction of the neutral nux that appears in a state of principal quantu numer $\mathrm{n}$ T.e. the rrection suned orer all angular mentur states of a (Aven $\mathrm{n}$ ). It is not the fraction of the total Plux including $\mathrm{H}^{+}, \mathrm{H}^{\circ}, \mathrm{H}^{-}$. It is found that the excited state Frection of the neutrel bean decresses as $\mathrm{n}^{-3}$. Thus it is convenient and conventional to quote the frection measured for a civen $\mathrm{n}$, altiplied by $a^{3}$; we adopt that convention here. It should be noted that the $\mathrm{n}^{-3}$ rule bolds onls for the hicher principal quantum number states and the date reproduced bere are deternined for $n \geq$ 10. Use of these date to estinate populations for sinell a nas bé subject to error. In order to deternine the fraction of excited neutrals of a diven state $n$, in the totel flux of particles, one siuply takes the value of $F_{0}$. for the reaction from the relevant preceding table, aultiplying by the flgure civen bere and dividing of the cube of the principal quantu number of interest.

(3) The reader is cautioned that an equilibriun excited state frection of the neutral bean is not the greatest fraction observed in the neutral bean; the greatest fraction occurs at a lower pressure than the equilibriu frection and as exceed the equilibriu fraction by a factor of three. See the references quoted here for deteils.

(4) Deuterons are expected to have the sane fractions as protons of equal relocity. 
0

A.7 Stoppine Grose section and Particle Bance

0 for Dens Particles in Caces and untels 
A.7.2

Stopping Grose Sections for Protons and I stons in Atonic fordrogen

\begin{tabular}{|c|c|c|}
\hline$\frac{\operatorname{mat}}{(\operatorname{man})}$ & \multicolumn{2}{|c|}{$\begin{array}{l}\text { Stogifing Croes Sections } \\
\text { (ev-e-O/aton) }\end{array}$} \\
\hline 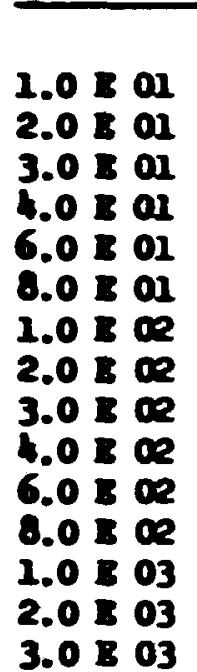 & $\begin{array}{ll} & \mathrm{I} \\
1.20 & \mathrm{~B}-14 \\
1.36 & \mathrm{E}-14 \\
1.22 & \mathrm{~B}-14 \\
1.10 & \mathrm{E}-14 \\
8.50 & \mathrm{E}-15 \\
7.00 & \mathrm{E}-15 \\
6.15 & \mathrm{E}-15 \\
3.74 & \mathrm{E}-15 \\
2.78 & \mathrm{E}-15 \\
2.21 & \mathrm{E}-15 \\
1.66 & \mathrm{E}-15 \\
1.32 & \mathrm{E}-15 \\
1.12 & \mathrm{E}-15 \\
6.50 & \mathrm{E}-16 \\
4.70 & \mathrm{E}-16\end{array}$ & \begin{tabular}{cc}
\multicolumn{1}{c}{$E$} \\
2.80 & $E-15$ \\
3.78 & $E-15$ \\
4.32 & $E-15$ \\
4.70 & $E-15$ \\
5.20 & $E-15$ \\
5.40 & $E-15$ \\
5.41 & $E-15$ \\
4.61 & $E-15$ \\
3.88 & $E-15$ \\
3.31 & $E-15$ \\
2.55 & $E-15$ \\
2.18 & $E-15$ \\
1.74 & $E-15$ \\
9.60 & $E-16$ \\
6.50 & $E-16$
\end{tabular} \\
\hline
\end{tabular}

\section{Dranes:}

$I^{+}+$I and I I Theoretical: A. Dalgarno and G.H. Grifring, Proc. Ros. 8oc. $\triangle$ 232, 123 (1955).

cences:

ser lote (4) at and of chapter. 
A.7.3

i)

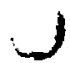

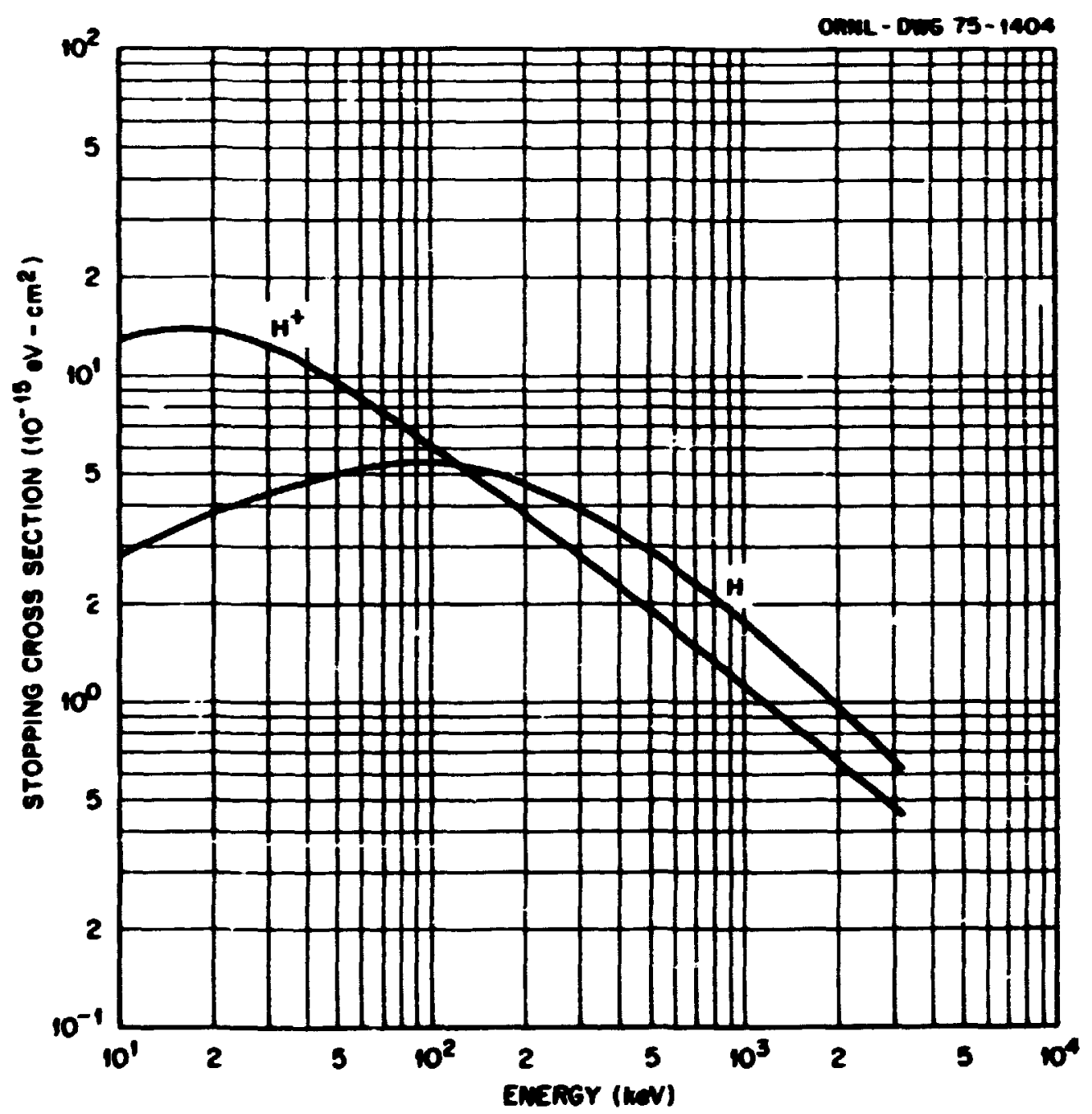

j) 
Stoping Crors Sections for Protons in Gases

$$
\left(\mathrm{H}_{2}, \mathrm{He}, \mathrm{H}_{2}\right)
$$

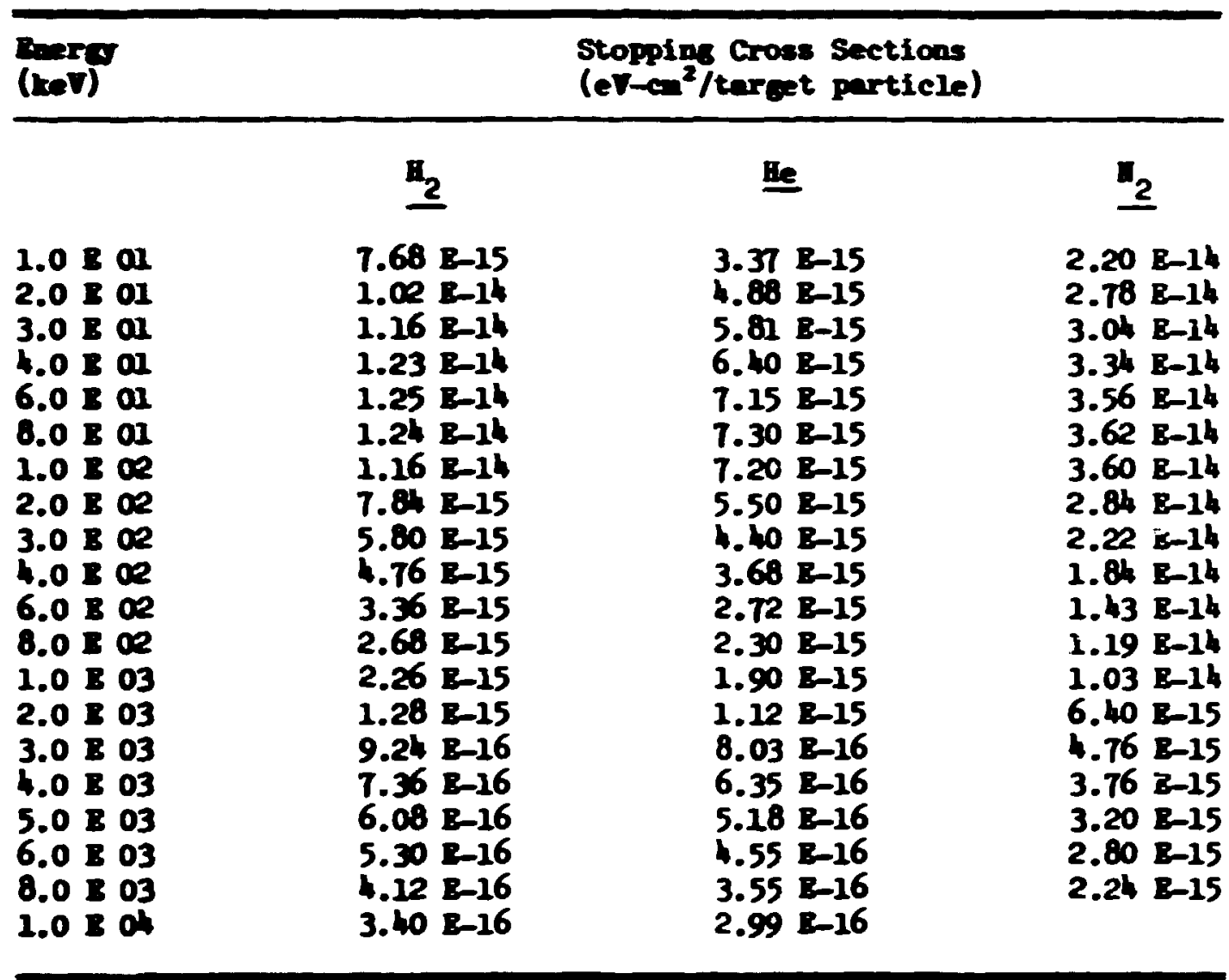

- [Data are in eV - $a^{2} /$ molecule for $\mathrm{H}_{2}$ and $\mathrm{H}_{2} ; \mathrm{eV}-\mathrm{c}^{2} / a t o$ for He].

Befernes:

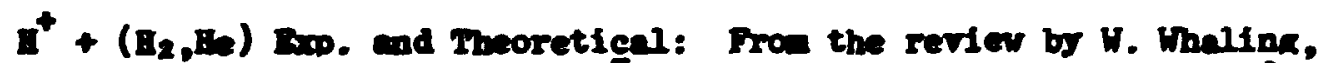
Eandioch der Puyil, a. 8. Mücre, Springer-Verles, Beriln 1958, Volve 34, pere 293.

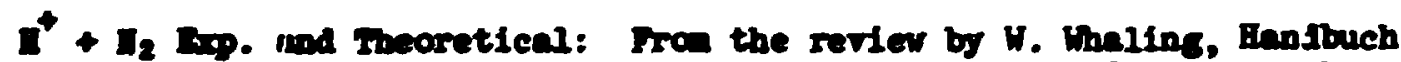
in Tupil, ad. 8. Mucre, Springer-Ver1es, Beriln 1958, Valune 34, pere 173.

\section{Lites:}

sac Wotas (1), (5), and (10) at and of chapter.

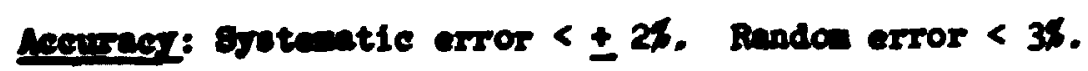


A.7.5

s

$\checkmark$

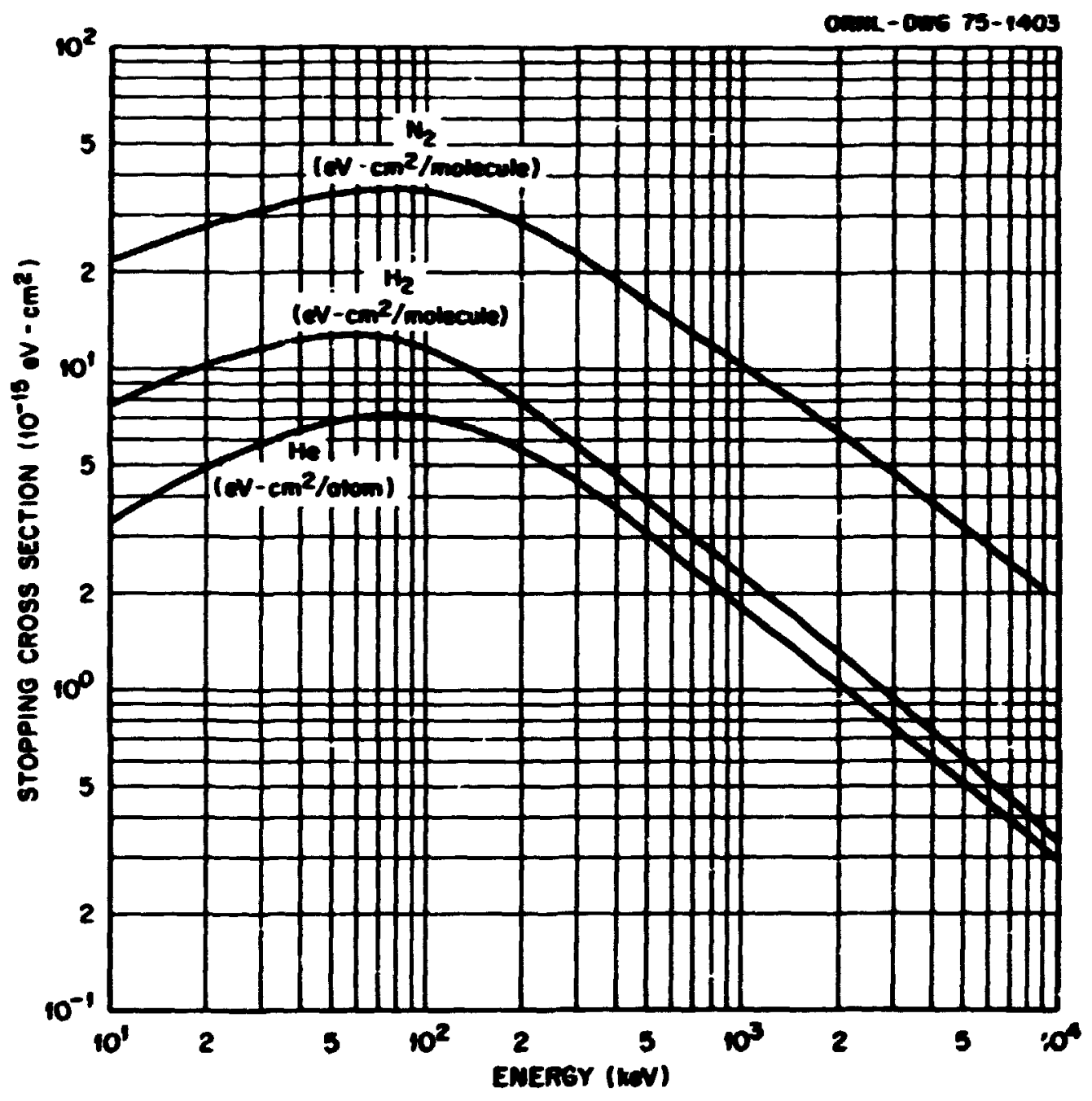


Stopping Cross Sections for Protons in Carbon and Iron

\begin{tabular}{|c|c|c|}
\hline \multirow[t]{2}{*}{$\begin{array}{l}\text { Beres } \\
\text { (keV) }\end{array}$} & \multicolumn{2}{|c|}{$\begin{array}{l}\text { Stopping Cross Sections } \\
\text { (ev-c } \mathrm{cm}^{2} / \text { aton) }\end{array}$} \\
\hline & $\underline{c}$ & Pe \\
\hline 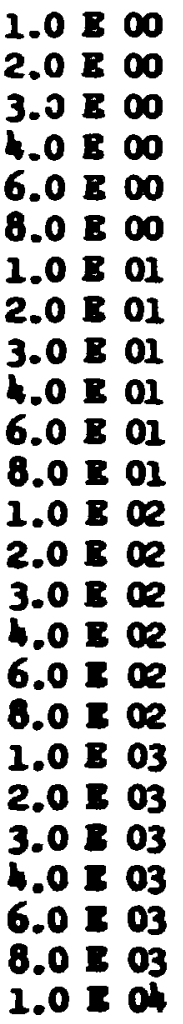 & 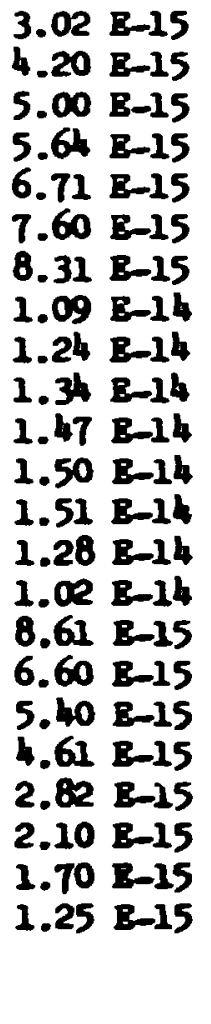 & $\begin{array}{ll}2.72 & \mathrm{~B}-14 \\
2.41 & \mathrm{E}-14 \\
2.17 & \mathrm{E}-14 \\
1.73 & \mathrm{E}-14 \\
1.47 & \mathrm{E}-14 \\
1.28 & \mathrm{E}-14 \\
8.00 & \mathrm{E}-15 \\
6.10 & \mathrm{E}-15 \\
3.00 & \mathrm{~B}-15 \\
3.78 & \mathrm{E}-15 \\
3.10 & \mathrm{~B}-15 \\
2.63 & \mathrm{E}-15\end{array}$ \\
\hline
\end{tabular}

\section{Drences:}

$E^{+}+$C In. And Theorotical: Trom the review by W. Wheling, Gendouch Cor ruyli, a. 8. Mive, Springer-Verles, Berlin 1958, Volume 3h, page 193; 3.1. Orod and I.E. Dockworth, Cen. J. Puvs. 4l, 1424 (i963); Z.P. Artajer an In. V. Cott, 2h. Dtop. Teor. Hz. 56, 1146 (1969) [Sortet rivies JaP 29,615 (1969)].

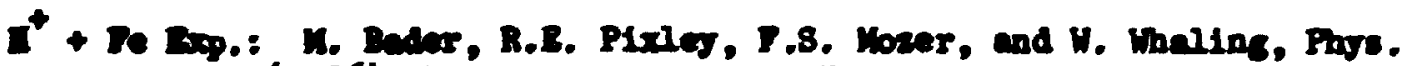
Rov. 103, 32 (1956); H.H. Laderreen, C.C. Hanke, H. Simoneen, H. Sorensen, and P. VJde, Prye. Bov. 175, 389 (1968).

\section{Exis:}

see lotes $(1),(2),(5),(10)$, and (11) at and of chapter.

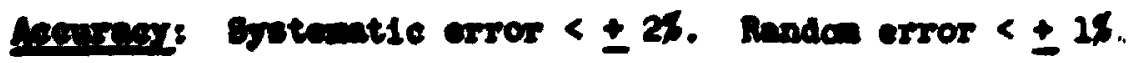




\section{A.7.7}

.j

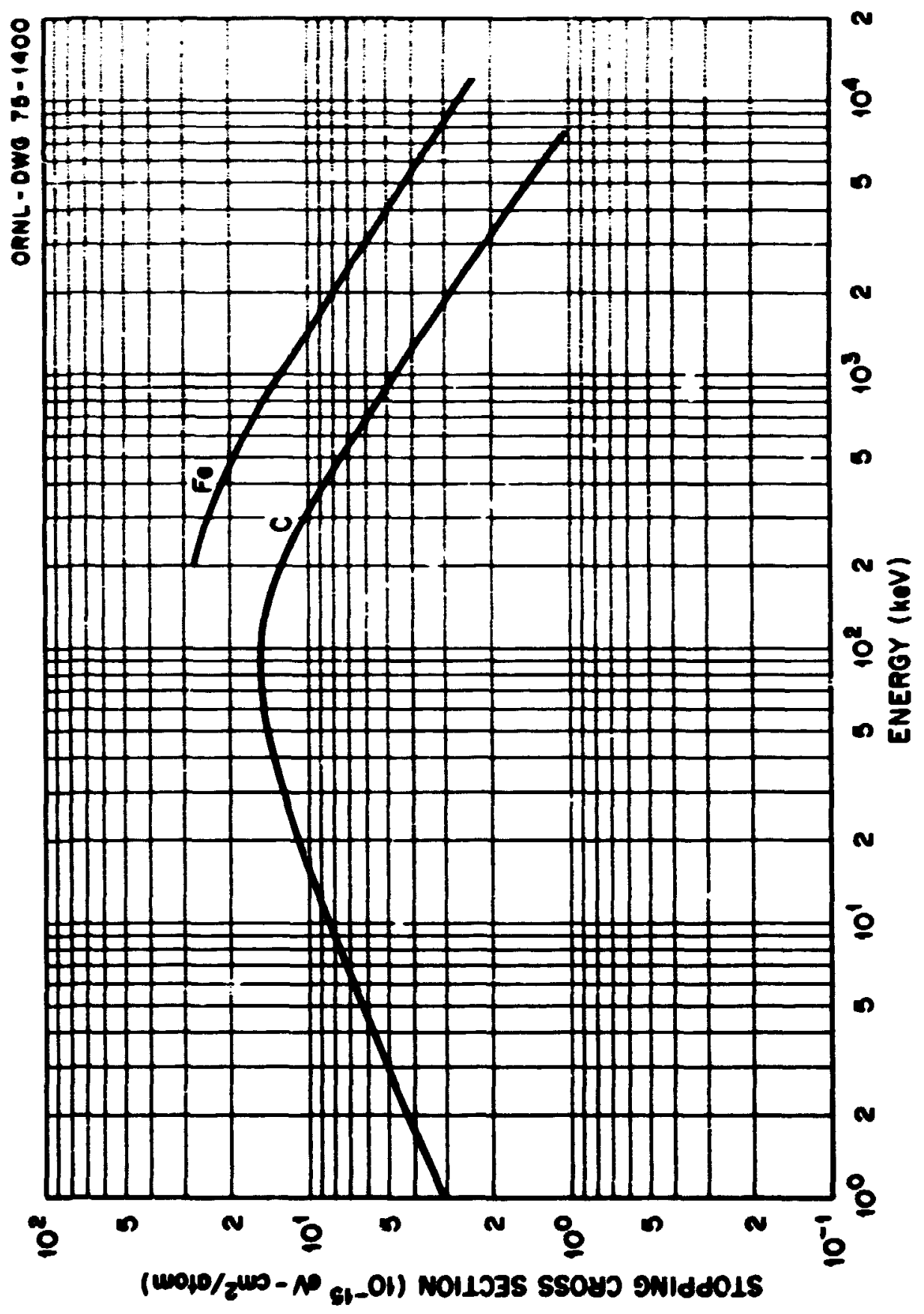




\section{A.T.8}

Stopping Crows Sections for Protons in

Copper, Molybdenun, Tungaten, and Coid

\begin{tabular}{|c|c|c|c|c|}
\hline \multirow[t]{2}{*}{$\begin{array}{l}\text { Bnery } \\
\text { (neVt) }\end{array}$} & \multicolumn{4}{|c|}{ 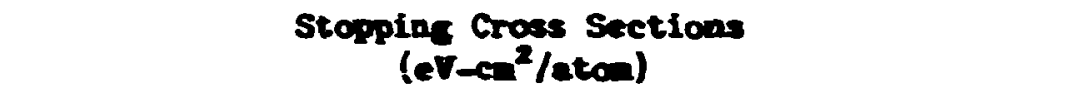 } \\
\hline & 으 & E & $\underline{U}$ & $\underline{\underline{A u}}$ \\
\hline 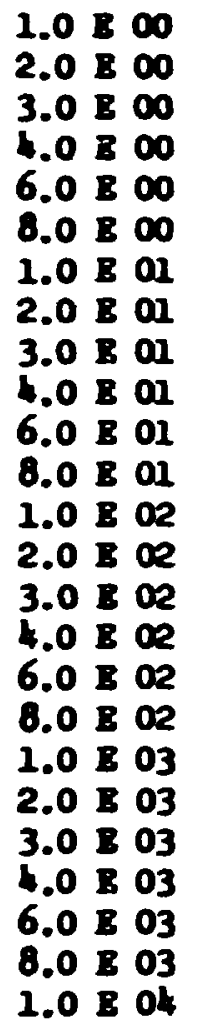 & 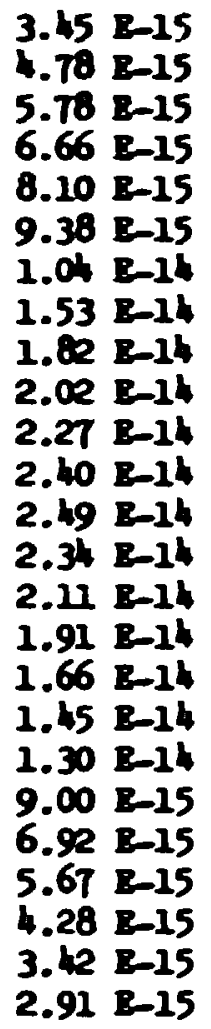 & $\begin{array}{ll}1.30 & B-14 \\
9.80 & B-15 \\
8.00 & B-15 \\
7.00 & B-15 \\
5.70 & B-15\end{array}$ & $\begin{array}{l}1.83 \mathrm{~B}-14 \\
1.38 \mathrm{~B}-14 \\
1.10 \mathrm{~B}-14 \\
9.40 \mathrm{~B}-15 \\
8.10 \mathrm{~B}-15\end{array}$ & $\begin{array}{ll}1.94 & \mathrm{E}-14 \\
2.60 & \mathrm{E}-14 \\
2.99 & \mathrm{E}-14 \\
3.28 & \mathrm{E}-14 \\
3.60 \mathrm{E}-14 \\
3.80 \mathrm{E}-14 \\
3.91 \mathrm{E}-14 \\
3.93 \mathrm{E}-14 \\
3.59 \mathrm{E}-14 \\
3.20 \mathrm{E}-14 \\
2.83 \mathrm{E}-14 \\
2.32 \mathrm{E}-14 \\
2.14 & \mathrm{E}-14 \\
1.54 & \mathrm{E}-14 \\
1.23 & \mathrm{E}-14 \\
1.93 & \mathrm{E}-14 \\
8.20 & \mathrm{E}-15 \\
6.97 & \mathrm{E}-15 \\
6.00 \mathrm{E}-15\end{array}$ \\
\hline
\end{tabular}

\section{Beferences:}

$\mathrm{B}^{+}+(\mathrm{Cu}, \mathrm{du}) \mathrm{Bg}$. and Theoretical: Pron the review by W. Whaling, Rendbuch der Phati, ed. S. Müce, Sprineer-Verlas, Berlin 1958, Volve 34, page 193. A. Veleazuels, H. Meckbach, A.J. Kestelnan, and J.C. Eckardt, Pws. Bov. B 6, 95 (1972) (normalized to Whalling). I. Norite, B. Nimine, and T. Butta, J. Prys. Soc. Jepan 25, 1525 (1968) (nowalled to Velenziele). B.P. Arthipor and Iu. V. Cott, zh. Drap.

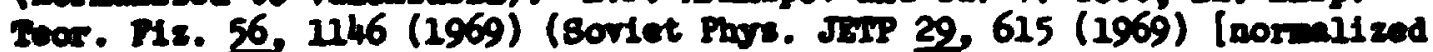
to Mosite].

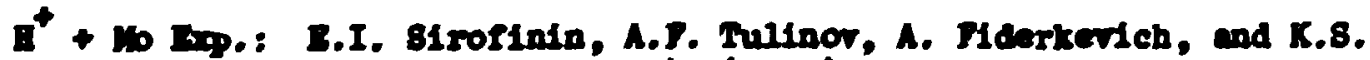
ompridn, Radetion Brfecte 15 , itg (1972).

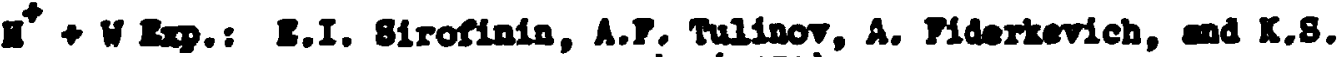
owhein, Radiation Bffects 15, 149 (1972).

Dotes: see notes (1), (2), (10), (12), and (13) at end of chepter. Sagreor: Sytemetic arror $< \pm 2 \%$. Randon error $< \pm 18$. 


\section{A.7.9}

3

0

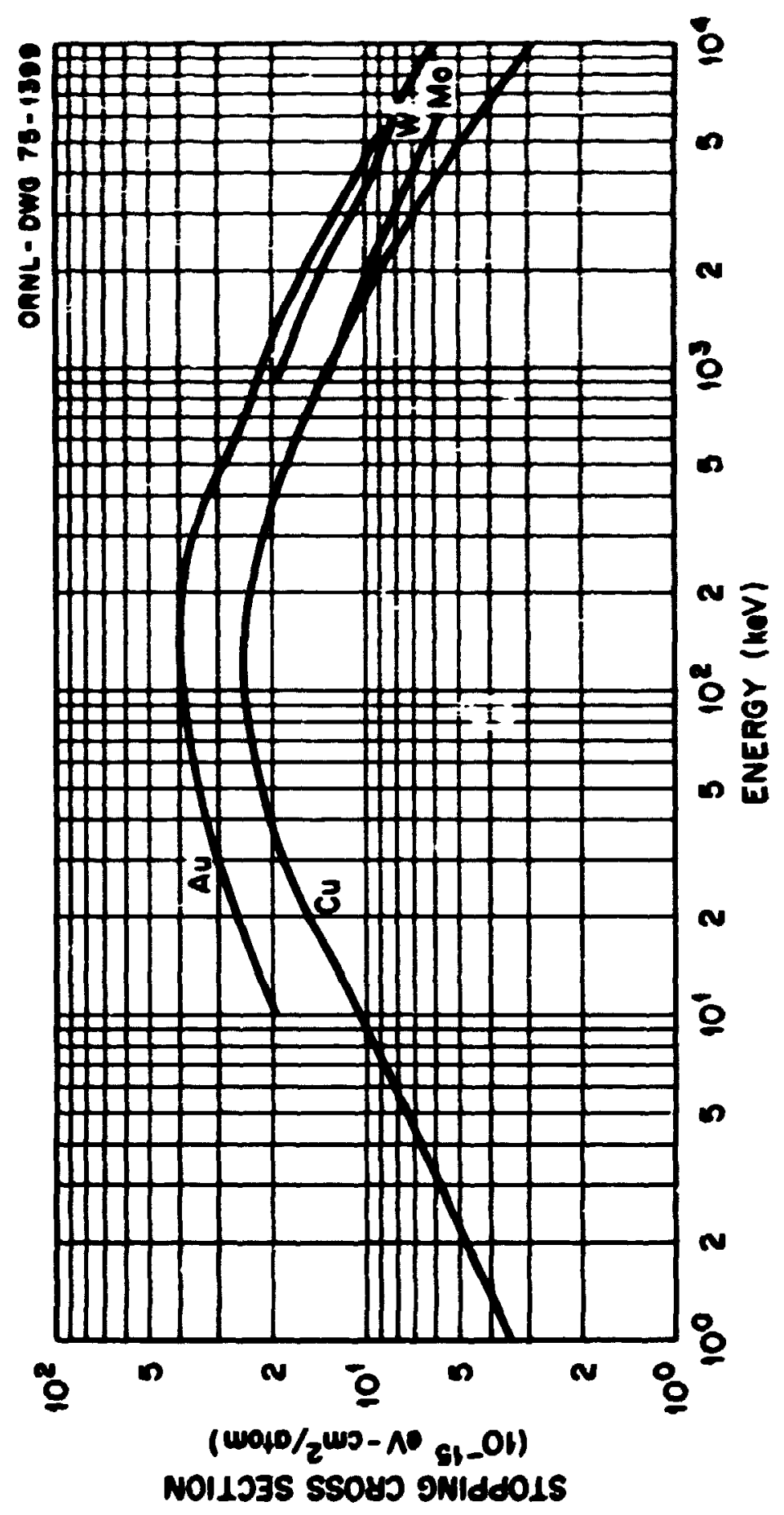

0 
Pance of $\mathrm{u}^{+}$in $\mathrm{u}_{2}$, fie, and $\mathrm{a}_{2}^{*}$

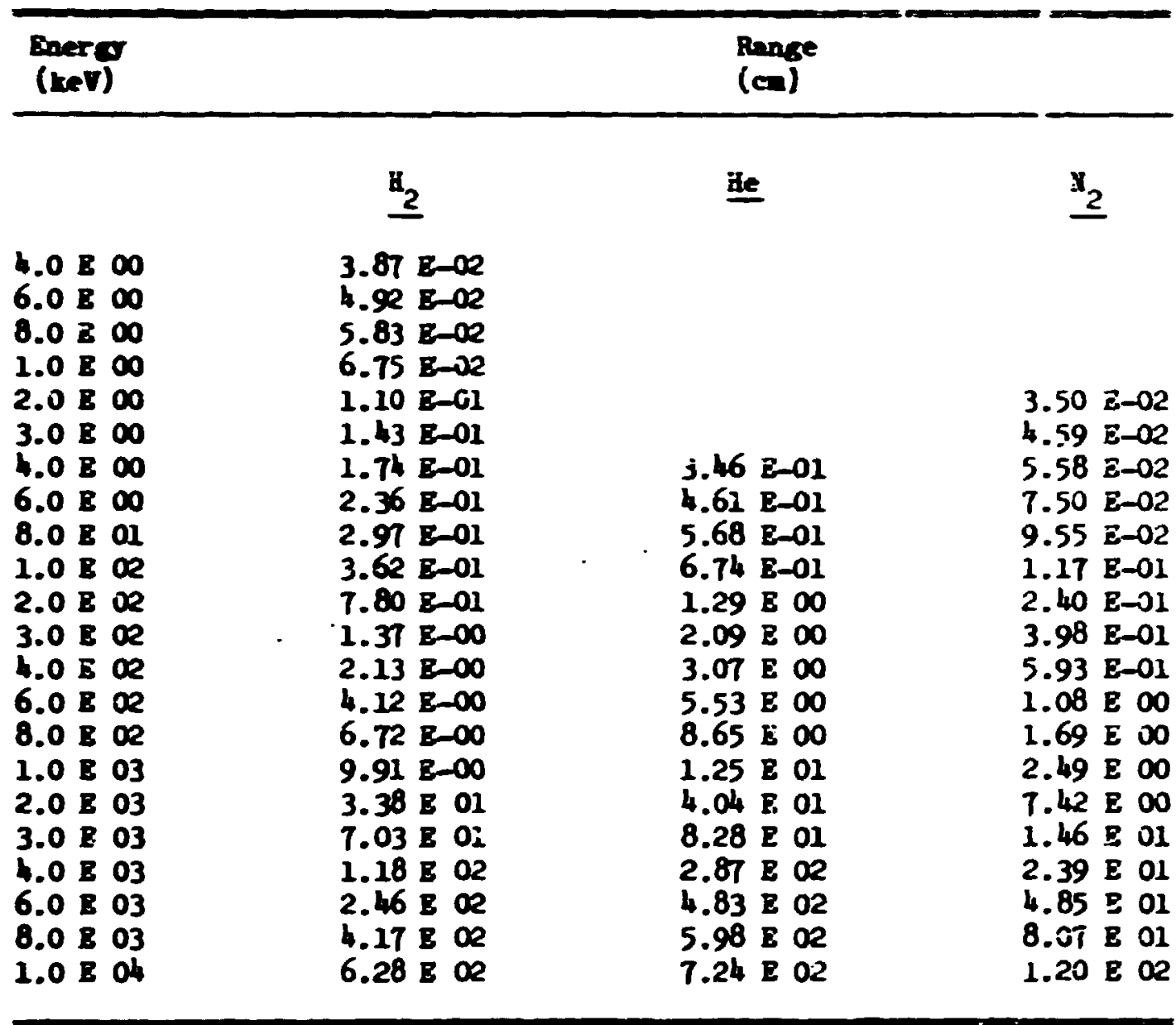

[For target at $760=$ H8 pressure and $15^{\circ} \mathrm{C}$ temperature].

\section{Deferences:}

$\mathrm{H}^{+}+\mathrm{H}_{2}$ Rxp.: Fron the review by $\mathrm{H}$. Thaling, Handbuch der Physik, ed. 8. Plürge, Springer-Verlag, Berlín 1958, Volume 34, page 193.

C.J. Cook, Z. Jones, Jr., and T. Jorcensen, Jr., Phys. Rev. 91,1417 (1953).

$\mathrm{A}^{+}+\left(\mathrm{Bl}, \mathrm{H}_{2}\right)$ Bep.: Pron the review by H. Whaling, Handbuch cier Physik, d. 8. Pilige, Springer-Verlag, Berlin 1958, Volune 34, page 193.

\section{Note:}

See lotes $(3),(5),(9),(10)$, and $(15)$ at end of chapter.

iccureg:

Systematic error $< \pm 2 \%$. Randon error $< \pm 1.5 \%$. 
A.7.11

.j

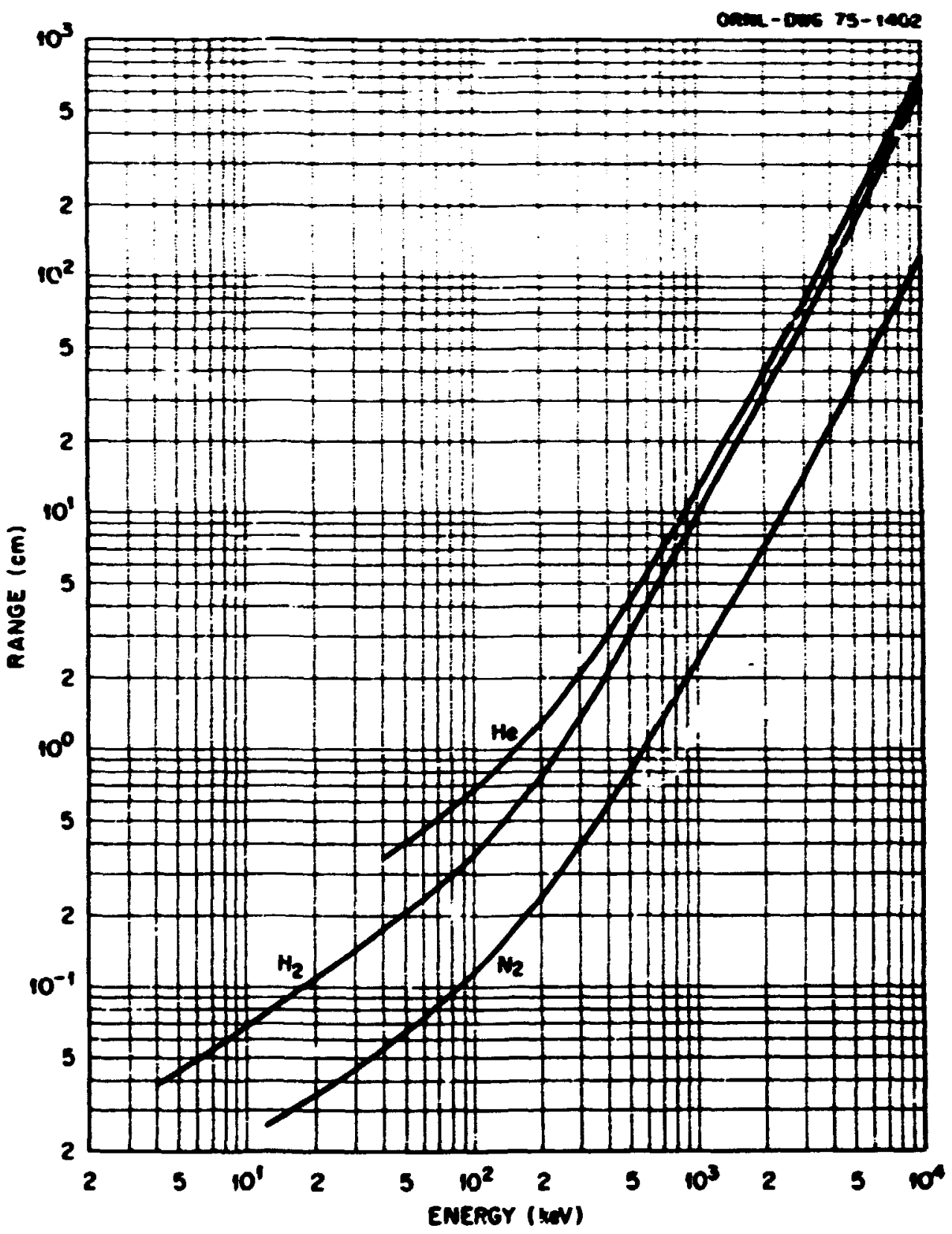

0 


\section{A.7.12}

Range of $\mathrm{H}^{+}$in Solid $\mathrm{C}, \mathrm{Cu}$, and Au

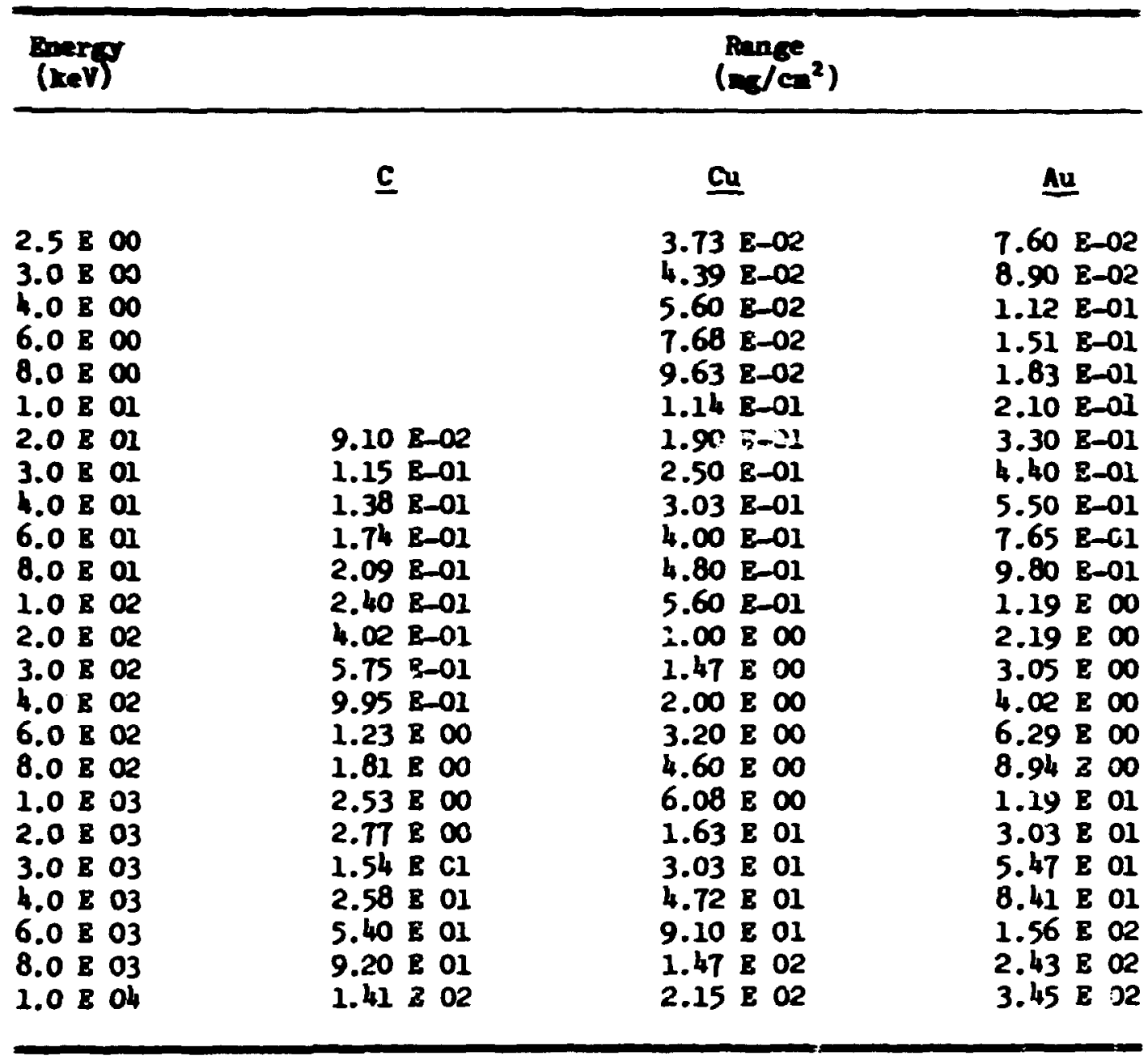

\section{Rererences}

$\mathrm{H}^{+}+\mathrm{C}$ Theoretical: L.C. Horthcliffe and R.F. Schilling, Nuclear Date Tables A I, 233 (1970).

$\mathrm{H}^{+}+(\mathrm{Cu}, \mathrm{Au})$ Theoretical: From the review by $\mathrm{W}$. Whaling, Handbuch der Phyalk, ed. S. Flüge, Springer-Verlas, Berlin 1958, Volune 34, page 193. Experimental ( $\mathrm{E}<10 \mathrm{keV}$ ) R.L. Hines, Phys. Rev. 132, 701 (1963).

\section{Moter:}

See Hotes $(3),(5),(7),(10)$, and $(24)$ at end of chapter.

Accurecy: See Note (6) at end of chapter. 


\section{A.7.13}

ij

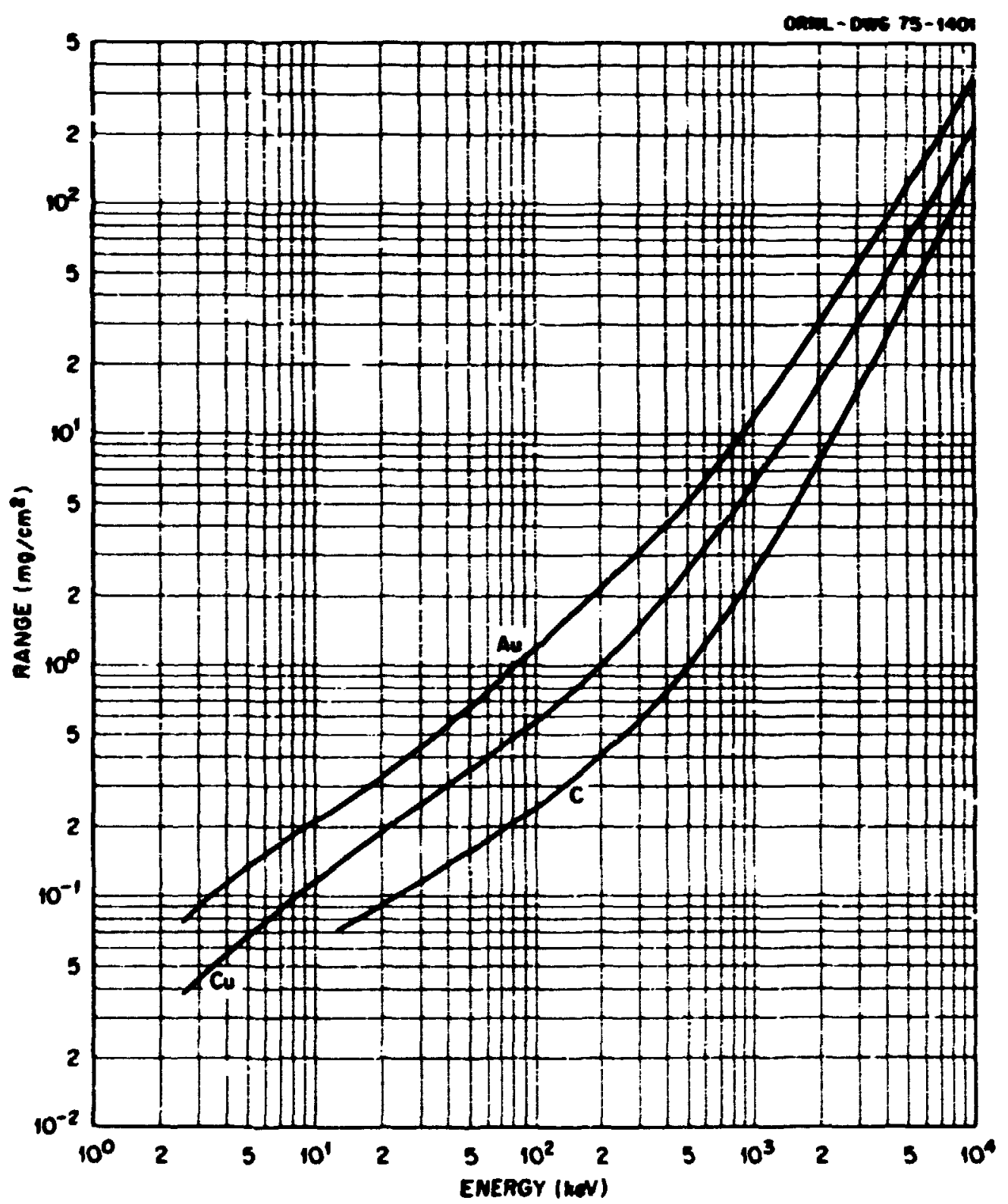


Ranges of Beliu Ions in Cases ( $\mathrm{H}_{2}, \mathrm{He}, \mathrm{H}_{2}$ )

\begin{tabular}{|c|c|c|c|}
\hline \multirow[t]{2}{*}{$\begin{array}{l}\text { Bera } \\
\text { (neV) }\end{array}$} & \multicolumn{3}{|c|}{$\begin{array}{c}\text { Rance (ca) [For target at } 760 \mathrm{~m} \text { Ho Pressure } \\
\text { and } 15^{\circ} \mathrm{C} \text { tengerature] }\end{array}$} \\
\hline & $\underline{B}_{2}$ & ㅂe & $\underline{\mathbf{B}_{2}}$ \\
\hline 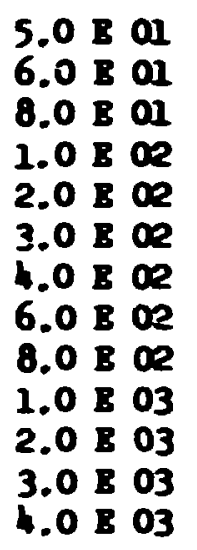 & 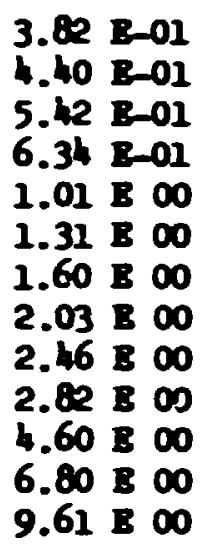 & 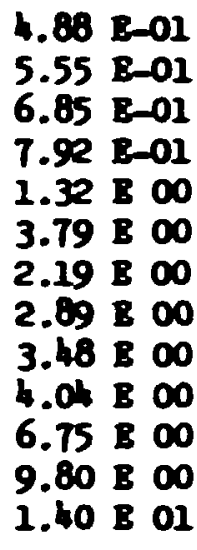 & $\begin{array}{ll}1.05 & \mathrm{E}-01 \\
1.20 \mathrm{E}-01 \\
1.45 \mathrm{E}-01 \\
1.69 \mathrm{E}-01 \\
2.68 \mathrm{E}-01 \\
3.49 \mathrm{E}-01 \\
4.20 \mathrm{E}-01 \\
5.40 \mathrm{E}-01 \\
6.44 \mathrm{~B}-01 \\
7.40 \mathrm{E}-01 \\
1.23 \mathrm{E} 00 \\
1.81 \mathrm{~B} 00 \\
2.64 \mathrm{~B} 00\end{array}$ \\
\hline
\end{tabular}

\section{Beferences:}

Buperinental and Theoretical: Pron the refiew by L.C. Morthcliffe and R.F. Schillins, Euclear Data Tables A I, 233 (1970).

Accurecs:

Iot specified. 


\section{A.7.15}

j)

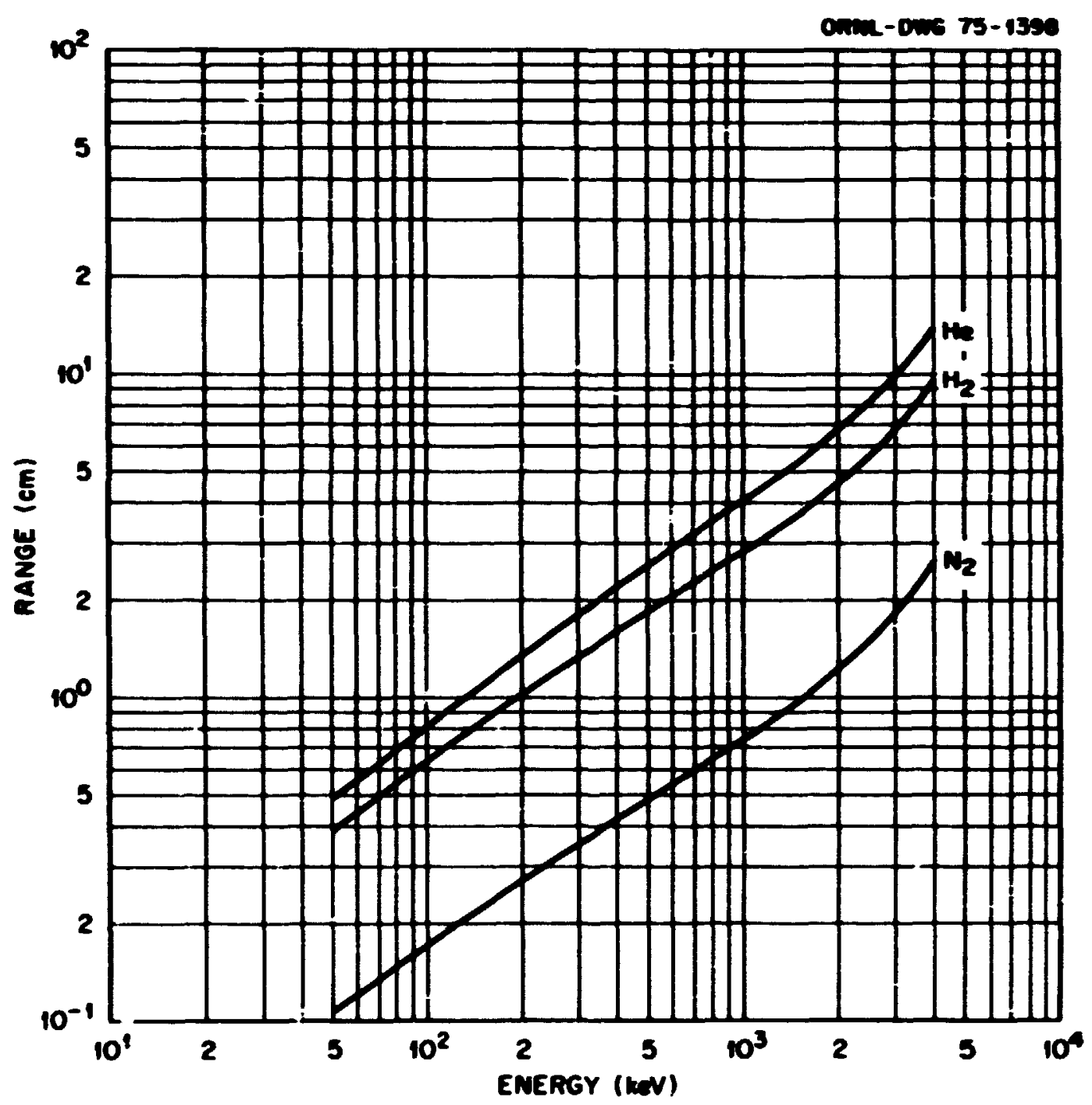




\section{A. $\mathbf{T} .16$}

Panges of Eeliun Ioss in Solids!:, Cu, Au)

\begin{tabular}{|c|c|c|c|}
\hline \multirow[t]{2}{*}{$\begin{array}{l}\text { Bherct } \\
\text { (keV) }\end{array}$} & & $\left(\operatorname{man} \cos ^{2}\right)$ & \\
\hline & $\underline{\mathbf{c}}$ & 으 & Ax \\
\hline 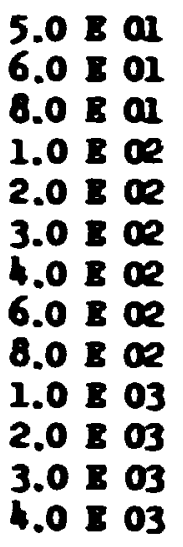 & $\begin{array}{ll}9.90 & \mathrm{E}-02 \\
1.11 \mathrm{E}-01 \\
1.35 \mathrm{E}-01 \\
1.55 \mathrm{E}-01 \\
2.35 \mathrm{E}-01 \\
2.99 \mathrm{E}-01 \\
3.56 \mathrm{E}-01 \\
1.59 \mathrm{E}-01 \\
5.59 \mathrm{E}-01 \\
6.50 \mathrm{E}-01 \\
1.72 \mathrm{E} 00 \\
1.90 \mathrm{~B} \quad 00 \\
2.85 \mathrm{~B} \quad 00\end{array}$ & 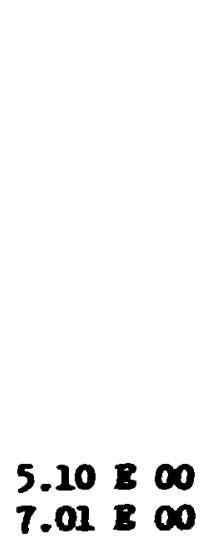 & 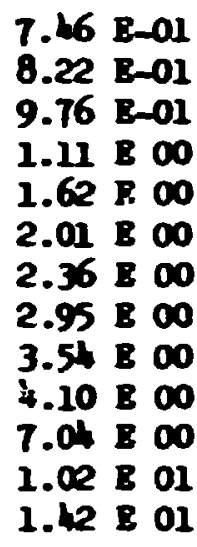 \\
\hline
\end{tabular}

\section{Daferencea:}

$\mathrm{E}^{+}+\mathrm{C}$ and Au, Bpperineatal and meoretical: Prow the reviev by I.C. Worthelffre, and R.P. Schllling, Ducleer Dete robles A I, 233 (1970).

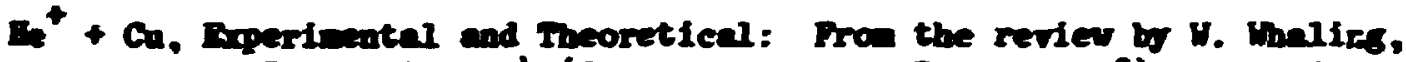
Bunduces der Puvelt Bend th (Springer Verles, Berlin 1958) pace 193.

securees:

Unopecifled. 


\section{A.T.17}

.3

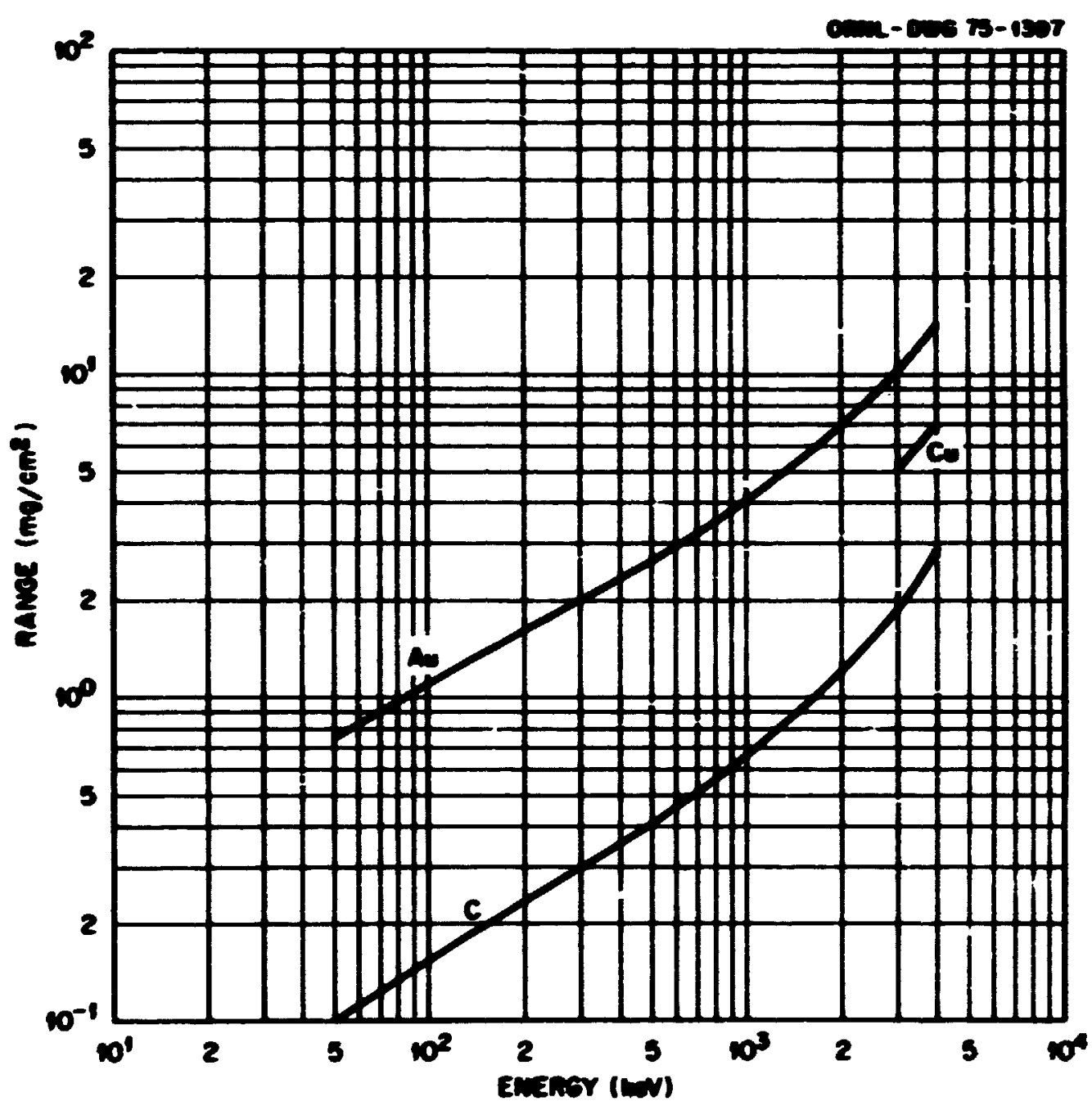




\section{A.T.18}

Conversica Pectors for Berry Loes Mesturenents

$$
\left(10^{-15} \mathrm{eV}-\mathrm{c}^{2}\right)=A\left(\frac{\mathrm{keV}-\mathrm{c}^{2}}{\mathrm{E}}\right)=\mathrm{B}\left(\frac{\mathrm{mV}}{\mathrm{c}}\right)
$$

\begin{tabular}{|c|c|c|c|c|}
\hline Exterial & $\Delta$ & $\mathbf{B}$ & old/ee at $\left.{ }^{\circ} \mathrm{C}-760 \mathrm{~m}\right)$ & $o\left(d / 1 t\right.$ at $\left.{ }^{\circ} \mathrm{c}-760 \mathrm{a}\right)$ \\
\hline$z_{2}$ & 500 & 0.0509 & & 0.090 \\
\hline Ex & 150 & 0.0255 & & 0.178 \\
\hline Ee & 66.8 & 124 & 1.8 & \\
\hline $\mathbf{B}$ & 55.7 & 130 & $3.33-2.34$ & \\
\hline C & 50.1 & 113 & $2.25-3552$ & \\
\hline$\omega_{2}$ & $\$ 3.0$ & 0.0509 & & 1.25 \\
\hline$o_{2}$ & 33.8 & 0.0509 & & 1.43 \\
\hline $\boldsymbol{\Lambda}$ & 22.3 & 60.3 & 2.7 & \\
\hline $\boldsymbol{\Lambda}$ & 15.1 & 0.02547 & & 1.78 \\
\hline re & 10.8 & 8.8 & $7.6-1.9$ & \\
\hline II & 10.3 & 91.3 & $8.6-8.9$ & \\
\hline$\infty$ & $9 .:-8$ & 8.7 & $8.3-8.9$ & \\
\hline 2 & 9.21 & 65.8 & 7.1 & \\
\hline pd & 5.64 & 68.6 & 12.2 & \\
\hline 4 & 5.58 & 58.6 & $10.4-10.6$ & \\
\hline 20 & 3.33 & 55.3 & 16.6 & \\
\hline $\mathbf{v}$ & 3.27 & 63.2 & $18.6-19.1$ & \\
\hline Pt & 3.09 & 65.9 & 21.4 & \\
\hline $\sin$ & 3.05 & 59.0 & 19.3 & \\
\hline $\mathbf{z}_{2} 0$ & 33.42 & 0.0764 & 1.0 & \\
\hline $\boldsymbol{\infty}_{2}$ & 13.68 & 0.0764 & & 1.98 \\
\hline
\end{tabular}




\section{Dotes}

(1) It is expasized that the stoppins crose exetion is for the eners loss of protons. Hodrocen ato no exiblt a meh anller eners lows. Unfortunatielf there Is only a sincle equerimeat were the projectile been is mintafined in a jarely atode state thronchout,

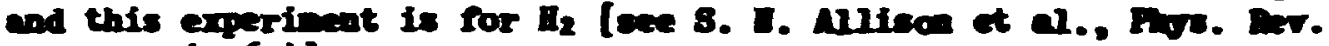

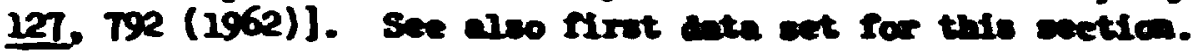

(2) The etopping eross section are for a solld tarct. There is no direct informetion as to whether the stopins erose section in a co

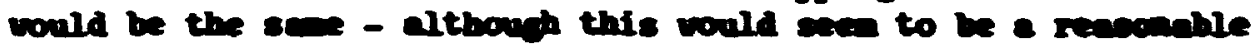
eserngtion.

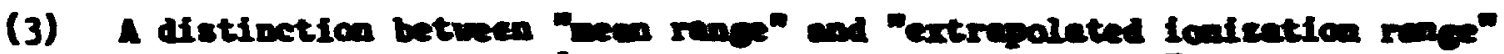

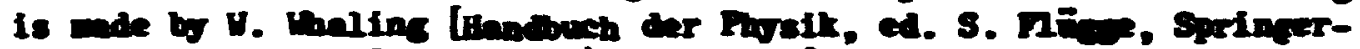

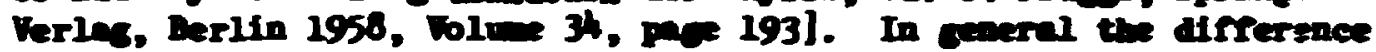
is seil, and the distinction bas not been observed bere.

(4) These date are theoretical ad beve not jet been confinged drectly is experinent. Thes are bowever in cood coreenent oith experineatel

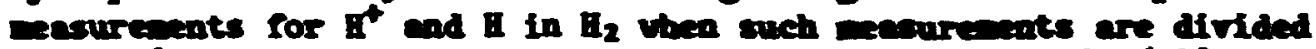
by two [see S. K. Allison et el., Puys. Det. 127, T92 (1962)].

(5) in extensive tabulation of senf-epirical data for variow projectiles and targets is to be found in the vort of L. C. Martheliffe and R. F. Schilling, tuclear Date Tbles A $I, 233$ (1970). Ints also provides prescriptions for interpolation to as male.

(6) These date are theoreticel and heve not jet been connred drectly bj experiment at these enerdes.

(7) Butensive tebulations of theoretical range-enerd relations for inpect enercies up to $10^{5} \mathrm{MeV}$ are to be found in the work of R. M. Stershisiner, Prys. Rev. 115, 137 (1959).

(8) The work of Hines quoted bere is ectually a mencurvent for $\mathrm{D}_{2}{ }^{\text {t }}$ ifpact. Pollowing Hines we have derived an equivalent velue for $\mathrm{H}^{t}$ by assuning $\mathrm{D}_{2}{ }^{t}$ behaves as two isolated $\mathrm{D}^{+}$loss of equal enerd. and the renge is turice that for protons of equal velocity.

(9) The references diven also contaln date for sany other cases.

(10) Deuterons behave the seme as protons of equal velocity.

(11) Cross sections for hicher enercles an be obtainat by the formale: $E=A / E[\ln (Z / 2)+B] 10^{-15}$. B is coero in MVV; 2 is tarpat etonic auber. The constants $A$ and $B$ for carbon are ce follows: $A=1$. h, $B=5.14$. 


\section{A.7.20}

(12) Cros setions ror hicher encries are the ane as in bote (II) Ith: $A=6.3 \%, B=5.21$ for an; $A=18.9 ; B=5.22$ for $\Delta$.

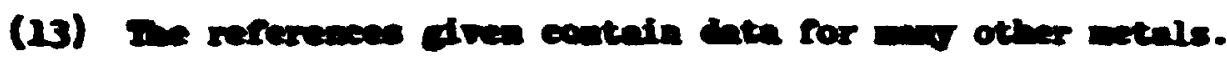

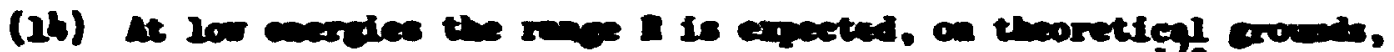

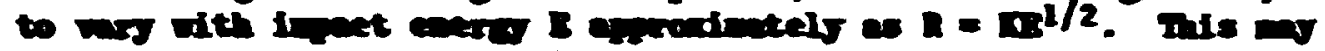

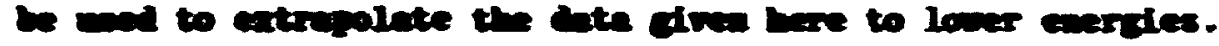

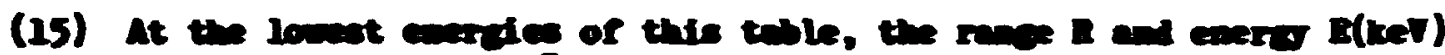

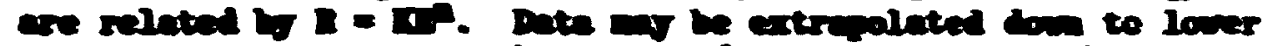

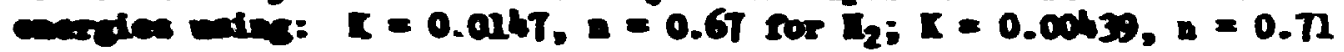
ore $\mathrm{I}_{2} \cdot$ 
$\mathbf{0}$

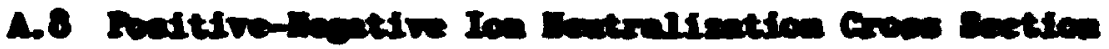

J 


\section{A.8.2}

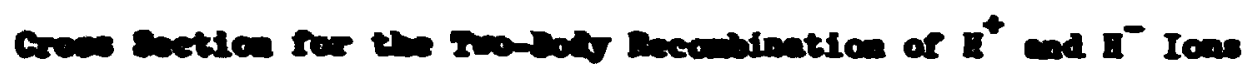

\begin{tabular}{|c|c|}
\hline (civ) & $\begin{array}{c}\text { Decoutination Croas Section } \\
\left(\mathrm{eq}^{2}\right)\end{array}$ \\
\hline 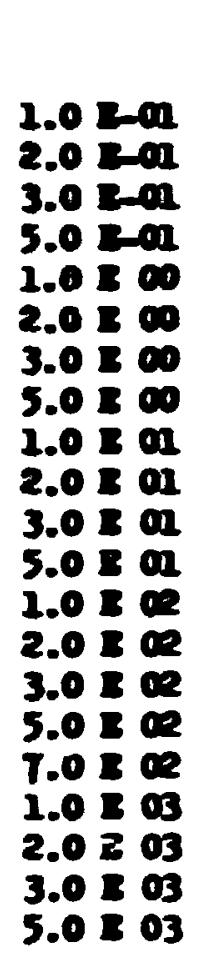 & 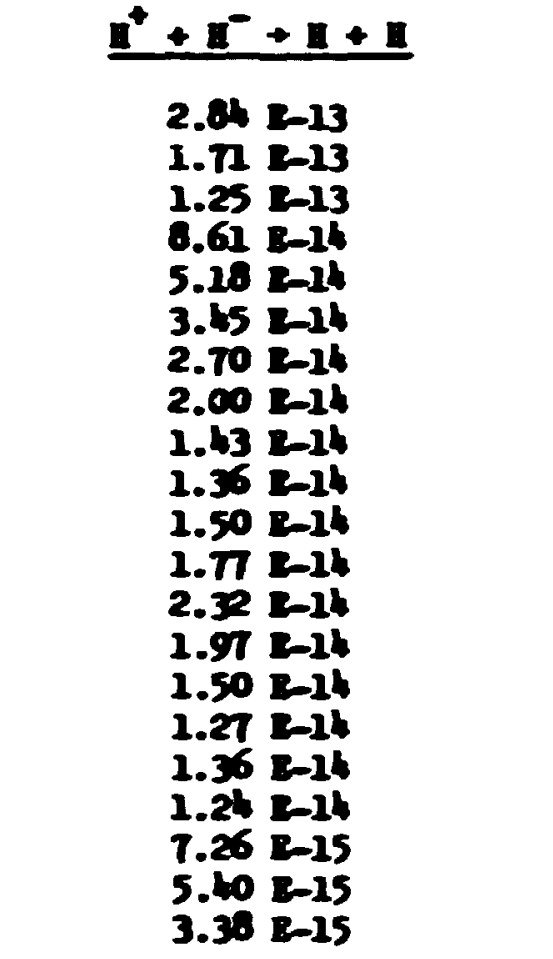 \\
\hline
\end{tabular}

\section{En in}

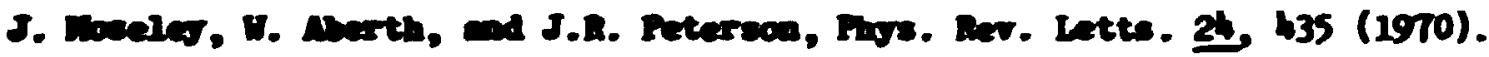

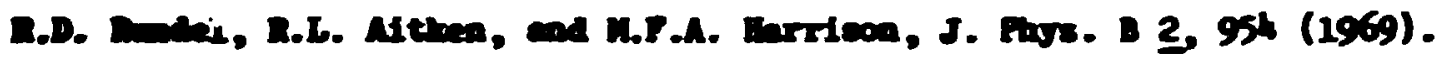
Stisc:

The total error is balievel not to exean $\$ 358$. 
A.6.3

u

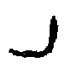

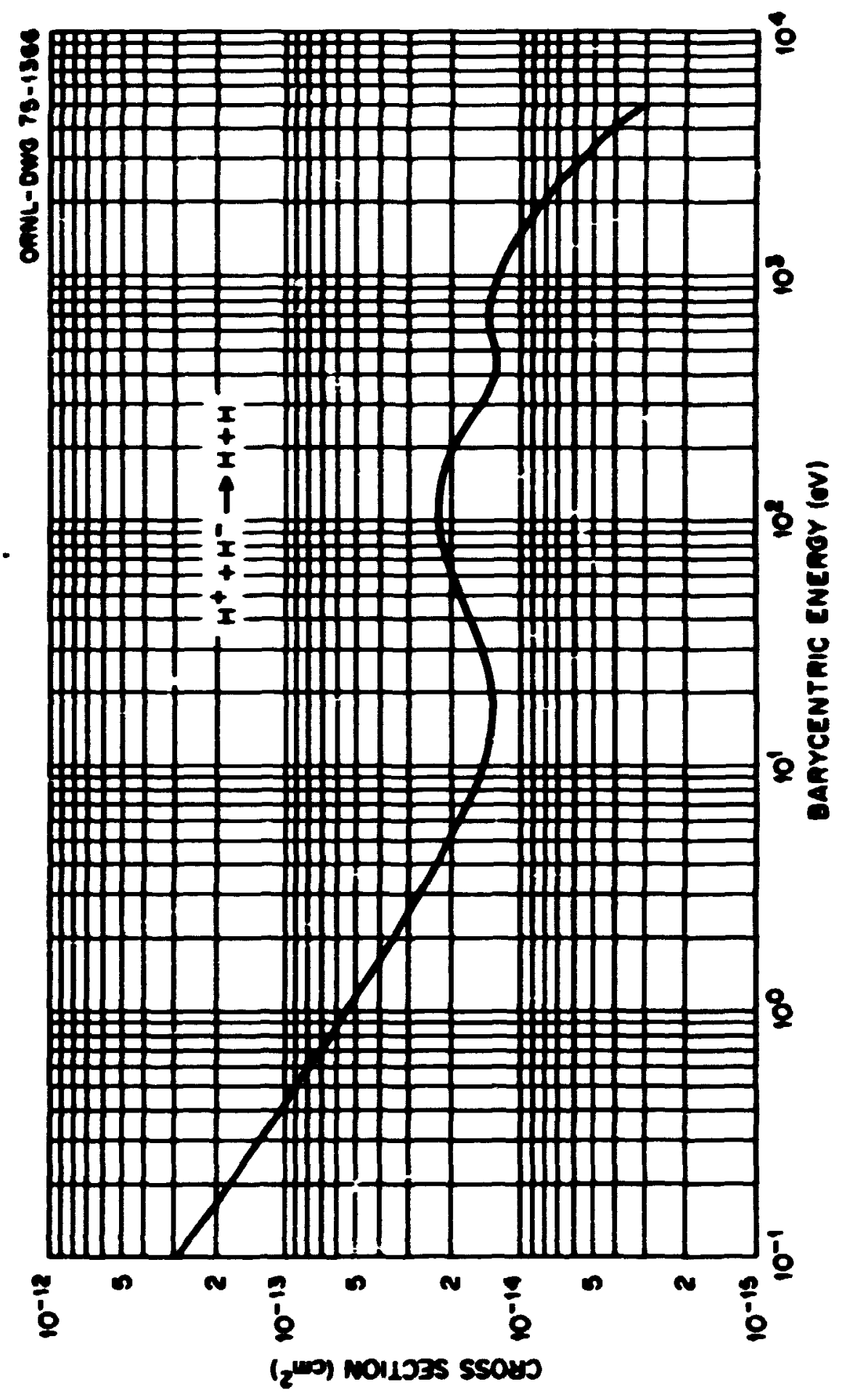

$J$ 


\section{A.8.6}

Cross Sections for the Two-Body Reconbination of He vith $\mathrm{H}^{-}$Ions and of $\mathrm{He}^{+}$vith $\mathrm{D}^{-}$Ions

\begin{tabular}{|c|c|c|}
\hline \multirow[t]{2}{*}{$\begin{array}{l}\text { Barjcentric } \\
\text { Berer } \\
\text { (ev) }\end{array}$} & \multicolumn{2}{|c|}{$\begin{array}{c}\text { Recombination Cross Section } \\
\left(\mathrm{cm}^{2}\right)\end{array}$} \\
\hline & $\mathrm{Be}^{+}+\mathrm{D}^{-}$ & $\underline{\mathrm{Be}^{+}+\mathrm{H}^{-}}$ \\
\hline $\begin{array}{l}1.0 \mathrm{E} 00 \\
2.0 \mathrm{E} 00 \\
3.0 \mathrm{E} 00 \\
5.0 \mathrm{E} 00 \\
1.0 \mathrm{E} 01 \\
2.0 \mathrm{E} 01 \\
3.0 \mathrm{E} 01 \\
5.0 \mathrm{E} 01 \\
1.0 \mathrm{E} 02 \\
2.0 \mathrm{E} 02 \\
3.0 \mathrm{E} 02 \\
5.0 \mathrm{E} 02 \\
9.0 \mathrm{E} 02 \\
1.5 \mathrm{E} 03 \\
2.0 \mathrm{E} 03 \\
4.0 \mathrm{E} 03 \\
6.0 \mathrm{E} 03\end{array}$ & $\begin{array}{ll}1.00 & \mathrm{E}-13 \\
7.36 & \mathrm{E}-14 \\
6.14 & \mathrm{E}-14 \\
5.09 & \mathrm{E}-14 \\
3.88 & \mathrm{E}-14 \\
2.96 & \mathrm{E}-14 \\
2.59 & \mathrm{E}-14 \\
2.18 & \mathrm{E}-14 \\
1.78 & \mathrm{E}-14 \\
1.53 & \mathrm{E}-14 \\
1.42 & \mathrm{E}-14 \\
1.25 & \mathrm{~B}-14\end{array}$ & $\begin{array}{l}1.4 h E-1 h \\
1.23 \text { E-14 } \\
1.09 \mathrm{E}-14 \\
9.06 \mathrm{E}-15 \\
9.27 \mathrm{E}-15 \\
4.42 \mathrm{E}-15 \\
1.93 \mathrm{E}-15\end{array}$ \\
\hline
\end{tabular}

\section{Beferences:}

$E^{+}+\mathrm{H}^{-}:$T.D. Gatles and M.P.A. Harrison, J. Fhys. B 3, 1098 (1970). $\mathrm{Be}^{+}+\mathrm{D}^{-}$: R.B. 01s00, J.R. Peterson, and J.T. Hoseley, J. Chea. Phrs. 53, 3391 (1970).

cecureg:

The total error is believed not to exceed \pm 358 . 


\section{A.8.5}

,

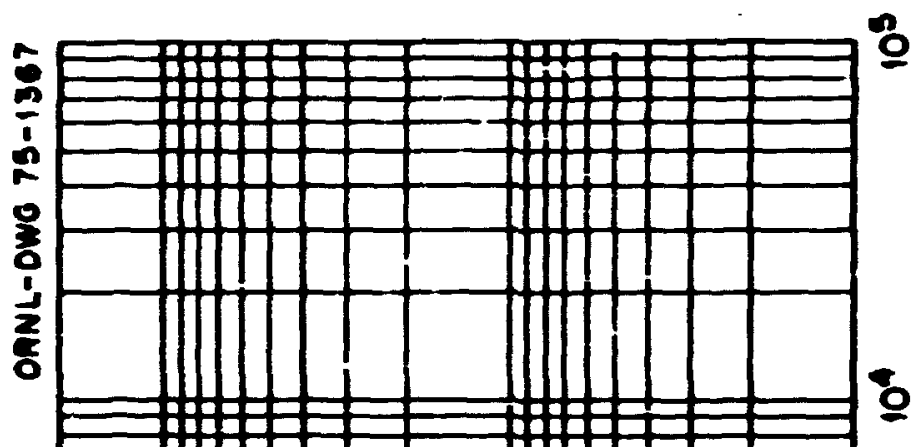

נ. 


\section{A.8.6}

Cross Sections for the Ro-Body Recombination of $\mathrm{x}^{+}$with $\mathrm{O}^{-}$Ions,

$$
\text { of } \mathrm{O}^{+} \text {vith } \mathrm{O}^{-} \text {Ions, and of } \mathrm{H}_{2}^{+} \text {with } \mathrm{D}^{-} \text {Ions }
$$

\begin{tabular}{|c|c|c|c|}
\hline \multirow[t]{2}{*}{$\begin{array}{c}\text { Barycentric } \\
\text { Energy } \\
(\mathrm{eV})\end{array}$} & \multicolumn{3}{|c|}{$\begin{array}{l}\text { Recombination Cross Section } \\
\qquad\left(\mathrm{cm}^{2}\right)\end{array}$} \\
\hline & $\mathrm{I}^{+}+\mathrm{O}^{-}$ & $0^{+}+0^{-}$ & $\underline{\mathrm{H}_{2}^{+}}+\mathrm{D}^{-}$ \\
\hline $\begin{array}{l}1.0 \mathrm{E}-01 \\
1.2 \mathrm{E}-01 \\
2.0 \mathrm{E}-0 \mathrm{I} \\
3.0 \mathrm{E}-01 \\
5.0 \mathrm{E}-01 \\
1.0 \mathrm{E} 00 \\
2.0 \mathrm{E} 00 \\
3.0 \mathrm{E} 00 \\
5.0 \mathrm{E} 00 \\
5.6 \mathrm{E} 00 \\
1.0 \mathrm{E} \mathrm{Ol} \\
1.5 \mathrm{E} 01 \\
2.0 \mathrm{E} 01 \\
3.0 \mathrm{E} 01 \\
4.0 \mathrm{E} 01 \\
5.0 \mathrm{E} 01 \\
6.0 \mathrm{E} 01\end{array}$ & $\begin{array}{ll}8.45 & \mathrm{E}-13 \\
5.50 & \mathrm{E}-13 \\
4.00 & \mathrm{E}-13 \\
2.74 & \mathrm{E}-13 \\
1.83 & \mathrm{E}-13 \\
1.21 & \mathrm{E}-13 \\
9.73 & \mathrm{E}-14 \\
7.41 & \mathrm{E}-14 \\
7.23 & \mathrm{E}-14 \\
5.62 & \mathrm{E}-14 \\
4.81 & \mathrm{E}-14\end{array}$ & $\begin{array}{ll}9.38 & E-13 \\
8.00 & E-13 \\
4.92 & E-13 \\
3.51 & E-13 \\
2.32 & E-13 \\
1.42 & E-13 \\
1.00 & E-13 \\
8.36 & E-14 \\
7.02 & E-14 \\
6.68 & E-14 \\
5.88 & E-14 \\
5.46 & E-14 \\
5.25 & E-14\end{array}$ & $\begin{array}{ll}7.21 & \mathrm{E}-13 \\
6.00 & \mathrm{E}-13 \\
3.72 & \mathrm{E}-13 \\
2.67 & \mathrm{E}-13 \\
1.86 & \mathrm{E}-13 \\
1.16 & \mathrm{E}-13 \\
7.41 & \mathrm{E}-14 \\
5.75 & \mathrm{E}-14 \\
4.51 & \mathrm{E}-14 \\
4.21 & \mathrm{E}-14 \\
4.39 & \mathrm{E}-14 \\
3.60 & \mathrm{E}-14 \\
2.77 & \mathrm{E}-14 \\
3.10 & \mathrm{E}-14 \\
2.94 & \mathrm{E}-14 \\
2.66 & \mathrm{E}-14 \\
2.53 & \mathrm{E}-14\end{array}$ \\
\hline
\end{tabular}

References:

$\mathrm{N}^{+}+0^{-}$: W. Aberth and J.R. Peterson, Phys. Rev. A 1 , 158 (1970).

$0^{+}+\mathrm{C}^{-}:$J.T. Moseley, W. Aberth, and J.R. Peterson, J. Geophys. Res. 77, 255 (1972).

$\mathrm{H}_{2}^{+}+\mathrm{D}-\mathrm{W}$. Aberth, J.T. Moseley, end J.R. Peterson, Two Body Ion-Ion Neutralization Cross Sections, AFCRL Report No. 71-0481, Air Porce Cambridge Research Laboratorles, Bedford, Mass. (1971); J.T. Moseley, R.E. Olson, and J.R. Peterson, Case Studies in Atomic Physics 2, 1 (1975).

\section{Aceuracy:}

The total error is believed not to exceed $+35 \%$ 


\section{A.8.7}

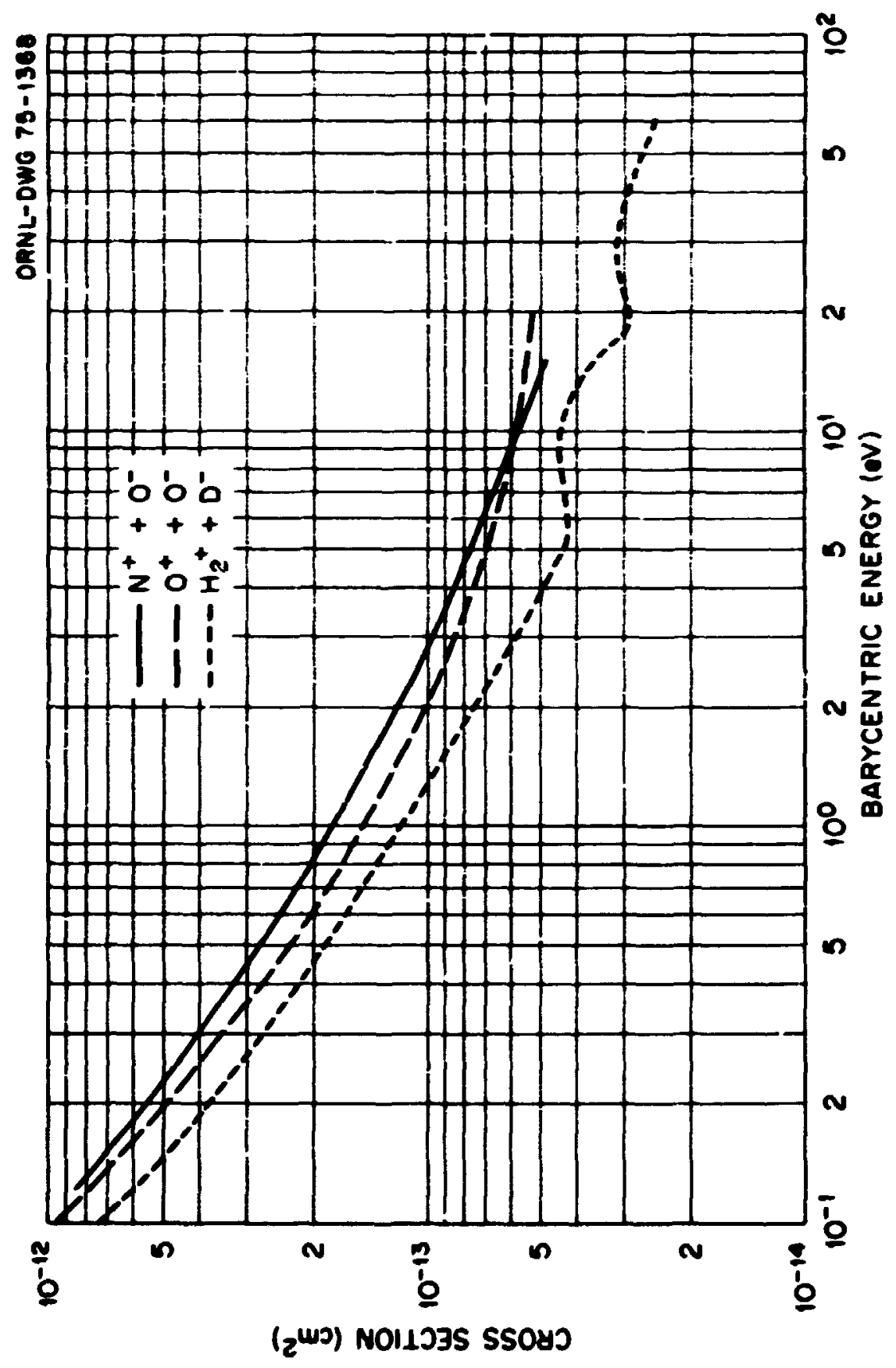




\section{A.8.8}

Cross Sections for the Two-Body Reconbination of $\mathrm{O}_{2}^{+}$with $\mathrm{O}_{2}^{-}$Ions and of $\mathrm{x}_{2}^{+}$with $\mathrm{O}_{2}^{-}$Ions

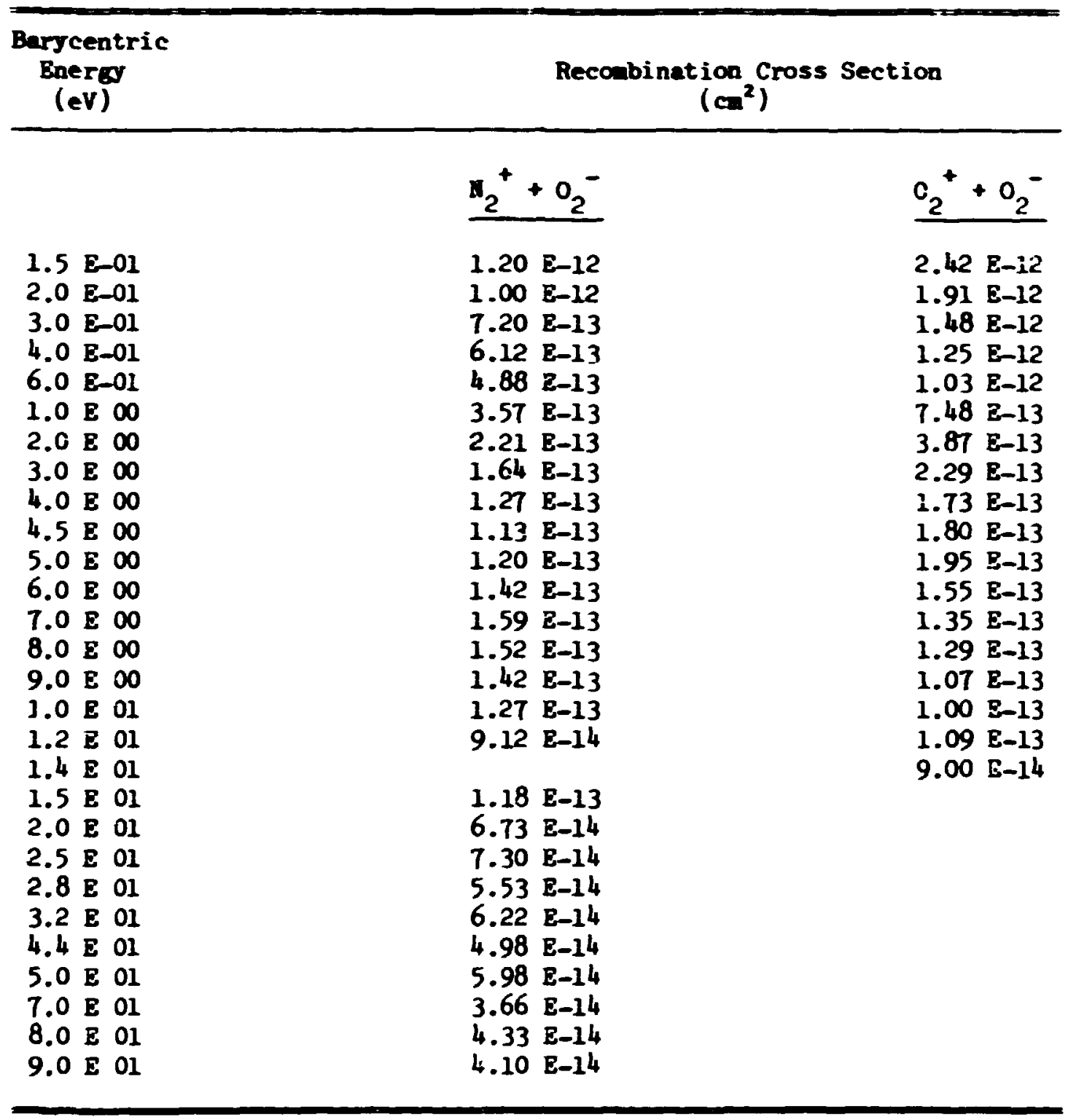

References:

$\mathrm{O}_{2}^{+}+\mathrm{O}_{2}^{-}:$J.R. Peterson, W. Aberth, J.T. Moseley, and J.R. Sheriden, Phys. Rev. A 3, 1651 (1971).

$\mathrm{N}^{+}+\mathrm{n}_{2}^{-}$: W. Aberth and J.R. Peterson, Phys. Rev. A I, 158 (1970).

Accuracy:

The totai error is believed not to exceed $\pm 35 \%$. 
A.8.9

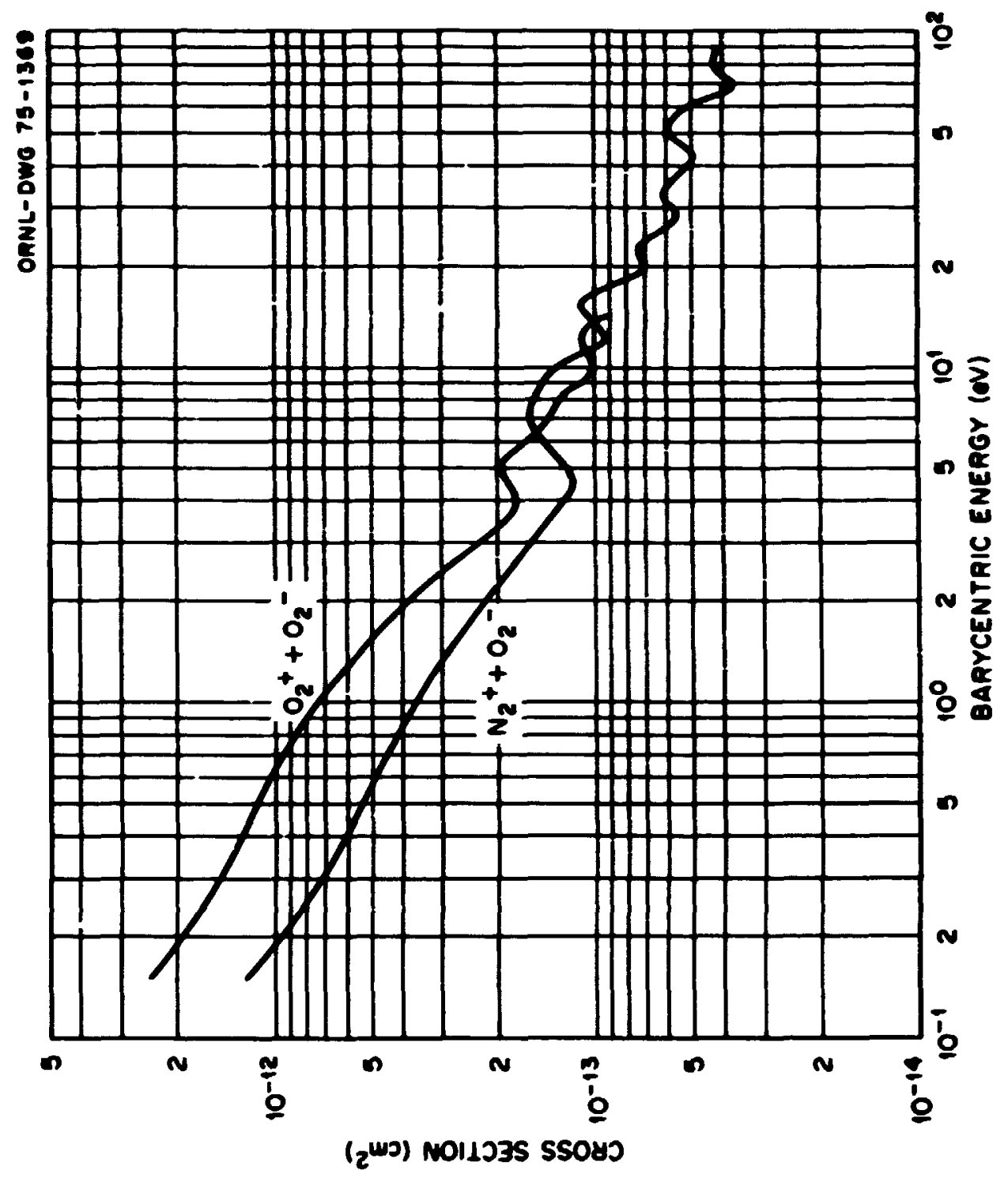




\subsubsection{0}

Cross Sections for the Tro-Body Recombination of

$$
\mathrm{O}_{2}^{+} \text {vith } \mathrm{O}^{-} \text {Ions and of } 10^{+} \text {vith } \mathrm{O}^{-} \text {ians }
$$

\begin{tabular}{|c|c|c|}
\hline $\begin{array}{c}\text { Barjcentric } \\
\text { Bherd } \\
\text { (eV) }\end{array}$ & $\begin{array}{c}\text { Recombination Cross } \\
\left(\mathrm{cm}^{2}\right)\end{array}$ & Section \\
\hline & $10^{+}+0^{-}$ & $\mathrm{O}_{2}^{+}+\mathrm{O}^{-}$ \\
\hline $\begin{array}{l}1.5 \mathrm{E}-01 \\
2.0 \mathrm{E}-01 \\
3.0 \mathrm{E}-01 \\
5.0 \mathrm{E}-01 \\
1.0 \mathrm{E} 00 \\
2.0 \mathrm{E} 00 \\
5.0 \mathrm{E} 00 \\
1.0 \mathrm{E} 01 \\
2.0 \mathrm{E} 01 \\
2.5 \mathrm{E} 01 \\
5.0 \mathrm{E} \text { ol } \\
8.0 \mathrm{E} \text { ol }\end{array}$ & $\begin{array}{ll}1.12 & E-12 \\
8.97 & E-13 \\
6.41 & E-13 \\
4.44 & E-13 \\
2.71 & E-13 \\
1.66 & E-13 \\
1.00 & E-13 \\
7.94 & E-14 \\
6.30 & E-14 \\
5.93 & E-14\end{array}$ & $\begin{array}{ll}4.17 & E-13 \\
3.44 & E-13 \\
2.53 & E-13 \\
1.83 & E-13 \\
1.19 & E-13 \\
8.53 & E-14 \\
5.88 & E-1 b \\
4.74 & E-14 \\
3.80 & E-14 \\
3.37 & E-14 \\
3.11 & E-14 \\
2.67 & E-14\end{array}$ \\
\hline
\end{tabular}

Reference:

J.T. Moseley, W. Aberth, and J.R. Peterson, J. Geophys. Res. 77, 255 (1972). Accuracy:

The total error is belfered not to exceed \pm 358 . 


\section{A.8.11}

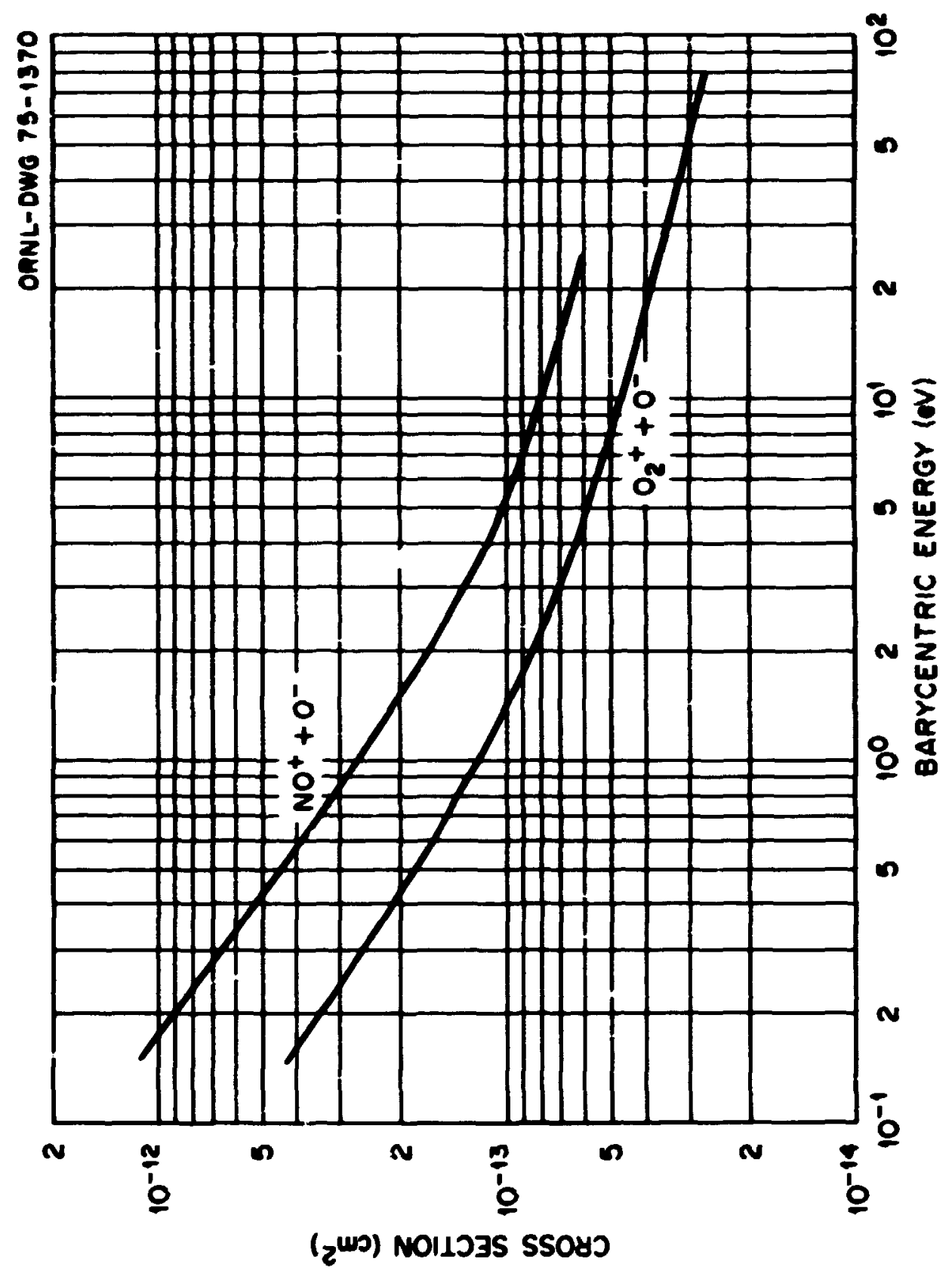




\section{A.9 Ion-Holecule and Atan-Holecule Interchnge Reactions}

1 
$\mathbf{4 . 9 . 2}$

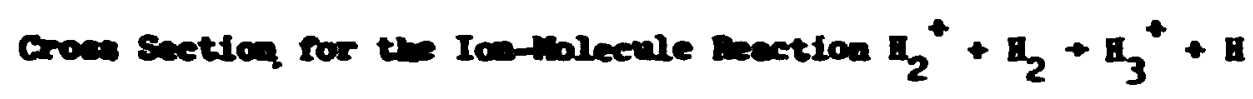

\begin{tabular}{|c|c|}
\hline $\begin{array}{c}\text { Interwetion Dero } \\
(\mathrm{eV})\end{array}$ & $\begin{array}{l}\text { Croses section } \\
\left(c^{2}\right)^{-}\end{array}$ \\
\hline 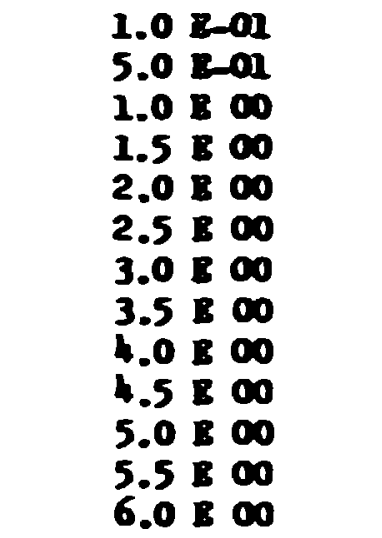 & $\begin{array}{ll}3.40 & \mathrm{E}-15 \\
2.03 & \mathrm{E}-15 \\
1.22 & \mathrm{E}-15 \\
7.82 & \mathrm{E}-16 \\
5.30 & \mathrm{E}-16 \\
3.65 & \mathrm{E}-16 \\
2.58 & \mathrm{E}-16 \\
1.88 & \mathrm{E}-16 \\
1.42 & \mathrm{E}-16 \\
1.10 & \mathrm{E}-16 \\
8.80 & \mathrm{E}-17 \\
7.20 & \mathrm{E}-17 \\
5.95 & \mathrm{E}-17\end{array}$ \\
\hline
\end{tabular}

\section{Dareces:}

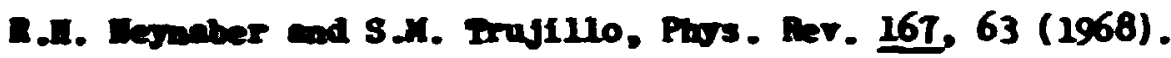

A.B. Leer and P.X. Bol, J. Chen. Phos. 61, Wh4t (197h).

\section{secteg:}

The totel error Is believed not to exceed $\pm 40 \mathrm{z}$.

Dote:

The states of the reactents are unknom. 
A.9.3

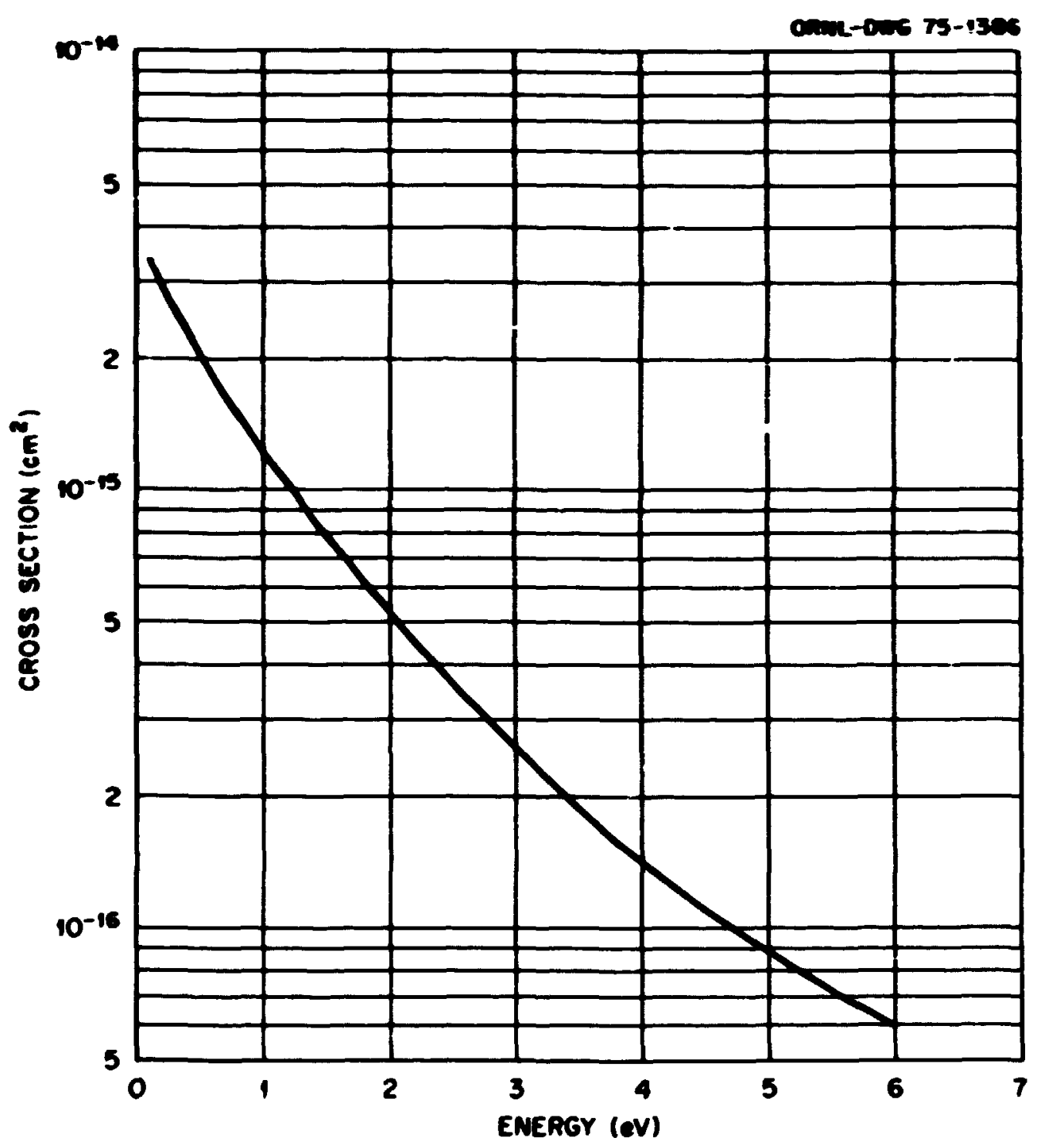




\section{A.9.4}

Cross Sections for the Proiuction of $\mathrm{C}^{+}$, i $\mathrm{HD}^{+}$, and $\mathrm{J}_{2}^{+}$

by Proton Inpect on $\mathrm{D}_{2}$

\begin{tabular}{|c|c|c|c|}
\hline $\begin{array}{l}\text { Bneres } \\
(e v)\end{array}$ & & $\begin{array}{c}\text { Oross Section } \\
\left(\mathrm{cs}^{2}\right)\end{array}$ & \\
\hline & $E^{*}+D_{2} \rightarrow D^{*}+(H D)$ & $\mathrm{I}^{+}+\mathrm{D}_{2}+\mathrm{DD}^{+}+(2)$ & $\ddot{a}^{*}+D_{2}+D_{2}+(\ddot{i})$ \\
\hline 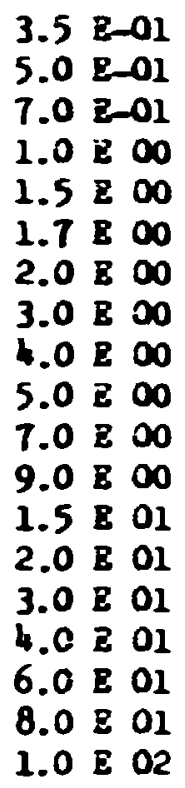 & $\begin{array}{ll}1.16 & E-15 \\
7.70 & E-16 \\
5.00 & \mathrm{E}-16 \\
3.10 & \mathrm{E}-16 \\
1.65 & \mathrm{z}-16 \\
1.36 & \mathrm{E}-16 \\
1.08 & \mathrm{E}-16 \\
5.50 & \mathrm{E}-17 \\
3.35 & \mathrm{E}-17 \\
2.35 & \mathrm{E}-17 \\
. .55 & \mathrm{E}-17 \\
1.25 & \mathrm{E}-17 \\
7.25 & \mathrm{E}-18 \\
8.10 & \mathrm{E}-18 \\
6.45 & \mathrm{E}-18 \\
5.10 & \mathrm{E}-18 \\
3.45 & \mathrm{E}-18 \\
2.43 & \mathrm{E}-18 \\
1.90 & \mathrm{E}-18\end{array}$ & 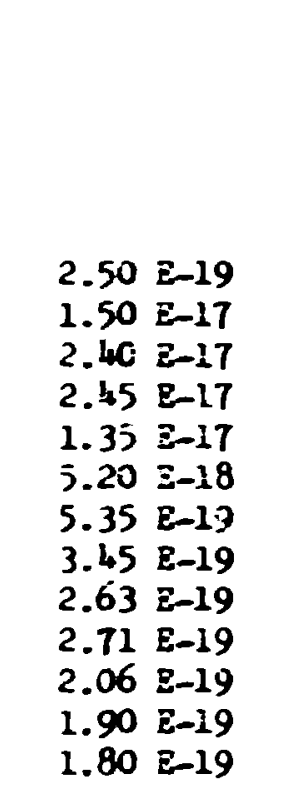 & $\begin{array}{ll}2.00 & \Xi-19 \\
7.00 & \Xi-19 \\
1.05 & \Xi-17 \\
2.90 & \Xi-17 \\
5.10 & \Xi-17 \\
6.50 & \Xi-17 \\
3.15 & \Xi-17 \\
1.65 & \Xi-17 \\
1.33 & \Xi-17 \\
1.00 & \Xi-17 \\
3.30 & \Xi-18 \\
6.20 & \Xi-18 \\
5.50 & \Xi-18 \\
4.85 & \Xi-18\end{array}$ \\
\hline
\end{tabular}

\section{Reference:}

W.B. Maier, J. Chem. Phys. 54, 2732 (1971).

\section{Accuracy:}

The total error is belleved not to exceed $\pm 30 \%$.

Note:

At the higher energfes, the undetected products may differ from the neutral products in parentheses. 


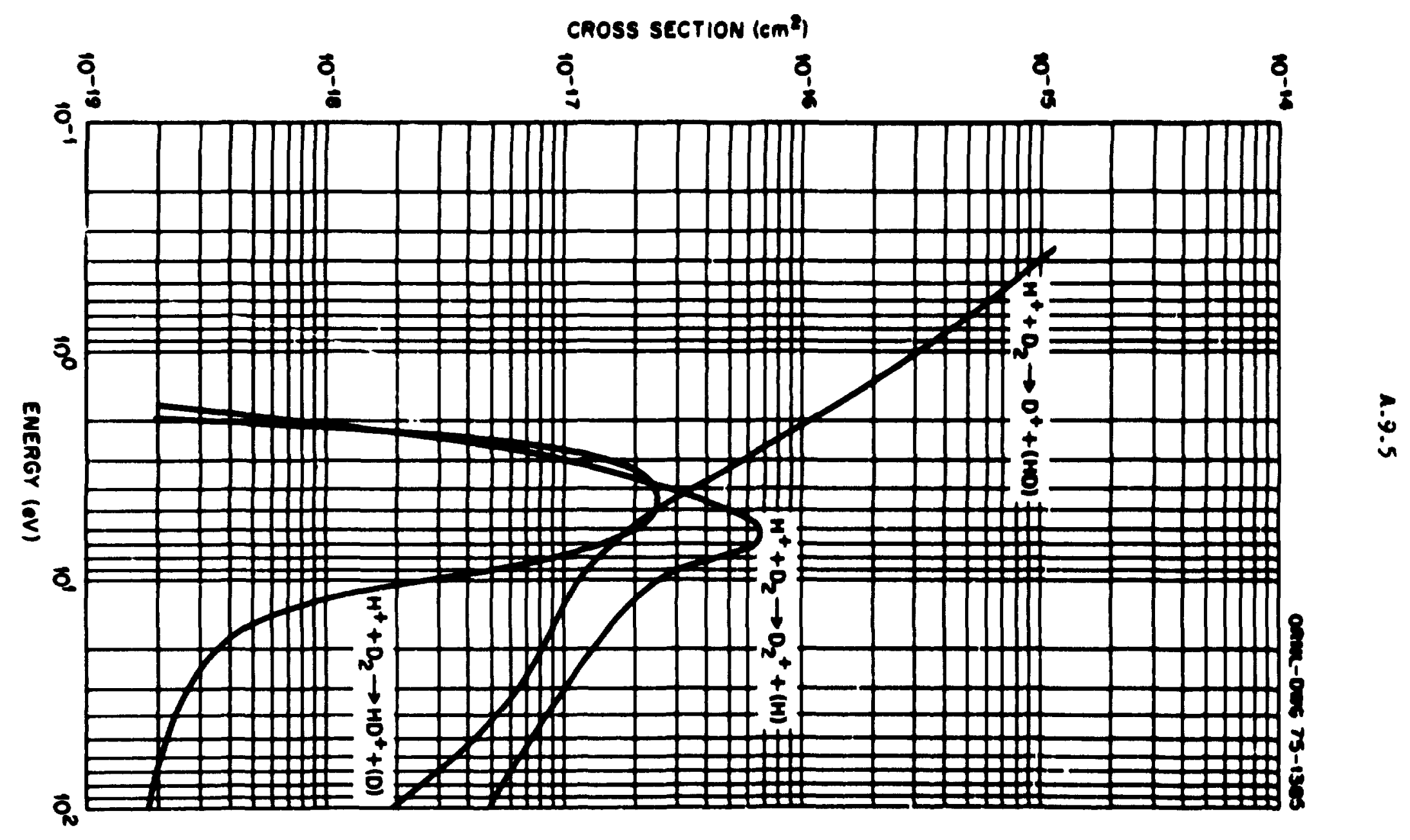




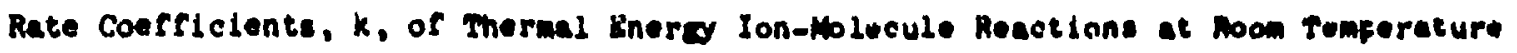

\begin{tabular}{|c|c|c|c|}
\hline Reaction & $k$ & Uncertalnty & Meference \\
\hline $\mathrm{H}_{2}^{+}+\mathrm{H}_{2}+\mathrm{H}_{3}^{+}+\mathrm{H}$ & $1.95 \times 10^{-9} \mathrm{~cm}^{3} / \mathrm{sec}$ & $\pm 20 s$ & $\begin{array}{l}\text { M.t. Bowers, D. D. 81lemen, J, Kine, J. Chem. } \\
\text { Phys. S0, } 4707 \text { (is69i. }\end{array}$ \\
\hline $\mathrm{HD}^{+}+\mathrm{HD} \rightarrow \mathrm{H}_{2} \mathrm{D}^{+}+\mathrm{D}$ & $0.75 \times 10^{-9} \mathrm{~cm}^{3} / \mathrm{sec}$ & \pm 208 & $\begin{array}{l}\text { M.T. Bowers, D.D, Edzemen, J, Klnc, J, Chem. } \\
\text { Phye. SO, W7B7 (1969). }\end{array}$ \\
\hline $\mathrm{HD}^{+}+\mathrm{HD}+\mathrm{HD}_{2}^{+}+\mathrm{H}$ & $1.05 \times 10^{-9} \mathrm{~nm}^{3} / \mathrm{sec}$ & \pm 208 & $\begin{array}{l}\text { M.T. Bowers, D.D. El2eman, \&, KInc, J, Chem. } \\
\text { Phys, 50, } 4787(2969) \text {. }\end{array}$ \\
\hline $\mathrm{D}_{2}^{+}+\mathrm{D}_{2}+\mathrm{D}_{3}^{+}+\mathrm{D}$ & $1.6 \times 10^{-9} \mathrm{~cm}^{3} / \mathrm{sec}$ & \pm 208 & 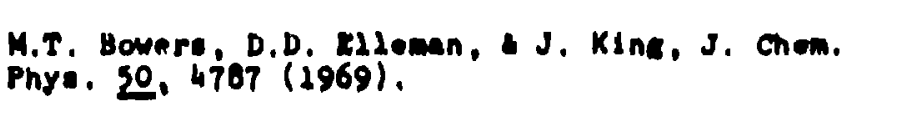 \\
\hline $\mathrm{H}_{3}^{+}+\mathrm{He} \rightarrow$ products & $<3.0 \times 10^{-12} \mathrm{~cm}^{3} / \mathrm{sec}$ & $v$. & 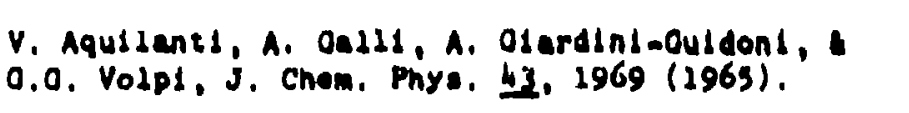 \\
\hline $\begin{array}{l}\mathrm{He}^{+}+\mathrm{H}_{2} \rightarrow \text { products } \\
\mathrm{He}^{+}+\mathrm{D}_{2} \rightarrow \text { products }\end{array}$ & $\begin{array}{l}1.2 \times 10^{-23} \mathrm{~cm}^{3} / \mathrm{sec} \\
5 \times 10^{-14} \mathrm{~cm}^{3 / \mathrm{sec}}\end{array}$ & $\begin{array}{l} \pm 108 \\
\pm 208\end{array}$ & $\begin{array}{l}\text { R. Johnsen and M.A. Blonds, J. Chen. Phys. } \\
61,2112(1974) \text {. }\end{array}$ \\
\hline $\mathrm{He}_{2}^{+}+\mathrm{H}_{2} \rightarrow$ products & $5.3 \times 10^{-20} \mathrm{~cm}^{3} / \mathrm{sec}$ & \pm 308 & 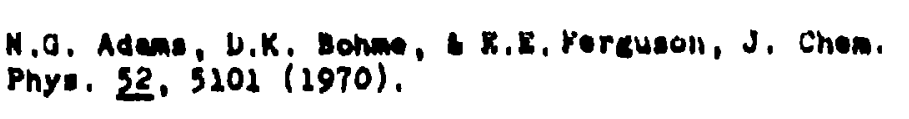 \\
\hline $\mathrm{H}^{-}+\mathrm{H}+\mathrm{H}_{2}+e$ & $1.8 \times 10^{-9} \mathrm{~cm}^{3} / \mathrm{sec}$ & Fictor of 2 & 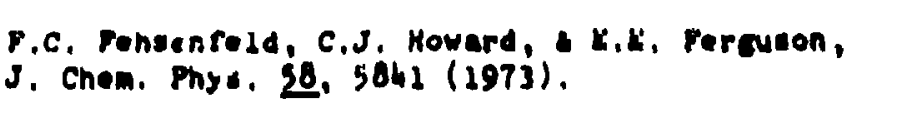 \\
\hline $\mathrm{He}^{+}+2 \mathrm{He} \rightarrow \mathrm{He}_{2}^{+}+\mathrm{He}$ & $10.2 \times 10^{-32} \mathrm{~cm}^{6} / \mathrm{sen}$ & \pm 108 & 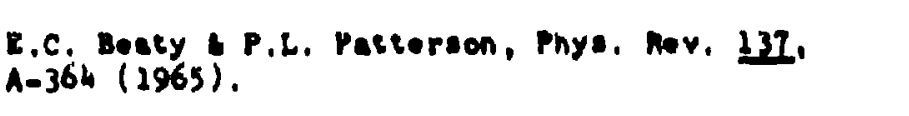 \\
\hline $\mathrm{H}^{+}+2 \mathrm{H}_{2} \rightarrow \mathrm{H}_{3}^{+}+\mathrm{H}_{2}$ & $3.05 \times 10^{-29} \mathrm{~cm}^{6} / \mathrm{sec}$ & \pm 58 & 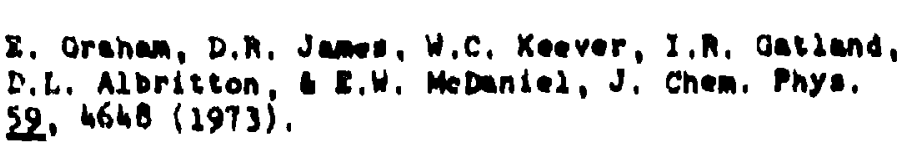 \\
\hline$D^{+}+2 D_{2}+D_{3}^{+}+D_{2}$ & $3.05 \times 10^{-29} \mathrm{~cm}^{6} / \mathrm{sec}$ & \pm 58 & 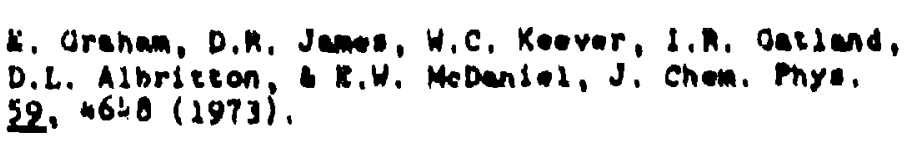 \\
\hline $\mathrm{HeH}^{+}+\mathrm{H}_{2}-\mathrm{H}_{3}^{+}+\mathrm{He}$ & $1.4 \times 10^{-9} \mathrm{~cm}^{3} / \mathrm{sec}$ & \pm 258 & 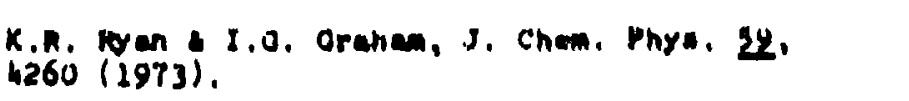 \\
\hline
\end{tabular}


Rate Coefrictents, $k$, of Thermad Inerg Neutral-Neutral Ragetione

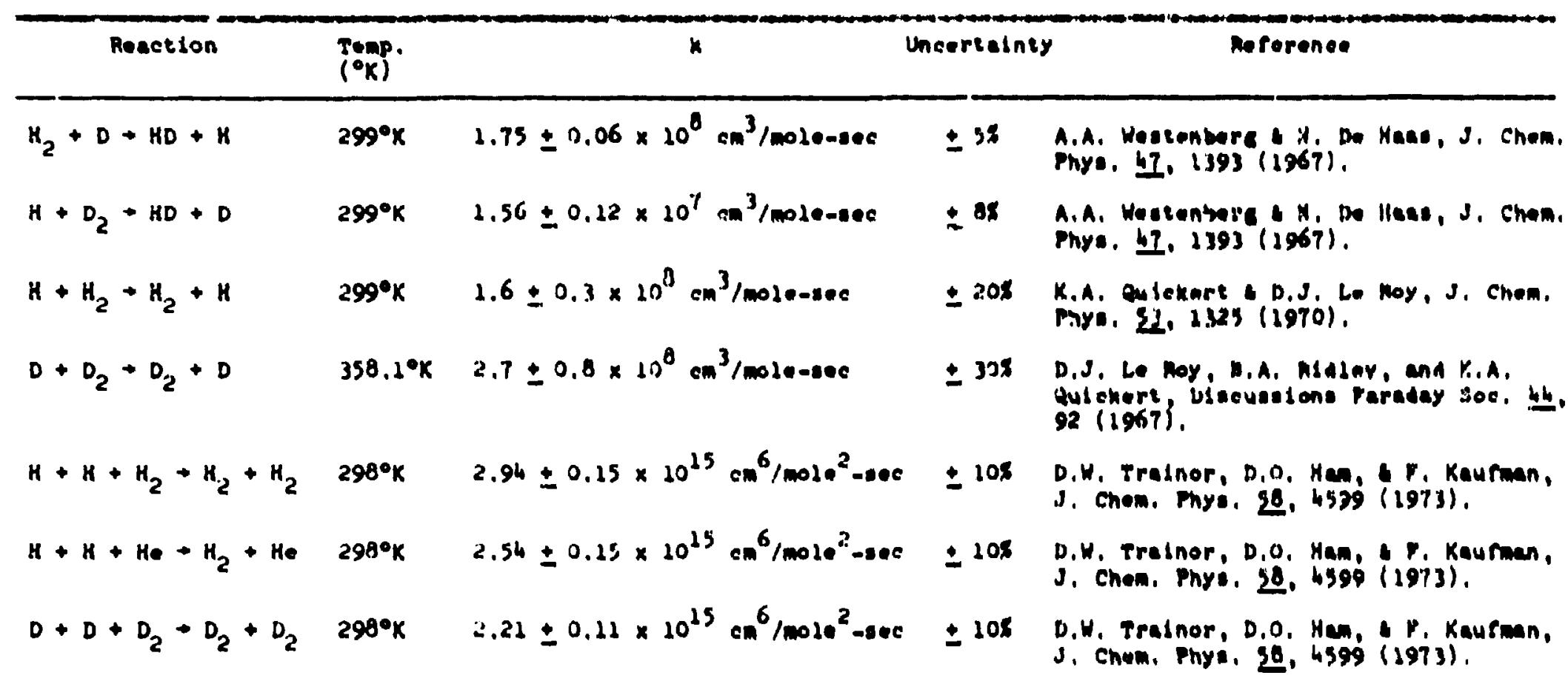


ᄂ

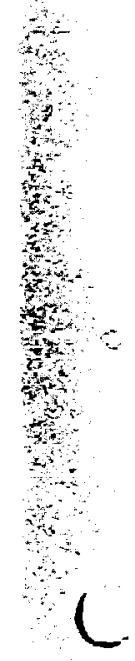

B. Photon collisions

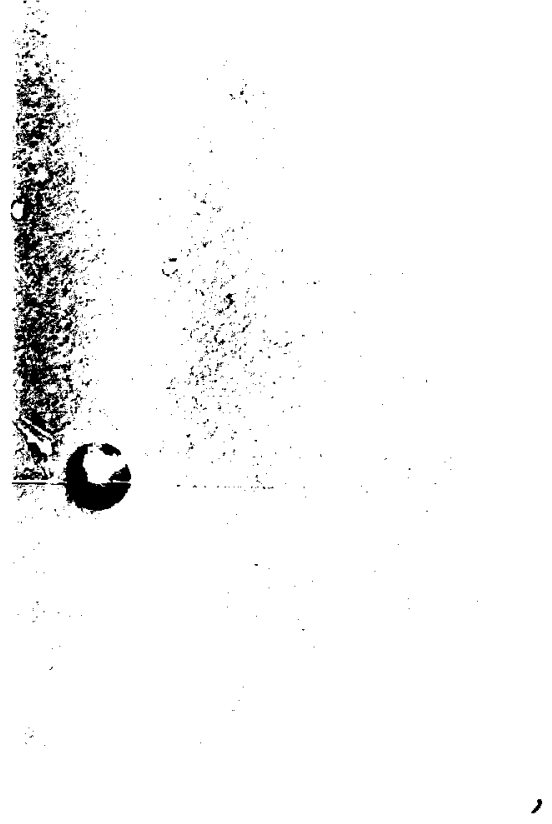


,

B.I Photuabsorption and Photoionization

3

$j$ 
B.1.4

Photolbsorption and Photoionization ic Atonic Hydrogen

hv + H

\begin{tabular}{|c|c|c|c|}
\hline \multirow{2}{*}{$\begin{array}{l}\text { Photon } \\
\text { Wavelength } \\
\text { (R) }\end{array}$} & \multirow{2}{*}{$\begin{array}{l}\text { Photon } \\
\text { Energy } \\
\text { (keV) }\end{array}$} & \multicolumn{2}{|c|}{$\begin{array}{c}\text { Cross Section } \\
\left(c^{2}\right)\end{array}$} \\
\hline & & Absorption & Ionization \\
\hline $\begin{array}{c}911.753 \\
\text { (Threshold) }\end{array}$ & $1.36 \mathrm{E}-02$ & $\begin{array}{l}\text { Same as } \\
\text { Ionization. }\end{array}$ & $6.31 \mathrm{E}-18$ \\
\hline 900 & $1.38 \mathrm{E}-02$ & See Note (1) & $6.12 \mathrm{E}-18$ \\
\hline 800 & $1.55 E-02$ & & $4.46 \mathrm{E}-18$ \\
\hline 700 & 1.77 E-02 & & $3.10 \mathrm{E}-18$ \\
\hline 600 & 2.07 E-02 & & $2.02 \mathrm{E}-18$ \\
\hline 500 & $2.48 \mathrm{E}-02$ & & $1.22 \mathrm{E}-18$ \\
\hline 400 & 3.10 E-02 & & 6.43 E-19 \\
\hline 300 & $4.13 \mathrm{E}-02$ & & $2.78 \mathrm{E}-19$ \\
\hline 200 & 6.20 E-02 & & $8.20 \mathrm{E}-20$ \\
\hline 100 & $1.24 \mathrm{E}-01$ & & $9.92 \mathrm{E}-21$ \\
\hline 80 & 1.55 E-01 & & $4.90 \mathrm{E}-21$ \\
\hline 60 & 2.07 E-01 & & 1.97 E-21 \\
\hline 50 & $2.48 \mathrm{E}-01$ & & $1.10 \mathrm{~B}-21$ \\
\hline 40 & $3.10 \mathrm{E}-02$ & & $5.39 \quad 8-22$ \\
\hline 30 & $4.13 E-02$ & & $2.10 \mathrm{E}-22$ \\
\hline 20 & $6.20 \mathrm{E}-02$ & & $5.56 \mathrm{~B}-23$ \\
\hline 10 & 1.24 E-00 & & $5.55 \mathrm{E}-24$ \\
\hline
\end{tabular}

Reference:

Theoretical: J.A.R. Samson, Advarces in Atonic \& Nolecular Physics $\underline{2}$, 177 (1966) [Publ. Academic Press, N.Y., Ed. D.R. Bates and I. Estermann].

Accuracy:

Unspecified.

Notes:

See Iotes (1), (2), and (3) at end of chapter. 
B.1.5

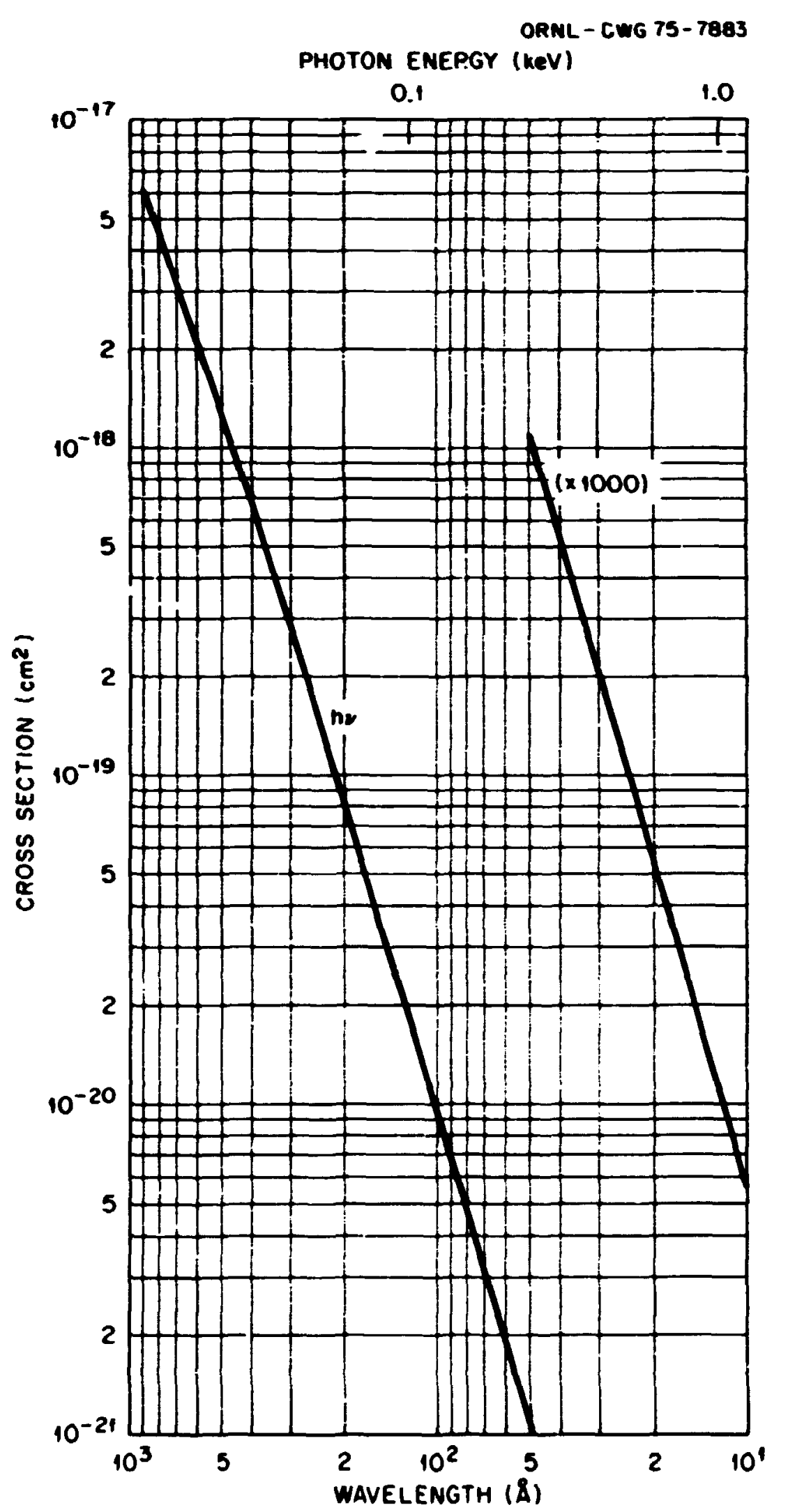




\section{B.1.6.}

Photoabsorption and Photoionization in Mlecular Hydrogen

$$
\begin{gathered}
\mathrm{hv}+\mathrm{H}_{2} \\
\mathrm{hv}+\mathrm{D}_{2} \text { (See Note is) }
\end{gathered}
$$

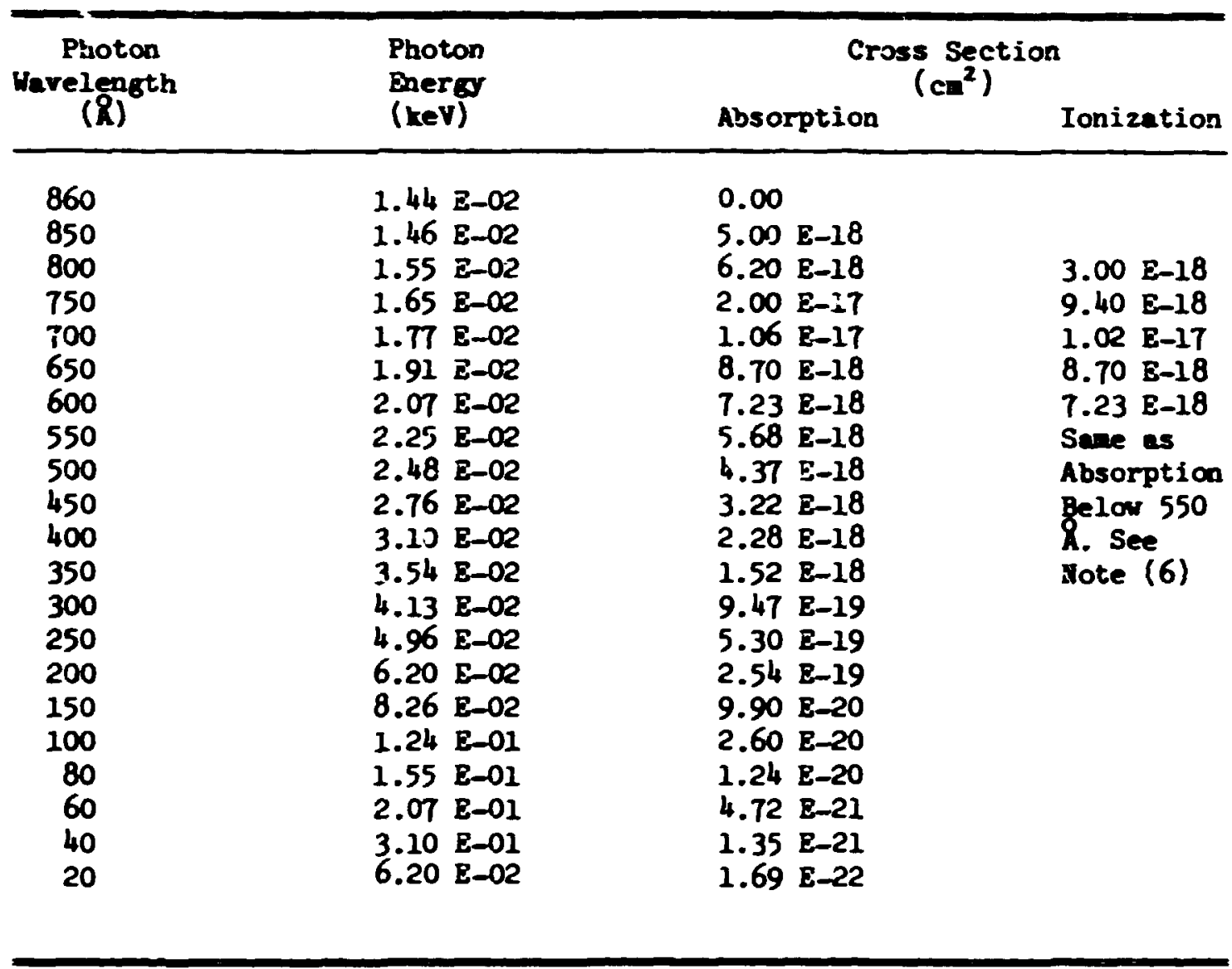

References:

Experimental ( 880 to 200 R): G.R. Cook and P.H. Metzger, J. Opt. Soc. Am. 54, 968 (1964). J.A.R. Semson and R.B. Cairns, J. Opt. Soc. Am. 55, 1035 (1965). Semiempirical (bel ow 60 \%). B.L. Henke, J. Appl. Phys. 28, 98 (1957).

Accuracy:

Total error $< \pm 8 \%$.

Notes:

See Notes $(4 ;,(5),(6),(7)$, and $(8)$ at end of chapter. 


\section{B.1.7}

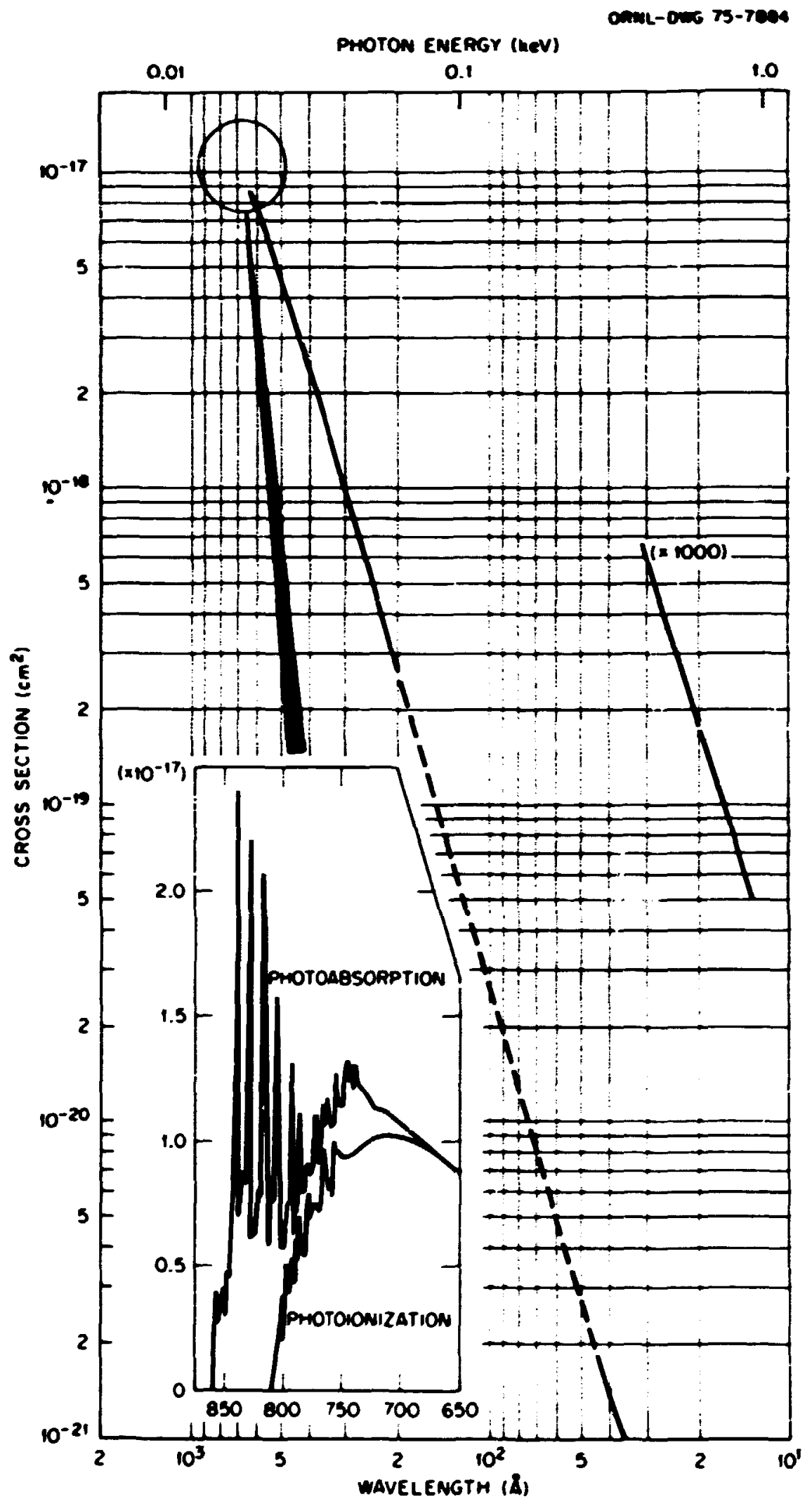




\section{1 .8}

Photoionization of Moleculer Hydrogen;

the Ratio of Dissoinated to Undissociated Product Ions

$$
\begin{aligned}
& r v+H_{2}-H^{+}+i+e\left(\text { Cross Section } \sigma_{1}\right) \\
& h v+H_{2} \rightarrow H_{2}^{+}+e\left(\text { Cross Section } \sigma_{2}\right) \\
& h v+D_{2} \rightarrow D^{+}+D+e\left(\text { Cross Section } \sigma_{3}\right) \\
& h v+D_{2}+D_{2}^{+}+e\left(\text { Cross Section } \sigma_{4}\right)
\end{aligned}
$$

\begin{tabular}{|c|c|c|c|}
\hline $\begin{array}{l}\text { Photon } \\
\text { uevelength } \\
\text { (R) }\end{array}$ & $\begin{array}{l}\text { Photon } \\
\text { Enerd } \\
\text { (kev) }\end{array}$ & $\sigma_{1} / \sigma_{2}$ & $\begin{array}{l}\text { Retios } \\
\sigma_{3} / \sigma_{4}\end{array}$ \\
\hline $\begin{array}{c}685 \\
\text { (Threshold) } \\
650 \\
600 \\
550 \\
500 \\
450\end{array}$ & $\begin{array}{ll}1.81 & E-02 \\
1.91 & \mathrm{E}-02 \\
2.04 & \mathrm{E}-02 \\
2.26 & \mathrm{E}-02 \\
2.48 & \mathrm{E}-02 \\
2.76 & \mathrm{E}-02\end{array}$ & $\begin{array}{ll}1.70 & E-02 \\
2.04 & E-02 \\
2.08 & E-02 \\
2.06 & E-02 \\
2.55 & E-02\end{array}$ & 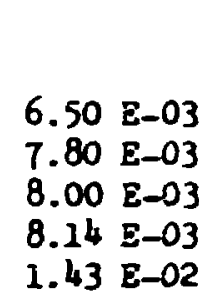 \\
\hline
\end{tabular}

Reference:

Experimentel: R. Browning and J. Pryar, J. Phys. B 6, 364 (1973).

Accuracy:

Systematic error $< \pm 8 \%$. Randon error $< \pm 10 \%$.

\section{Motes:}

See Hote (9) at end of chapter. 


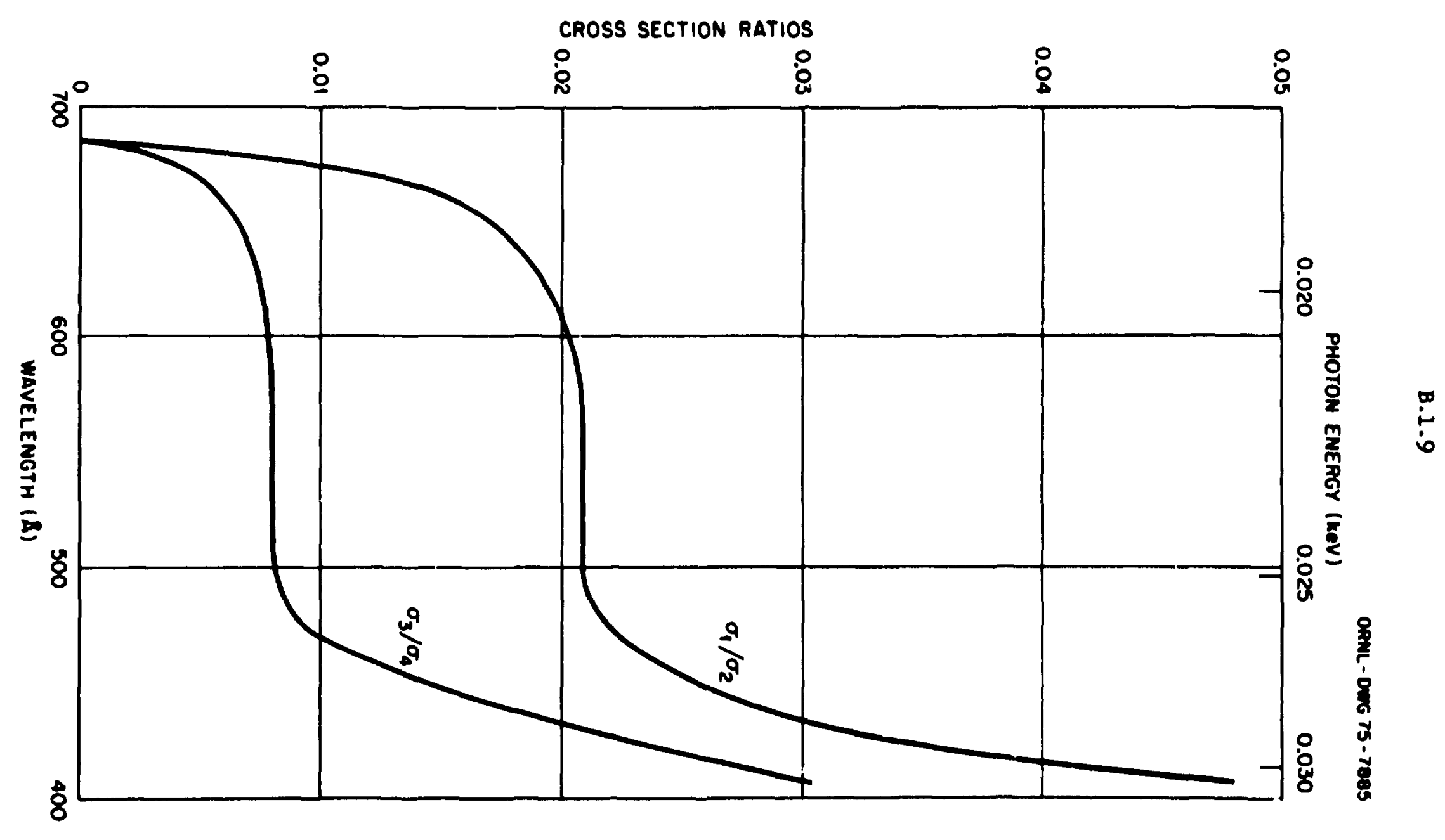




\section{B.1.10}

Photoabsorption and Photoionization in Helium

$$
\text { hv + He }
$$

\begin{tabular}{|c|c|c|c|}
\hline \multirow{2}{*}{$\begin{array}{l}\text { Photon } \\
\text { Nevefength } \\
\text { (R) }\end{array}$} & \multirow{2}{*}{$\begin{array}{l}\text { Photon } \\
\text { Bnergy } \\
\text { (keV) }\end{array}$} & \multicolumn{2}{|c|}{$\begin{array}{c}\text { Cross Section } \\
\left(\mathrm{cm}^{2}\right)\end{array}$} \\
\hline & & Absorption & Ionizaticy \\
\hline $\begin{array}{c}504.26 \\
\text { (Threshold) } \\
500 \\
450 \\
400 \\
350 \\
300 \\
250 \\
200 \\
150 \\
100 \\
80 \\
60 \\
50 \\
40 \\
30 \\
20\end{array}$ & $\begin{array}{ll}2.46 & E-02 \\
& \\
2.48 & E-02 \\
2.76 & E-02 \\
3.10 & E-02 \\
3.54 & E-02 \\
4.13 & E-02 \\
4.96 & E-02 \\
6.20 & E-02 \\
8.26 & E-02 \\
1.24 & E-01 \\
1.55 & E-01 \\
2.07 & E-01 \\
2.48 & E-01 \\
3.10 & E-01 \\
4.13 & E-01 \\
6.20 & E-01\end{array}$ & $\begin{array}{ll}7.36 & E-18 \\
7.20 & E-18 \\
6.20 & E-18 \\
5.00 & E-18 \\
3.84 & E-18 \\
2.85 & E-18 \\
1.98 & E-18 \\
1.25 & E-18 \\
6.20 & E-19 \\
2.00 & E-19 \\
1.10 & E-19 \\
5.21 & E-20 \\
3.25 & E-20 \\
i .86 & E-20 \\
8.56 & E-21 \\
2.61 & E-21\end{array}$ & $\begin{array}{l}\text { Same as } \\
\text { Absorption. } \\
\text { See Note (i). }\end{array}$ \\
\hline
\end{tabular}

References:

Bxperinental: J.A.R. Sanson, Advances in Atonic and :tolecular Physics 2 , 177 (1966) [publ. Acadenic Press H.Y., Ed. D.R. Rates and I. Estermann].

W.S. Hatson, J. Phys. B S, 2292 (1972).

Accuracy:

Systematic error $< \pm 5 \%$. Randa error $< \pm 3 \%$.

Sotes:

See Jotes (1); (3), (14), (15), and (16) at end of chapter. 
8.1 .11

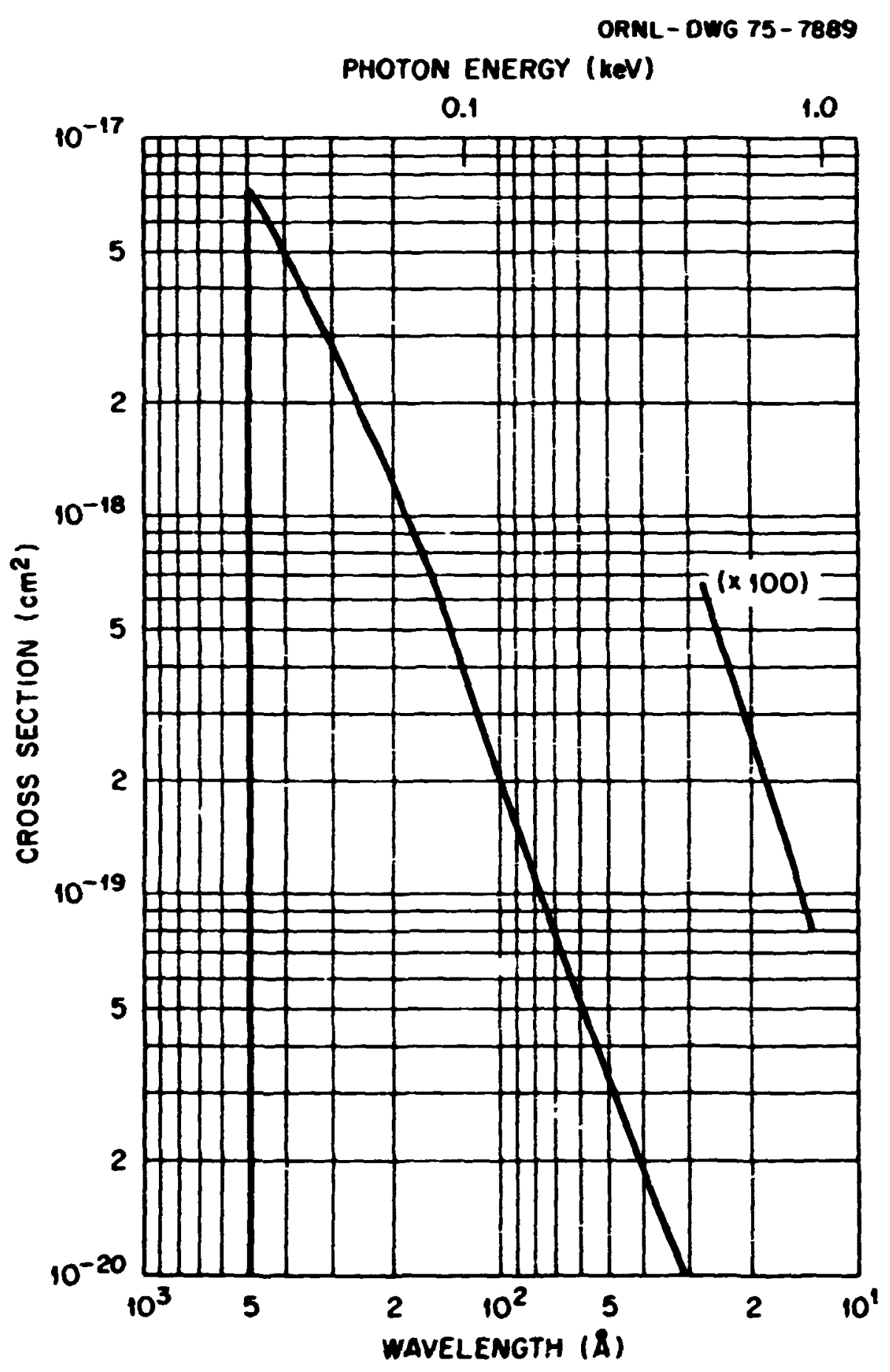


Photoionization and Photoabsorption in Atomic $C$ and $c^{3+}$

$$
\text { hv }+c, c^{3+}
$$

\begin{tabular}{|c|c|c|c|}
\hline $\begin{array}{l}\text { Photon } \\
\text { Wevelength } \\
\text { (R) }\end{array}$ & $\begin{array}{l}\text { Photon } \\
\text { Dherco } \\
\text { (keV) }\end{array}$ & C & $c^{3+}$ \\
\hline $\begin{array}{r}190 \\
180 \\
140 \\
125 \\
100 \\
80 \\
60 \\
50 \\
40 \\
20 \\
10\end{array}$ & 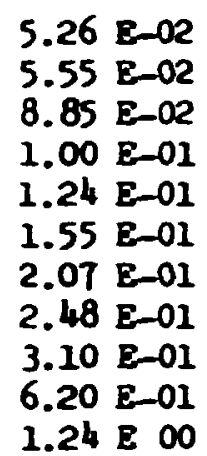 & 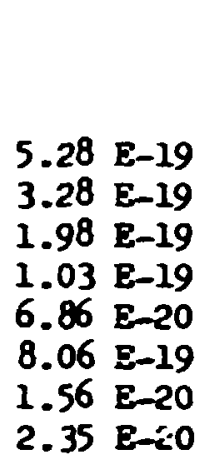 & $\begin{array}{ll}3.22 & E-18 \\
3.00 & E-18 \\
2.05 & E-18 \\
1.70 & E-18 \\
1.15 & E-18 \\
7.54 & E-19 \\
4.11 & E-19 \\
2.75 & E-19 \\
1.00 & E-19\end{array}$ \\
\hline
\end{tabular}

References:

hv + C; Theoretical - T. L. John and D. J. Norban, Phys. Letts. 45A, 135 (1973).

hv + $c^{3+}$; Seniempirical - Wn. J. Viegele, Atonic Date Tables 5,51 (1973).

Accuracy:

hv $+C-$ Totnl error $< \pm 5 \%$

bv $+c^{3+}-$ Unknown

Eotes:

Por photoabsorption of C see lotes 1 and 17 at end of chapter. 
B.1.13

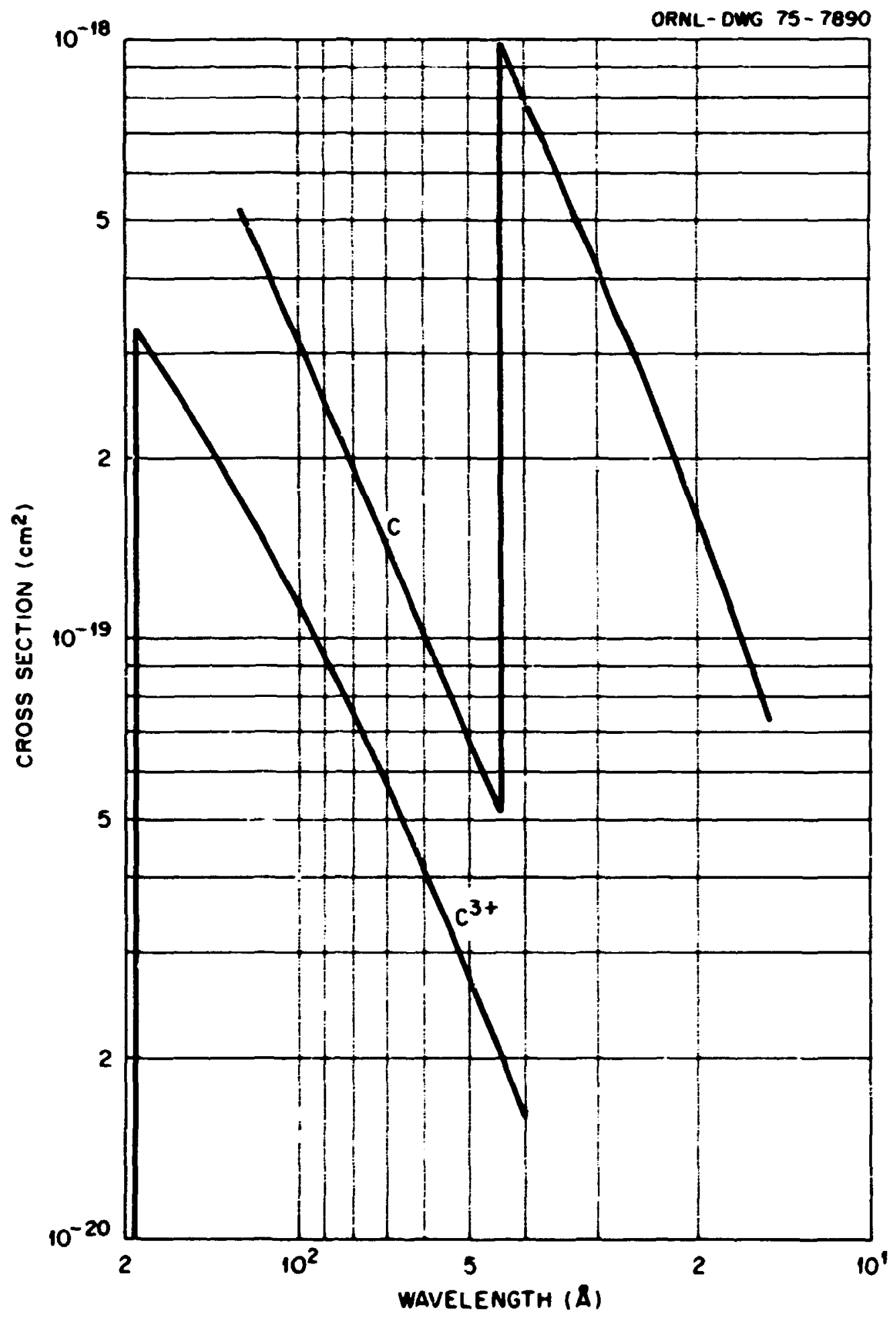




\section{B.1.14}

Photoabsorption and Photoionization of

Atonic Molybdenum, Tungsten and Gold

$$
\text { bu }+(\mathrm{No}, W, \mathrm{Au})
$$

\begin{tabular}{|c|c|c|c|c|}
\hline $\begin{array}{l}\text { Photan } \\
\text { Wavelength } \\
\text { (R) }\end{array}$ & $\begin{array}{l}\text { Photon } \\
\text { Eherox } \\
\text { (keV) }\end{array}$ & D & $\begin{array}{c}\text { Cross Se } \\
{ }_{W}^{(\mathrm{cm}}\end{array}$ & $\mathrm{Au}$ \\
\hline $\begin{array}{r}125 \\
100 \\
90 \\
80 \\
70 \\
60 \\
50 \\
40 \\
30 \\
20 \\
15 \\
10\end{array}$ & 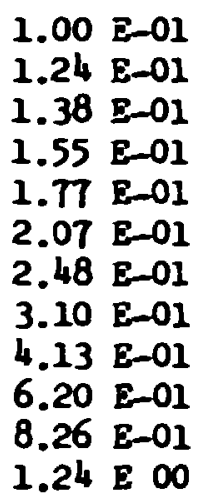 & 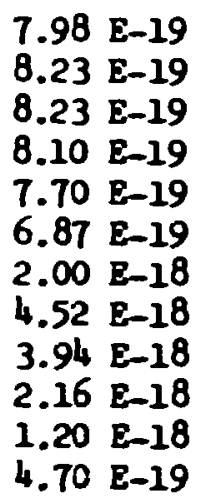 & $\begin{array}{ll}3.56 & E-18 \\
4.87 & E-18 \\
5.31 & E-18 \\
5.67 & E-18 \\
5.90 & E-18 \\
5.97 & E-18 \\
5.71 & E-18 \\
5.16 & E-18 \\
3.91 & E-18 \\
3.77 & E-18 \\
2.73 & E-18 \\
1.72 & E-18\end{array}$ & $\begin{array}{ll}2.07 & E-18 \\
1.61 & E-18 \\
1.66 & E-18 \\
1.80 & E-18 \\
2.25 & E-18 \\
3.90 & E-18 \\
4.91 & E-18 \\
5.21 & E-18 \\
4.80 & E-18 \\
3.41 & E-18 \\
2.30 & E-18 \\
1.07 & E-18\end{array}$ \\
\hline
\end{tabular}

Reference:

Sendepirical: Wh. J. Viegele, Atouic Data Tables S, 51 (1973).

\section{Accurac:}

Total error $< \pm 57$

Sotes:

See notes (1) and (3) at end of chapter. 
B.1.15

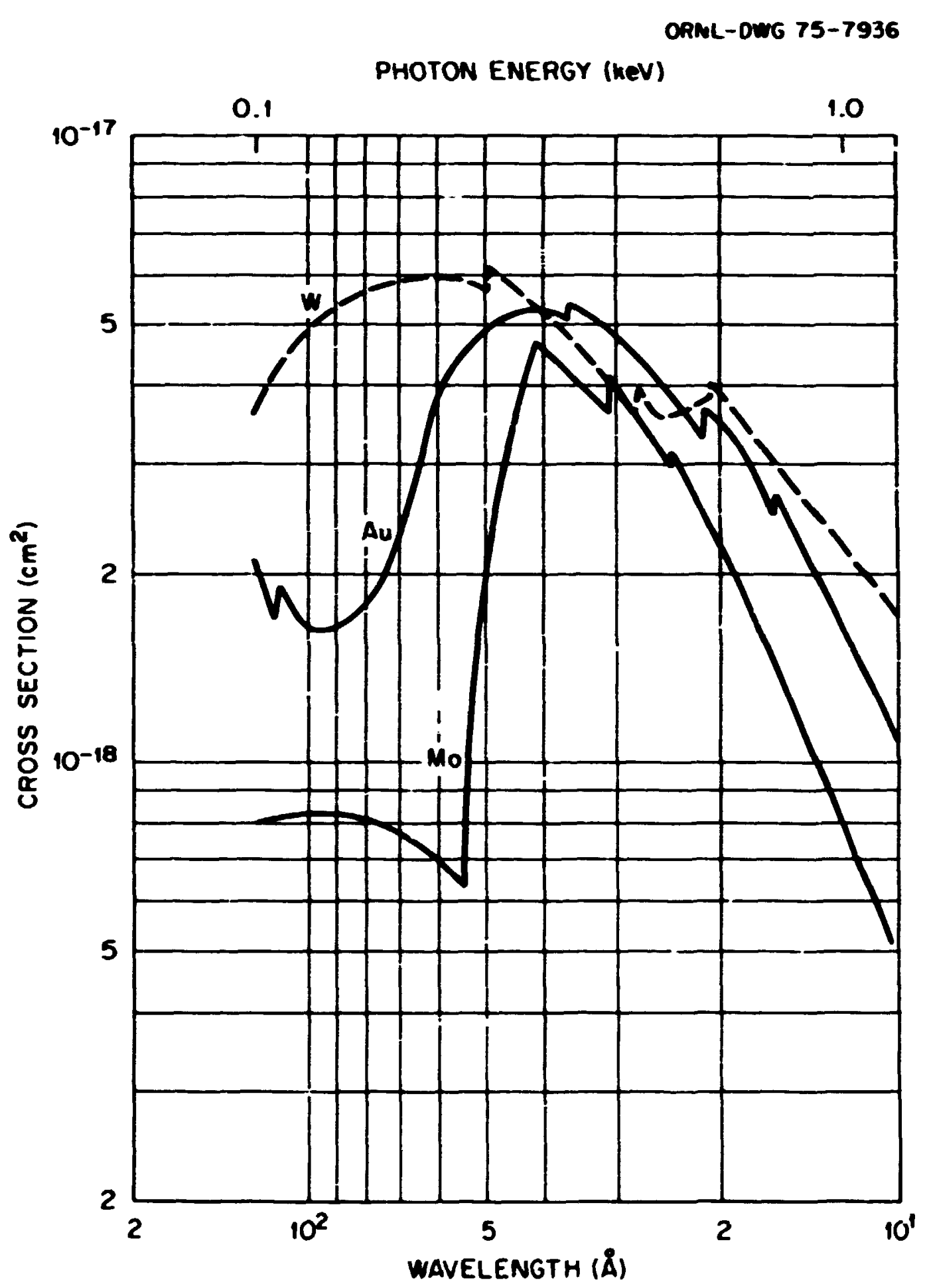


ilotes

(1) For atomic targets such as this, the photoabsorption cross section at wavelengths below the ionization limit (indicated by the word "threshold" in the tablel is confidently expected to be equal to the photoionization cross section. [See J.A.R. Samson, "Advances in Atomic and Molecular Physics," Academic Press, N.Y. Ed3. D.R. Bates and I.M. Estermann 2, 177 (1966)]. We show here only the eruss section (absorption or ionization) actuaily measured or calculated; the cther cross section (ionization or absorption respectively) is expected to be the same. Exceptions to this may occur at discrete structure in the absorption curve; such structure is however of only few $\&$ width and its influence is irrelevant when absorptioc of broad radiation continua is being considered.

The general equality of ionization and attenuation cross sections breaks down at very high photon energies ( $E>1 \mathrm{keV}$ ) because appreciable attenuation oceurs through scattering. We have terminated our tabulation at $10 \AA$ and scattering is insignificant for the situations we cover. For tabulations of separate cross sections for ionization and absorption at photon energies from $100 \mathrm{eV}$ to $1 \mathrm{MeV}$ see Wm. J. Veigele, Atomic Data 5, 51 (1973).

(2) Although these dats are theoretical, it is expected that their accuracy will be very high. They have heen confirmed by experiment at a single wavelength. For details see reference cited.

(3) For information on cross sections at higher photon energies, see the extensive tabular data of Ho. J. Viegele, Atomic Data $\underline{5}, 51$ (1973).

(4) The cross sections for absorption and ionization in $D_{2}$ have been given by G.R. Cook and P.H. Metzger, J. Opt. Soc. Am. 54, 968 (1964). They are almost identical to those for $\mathrm{H}_{2}$ except for a wavelength shift of the absorption bands according to the isotope rule.

(5) It should be noted that the structure in these cross sections is too fine to be reprodured adequately in a table of any reasonabje length. In the region of structure, the data we tabulate here represent a smooth line that represents roughly the behavior of the average structure. In any application where the structure is of importance the reader must refer to the original paper of G.R. Cook and P.H. Metzger that is cited. For a very ine resolution study of these structure with detailed identification of the transitions from which they arise, see W.A. Chupka and

J. Berkowitz, J. Chem. Phys. 51, 4244 (1969). 
(6) For wavelengths below $650 \&$ the ionization and absorption cross s.ctions become equal. At wavelengths below $550 \AA$ there have been no direct measurements of the ionization cross section, but it seens reasonable to take it as being equal to the mensured absorption cross section.

(7) The cross sections shown here are for the formation of both atouic and wolecular ions. Date on the following graph shous that, for wavelengths between 600 and $400 \AA$, at least $96 \%$ of the ions are undissociated (i.e. $\mathrm{H}_{2}^{+}$or $\mathrm{D}_{2}^{+}$ions).

(a) At wavelengths below $680 \&$ the photosbsorption gives rise entirely to ian formation; above 680 much of the absorption is to excited molecular states and to dissociation of the neutral alecule into two neutral atoms. An extensive tabulation of theoretical cross sections for photodissociation into two neutral atons is given in the work of A.C. Allison and A. Dalgarno, Atomic Data Thbles 1 , 91 (1969); there are no experimental studies of this cross section.

(9) These data are cross section ratios as indicated. At vavelengths above $682 \AA$ it is not energetically possible to cause dissociative photoionization. Combining these data with the measured cross sections for ion formation (shown on the preceding araph) one could generate separete cross sections for formation of the atomic and molecular ions.

(10) It is important to note that this cross section is greatly dependent on the vibrational state of the molecular ion. The data presented here are for a particilar distribution among vibrational levels of the electronic ground state; this distribution will be appropriate to a low pressure source using an electron beam of energy that is high compared with threshold energies for the rarious excited stetes. For $\mathrm{H}_{2}{ }^{+}$(or $\mathrm{D}_{2}{ }^{+}$) ions produced by any other means the cross sections may be substantially different from those given here. There are no experimental measurements for dissociation from a single, knom, vibrational state, although such measurements are now possible. [N.P.F.B. Van Asselt, J.C. Mas, and J. Los, Chem. Phys, Letts. 24, 555 (1974)].

Theoretical ross sections are available and are discussed in the following table. With these one can generate a cross section for any known initial distribution among vibrational states.

(11) The experimental data extend only down to $2500 \&$. They are however completely consistent with a theoretical calculation given in the reference cited, and we have used that calculation to extend the data to lower wavelengths.

(12) It seems likeiy that photoabsorption cross sections will be the same as the photodissncietion cross sections given here; there is, however, no proof of this. 
(13) The cross sections given here are for the first three vibrational states. Dunn has described the celculation in Phys. Rev. 172, 1 (1968), but presents there only representative data. The unpublished report cited contains calculated cross sections for all of the 19 vibrational states of $\mathrm{H}_{2}^{+}$and the 27 vibrational states of $\mathrm{D}_{2}{ }^{7}$; the complete tabulation is far toc extensive to reproduce here.

(14) Absorption will occur at discrete wavelent-ihs above the ionization threshold that correspond to excitation of excited states of the target aton. This represents photoexcitation. There appear to be no messurements of cross sections for such processes in this case.

(15) Discrete structure in the photonbsorption curve has been observed at verelengths around 206 \& [R. Madden, K. Codling, Astrophys. J. 141, 364 (1965)]. The structure is, however, of the order an Wostros in vidth and ve have caitted it rom the table and oraph. The structure arises by two electron excitation to an autoionizing state.

(16) In addition to the cross section for photoionization, there is aiso infornation on the cross section for removal of one electron and excitation of the other. For details see the experiment of M.O. Krause and P. Huillewnier, J. Phys. B. S, L143 (1972); and the theoretical work of K.L. Bell, A.B. Kingston, and I.R. Taylor, J. Phys. B 6,1228 (1973).

(17) In addition to the data shown here, there are some predictions and neasurements of absorption and ioni iation cross sections, close to the iocization threshold of $11.3 \mathrm{eV}$. The predictions show considerable differences and do not agree with the single experinental measurement too well; moreover the experiment is based on shock tube date and any not be reliable. Por rurther details the reader is referred to the following references. W. Holrmann and G.L. Heissler, J. Opt. Soc. An. 61, 223 (1971); D.R. Bates and M.J. Seaton, Mon. Notes. Roy. Astron. Soc. 109, 698 (1949); F. Praderie, Ann. D'Astrophys. 27, 129 (1964). 
1

B.2 Photodissociation

1 


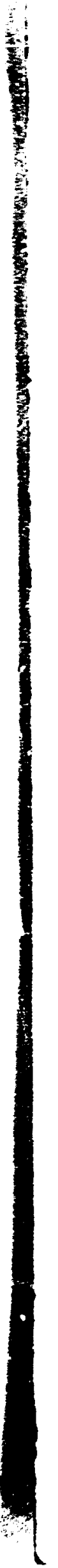

BLANK PAGE 
Photodissociation of $\mathrm{B}_{2}^{+}$and $\mathrm{D}_{2}^{+}$

hv $+\left(\mathrm{H}_{2}^{+}, \mathrm{D}_{2}^{+}\right) \rightarrow(\mathrm{H}, \mathrm{D})+\left(\mathrm{A}^{+}, \mathrm{D}^{+}\right)$

\begin{tabular}{|c|c|c|c|}
\hline $\begin{array}{l}\text { Puotad } \\
\text { unefensth } \\
\text { (i) }\end{array}$ & $\begin{array}{l}\text { Photon } \\
\text { Berey } \\
\text { (kev) }\end{array}$ & $\mathrm{H}_{2}^{+}$ & $\mathrm{D}_{2}^{+}$ \\
\hline $\begin{array}{l}1.5 \\
1.5\end{array}$ & $\begin{array}{ll}8.26 & \mathrm{Z}-04 \\
8.85 & \mathrm{E}-04 \\
9.54 & \mathrm{E}-04 \\
1.03 & \mathrm{E}-03 \\
1.13 & \mathrm{E}-03 \\
1.24 & \mathrm{E}-03 \\
1.36 & \mathrm{E}-03 \\
1.55 & \mathrm{E}-03 \\
1.77 & \mathrm{E}-03 \\
2.07 & \mathrm{E}-03 \\
2.48 & \mathrm{E}-03 \\
3.10 & \mathrm{E}-03 \\
4.13 & \mathrm{E}-03 \\
6.20 & \mathrm{E}-03 \\
8.27 & \mathrm{E}-03 \\
1.24 & \mathrm{E}-02 \\
1.38 & \mathrm{E}-02 \\
1.55 & \mathrm{E}-02 \\
1.77 & \mathrm{E}-02 \\
2.07 & \mathrm{E}-02 \\
2.46 & \mathrm{E}-02\end{array}$ & $\begin{array}{ll}2.00 & E-19 \\
2.18 & E-19 \\
2.35 & E-19 \\
2.60 & E-19 \\
2.80 & E-19 \\
3.15 & E-19 \\
3.75 & E-19 \\
4.30 & E-19 \\
5.25 & E-19 \\
6.60 & E-19 \\
9.00 & E-19 \\
1.08 & E-18 \\
2.00 & E-18 \\
2.95 & E-18 \\
2.80 & E-18 \\
1.82 & E-18 \\
1.80 & E-18 \\
1.60 & E-18 \\
6.00 & E-19 \\
6.00 & E-20 \\
0.00 & E-20\end{array}$ & $\begin{array}{ll}1.15 & E-19 \\
1.26 & E-19 \\
1.40 & E-19 \\
1.57 & E-19 \\
1.80 & E-19 \\
2.12 & E-19 \\
2.54 & E-19 \\
3.16 & E-19 \\
4.09 & E-19 \\
5.53 & E-19 \\
8.00 & E-19 \\
1.25 & E-18 \\
2.10 & E-18 \\
3.25 & E-18 \\
2.11 & E-18 \\
1.63 & E-18 \\
1.60 & E-18 \\
1.68 & E-18 \\
9.00 & E-19 \\
1.10 & E-19 \\
0.00 & E-19\end{array}$ \\
\hline
\end{tabular}

References:

hu $+H_{2}^{+}, D_{2}^{+}$: F. Von Busch and G. H. Dunn, Phss. Rer. A S, 1726 (1972).

Accuras:

Systemtic error $< \pm 58$

findon error $<+10 z$

Dotex:

See Notes (10), (11) and (12) at end of section. 
B.2.3

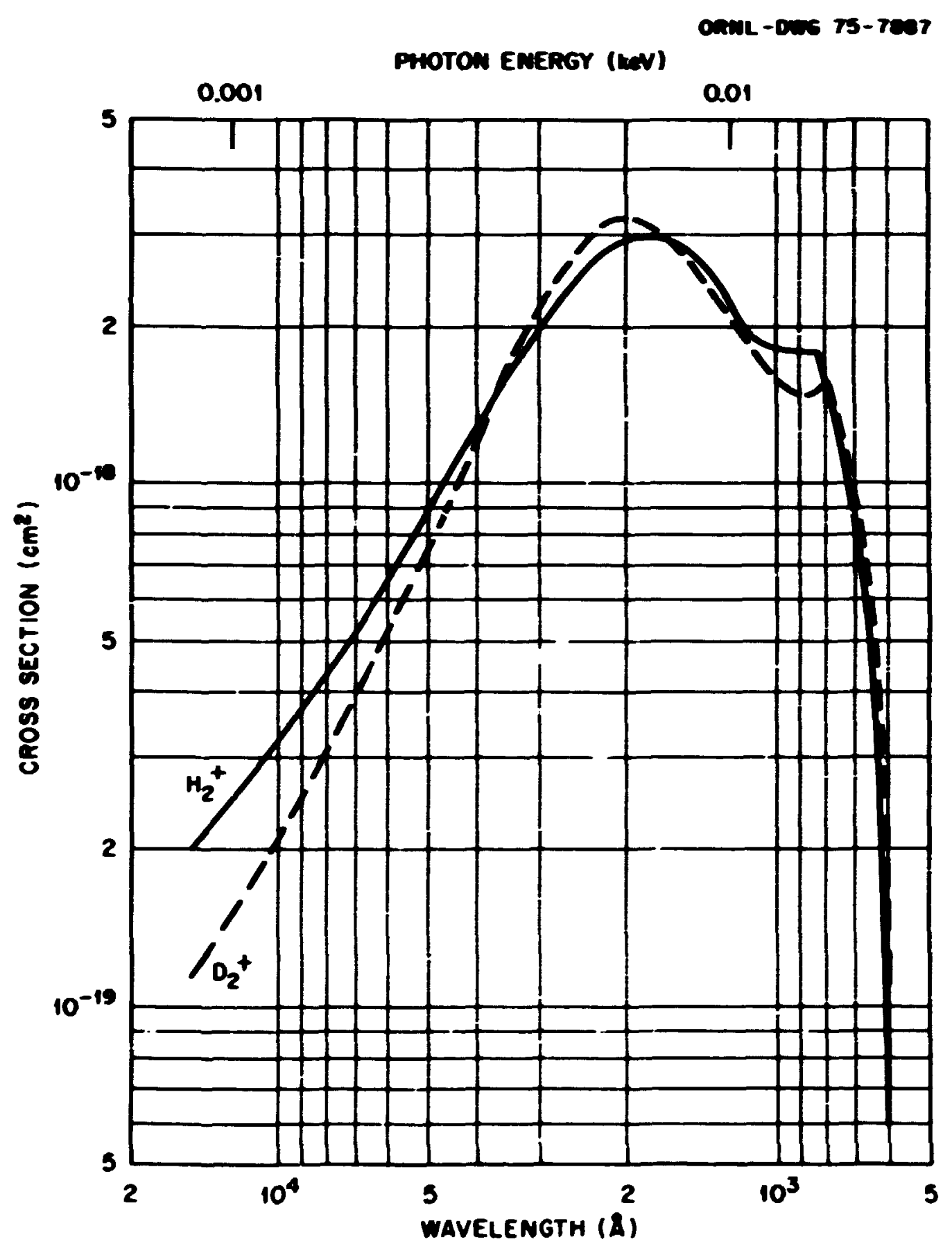




\section{B.2.4}

Photodissociatioa of $\mathrm{H}_{2}^{+}$tron Seiected Vibrational states (Theoretical)

\begin{tabular}{|c|c|c|c|c|}
\hline $\begin{array}{l}\text { Photon } \\
\text { invelength } \\
\text { (R) }\end{array}$ & $\begin{array}{l}\text { Photon } \\
\text { Deres } \\
\text { (keV) }\end{array}$ & $V=0$ & $\begin{array}{c}\text { Cross Section } \\
\left(\mathrm{cm}^{2}\right) \\
T=1\end{array}$ & $\nabla=2$ \\
\hline $\begin{array}{r}1000 \\
3000 \\
2000 \\
1500 \\
1000 \\
900 \\
800 \\
700 \\
600 \\
500\end{array}$ & $\begin{array}{ll}3.10 & E-03 \\
4.13 & E-03 \\
6.20 & E-03 \\
8.27 & E-03 \\
1.24 & E-02 \\
1.38 & E-02 \\
1 . j 5 & E-02 \\
1.77 & E-02 \\
2.07 & E-02 \\
2.48 & E-02\end{array}$ & $\begin{array}{ll}2.390 & E-20 \\
1.807 & E-18 \\
6.058 & E-18 \\
3.078 & E-18 \\
8.977 & z-19 \\
1.069 & z-19 \\
3.440 & E-21 \\
1.020 & E-23\end{array}$ & 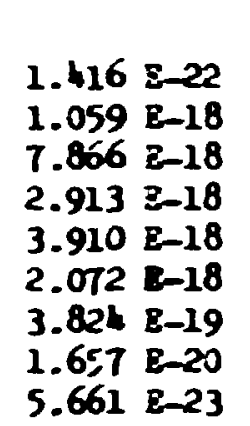 & $\begin{array}{ll}2.801 & 3-24 \\
2.504 & \Xi-20 \\
1.384 & z-18 \\
2.677 & \Xi-19 \\
1.248 & E-19 \\
2.256 & E-18 \\
2.616 & \Xi-18 \\
7.538 & \Xi-19 \\
4.367 & E-20 \\
1.806 & z-22\end{array}$ \\
\hline
\end{tabular}

\section{Reference:}

Theoretical: G.H. Dunn, Phys. Rer. 172, I (1968); G.H. Dunn, JILA Report 50. 92, (1968) (unpublisbed).

Accurect:

Besentially exact.

Notes:

See wotes (12) and (13) at end of chapter. 


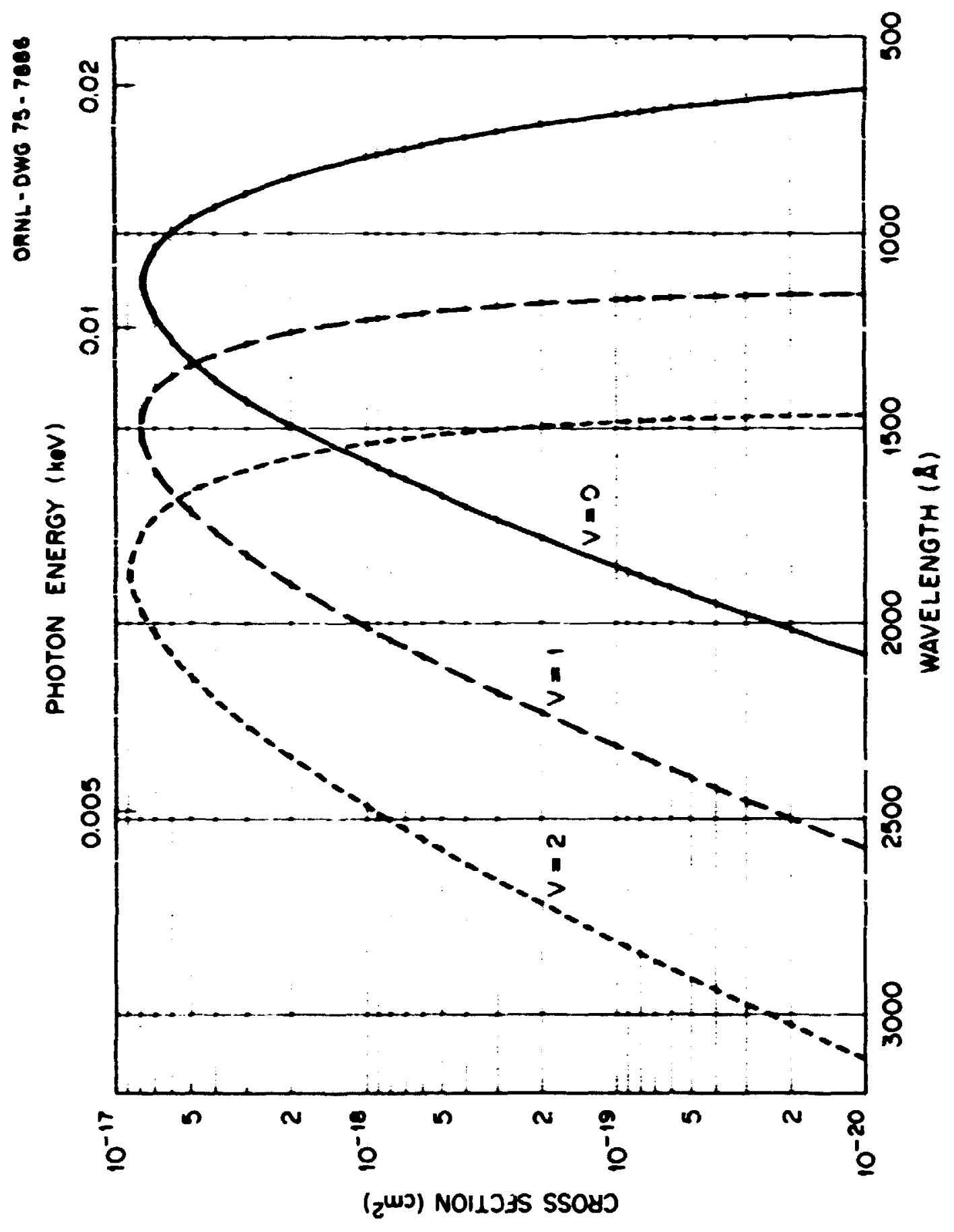




\subsection{6}

Photodissociation of $\mathrm{D}_{2}{ }^{+}$fron Selected Vibretioan Stetes (Theoretical)

$$
\text { hu }+D_{2}^{+}(150, \nabla=0,1,2)+D_{2}^{+}\left(2 \mathrm{po}_{\mathrm{u}}\right) \rightarrow \mathrm{D}+\mathrm{D}^{+}
$$

\begin{tabular}{|c|c|c|c|c|}
\hline $\begin{array}{l}\text { Photon } \\
\text { Wevelength } \\
\text { (R) }\end{array}$ & $\begin{array}{l}\text { Photon } \\
\text { anery } \\
\text { (her) }\end{array}$ & $\nabla=0$ & $\begin{array}{l}\text { Oross Section } \\
\left(\mathrm{cm}^{2}\right) \\
r=1\end{array}$ & $V=2$ \\
\hline $\begin{array}{r}3500 \\
3000 \\
2000 \\
1500 \\
1000 \\
900 \\
800 \\
700 \\
600 \\
500\end{array}$ & $\begin{array}{ll}3.54 & E-03 \\
4.13 & E-03 \\
6.20 & \mathrm{E}-03 \\
8.27 & \mathrm{E}-03 \\
1.24 & \mathrm{E}-02 \\
1.38 & \mathrm{E}-\infty 2 \\
1.55 & \mathrm{E}-02 \\
1.77 & \mathrm{E}-02 \\
2.07 & \mathrm{E}-02 \\
2.48 & \mathrm{E}-02\end{array}$ & $\begin{array}{ll}1.326 & \Sigma-21 \\
3.599 & E-19 \\
7.134 & E-18 \\
3.053 & E-18 \\
6.164 & E-19 \\
3.117 & \varepsilon-20 \\
3.744 & E-22 \\
3.107 & E-25\end{array}$ & 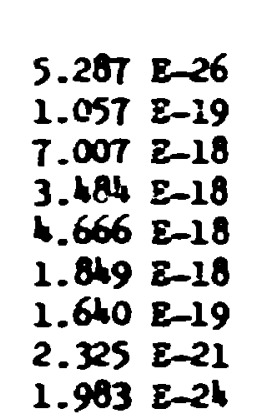 & 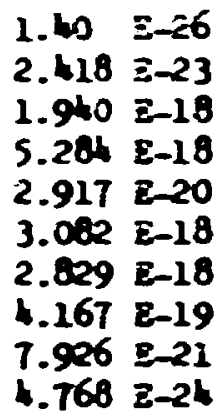 \\
\hline
\end{tabular}

\section{Reference:}

Treoreticel: G.H. Duan, Pays. Rer. 172, 1 (:968); G.4. Dunn, Joint

Institute for Laboratory Astrophysi=s Report $10.92,(1368$ ) (unpublished).

\section{Mecureg:}

Essentially exact.

\section{Wotes:}

See wotes (12) and (13) at end of chapter. 


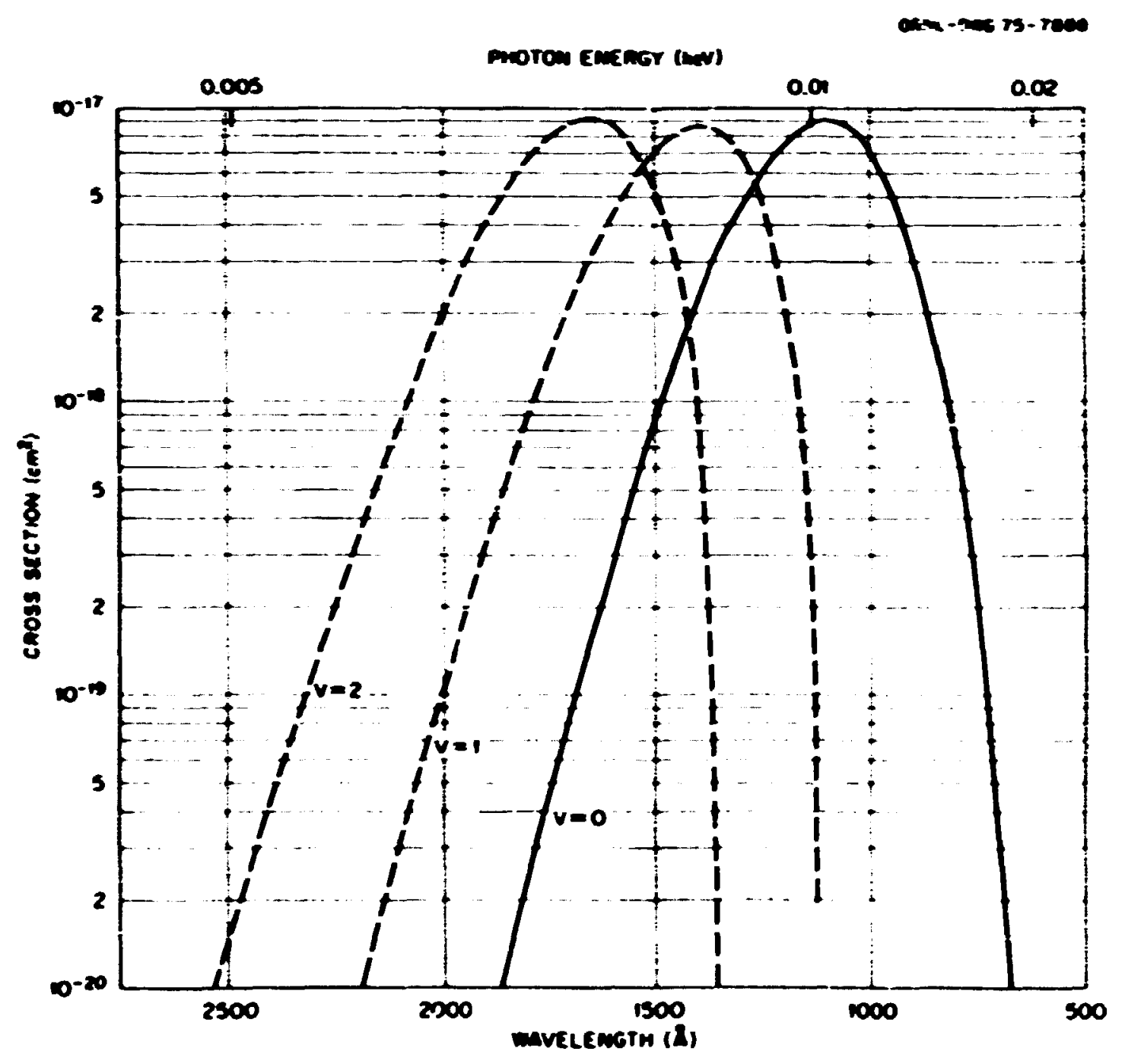


1

\section{B. 3 Photodetachent}

1 
$r-\cdots$

1

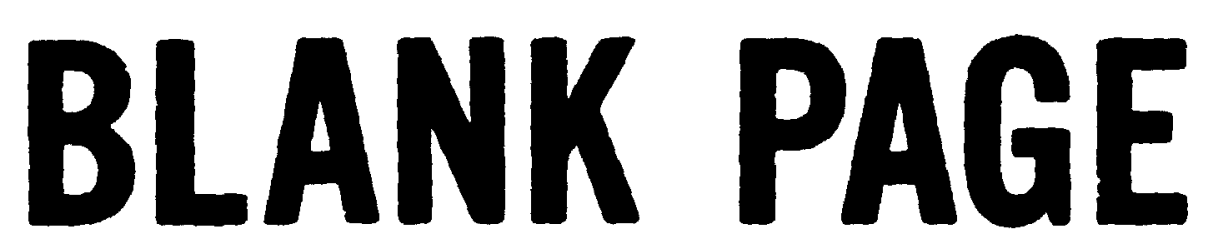


B.3.2

Cross Sections for Photodetechent of an Electron

rom $\mathrm{i}^{-}$and $\mathrm{C}^{-}$

\begin{tabular}{|c|c|c|c|}
\hline \multirow[t]{2}{*}{$(\hat{\boldsymbol{R}})$} & \multirow[t]{2}{*}{$\begin{array}{c}\text { Energy } \\
(\mathrm{ev})\end{array}$} & \multicolumn{2}{|c|}{$\begin{array}{c}\text { Cross Sections } \\
\left.\text { (con }{ }^{2}\right)\end{array}$} \\
\hline & & $\mathrm{H}^{-}$ & $c^{-}$ \\
\hline $\begin{array}{lll}5.0 & E & 02 \\
6.0 & E & 02 \\
8.0 & E & 02 \\
9.0 & E & 02 \\
y .7 & E & 02 \\
1.0 & E & 03 \\
1.5 & E & 03 \\
2.0 & E & 03 \\
3.0 & E & 03 \\
4.0 & E & 03 \\
6.0 & E & 03 \\
8.0 & E & 03 \\
1.0 & E & 04 \\
1.2 & E & 04 \\
1.4 & E & 04 \\
1.6 & E & 04\end{array}$ & $\begin{array}{lll}2.48 & E & 01 \\
2.07 & E & 01 \\
1.55 & E & 01 \\
1.38 & E & 01 \\
1.28 & E & 01 \\
1.24 & E & 01 \\
8.27 & E & 00 \\
6.20 & E & 00 \\
4.13 & E & 00 \\
3.10 & E & 00 \\
2.07 & E & 00 \\
1.55 & E & 00 \\
1.24 & E & 00 \\
1.03 & E & 00 \\
8.86 & E-01 \\
7.75 & E-01\end{array}$ & $\begin{array}{ll}8.1 & \mathrm{E}-19 \\
1.2 & \mathrm{E}-18 \\
3.9 & \mathrm{E}-18 \\
5.3 & \mathrm{E}-18 \\
2.7 & \mathrm{E}-17 \\
2.2 & \mathrm{E}-17 \\
6.9 & \mathrm{E}-18 \\
9.9 & \mathrm{E}-18 \\
1.6 & \mathrm{E}-17 \\
2.2 & \mathrm{E}-17 \\
3.4 & \mathrm{E}-17 \\
4.1 & \mathrm{E}-17 \\
3.7 & \mathrm{E}-17 \\
2.5 & \mathrm{E}-17 \\
1.1 & \mathrm{E}-17 \\
5.9 & \mathrm{E}-19\end{array}$ & $\begin{array}{ll}1.3 & \mathrm{E}-17 \\
1.4 & \mathrm{E}-17 \\
1.4 & \mathrm{E}-17 \\
2.9 & \mathrm{E}-19\end{array}$ \\
\hline
\end{tabular}

References:

hv $+H^{-}$: L. M. Branscomb and S. J. Sxith, Phys, Rev. 98, 1028 (1955); S. J. Smith and D. S. Burch, Phys. Rev. 116, 1125 (1959); D. Feldanan, Z. Naturforsah. 25-8, 621 (1970); J. Macek, Proc. Phys. Soc. 92, 365 (1967); L. M. Branscomb,"Physics of the One- and Wo-Electron Atcoms", pp.609-699, North-Holland Publishing Co. (Amsterdam) 1969).

hv $+C^{-}$: M. L. Seran and L. M. Brars comb, Phys. Rev. 125, 1602 (1962); D. Peldman, 2. Naturforsch.25-a, 621 (1970).

Accuracy:

The total error is believed not to exceed $\pm 10 \%$. 


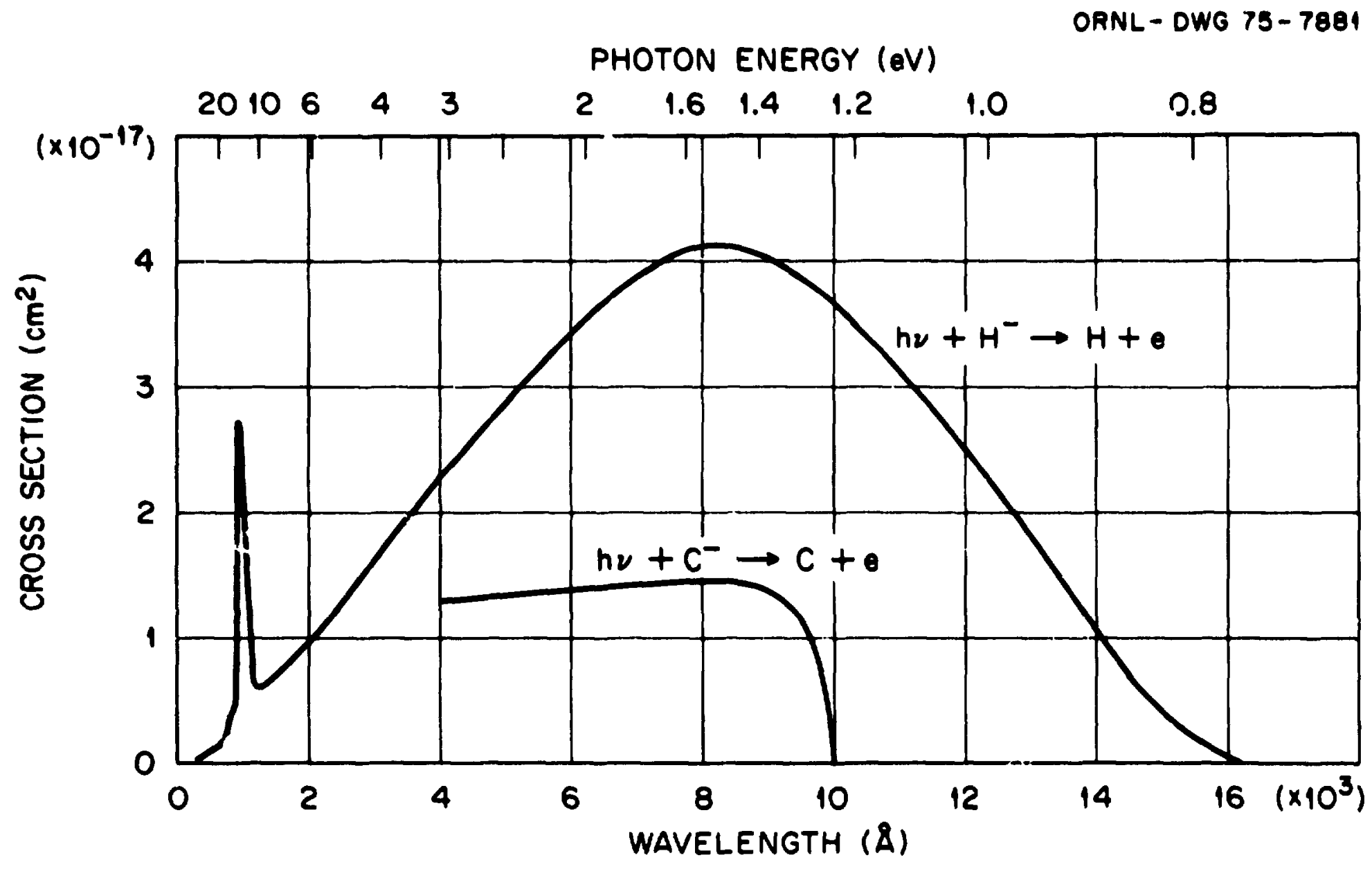




\section{B. 3.4}

Cross Sections, $\sigma$, for Photodetachment of an Electron

$$
\text { fron the } \mathrm{O}^{-} \text {and } \mathrm{O}_{2}^{-} \text {Ions }
$$

\begin{tabular}{|c|c|c|c|}
\hline$(\hat{\boldsymbol{R}})$ & $\begin{array}{c}\text { Bnergy } \\
(\mathrm{ev})\end{array}$ & $\begin{array}{l}\text { hur }{ }_{2}^{-}+\mathrm{O}_{2}+\mathrm{t} \\
\text { Cross Section } \\
\left(\mathrm{cm}^{2}\right)\end{array}$ & $\begin{array}{c}\text { hut } 0^{-}+\mathrm{pte} \\
\text { Cross Section } \\
\left(\mathrm{cm}^{2}\right)\end{array}$ \\
\hline $\begin{array}{lll}4.2 & E & 03 \\
4.4 & E & 03 \\
4.8 & E & 03 \\
5.2 & E & 03 \\
5.6 & E & 03 \\
6.0 & E & 03 \\
6.4 & E & 03 \\
6.8 & E & 03 \\
7.2 & E & 03 \\
8.0 & E & 03 \\
8.4 & E & 03 \\
8.6 & E & 03 \\
9.0 & E & 03 \\
1.0 & E & 04 \\
1.2 & E & 04 \\
1.6 & E & 04 \\
2.0 & E & 04\end{array}$ & $\begin{array}{lll}2.95 & E & 00 \\
2.82 & E & 00 \\
2.58 & \Xi & 00 \\
2.38 & E & 00 \\
2.21 & E & 00 \\
2.07 & E & 00 \\
1.94 & E & 00 \\
1.82 & E & 00 \\
1.72 & E & 00 \\
1.55 & E & 00 \\
1.48 & E & 00 \\
1.44 & E & 00 \\
1.38 & E & 00 \\
1.24 & E & 00 \\
1.03 & E & 00 \\
7.75 & E & 01 \\
6.20 & E & 0\end{array}$ & $\begin{array}{ll}2.4 & E-13 \\
2.3 & E-18 \\
2.0 & E-18 \\
1.7 & E-18 \\
1.5 & E-18 \\
1.3 & E-18 \\
1.1 & E-18 \\
1.0 & E-18 \\
9.0 & E-19 \\
7.3 & E-19 \\
6.6 & E-19 \\
6.0 & E-19 \\
5.6 & E-19 \\
4.3 & E-19 \\
2.7 & E-19 \\
1.4 & E-19 \\
4.3 & E-20\end{array}$ & $\begin{array}{ll}6.4 & \mathrm{E}-18 \\
6.4 & \mathrm{E}-18 \\
6.4 & \mathrm{E}-18 \\
6.4 & \mathrm{E}-18 \\
6.4 & \mathrm{E}-18 \\
6.3 & \mathrm{E}-18 \\
6.1 & \mathrm{E}-18 \\
5.8 & \mathrm{E}-18 \\
5.3 & \mathrm{E}-18 \\
3.6 & \mathrm{E}-18 \\
1.3 & \mathrm{E}-18 \\
1.4 & \mathrm{E}-19\end{array}$ \\
\hline
\end{tabular}

\section{References:}

$0^{-}$: L.M. Branscomb and S.J. Smith, Phys. Kev. 98, 1127 (1955); L.M. Branscomb, D.S. Burch, S.J. Smith, and S. Geltman, Phys. Rev. III, 504 (1958).

$\mathrm{O}_{2}{ }^{-}$: D.S. Burch, S.J. Smith, and L.M. Branscomb, Phys. Rev. 112, 171 (1958); Phys. Rev. 114, 1652 (1959).

\section{Accuracy:}

$0^{-}:$The total error is believed not to exceed $\pm 10 \%$.

$0_{2}^{-}:$The total error is believed not to exceed $\pm 15 \%$. 


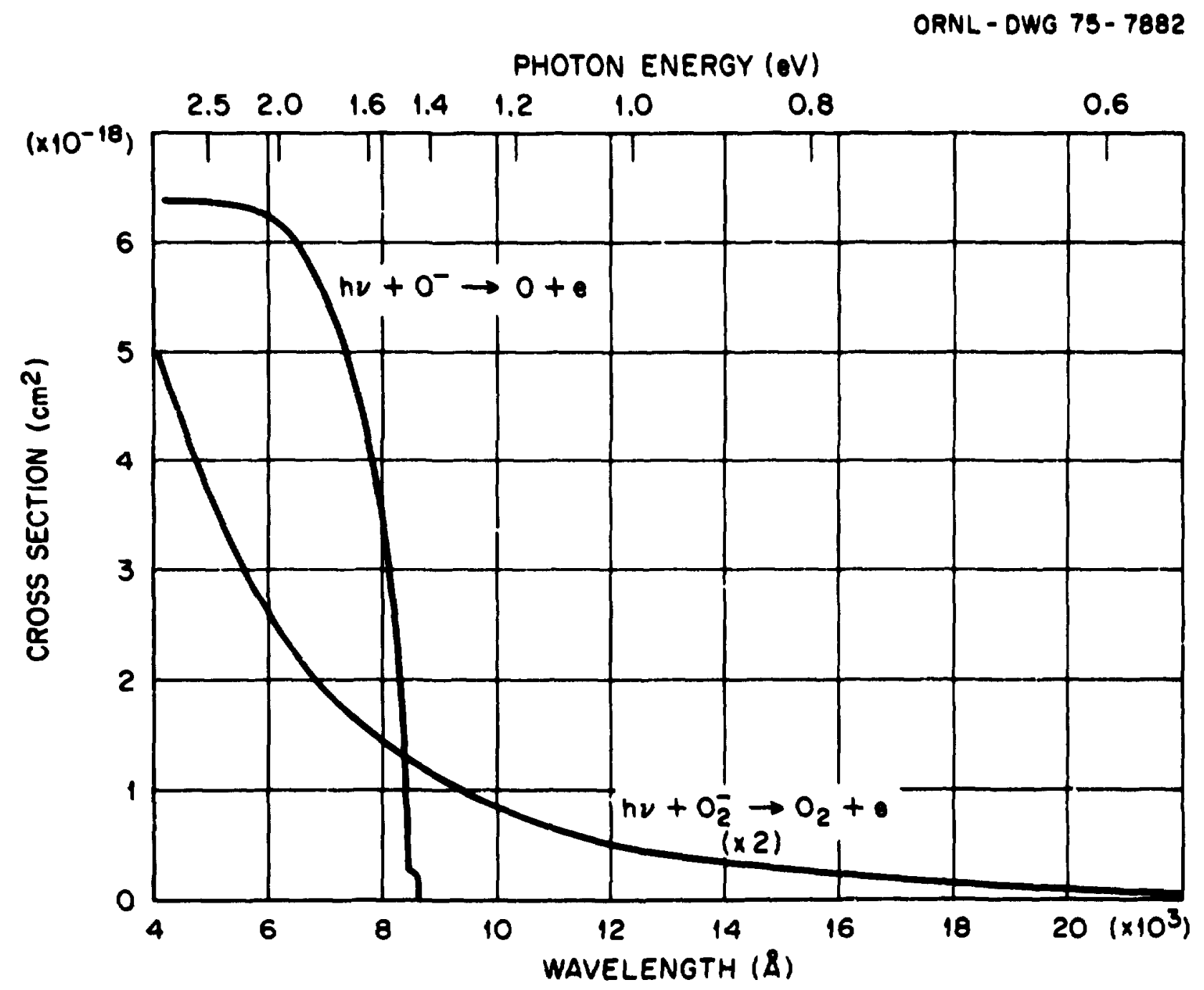

